SANDIA REPORT

SAND94-0472/3 • UC-721

Unlimited Release

Printed August 1997

RECEIVED

Sit) 151997

OSTI

\title{
Porosity, Single-Phase Permeability, and Capillary Pressure Data from Preliminary Laboratory Experiments on Selected Samples from Marker Bed 139 at the Waste Isolation Pilot Plant
}

\section{Volume 3 of 3: Appendices C, D, E, and F}

Súsan M. Howarth, Tracy Christian-Frear

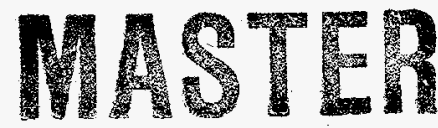

Prepared by

Sandia National Laboratories

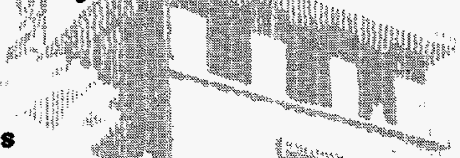

Albuquerque, New Mexico 87185 and Livermoro, California 94550

Sandia is a multiphogram laboratory oporated by Sandia

Corporation, a Lockheed Martin Company, for the United States

Department of Energy under Contract DE-AC04-94AL85000.

Approved for public release; distribution is unififfed.

G Sandia National Laboratories 
Issued by Sandia National Laboratories, operated for the United States Department of Energy by Sandia Corporation.

NOTICE: This report was prepared as an account of work sponsored by an agency of the United States Government. Neither the United States Government nor any agency thereof, nor any of their employees, nor any of their contractors, subcontractors, or their employees, makes any warranty, express or implied, or assumes any legal liability or responsibility for the accuracy, completeness, or usefulness of any information, apparatus, product, or process disclosed, or represents that its use would not infringe privately owned rights. Reference herein to any specific commercial product, process, or service by trade name, trademark, manufacturer, or otherwise, does not necessarily constitute or imply its endorsement, recommendation, or favoring by the United States Government, any agency thereof, or any of their contractors or subcontractors. The views and opinions expressed herein do not necessarily state or reflect those of the United States Government, any agency thereof, or any of their contractors.

Printed in the United States of America. This report has been reproduced directly from the best available copy.

Available to DOE and DOE contractors from

Office of Scientific and Technical Information

P.O. Box 62

Oak Ridge, TN 37831

Prices available from (615) 576-8401, FTS 626-8401

Available to the public from

National Technical Information Service

U.S. Department of Commerce

5285 Port Royal Rd

Springfield, VA 22161

NTIS price codes

Printed copy: A14

Microfiche copy: A01 


\title{
Porosity, Single-Phase Permeability, and Capillary Pressure Data from Preliminary Laboratory Experiments on Selected Samples from Marker Bed 139 at the Waste Isolation Pilot Plant
}

\author{
Volume 3 of 3: Appendices C, D, E and F
}

\author{
Susan M. Howarth \\ Tracy Christian-Frear \\ Geohydrology Department 6115 \\ Sandia National Laboratories \\ Albuquerque, NM 87185
}

\begin{abstract}
This volume contains the mineralogy, porosity, and permeability results from the Marker Bed 139 specimens evaluated by TerraTek, Inc. This volume also documents the brine recipe used by RE/SPEC, Inc., the parameter package submitted to Performance Assessment based on all the data, and a memo on the mixed Brooks and Corey two-phase characteristic curves.
\end{abstract}


Appendix C.

Data Report: TerraTek Inc.

The following appendix section includes Appendix C and Appendices C-A through C-E. 
C-2 


\section{DISCLAWMIER}

Portions of this document may be illegible in electronic image products. Images are produced from the best available original document. 


\section{Appendix C}

\section{Characterization of MB139 at WIPP}

\section{Errata Sheet}

Total porosity data for sample EP2 is not included in the data report because the mass loss during crushing was excessive.

The liquid permeability data are included in the data report as "scoping only" because the saturation data (Table 8) were characterized as scoping only in the laboratory notebooks.

The following errors in the Appendix, Characterization of MB139 at WIPP, are noted:

1. Table 3: Sample designations should not have a "-" separating the letters from the numerals (e.g., PX-1 should be PX1).

2. Table 4: Rows 8 and 9 o the data indicate PX4 should be PX3.

3. Table 6: Sample B's grain volume $\left(V_{g \text { eff }}\right)$ should be 773.00 , not $723.00 \mathrm{cc}$.

4. Table 6: Sample E's effective porosity $\left(\phi_{\text {eff }}\right)$ should be 1.45 , not $1.55 \%$.

The following modifications should be made to the references on page C-82 in Appendix C.

\begin{tabular}{|l|l|}
\hline Ref. No. & \multicolumn{1}{|c|}{ Comment } \\
\hline 1 & copy of Davies, 1991 on file in SWCF as WPO\#26169 \\
\hline 2 & $\begin{array}{l}\text { authors are A.M. Petrovic, J.E. Siebert, and P.E. Rieke; journal title is Soil Science } \\
\text { Society of America Journal Vol. 46, no. 3; copy on file in SWCF as WPO\#42627 }\end{array}$ \\
\hline 3 & $\begin{array}{l}\text { authors are P.K. Hunt, P. Engler, and C. Bajsarowicz; journal title is Journal of } \\
\text { Petroleum Technology Vol. 40, no. 9; copy on file in SWCF as WPO\#45565 }\end{array}$ \\
\hline 4 & $\begin{array}{l}\text { authors are G.O. Brown, M.L. Stone, and J.E. Gazin; journal title is Water Resources } \\
\text { Research Vol. 29, no. 2; copy on file in SWCF as WPO\#42086 }\end{array}$ \\
\hline 5 & $\begin{array}{l}\text { authors are R.A. Johns, J.S. Steude, L.M. Castanier, and P.V. Roberts; journal title is } \\
\text { Journal of Geophysical Research Vol. 98, no. B2; copy on file in SWCF as WPO\#40567 }\end{array}$ \\
\hline 6 & $\begin{array}{l}\text { the existence of Vinegar and Hill could not be verified (company confidential } \\
\text { document); cited on p. C-14. }\end{array}$ \\
\hline 8 & the publication date for this ISRM method is 1981 \\
\hline 9 & correct publisher of Taylor, 1982 is University Science Books, Mill Valley, CA \\
\hline
\end{tabular}

The following modifications should be made to the references in Appendix C-A: Procedures.

\begin{tabular}{|l|l|}
\hline Page No. & \multicolumn{1}{|c|}{ Change } \\
\hline C-86 & reference 1: existence of Operator's Manual 961036 could not be verified \\
\hline C-87 & reference 2: publisher location is Swarthmore, PA \\
\hline C-87 & reference 4: correct name of second author is R.C. Reynolds, Jr. \\
\hline C-91 & reference 9: cited pages are on file in SWCF \\
\hline C-91 & reference 10: cited pages are on file in SWCF \\
\hline
\end{tabular}


C-4 


\title{
CHARACTERIZATION OF MARKER BED 139 AT WIPP
}

\author{
Final Report \\ Contract AD-3656 \\ Nancy S. Davis, Org. 7216 \\ Sandia Contracting Representative
}

Submitted to:

Sandia National Laboratories

P.O Box 5800

Albuquerque, NM 87185-5800

Attention: Dr. Susan Howarth, Org. 6115

Sandia Delegated Representative

Submitted by:

Joanne T. Fredrich

Principal Investigator

TERRATEK, INC.

University Research Park

400 Wakara Way

Salt Lake City, Utah 84108

TR94-20

December, 1993 


\section{Table of Contents}

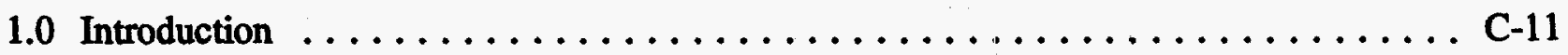

2.0 Core Receipt and Inspection $\ldots \ldots \ldots \ldots \ldots \ldots \ldots \ldots \ldots \ldots \ldots \ldots \ldots \ldots \ldots \ldots$

3.0 X-Ray CT Non-Destructive Core Analysis . . . . . . . . . . . . . C-14

3.1 Application of a Second Generation CT Scanner .............. C-14

3.2 Exploratory Application of a Third Generation CT Scanner ......... C-23

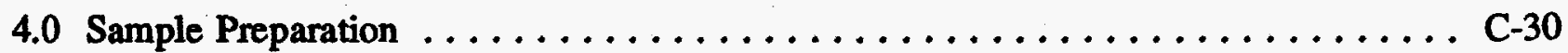

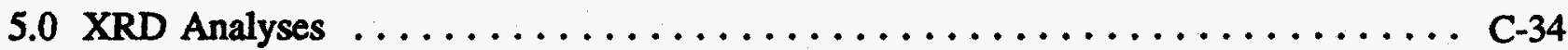

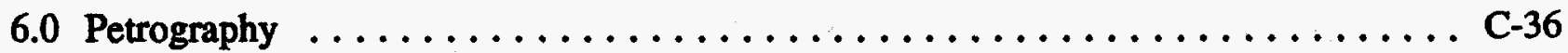

6.1 Overview ............................

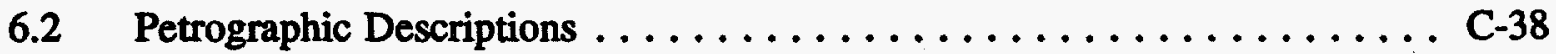

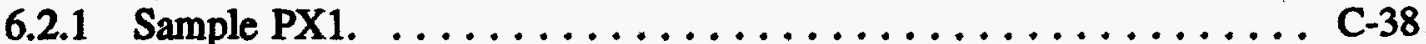

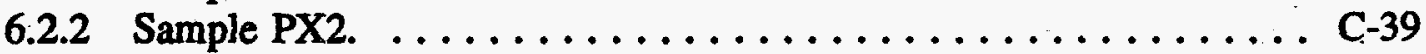

6.2.3 Sample PX3. ........................ C-39

6.2.4 Sample PX4. ........................ C-39

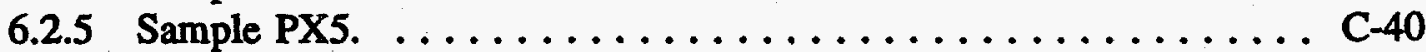

6.2.6 Sample PX6. ....................... C-40

7.0 Effective Porosity, Total Porosity, and Saturation $\ldots \ldots \ldots \ldots \ldots \ldots$ C-71

8.0 Single Phase Permeability $\ldots \ldots \ldots \ldots \ldots \ldots \ldots \ldots \ldots \ldots \ldots \ldots \ldots \ldots \ldots \ldots \ldots$ C-74

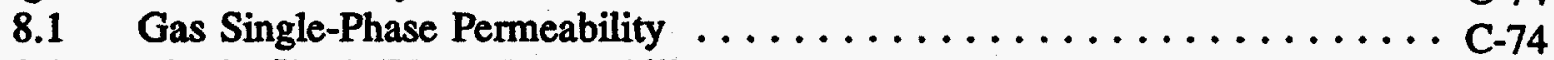

8.2 Liquid Single-Phase Permeability $\ldots \ldots \ldots \ldots \ldots \ldots \ldots \ldots \ldots$ C-74

9.0 Summary and Recommendations $\ldots \ldots \ldots \ldots \ldots \ldots \ldots \ldots \ldots \ldots$ C-80

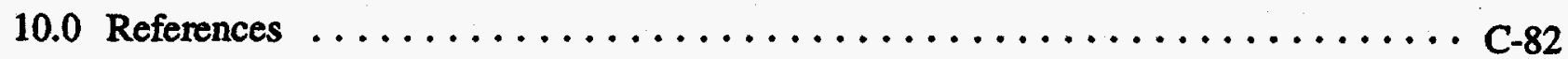

APPENDIX C-A: Procedures $\ldots \ldots \ldots \ldots \ldots \ldots \ldots \ldots \ldots \ldots \ldots \ldots \ldots$ C-83

A.1 X-Ray Computerized Tomography $\ldots \ldots \ldots \ldots \ldots \ldots \ldots \ldots \ldots$ C-84

A.1.1 Background. ........................ C-84

A.1.2 TerraTek's CT Facility. $\ldots \ldots \ldots \ldots \ldots \ldots \ldots \ldots \ldots \ldots \ldots \ldots \ldots \ldots$ C-84

A.1.3 Operating Procedures. .................. C-85

A.1.4 Imaging of fluid flow through cores. $\ldots \ldots \ldots \ldots \ldots \ldots \ldots$, C-86

A.2 X-Ray Diffraction (XRD) Analyses $\ldots \ldots \ldots \ldots \ldots \ldots \ldots \ldots \ldots \ldots \ldots \ldots$ C-87

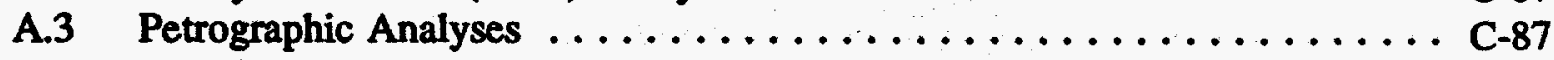

A.4 Porosity Measurement $\ldots \ldots \ldots \ldots \ldots \ldots \ldots \ldots \ldots \ldots \ldots \ldots$ C-88 
Characterization of MB139 at WIPP

December 10, 1993

Final Report, Contract No. AD-3656

A.4.1 Gas porosimetry. . . . . . . . . . . . . . . . C-91

A.4.2 Bulk volume. . . . . . . . . . . . . . . . . C C-91

A.4.3 Powdering for measurement of total porosity. . . . . . . . . . . . C-91

A.5 Liquid Saturation . . . . . . . . . . . . . . . . . . . . C-92

A.6 Gas Single-Phase Permeability . . . . . . . . . . . . . C-92

A.7 Liquid Single-Phase Permeability $\ldots \ldots \ldots \ldots \ldots \ldots \ldots \ldots \ldots \ldots$ C-93

A.8 Calibration Facilities and Instrumentation $\ldots \ldots \ldots \ldots \ldots \ldots \ldots \ldots$

APPENDIX C-B: Index of CT Cross-sectional Images from Whole Core Scan (E1X-08) . C C-97

APPENDIX C-C: Drying History of Samples A-F \& EP1-8

C-99

APPENDIX C-D: Gas Permeability Spreadsheets

C-105

APPENDIX C-E: Liquid Permeability Spreadsheets

C-179

C-7 


\section{List of Tables}

Table 1. Sample Identification $\ldots \ldots \ldots \ldots \ldots \ldots \ldots \ldots \ldots \ldots \ldots \ldots \ldots \ldots \ldots$

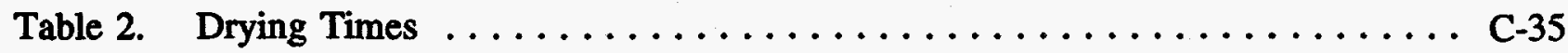

Table 3. XRD Semi-Quantitative Mineralogic Analyses $\ldots \ldots \ldots \ldots \ldots \ldots \ldots$ C-35

Table 4. Modal Analyses $\ldots \ldots \ldots \ldots \ldots \ldots \ldots \ldots \ldots \ldots \ldots \ldots \ldots \ldots \ldots \ldots \ldots \ldots \ldots$

Table 5. Grain Size, Sorting, and Porosity from 300-Point Count . . . . . . . . C-37

Table 6. Bulk Volume, Effective Grain Volume, and Effective Porosity . . . . . . C-72

Table 7. Bulk Density, Grain Density, and Total Porosity . . . . . . . . . C C-73

Table 8. Liquid (OMS) Saturation $\ldots \ldots \ldots \ldots \ldots \ldots \ldots \ldots \ldots \ldots \ldots \ldots \ldots \ldots \ldots \ldots \ldots$

Table 9. Single Phase Gas Permeability $\ldots \ldots \ldots \ldots \ldots \ldots \ldots \ldots \ldots \ldots \ldots$ C-75

Table 9. Single Phase Gas Permeability (continued) $\ldots \ldots \ldots \ldots \ldots \ldots \ldots$ C-76

Table 9. Single Phase Gas Permeability (continued) $\ldots \ldots \ldots \ldots \ldots \ldots \ldots \ldots$ C-77

Table 10. Single Phase Klinkenberg Gas Permeability $\ldots \ldots \ldots \ldots \ldots \ldots \ldots$ C-78

Table 11. Single Phase Liquid Permeability vs Calculated Klinkenberg Permeability ... C-79

Table B1. Index of CT Images for E1X08 $\ldots \ldots \ldots \ldots \ldots \ldots \ldots \ldots \ldots \ldots \ldots$ C-98 


\section{List of Figures}

Figure 1. As-received MB139 Core E1X07 $\ldots \ldots \ldots \ldots \ldots \ldots \ldots \ldots \ldots \ldots \ldots$

Figure 2. As-received MB139 Core $\mathrm{E} 1 \mathrm{X} 08 \ldots \ldots \ldots \ldots \ldots \ldots \ldots \ldots \ldots \ldots \ldots \ldots$

Figure 3. Representative cross-sectional images of E1X08-3. . . . . . . . C-15

Figure 4. Representative cross-sectional images of E1X08-4. . . . . . . . C-16

Figure 5. Representative cross-sectional images of E1X08-5. $\ldots \ldots \ldots \ldots \ldots, C-17$

Figure 6. Representative cross-sectional images of E1X08-6. . . . . . . .

Figure 7a. Longitudinal reconstruction of E1X08-4 (3.55-4.13 feet). . . . . . C-19

Figure 7b. Longitudinal reconstruction of E1X08-4 (4.14-4.72 feet). . . . . . C-20

Figure 7c. Longitudinal reconstruction of E1X08-4 (4.73-5.33 feet). . . . . . . C-21

Figure 7d. Longitudinal reconstruction of E1X08-4 (5.34-5.94 feet). . . . . . . C-22

Figure 8. Examples of coring-induced damage as evidenced by CT crosssectional images in tuff cores from the tunnel beds in Rainer Mesa, Nevada Test Site. (Images furnished with permission of Dr. B.L. Ristvet, Defense Nuclear Agency). . . . . . . . . . . . . C-24

Figure 9. CT cross-sectional images from E1X08-4 showing linear features of comparatively low density. Such features, possibly pre-existing healed fractures, were very rare in the E1X08 images. . . . . . . C-25

Figure 10a. Raw image from E1X08-5 (salt) acquired with a third-generation Philips 60/TX CT scanner. Beam-hardening, as evidenced by the bright ring on the sample perimeter, is present. ........... C-26

Figure 10b. Image shown in Figure 10a following post-processing with CORESCAN $^{\mathrm{TM}}$ to eliminate beam-hardening artifacts. . . . . . . C -27

Figure 11a. Raw image from E1X08-4 (anhydrite with mudrock) acquired with a third-generation Philips 60/TX CT scanner. Beam-hardening, as evidenced by the bright ring on the sample perimeter, is present. 
Figure 11b. Image shown in Figure 11a following post-processing with

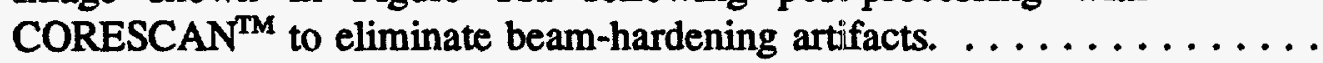

Figure 12. Experimental program. $\ldots \ldots \ldots \ldots \ldots \ldots \ldots \ldots \ldots \ldots \ldots \ldots \ldots$

Figure 13. Documentation of sample preparation from core E1X08-4. ....... C-32

Figure 14. Documentation of sample preparation from core E1X07-4. $\ldots \ldots \ldots \ldots$ C-33

Figure 15. Schematic illustrating PX sample preparation. Thin sections were prepared parallel to O1-03. 01 is horizontal (parallel to bedding) and $\mathrm{O} 2$ and $\mathrm{O} 3$ are vertical and mutually perpendicular. XRD analyses were conducted on half of the $\mathrm{O} 2$ split. . . . . . . . . C-34

Figure 16. Grain size histogram for PX1 $\ldots \ldots \ldots \ldots \ldots \ldots \ldots \ldots \ldots \ldots \ldots$

Figure 17. Grain size histogram for $\mathrm{PX} 2 \ldots \ldots \ldots \ldots \ldots \ldots \ldots \ldots \ldots$ C-44

Figure 18. Grain size histogram for $\mathrm{PX} 3 \ldots \ldots \ldots \ldots \ldots \ldots \ldots \ldots \ldots \ldots \ldots$

Figure 19. Grain size histogram for $\mathrm{PX} 4 \ldots \ldots \ldots \ldots \ldots \ldots \ldots \ldots \ldots \ldots \ldots \ldots$

Figure 20. Grain size histogram for PX5 $\ldots \ldots \ldots \ldots \ldots \ldots \ldots \ldots \ldots \ldots \ldots$

Figure 21. Grain size histogram for PX6 $\ldots \ldots \ldots \ldots \ldots \ldots \ldots \ldots \ldots \ldots \ldots \ldots$

Figure Ala. Documentation of thin section preparation from PX samples from core E1X08-4.

Figure A1b. Documentation of thin section preparation from PX samples from core E1X07-4.

Figure A2. Schematic of experimental system for gas permeability measurement.

Figure A3. Schematic of experimental system for liquid permeability measurement. . C-95 


\subsection{Introduction}

The Waste Isolation Pilot Plant (WIPP) is the U.S. Department of Energy's planned repository for transuranic waste generated by defense programs. The WIPP is located $660 \mathrm{~m}$ underground in the Salado Formation which consists of thick halite with interbeds of minerals such as clay and anhydrite. The polycrystalline Salado salt contains 0.1 to $1 \%$ brine in intragranular fluid inclusions and as an intergranular pore fluid. The anhydrite interbed layers are expected to contain similar quantities. Quantification of the amount of brine, and its mobility and flow rate are critical for accurate assessment of the long-term performance of the repository. Field tests indicate that the permeability of the Salado anhydrite interbeds is 1 to 2 orders of magnitude greater than that of the halite intervals and sensitivity analyses show that the anhydrite beds would be the favored path for fluid flow [1].

The effort described here focused on characterization of the anhydrite bed known as Marker Bed 139 (MB139) which occurs beneath the planned waste-storage rooms. Laboratory investigations were performed on two cores from MB139 to (1) characterize the lithology and mineralogy; (2) determine total and effective porosity; (3) measure maximum achievable liquid saturation; (3) determine single-phase (both gas and liquid) permeability under varying stress conditions; and (4) explore the use of X-ray computerized axial tomography (CT) for identification and characterization of natural and coring-induced fractures and for tracking fluid flow through cores.

\subsection{Core Receipt and Inspection}

Two core samples identified as E1X07 and E1X08 were received at TerraTek on November 23, 1992. The core containers were photographed immediately upon receipt and opened for examination on November 25,1992 . The core pieces were sheathed individually in saran wrap, packed in bubble wrap, and sealed in 8 " diameter PVC tubes. The first tube contained three 5\%" diameter cores with identification and length as follows: E1X07-3 (3'8" to 4'); EIX07-4 (4' to 6'2"); and E1X07-5 (6'2" to 7'5"). The second tube contained four 5\%" diameter cores with identification and length as follows: E1X08-3 (2'8" to 3'6"); EIX08-4 (3'6" to 6'); E1X08-5 (6' to 6'4"); and E1X08-6 (6'4" to 7'41/2"). Photographs were taken to document conditions during each stage of unwrapping and the cores were noted to be in good condition (Figures 1 and 2).

Bedding was approximately perpendicular to the core axis. Significant lithologic discontinuities were apparent at each of the break points (Figures 1 and 2). Both the upper and lower core pieces from E1X07 and E1X08 (E1X07-3 and E1X07-5; E1X08-3 and E1X08-6) were identified as halite and thus not considered to be part of the marker bed. The lower portion of the marker bed in both cores ( -5.8 to 6.2 feet for E1X07; -6.0 to 6.3 feet for E1X08) was identified as mudrock. The remaining material was identified as anhydrite mixed with mudrock. Core E1X08 was separated into two pieces (identified as E1X08-4 and E1X08-5) at the interface between the mixed anhydrite/mudrock and mudrock zones. Core E1X07 contained no throughgoing fissure at this lithologic discontinuity; however, some partial separation along the bedding plane was apparent along a portion of the circumference (Figure 1). 


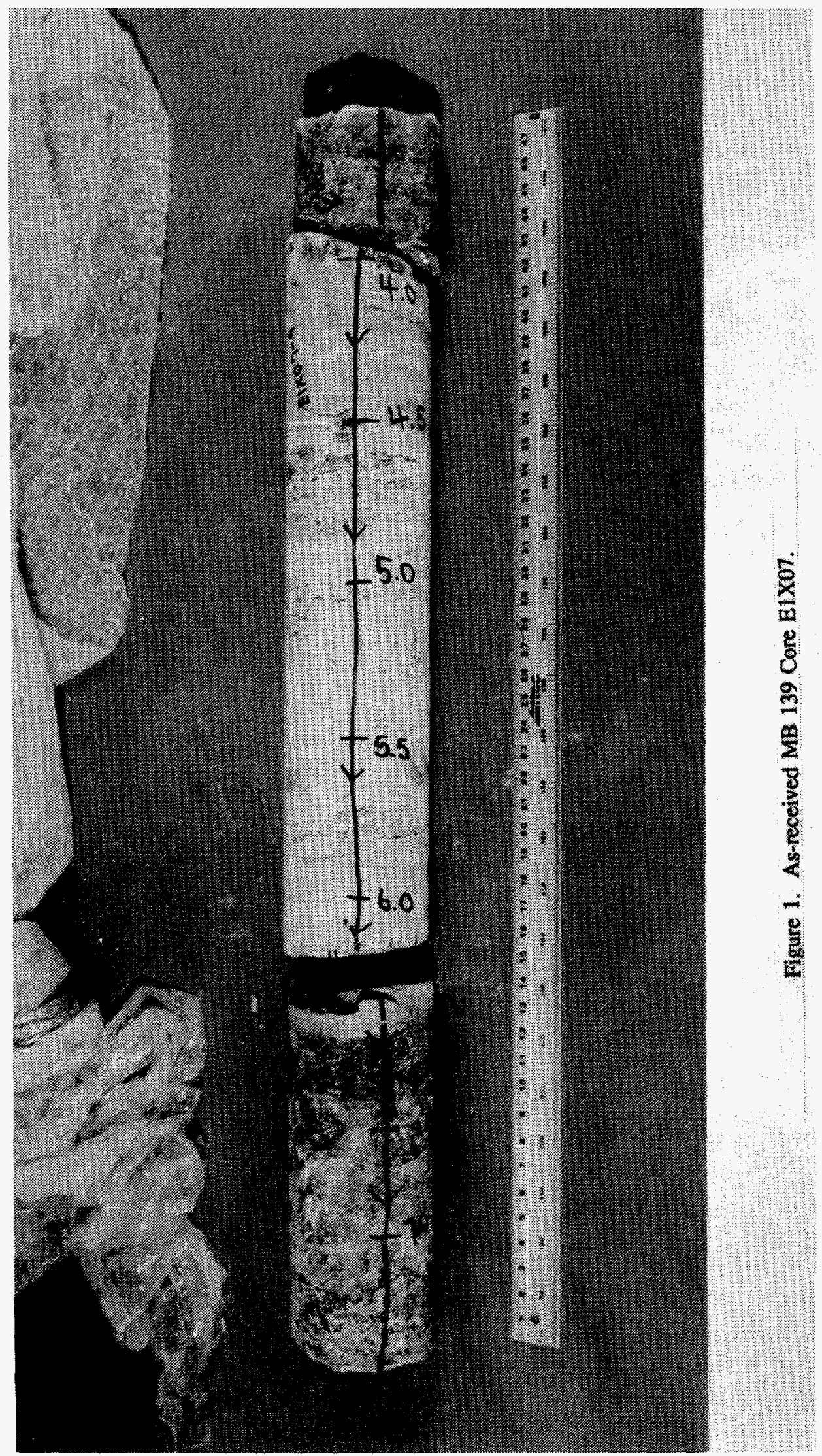

C-12 


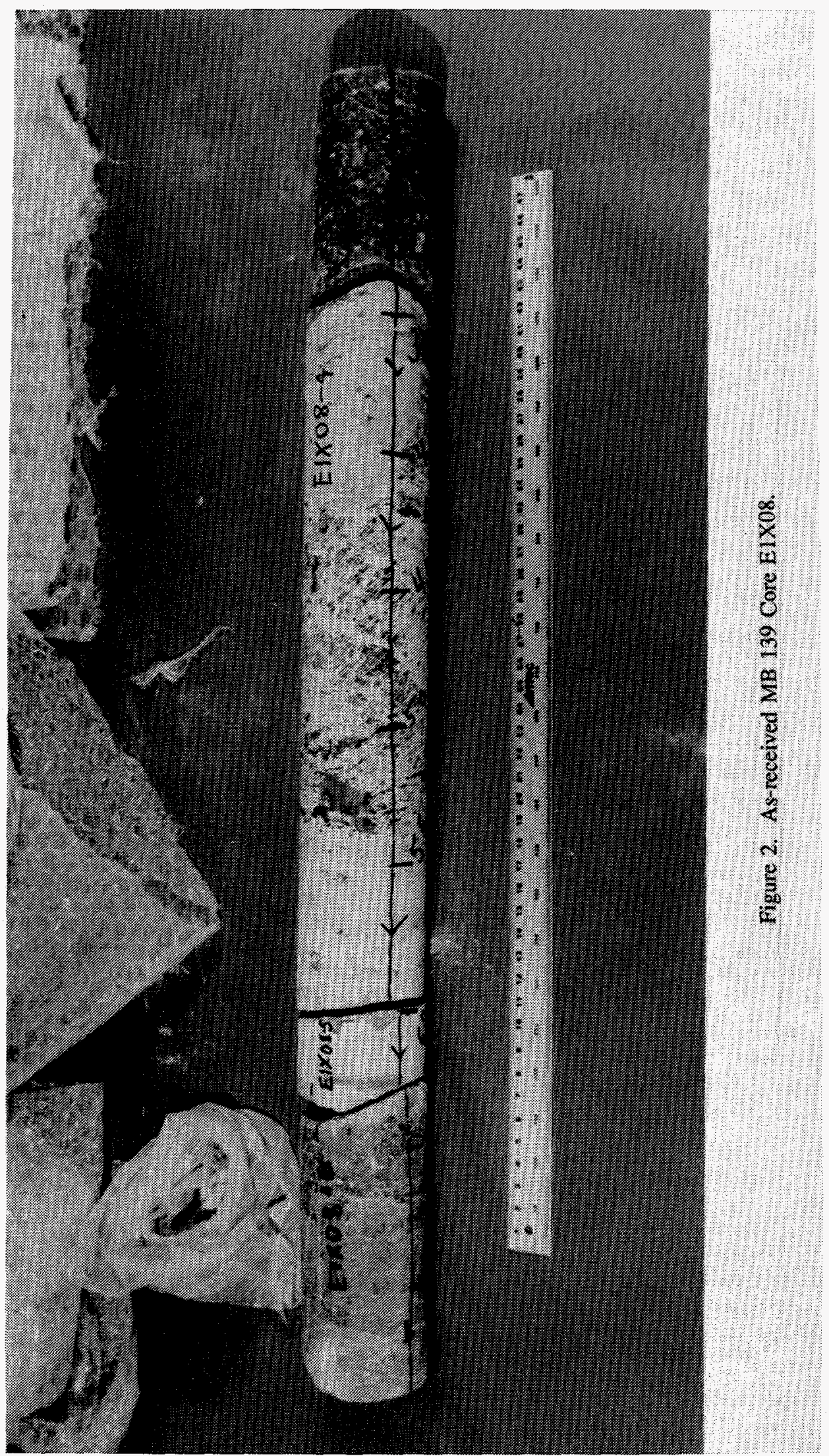

C-13 


\subsection{X-Ray CT Non-Destructive Core Analysis}

\subsection{Application of a Second Generation CT Scanner}

A whole core X-ray computerized tomography (CT) scan was performed on core E1X08 in its as-received condition to investigate the applicability of CT for the identification of natural and coring-induced fractures. The four core pieces (E1X08-3, E1X08-4, E1X08-5, E1X08-6; previously identified as halite, anhydrite with mudrock, mudrock, and halite, respectively) were scanned continuously in TerraTek's Second Generation CT laacility using a beam width of $3 \mathrm{~mm}$ and energy of $120 \mathrm{KV}$ (see Appendix A for facility description and operating procedures).

Representative cross-sectional images from each of the core pieces are shown in Figures 3-6 (see Appendix B for an index of cross-sectional images with respect to depth). A vertical reconstruction through core E1X08-4, which traverses the bulk of MB139, is shown in Figure 7. The images from the two cores identified as halite (E1X08-3 and E1X08-6) are of acceptable quality (Figures 3 and 6); however, the images from cores E1X08-4 and E1X08-5 contain severe beam-hardening artifacts (Figures 4 and 5), as evidenced by apparent concentric increases in density.

"Beam hardening" is a term used to describe the selective filtration of the lower energy component of the X-ray beam which occurs as the beam penetrates the sample. Beam-hardening causes the effective energy of the $\mathrm{x}$-ray beam to increase as the beam penetrates the sample; this leads to an artificially high CT number on the sample circumference, which implies an artificially high density. Beam-hardening is a well known characteristic of all $\mathrm{x}$-ray CT scanners since they employ polychromatic $x$-ray sources [2-5]; however, its effect is usually small for standard geologic cores (i.e., NX- or HQ- size) with low-to-medium densities (i.e., oil/gas reservoir rock).

In second-generation CT scanners such as TerraTek's Ohio Nuclear DeltaScan 100, beamhardening corrections may be performed using either (1) single energy pre-reconstruction corrections or (2) by "pre-filtering" the x-ray beam to absorb the low-energy portion prior to penetration of the sample [6]. TerraTek's CT facility employs the first technique. A fused quartz sample with a nominal diameter of four inches and density of $2.20 \mathrm{~g} / \mathrm{cc}$ is scanned and an algorithm which is part of the DeltaScan 100's progranming is used to calculate non-linear coefficients which are later used in the DeltaScan's reconstruction algorithm to perform beamhardening corrections during scanning of the test specimen.

The severe beam hardening which occurred during CT scanning of Core E1X08 was apparently caused by (1) the very large diameter of the core (6 inch); and (2) the relatively high density of the material $\left(\rho_{\text {anhydrite }}=2.9-3.0 \mathrm{~g} / \mathrm{cc}\right)$. The standard beam-hardening correction procedure was therefore inadequate. Attempts to "pre-filter" the x-ray beam by either encasing the core in a hollow aluminum tube (with a nominal wall thickness of $1 / 6$ inch) or by placing thin aluminum shields (nominally $\%$ inch) over the $x$-ray source did not yield significant improvements. Generation of the non-linear coefficients by scanning a 5 inch diameter aluminum sample with a density of $2.70 \mathrm{~g} / \mathrm{cc}$ also did not yield significant improvements. 


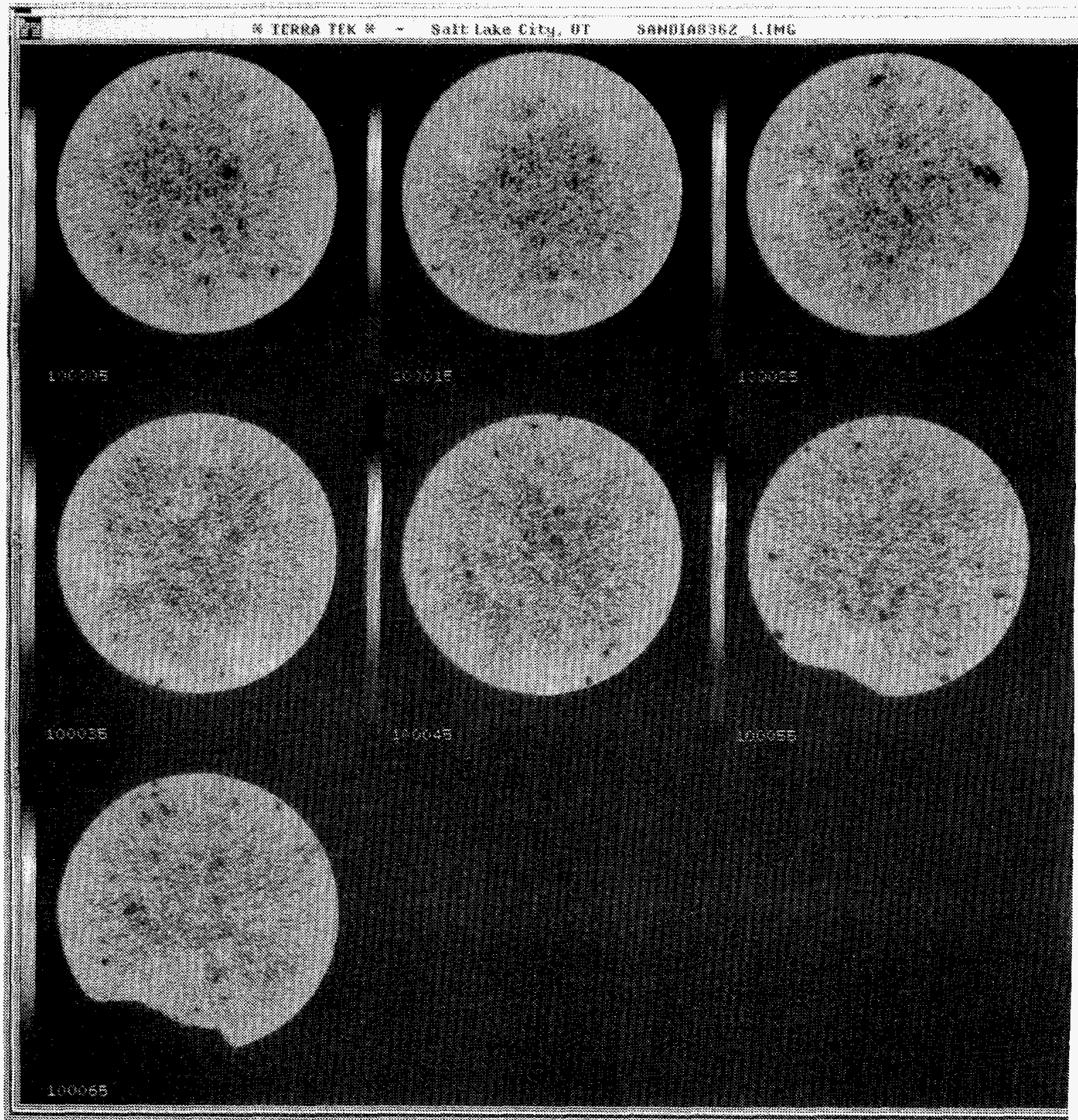

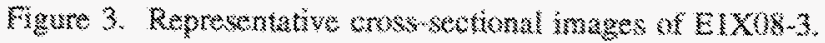




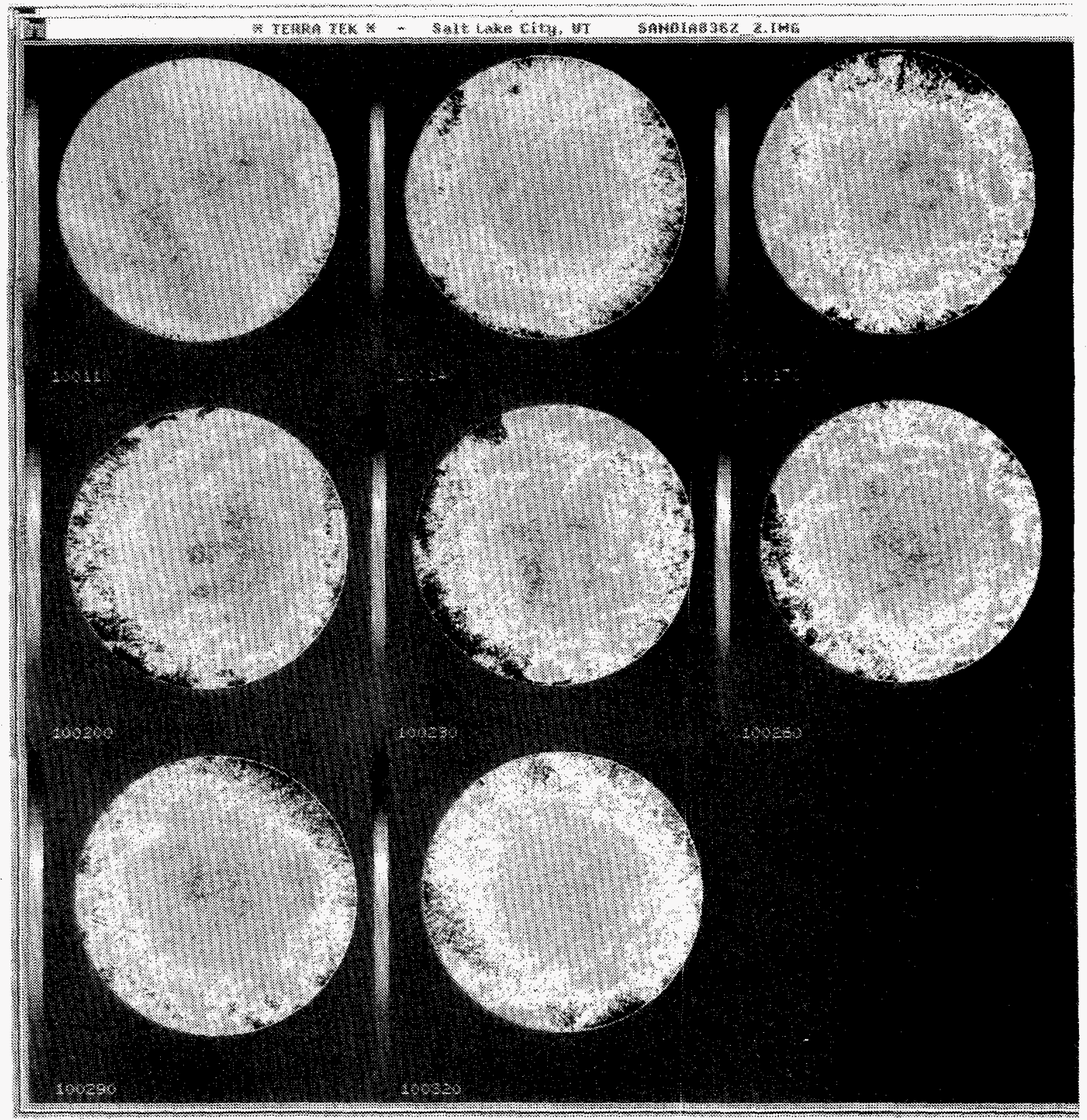

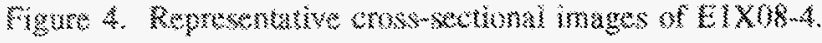




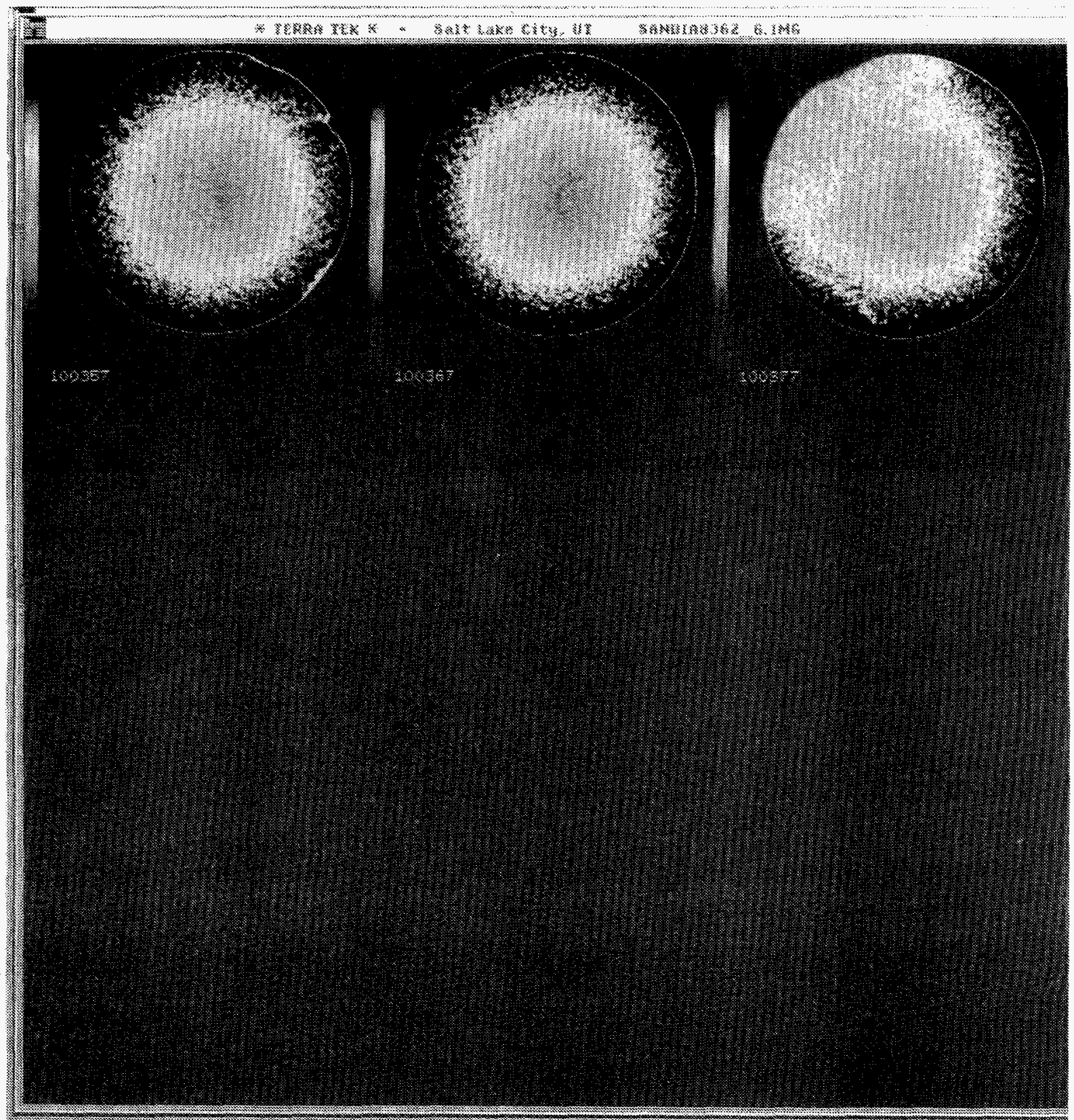

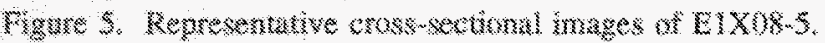




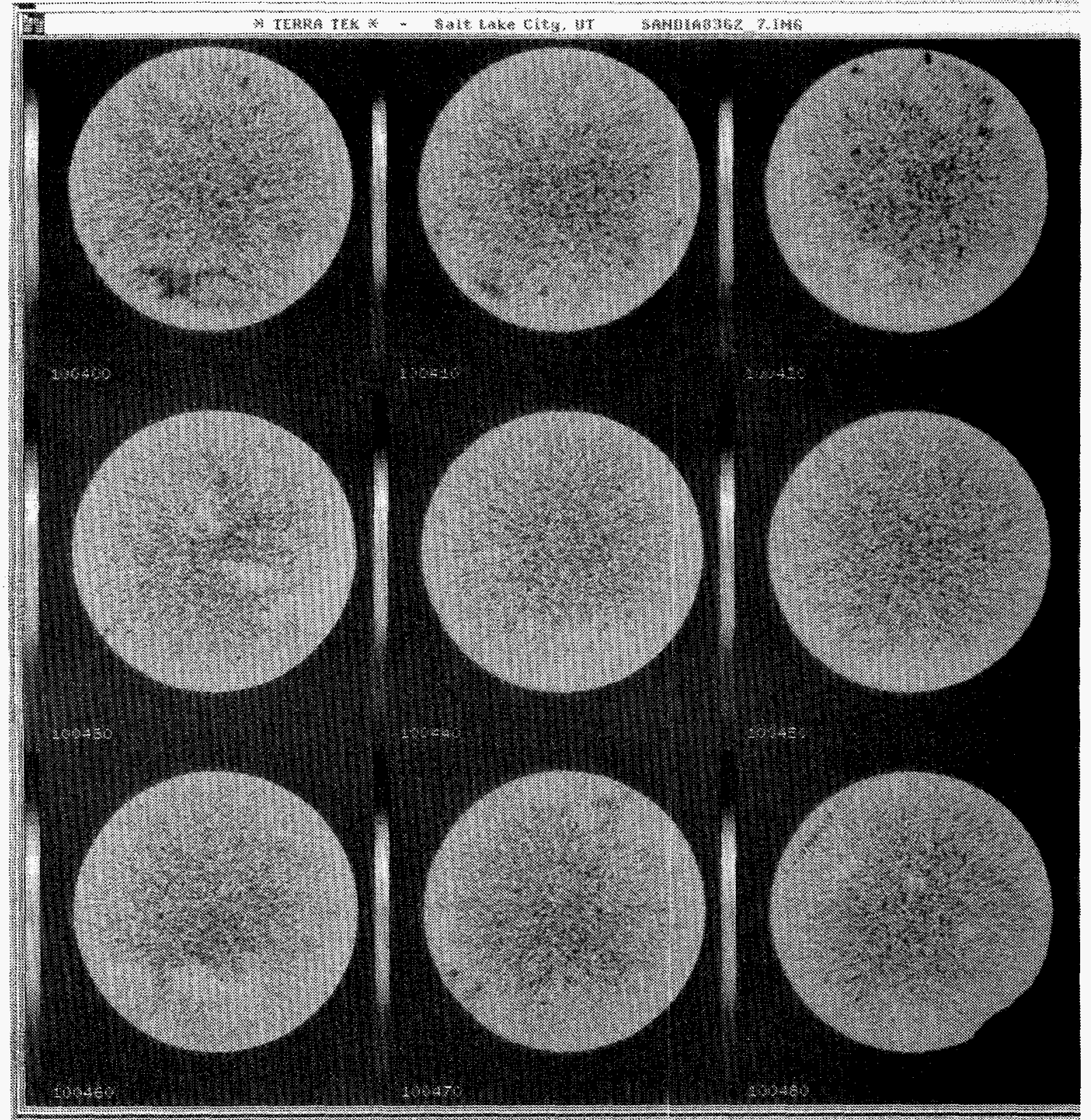

Figute 6. Representutive cross-sectonal images of $\mathrm{E}$ X0845. 


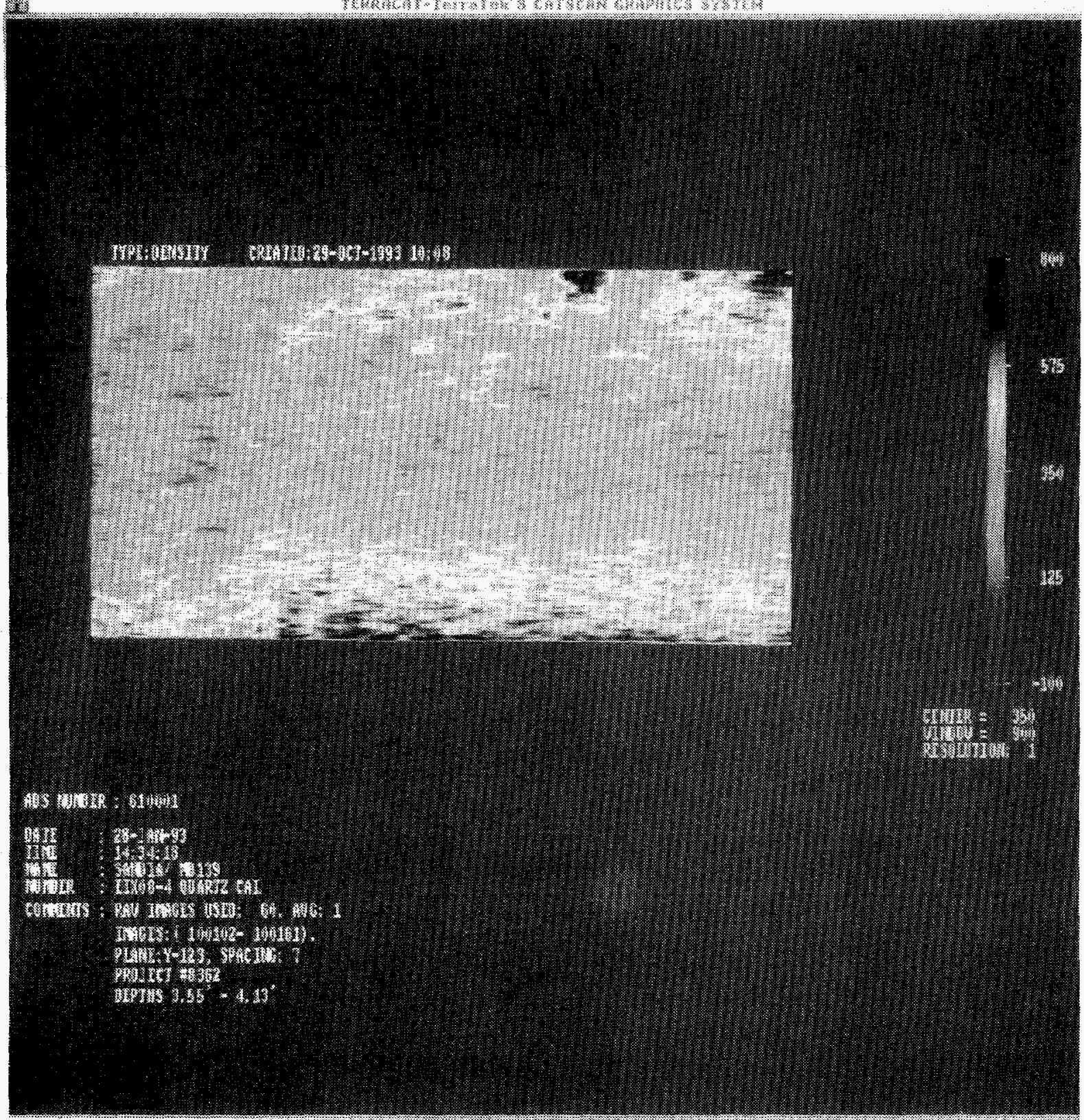

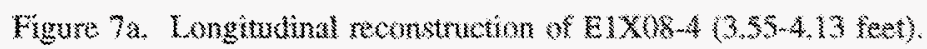




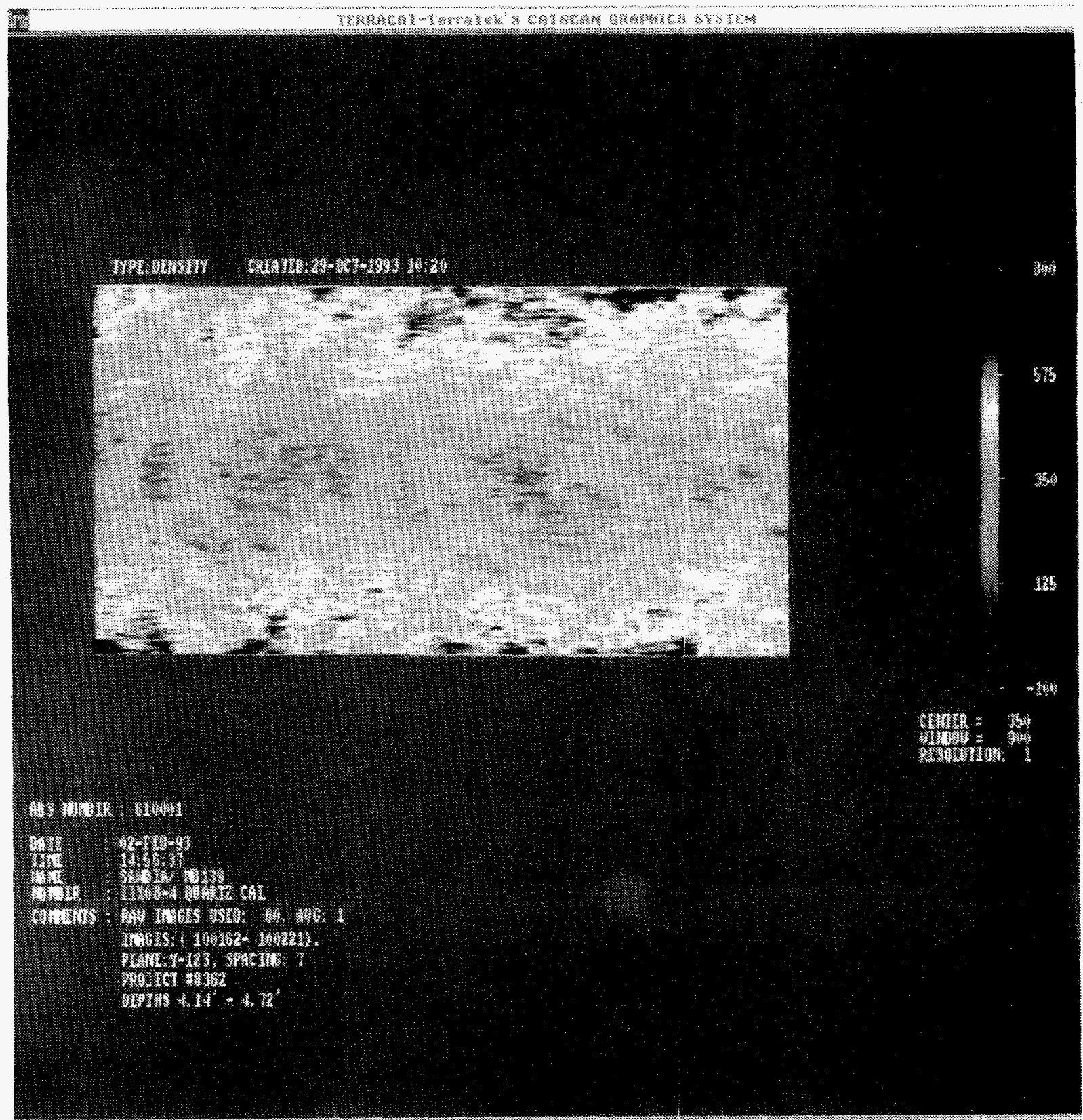

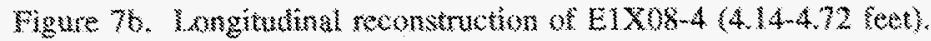




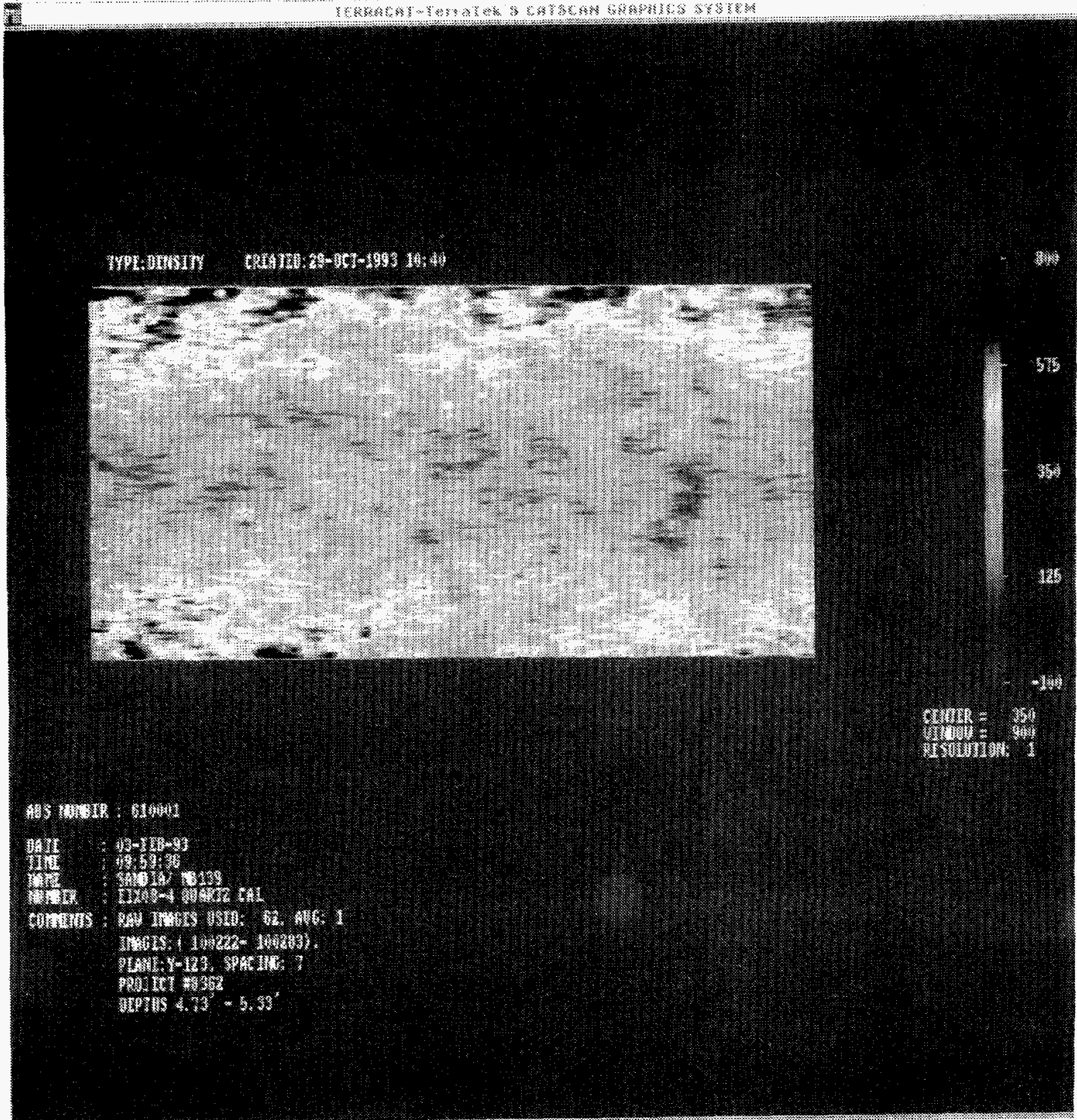

Figure 7c. Longitudinal reconstrution of E $1 \times 08-4(4,73,533$ feot). 


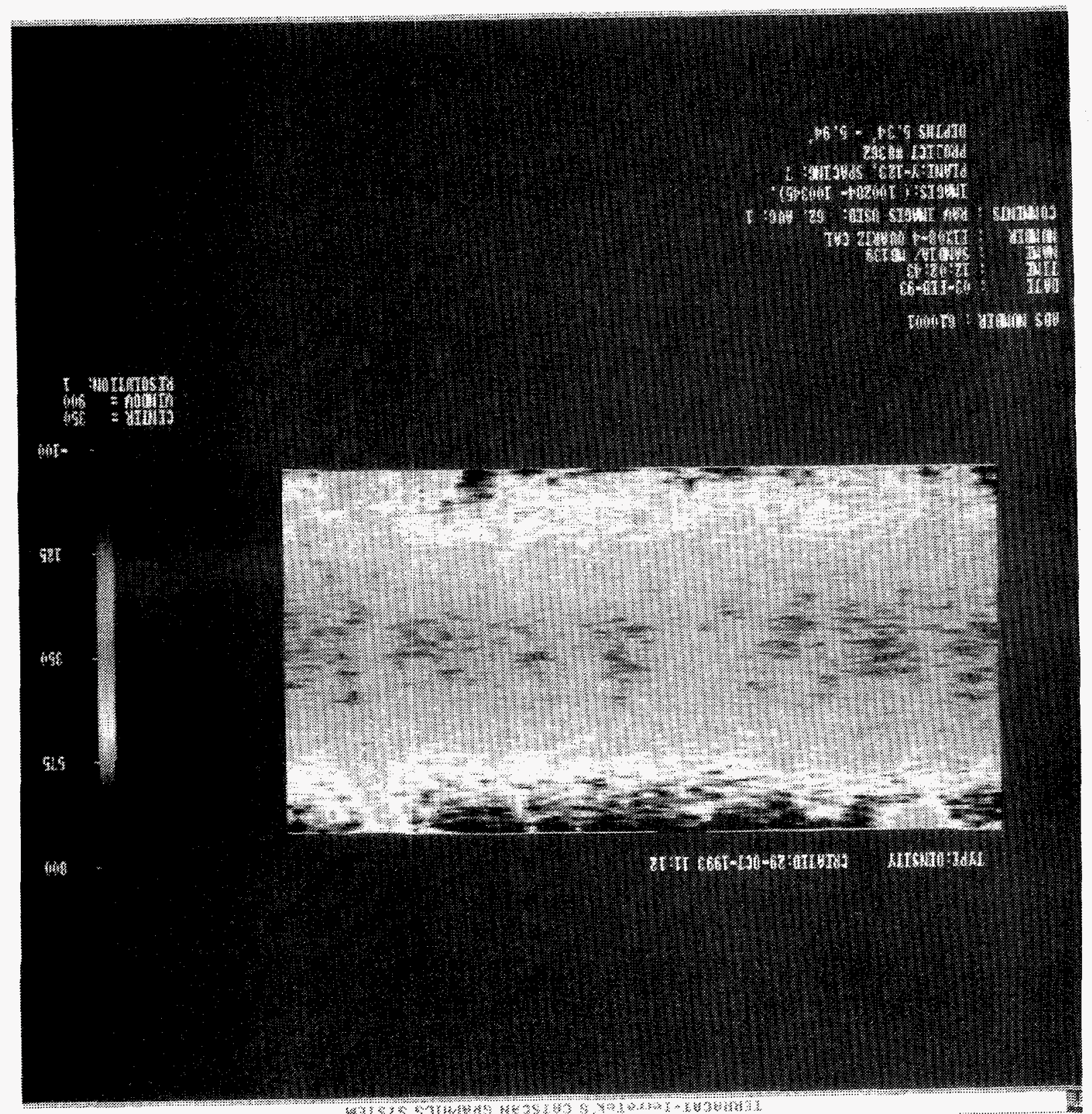


A number of observations may nevertheless be made. First, there is no evidence for substantial coring-induced damage (in the form of circumferential fractures around the core perimeter) in core E1X08 (Figures 3-7). Figure 8 shows several examples where CT scans have successfully identified coring-induced damage in HQ-sized ( $21 / 4$ inch diameter) core from the tunnel beds in Rainer Mesa, Nevada Test Site (images furmished with permission of Dr. B.L. Ristvet, Defense Nuclear Agency). Furthermore, the occasional chips which occur along the core axis of E1X08 do not appear to be marked by damage zones extending into the intact/core. (Note that sample E1X08-5 (6.0 to $6.4 \mathrm{ft}$ ), which is classified as mudrock, ultimately fractured into several pieces during handling. The sample broke along what was possibly either a pre-existing healed fracture or bedding plane which may have been unduly stressed during either coring, shipping, and/or handling.) Second, the two whole-core pieces identified as halite, E1X08-3 (2.7 to $3.5 \mathrm{ft}$ ) and E1X08-6 (6.3 to $7.4 \mathrm{ft}$ ) appear to be very homogeneous both laterally and vertically (Figures 3 and 6). These two core pieces appear to be of substantially lower density than either E1X08-4 (anhydrite/mudrock) and E1X08-5 (mudrock). Third, core piece E1X08-5 (6.0 to $6.4 \mathrm{ft}$ ), classified as mudrock, appears to be the densest portion of core E1X08 overall (Figure 5). Images from this core exhibit the grossest beam-hardening. This core piece appears to be remarkably homogeneous both laterally and vertically in the upper section; however, the lower interval exhibits substantial lateral and vertical heterogeneity. Finally, core piece E1X08-4 (3.6 to $6.0 \mathrm{ft}$ ) exhibits substantial lateral and cross-sectional heterogeneity across the entire core length (Figure 7). The 5.5 to $6.0 \mathrm{ft}$ interval appears to be highest, and the 3.6 to $3.7 \mathrm{ft}$ interval lowest, in overall density. Narrow linear features of comparatively low density which appear in a few images at both the top and bottom of the core (Figure 9) may be healed fractures.

\subsection{Exploratory Application of a Third Generation CT Scanner}

Midway through the contract performance period, TerraTek acquired a more advanced third generation Philips 60/TX CT scanner. Advantages of the Philips scanner include a higher maximum operating voltage (140 KV vs $120 \mathrm{KV}$ for the DeltaScan 100 ) and current (250 mA versus $25 \mathrm{~mA}$ for the DeltaScan 100) for the x-ray tube. TerraTek at this time also completed development of a new software package which includes a capability to perform automatic numerical correction for beam-hardening artifacts. Several scans were performed through intact pieces of cores E1X08-5 (halite) and E1X08-4 (anhydrite mixed with mudrock) to investigate whether the more powerful scanner would yield high quality images of very large, dense samples such as the MB139 cores.

The raw images from E1X08-5 and E1X08-4 are shown in Figures 10:a and 11a, and the images following processing with CORESCAN ${ }^{\mathrm{TM}}$ to correct for beam-hardening are shown in Figures $10 \mathrm{~b}$ and $11 \mathrm{~b}$. The images obtained with the Philips 60/TX scanner are excellent, and many details such as scattered low density inclusions and occasional pockets of a higher density mineral are visible in the halite core (Figure 10b). Similarly, the images from the E1X08-4 (Figure 11b) suggest two dominant mineral phases, possibly anhydrite and halite. 


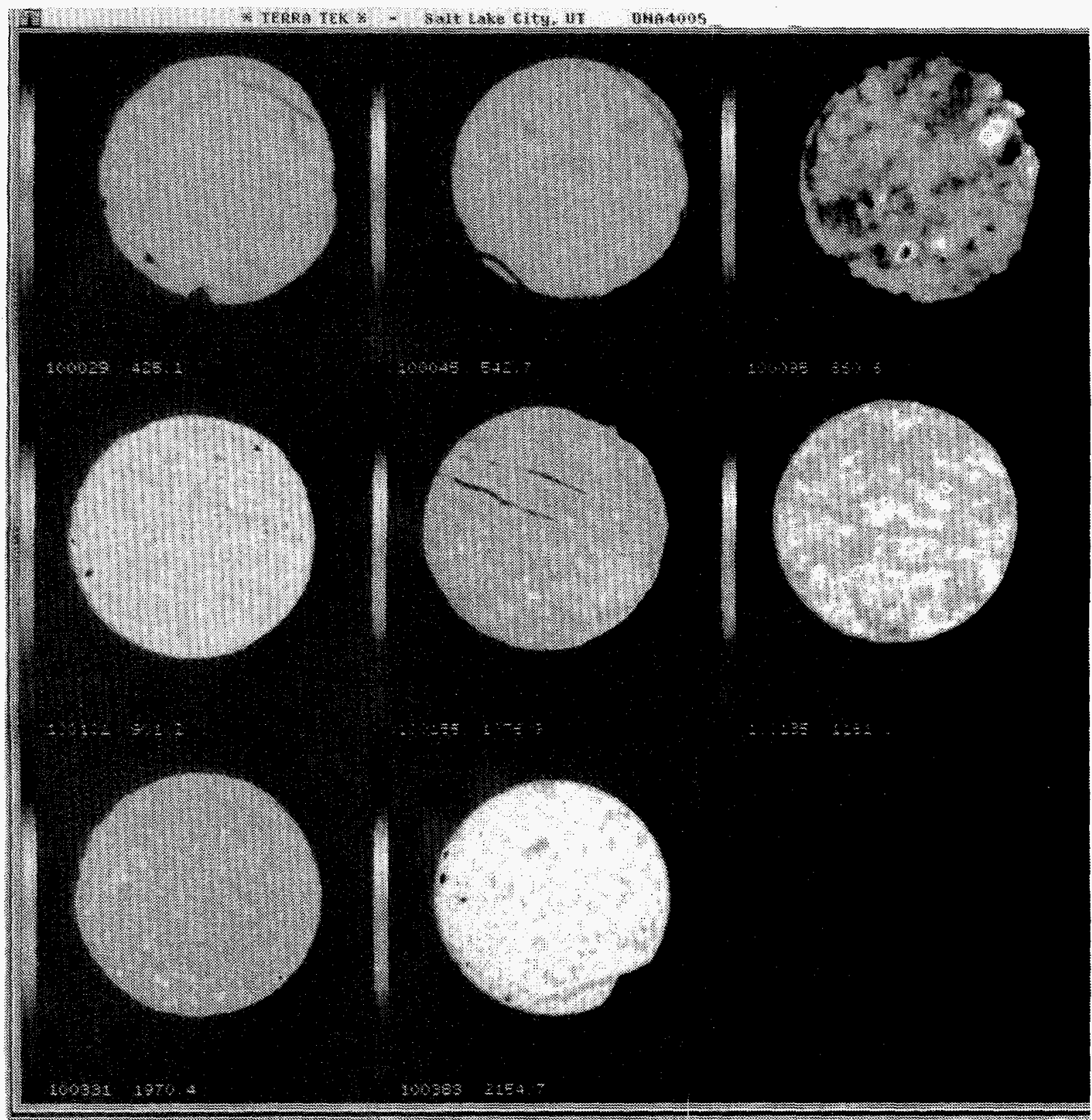

Figure 8 . Examples of cortig induced damage as evidenced by CT cross-sectional inages in tuff cores from the fumel beds in Rainer Mesa, Nevada Test Site. Images furnished with permission of Dr. B... Ratret, Defonge Nucters Agency). 


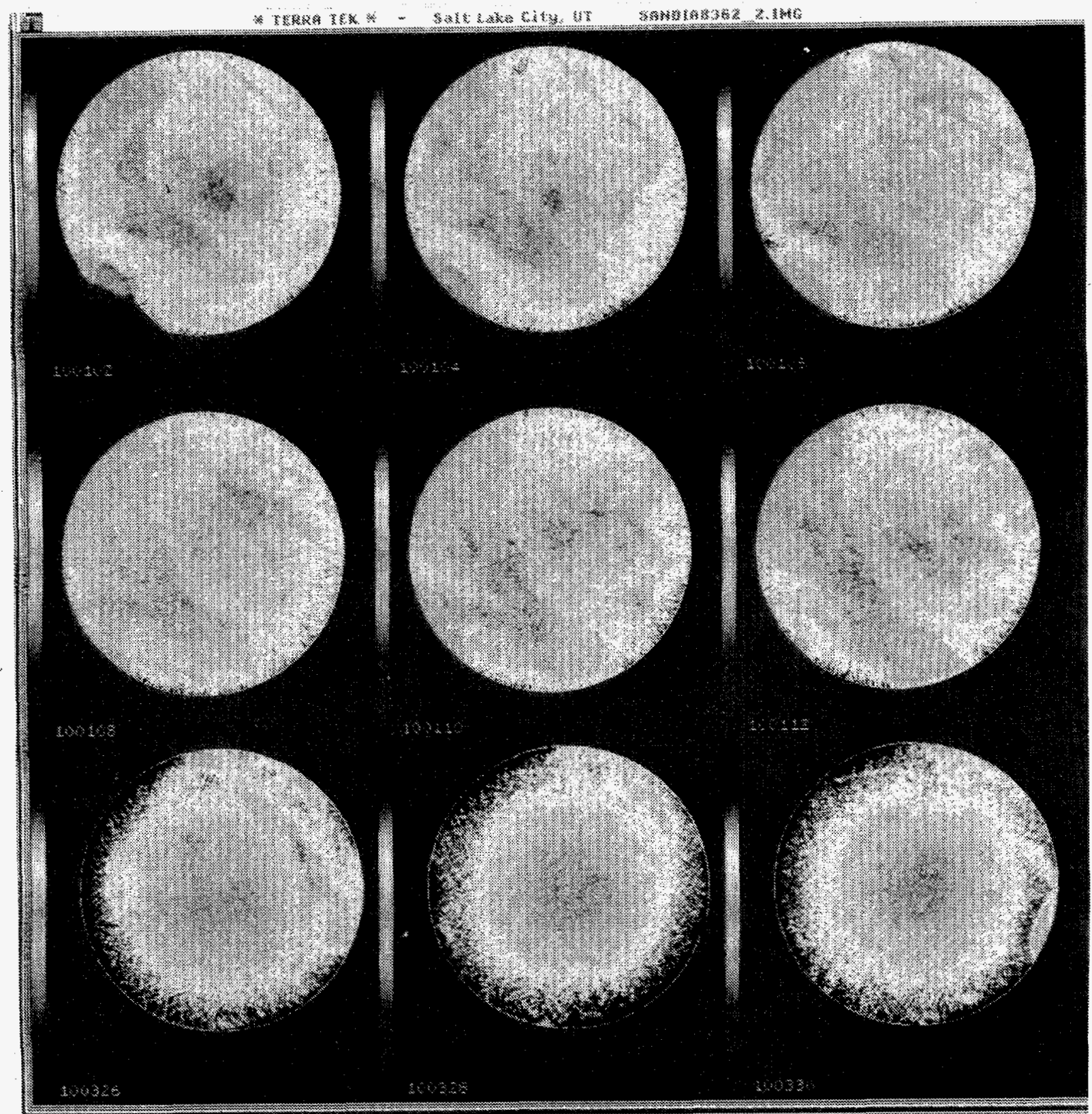

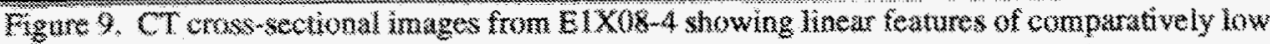

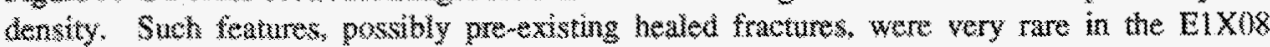
images. 


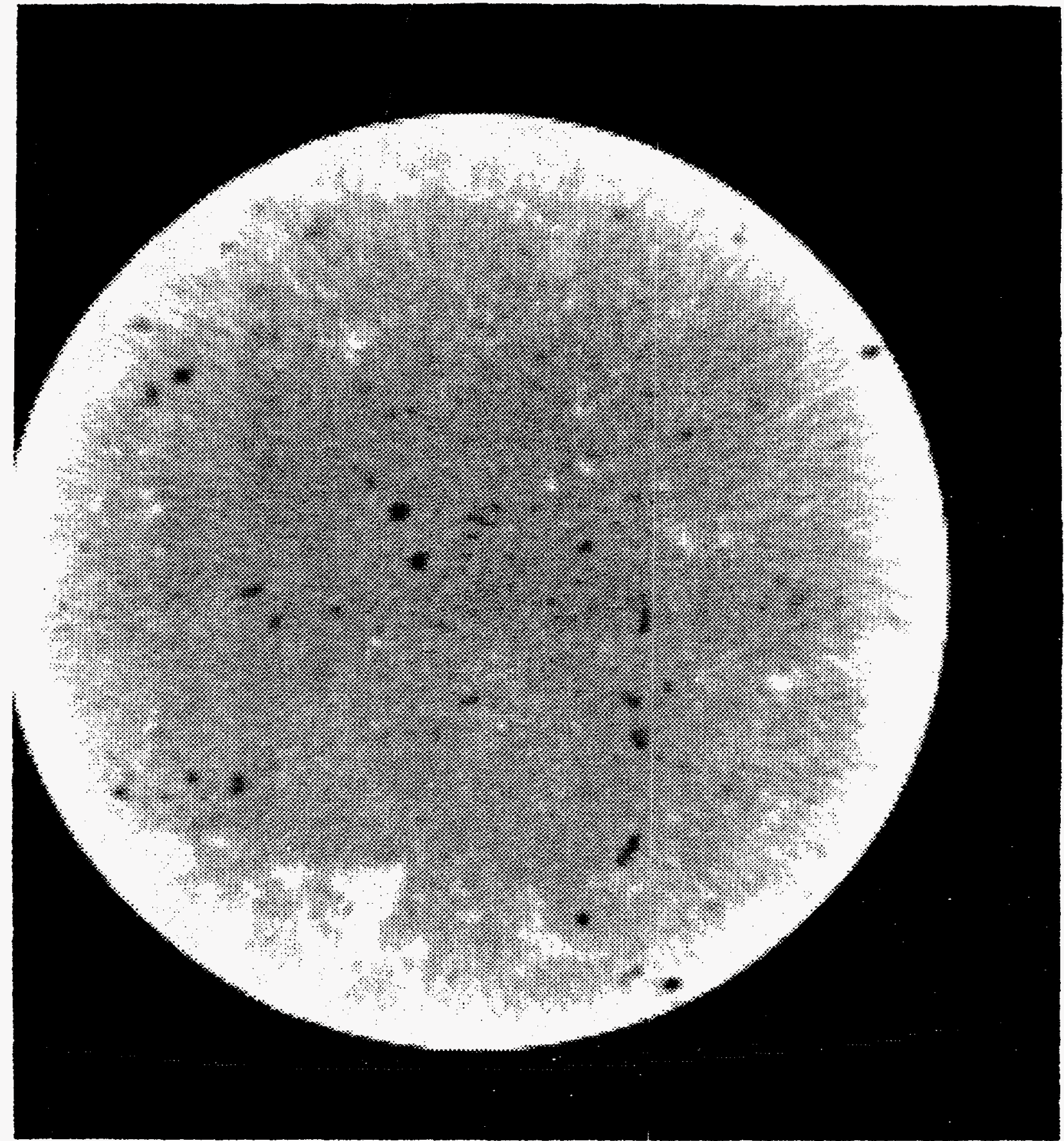

Figure 10a. Raw image from E1X08-5 (halite) acquired with a third-generation Philips 60/TX CT scanner. Beam-hardening, as evidenced by the bright ring on the sample perimeter, is present. 


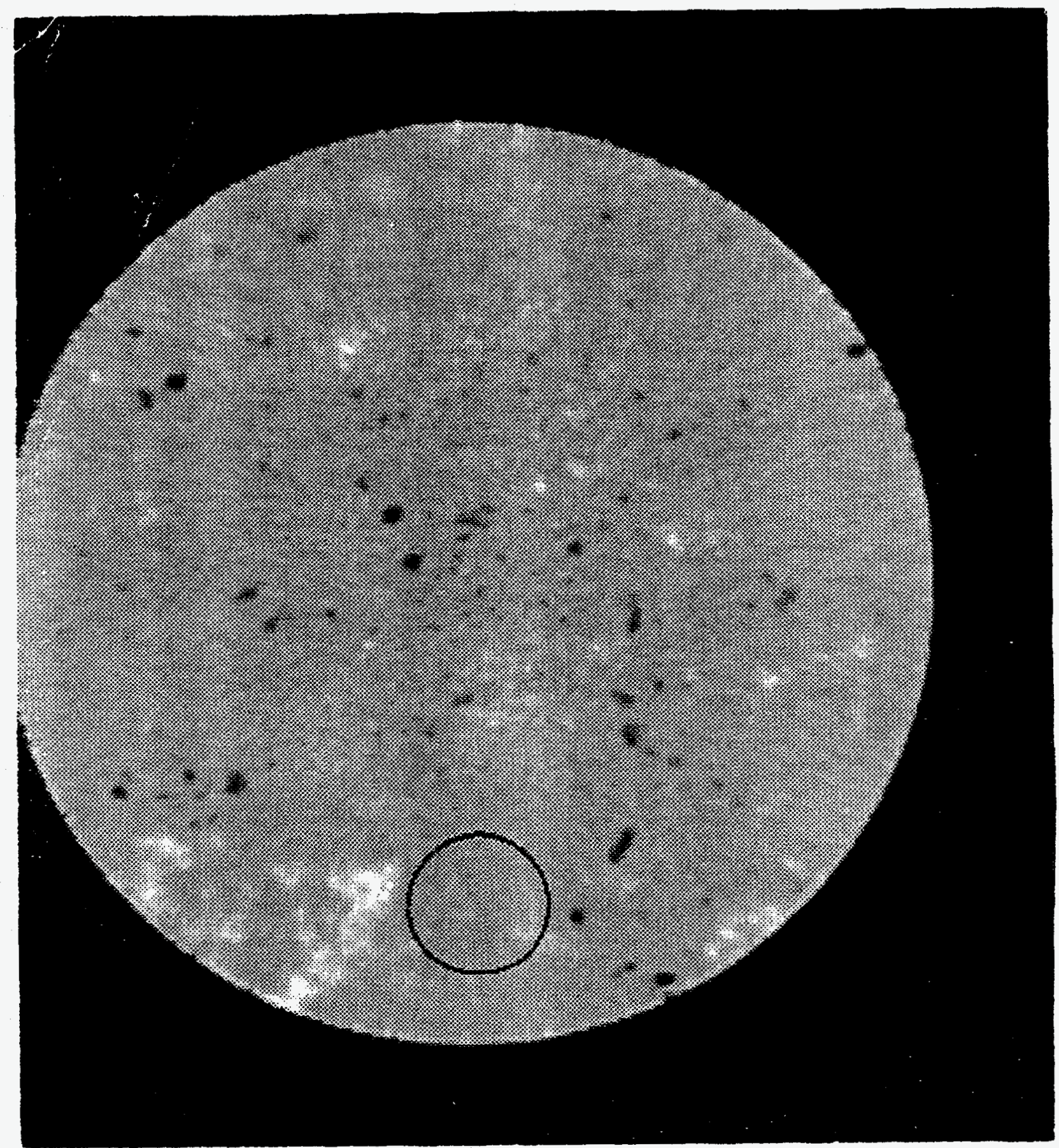

Figure 10b. Image shown in Figure $10 \mathrm{a}$ following post-processing with CORESCAN ${ }^{\mathrm{TM}}$ to eliminate beam-hardening artifacts. 


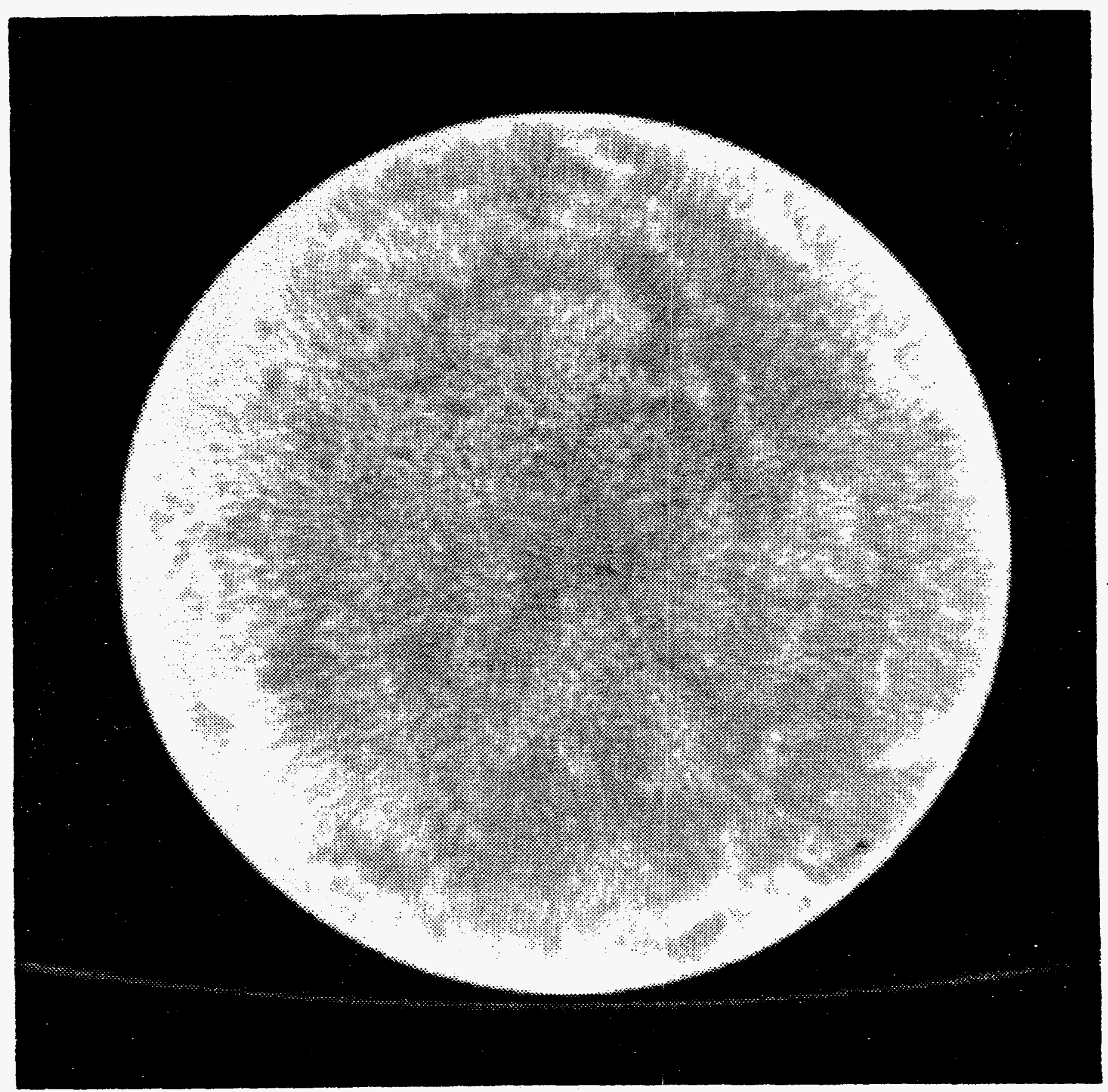

Figure 11a. Raw image from E1X08-4 (anhydrite with mudrock) acquired with a third-generation Philips 60/TX CT scanner. Beam-hardening, as evidenced by the bright ring on the sample perimeter, is present. 


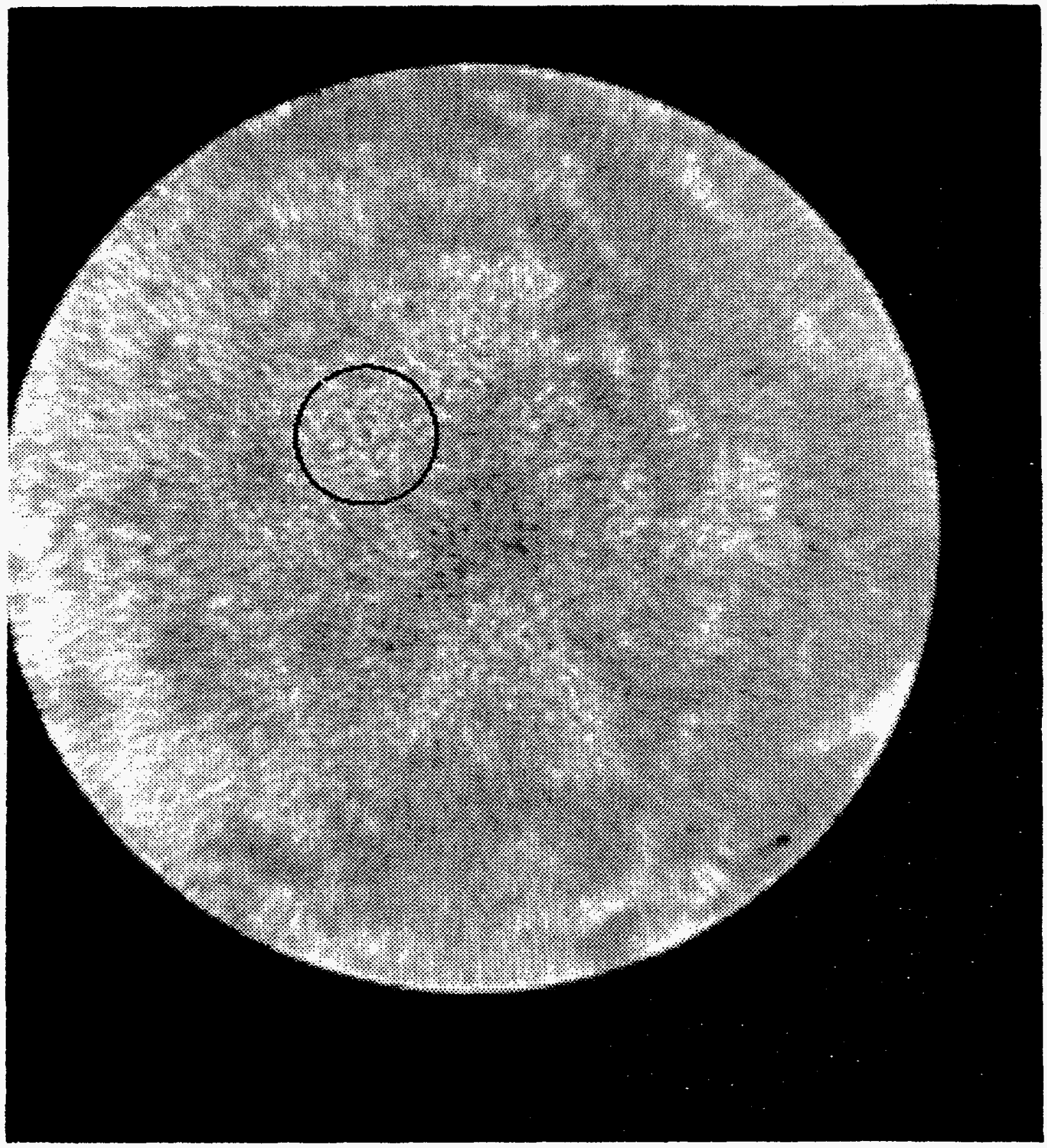

Figure 11b. Image shown in Figure 11a following post-processing with $\operatorname{CORESCAN}^{\mathrm{TM}}$ to eliminate beam-hardening artifacts. 


\subsection{Sample Preparation}

Sample preparation focused on core intervals E1X07-4 and E1X08-4. MB139 was considered to include core interval E1X08-5; however, this section was too small for complete characterization. A total of six four-inch diameter samples (hereafter referred to as A-F) and fourteen twoinch diameter samples (hereafter referred to as EP1-8 and PX1-6) were diamond-cored from E1X08-4 and E1X07-4 in an orientation perpendicular to the original core axis. The samples were located such that complete characterization would be performed on three separate intervals of each of the MB139 cores. The experimental program is detailed in Figure 12. The identification of each sample is documented in Figures 13 and 14 and cataloged in Table 1.

Table 1. Sample Identification

\begin{tabular}{ccc}
\hline Sample & Core & $\begin{array}{c}\text { Depth } \\
\text { (feet) }\end{array}$ \\
\hline A & E1X08 & $3.63-4.00$ \\
B & E1X08 & $4.47-4.84$ \\
C & E1X08 & $5.34-5.71$ \\
D & E1X07 & $4.14-4.50$ \\
E & E1X07 & $4.64-5.00$ \\
F & E1X07 & $5.19-5.56$ \\
& & \\
EP1 & E1X08 & $3.50-3.63$ \\
EP2 & E1X08 & $4.33-4.47$ \\
EP3 & E1X08 & $5.05-5.19$ \\
EP4 & E1X08 & $5.85-6.00$ \\
EP5 & E1X07 & $4.00-4.14$ \\
EP6 & E1X07 & $4.50-4.64$ \\
EP7 & E1X07 & $5.00-5.14$ \\
EP8 & E1X07 & $5.59-5.73$ \\
& & \\
PX1 & E1X08 & $4.00-4.14$ \\
PX2 & E1X08 & $4.86-5.00$ \\
PX3 & E1X08 & $5.71-5.85$ \\
PX4 & E1X07 & $4.00-4.14$ \\
PX5 & E1X07 & $5.00-5.14$ \\
PX6 & E1X07 & $5.73-5.87$ \\
\hline & & \\
\hline
\end{tabular}




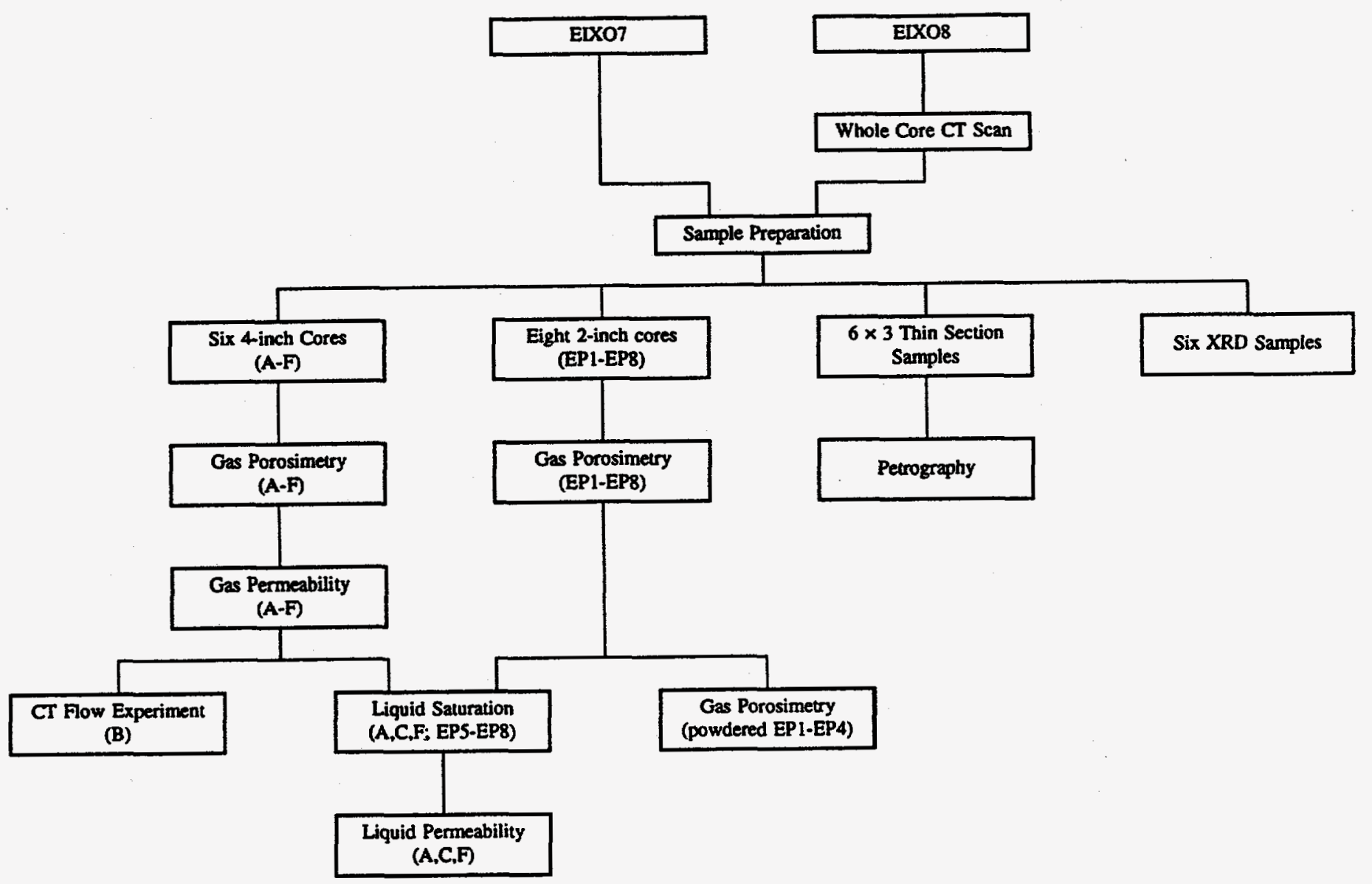

Figure 12. Experimental program. 


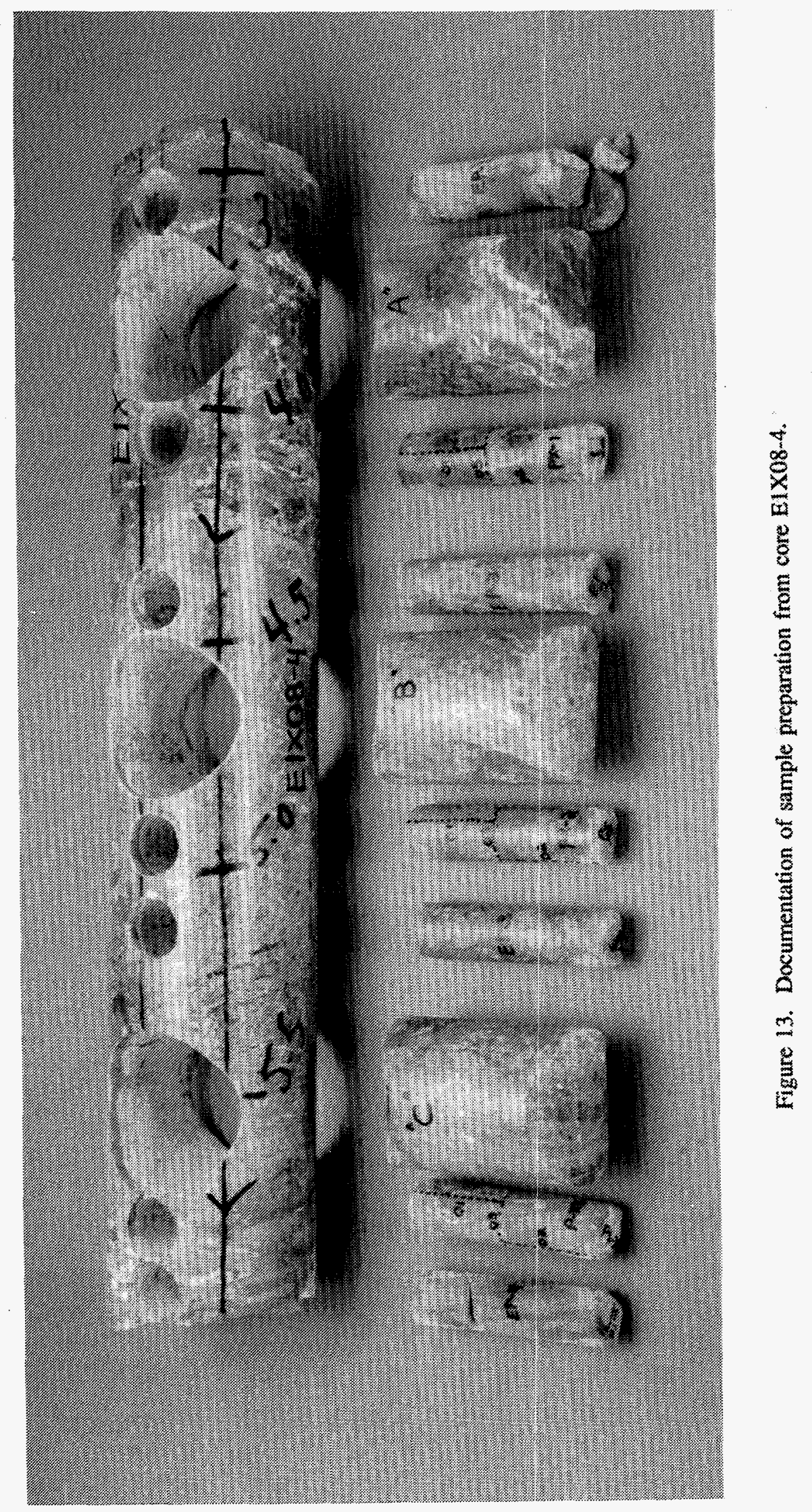

C- 32 


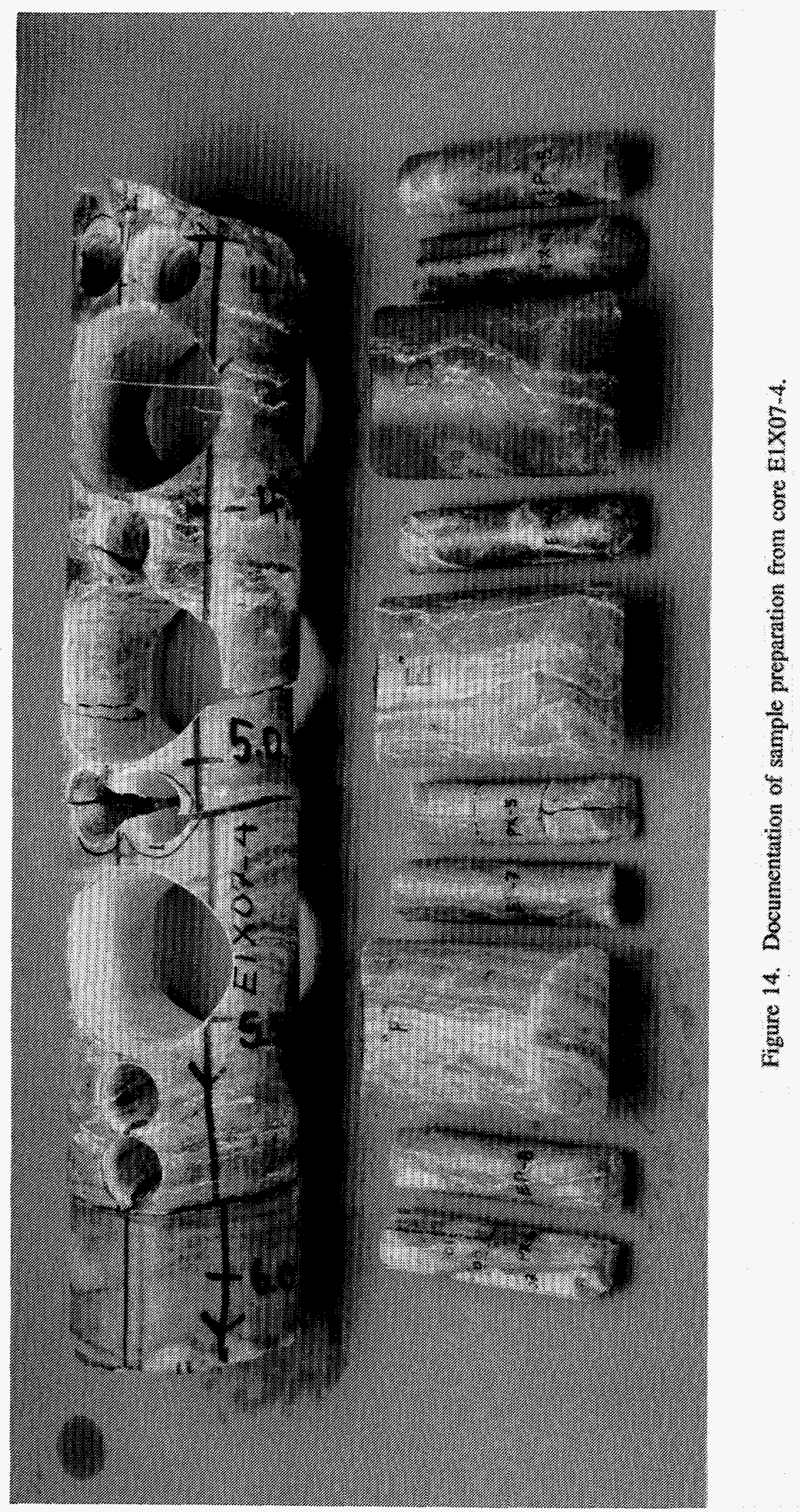


The ends of the six four-inch samples were cut with a diamond saw and endground flat and parallel. The ends of eight of the twoinch samples (EP1-8) were cut approximately perpendicular to the core axis with a diamond saw. The remaining six two-inch cores (PX16) were cut with a diamond saw into several pieces for petrographic study and X-Ray Diffraction (XRD) analysis (Figure 15, also see Figure Al Appendix C-A). To avoid any possible reaction with water-sensitive minerals, odorless mineral spirits (OMS) were used as a coolant during diamond-coring and endgrinding. Petrographic samples were vacuumimpregnated with a blue-dye epoxy and used to prepare oversized (approximately $2 \times 1 \frac{1}{2}$ inch) thin $(30 \mu \mathrm{m})$ sections by an outside agent.

Samples A-F and EP1-8 were dried to constant mass in a constant-humidity oven. Criteria for weight stabilization exceeded ISRM recommendations [7], which define stability as successive mass determinations (4 $\mathrm{hr}$ intervals) differing by less than $0.1 \%$ of the sample mass. The MB139 samples were dried until no observable systematic mass change occurred. Temperature ranged from approximately $55-66^{\circ} \mathrm{C}$ and approximate relative humidity varied from $44-54 \%$ over the more than 2500 hours required to dry all samples. Total drying times are listed in Table 2 and the drying histories are shown graphically (Appendix C-C) (note that samples were initially removed from the oven after the ISRM criteria [7] were satisfied (at -200 hours) and then returned to the oven after analysis of the mass measurements suggested that complete drying had not yet been achieved).

\subsection{XRD Analyses}

XRD analyses for determination of semi-quantitative mineralogy were performed on samples PX1-3 from E1X08-4 and PX4-6 from E1X07-4 and are reported in Table 3. 
Table 2. Drying Times

\begin{tabular}{cc}
\hline Sample & $\begin{array}{c}\text { Time } \\
\text { (hours) }\end{array}$ \\
\hline A & 1546 \\
B & 366 \\
C & 918 \\
D & 2532 \\
E & 558 \\
F & 1787 \\
& \\
EP1 & 918 \\
EP2 & 366 \\
EP3 & 366 \\
EP4 & 366 \\
EP5 & 918 \\
EP6 & 918 \\
EP7 & 558 \\
EP8 & 366 \\
\hline
\end{tabular}

Table 3. XRD Semi-Quantitative Mineralogic Analyses

\begin{tabular}{ccccccc}
\hline Sample & $\begin{array}{c}\text { Anhydrite } \\
\text { (wt\%) }\end{array}$ & $\begin{array}{c}\text { Halite } \\
(\mathrm{wt} \%)\end{array}$ & $\begin{array}{c}\text { Ferroan } \\
\text { Dolomite* } \\
\text { (wt\%) }\end{array}$ & $\begin{array}{c}\text { Polyhalite } \\
\text { (wt\%) }\end{array}$ & $\begin{array}{c}\text { Aragonite } \\
\text { (wt\%) }\end{array}$ & $\begin{array}{c}\text { Quartz } \\
\text { (wt\%) }\end{array}$ \\
\hline PX-1 & 70 & 30 & trace & trace & - & - \\
PX-2 & 32 & 68 & - & - & - & - \\
PX-3 & 98 & 2 & - & - & - & - \\
PX-4 & 7 & 23 & $\approx 30$ & $\approx 28$ & 12 & trace \\
PX-5 & 62 & 38 & - & - & - & - \\
PX-6 & 81 & 19 & - & - & - & - \\
\hline
\end{tabular}

* Or another cation-disordered $\mathrm{Ca}-\mathrm{Mg}$-Fe carbonate. May be well crystalline.

$\S$ No standards available for quantification. Accuracy estimated at $\pm 20 \%$.

$\dagger \mathrm{K}_{2} \mathrm{Ca}_{2} \mathrm{Mg}\left(\mathrm{SO}_{4}\right)_{4} \cdot 2 \mathrm{H}_{2} \mathrm{O}$. According to Dana's System of Mineralogy, the salmon-pink color of samples containing this mineral may be due to finely divided inclusions of iron oxide.

$\ddagger$ Tentative identification. 


\subsection{Petrography}

Petrographic analyses, including a 300-point count for mineral identification and grain (crystal) size measurement, were conducted on eighteen thin sections (see Figure 15 for schematic of the three mutually perpendicular sections prepared from each PX sample and Appendix C-D for documentation of thin-section preparation). Point count data are given in Tables 4 and 5 and histograms of grain size distribution are shown in Figures 16-21. Micrographs illustrating the characteristic mineralogy and texture of each PX sample are shown in Plates 1 to 6.

Table 4. Modal Analyses

\begin{tabular}{|c|c|c|c|c|c|}
\hline Sample & $\begin{array}{c}\text { Anhydrite } \\
\text { (vol\%) }\end{array}$ & $\begin{array}{l}\text { Halite } \\
\text { (vol\%) }\end{array}$ & $\begin{array}{l}\text { Carbonate* } \\
\text { (vol\%) }\end{array}$ & $\begin{array}{c}\text { Polyhalite } \\
\text { (vol\%) }\end{array}$ & $\begin{array}{l}\text { Pyrite } \\
\text { (vol\%) }\end{array}$ \\
\hline PX1-01 & 6 & $<1$ & 5 & 87 & 1 \\
\hline PX1-O2 & 67 & 20 & 6 & 7 & - \\
\hline PX1-O3 & 33 & 2 & 7 & 56 & 1 \\
\hline PX2-01 & 62 & 37 & 2 & - & - \\
\hline PX2-O2 & 48 & 51 & 2 & - & - \\
\hline PX2-O3 & 67 & 30 & 4 & - & - \\
\hline PX3-O1 & 80 & 3 & 16 & - & - \\
\hline PX4-O2 & 87 & 1 & 12 & - & - \\
\hline PX4-O3 & 86 & 2 & 10 & - & 1 \\
\hline PX4-01 & 50 & 44 & 1 & 5 & - \\
\hline PX4-O2 & 20 & 12 & 13 & 55 & - \\
\hline PX4-O3 & 27 & 14 & 3 & 57 & - \\
\hline PX5-01 & 66 & 26 & 9 & - & - \\
\hline PX5-O2 & 79 & 18 & 3 & - & - \\
\hline PX5-O3 & 75 & 21 & 4 & - & - \\
\hline PX6-01 & 82 & 17 & $<1$ & - & $<1$ \\
\hline $\mathrm{PX} 6-\mathrm{O} 2$ & 81 & 16 & 3 & - & - \\
\hline PX6-O3 & 92 & 4 & 4 & - & - \\
\hline
\end{tabular}




\subsection{Overview}

The dominant minerals in MB 139 are anhydrite, halite, and polyhalite; small amounts of very finely crystalline carbonate (tentatively identified as dolomite) are also present (typically about $5 \mathrm{vol} \%$ ). The distribution of the major mineral phases, as the marker bed is traversed both laterally and vertically, is highly variable.

Anhydrite is typically finely crystalline, although textural alterations are observed when it is closely associated with halite or polyhalite. Anhydrite adjacent to and within halite crystals is coarser, whereas anhydrite intergrown with polyhalite tends to occur as elongate, tabular crystals. Halite crystals and nodules are usually impure, containing anhydrite and/or carbonate inclusions. Halite occurs as nodules, up to $2-3 \mathrm{~cm}$ in diameter, which likely grew displacively in the sediment within anhydrite laminae. Halite also fills space between intergrown polyhalite and

Table 5. Grain Size, Sorting, and Porosity from 300-Point Count

\begin{tabular}{cccc}
\hline Sample & $\begin{array}{c}\text { Median Grain Size } \\
(\mu \mathrm{m})\end{array}$ & $\begin{array}{c}\text { Sorting } \\
(\mathrm{phi})\end{array}$ & $\begin{array}{c}\text { Porosity } \\
(\%)\end{array}$ \\
\hline PX1-01 & 15 & 0.98 & - \\
PX1-02 & 23.5 & 2.00 & - \\
PX1-03 & 20 & 0.91 & - \\
& & & - \\
PX2-01 & 66 & 2.06 & - \\
PX2-02 & 64 & 1.96 & - \\
PX2-03 & 39 & 2.15 & - \\
& & & \\
PX3-01 & 16.5 & 0.85 & $<1$ \\
PX3-02 & 13.5 & 0.83 & - \\
PX3-03 & 12.5 & 0.92 & - \\
& & & \\
PX4-01 & 128 & 1.91 & - \\
PX4-02 & 26 & 1.54 & - \\
PX4-03 & 38 & 1.34 & - \\
& & & \\
PX5-01 & 64 & 1.80 & - \\
PX5-02 & 45 & 2.02 & - \\
PX5-03 & 62.5 & 1.86 & - \\
& & & - \\
PX6-01 & 17 & 1.51 & - \\
PX6-02 & 17 & 1.56 & - \\
PX6-03 & 16 & 1.02 & - \\
\hline
\end{tabular}


anhydrite structures. Polyhalite, which dominates specific areas of samples PX1 and PX4 but is absent in the other samples, occurs as narrow, needle-like crystals which form spherulitic to pseudocross-like structures (see Plates 1 and 4). Finely crystalline $(<8 \mu \mathrm{m}$ grain size) carbonate is a minor phase in all samples and occurs in scattered patches and as thin wisps, which commonly define bedding. This material, which appears dolomitic, is typically microporous, as evidenced by a light blue color in thin section. Very minor pyrite and possible clay minerals are observed in some thin sections, but always constitute $\leq 1 \%$ of the bulk composition.

Total porosity in all six samples appears very low ( $\leq 2 \%)$ and poorly connected when observed in 2-D. Microporosity associated with carbonate minerals is the most commonly observed porosity type. Fractures, at angles between $45^{\circ}$ and $90^{\circ}$ to bedding and with apparent apertures ranging from 10 to $30 \mu \mathrm{m}$, are present in several samples. (Note that since thin sections were prepared at ambient conditions, in situ apertures are probably lower.) A number of bedding parallel fractures (with apertures up to tens of microns) are also observed. High-angle fractures are commonly confined to individual laminae and often terminate at the interface between laminae. Several healed fractures (sometimes filled by anhydrite) were observed.

\subsection{Petrographic Descriptions (see Figure 15 for sample locations)}

6.2.1 Sample PX1. Mineral distribution varies significantly amongst the three sections. $O 1$ and $\mathrm{O} 3$ are dominated by polyhalite, but contain lesser amounts of halite, anhydrite, carbonate (dolomite?), and pyrite. $\mathrm{O} 2$ is dominated by anhydrite and halite; polyhalite is present in only a small area of the section.

Polyhalite generally occurs as small spherulites composed of tiny, radiating, needle-like crystals which are intergrown to form a tightly interlocked crystal network. Singular elongate and tabular anhydrite crystals are commonly scattered throughout the polyhalite. Anhydrite is also intergrown with polyhalite in the outer portions of spherulites. Finely crystalline carbonate, probably dolomite, is also intergrown with anhydrite and polyhalite in the outer portions of spherulites. Finely crystalline carbonate also fills spaces between spherulites and occurs in somewhat randomly distributed patches. Halite fills areas between spherulites. Minor pyrite is scattered throughout these areas.

Most of section $\mathrm{O} 2$ and one edge of section $\mathrm{O} 3$ are clominated by anhydrite and halite. Anhydrite is generally finely crystalline, except when it is located adjacent to halite crystals where an increase in crystal size is apparent. Halite within anhydrite forms irregular nodules which appear to be aligned along bedding planes, which are very irregular. Finely crystalline carbonate material in these areas occurs in small randomly distributed patches and as thin wisps (which define bedding). Some carbonate patches exhibit a somewhat peloidal texture.

Minor microporosity is apparent within some of the finely crystalline carbonate patches and is most abundant in $\mathrm{O} 3$, which contains more finely crystalline carbonate than $\mathrm{O} 2$ or $\mathrm{O} 1$. Both open and healed fractures, with apertures of 10-30 $\mu \mathrm{m}$, are present in anhydrite laminae in $\mathrm{O}$. 
6.2.2 Sample $P X 2$. All sections are very similar and dominated by intergrown anhydrite and halite with minor finely crystalline carbonate. Halite crystals are very large, particularly in $O 2$, and range up to several centimeters in diameter. Note that such large crystals cannot be measured concurrently with the fine-grained material, and histograms constructed for these three sections (Figures 17a-c) are for "matrix" only. Anhydrite ranges from fine to medium crystalline and is generally coarser than in most of the other PX samples. Anhydrite crystals adjacent to halite are typically coarser than those residing in the fine-grained matrix. Carbonate material is finely crystalline and occurs as scattered patches, and in $\mathbf{0 3}$ as thin wisps which define bedding. Bedding is not apparent in $\mathrm{O} 1$ or $\mathrm{O} 2$.

Minor microporosity is seen within patches of finely crystalline carbonate. Several narrow fractures are present on one edge of 03 . These fractures are partially within halite crystals and parallel to cleavage. Note that their proximity to the sample edge may suggest a relationship to sample preparation.

6.2.3 Sample PX3. PX3 is dominated by finely crystalline anhydrite which occurs in thin irregular laminae in all sections. Anhydrite appears more finely crystalline in this sample than in any of the other samples: this may be due to the low abundance of halite given the previously noted observation that anhydrite associated with halite is commonly more coarsely crystalline. Finely crystalline carbonate material is second in abundance, occurring in thin wisps along bedding planes and as irregular patches in the anhydrite matrix. Halite is least abundant and forms small irregular nodules within certain anhydrite laminae in $\mathrm{O} 2$ and $\mathrm{O} 3$, and in the central portion of 01 .

Bedding in all three samples is defined by thin, finely crystalline carbonate laminae and appears oriented at about $30^{\circ}$ to the core axis in $\mathrm{O} 2$. Several thin fractures (partings) parallel to bedding were observed.

Minor microporosity is present within the finely crystalline carbonate material in all sections. Fractures, oriented at about $45^{\circ}$ to bedding and with somewhat irregular traces, are present on one end of $\mathrm{O} 1$. $\mathrm{O} 3$ contains a healed, very narrow fracture which appears confined to several laminae and is oriented at about $80^{\circ}$ to vertical.

6.2.4 Sample PX4. PX4 is composed of intergrown polyhalite, halite, anhydrite, and finely crystalline carbonate. Polyhalite occurs as needle-like crystals in spherulitic to pseudo-cross type structures. Elongate anhydrite crystals are intergrown in the outer portions of the spherulites and halite fills space between spherulites. Polyhalite spherulites, containing very little halite or anhydrite, dominate portions of $\mathrm{O} 2$ and $\mathrm{O3}$. Anhydrite, in addition to being intergrown with polyhalite, also occurs as equant crystals in the matrix. Finely crystalline carbonate material, which is most abundant in 02 , occurs in scattered patches and as thin wisps, which may define bedding. Crystal size is highly variable, and the high sorting ( $2.94 \mathrm{phi}$ ) found for $\mathrm{O} 1$ reflects the abundance of elongate crystals. 
Only hints of bedding are observed in the PX4 sections. The irregular interface between mixed mineralogy and the polyhalite-dominated areas may represent bedding. Thin wisps of finely crystalline carbonate may define bedding in $\mathrm{O} 2$.

Very little porosity, consisting of minor microporosity associated with finely crystalline carbonate, was observed in the PX4 sections.

6.2.5 Sample PX5. PX5 is dominated by admixed anhydrite and halite. Anhydrite occurs as both individual laminae and combined with halite in other laminae. Grains are fine to medium crystalline and appear much coarser than in the underlying sample PX6. As observed previously, anhydrite in close association with halite is usually more coarsely crystalline than in laminae containing only anhydrite. Halite forms nodules within specific laminae in $\mathrm{O} 2$ and $\mathrm{O} 3$, but appears somewhat randomly oriented in $\mathrm{Ol}(\mathrm{O}$, which is parallel to bedding, may possibly have been prepared from a lamination rich in halite). Carbonate material is finely crystalline and occurs as scattered patches and wisps parallel to laminae.

Bedding is easily recognizable in $\mathrm{O} 2$ and $\mathrm{O} 3$, but less distinct in thin section $\mathrm{O} 1$. Wisps of carbonate material commonly define bedding in this PX sarnple.

Minor microporosity is present within the finely crystalline carbonate material. One high-angle (to bedding) fracture was observed in an anhydrite lamination in 02 . Total porosity appears quite low.

6.2.6 Sample PX6. Sample PX6 is characterized by bedded to laminated anhydrite containing nodular halite. Halite nodules are relatively impure and contain abundant anhydrite inclusions, which may indicate displacive growth in an anhydrite mush prior to lithification. Anhydrite is finely crystalline, except where closely associated with halite. Minor finely crystalline carbonate occurs as wisps parallel to laminations. Minor clay may also be present along some bedding planes. Scattered pyrite framboids were observed in 01 .

Bedding is best developed in this sample, although somewhat wavy and discontinuous. Local bedding appears oriented at about $20^{\circ}$ to the long dimension (vertical) of thin section P2-O2. Some laminae contain exclusively anhydrite, whereas others contain a mixture of anhydrite and halite. Growth of halite nodules has disrupted some of the original depositional texture.

Total porosity appears low. Microporosity associated with tinely crystalline carbonate wisps and patches is most common. Several narrow fractures oriented between $70^{\circ}$ and $90^{\circ}$ to bedding and with apertures less than $20 \mu \mathrm{m}$ are present in $\mathrm{O} 2$ and 03 . Bedding-parallel fractures are present in $\mathrm{O} 2$. 
FIGUAE 16A. GRAIN/CAYSTAL SIZE HISTOGRAM FOR SAMPLE PX1-01.

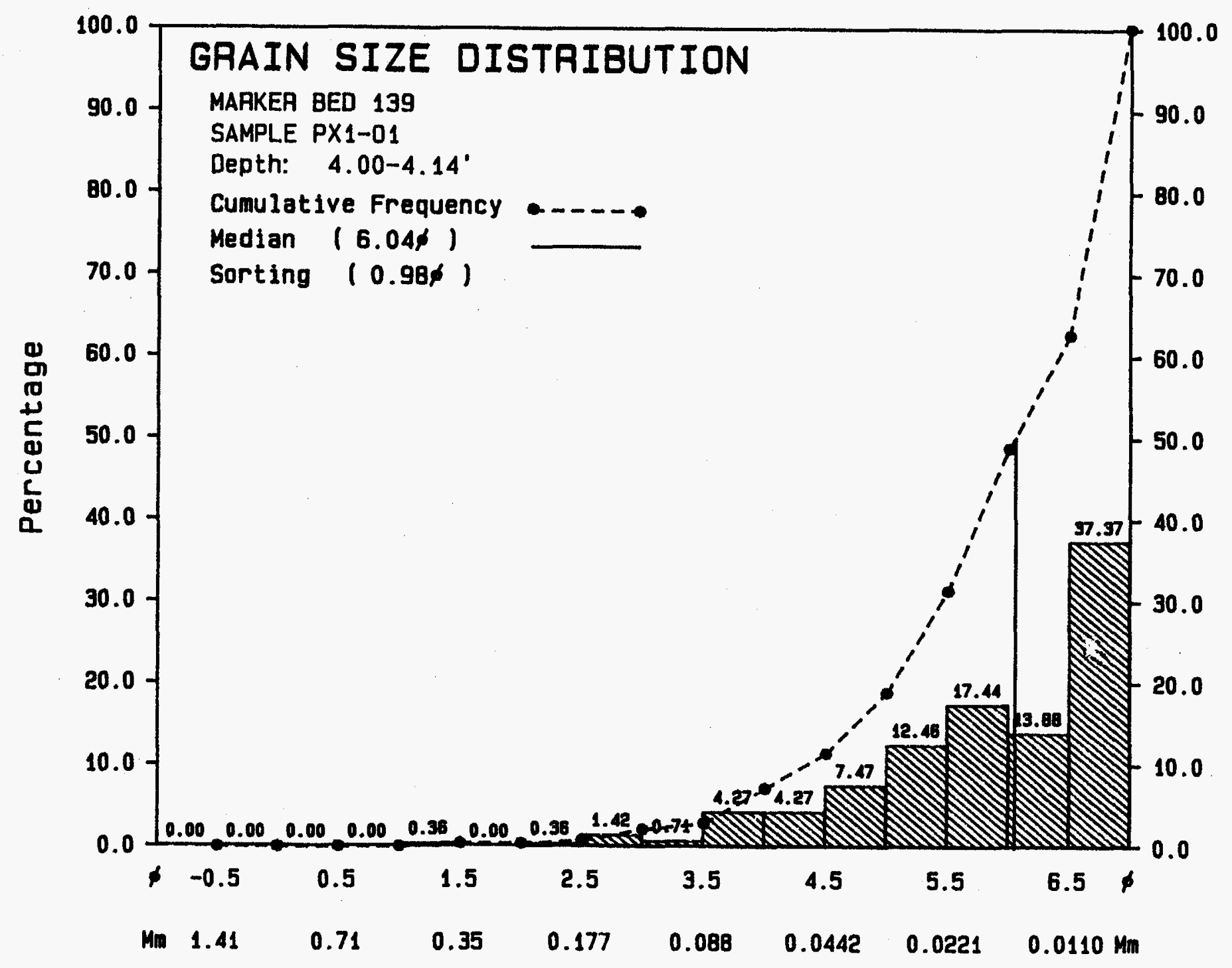


FIGUAE 168. GAAIN/CAYSTAL SIZE HISTOGRAM FOR SAMPLE PX1-02.

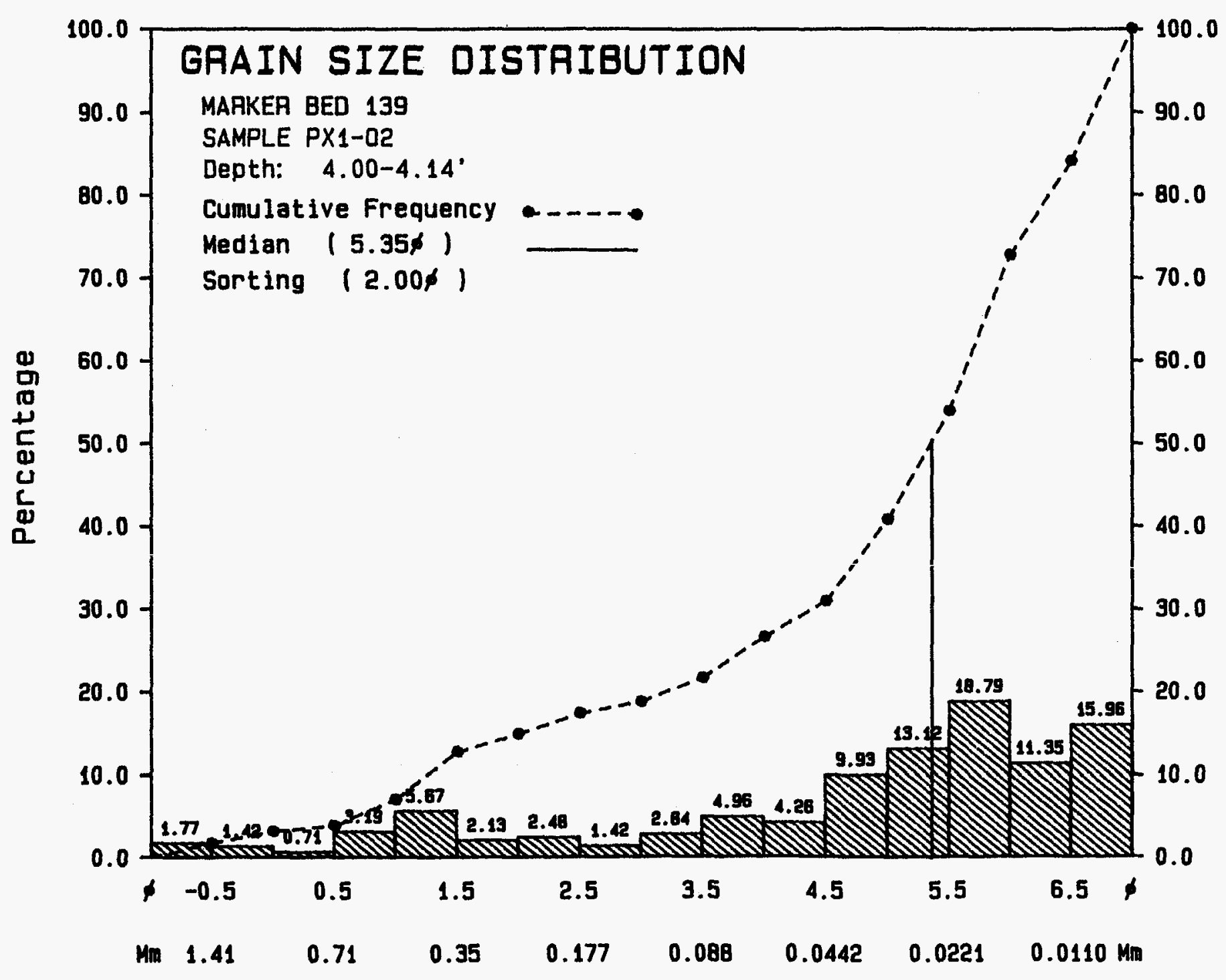




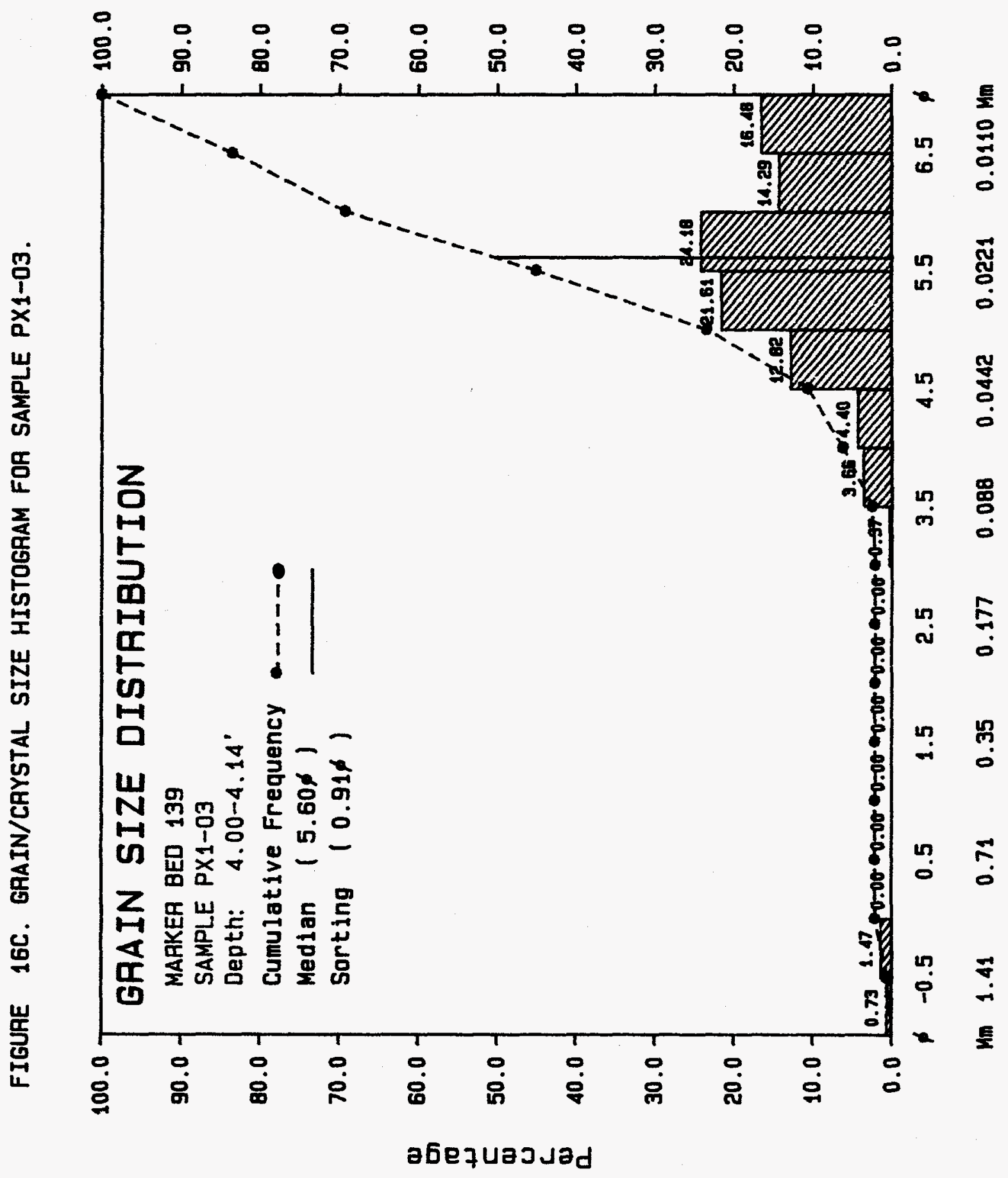


FIGUAE 17A. GRAIN/CAYSTAL SIZE HISTOGAAM FOA SAMPLE PX2-01.

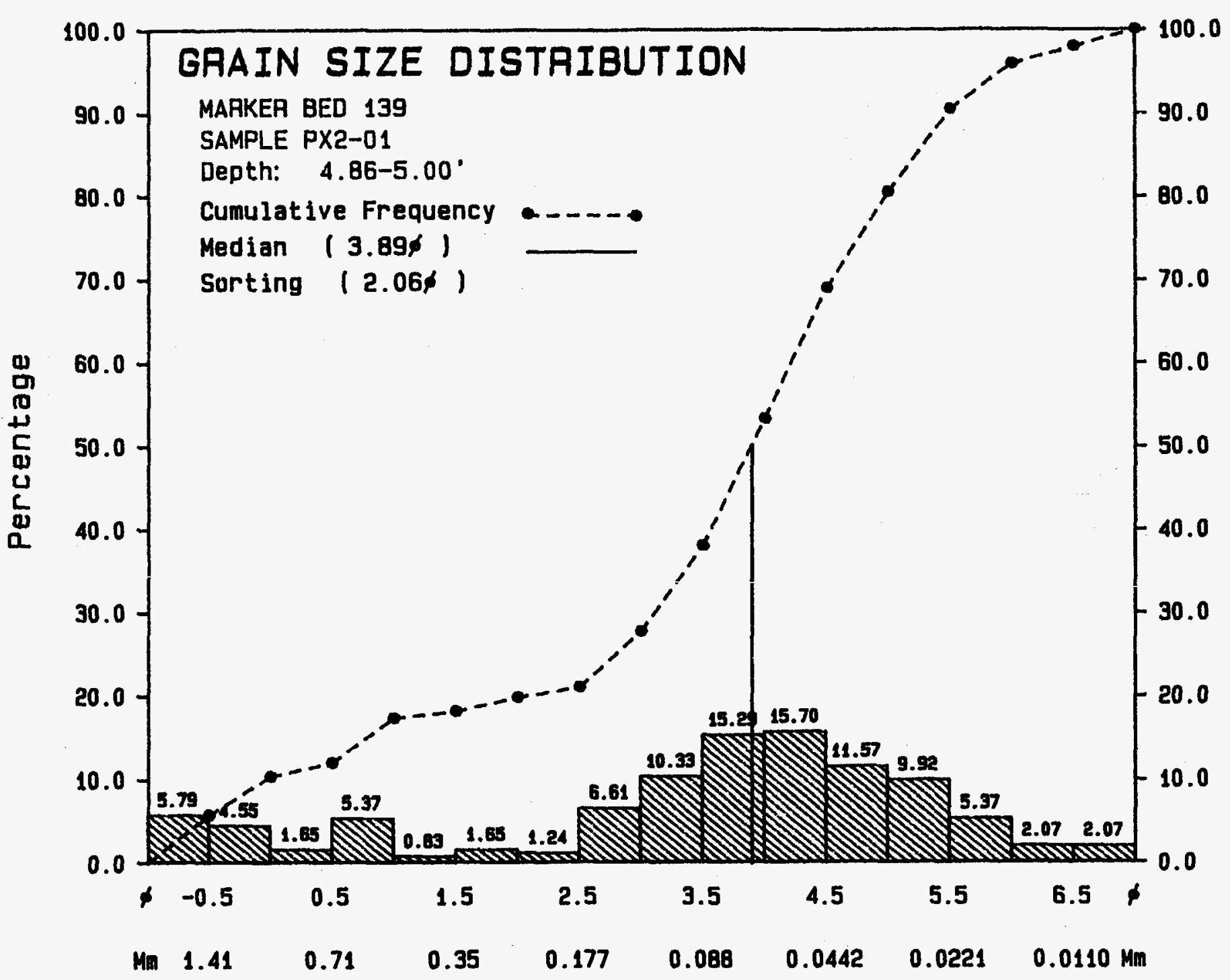




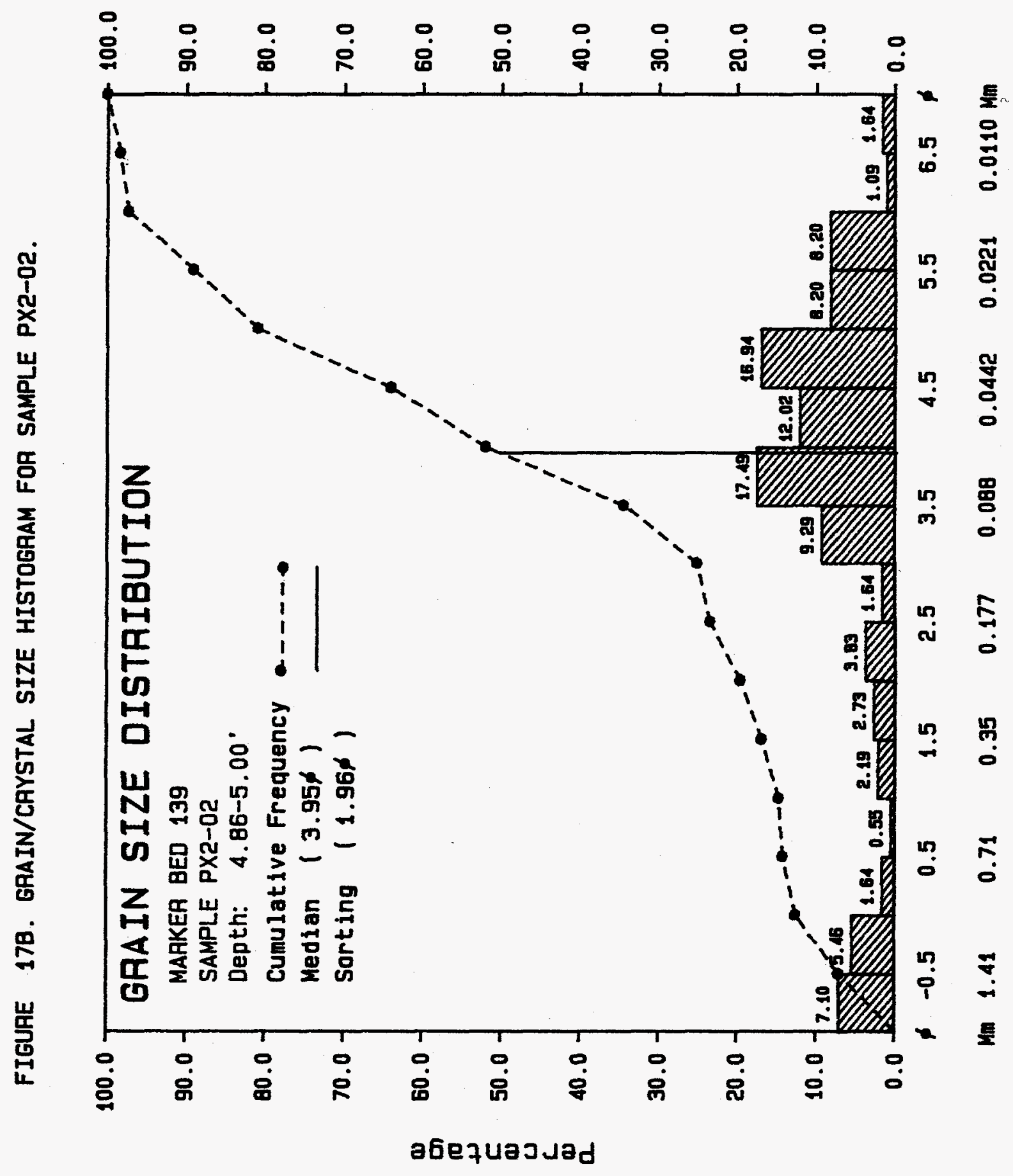


FIGUAE 17C. GRAIN/CAYSTAL SIZE hISTOGRAM OF SAMPLE PX2-03.

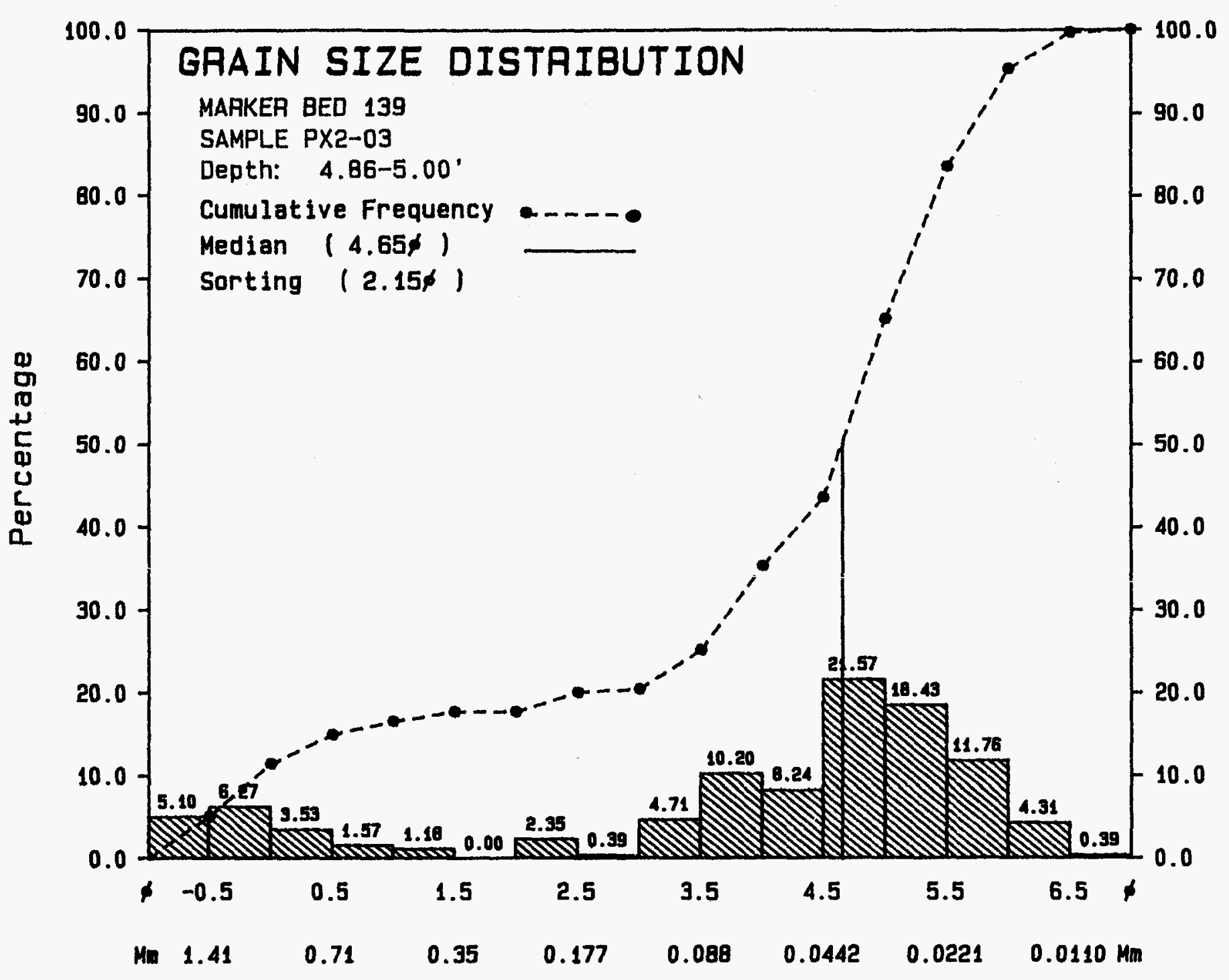


FIGURE 18A. GRAIN/CAYSTAL SIZE HISTOGRAM FOR SAMPLE PX3-01.

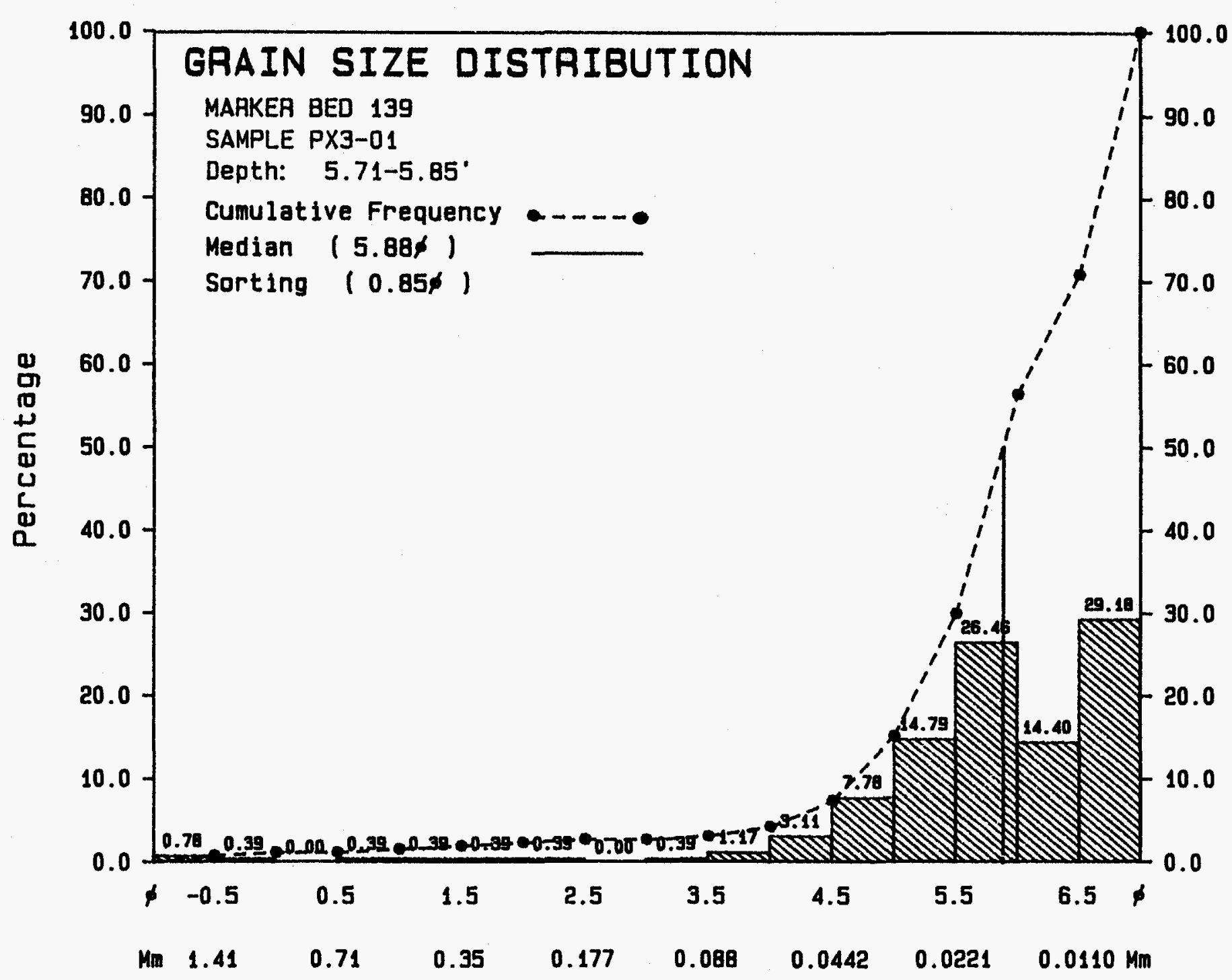


FIGURE 188. GRAIN/CAYSTAL SIZE HISTOGRAM OF SAMPLE PX3-02.

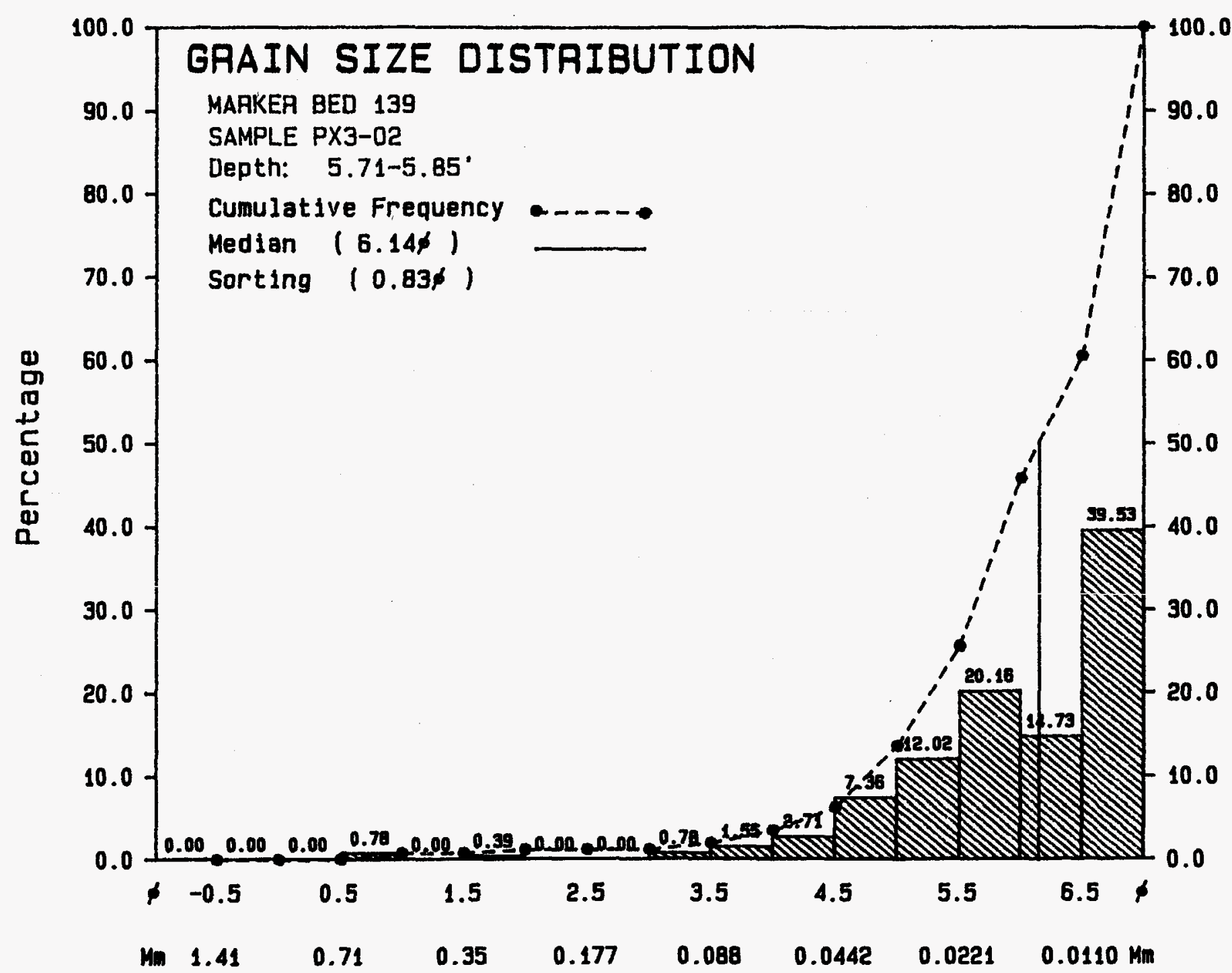


FIGURE 18C. GRAIN/CRYSTAL SIZE HISTOGRAM OF SAMPLE PX3-03.

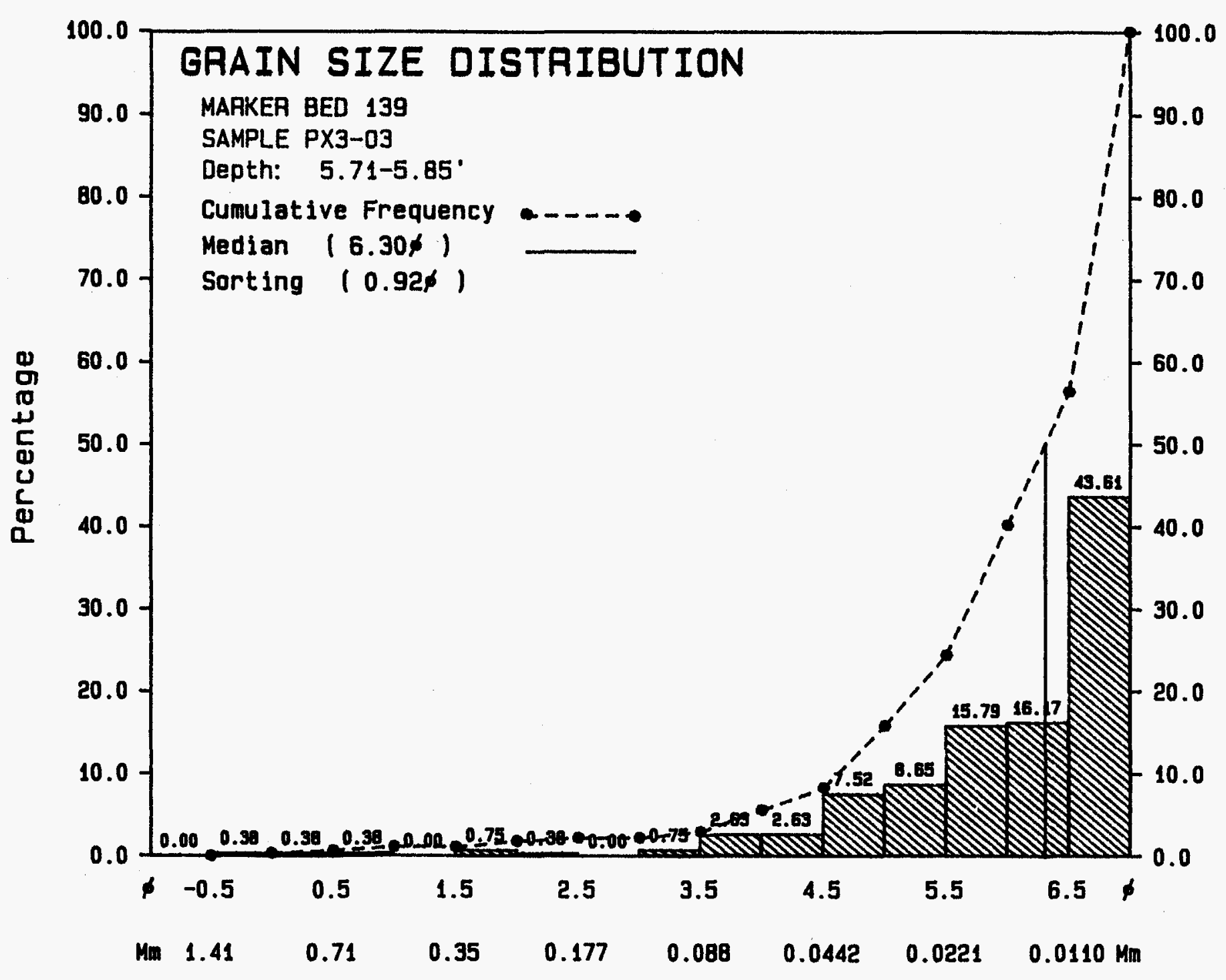




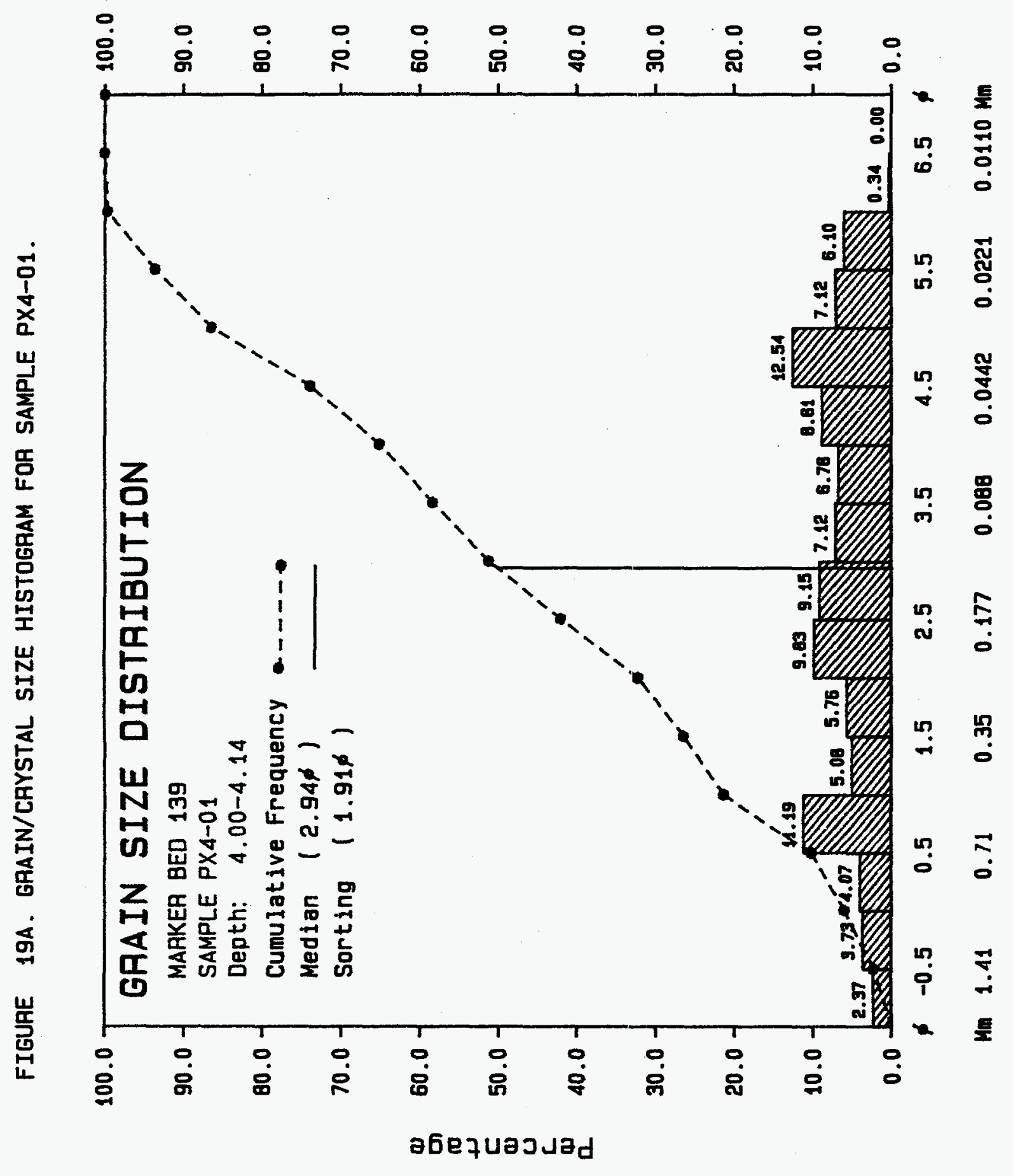


FIGURE 198. GRAIN/CAYSTAL SIZE HISTOGAAM FOR SAMPLE PX4-02.

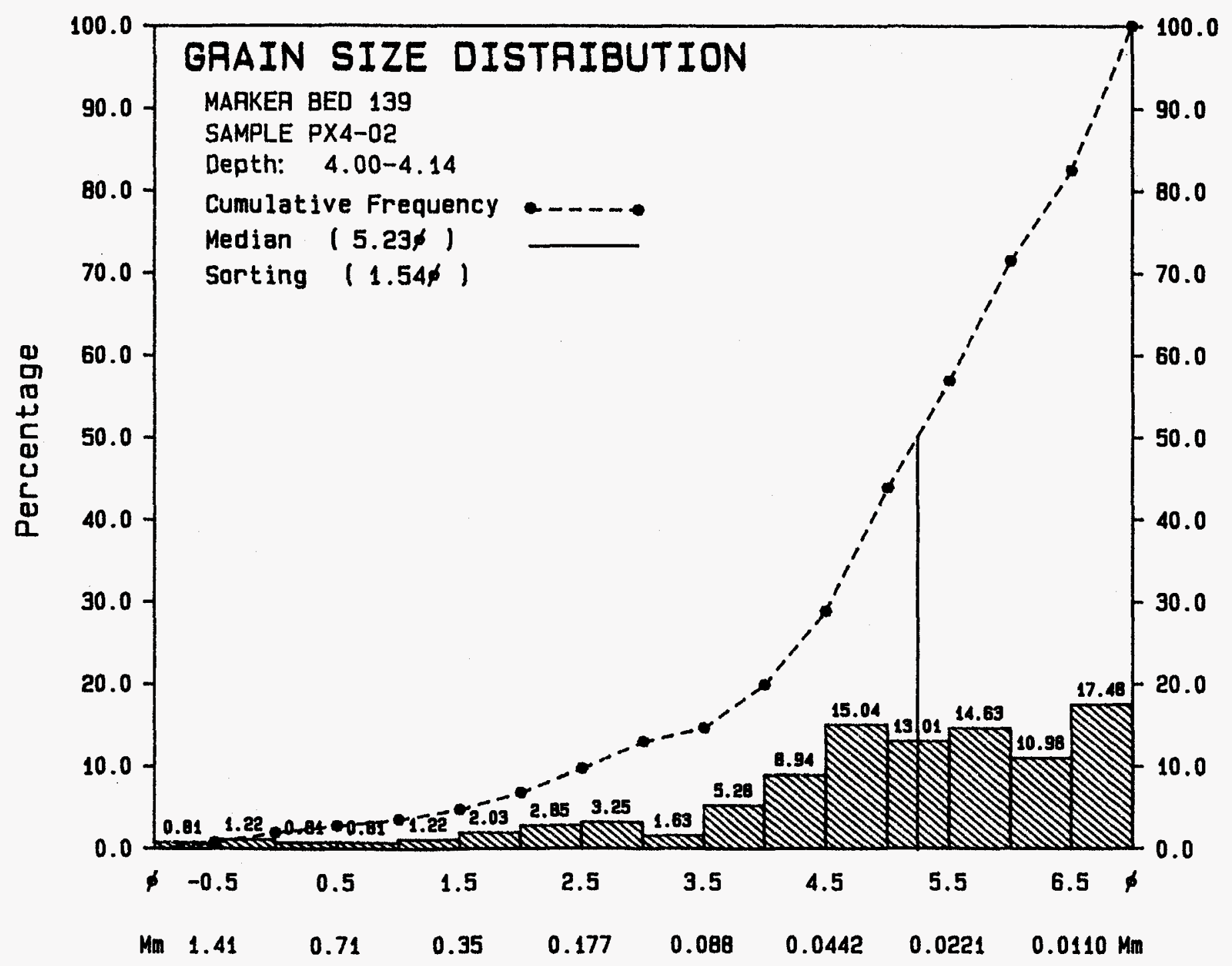


FIGURE 19C. GRAIN/CAYSTAL SIZE HISTOGRAM FOR SAMPLE PX4-03.

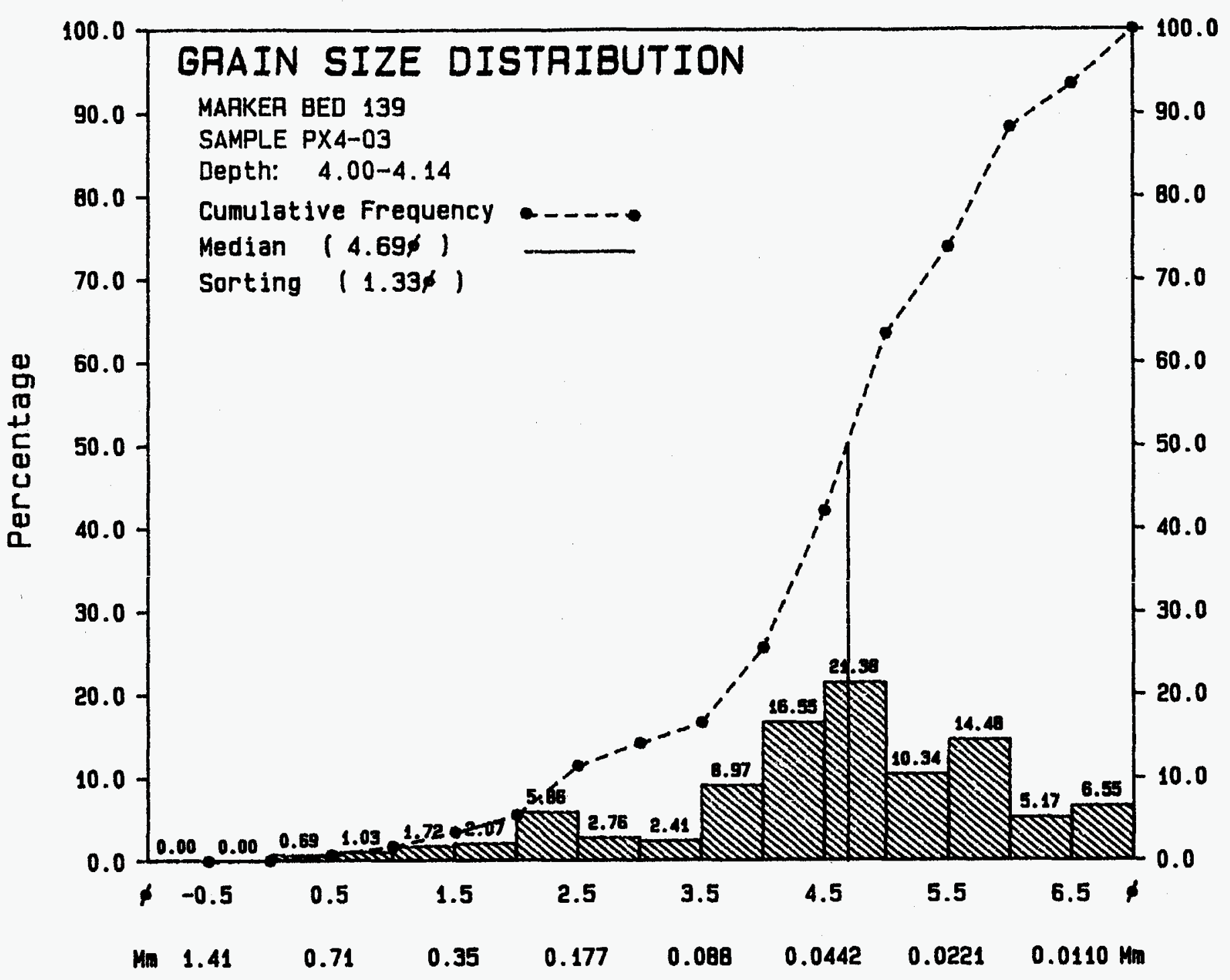


FIGUAE 20A. GRAIN/CAYSTAL SIZE HISTOGRAM FOR SAMPLE PX5-01.

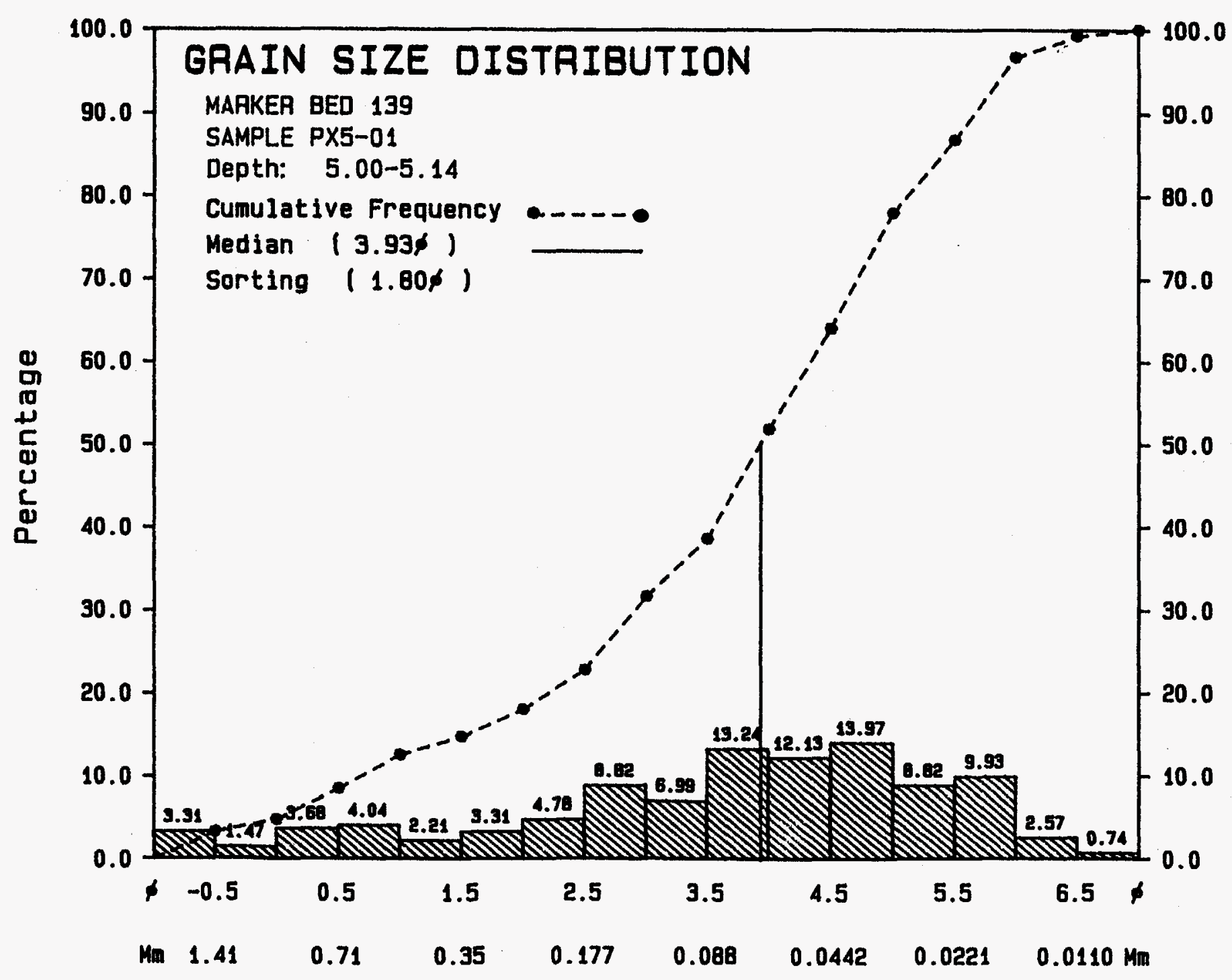


FIGUAE 20B. GRAIN/CAYSTAL SIZE HISTOGRAM FOR SAMPLE PX5-02.

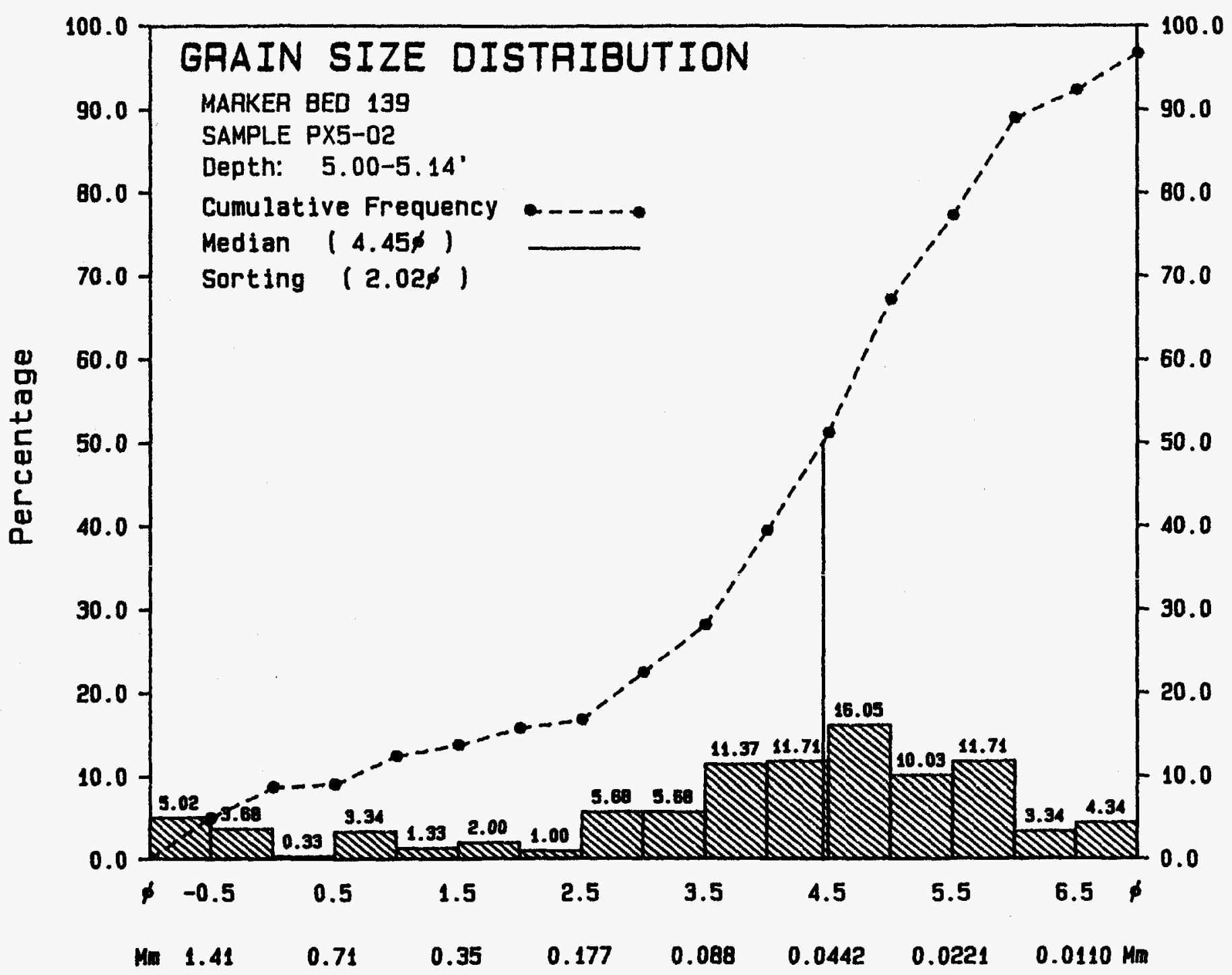


FIGUAE 20C. GRAIN/CAYSTAL SIZE HISTOGRAM FOR SAMPLE PX5-03.

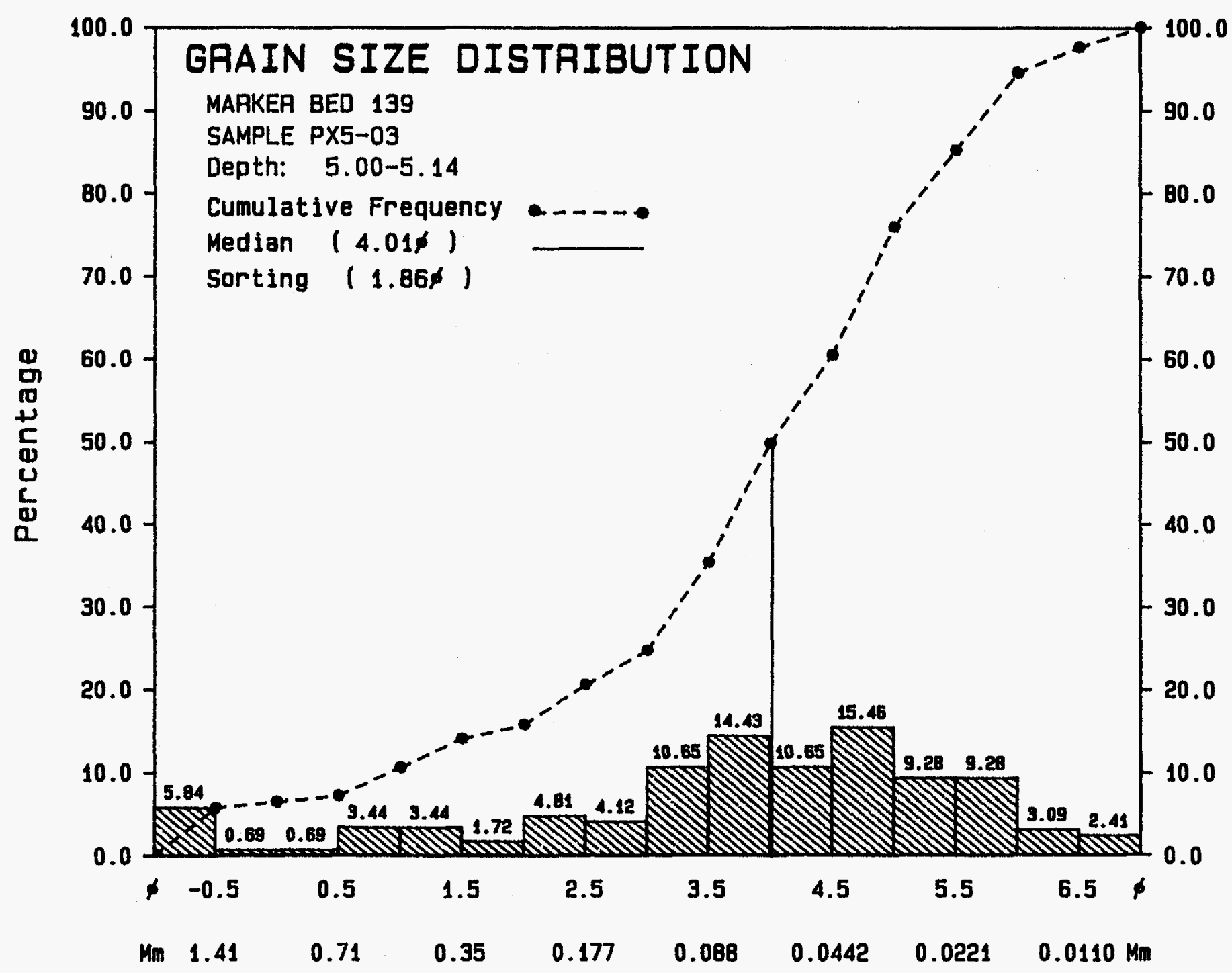


FIGUAE 21A. GRAIN/CRYSTAL SIZE hISTOGAAM FOR SAMPLE PX6-01.

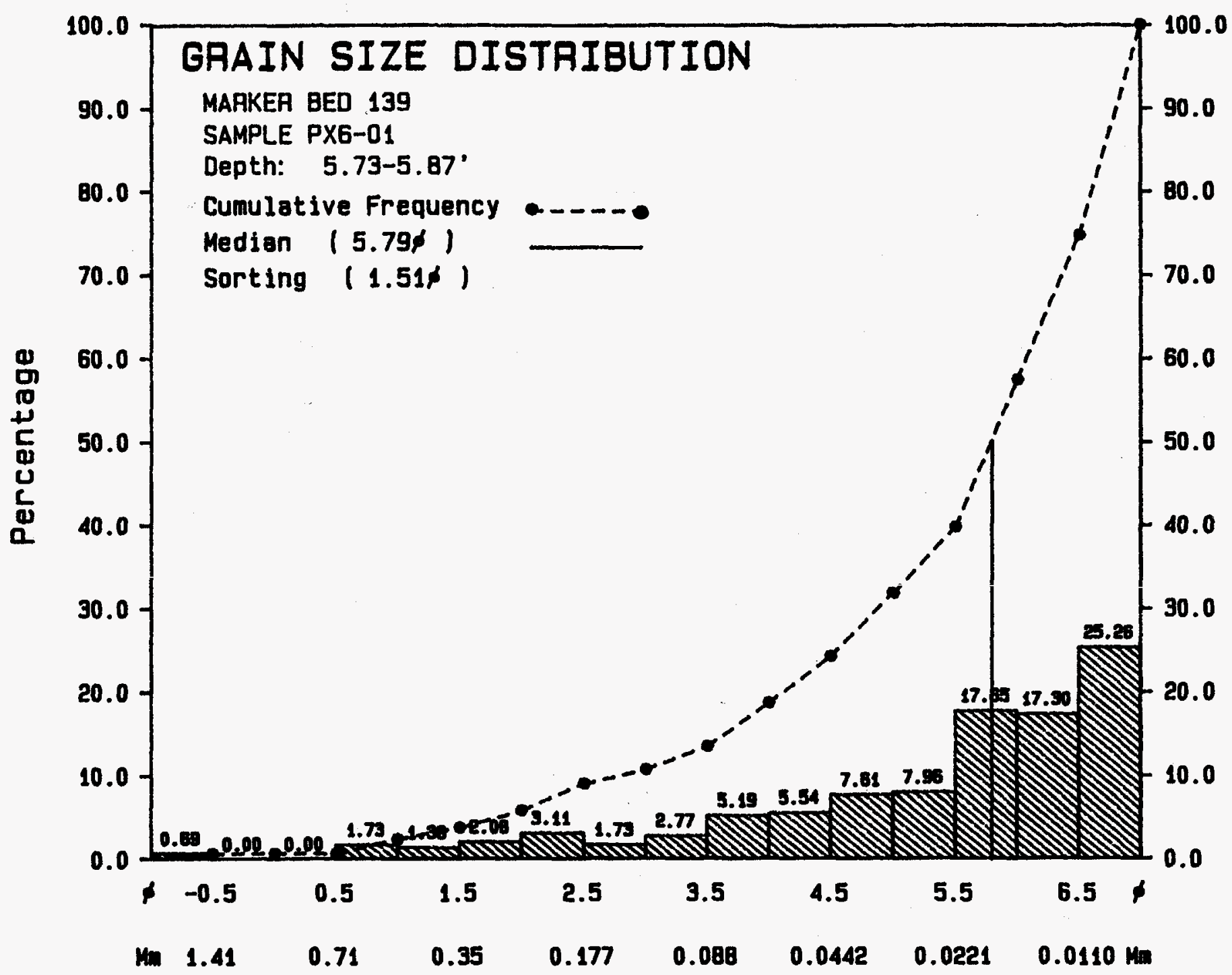


FIGURE 218. GRAIN/CRYSTAL SIZE hISTOGRAM FOR SAMPLE PX6-02.

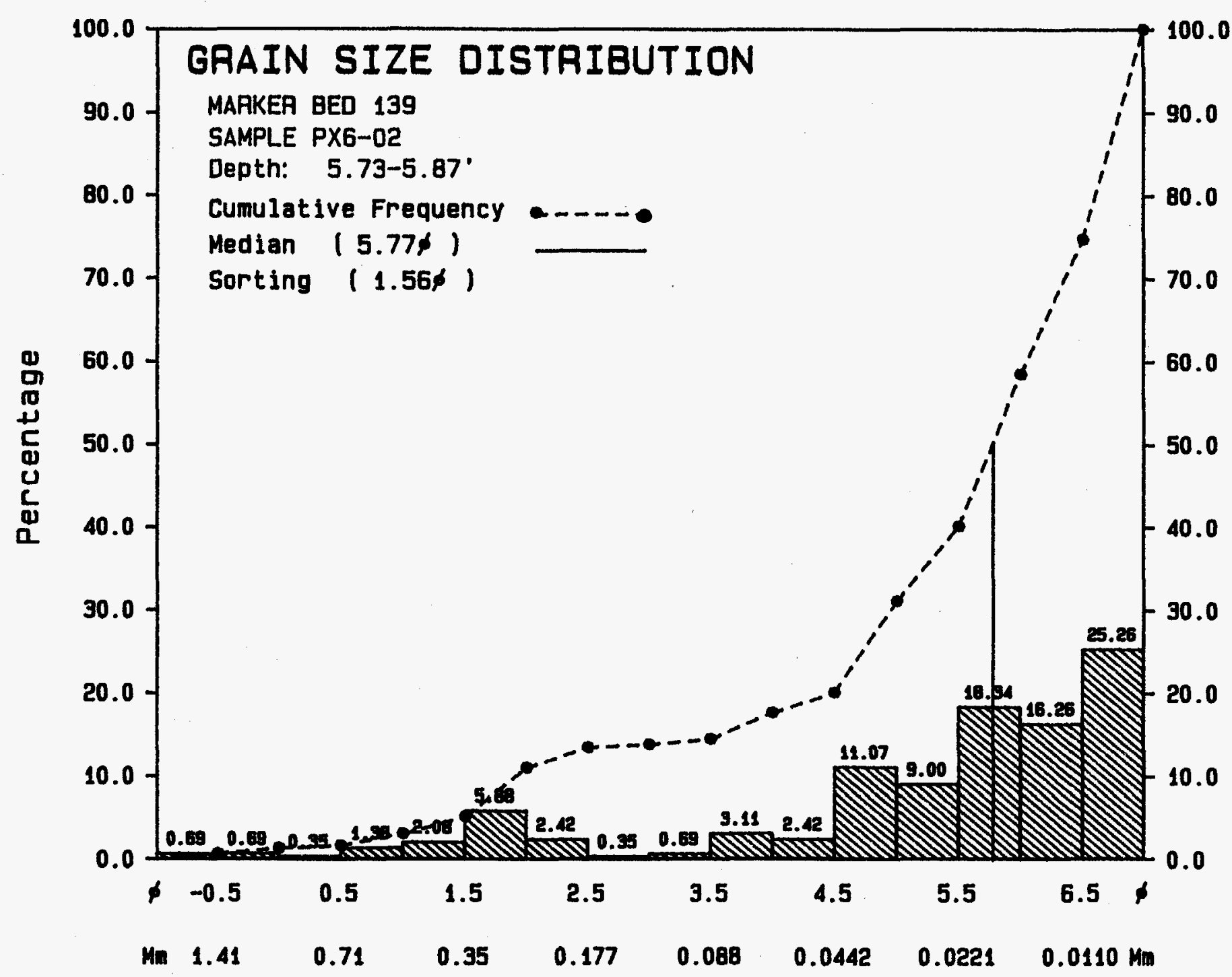


FIGUAE 21C. GRAIN/CAYSTAL SIZE HISTOGAAM FOR SAMPLE PX6-03.

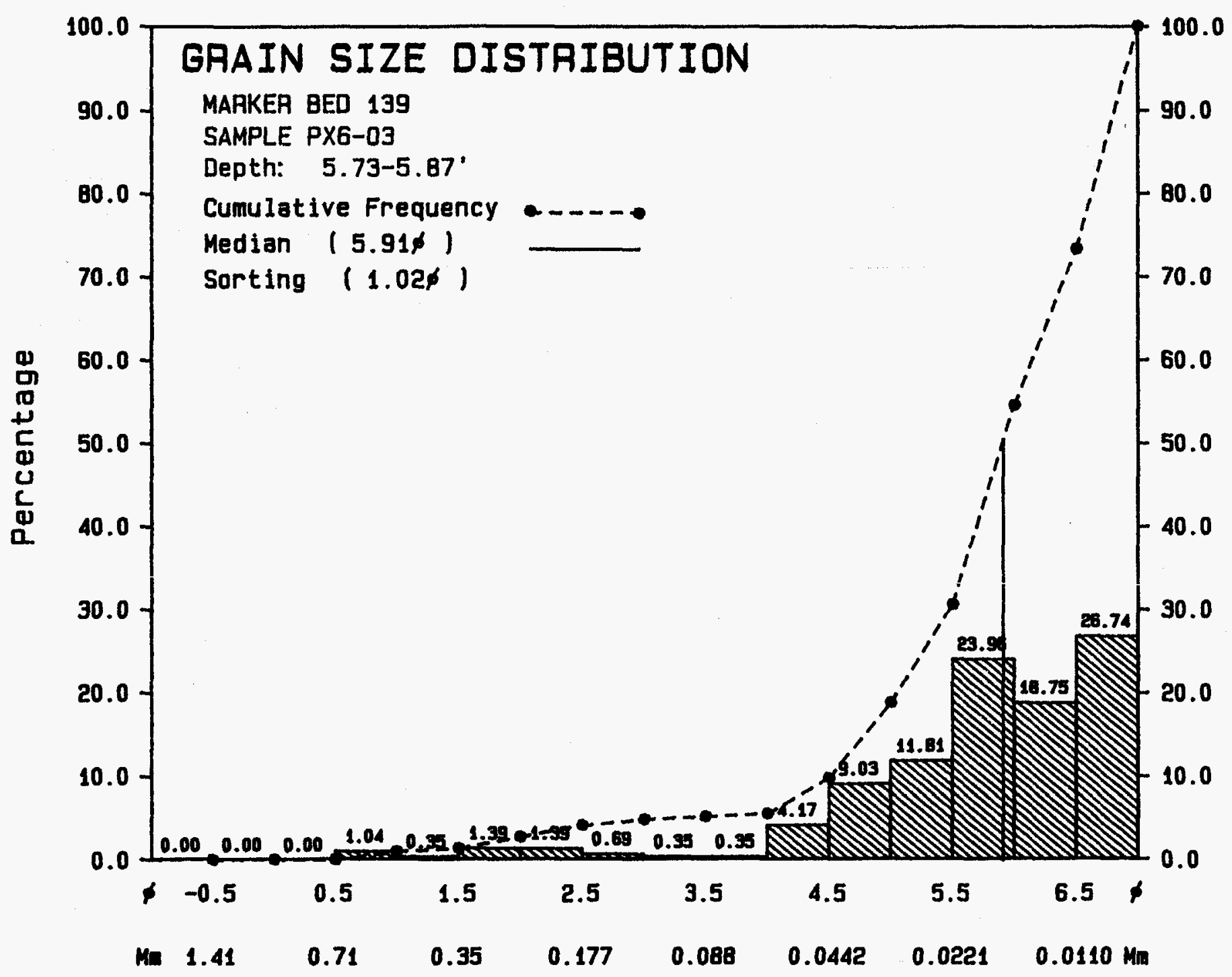




\section{Plate 1}

Sample PX1

A. General view. of thin section PX1-01 showing intergrown polyhalite spherulites. Each spherulite is composed of tiny, radiating, needle-like crystals. Elongate tabular crystals scattered throughout the view are anhydrite. Finely crystalline carbonate (dolomite?) is also present and appears as dark patches. Plane-polarized light. (40x)

B. Low magnification view of an open fracture in thin section PX1-O2. This fracture dips at approximately $45^{\circ}$ and extends across one corner of the thin section. Anhydrite is the dominant mineral in this portion of the sample. Minor halite (white patches) is also present. Plane-polarized light. (20x)

C. View of the interface between polyhalite and anhydrite in thin section PX1-O2. The upper portion of the photomicrograph is dominated by anhydrite whereas the lower portion contains more abundant polyhalite. Black patches represent halite (isotropic under crossed nicols). Finely crystalline carbonate (dolomite?) occurs in irregular brownish patches throughout the central portion of the view. Crossednicols. (20x)

D. Overview of thin section PX1-O3 showing abundant polyhalite. Minor amounts of finely crystalline carbonate (dolomite?) are also present. Iron staining (reddish brown color) is present in the lower portion of the view. Plane-polarized light. $(40 x)$ 
plate 1

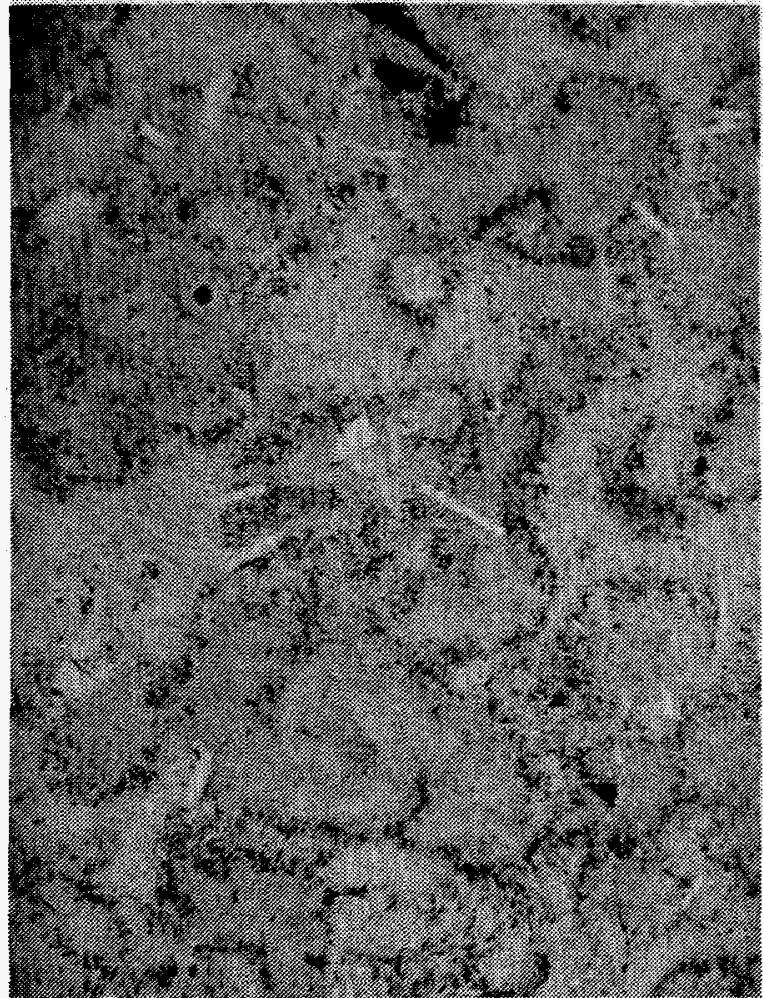

A

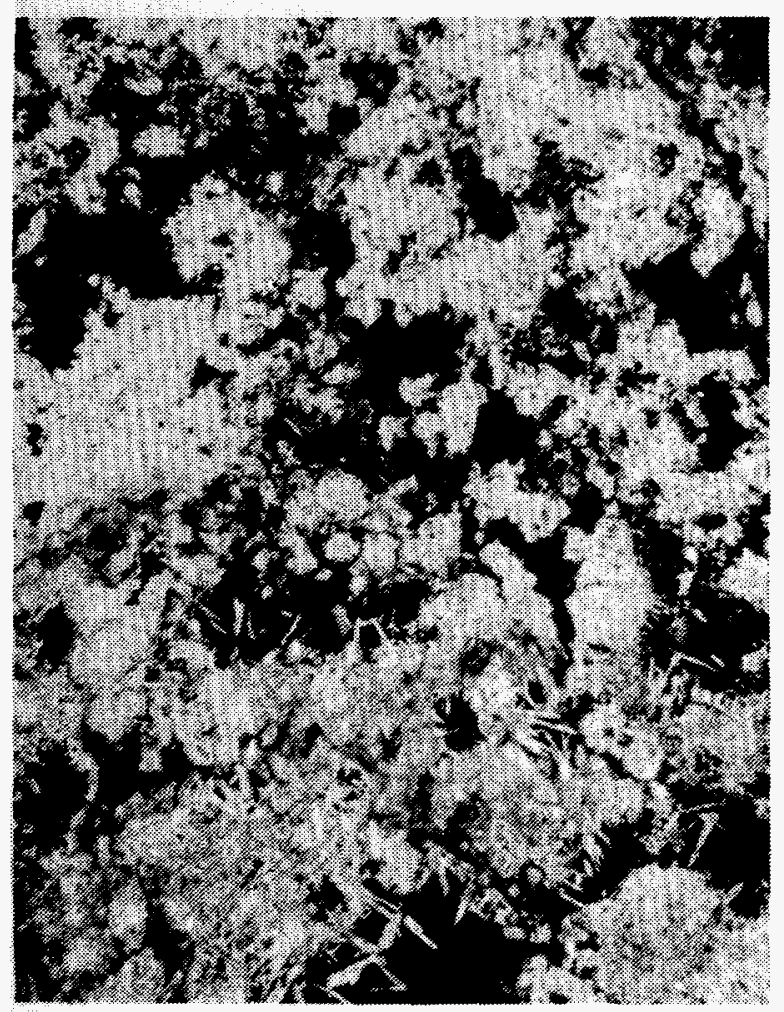

c

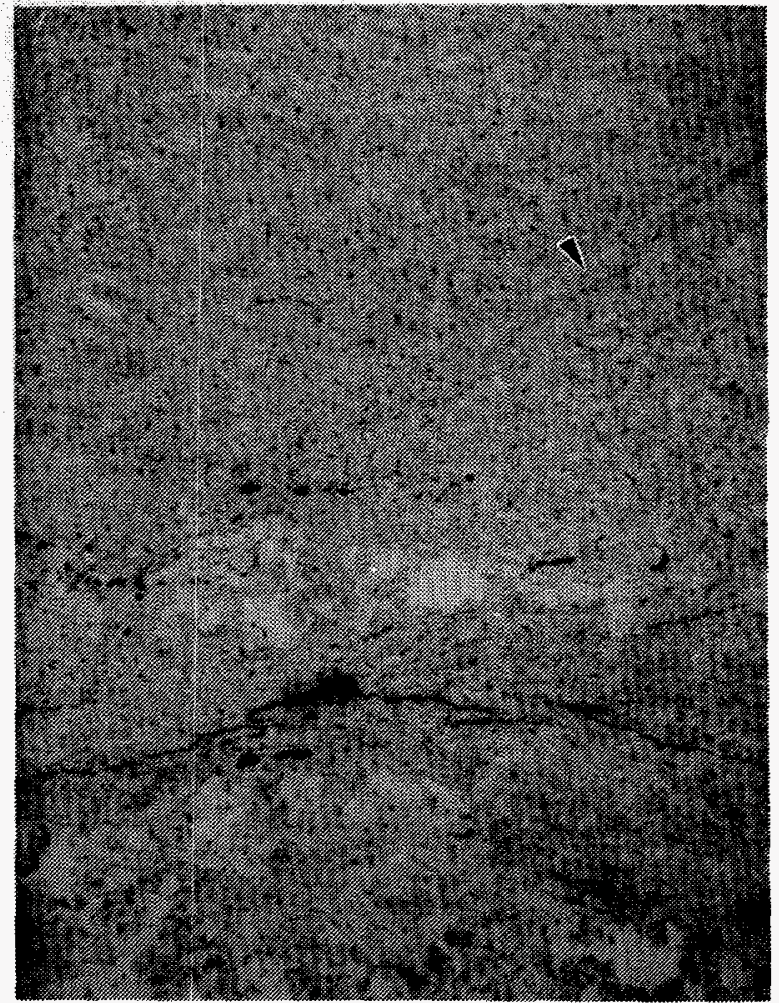

B

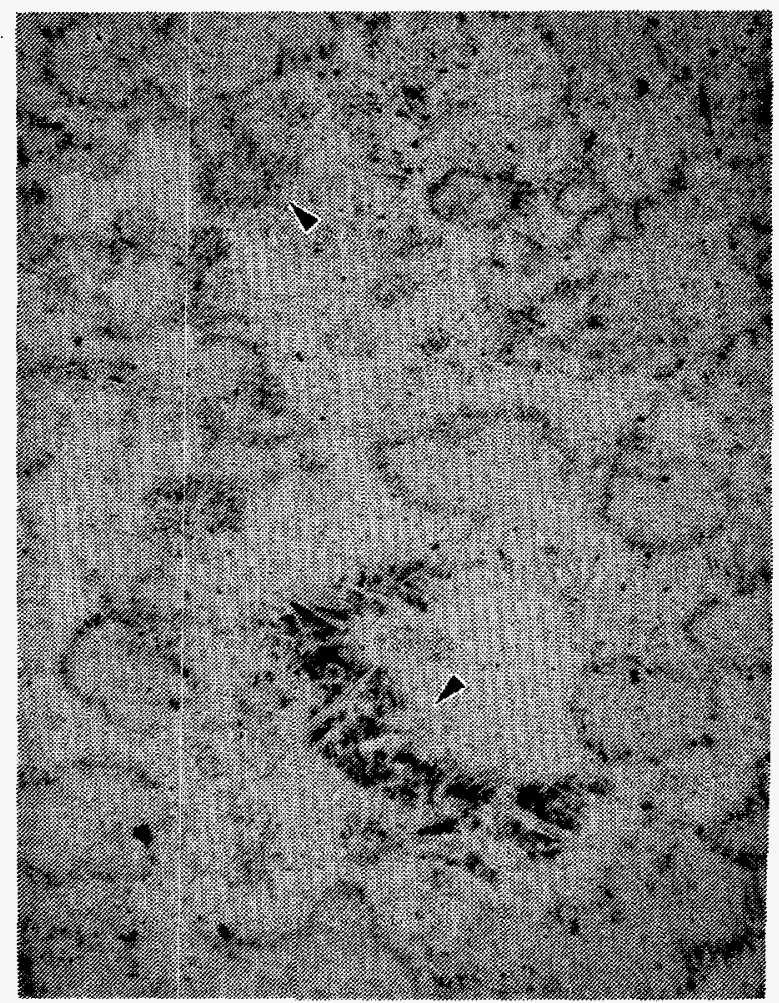

D 
Plate 2

Sample PX2

A. Low.magnification view of thin section PX2-01 showing large halite crystals (lower left) surrounded by more finely crystalline anhydrite. Minor carbonate (dolomite?) appears as dark brown patches within both halite and anhydrite. Many of the halite crystals in this sample are too large to photograph. Planepolarized light. (20x)

B. Same view as the previous photomicrograph more clearly showing halite distribution. Significant variation in anhydrite crystal size is also evident. Note high birefringence which is characteristic of anhydrite. Halite is isotropic and appears black. Crossed-nicols. (20x)

C. Low magnification view of thin section PX2-O2 showing large halite crystals with minor anhydrite around crystal edges and as inclusions. Halite crystals in this thin section are larger than in the other two thin sections from this sample. Crossednicols. (20x)

D. Overview of thin section PX2-03 showing fractures developed along cleavage planes in relatively large halite crystals. Note fluid inclusion trains along fractures, indicating earlier fractures which have healed. Plane-polarized light. (40x) 
Plate 2

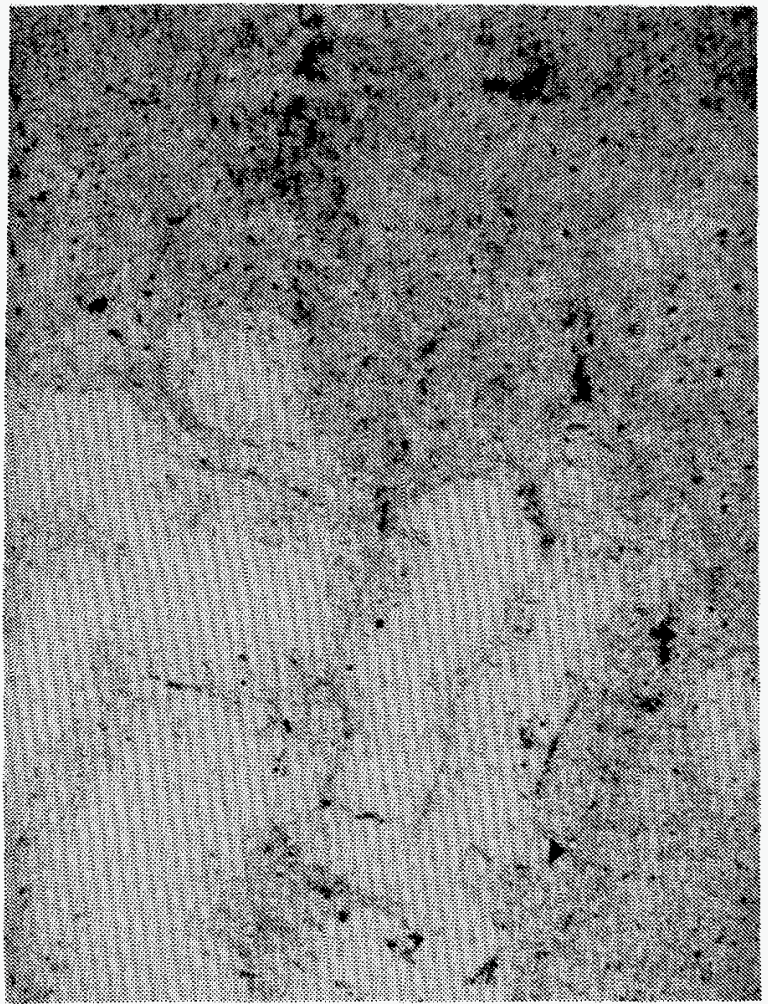

A

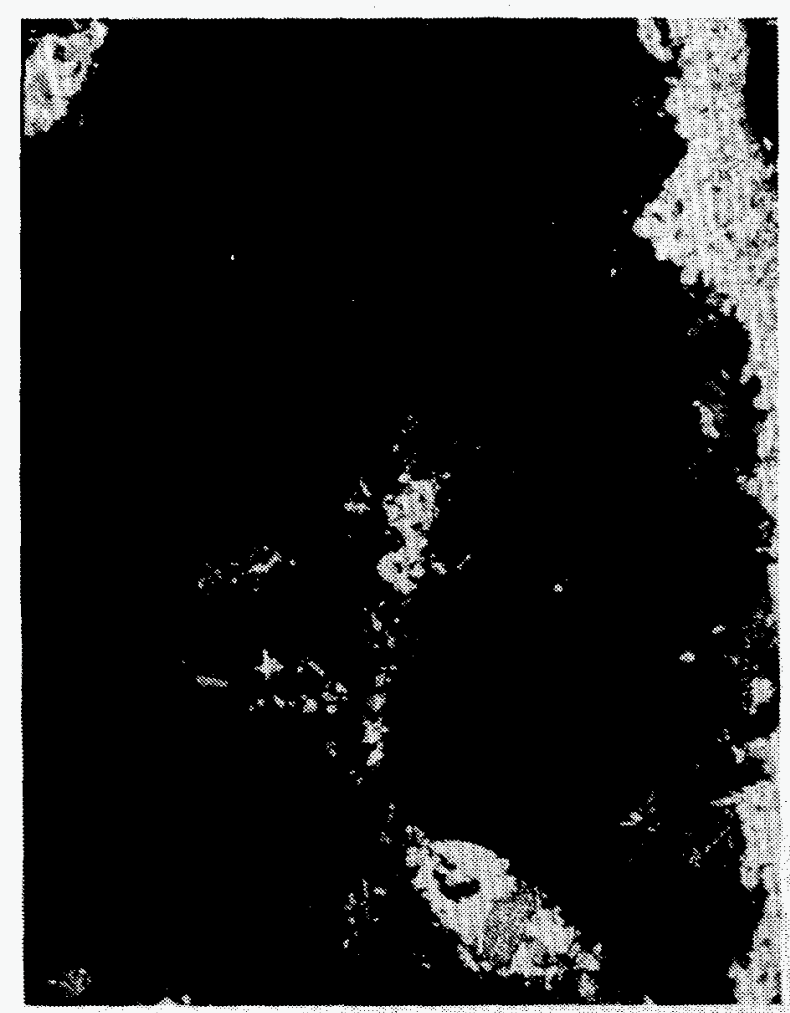

c

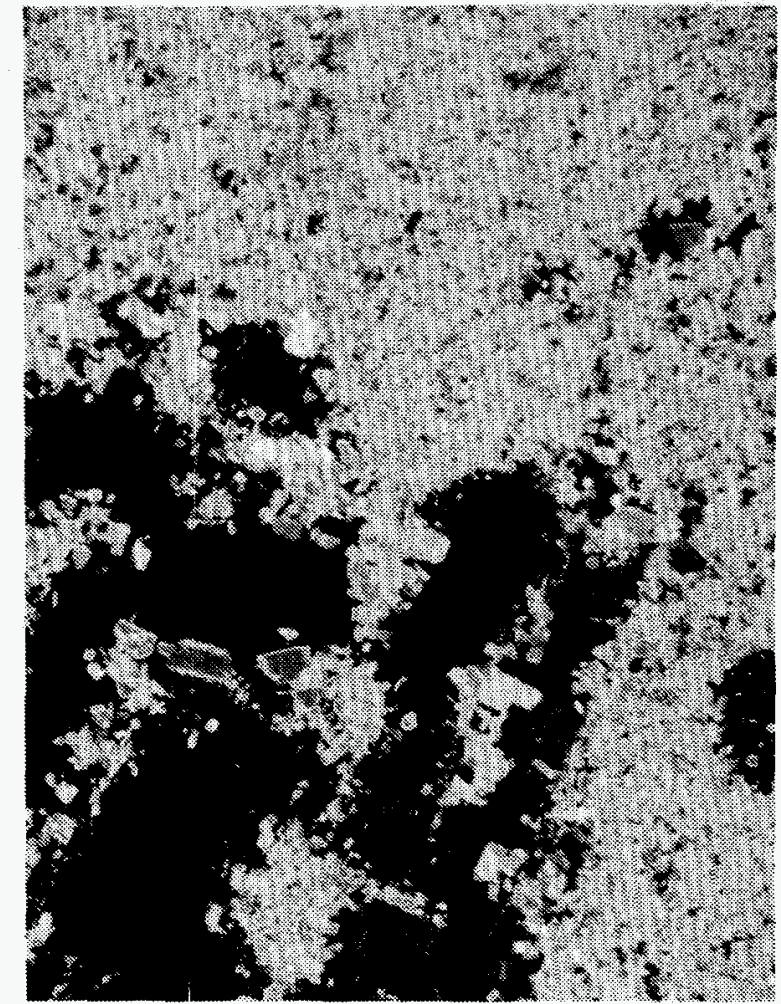

B

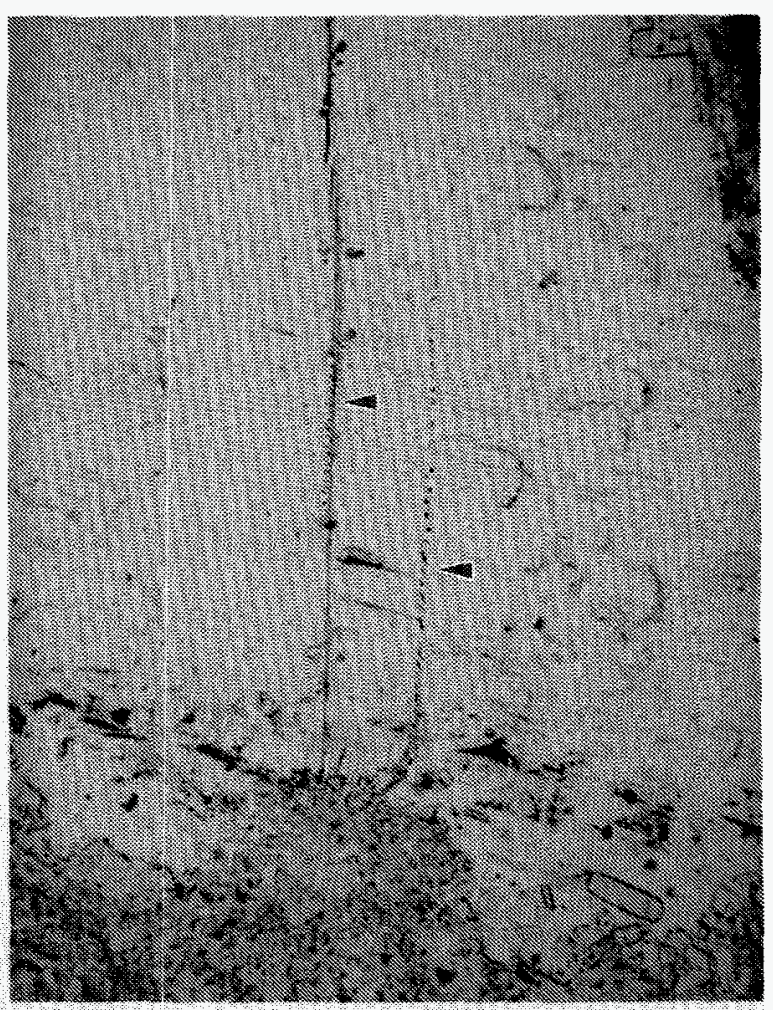

D 


\section{Plate 3}

Sample PX3

A. Overview of thin section PX3-01 showing low angle fractures in a matrix composed of anhydrite, carbonate (dolomite?), and halite. Plane-polarized light. $(40 x)$

B. Low magnification view of thin section PX3-01 showing the distribution of various mineral components. Halite appears black and forms nodules in the upper portion of the view. Anhydrite and carbonate (dolomite?) occur as inclusions in halite and comprise most of the matrix. Note variation in crystal size. Crossednicols. (20x)

C. Overview of thin section PX3-O2 showing interlaminated halite, anhydrite, and carbonate (dolomite?). Halite appears black and occurs in much larger crystals than either anhydrite or dolomite. Crossed-nicols. (40x)

D. Low magnification view of thin section PX3-O3 showing irregular anhydrite (white) and carbonate (dolomite?) (dark) laminae. The fracture running from left to right through the central portion of the view is generally parallel to laminations. Plane-polarized light. (20x) 
Pate 3

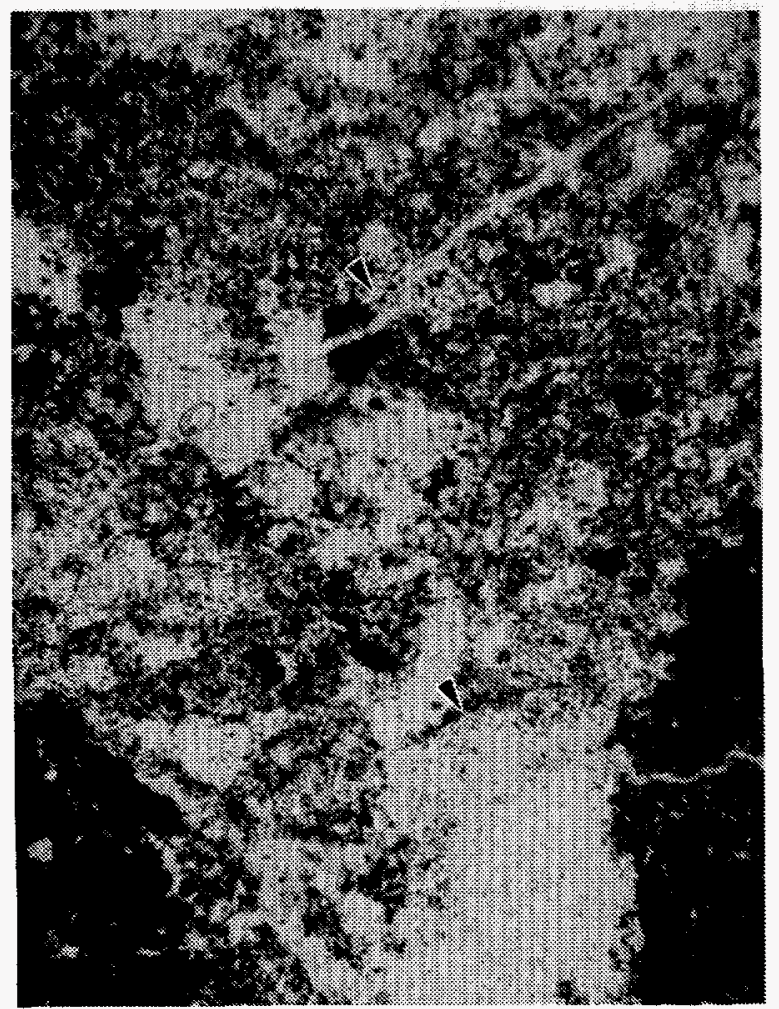

A

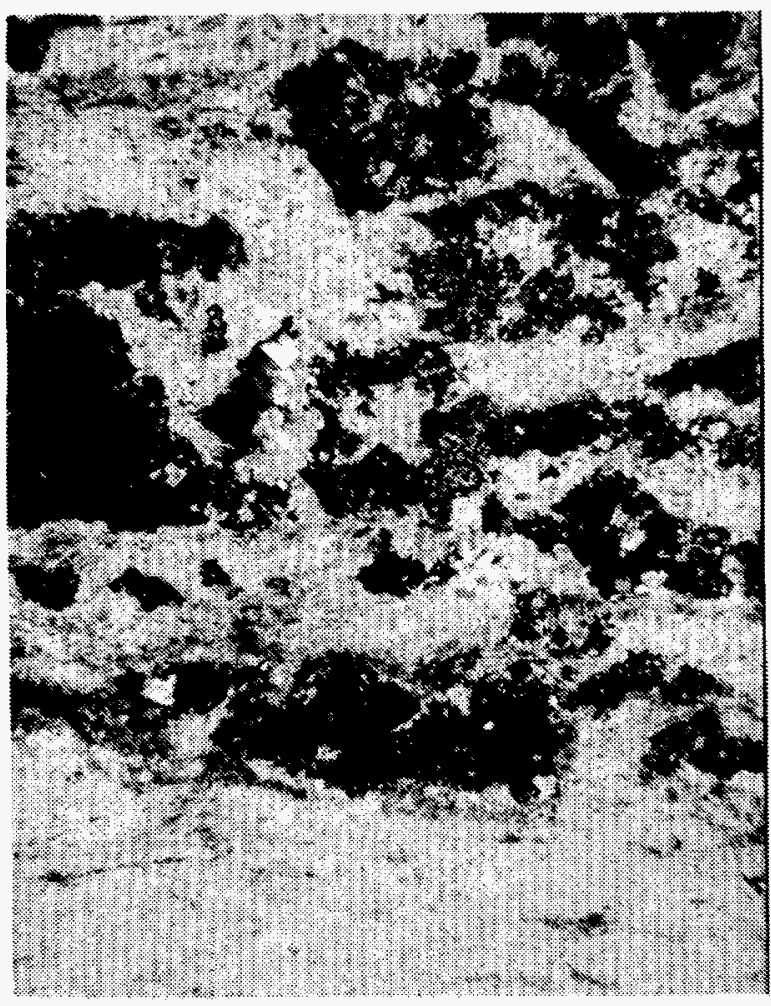

C

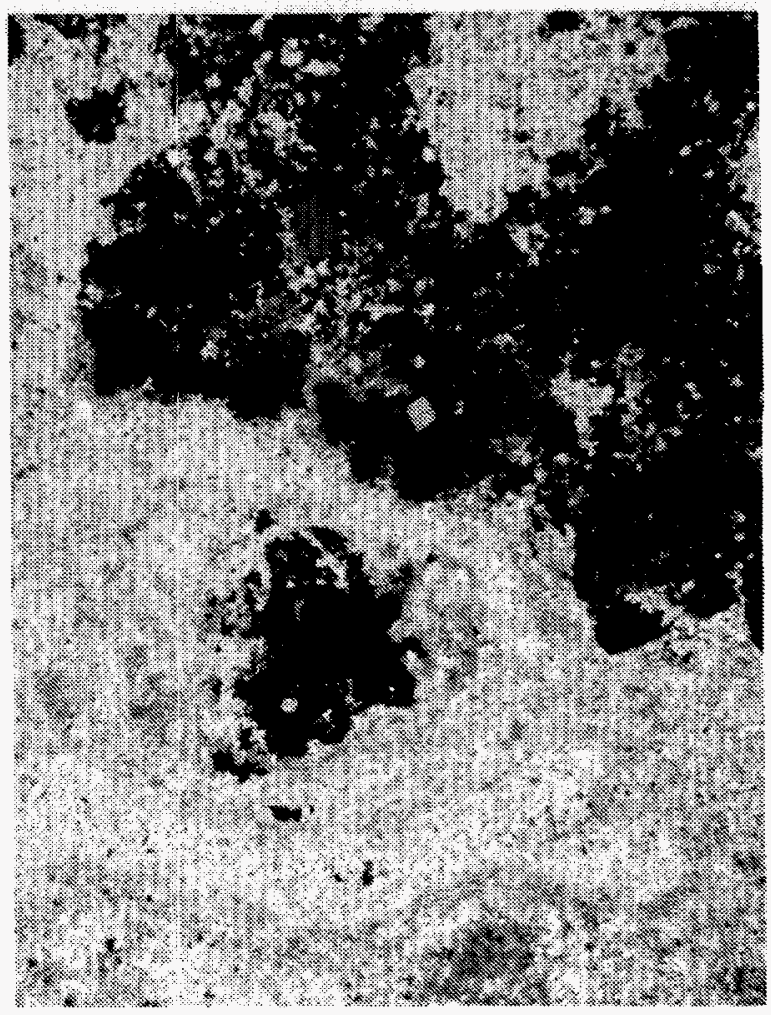

B

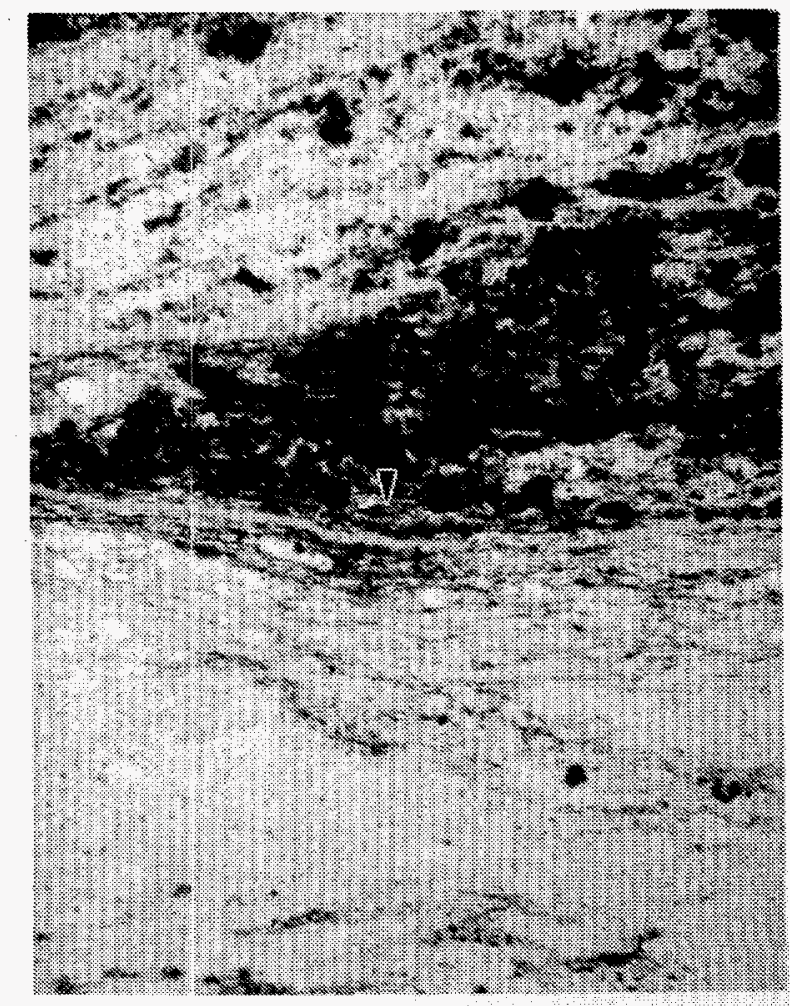

D 


\section{Plate 4 \\ Sample PX4}

A. Low magnification view of thin section PX4-01 showing intergrown halite, polyhalite, and anhydrite. Halite appears white under plane light. Polyhalite along with anhydrite forms relatively large radiating structures. Anhydrite also dominates the matrix between halite and polyhalite crystals. Plane-polarized light. (20x)

B. More detailed view of thin section PX4-O1. Isotropic halite appears black. Anhydrite occurs as small equant crystals around halite nodules and as elongate needles intergrown with polyhalite. Central portions of the cross-like structures are composed primarily of polyhalite. Crossed-nicols. (40x)

C. Overview of thin section PX4-O2 showing abundant fibrous polyhalite. Small halite patches (black) are also present on the left side of the view. Minor anhydrite is also present. This thin section is dominated by polyhalite. Crossednicols. (40x)

D. View of intergrown halite, polyhalite, and anhydrite characteristic of thin section PX4-03. Anhydrite appears in shades of red, yellow, and blue. Note crystal size variation in anhydrite. Polyhalite occurs as needle-like crystals in the central portions of radiating structures. Halite (black) fills space between the other minerals. Crossed-nicols. (40x) 
Mate 4

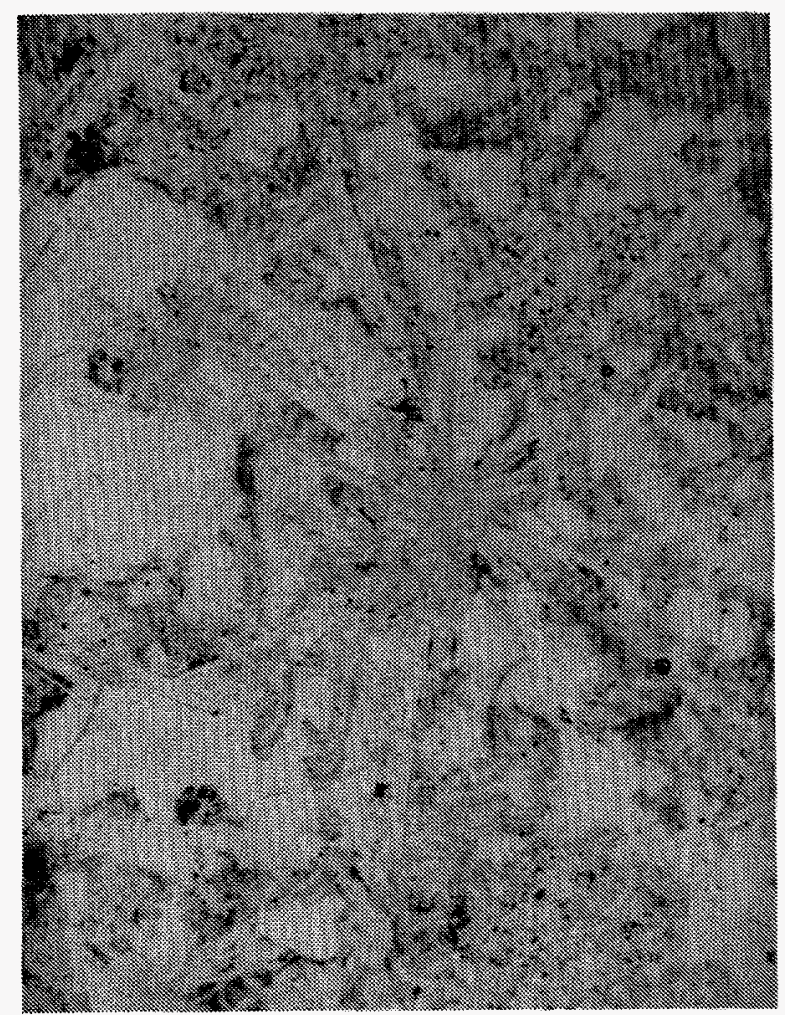

A

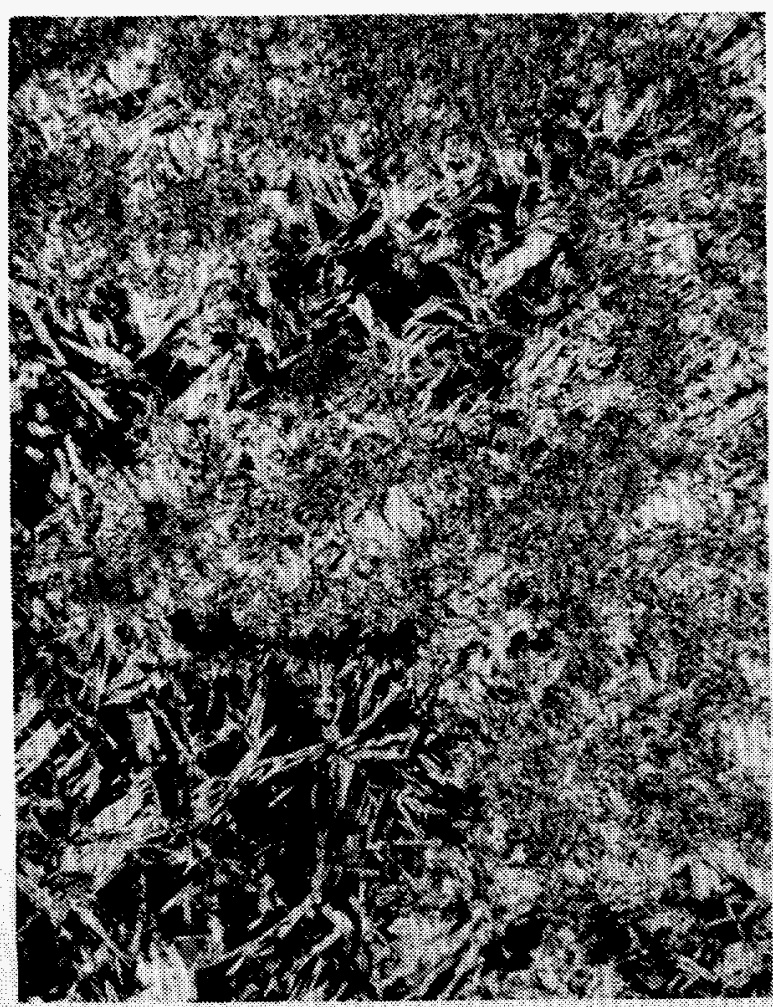

c

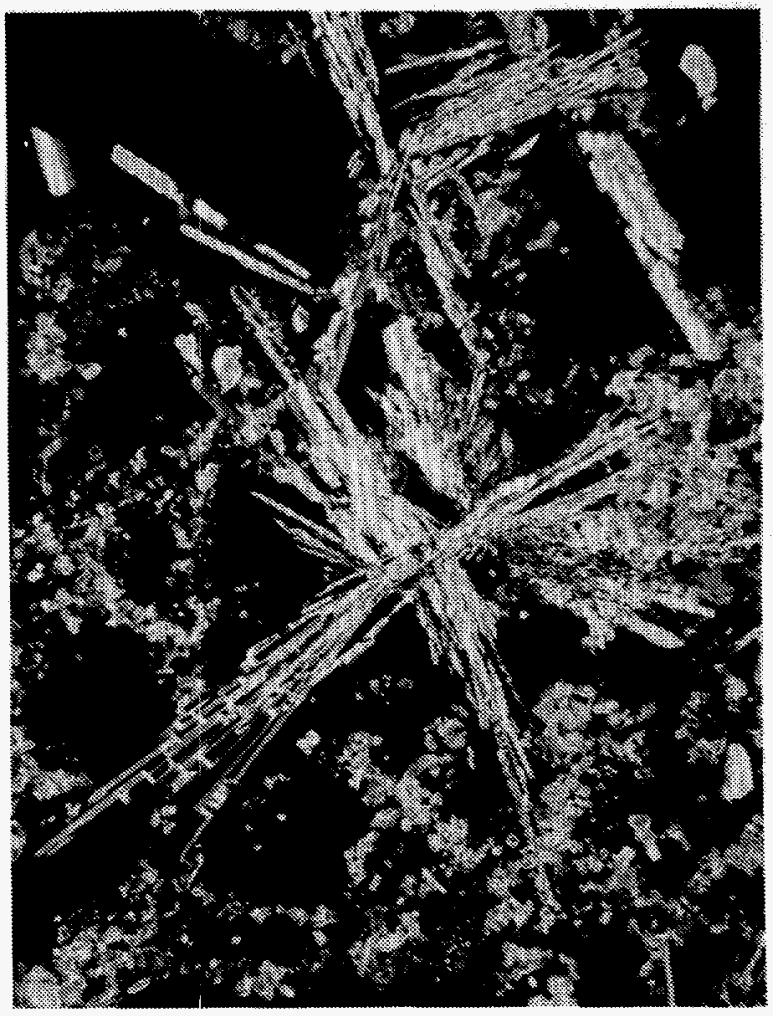

B

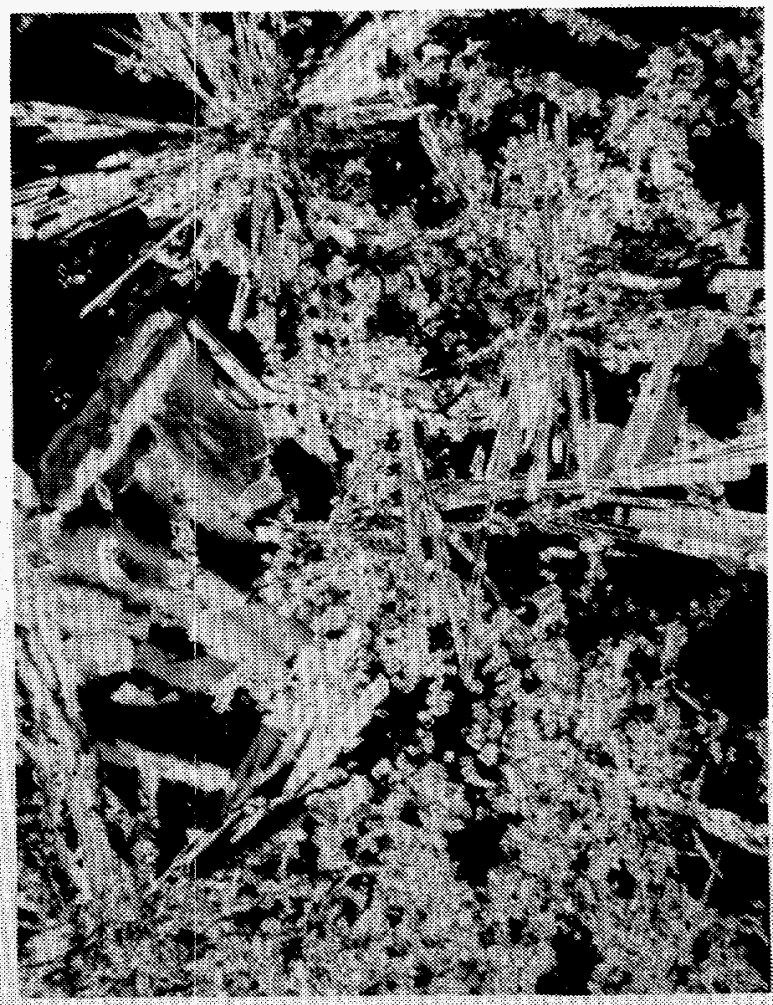

D 


\section{Plate 5}

Sample PX5

A. Low magnification view of relatively coarsely crystalline anhydrite with minor halite. Anhydrite in this sample appears coarser than in the overlying or underlying sample. Minor carbonate (dolomite?) appears dark in the lower left. Crossed-nicols. (20x)

B. Low magnification view of high-angle fractures in thin section PX5-O2. Fractures are near vertical and commonly terminate at the interface between different minerals. Anhydrite and halite are the dominant mineral constituents. Minor carbonate (dolomite?), which appears as small dark patches, is scattered throughout. Plane-polarized light. (20x)

C. Overview showing the distribution of mineral components in thin section PX5-O3. Halite and anhydrite dominate the upper portion of the view. Many anhydrite crystals appear tabular in this view. Halite fills space between anhydrite crystals. Finely crystalline carbonate, probably dolomite, appears dark and is most abundant in the lower portion of the view. Plane-polarized light. (40x)

D. Same view as the previous photomicrograph showing mineral distribution. Crossed-nicols. (40x) 
Waxe 5

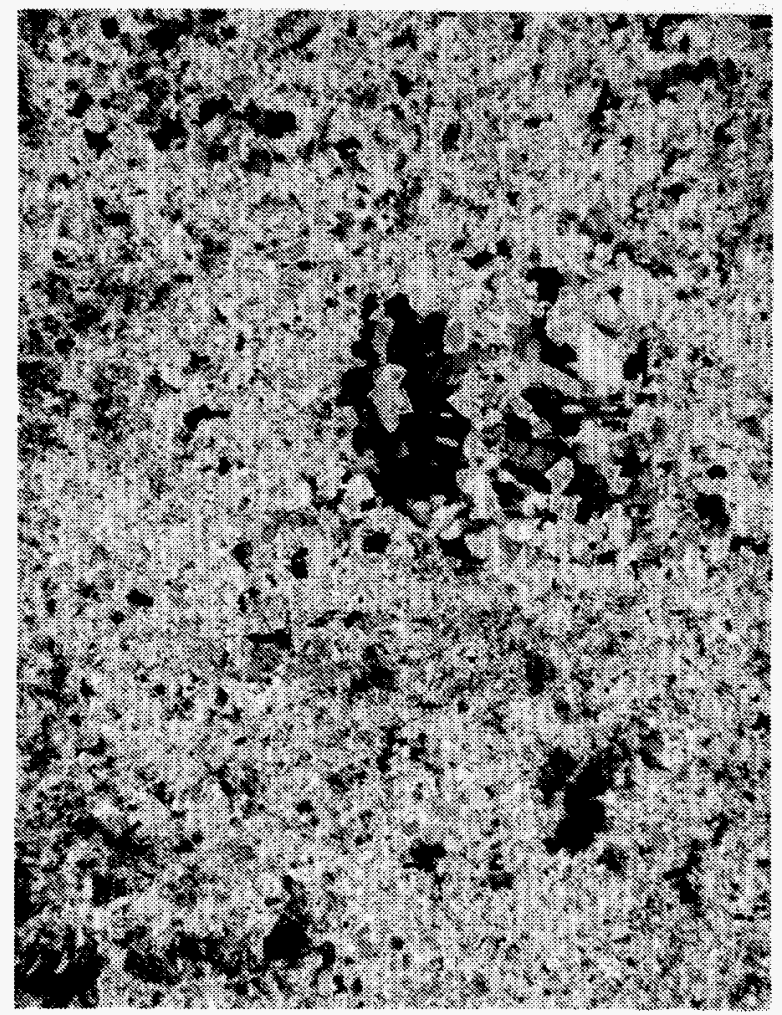

A

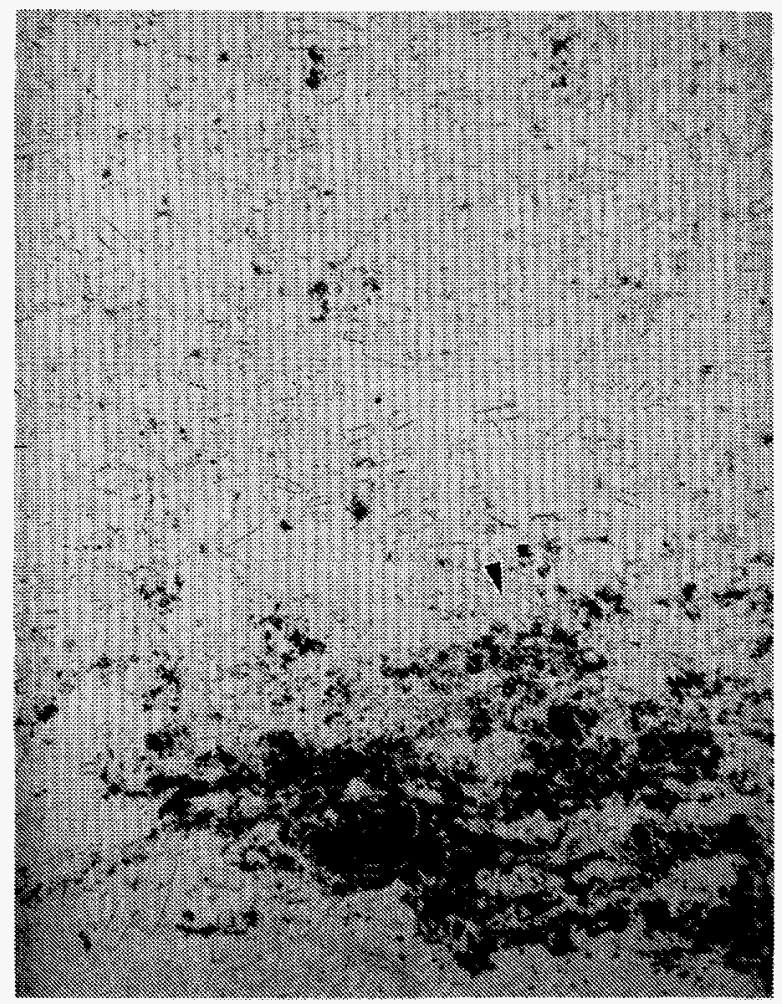

c

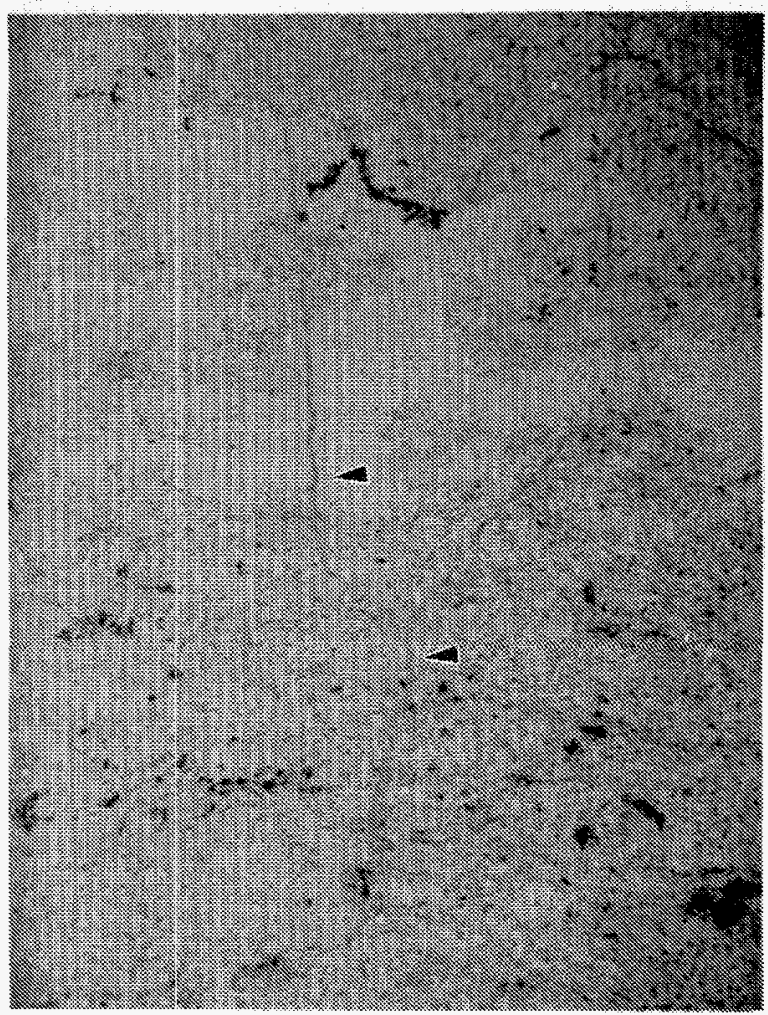

$\mathbf{B}$

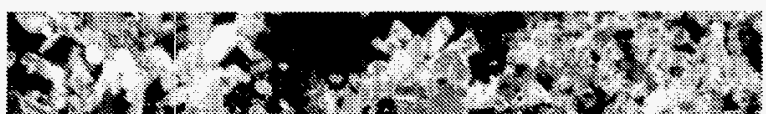

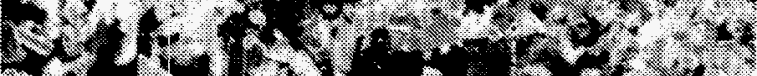

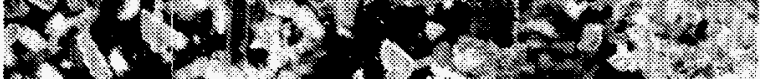

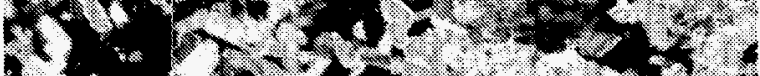

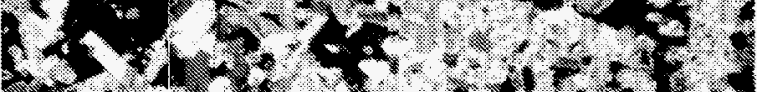

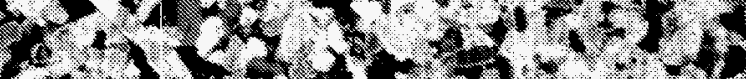

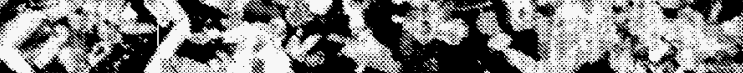

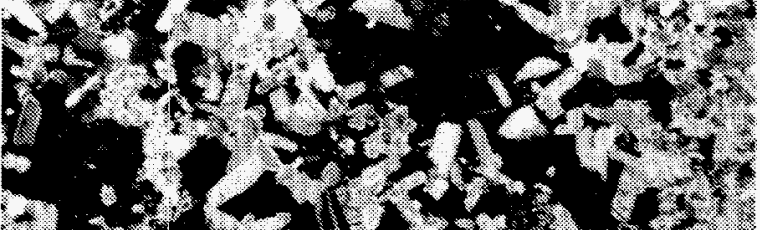

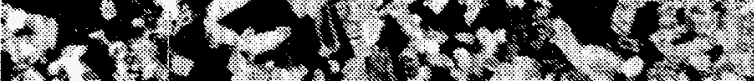

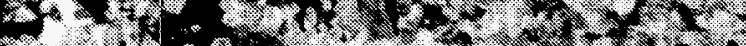

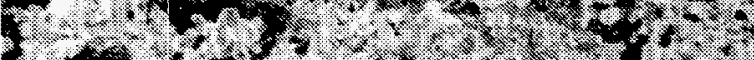

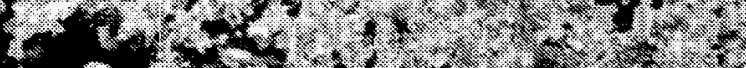

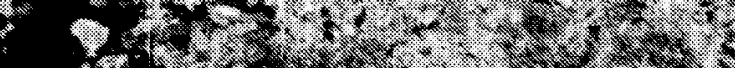

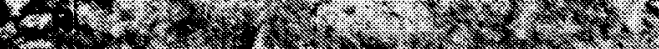

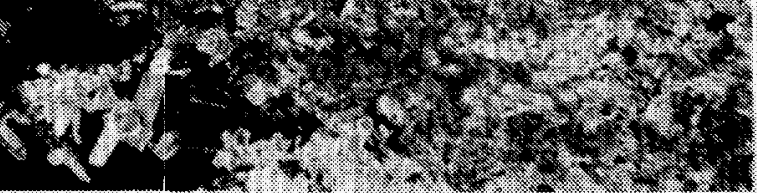
D 


\section{Plate 6}

Sample PX6

A. Low magnification view of thin section PX6-O1. A narrow high-angle fracture (arrow) in the upper portion of the view terminates at a thin carbonate lamination. Intergrown anhydrite and halite dominate the matrix. Minor pyrite (black patches) is seen in the carbonate lamination in the upper portion of the view. Planepolarized light. (20x)

B. Low magnification view showing a near vertical fracture in thin section PX6-O2. A fracture oriented subparallel to bedding is also seen in the upper portion of the view. Some laminae in this sample are dominated by anhydrite, whereas others contain both anhydrite and halite. Crossed-nicols. (20x)

C. Overview of a lamination containing both halite and anhydrite in thin section PX6-02. Anhydrite around halite crystals appears more coarsely crystalline than in the matrix away from halite nodules. Crossed-nicols. (40x)

D. Overview of a healed fracture (arrow) in thin section PX6-03. This fracture is primarily filled by anhydrite, but a very narrow aperture is present in the center. Anhydrite dominates the matrix. This fracture also appears parallel to narrow open fractures in other portions of the thin section. Plane-polarized light. (40x) 
Plate 6

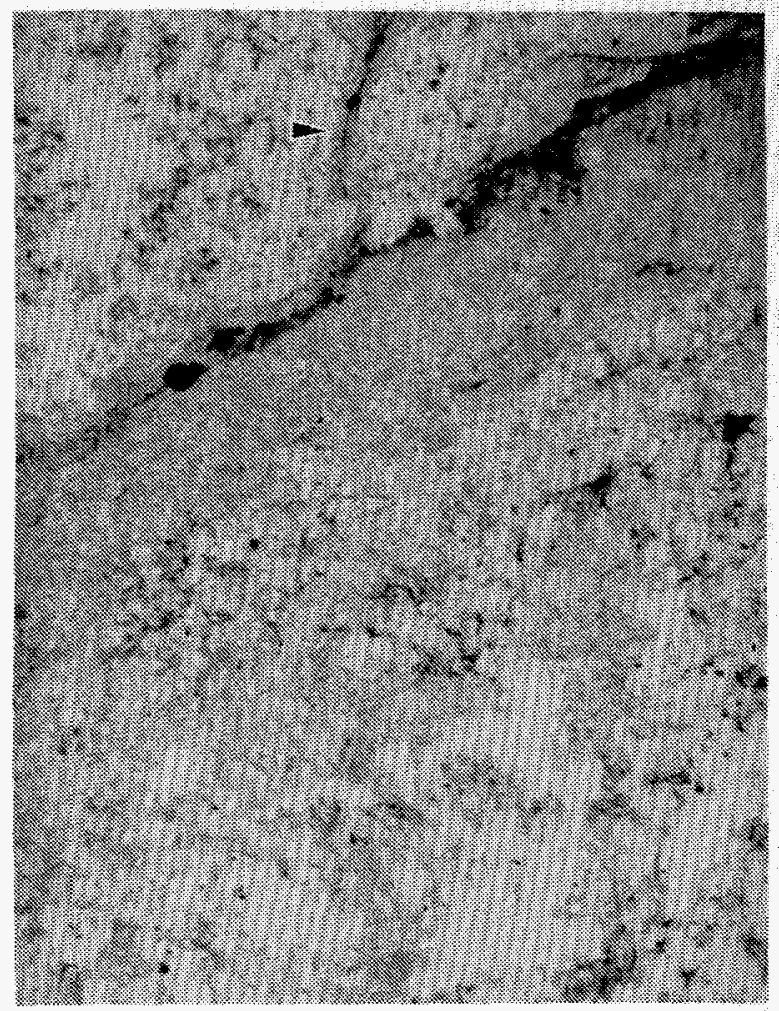

A

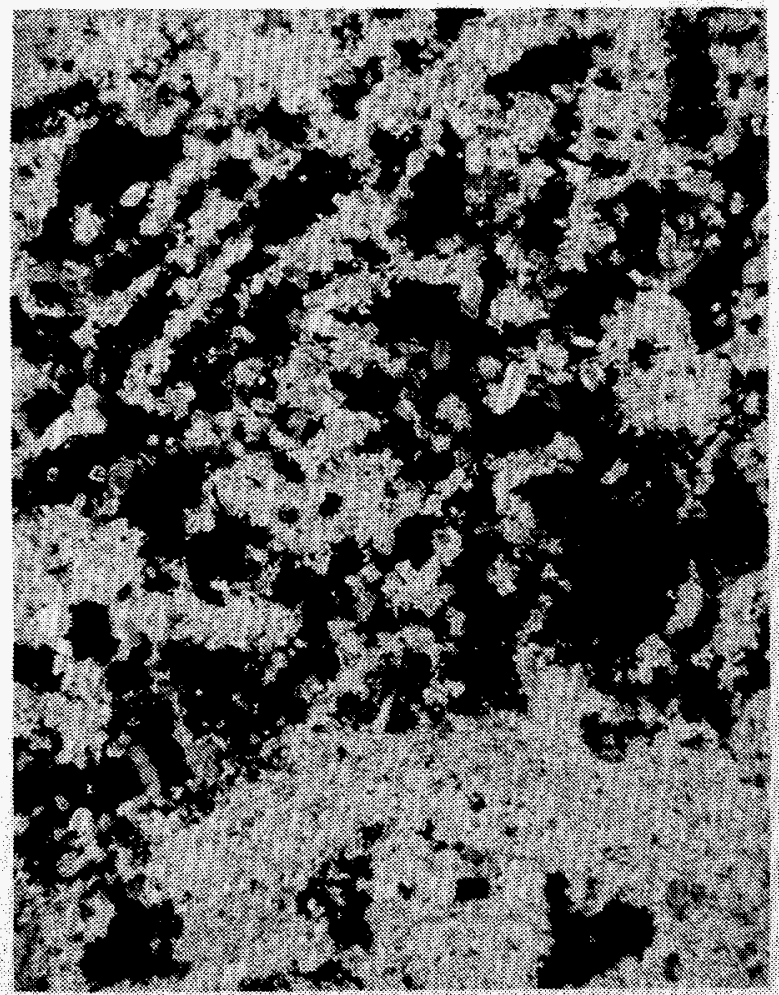

c

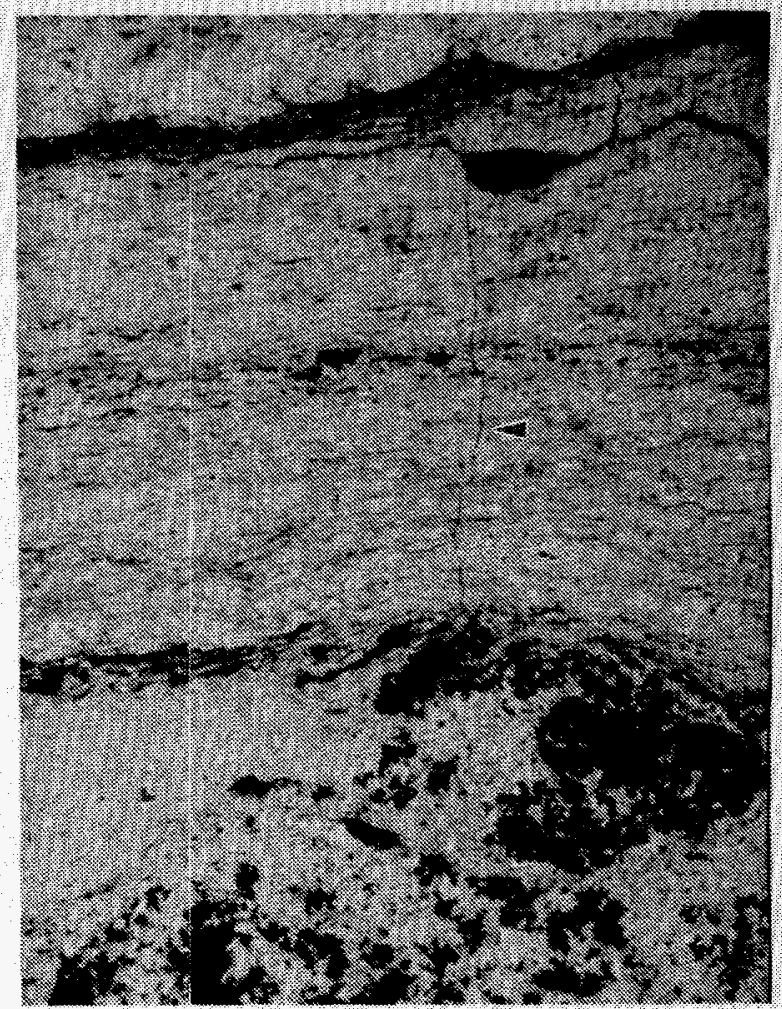

B

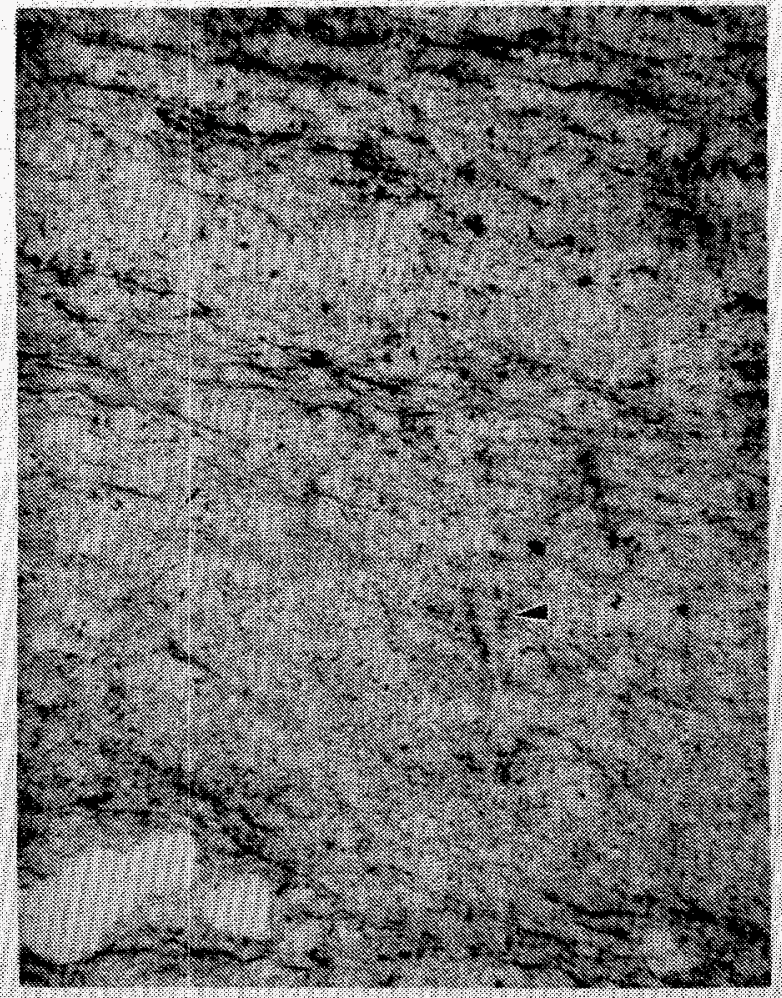

D 


\subsection{Effective Porosity, Total Porosity, and Saturation}

The bulk volumes $V_{b}$ of the intact samples (A-F and EP1-8) were determined from the volume of fluid displaced when the samples were immersed in water (see Appendix C-A).Samples were first coated with wax to prevent the water from penetrating the sample and the data were corrected for the amount of fluid volume displaced by the wax. (Note that for several samples (A-C, F, and EP4-8) which were later saturated with Odorless Mineral Spirits (OMS), $V_{b}$ was remeasured by directly submerging the saturated sample in OMS and measuring the mass of fluid displaced. These measurements are believed to be more accurate than the waxed measurements (since they do not require a correction for the amount of fluid displaced by the wax); however, these data are not used in subsequent calculations since they were not available for all samples. We simply make note of the fact that the two sets of measurements for the seven samples all agreed to within $0.30 \%$.)

Boyle's law helium gas porosimetry was used to determine the effective grain volume $V_{g \text { eff }}$ of the intact samples A-F and EP1-EP8 (Appendix C-A). Data are reported in Table 6, where the effective (i.e. interconnected) porosity $\phi_{\text {eff }}$ is defined by

$$
\phi_{\text {eff }}=1-\frac{V_{\text {geff }}}{V_{\text {bulk }}}
$$

Samples EP4-8 were subsequently powdered for determination of total porosity (see Appendix C-A).Because mass is not fully conserved during the (initially violent) powdering process, data are normalized with respect to mass to obtain the bulk density $\rho_{b}$ (for the intact sample) and the (true) grain density $\rho_{g}$ for the powdered sample. Data are reported in Table 7, where the total porosity $\phi$ is defined by

$$
\phi=1-\frac{\rho_{b}}{\rho_{g}}
$$

The largest difference between the effective and total porosity for the four samples on which both measurements were performed is $0.2 \%$; however, no discrepancy may be formally identified since the two measurements for all samples agree within the experimental errors. Note that the data for sample EP2 suggests that the total porosity may be somewhat less than the effective porosity (again, the two measurements technically agree within the experimental errors). Sample EP2 experienced the largest loss of material loss during the powdering process $(19 \mathrm{~g}$, or $8 \%$ of the original sample mass). It is likely that this loss of $8 \mathrm{wt} \%$ of the original sample is the cause for the slight discrepancy between the measurement of effective versus total porosity for EP2. This inference is consistent with the pervasive heterogeneity which characterizes the MB 139 samples (e.g., Figures 13 and 14 and Section 9).

Samples EP4-8 and samples A, C, and F were vacuum saturated with a non-reactive fluid (odorless mineral spirits) for determination of maximum achievable saturation (Appendix C-A). 
Table 6. Bulk Volume, Effective Grain Volume, and Effective Porosity*

\begin{tabular}{|c|c|c|c|}
\hline Sample & $\begin{array}{c}V_{b} \\
\text { (cc) }\end{array}$ & $\begin{array}{l}V_{\text {seff }} \\
\text { (cc) }\end{array}$ & $\begin{array}{l}\phi_{\text {eff }} \\
(\%)\end{array}$ \\
\hline $\mathbf{A}$ & $822.84 \pm 4.94$ & $807.47 \pm 3.23$ & $1.87 \pm 0.71$ \\
\hline B & $776.83 \pm 4.66$ & $723.00 \pm 3.09$ & $0.49 \pm 0.72$ \\
\hline C & $819.62 \pm 4.92$ & $811.31 \pm 3.25$ & $1.01 \pm 0.71$ \\
\hline D & $803.84 \pm 4.82$ & $798.00 \pm 3.19$ & $0.73 \pm 0.72$ \\
\hline $\mathbf{E}$ & $843.08 \pm 5.06$ & $829.98 \pm 3.32$ & $1.55 \pm 0.71$ \\
\hline $\mathbf{F}$ & $815.25 \pm 4.89$ & $807.09 \pm 3.23$ & $1.00 \pm 0.71$ \\
\hline EP1 & $83.47 \pm 0.50$ & $82.39 \pm 0.21$ & $1.29 \pm 0.64$ \\
\hline EP2 & $84.39 \pm 0.51$ & $83.75 \pm 0.21$ & $0.75 \pm 0.64$ \\
\hline EP3 & $83.54 \pm 0.50$ & $83.19 \pm 0.21$ & $0.42 \pm 0.64$ \\
\hline EP4 & $83.35 \pm 0.50$ & $82.01 \pm 0.21$ & $1.60 \pm 0.64$ \\
\hline EP5 & $84.85 \pm 0.51$ & $83.22 \pm 0.21$ & $1.92 \pm 0.64$ \\
\hline EP6 & $84.04 \pm 0.50$ & $81.74 \pm 0.20$ & $2.73 \pm 0.63$ \\
\hline EP7 & $83.73 \pm 0.50$ & $83.27 \pm 0.21$ & $0.55 \pm 0.64$ \\
\hline EP8 & $84.52 \pm 0.51$ & $83.19 \pm 0.21$ & $1.57 \pm 0.64$ \\
\hline \multicolumn{4}{|c|}{$\begin{array}{l}\text { Quoted uncertainties are derived from formal propagation of } \\
\text { random and systematic errors [8]. Sources for systematic } \\
\text { error in } V_{8} \text { include pressure transducer accuracy, porosimeter } \\
\text { calibration (two constants), and random error is calculated } \\
\text { from duplicate measurements. Error in } V_{b} \text { is estimated at } \\
0.6 \%, \text { based upon comparison of waxed-buoyant measure- } \\
\text { ments with those determined from direct OMS buoyant mea- } \\
\text { surements (see text) and includes an allowance for systematic } \\
\text { errors in OMS measurements due to accuracy of digital bal- } \\
\text { ances and uncertainty in density of OMS (which was mea- } \\
\text { sured directly). }\end{array}$} \\
\hline
\end{tabular}

for procedures). Data are reported in Table 8. Calculated saturations for the four smaller samples (EP5-8) are in excess of 100\%; however, the associated uncertainties are substantial. The largest source of error, which is not readily quantifiable, apparently results from the moisture-sensitivity of the samples. The samples were moisture-equilibrated in a constant temperature and humidity oven at conditions $\left(60^{\circ} \mathrm{C}, 45 \%\right.$ R.H. $)$ which deviated substantially from the ambient conditions. The four EP samples were all found to have experienced some weight gain during the period following the gas porosimetry measurement of effective grain volume and prior to the liquid saturation, even though the samples were plastic-wrapped and stored in ziploc bags during the interim. An additional complication is the change in pore volume, due to a loss of absorbed and/or adsorbed water, which probably occurred during the evacuation process preceding liquid saturation. It is believed that the resultant changes in pore volume, while small in the absolute sense are nevertheless significant relative to the very small pore volume of the EP samples, and the cause of the calculated unphysical $(>100 \%)$ saturations. Additional support for this hypothesis is offered by the observed inverse relationship between over-saturation and 
Table 7. Bulk Density, Grain Density, and Total Porosity*

\begin{tabular}{ccccc}
\hline Sample & $\begin{array}{c}\rho_{b} \\
(\mathrm{~g} / \mathrm{cc})\end{array}$ & $\begin{array}{c}\rho_{g} \\
(\mathrm{~g} / \mathrm{cc})\end{array}$ & $\begin{array}{c}\phi \\
(\%)\end{array}$ & $\begin{array}{c}M_{\text {orisinal }} \dagger \\
(\%)\end{array}$ \\
\hline EP1 & $2.530 \pm 0.006$ & $2.566 \pm 0.005$ & $1.40 \pm 0.30$ & 98 \\
EP2 & $2.643 \pm 0.006$ & $2.658 \pm 0.005$ & $0.56 \pm 0.29$ & 92 \\
EP3 & $2.574 \pm 0.006$ & $2.585 \pm 0.005$ & $0.43 \pm 0.30$ & 98 \\
EP4 & $2.836 \pm 0.006$ & $2.882 \pm 0.005$ & $1.60 \pm 0.49$ & 94 \\
\hline
\end{tabular}

* Quoted uncertainties are derived from formal propagation of random and systematic errors [8]. Error in mass determination includes random errors derived from duplicate measurements and systematic errors due to balance accuracy. Sources for systematic error in $V_{z}$ include pressure transducer accuracy, porosimeter calibration (two constants), and random error calculated from duplicate measurements. Error in $V_{b}$ is based upon comparison of waxed-buoyant measurements with those determined from direct OMS buoyant measurements (see text) and includes an allowance for systematic errors in OMS measurements due to accuracy of digital balances and uncertainty in density of OMS (which was measured directly).

$\dagger$ Percentage of original sample mass used for determination of total porosity. (Reduction is due to material losses which occur during the powdering process.)

Table 8. Liquid (OMS) Saturation*

\begin{tabular}{cccccc}
\hline Sample & $\begin{array}{c}\mathrm{m}_{s} \\
(\mathrm{~g})\end{array}$ & $\begin{array}{c}\mathrm{m}_{d} \\
(\mathrm{~g})\end{array}$ & $\begin{array}{c}V_{b} \\
(\mathrm{cc})\end{array}$ & $\begin{array}{c}V_{8} \text { aff } \\
(\mathrm{cc})\end{array}$ & $S$ \\
\hline EP5 & $220.998 \pm 0.002$ & $219.596 \pm 0.002$ & $84.85 \pm 0.51$ & $83.22 \pm 0.21$ & $1.2 \pm 0.3$ \\
EP6 & $222.678 \pm 0.004$ & $220.796 \pm 0.002$ & $84.04 \pm 0.50$ & $81.74 \pm 0.20$ & $1.1 \pm 0.2$ \\
EP7 & $234.143 \pm 0.003$ & $233.502 \pm 0.002$ & $83.73 \pm 0.50$ & $83.27 \pm 0.21$ & $1.9 \pm 1.2$ \\
EP8 & $230.016 \pm 0.005$ & $228.722 \pm 0.002$ & $84.52 \pm 0.51$ & $83.19 \pm 0.21$ & $1.3 \pm 0.4$ \\
& & & & & \\
A & $2151.88 \pm 0.04$ & $2140.18 \pm 0.04$ & $822.84 \pm 4.94$ & $807.47 \pm 3.23$ & $1.0 \pm 0.4$ \\
C & $2214.84 \pm 0.04$ & $2208.66 \pm 0.04$ & $819.62 \pm 4.92$ & $811.31 \pm 3.25$ & $1.0 \pm 0.7$ \\
F & $2333.49 \pm 0.04$ & $2327.39 \pm 0.04$ & $815.25 \pm 4.89$ & $807.09 \pm 3.23$ & $1.0 \pm 0.7$ \\
\hline
\end{tabular}

* Quoted uncertainties are derived from formal propagation of random and systematic errors [8]. Sources for systematic error in $m_{e}$ and $m_{d}$ include digital balance accuracy and random error (for $m_{s}$ ) is calculated from duplicate measurements. The density of the saturant (OMS) was measured directly with a calibrated pycnometer and digital balance and is $0.753 \pm 0.011 \mathrm{~g} / \mathrm{cc}$. Error in fluid density includes uncertainty caused by small temperature fluctuations. Sources for systematic error in $V_{8,}$ include pressure transducer accuracy, porosimeter calibration (two constants), and random error calculated from duplicate measurements. Error in $V_{b}$ is estimated at $0.6 \%$, based upon comparison of waxed-buoyant measurements with those determined from direct OMS buoyant measurements (see text) and includes an allowance for systematic errors in OMS measurements due to accuracy of digital balances and uncertainty in density of OMS (which was measured directly). 
pore volume. (Note that for consistency, the "dry" mass used in the saturation calculation is that determined at the time of the effective porosity measurement. Although it is known that the "dry" mass immediately prior to saturation differs, it would be inconsistent to apply this data since the porosity to go along with this second "dry" state is unknown.)

\subsection{Single Phase Permeability}

\subsection{Gas Single-Phase Permeability}

Single-phase gas (nitrogen) permeabilities were measured for samples A-F at three effective stress conditions $(2,6$, and $10 \mathrm{MPa})$ using the steady state technique (seeAppendix C-A for a schematic of the experimental assembly and procedures). Apparent gas permeabilities $k_{g}$ were measured at four different mean pore pressures at each effective stress condition (Table 9) and used to calculate Klinkenberg (a.k.a. equivalent liquid) permeabilities $k_{\infty}$ (Table 10). (Raw data are compiled in Appendix C-D).

\subsection{Liquid Single-Phase Permeability}

Single-phase liquid permeabilities $k_{l}$ were measured at three effective stress conditions $(2,6$, and $10 \mathrm{MPa}$ ) using the steady state technique (see Appendix C-A. for a schematic of the experimental assembly and procedures) for samples A, C, and F (see Section 7.0 and Table 8 above for saturation data). The calculated permeabilities are reported in Table 11. (Raw data are included inAppendix C-E).

The measurements of liquid permeability agree well with the calculated Klinkenberg permeabilities. The differences for seven out of the nine data sets fall within the experimental errors, although the liquid permeabilities do appear, on average, to be systematically lower than the extrapolated Klinkenberg permeabilities. 
Table 9. Single Phase Gas Permeability*

\begin{tabular}{|c|c|c|c|}
\hline Sample & $\begin{array}{l}P_{c \text { edf }} \\
\text { (MPa) }\end{array}$ & $\begin{array}{l}P_{p \text { max }} \\
(\mathrm{MPa})\end{array}$ & $\begin{array}{c}k_{\boldsymbol{g}} \\
(\mu \mathrm{Da})\end{array}$ \\
\hline \multirow[t]{12}{*}{$\mathbf{A}$} & 2 & 0.2725 & $12.3 \pm 0.2$ \\
\hline & & 0.3405 & $11.6 \pm 0.2$ \\
\hline & & 0.4090 & $11.0 \pm 0.2$ \\
\hline & & 0.4779 & $10.6 \pm 0.2$ \\
\hline & 6 & 0.2728 & $8.88 \pm 0.14$ \\
\hline & & 0.3397 & $8.40 \pm 0.13$ \\
\hline & & 0.4105 & $7.82 \pm 0.12$ \\
\hline & & 0.4787 & $7.56 \pm 0.12$ \\
\hline & 10 & 0.2716 & $7.29 \pm 0.11$ \\
\hline & & 0.3421 & $6.84 \pm 0.11$ \\
\hline & & 0.4100 & $6.55 \pm 0.10$ \\
\hline & & 0.4795 & $6.31 \pm 0.10$ \\
\hline \multirow[t]{12}{*}{ B } & 2 & 0.2797 & $19.8 \pm 0.3$ \\
\hline & & 0.3475 & $18.5 \pm 0.3$ \\
\hline & & 0.4176 & $17.5 \pm 0.3$ \\
\hline & & 0.4881 & $16.8 \pm 0.3$ \\
\hline & 6 & 0.2758 & $13.2 \pm 0.2$ \\
\hline & & 0.3464 & $12.1 \pm 0.2$ \\
\hline & & 0.4166 & $11.3 \pm 0.2$ \\
\hline & & 0.4878 & $10.7 \pm 0.2$ \\
\hline & 10 & 0.2755 & $8.23 \pm 0.13$ \\
\hline & & 0.3452 & $7.54 \pm 0.11$ \\
\hline & & 0.4159 & $7.02 \pm 0.11$ \\
\hline & & 0.4867 & $6.69 \pm 0.10$ \\
\hline
\end{tabular}

* Quoted uncertainties are derived from formal propagation of random and systematic errors [8]. Sources for systematic error in $\boldsymbol{k}_{\boldsymbol{g}}$ include pressure transducer accuracy (differential, gauge, and barometric), accuracy of flow rate measurement (volume and time), caliper accuracy, and uncertainty in temperature measurements. Random error included in flow rate error is calculated from $4 \mathrm{du}$ plicate measurements. Gas viscosity is $0.0176 \mathrm{cp}$ [9]. 
Table 9. Single Phase Gas Permeability* (continued)

\begin{tabular}{|c|c|c|c|}
\hline Sample & $\begin{array}{c}P_{c \text { cat }} \\
(\mathrm{MPa})\end{array}$ & $\begin{array}{l}P_{p \text { man }} \\
(\mathrm{MPa})\end{array}$ & $\begin{array}{c}k_{\boldsymbol{g}} \\
(\mu \mathrm{Da})\end{array}$ \\
\hline \multirow[t]{12}{*}{ C } & 2 & 0.2871 & $7.84 \pm 0.12$ \\
\hline & & 0.3559 & $7.26 \pm 0.11$ \\
\hline & & 0.4224 & $6.81 \pm 0.10$ \\
\hline & & 0.4940 & $6.50 \pm 0.10$ \\
\hline & 6 & 0.3337 & $5.41 \pm 0.08$ \\
\hline & & 0.3552 & $4.86 \pm 0.07$ \\
\hline & & 0.4236 & $4.49 \pm 0.07$ \\
\hline & & 0.4787 & $4.25 \pm 0.07$ \\
\hline & 10 & 0.3361 & $4.09 \pm 0.06$ \\
\hline & & 0.3545 & $3.67 \pm 0.06$ \\
\hline & & 0.4248 & $3.41 \pm 0.05$ \\
\hline & & 0.4940 & $3.21 \pm 0.05$ \\
\hline \multirow[t]{12}{*}{ D } & 2 & 0.4686 & $0.275 \pm 0.014$ \\
\hline & & 0.5453 & $0.261 \pm 0.013$ \\
\hline & & 0.6224 & $0.238 \pm 0.012$ \\
\hline & & 0.6787 & $0.239 \pm 0.012$ \\
\hline & 6 & 0.4743 & $0.127 \pm 0.007$ \\
\hline & & 0.5439 & $0.120 \pm 0.006$ \\
\hline & & 0.6142 & $0.113 \pm 0.006$ \\
\hline & & 0.6875 & $0.106 \pm 0.005$ \\
\hline & 10 & 0.4743 & $0.088 \pm 0.004$ \\
\hline & & 0.5431 & $0.083 \pm 0.004$ \\
\hline & & 0.6111 & $0.080 \pm 0.005$ \\
\hline & & 0.6856 & $0.078 \pm 0.004$ \\
\hline
\end{tabular}

* Quoted uncertainties are derived from formal propagation of random and systematic errors [8]. Sources for systematic error in $k_{g}$ include pressure transducer accuracy (differential, gauge, and barometric), accuracy of flow rate measurement (volume and time), caliper accuracy, and uncertainty in temperature measurements. Random error included in flow rate error is calculated from 4 duplicate measurements. Gas viscosity is $0.0176 \mathrm{cp}$ [9]. 
Table 9. Single Phase Gas Permeability* (continued)

\begin{tabular}{|c|c|c|c|}
\hline Sample & $\begin{array}{c}P_{c \text { Gf }} \\
(\mathrm{MPa})\end{array}$ & $\begin{array}{l}P_{p \text { mean }} \\
(\mathrm{MPa})\end{array}$ & $\begin{array}{c}k_{g} \\
(\mu \mathrm{Da})\end{array}$ \\
\hline \multirow[t]{12}{*}{$\mathbf{E}$} & 2 & 0.1023 & $1168 \pm 19$ \\
\hline & & 0.1711 & $1054 \pm 17$ \\
\hline & & 0.2401 & $997 \pm 16$ \\
\hline & & 0.3086 & $927 \pm 15$ \\
\hline & 6 & 0.1192 & $497 \pm 8$ \\
\hline & & 0.1980 & $426 \pm 7$ \\
\hline & & 0.2660 & $398 \pm 6$ \\
\hline & & 0.3345 & $382 \pm 6$ \\
\hline & 10 & 0.1290 & $292 \pm 5$ \\
\hline & & 0.1968 & $253 \pm 4$ \\
\hline & & 0.2671 & $218 \pm 4$ \\
\hline & & 0.3342 & $208 \pm 3$ \\
\hline \multirow[t]{12}{*}{ F } & 2 & 0.4510 & $1.77 \pm 0.03$ \\
\hline & & 0.5233 & $1.69 \pm 0.02$ \\
\hline & & 0.5908 & $1.62 \pm 0.02$ \\
\hline & & 0.6581 & $1.57 \pm 0.02$ \\
\hline & 6 & 0.4524 & $1.23 \pm 0.02$ \\
\hline & & 0.5193 & $1.15 \pm 0.02$ \\
\hline & & 0.5921 & $1.11 \pm 0.02$ \\
\hline & & 0.6597 & $1.06 \pm 0.02$ \\
\hline & 10 & 0.4525 & $1.06 \pm 0.016$ \\
\hline & & 0.5176 & $0.99 \pm 0.01$ \\
\hline & & 0.5911 & $0.95 \pm 0.01$ \\
\hline & & 0.6636 & $0.91 \pm 0.01$ \\
\hline
\end{tabular}

* Quoted uncertainties are derived from formal propagation of random and systematic errors [8]. Sources for systematic error in $k_{z}$ include pressure transducer accuracy (differential, gauge, and barometric), accuracy of flow rate measurement (volume and time), caliper accuracy, and uncertainty in temperature measurements. Random error included in flow rate error is calculated from 4 duplicate measurements. Gas viscosity is $0.0176 \mathrm{cp} \mathrm{[9]}$. 
Table 10. Calculated Klinkenberg Perneability

\begin{tabular}{|c|c|c|}
\hline Sample & $\begin{array}{c}P_{\text {c edf }} \\
\text { (MPa) }\end{array}$ & $\begin{array}{c}k_{\infty} \\
(\mu \mathrm{Da})\end{array}$ \\
\hline \multirow[t]{3}{*}{$\mathbf{A}$} & 2 & $8.36 \pm 0.12$ \\
\hline & 6 & $5.76 \pm 0.20$ \\
\hline & 10 & $5.05 \pm 0.03$ \\
\hline \multirow[t]{3}{*}{ B } & 2 & $12.76 \pm 0.09$ \\
\hline & 6 & $7.50 \pm 0.12$ \\
\hline & 10 & $4.67 \pm 0.04$ \\
\hline \multirow[t]{3}{*}{ C } & 2 & $4.63 \pm 0.06$ \\
\hline & 6 & $2.63 \pm 0.03$ \\
\hline & 10 & $2.01 \pm 0.02$ \\
\hline \multirow[t]{3}{*}{ D } & 2 & $0.147 \pm 0.019$ \\
\hline & 6 & $0.060 \pm 0.004$ \\
\hline & 10 & $0.055 \pm 0.008$ \\
\hline \multirow[t]{3}{*}{$\mathbf{E}$} & 2 & $836 \pm 28$ \\
\hline & 6 & $307 \pm 19$ \\
\hline & 10 & $155 \pm 8$ \\
\hline \multirow[t]{3}{*}{$\mathbf{F}$} & 2 & $1.13 \pm 0.02$ \\
\hline & 6 & $0.70 \pm 0.03$ \\
\hline & 10 & $0.58 \pm 0.01$ \\
\hline
\end{tabular}


Table 11. Single Phase Liquid Permeability* vs Calculated Klinkenberg Permeability

\begin{tabular}{cccc}
\hline Sample & $\begin{array}{c}P_{\text {e eff }} \\
(\mathrm{MPa})\end{array}$ & $\begin{array}{c}k_{l} \\
(\mu \mathrm{Da})\end{array}$ & $\begin{array}{c}k_{\infty} \\
(\mu \mathrm{Da})\end{array}$ \\
\hline $\mathrm{A}$ & 2 & $6.75 \pm 1.36$ & $8.36 \pm 0.12$ \\
& 6 & $5.76 \pm 0.65$ & $5.76 \pm 0.20$ \\
& 10 & $5.37 \pm 0.60$ & $5.05 \pm 0.03$ \\
$\mathrm{C}$ & 2 & $3.66 \pm 0.40$ & $4.63 \pm 0.06$ \\
& 6 & $2.43 \pm 0.27$ & $2.63 \pm 0.03$ \\
& 10 & $1.83 \pm 0.20$ & $2.01 \pm 0.02$ \\
$\mathrm{~F}$ & 2 & $1.06 \pm 0.12$ & $1.13 \pm 0.02$ \\
& 6 & $0.62 \pm 0.07$ & $0.70 \pm 0.03$ \\
& 10 & $0.52 \pm 0.06$ & $0.58 \pm 0.01$ \\
\hline
\end{tabular}

* Quoted uncertainties are derived from formal propagation of random and systematic errors [8]. Sources for systematic error in $k_{l}$ include differential pressure transducer accuracy, accuracy of flow rate measurement (volume and time), caliper accuracy, and uncertainty in fluid viscosity. Random error is calculated from at least 5 duplicate measurements. Experimental measurement of OMS viscosity is $1.29 \mathrm{cp}$ with an assumed maximum uncertainty of $10 \%$. 


\subsection{Summary and Recommendations}

The laboratory investigations demonstrate that Marker Bed 139 is characterized by significant and pervasive heterogeneity. Remarkable heterogeneity occurs as the marker bed is traversed vertically, and some variability is also apparent as the marker bed is traversed laterally. Mineralogy varies dramatically, and although porosity is typically $-0.5-3.0 \%$, permeability varies by approximately four orders of magnitude (e.g., from tenths of microdarcies to hundreds of microdarcies at an effective pressure of $2 \mathrm{MPa}$ ).

Attempts to use a second-generation x-ray CT scanner to identify natural and coring-induced fractures were unsuccessful, due to the large diameter of the core material (6 inches) and high bulk density (close to $3 \mathrm{~g} / \mathrm{cc}$ ). Several attempts to compensate and/or correct for pronounced image artifacts caused by excessive beam-hardening were unsuccessful. However, preliminary scoping tests which were performed with a more powerful and advanced third-generation CT scanner (acquired midway through the contract performance period) yielded promising results. Thus, with the newer CT technology it may be possible to perform highly detailed nondestructive lithologic evaluations. Also, fractures with apertures on the order of tenths of millimeters should be resolvable with the more advanced scanner.

The marker bed exhibits four distinct mineralogic zones. The upper portion is rich in polyhalite, which is manifested by a conspicuous salmon-pink color. Halite and anhydrite are also major mineral phases in this section, as are dolomite and aragonite. The central region contains both anhydrite and halite, which occur in varying proportions and account for nearly all of the solid phase. The lower portion of this central region is dominated by anhydrite with markedly smaller $(<20 \%)$ amounts of halite. The very bottom of the marker bed is significantly more fine-grained than the rest of the marker bed; unfortunately, this section is limited in length (4-5") and thus no samples for detailed testing were prepared from this region. CT scans, however, indicate that this region is the densest part of the entire marker bed. Thus, this region may contain nearly $100 \%$ anhydrite, or possibly small amounts of very dense impurities such as pyrite (which was identified in thin-section).

The petrographic studies corroborate the general observations above and, moreover, indicate that marked heterogeneity can exist at even the $\mathrm{cm}$ scale. For example, the volumetric percentage of various minerals may vary by as much as 20 to $30 \%$ for samples prepared from locations separated by only two or three centimeters. The modal analyses based on 300-point counts consistently suggest the occurrence of several vol\% (and sometimes as much as $16 \mathrm{vol} \%$ ) of a carbonate phase, tentatively identified as dolomite, in each of the eighteen thin sections examined. Although the XRD analyses are recognized as only semi-quantitative in nature, the apparent absence of carbonate in four of the six samples tested is conspicuous. Although it is possible that the carbonate material is present only locally, it is more likely that the XRD analyses fail to identify carbonate in the MB139 samples because of variations in crystallanity and possibly $\mathrm{mg} / \mathrm{fe} / \mathrm{ca}$ cation ratios (as compared to the reference stanclards used to calibrate the diffractometer). Thus, depending upon the level of investigations to be performed in the future, it may be worthwhile to restrict further XRD analyses to a single institution and to invest in the development of a set of standards specific to the WIPP. 
Measurements of effective and total porosity consistently indicate that the bulk porosity of the marker bed is quite low, and varies from $0.4 \%$ to $2.7 \%$. The measurements of effective versus total porosity agree within the experimental errors. Permeability, however, varies by approximately four orders of magnitude (e.g., from tenths of microdarcies to hundreds of microdarcies at an effective pressure of $2 \mathrm{MPa}$ ). A permeability on the order of millidarcies was measured for one sample at an effective stress of $2 \mathrm{MPa}$ and may represent fracture permeability. The other extreme is embodied by a single sample with a permeability on the order of tenths of microdarcies (i.e., hundreds of nanodarcies) at an effective stress of $2 \mathrm{MPa}$. Four other samples are characterized by permeabilities on the order of microdarcies. The measurements of liquid permeability agree well with calculated Klinkenberg (a.k.a equivalent liquid) permeabilities. The differences for seven out of the nine data sets fall within the experimental errors, although the liquid permeabilities do appear, on average, to be systematically lower than the extrapolated Klinkenberg permeabilities.

Calculations of achievable saturation suggest that complete saturation is probably attained by routine vacuum saturation; however, the experimental errors are significant given the excessively small pore volumes of the samples. Interpretation is further complicated by possible changes in pore volume caused by the apparent adsorption and/or absorption of moisture from the atmosphere which occurred while samples were between various parts of the testing program. Although very small in the absolute sense, the hypothesized changes in pore volume are significant in the relative sense. Thus, it may be advisable to either equilibrate samples to local atmospheric conditions or else make provisions to acquire a constant temperature and constant humidity oven dedicated exclusively to the test program. 


\subsection{References}

1. Davies, P. B., Evaluation of the role of threshold pressure in controlling flow of wastegenerated gas into bedded salt at the Waste Isolation Pilot Plant, SAND90-3246, Sandia National Laboratories, Albuquerque, New Mexico, 1991.

2. A. M. Petrovic et al., Soil bulk density analysis in three dimensions by computed tomographic scanning, Am. J. Soil Sci. Soc., v. 46, p. 445-450, 1982.

3. P. K. Hunt et al., Computed tomography as a core analysis tool: Applications, instrument evaluation, and image improvement techniques, J. Pet. Tech., 1203-1210, (Sept.) 1988.

4. Brown et al., Accuracy of gamma ray computerized tomography in porous media, Water Resour. Res., 29, 479-486, 1993.

5. Johns et al., Nondestructive measurements of fracture aperture in crystalline rock cores using X ray computed tomography, J. Geophys. Res., 98, 1889-1900, 1993.

6. H. J. Vinegar \& G. E. Hill, Beam-hardening corrections on the DeltaScan 100 CT Scanner, Report No. BRC-2044, Shell Development Company, Houston, 1988 (Company Confidential).

7. Rock Characterization, Testing, and Monitoring: ISRM Suggested Methods, E. T. Brown (Ed.), 211 p., Pergamon Press, New York.

8. J. R. Taylor, An Introduction to Error Analysis: The Study of Uncertainties in Physical Measurements, Oxford University Press, 1982.

9. Handbook of Chemistry and Physics, 66th ed., CRC Press, Inc., Florida, 1985. 


\section{APPENDIX C-A: Procedures}




\section{A.1 X-Ray Computerized Tomography}

\section{A.1.1 Background. Computerized tomography (CT) is a non-destructive technique for the} evaluation of the internal structure of a material. Imaging technology is based on the analysis of the attenuation of $\mathrm{x}$-rays as they pass through a material. This attenuation is due to scattering and adsorption and is characterized by Beer's law:

$$
I=I_{0} e^{-\alpha x}
$$

where $I_{0}$ and $I$ are the incident and attenuated $\mathrm{x}$-ray intensity, $x$ is the thickness of the material, and $\alpha$ is the linear attenuation coefficient.

The attenuation of the $\mathrm{x}$-rays is dependant on photoelectric adsorption and Compton scattering. Photoelectric adsorption is dependent on the electron density or the effective atomic number of the material and is a predominant term at $\mathrm{x}$-ray energies below $100 \mathrm{kV}$. Compton scattering is dependent on the density of the material and becomes a more predominant term at energy levels above $100 \mathrm{kV}$. Thus, the attenuation coefficient for a material depends upon both the density and effective atomic number of the material as well as the energy level of the $\mathrm{x}$-ray:

$$
\alpha=\rho\left(a+\frac{b Z^{3.8}}{E^{3.2}}\right)
$$

where $\rho$ is the mass density of the material, $Z$ is the effective atomic number, $E$ is the $x$-ray energy level, $a$ is the Klein-Nishina coefficient, and $b$ is a constant. At high energy levels, and for materials with similar chemical composition, differences in the effective atomic number are small. Differences in attenuation are thus due primarily to differences in mass density.

A.1.2 TerraTek's CT Facility. At the start of the performance period for this contract, TerraTek's CT facility employed an Ohio Nuclear DeltaScan 100 . The DeltaScan 100 is a second generation medical CT imaging machine and TerraTek's scanner was originally built in 1980 for use as a head scanner. No hardware modifications were made to the scanner for use as a material imager. When a test sample is inserted in the scanner, an $\mathrm{x}$-ray source and detector are passed in parallel planes past the sample (called a traverse). The tube and detector are then rotated through a specified number of degrees and another traverse occurs. This is repeated through a 180-degree rotation. A cross-sectional image of the test sample can then be generated by dividing the sample up into small discrete elements (or pixels) and solving a set of linear equations. The linear attenuation coefficient for each element can be determined through reconstruction algorithms intrinsic to the scanner's computer.

The x-ray beam is collimated into three fan shaped beams which are attenuated as they pass through the sample. The width of the beam, which determines the volume of material (voxel) for which linear attenuation coefficients are calculated, can be adjusted from 3 to $10 \mathrm{~mm}$. The attenuated x-rays are measured by three detectors with measurements recorded every $3^{\circ}$ in a $180^{\circ}$ rotation. Each image requires two minutes to complete the 60 scans. The maximum diameter of a sample is limited to $305 \mathrm{~mm}$. However, with large samples resolution is decreased since 
the pixel array is fixed at $256 \times 256$. Also, for some materials the maximum diameter must be reduced to insure successful $\mathrm{X}$-ray penetration.

The data array is composed of CT numbers which are defined as normalized attenuation coefficients for the material being scanned. Cross-sectional images may reveal the presence of open fractures, filled fractures, vugs, and mineral inclusions. Broken or crushed sections of core can be identified before the sample is removed from its packaging, aluminum casing, or rubber sleeves. Vertical reconstructions are built from a series of cross-sectional images. Six contiguous rows or columns (in either the $x$ or $y$ direction) of data elements for each cross section in the series are averaged. For discontinuous scanning, the image is completed by interpolating between scans. The resultant image represents the core as it would appear if it were slabbed through the $\mathrm{x}$ or $\mathrm{y}$ plane as defined above. The software utilized to process the CT data is Shell Development Company's CATPIX programming, which was licensed to TerraTek in 1987. Although modifications were made to the graphics display software, no modifications were made to the fundamental algorithms used to process the data.

During the performance period for this contract, TerraTek constructed a new, more advanced CT facility employing a third generation Philips 60/TX CT scanner. The Philips 60/TX provides significantly greater resolution and imaging capabilities and TerraTek has since discontinued use of its original second generation CT facility. TerraTek has also developed a software for advanced CT analysis which provides automatic numerical correction for beam-hardening, density and atomic number determination, and many other features. The $\mathrm{C}++$ software is implemented on a Sun SparcStation 10 and marketed under the name CORESCAN ${ }^{\mathrm{TM}}$. The new CT facility was used only for exploratory (scoping) work under the present contract, and the operating procedures described below refer to the DeltaScan 100.

A.1.3 Operating Procedures. The scan tube was mounted on to a table designed by Shell Development Company which provides positioning control of the sample to within 0.002 inch. Positions were measured by an Acu-Rite III scale assembly which is mounted onto the table and the scanning sequence (consisting of the number of scans, the distance which the scan table moves between scans, and the feed rate) were programmed into an Aerotech Unidex IIIa Motion Controller. The position of the first scan was indicated by a fixed light source. The gantry system and data acquisition were controlled by a VAX PDP 11/04 computer system. Scan data acquired with the VAX PDP 11/04 was transferred to magnetic tape, and then to a Microvax II GPX color graphics work station.

The data array generated by the scanner is composed of CT numbers which are defined as normalized attenuation coefficients for the material being scanned. CT numbers are defined as follows:

$$
C T \#=K \frac{\mu_{P X X}-\mu_{P H A}}{\mu_{P H A}}
$$

where $\mu_{P X}$ is the attenuation coefficient of the pixel being measured, $\mu_{P H A}$ is the attenuation coefficient of the "phantom" used to generate the non-linear coefficients for the back projection 
algorithm, and $K$ is a constant. The phantom used for calibration depends upon the material to be scanned. For example, for medical scanning of human subjects, a phantom filled with distilled, deionized water is used to calibrate the CT number scale so that the CT number of water equals zero. For scanning of geologic materials, TerraTek uses a 4.17 inch diameter fused quartz standard. The fused quartz standard is mounted on a platform so that the standard extends beyond the platform into the plane of the CT scan traverse; essentially, the quartz standard is suspended in air. The nonlinear coefficients are generated by software which is part of the Ohio Nuclear DeltaScan 100 programming such that the CT number of the fused quartz standard is equal to zero.

Consistency is maintained by scanning a second 0.978 inch diameter fused quartz standard in the scan tube immediately after the nonlinear coefficients are obtained. The average CT number of this standard is calculated based upon a Region of Interest (ROI) equal to approximately $80 \%$ of the sample diameter (to avoid any data distortion at the circumference of the standard). This standard is then scanned before each programmed sequence of scans and the average CT number is compared to the initial CT value obtained for the standard. Any variance can be corrected for using another routine in the DeltaScan 100 programming.

The manufacturer's general operating procedures ${ }^{1}$ for the CT Scanner were adhered to during use.

A.1.4 Imaging of fluid flow through cores. Dynamic flow tests may be performed in which a non-reactive highly attenuating liquid is injected into a core sample and scanned as the fluid front penetrates the sample. A common dopants is sodium iodide. For flow tests which are conducted under elevated hydrostatic pressures, grooved end plugs and mesh screens are mounted on each end of the sample to insure that flow is homogeneous across the sample end faces. The sample and end plugs are then jacketed in a viton sleeve and pressure taps are located at each end of the sample. The pressure taps are connected to a differential pressure transducer and the pressure difference between the upstream and downstream ends is recorded on an X-Y plotter. The sample assembly is then placed in an aluminum pressure vessel and mounted in the scanner.

Before beginning fluid flow, the sample is scanned under hydrostatic stress conditions to establish the initial test condition. This condition is assumed to represent zero saturation. Once the initial conditions have been established, fluid flow begins at a constant rate until a stable differential pressure is achieved. Scanning can be performed internittently to investigate saturation phenomena such as fingering. Under steady-state flow, the core can be scanned continuously to establish saturation profiling and liquid content distribution. A series of cross-sectional images at steady-state can be displayed perpendicular to the axis of the core and pore fluid imbition can be identified by variations in the color assignments. The saturation for each location can also be determined along the length of the core.

\footnotetext{
'Operator's Manual Delta Scan 100 Series Brain Scanner, Manual No. 961036 Revision B, Technicare Corporation, Ohio, 1980.
} 


\section{A.2 X-Ray Diffraction (XRD) Analyses}

XRD analyses were performed by the University Of Utah Research Institute in accordance with guidelines provided in reference texts. ${ }^{2,3,4}$ In preparation for bulk XRD analysis, an approximately 50 gram sample was crushed to $<18$ mesh $(<1 \mathrm{~mm})$ and thoroughly homogenized to ensure that the portion to be analyzed was representative of the whole sample. A one gram split of the crushed sample was then ground in acetone in an agate mortar to $<325$ mesh $(<45 \mu \mathrm{m})$. The powdered split was then completely mixed by rolling it on weighing paper. The powder was then $x$-rayed at $2^{\circ} 2 \theta$ per minute from $10-65^{\circ} 2 \theta$ and $1^{\circ} 2 \theta$ per minute from $2-10^{\circ} 2 \theta$ using a Phillips XRG-3100 diffractometer. ${ }^{5}$ A quartz standard ${ }^{6}$ was x-rayed at the completion of each sample to monitor drift in $x$-ray intensity. Diagnostic peaks of minerals identified on the resulting diffractograms were rescanned on duplicate samples. Approximate weight percentages of mineral phases were determined by comparing diagnostic peak intensities with those generated by standard pure phases mixed in various known proportions. Results are reported in weight percent and are the average of three duplicate samples. Variables that can effect calculation of the proportion of each mineral phase in a sample include: matrix absorption, peak overlap, crystallinity and crystal size, amorphous or organic content, absorption factors, chemical substitution, preparation techniques, and detection limits. Although many of these variables can be controlled, some cannot; hence, the results of XRD analysis are semi-quantitative.

\section{A.3 Petrographic Analyses}

Petrography was conducted with a Leitz Laborlux 12 Pol polarizing microscope equipped with objectives of $2.5 \times(0.08$ numerical aperture (NA)), $6.3 \times(0.2 \mathrm{NA}), 25 \times(0.55 \mathrm{NA})$, and $40 \times(0.70$ NA). Eye pieces were 10x, yielding possible magnifications $25 x, 63 x, 250 x$, and $400 x$. Photomicrographs were taken with a Nikon Labophot-pol polarizing microscope equipped with objectives of $2 \times(0.08 \mathrm{NA}), 4 \times(0.2 \mathrm{NA}), 10 \times(0.50 \mathrm{NA})$, and $20 \times(0.04 \mathrm{NA})$. Eye pieces were $10 \mathrm{x}$, yielding possible magnifications of $20 \mathrm{x}, 40 \mathrm{x}, 100 \mathrm{x}$, and $200 \mathrm{x}$. The camera system was a Nikon UFX automatic system with a Nikon FX-35A camera body.

\footnotetext{
${ }^{2}$ Methods \& Practices in X-Ray Powder Diffraction, R. Jenkins (Ed.), JCPDS- International Centre for Diffraction Data, 1986.
}

${ }^{3}$ Starkey, H. C., P. D. Blackmon, and P. L. Hauff, The routine mineralogical analysis of clay-bearing samples, U.S. Geol. Surv. Bull. 1563, 32p., 1984.

'Moore, D. M., and R. C. Reynolds, X-ray Diffraction and the Identification and Analysis of Clay Minerals, Oxford University Press, 332p., New York, 1989.

SThe UURI maintains a service contract with Philips Electronic Instruments which includes two preventive maintenance checks per year.

'The quartz standard was supplied as part of the standard stock package when the XRD machine was purchased from Philips Electronic Instruments. 
Three mutually perpendicular thin sections were prepared from each PX sample (Figure A1). Sections were mounted on a Leitz \#553428 mechanical stage for point count analysis (300 points). Grain/crystal size was measured using a micrometer reticle in the eyepiece which was calibrated to a Zeiss $5+100 / 100$ calibration plate. Size determinations are accurate to approximately $10 \mu \mathrm{m}$. Imaging problems necessarily arise with objects whose dimensions are less than the thickness of the thin section.

Minerals were identified using standard petrographic techniques, such as appearance, extinction, birefringence, cleavage, crystal habit, pleochroism, and interference, following reference texts.? Textural parameters follow Blatt et al. $^{8}$ Sample composition and grain/crystal size were determined by point counts of 300 discrete points. Spacing between points was approximately $0.9 \mathrm{~mm}$ for the most commonly used objective. As the mechanical stage was moved to each point the object under the cross hairs was identified and diameter (defined as the longest axis for non-spherical objects) was measured for grains and crystals using the micrometer in the eyepiece. Histograms of grain size distribution were constructed using software developed by TerraTek.

\section{A.4 Porosity Measurement}

Gas porosimetry was used to determine the bulk ("effective") grain volume $V_{8 \text { eff }}$ of intact samples and the "total" grain volume $V_{g}$ of powdered samples. The bulk volume $V_{b}$ of intact samples was calculated from the fluid volume displaced by the submerged test sample (Archimedes principle). Masses of intact or powdered sarnples are determined with electronic balances. These data were used to calculated the interconnected ("effective") porosity $\phi_{\text {eff }}$ and total porosity $\phi$ :

$$
\begin{gathered}
\phi_{\text {eff }}=1-\frac{V_{\text {geff }}}{V_{b}} \\
\phi=1-\frac{\rho_{b}}{\rho_{g}}
\end{gathered}
$$

where the bulk density $\rho_{b}$ and grain density $\rho_{g}$ are given by

$$
\rho_{b}=\frac{m_{d}}{V_{b}}
$$

\footnotetext{
'Deer, W. A., R. A. Howie, and J. Zussman, An Introduction to the Rock Forming Minerals, Longmans, Green, and Co., Ltd., London, 1966.

'Blatt, H., G. Middleton, and R. Murray, Origin of Sedimentary Rocks, Prentice Hall, Inc., New Jersey, 1980.
} 


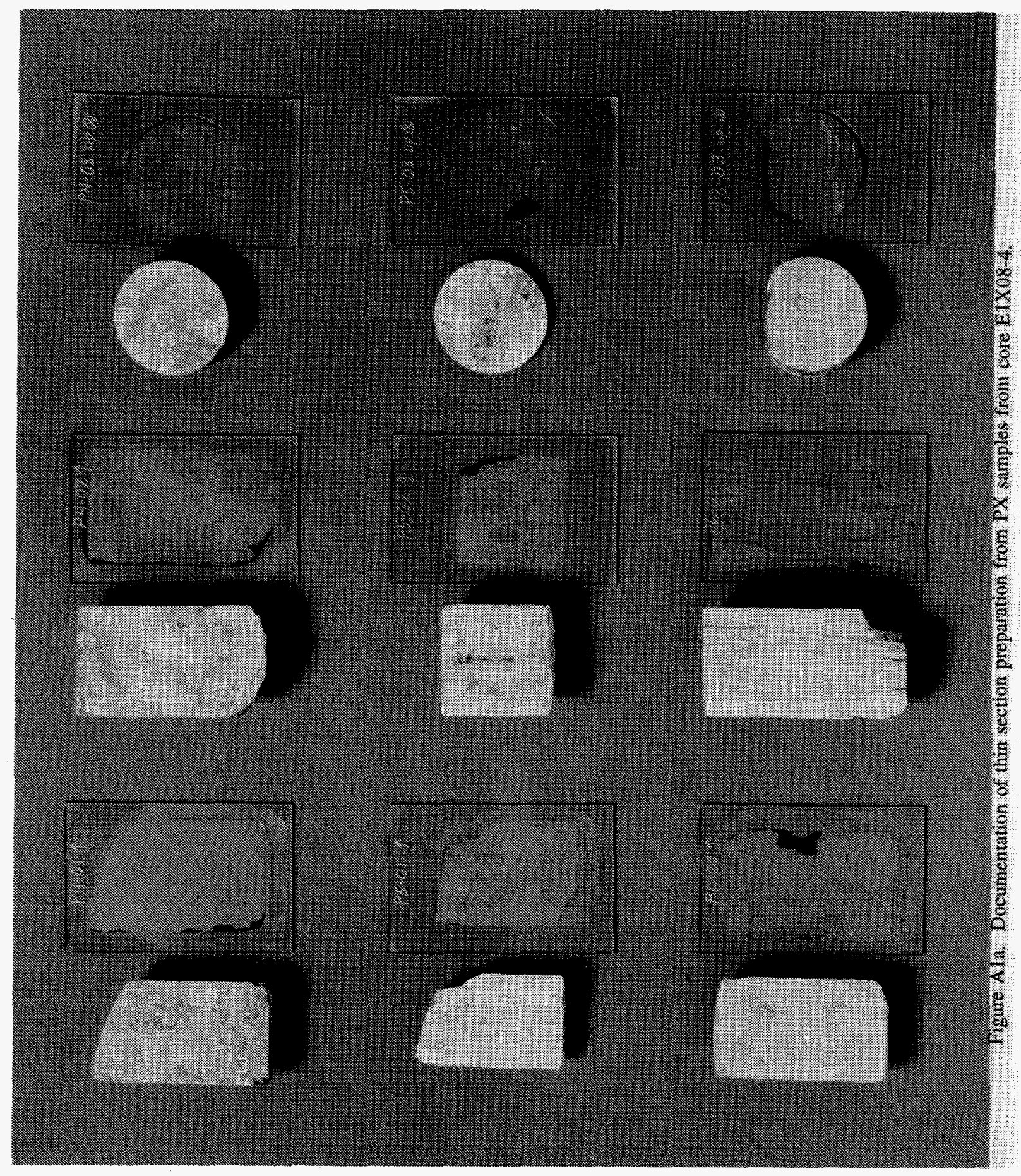




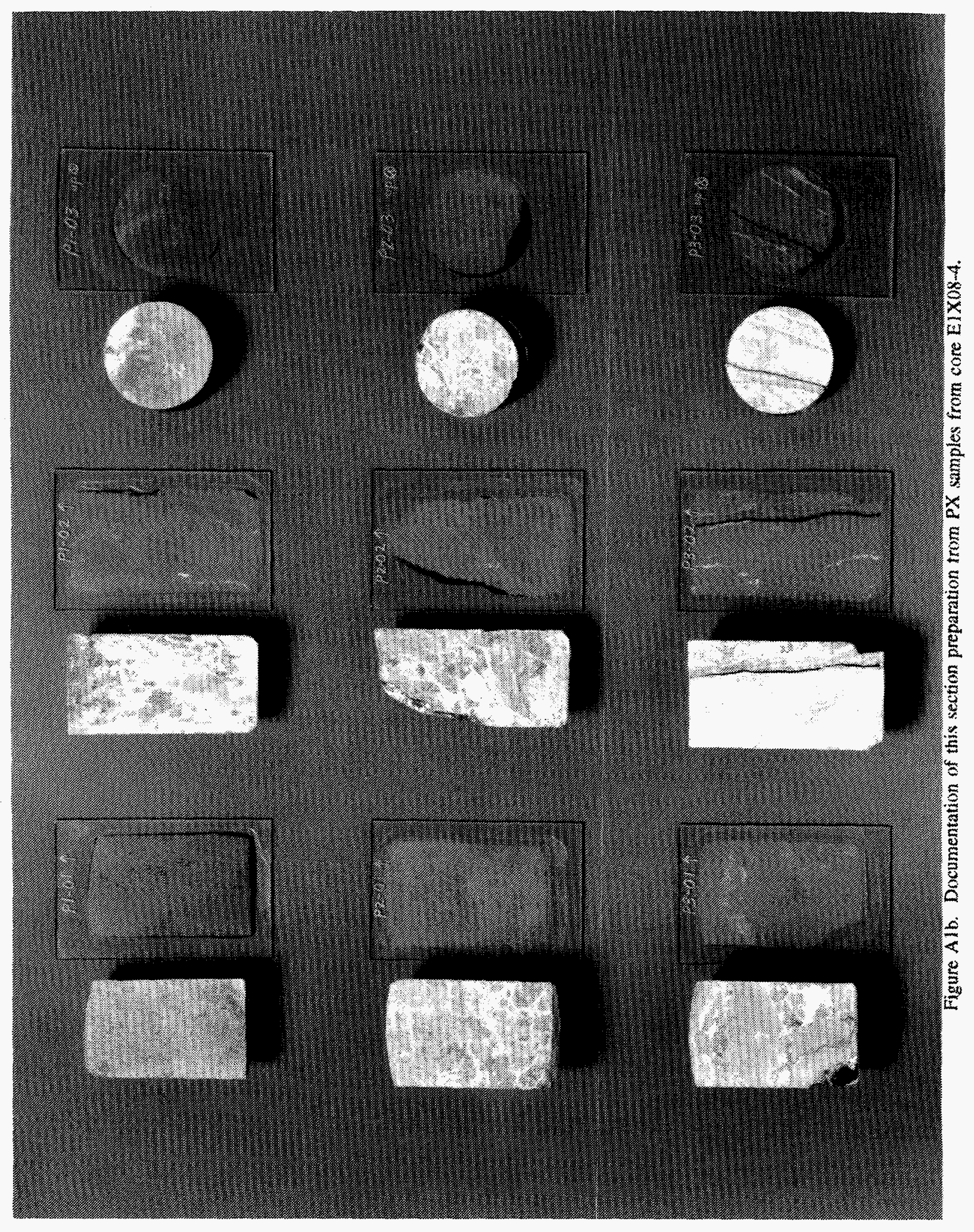




$$
\rho_{g}=\frac{m_{d}}{V_{g}}
$$

and $m_{d}$ is the mass of either the dry intact or dry powdered sample. Note that because mass is not fully conserved during the powdering of the sample for determination of total porosity, the ratio of bulk and grain density is used to calculate porosity rather than the ratio of bulk and grain volumes.

A.4.1 Gas porosimetry. Gas porosimetry is based on Boyle's law which holds that for an ideal gas at constant temperature the volume of the gas will vary inversely with pressure:

$$
\frac{P_{1}}{P_{2}}=\frac{V_{2}}{V_{1}}
$$

where $P_{1}$ is the initial pressure in the initial volume $V_{1}$ and $P_{2}$ is the final pressure in the final volume $V_{2}$. Calibration of TerraTek's custom-made gas expansion porosimeter was performed using a series of steel billets of known volume. A linear regression was performed to determine the relationship between the measured ratio $P_{1} / P_{2}$ and the sample volume. The measured pressure ratio with the sample in the porosimeter was then used to determine the grain volume $V_{8 \text { eff }}$ of the sample. Two duplicate runs were performed.

A.4.2 Bulk volume. Bulk volume was calculated from the fluid volume displaced when the test sample was submerged in fluid (Archimedes' principle). ${ }^{9}$ The volume of fluid displaced, which is equal to the sample bulk volume, is given by the difference between the weight in air and the buoyant weight divided by the temperature corrected density of the fluid. Samples were coated with wax so that fluid could not penetrate the sample (measurements were corrected for the volume of the wax). (Alternatively, saturated samples may be immersed directly.) The weight measurements are performed with appropriately ranged electronic balances. Temperature of the immersion fluid was measured to $0.1^{\circ} \mathrm{C}$ immediately following the weight measurements using a thermometer. As a check, the bulk volume of regularly shaped samples was also measured with calipers. ${ }^{10}$

A.4.3 Powdering for measurement of total porosity. Samples were crushed and pulverized to 350 mesh (approximately <46 microns) using a shatter box and then dried in a humiditycontrolled oven for an additional 24 hours to drive off any adsorbed water the powder may have imbibed (e.g. from atmospheric humidity) during the pulverizing process. Following the second drying period, the powder was allowed to reach ambient temperature in a vacuum chamber.

'Rock Characterization, Testing, \& Monitoring: ISRM Suggested Methods, E. T. Brown (Ed.), 211p., Pergamon Press, New York. Procedures for water displacement method for determination of bulk volume of solid and porous samples are outlined on p. 82.

${ }^{10}$ Rock Characterization, Testing, \& Monitoring: ISRM Suggested Methods, E. T. Brown (Ed.), 211p., Pergamon Press, New York. Procedure for determination of bulk volume using the caliper method is given on p. 82 . 


\section{A.5 Liquid Saturation}

Cores were vacuum saturated with a non-reactive liquid, odorless mineral spirits (OMS). Samples were first subjected to vacuum, flooded with $\mathrm{CO}_{2}$ (to facilitate displacement of any residual air), re-evacuated, and then flooded with OMS while under vacuum. Mass of the samples was measured using an appropriately ranged digital mass balances prior to and following saturation. The saturation $S$ of the samples is given by:

$$
S=\frac{\rho_{s}-\rho_{d}}{\phi_{\text {eff }} \rho_{f}}=\frac{\frac{\left(m_{s}-m_{d}\right)}{V_{b}}}{\frac{V_{\text {teff }}}{V_{b}} \rho_{f}}=\frac{m_{s}-m_{d}}{V_{\phi e f f} \rho_{f}}
$$

where $\rho_{s}$ and $\rho_{d}$ are the saturated and dry bulk densities, $m_{s}$ and $m_{d}$ are the saturated and dry masses, $\phi_{e f f}$ is the effective porosity, $V_{\text {teff }}$ is the effective pore volume, $\rho_{f}$ is the density of the pore fluid, and $V_{b}$ is the bulk volume. Density of the saturating fluid was measured directly using a calibrated precision pycnometer and appropriately ranged digital balance.

\section{A.6 Gas Single-Phase Permeability}

Gas single-phase permeability was measured under hydrostatic confining pressures using the steady-state flow method (Figure A2). The pore fluid was nitrogen gas and samples were jacketed in viton tubing ( 70 durometer) to prevent bypassing of the sample by the fluid.

Because of the compressibility of gases, the differential forn of Darcy's law must be integrated using the condition appropriate for gas flow (i.e. at constant temperature and steady state the product (pressure $x$ velocity) is constant throughout the sample) to determine the permeability $k_{g}$. The gas permeability $k_{g}$ was calculated from the following equation ${ }^{11}$ :

$$
k_{s}=-\frac{Q_{2}}{A}\left(\frac{2 P_{2} L}{P_{2}^{2}-P_{1}^{2}}\right) \mu=v_{2}\left(\frac{P_{2}}{P_{m}}\right)\left(\frac{L}{\Delta P}\right) \mu
$$

where $v_{2}=Q_{2} / A$, and $Q_{2}$ is the volumetric flow rate (or "discharge") at the downstream end, $A$ is the cross-sectional area of the sample, $P_{1}$ and $P_{2}$ are the gas pressures at the upstream and downstream reservoirs, $\mu$ is the gas viscosity, $L$ is the length of the sample in the macroscopic flow direction, and $P_{\mathrm{m}}=1 / 2\left(P_{1}+P_{2}\right)$.

Gas permeability varies with the pressure of the gas due to the so-called "slip" effect. The "Klinkenberg permeability" $k_{\infty}$ (a.k.a. equivalent liquid permeability) was calculated from the following relation":

\footnotetext{
11 e.g., Dullien, F. A. L., Porous Media-Fluid Transport and Pore Structure, 2nd ed., Academic Press, New York, 1992.
} 


$$
\frac{v_{2} P_{2} L \mu}{\Delta P P_{m}}=k_{\infty}\left(1+\frac{b}{P_{m}}\right)
$$

where $b$ is a constant characteristic of both the gas and porous medium and the permeability. The left hand side of equation 5 was plotted against $1 / P_{\mathrm{m}}$ and a straight line was fit to the data; the slope of the line is $\mathrm{b} k_{\infty}$ and the intercept is $k_{\infty}$.

Mean pore pressures were minimized, but sufficient to cause fluid flow along the sample length. Measurements were made at four different mean pore pressures (increments of approximately $0.07 \mathrm{MPa}$ ) to allow for accurate determination of the Klinkenberg permeability. Measurements were repeated four times at each mean pore pressure.

Pressure drops across the core were chosen such that laminar flow was favored. Analysis to verify the existence of Darcian (i.e., laminar viscous) flow was performed for verification. The analysis followed standard industry practice of relating the difference of the squared upstream and downstream pressures $\left(P_{1}^{2}-P_{2}^{2}\right)$ to the basis flow rate $Q_{\text {basir }}{ }^{12}$ A strong linear correlation $(R>0.99)$ is evidence of laminar viscous flow.

\section{A.7 Liquid Single-Phase Permeability}

Liquid permeabilities were measured for saturated samples at hydrostatic confining pressures using the steady state technique (Figure A3). Permeability $k_{l}$ is calculated using Darcy's law:

$$
Q=\left(\frac{k_{l} A}{\mu}\right)\left(\frac{\Delta P}{L}\right)
$$

where $Q$ is the volumetric flow rate (or "discharge"), $A$ is the cross-sectional area of the sample, $L$ is the length of the sample in the macroscopic flow direction, $\Delta P=P_{1}-P_{2}$ is the hydrostatic pressure drop across the sample length, and $\mu$ is the viscosity of the fluid.

Pressure drops across the core were chosen such that laminar flow was assured. The fluid pressure differential was minimized, but sufficient to cause fluid flow along the sample length. Flow measurements were performed at least 5 times.

\section{A.8 Calibration Facilities and Instrumentation}

TerraTek operates a calibration lab used to support the various testing groups within the company. The lab maintains calibration standards for force, pressure, mass, displacement, temperature, and voltage. These standards are wholly owned by the Company and are traceable

\footnotetext{
12e.g., Handbook of Natural Gas Engineering, D. L. Katz et al., McGraw-Hill Book Company, New York, 1959.
} 


\section{GAS PERMEAMETER}

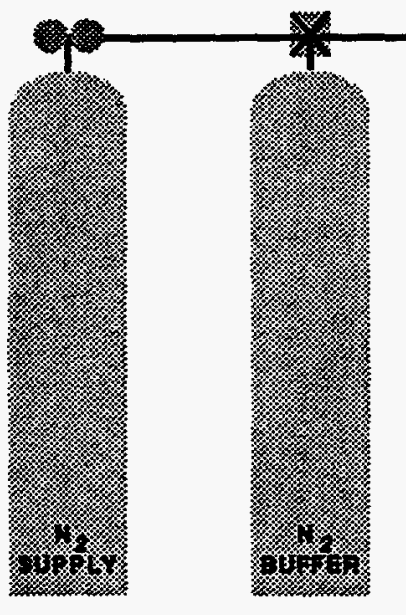

$N_{2}=$ NITROGEN GAS

(STANDAAD 304 CU. FT. BOTTLES)

SNOOP = LEAK DETECTION SOAP SOLUTION
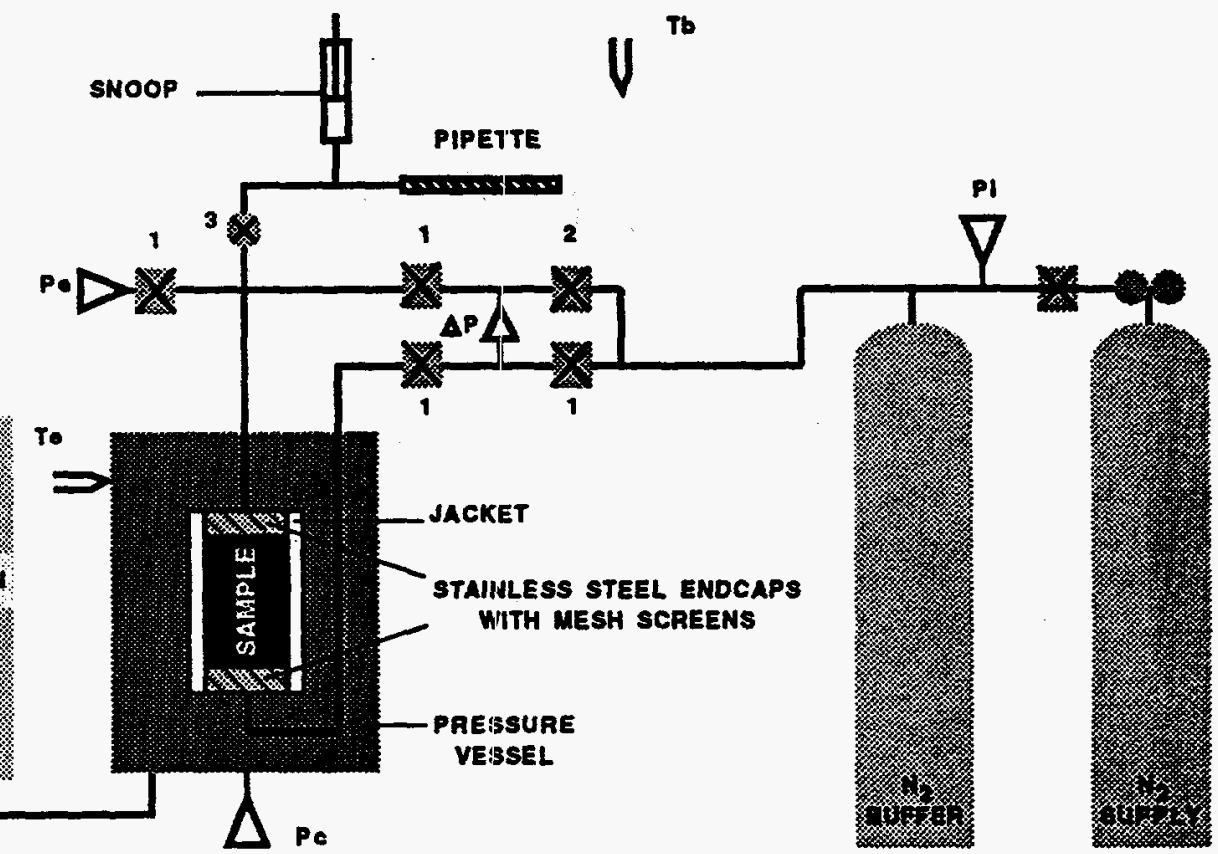

PI - INJECTION PRESSURE TRANSDUCER

Pe = EXIT PRESSURE TRAMSdiJcer

Pe = confInING PREsSURE tRansducea

AP * DIFFERENTIAL PRESSURE TRANSDUCER

To - VESSEL TEMPERATURE (IFLOW

Tb = ROOM TEMPERATURE (BASIS)
1 - isolation valve

2 - "shorting" valve (isolates INJECTION LINE FRON EXIT LINE)
3 - MICRO-METERING VALYE

Figure A2. Schematic of experimental system for gas permeability measurement. 


\section{LIQUID PERMEAMETER}

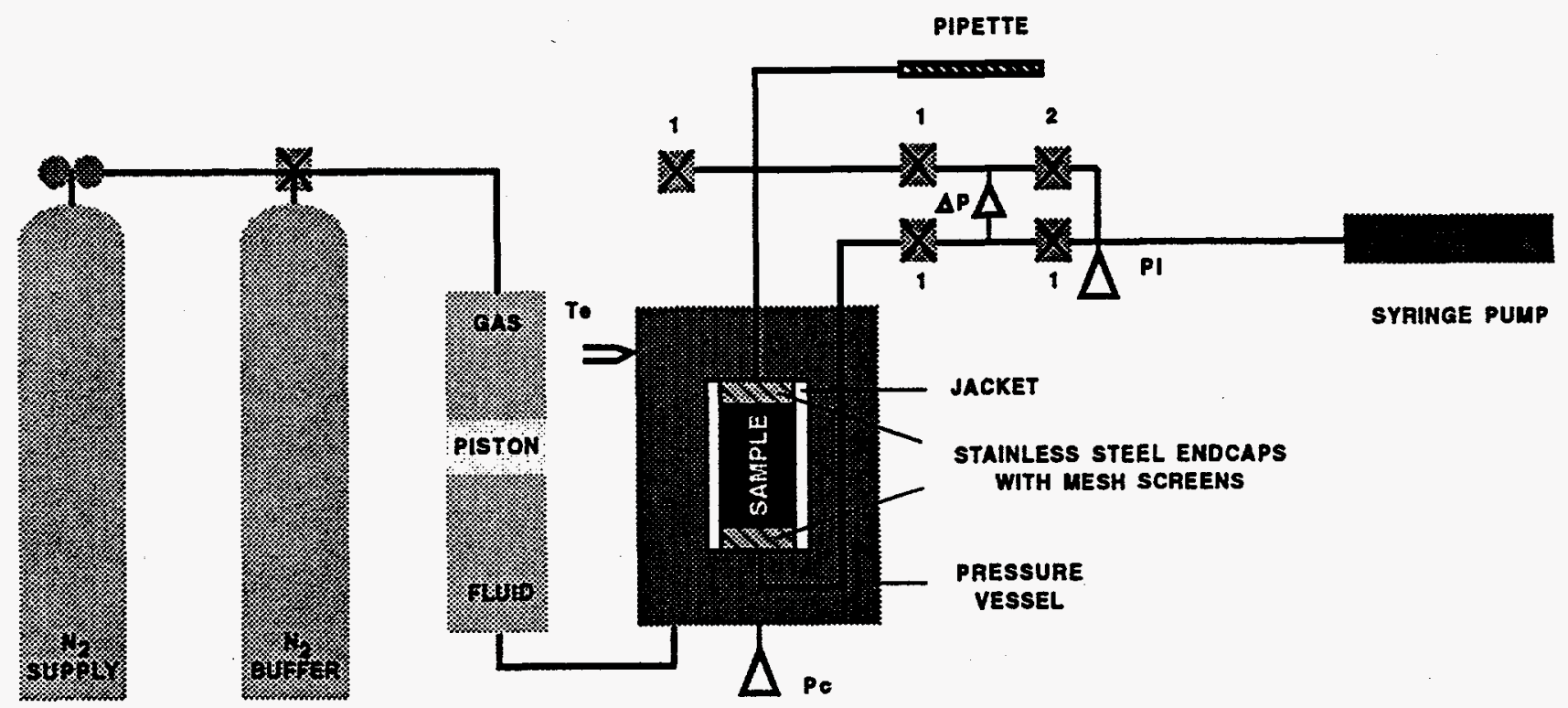

$\mathrm{N}_{2}=$ NITROGEN QAS

(STANDARD 304 CU. FT. BOTTLES)
PI = INJECTION PRESSURE TRANSDUCER

PC = CONFINING PRESSURE TRANSDUCER

$\Delta P=$ DIFFERENTIAL PAESSURE TRANSDUCER

Te = VESSEL TEMPERATUAE (FLOW)
1 = ISOLATION VALVE

2 a "ShoRtiNa" VALVE (ISOLATES WNECTION LINE FROU EXIT UNE)

3 = MICRO-METERING VALVE

Figure A3. Schematic of experimental system for liquid permeability measurement. 
to the National Institute of Standards and Technology. Calibration records consisting of certificates, data sheets, reports, and calibration schedules are naaintained. 


\section{APPENDIX C-B: Index of CT Cross-sectional Images from Whole Core Scan (E1X-08)}


Table B1. Index of CT Images for E1X08

\begin{tabular}{ll}
\hline Image No. & $\begin{array}{c}\text { Depth } \\
\text { (feet) }\end{array}$ \\
\hline 100005 & 2.706 \\
100015 & 2.805 \\
100025 & 2.903 \\
100035 & 3.002 \\
100045 & 3.100 \\
100055 & 3.198 \\
100065 & 3.297 \\
100110 & 3.686 \\
100140 & 3.981 \\
100170 & 4.277 \\
100200 & 4.572 \\
100230 & 4.867 \\
100260 & 5.163 \\
100290 & 5.458 \\
100320 & 5.753 \\
100357 & 6.029 \\
100367 & 6.128 \\
100377 & 6.226 \\
100400 & 6.452 \\
100410 & 6.551 \\
100420 & 6.649 \\
100430 & 6.747 \\
100440 & 6.846 \\
100450 & 6.944 \\
100460 & 7.043 \\
100470 & 7.141 \\
100480 & 7.240 \\
\hline &
\end{tabular}




\section{APPENDIX C-C: Drying History of Samples A-F \& EP1-8}



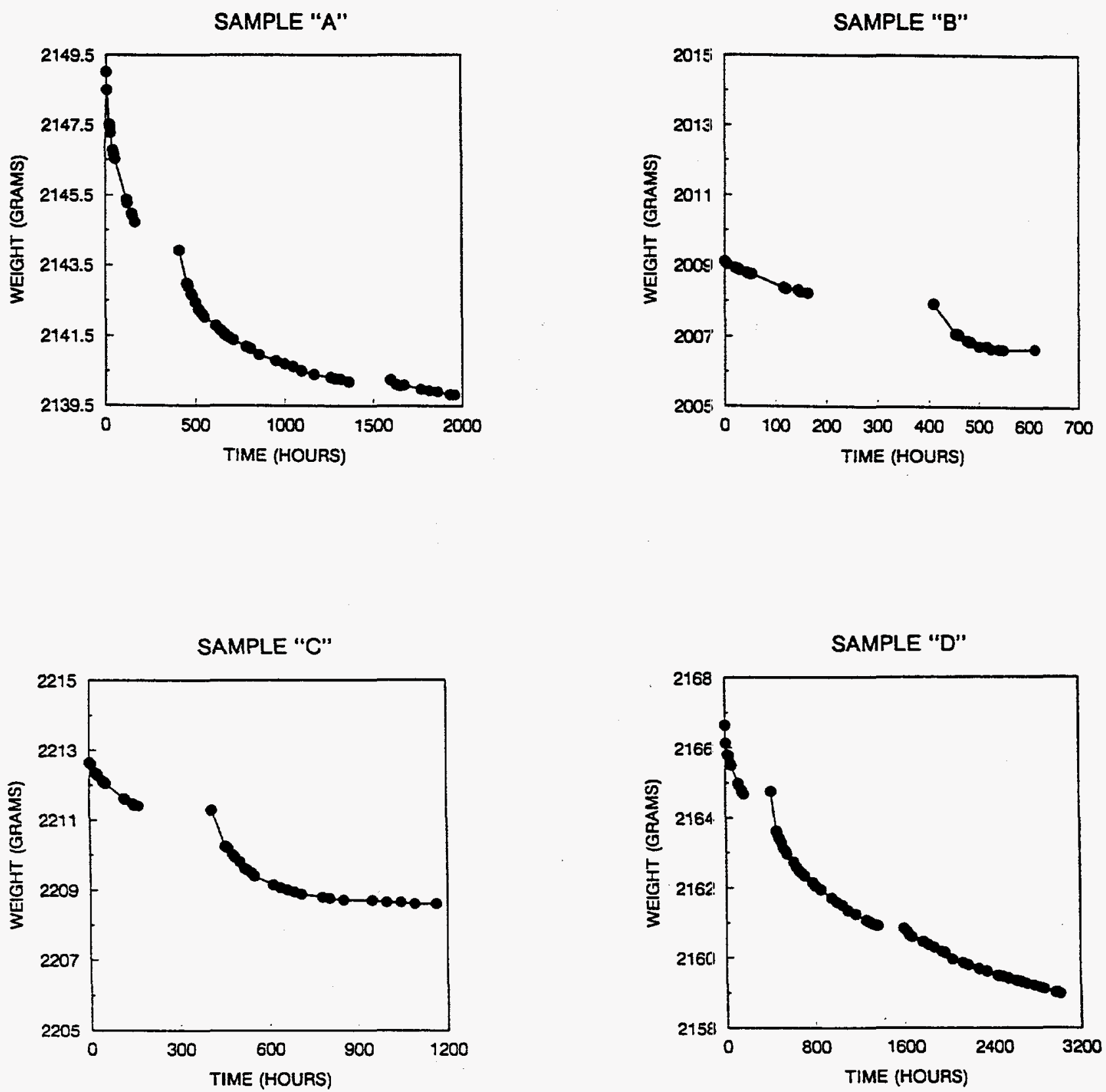

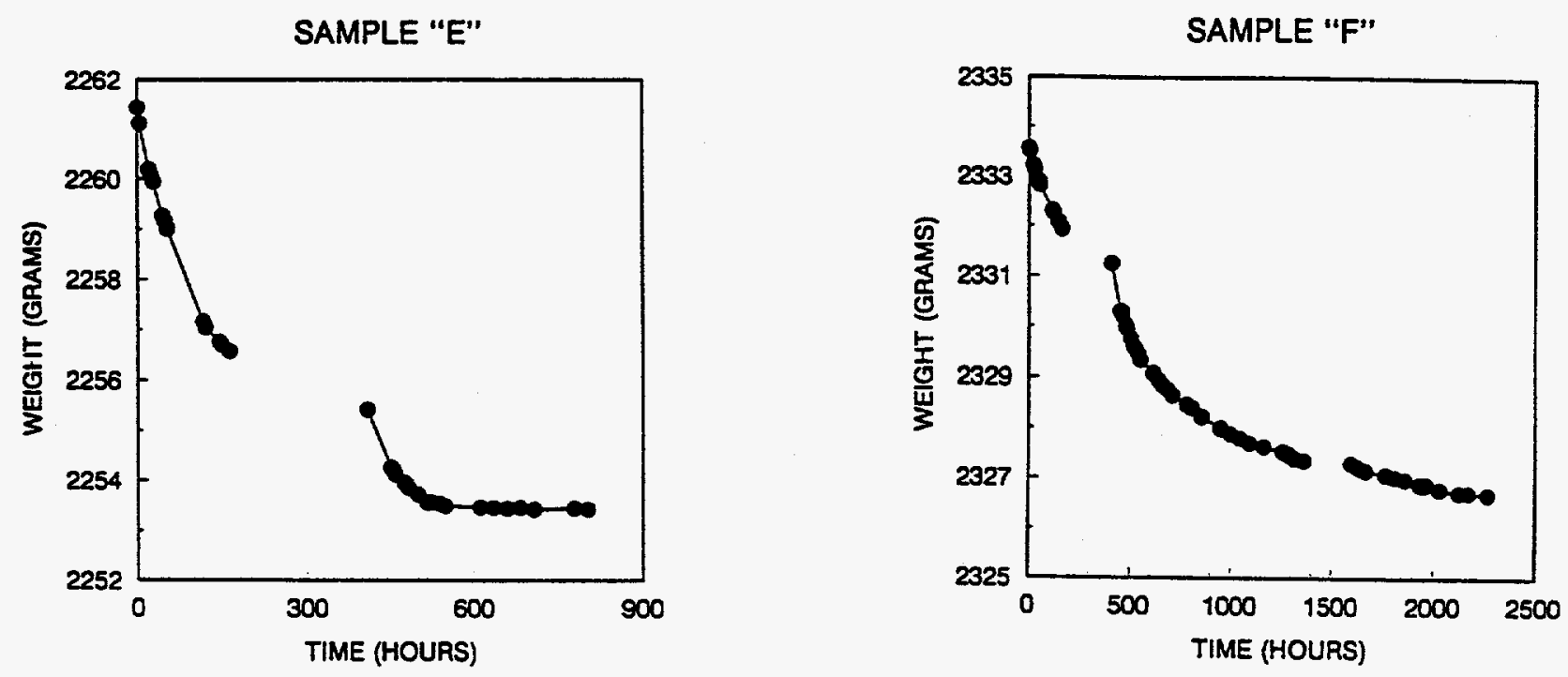

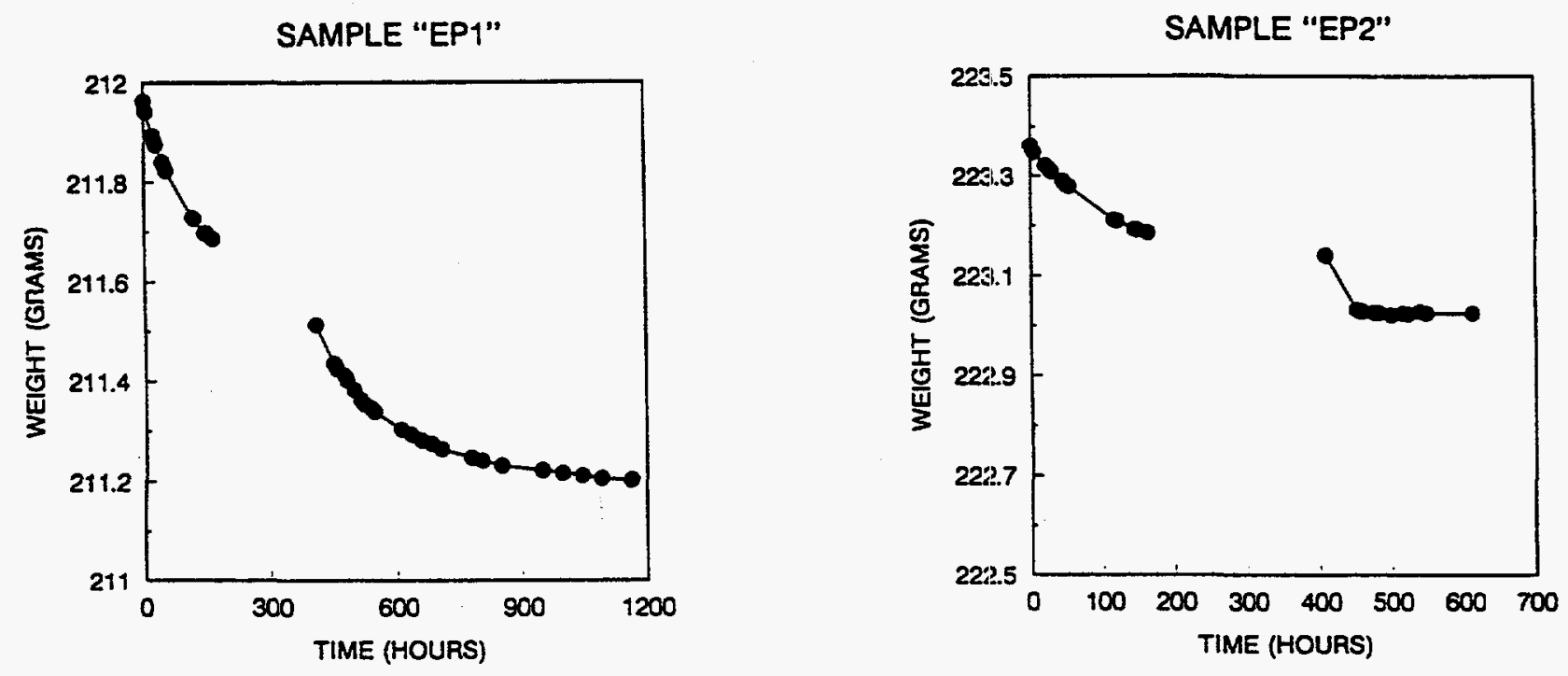

SAMPLE "EP3"
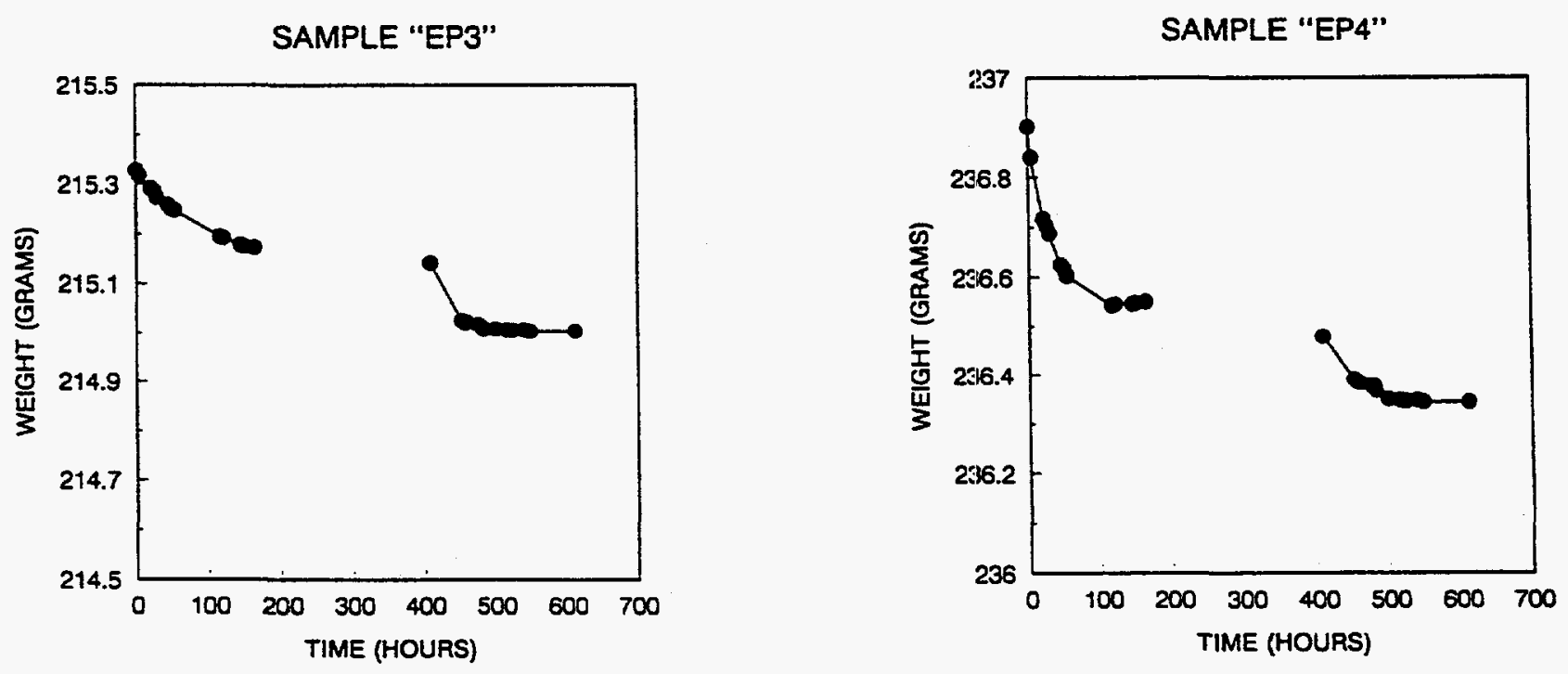
SAMPLE "EP5"

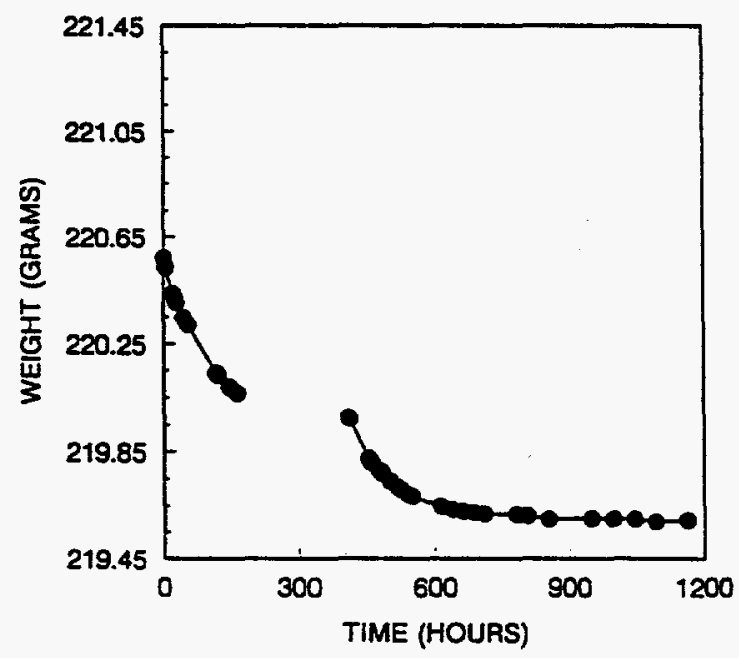

SAMPLE "EP7"

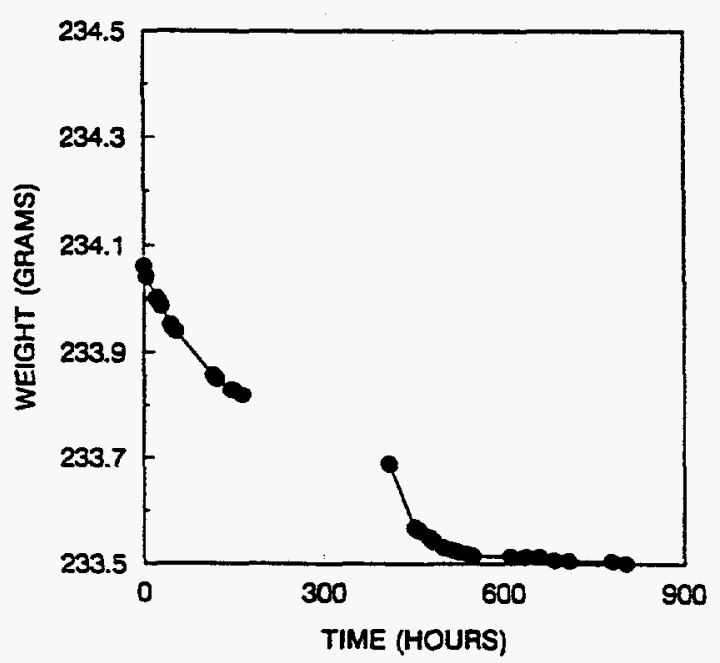

SAMPLE "EP6"

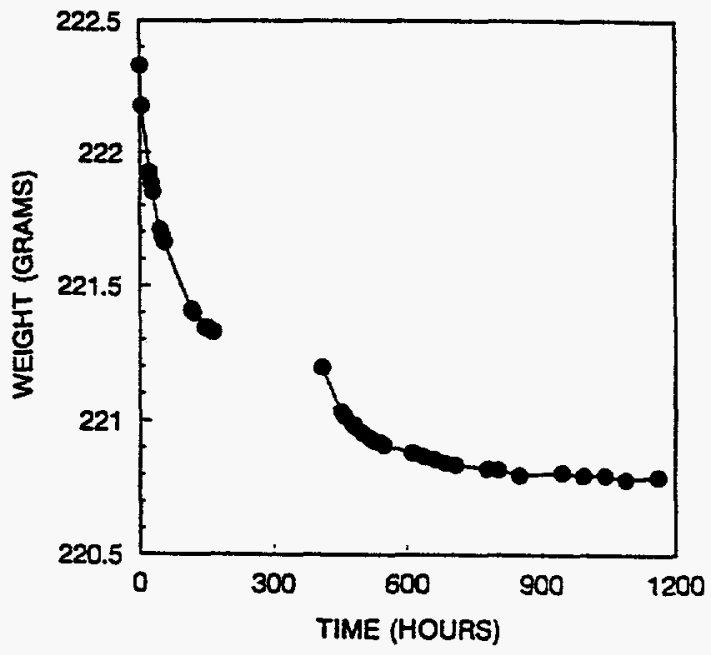

SAMPLE "EP8"

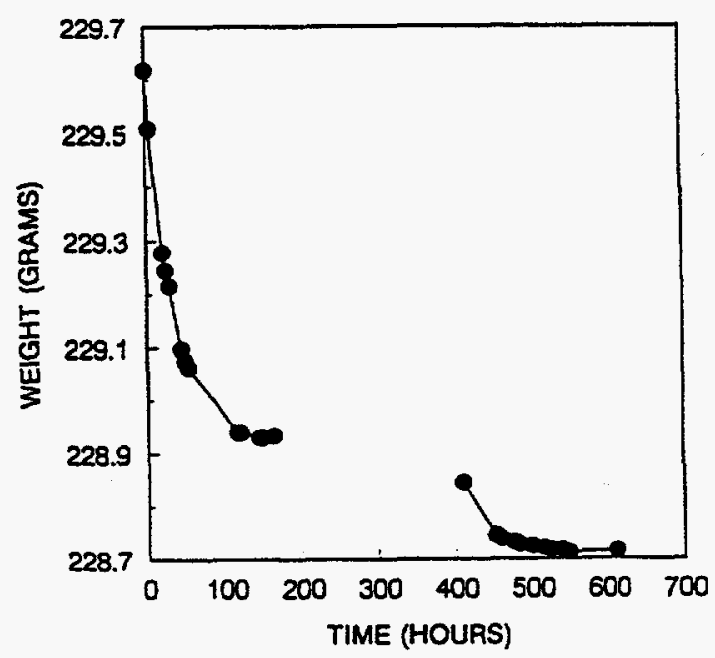


C-104 


\section{APPENDIX C-D: Gas Permeability Spreadsheets}




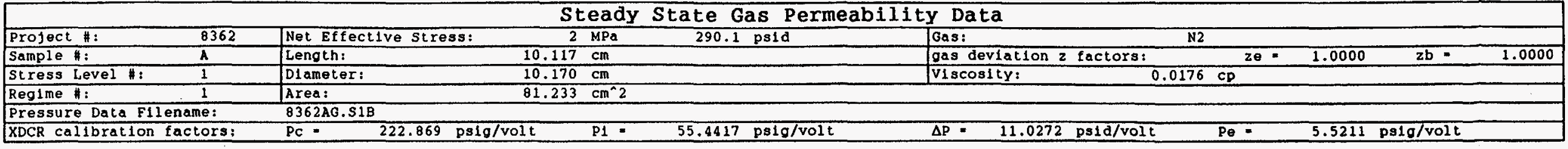

\begin{tabular}{|c|c|c|c|c|c|c|c|c|c|c|c|c|}
\hline Date & $\begin{array}{l}\text { Time } \\
\text { of } \\
\text { Day }\end{array}$ & $\begin{array}{l}\text { File } \\
\text { Time } \\
\text { (min) }\end{array}$ & $\underset{*}{\text { Reglme }}$ & \begin{tabular}{|l} 
Pb \\
Barometric \\
Pressure
\end{tabular} & $\begin{array}{l}\text { Pc } \\
\text { Confining } \\
\text { Pressure }\end{array}$ & $\begin{array}{l}\text { P1 } \\
\text { Inlet } \\
\text { Pressure }\end{array}$ & \begin{tabular}{|l}
$\Delta P$ \\
Differential \\
Pressure
\end{tabular} & $\begin{array}{l}\text { Pm } \\
\text { Mean pore } \\
\text { Pressure } \\
\text { Pe }+\Delta P / 2\end{array}$ & \begin{tabular}{|l} 
Pe \\
Ex1t \\
Pressure
\end{tabular} & $\begin{array}{l}\text { Te } \\
\text { Flow } \\
\text { Temp } \\
\left({ }^{\circ} \mathrm{C}\right)\end{array}$ & $\begin{array}{c}\text { Tb } \\
\text { Amblent } \\
\text { Temp } \\
\left({ }^{\circ} \mathrm{C}\right)\end{array}$ & $\begin{array}{c}\text { Qb } \\
\text { Flow Rate } \\
\text { opb\&Tb } \\
\text { (ml/sec) }\end{array}$ \\
\hline 26 May 93 & $12: 51$ & 108 & ASIR1a & 12.33 ps1a & volts & 0.870 volts & 3.874 volts & & volts & 23 & 22 & 0.05232 \\
\hline 26 May 93 & $12: 54$ & 108 & ASIR1b & $12.33 \mathrm{ps} 1 \mathrm{a}$ & volts & 0.870 & 3.874 volts & & volts & 23 & 22 & 0.05232 \\
\hline 26 May 93 & $12: 56$ & 113 & AS1R1C & $12.33 \mathrm{pgla}$ & 1.423 volts & 0.870 & 3.874 volts & & volts & 23 & 22 & 0.05219 \\
\hline 26 MaY 93 & $12: 58$ & 113 & AS1R1d & $12.33 \mathrm{ps} 1 \mathrm{a}$ & 1.423 vol ts & 0.870 & 3.874 volts & & volts & 23 & 22 & 0.05229 \\
\hline \multirow{9}{*}{ AVERAGES } & & & \multirow{9}{*}{ AS1R1 } & & OUAOE & OOUAOE & DIFEEREMT XAI & OQXGE: & GUAGE & (क) & (oc) & \\
\hline & & & & & 1.423 volts & 0.870 volts & 3.874 volts & & volts & \multirow{4}{*}{23} & \multirow{4}{*}{22} & \\
\hline & & & & & 317.1 & 48.23 & 42.72 ps1d & 27.20 ps1g & 5.836 & & & \\
\hline & & & & & 21.58 & 3.282 & 2.907 & $1.851 \mathrm{~atm}$ & 0.3971 & & & \\
\hline & & & & & 2.187 & 0.3326 & $0.2945 \quad \mathrm{Mpa}$ & $0.1875 \mathrm{Mpa}$ & 0.04024 & & & \\
\hline & & & & ABSOLUTE & ABSOLUTE & ABSOLUTE & DIFFERENT IL & \multicolumn{2}{|l|}{ ABSOLUTE } & (OK) & (OK) & (nT $/ \mathrm{sec}$ \\
\hline & & & & 12.33 psla & 329.5 psia & 60.56 pala & 42.72 ps1d & $39.53 \mathrm{ps} 1 \mathrm{a}$ & 18.17 & \multirow{3}{*}{296} & \multirow{3}{*}{295} & \multirow{3}{*}{0.05228} \\
\hline & & & & $0.8390 \mathrm{~atm}$ & 22.42 & 4.121 & 2.907 & 2.690 atm & 1.236 & & & \\
\hline & & & & 0.08501 & 2.272 & 0.4176 & 0.2945 & 0.2725 & 0.1252 & & & \\
\hline
\end{tabular}

8

Apparent gas permeab1l1ty:

$K a=\left(v e^{\star P e}{ }^{\star} u^{\star L}\right) /(P m \star \Delta P)$

Boyle's Law:

$\mathrm{Ve}=(\mathrm{Pb} / \mathrm{Pe}) *(\mathrm{Te} / \mathrm{Tb}) *(\mathrm{ze} / \mathrm{zb}) * \mathrm{Vb}$

$\Omega=(D b / D e) *(T e / T b) *(z e / z b) * o b$

$v e=Q e / A=(\mathrm{Pb} / \mathrm{Pe}) *(\mathrm{Te} / \mathrm{Tb}) \cdot(\mathrm{ze} / \mathrm{zb}) *(\mathrm{Qb} / \mathrm{A})$

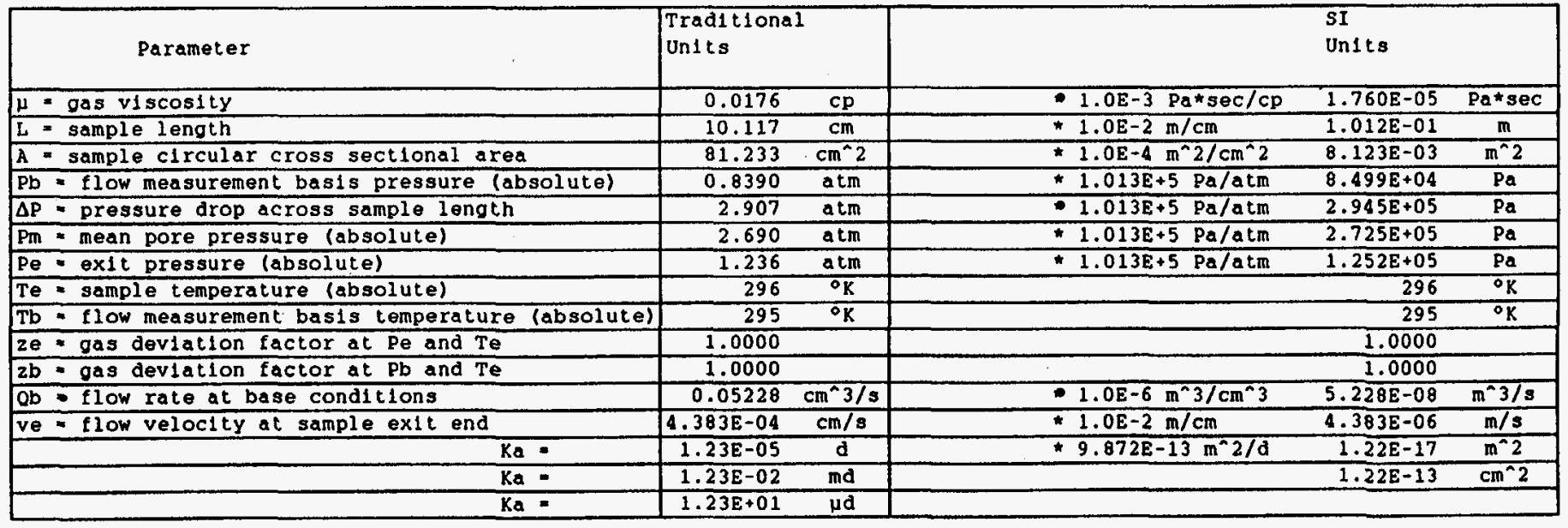


Steady State Gas Permeability Data

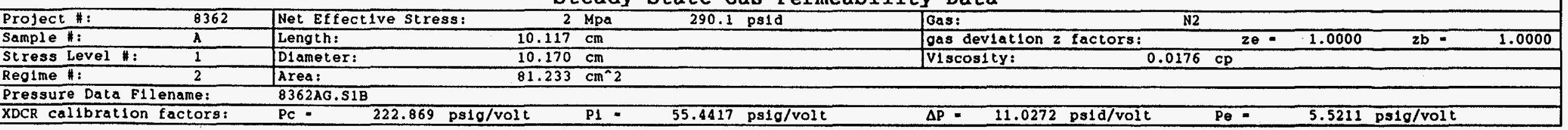

\begin{tabular}{|c|c|c|c|c|c|c|c|c|c|c|c|c|}
\hline Date & $\begin{array}{l}\text { Time } \\
\text { of } \\
\text { Day }\end{array}$ & $\begin{array}{l}\text { File } \\
\text { Time } \\
\text { (min) }\end{array}$ & $\begin{array}{c}\text { Reglme } \\
\#\end{array}$ & $\begin{array}{l}\text { Pb } \\
\text { Barometric } \\
\text { Pressure }\end{array}$ & $\begin{array}{l}\text { Pc } \\
\text { Confining } \\
\text { Pressure }\end{array}$ & $\begin{array}{l}\text { P1 } \\
\text { Inlet } \\
\text { Pressure }\end{array}$ & $\begin{array}{l}\Delta P \\
D 1 f \text { ferentlal } \\
\text { Pressure }\end{array}$ & $\begin{array}{l}\text { Pm } \\
\text { Mean Pore } \\
\text { Pressure } \\
\text { Pe+ } \Delta P / 2\end{array}$ & $\begin{array}{l}\text { Pe } \\
\text { Ex1t } \\
\text { Pressure }\end{array}$ & $\begin{array}{l}\text { Te } \\
\text { Flow } \\
\text { Temp } \\
\left({ }^{\circ} \mathrm{C}\right)\end{array}$ & $\begin{array}{c}\text { Tb } \\
\text { Amblent } \\
\text { Temp } \\
\left({ }^{\circ} \mathrm{C}\right)\end{array}$ & $\begin{array}{c}\text { Qb } \\
\text { Flow Rate } \\
\text { OPb\&Tb } \\
\text { (ml/sec) }\end{array}$ \\
\hline 26 May 93 & $15: 41$ & 278 & AS1R2a & $12.30 \mathrm{psta}$ & volts & volts & 3.851 volts & & volts & 23 & 23 & 0.06131 \\
\hline 26 May 93 & $15: 45$ & 283 & AS1R2b & $12.30 \mathrm{ps} 1 \mathrm{a}$ & volts & volts & 3.851 volts & & rolts & 23 & $\frac{5}{23}$ & 0.06122 \\
\hline 26 May 93 & $15: 48$ & 283 & AS1R2C & $12.30 \mathrm{ps} 1 \mathrm{a}$ & volts & volts & 3.851 volts & & volts & 23 & 23 & 0.06125 \\
\hline 26 MaY 93 & $15: 50$ & 288 & AS1R2d & 12.30 ps1a & volts & volts & 3.851 volts & & volts & 23 & 23 & 0.06133 \\
\hline \multirow{9}{*}{ AVERAGES } & & & \multirow{9}{*}{ AS1R2 } & & OUUAGE & GUAGD & DTEFEREKTM & OUXGE & OUROE & क्वा & क्टा & \\
\hline & & & & & 1.466 volts & 1.045 volts & 3.851 volts & & 2.872 & \multirow{4}{*}{23} & \multirow{4}{*}{23} & \\
\hline & & & & & 326.6 & 57.94 & 42.47 psid & 37.09 ps1g & 15.854 & & & \\
\hline & & & & & 22.22 & 3.942 & 2.890 & 2.524 atm & 1.0788 & & & \\
\hline & & & & & 2.252 & 0.3995 & 0.2928 & 0.2557 & 0.10931 & & & \\
\hline & & & & ABSOLOTE & ABSOLOTE & ABSOLUTE & DREEERENTIAL & AESOLUTE & ABSOLUTE & (ox) & (OK) & (DFoc) \\
\hline & & & & $12.3 \mathrm{ps} 1 \mathrm{a}$ & 338.9 ps1a & 70.24 ps1a & 42.47 psid & $49.39 \mathrm{pgla}$ & 28.15 & \multirow{3}{*}{296} & \multirow{3}{*}{296} & \multirow{3}{*}{0.06128} \\
\hline & & & & 0.8370 atm & 23.06 & 4.779 & 2.890 & $3.361 \mathrm{~atm}$ & 1.916 & & & \\
\hline & & & & 0.08481 & 2.337 & 0.4843 & 0.2928 & 0.3405 & 0.1941 & & & \\
\hline
\end{tabular}

\section{Apparent gas permeab111ty: $\quad K a=\left(v e^{\star P} e^{\star} u^{\star L} L\right) /(P m \star \Delta P)$}

Boyle's Law

$\mathrm{Ve}=(\mathrm{Pb} / \mathrm{Pe}) *(\mathrm{Te} / \mathrm{Tb}) *(\mathrm{ze} / \mathrm{zb}) * \mathrm{Vb}$

$\mathrm{Qe}^{-}(\mathrm{Pb} / \mathrm{Pe}) *(\mathrm{Te} / \mathrm{Tb}) \cdot(2 \mathrm{e} / \mathrm{zb}) * \mathrm{Qb}$

$v_{e}=Q e / A=(\mathrm{Pb} / \mathrm{Pe}) *(\mathrm{Te} / \mathrm{Tb}) *(2 \mathrm{e} / 2 \mathrm{~b}) *(\mathrm{Ob} / \mathrm{A})$

\begin{tabular}{|c|c|c|c|c|}
\hline Parameter & $\begin{array}{l}\text { Traditlonal } \\
\text { Units }\end{array}$ & & $\begin{array}{l}\text { SI } \\
\text { Units }\end{array}$ & \\
\hline$\mu=g a s$ viscosity & 0.0176 & *1.0E-3 Pa*sec/cp & $1.760 \mathrm{E}-05$ & $\mathrm{~Pa} * \mathrm{sec}$ \\
\hline L = sample length & 10.117 & $\approx 1.0 \mathrm{E}-2 \mathrm{~m} / \mathrm{cm}$ & $1.012 \mathrm{E}-01$ & $\bar{m}$ \\
\hline$A=$ sample clrcular cross sectional area & 81.233 & $\pitchfork 1.0 \mathrm{E}-4 \mathrm{~m}^{\wedge} 2 / \mathrm{cm}^{\wedge} 2$ & $8.123 \mathrm{E}-03$ & $m^{\star 2} 2$ \\
\hline $\mathrm{Pb}=$ flow measurement basls pressure (absolute) & 0.8370 & $\star 1.013 \mathrm{E}+5 \mathrm{~Pa} / \mathrm{atm}$ & $8.478 E+04$ & $\mathrm{~Pa}$ \\
\hline$\Delta \mathrm{P}=$ pressure drop across sample length & 2.890 & $\$ 1.013 \mathrm{E}+5 \mathrm{~Pa} / \mathrm{atm}$ & $2.927 \varepsilon+05$ & $\mathrm{~Pa}$ \\
\hline $\mathrm{Pm}=$ mean pore pressure (absolute) & 3.361 & $\star 1.013 \mathrm{E}+5 \mathrm{~Pa} / \mathrm{atm}$ & $3.404 E+05$ & $\mathrm{~Pa}$ \\
\hline $\mathrm{Pe}=$ exit pressure (absolute) & 1.916 & $-1.013 \mathrm{E}+5 \mathrm{~Pa} / \mathrm{atm}$ & $1.941 \mathrm{E}+05$ & Pa \\
\hline Te = sample temperature (absolute) & 296 & & 296 & ${ }^{\circ} \mathrm{K}$ \\
\hline $\mathrm{Tb}=$ flow measurement basls temperature (absolute) & 296 & & 296 & ${ }^{\circ} \mathbf{K}$ \\
\hline $2 e=$ gas devlation factor at $\mathrm{Pe}$ and $\mathrm{Te}$ & 1.0000 & & 1.0000 & \\
\hline $\mathrm{zb}$ - gas deviation factor $\mathrm{at} \mathrm{Pb}$ and $\mathrm{Te}$ & 1.0000 & & 1.0000 & \\
\hline $\mathrm{Qb}$ - flow rale at base conditions & 0.06128 & $\star 1.0 \mathrm{E}-6 \mathrm{~m}^{\wedge} 3 / \mathrm{cm}^{\wedge} 3$ & $6.128 E-08$ & $\mathrm{~m} \wedge^{\wedge} 3 / \mathrm{s}$ \\
\hline ve $=$ flow velocity at sample exit end & $3.296 \mathrm{E}-04$ & $1.0 \mathrm{E}-2 \mathrm{~m} / \mathrm{cm}$ & $3.296 \mathrm{E}-06$ & $\mathrm{~m} / \mathrm{s}$ \\
\hline $\mathrm{Ka}=$ & $1.16 \mathrm{E}-05$ & $* 9.872 \mathrm{E}-13 \mathrm{~m} 2 / \mathrm{d}$ & $1.14 \mathrm{E}-17$ & $m^{\wedge} 2$ \\
\hline $\mathrm{Ka}=$ & $1.16 \mathrm{E}-02$ & & $1.14 \mathrm{E}-13$ & $\mathrm{~cm}^{n} 2$ \\
\hline Ka * & $1.16 \mathrm{E}+01$ & & & \\
\hline
\end{tabular}




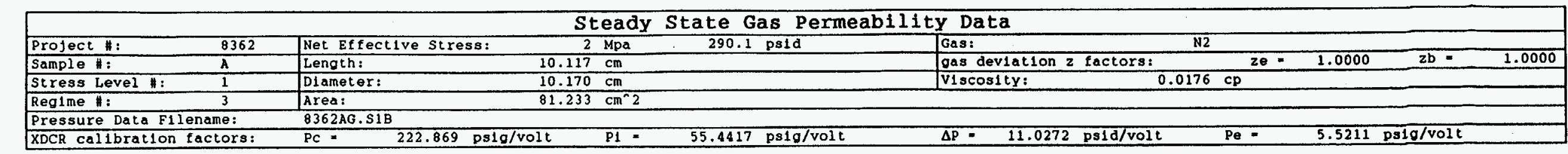

\begin{tabular}{|c|c|c|c|c|c|c|c|c|c|c|c|c|}
\hline Date & $\begin{array}{l}\text { Time } \\
\text { of } \\
\text { Day }\end{array}$ & $\begin{array}{l}\text { File } \\
\text { Time } \\
(m 1 n)\end{array}$ & $\begin{array}{c}\text { Reglme } \\
*\end{array}$ & $\begin{array}{l}\mathrm{Pb} \\
\text { Barometr1c } \\
\text { Pressure }\end{array}$ & $\begin{array}{l}\text { Pc } \\
\text { Confining } \\
\text { Pressure }\end{array}$ & $\begin{array}{l}\text { P1 } \\
\text { Inlet } \\
\text { Pressure }\end{array}$ & $\begin{array}{l}\Delta \mathrm{P} \\
\mathrm{D} 1 \text { fferentlal } \\
\text { Pressure }\end{array}$ & $\begin{array}{l}\text { Pm } \\
\text { Mean Pore } \\
\text { Pressure } \\
\text { Pe }+\Delta P / 2\end{array}$ & $\begin{array}{l}\text { Pe } \\
\text { Ex1t } \\
\text { Pressure }\end{array}$ & $\begin{array}{l}\text { Te } \\
\text { Flow } \\
\text { Temp } \\
\left({ }^{\circ} \mathrm{C}\right)\end{array}$ & $\begin{array}{c}\text { Tb } \\
\text { Amblent } \\
\text { Temp } \\
\left({ }^{\circ} \mathrm{C}\right)\end{array}$ & $\begin{array}{c}\text { Qb } \\
\text { Flow Rate } \\
\text { OPb\&Tb } \\
\text { (ml/sec) }\end{array}$ \\
\hline 27 May 93 & $10: 51$ & 1428 & AS1R3a & 12.30 psia & volts & volts & 3.904 volts & & volts & 23 & 22 & 0.07055 \\
\hline 27 May 93 & $10: 54$ & 1428 & AS1R3b & 12.30 psla & volts & volts & 3.905 volts & & volts & 23 & 22 & 0.07050 \\
\hline 27 May 93 & $10: 57$ & 1433 & AS1R3C & $12.30 \quad$ p91a & volts & volts & 3.905 vol ts & & volts & 23 & 22 & 0.07053 \\
\hline 27 May 93 & $10: 59$ & 1433 & ASIR3d & $12.30 \mathrm{ps} 1 \mathrm{a}$ & 1.512 volts & volts & 3.905 volts & & volts & 23 & 22 & 0.07056 \\
\hline \multirow{9}{*}{ AVERAGES } & & & \multirow{9}{*}{ AS1R3 } & & GUAAE & OUAGE & DIFFERENTIALI & OOAGE & SUROE & (c) & (6्c) & \\
\hline & & & & & 1.512 volts & 1.228 voltg & 3.905 volts & & 4.618 & \multirow{4}{*}{23} & \multirow{4}{*}{22} & \\
\hline & & & & & $337.0 \quad \mathrm{ps1g}$ & 68.08 & 43.06 psid & $47.03 \quad \mathrm{psig}$ & 25.496 & & & \\
\hline & & & & & 22.93 & 4.633 & 2.930 & 3.200 atm & 1.7349 & & & \\
\hline & & & & & $2.323 \quad \mathrm{Mpa}$ & 0.4694 & $0.2969 \quad \mathrm{Mpa}$ & $0.3242 \quad \mathrm{Mpa}$ & 0.17579 & & & \\
\hline & & & & ABSOLUTE & ABSOLUTE & ABSOLUTE $\bigcirc$ & DFEFERENTHAL & \multirow{2}{*}{\multicolumn{2}{|c|}{ TASOLUTRE }} & (OK) & $8 \mathrm{x}$ & (nI/soc) \\
\hline & & & & $12.3 \mathrm{psla}$ & 349.3 psia & $80.38 \quad \mathrm{ps} 1 \mathrm{a}$ & $43.06 \mathrm{ps1d}$ & $59.33 \mathrm{ps} 1 \mathrm{a}$ & & \multirow{3}{*}{296} & \multirow{3}{*}{295} & \multirow{3}{*}{0.07053} \\
\hline & & & & 0.8370 atm & 23.77 & 5.470 & 2.930 & $4.037 \mathrm{~atm}$ & 2.572 & & & \\
\hline & & & & $0.08481 \mathrm{MPa}$ & 2.408 & 0.5542 & 0.2969 & 0.4090 & 0.2606 & & & \\
\hline
\end{tabular}

\begin{abstract}
Apparent gas permeabillty: $K a=\left(v e^{*} P e^{*} u^{* L}\right) /(P m \star \Delta P)$
\end{abstract}
Boyle's Law:

$\mathrm{Ve}=(\mathrm{Pb} / \mathrm{Pe}) \cdot(\mathrm{Te} / \mathrm{Tb}) *(\mathrm{ze} / \mathrm{zb}) \cdot \mathrm{Vb}$

$\mathrm{Qe}=(\mathrm{Pb} / \mathrm{Pe}) *(\mathrm{Te} / \mathrm{Tb}) *(z e / 2 \mathrm{~b}) * \mathrm{Qb}$

ve $=Q \mathrm{Qe} / \mathrm{A}=(\mathrm{Pb} / \mathrm{Pe}) *(\mathrm{Te} / \mathrm{Tb}) *(2 \mathrm{e} / 2 \mathrm{C}) *(\mathrm{Qb} / \mathrm{A})$

\begin{tabular}{|c|c|c|c|c|}
\hline Parameter & \begin{tabular}{|l} 
Traditional \\
Units
\end{tabular} & & $\begin{array}{l}\text { SI } \\
\text { Units }\end{array}$ & \\
\hline$\mu=\operatorname{gas} v 1 \operatorname{scos} 1 \mathrm{t} y$ & 0.0176 & $\star 1.0 \mathrm{E}-3 \mathrm{~Pa} * \mathrm{sec} / \mathrm{cp}$ & $1.760 \mathrm{E}-05$ & Pa*sec \\
\hline$L=$ sample length & 10.117 & $1.0 \mathrm{E}-2 \mathrm{~m} / \mathrm{cm}$ & $1.012 \mathrm{E}-01$ & $\mathrm{~m}$ \\
\hline A - sample clrcular cross sectional area & 81.233 & $* 1.0 \mathrm{E}-4 \mathrm{~m}^{\wedge} 2 / \mathrm{cm}^{\wedge} 2$ & $8.123 \mathrm{E}-03$ & $m^{\wedge} 2$ \\
\hline $\mathrm{pb}=$ flow measurement bas1s pressure (absolute) & 0.8370 & $\approx 1.013 \mathrm{E}+5 \mathrm{~Pa} / \mathrm{atm}$ & $8.478 \mathrm{E}+04$ & $\mathrm{~Pa}$ \\
\hline$\Delta \mathrm{P}=$ pressure drop across sample length & 2.930 & $1.013 \mathrm{E}+5 \mathrm{~Pa} / \mathrm{atm}$ & $2.968 \mathrm{E}+05$ & Pa \\
\hline$P_{m}=$ mean pore pressure (absolute) & 4.037 & * $1.013 \mathrm{E}+5 \mathrm{~Pa} / \mathrm{atm}$ & $4.089 E+05$ & $\mathrm{~Pa}$ \\
\hline $\mathrm{Pe}=$ exit pressure (absolute) & 2.572 & $1.013 \mathrm{E}+5 \mathrm{~Pa} / \mathrm{atm}$ & $2.605 \mathrm{E}+05$ & Pa \\
\hline $\mathrm{Te}=$ sample temperature (absolute) & 296 & & 296 & ${ }^{\circ} \mathrm{K}$ \\
\hline $\mathrm{Tb}=$ flow measurement basis temperature (absolute) & 295 & & 295 & ${ }^{\circ} \mathrm{K}$ \\
\hline$z e=$ gas deviation factor at Pe and $\mathrm{Te}$ & 1.0000 & & 1.0000 & \\
\hline $\mathrm{zb}$ - gas deviation factor at $\mathrm{Pb}$ and $\mathrm{Te}$ & 1.0000 & & 1.0000 & \\
\hline$a b=$ flow rate at base conditions & $0.07053 \mathrm{~cm}^{\wedge} 3 / \mathrm{s}$ & $1.0 \mathrm{E}-6 \mathrm{~m}^{n} 3 / \mathrm{cm}^{2} 3$ & $7.053 \mathrm{E}-08$ & $m^{2} 3 / s$ \\
\hline ve = flow velocity at sample exit end & $2.835 \mathrm{E}-04 \mathrm{~cm} / \mathrm{s}$ & $1.0 \mathrm{E}-2 \mathrm{~m} / \mathrm{cm}$ & $2.835 \mathrm{E}-06$ & $\mathrm{~m} / \mathrm{s}$ \\
\hline $\mathrm{Ka}=$ & $1.10 \mathrm{E}-05$ & $9.872 \mathrm{E}-13 \mathrm{~m}^{2} 2 / \mathrm{d}$ & $1.08 E-17$ & $m^{\wedge} 2$ \\
\hline Ka - & $1.10 E-02$ & & $1.08 E-13$ & $\mathrm{~cm}^{2} 2$ \\
\hline $\mathrm{Ka}=$ & $1.10 \mathrm{E}+01$ & & & \\
\hline
\end{tabular}


Steady State Gas Permeability Data

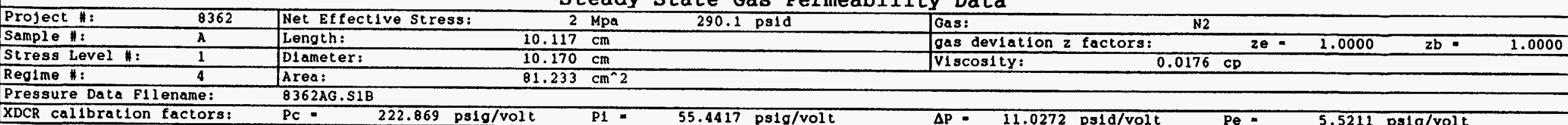

\begin{tabular}{|c|c|c|c|c|c|c|c|c|c|c|c|c|}
\hline Date & $\begin{array}{l}\text { Time } \\
\text { of } \\
\text { Day }\end{array}$ & $\begin{array}{l}\text { File } \\
\text { Time } \\
(m 1 n)\end{array}$ & $\begin{array}{c}\text { Regime } \\
\|\end{array}$ & $\begin{array}{l}\text { Pb } \\
\text { Barometric } \\
\text { Pressure }\end{array}$ & $\begin{array}{l}\text { Pc } \\
\text { Confining } \\
\text { Pressure }\end{array}$ & $\begin{array}{l}\text { Pi } \\
\text { Inlet } \\
\text { Pressure }\end{array}$ & $\begin{array}{l}\Delta P \\
\text { Differential } \\
\text { Pressure }\end{array}$ & $\begin{array}{l}\text { Pm } \\
\text { Mean Pore } \\
\text { Pressure } \\
\text { Pe }+\Delta \mathrm{P} / 2\end{array}$ & $\begin{array}{l}\text { Pe } \\
\text { Ex1t } \\
\text { Pressure }\end{array}$ & $\begin{array}{l}\text { Te } \\
\text { Flow } \\
\text { Temp } \\
\left({ }^{\circ} \mathrm{C}\right)\end{array}$ & $\begin{array}{c}\text { Tb } \\
\text { Amb1 ent } \\
\text { Temp } \\
\left({ }^{\circ} \mathrm{C}\right)\end{array}$ & $\begin{array}{c}\text { Ob } \\
\text { Flow Rate } \\
\text { OPb ETb } \\
\text { (m1/sec) }\end{array}$ \\
\hline 27 MaY 93 & $14: 50$ & 1668 & AS1R4a & 12.29 ps1a & 1.558 & volts & 3.920 volts & & volts & 23 & 23 & 0.08047 \\
\hline 27 MaY 93 & $14: 53$ & 1668 & AS1R4b & 12.29 psta & volts & volts & 3.920 volts & & volts & 23 & 23 & 0.08060 \\
\hline 27 May 93 & $14: 55$ & 1673 & AS1R4C & $12.29 \mathrm{ps} 1 \mathrm{a}$ & volts & 1.409 & 3.920 volts & & volts & 23 & 23 & 0.08053 \\
\hline $27 \operatorname{May} 93$ & $14: 57$ & 1673 & AS1RGd & 12.29 ps1a & 1.559 volts & 1.409 volts & 3.920 volts & & volts & 23 & 23 & 0.08053 \\
\hline \multirow{9}{*}{ AVERAGES } & & & \multirow{9}{*}{ AS1R4 } & & GUAGE & OUURAE & DIFFERENTI XIP & SUXGE & GUAिOE & का & (क्C) & \\
\hline & & & & & 1.559 volts & 1.409 volts & 3.920 volts & & 6.415 & \multirow{4}{*}{23} & \multirow{4}{*}{23} & \\
\hline & & & & & 347.3 & 78.12 & 43.23 ps1d & $57.03 \mathrm{psig}$ & 35.415 & & & \\
\hline & & & & & 23.64 & 5.316 & 2.941 & $3.881 \mathrm{~atm}$ & 2.4098 & & & \\
\hline & & & & & $2.395 \quad \mathrm{Mpa}$ & 0.5386 & $0.2980 \quad \mathrm{Mpa}$ & $0.3932 \mathrm{Mpa}$ & 0.24418 & & & \\
\hline & & & & ABSOLUTE & ABSOLUTE & AESOLUTE & DIFFERENTIXI & \multirow{2}{*}{\multicolumn{2}{|c|}{$\frac{\text { ABSOLUTE }}{69.32 \text { psla }}$}} & (OK) & (\%k) & $(\mathrm{m} 17 \mathrm{sec})$ \\
\hline & & & & $12.29 \quad \mathrm{Ds} 1 \mathrm{a}$ & 359.6 ps1a & $90.41 \quad$ ps1a & $43.23 \quad$ ps1d & & & \multirow{3}{*}{296} & \multirow{3}{*}{296} & \multirow{3}{*}{0.08053} \\
\hline & & & & $0.8363 \mathrm{~atm}$ & 24.47 & 6.152 & 2.941 & 4.717 atm & 3.246 & & & \\
\hline & & & & 0.08474 & 2.480 & 0.5233 & 0.2980 & 0.4779 & 0.3289 & & & \\
\hline
\end{tabular}

Apparent gas permeab111ty: Ka $=\left(v^{*} e^{\star} U^{\star} L\right) /(P m * \Delta P)$

Boyle's Law:

$V e=(\mathrm{Pb} / \mathrm{Pe}) *(\mathrm{Te} / \mathrm{Tb}) *(2 \mathrm{ze} / \mathrm{Zb}) * \mathrm{Vb}$

$\mathrm{Qe} *(\mathrm{~Pb} / \mathrm{Pe}) *(\mathrm{Te} / \mathrm{Tb}) *(2 \mathrm{e} / \mathrm{Zb}) * \mathrm{Qb}$

ve $\mathrm{Qe}^{\mathrm{A}}=(\mathrm{Pb} / \mathrm{Pe}) *(\mathrm{Te} / \mathrm{Tb}) *(\mathrm{ze} / \mathrm{zb}) *(\mathrm{Qb} / \mathrm{A})$

\begin{tabular}{|c|c|c|c|c|}
\hline Parameter & $\begin{array}{l}\text { Traditional } \\
\text { Units }\end{array}$ & & $\begin{array}{l}\text { SI } \\
\text { Un1ts }\end{array}$ & \\
\hline$\mu=$ gas viscosity & 0.0176 & $\star 1.0 \mathrm{E}-3 \mathrm{~Pa} \mathrm{SeC}_{\mathrm{S}} \mathrm{Cp}$ & $1.760 E-05$ & Pa\#sec \\
\hline$L$ = sample length & 10.117 & $1.0 \mathrm{E}-2 \mathrm{~m} / \mathrm{cm}$ & $1.012 \mathrm{E}-01$ & $\mathrm{~m}$ \\
\hline A - sample clrcular cross sectional area & 81.233 & $1.0 \mathrm{E}-4 \mathrm{~m}^{\wedge} 2 / \mathrm{cm}^{\wedge} 2$ & $8.123 \mathrm{E}-03$ & $\mathbf{m}^{\wedge} 2$ \\
\hline $\mathrm{Pb}=$ flow measurement basis pressure (absolute) & 0.8363 & $1.013 \mathrm{E}+5 \mathrm{~Pa} / \mathrm{atm}$ & $8.472 E+04$ & Pa \\
\hline$\Delta \mathrm{P}=$ pressure drop across sample length & 2.941 & $* 1.013 \mathrm{E}+5 \mathrm{~Pa} / \mathrm{atm}$ & $2.980 \mathrm{E}+05$ & Pa \\
\hline $\mathrm{Pm}=$ mean pore pressure (absolute) & 4.717 & $\star 1.013 \mathrm{E}+5 \mathrm{~Pa} / \mathrm{atm}$ & $4.778 E+05$ & $\mathrm{~Pa}$ \\
\hline$P_{e}=$ exit pressure (absolute) & 3.246 & $1.013 \mathrm{E}+5 \mathrm{~Pa} / \mathrm{atm}$ & $3.288 \mathrm{E}+05$ & Pa \\
\hline Te = sample temperature (absolute) & 296 & & 296 & ${ }^{\circ} \mathrm{K}$ \\
\hline $\mathrm{Tb}=$ flow measurement basls temperature (absolute) & 296 & & 296 & ${ }^{\circ} \mathrm{K}$ \\
\hline$z e=$ gas deviation faclor ot $\mathrm{Pe}$ and $\mathrm{Te}$ & 1.0000 & & 1.0000 & \\
\hline $2 b=$ gas deviation factor at $\mathrm{Pb}$ and $\mathrm{Te}$ & 1.0000 & & 1.0000 & \\
\hline$Q b=$ flow rate at base conditions & 0.08053 & $1.0 \mathrm{E}-6 \mathrm{~m}^{\wedge} 3 / \mathrm{cm}^{\wedge} 3$ & $8.053 E-08$ & $m^{2} 3 / s$ \\
\hline ve - flow velocity at sample exit end & $2.554 \mathrm{E}-04$ & $1.0 \mathrm{E}-2 \mathrm{~m} / \mathrm{cm}$ & $2.554 \mathrm{E}-06$ & $\mathrm{~m} / \mathrm{s}$ \\
\hline $\mathrm{K}_{a}=$ & $1.06 \mathrm{E}-05$ & * $9.872 \mathrm{E}-13 \mathrm{~m}^{2} 2 / \mathrm{d}$ & $1.05 \mathrm{E}-17$ & $m^{2} 2$ \\
\hline $\mathrm{Ka}=$ & $1.06 \mathrm{E}-02$ & & $1.05 E-13$ & $\mathrm{~cm}^{2} 2$ \\
\hline $\mathrm{K}_{a}=$ & $1.06 \mathrm{E}+01$ & & & \\
\hline
\end{tabular}




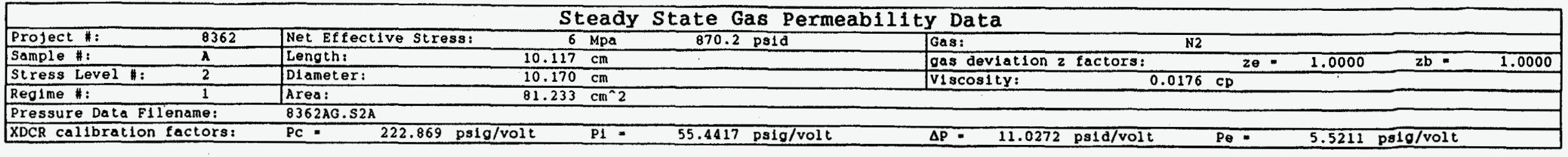

\begin{tabular}{|c|c|c|c|c|c|c|c|c|c|c|c|c|}
\hline Date & $\begin{array}{l}\text { Time } \\
\text { of } \\
\text { Day }\end{array}$ & $\begin{array}{l}\text { File } \\
\text { Time } \\
\text { (min) }\end{array}$ & $\underset{\#}{\text { Regime }}$ & $\begin{array}{l}\text { Pb } \\
\text { Barometric } \\
\text { Pressure }\end{array}$ & $\begin{array}{l}\text { Pc } \\
\text { Confining } \\
\text { Pressure }\end{array}$ & $\begin{array}{l}\text { P1 } \\
\text { Inlet } \\
\text { Pressure }\end{array}$ & $\begin{array}{l}\Delta P \\
\text { D1fferential } \\
\text { Pressure }\end{array}$ & $\begin{array}{l}\text { Pm } \\
\text { Mean Pore } \\
\text { Pressure } \\
\text { Pe+ } \Delta P / 2\end{array}$ & $\begin{array}{l}\text { Pe } \\
\text { Exit } \\
\text { Pressure }\end{array}$ & $\begin{array}{l}\text { Te } \\
\text { Flow } \\
\text { Temp } \\
\left({ }^{\circ} \mathrm{C}\right)\end{array}$ & $\begin{array}{c}\text { Tb } \\
\text { Ambient } \\
\text { Temp } \\
\left({ }^{\circ} \mathrm{C}\right)\end{array}$ & $\begin{array}{c}\text { Qb } \\
\text { Flow Rate } \\
\text { PpbTb } \\
\text { (ml/sec) }\end{array}$ \\
\hline 28 MaY 93 & $12: 17$ & 272 & AS2R1a & 12.32 psia & volts & volts & 3.833 volts & & volts & 23 & 23 & 0.03740 \\
\hline 28 MaY 93 & $12: 20$ & 277 & AS2R1b & 12.32 ps1a & volts & 0.866 & 3.833 volts & & volts & 23 & 23 & 0.03745 \\
\hline 28 May 93 & $12: 23$ & 277 & AS2R1C & 12.32 psia & volts & 0.866 & 3.833 volts & & volts & 23 & 23 & 0.03739 \\
\hline 28 MaY 93 & $12: 25$ & 282 & AS2R1d & $12.32 \mathrm{ps} 1 \mathrm{a}$ & volts & 0.866 & 3.833 volts & & volts & 23 & 23 & 0.03743 \\
\hline \multirow{9}{*}{ AVERAGES } & & & \multirow{9}{*}{ AS2R1 } & & P४GUAGE & OUAGD & DTRFEREAT TAI & OUNGE & ounoet & एका & (क्ट) & \\
\hline & & & & & 4.027 volts & 0.866 volts & 3.833 volts & & 1.107 & \multirow{4}{*}{23} & \multirow{4}{*}{23} & \\
\hline & & & & & 897.5 & 48.01 & 42.27 psid & $27.25 \mathrm{pgig}$ & 6.112 & & & \\
\hline & & & & & 61.07 & 3.267 & 2.876 & 1.854 atm & 0.4159 & & & \\
\hline & & & & & $6.188 \quad \mathrm{Mpa}$ & 0.3310 & $0.2914 \quad \mathrm{MpQ}$ & $0.1879 \mathrm{Mpa}$ & 0.04214 & & & \\
\hline & & & & ABSOLUTE & ABSOLUTE & ABSOLUTE & DIFFERENT XAL & ABSOLUTE & ABSOLUTE & (बx) & का & $(\mathrm{ml} /: \Delta \theta \mathrm{C})$ \\
\hline & & & & 12.32 psia & 909.8 ps1a & $60.33 \quad \mathrm{ps} 1 \mathrm{a}$ & 42.27 psid & $39.57 \mathrm{psia}$ & $18.43 \quad \mathrm{ps} 1 \mathrm{a}$ & \multirow{3}{*}{296} & \multirow{3}{*}{296} & \multirow{3}{*}{0.03742} \\
\hline & & & & $0.8383 \mathrm{~atm}$ & 61.91 & 4.105 & 2.876 & $2.692 \quad \mathrm{~atm}$ & 1.254 & & & \\
\hline & & & & $0.08494 \mathrm{Mpa}$ & 6.273 & 0.4160 & 0.2914 & 0.2728 & 0.1271 & & & \\
\hline
\end{tabular}

Apparent gas permeab1l1ty: $K a=\left(v v^{*} P e^{\star} u * L\right) /(P m * \Delta P)$

Boyle's Law:

$\mathrm{Ve} \cdot(\mathrm{Pb} / \mathrm{Pe}) *(\mathrm{Te} / \mathrm{Tb}) *(\mathrm{ze} / \mathrm{zb}) * \mathrm{Vb}$

$\mathrm{Qe}=(\mathrm{Pb} / \mathrm{Pe}) *(\mathrm{Te} / \mathrm{Tb}) *(z e / 2 b) * \mathrm{Qb}$

$v e=Q_{e} / \mathrm{A}=(\mathrm{Pb} / \mathrm{Pe}) \cdot(\mathrm{Te} / \mathrm{Tb}) *(z e / 2 \mathrm{~b}) *(\mathrm{Qb} / \mathrm{A})$

\begin{tabular}{|c|c|c|c|c|}
\hline Parameter & $\begin{array}{l}\text { Traditional } \\
\text { Un1ts }\end{array}$ & & $\begin{array}{l}\text { SI } \\
\text { Undts }\end{array}$ & \\
\hline$\mu=\operatorname{gas} v 1 \mathrm{scos} 1 \mathrm{ty}$ & 0.0176 & $\star 1.0 \mathrm{E}-3 \mathrm{~Pa} * \mathrm{sec} / \mathrm{cp}$ & $1.760 \mathrm{E}-05$ & $P a * \sec$ \\
\hline$L=$ sample length & 10.117 & $* 1.0 \mathrm{E}-2 \mathrm{~m} / \mathrm{cm}$ & $1.012 \mathrm{E}-01$ & $\mathrm{~m}$ \\
\hline$A$ - sample clrcular cross sectional area & 81.233 & $1.0 \mathrm{E}-4 \mathrm{~m}^{\wedge} 2 / \mathrm{cm}^{\wedge} 2$ & $8.123 E-03$ & $m^{2} 2$ \\
\hline $\mathrm{Pb}=$ flow measurement basis pressure (absolute) & 0.8383 & $* 1.013 \mathrm{E}+5 \mathrm{~Pa} / \mathrm{atm}$ & $8.492 \mathrm{E}+04$ & $\mathrm{~Pa}$ \\
\hline$\Delta P$ = pressure drop across sample length & 2.876 & $1.013 \mathrm{E}+5 \mathrm{~Pa} / \mathrm{atm}$ & $2.913 \mathrm{E}+05$ & Pa \\
\hline$P_{m}=$ mean pore pressure (absolute) & 2.692 & $\$ 1.013 \mathrm{E}+5 \mathrm{~Pa} / \mathrm{atm}$ & $2.727 \mathrm{E}+05$ & Pa \\
\hline$P_{e}=$ exit pressure (absolute) & 1.254 & $1.013 \mathrm{E}+5 \mathrm{~Pa} / \mathrm{atm}$ & $1.271 \mathrm{E}+05$ & $\mathrm{~Pa}$ \\
\hline Te - sample temperature (absolute) & 296 & & 296 & ${ }^{\circ} \mathrm{K}$ \\
\hline$T b=$ flow measurement basis temperature (absolute) & 296 & & 296 & ${ }^{\circ} \mathrm{K}$ \\
\hline $2 e$ - gas deviation factor at $\mathrm{Pe}$ and $\mathrm{Te}$ & 1.0000 & & 1.0000 & \\
\hline $2 \mathrm{~b}$ - gas deviation factor at $\mathrm{Pb}$ and $\mathrm{Te}$ & 1.0000 & & 1.0000 & \\
\hline$Q b=$ flow rate at base conditlons & 0.03742 & $1.0 \mathrm{E}-6 \mathrm{~m}^{\wedge} 3 / \mathrm{cm}^{\wedge} 3$ & $3.742 \mathrm{E}-08$ & $\mathrm{~m}^{\wedge} 3 / \mathrm{s}$ \\
\hline ve = flow velocity at sample exit end & $3.079 E-04$ & $\$ 1.0 \mathrm{E}-2 \mathrm{~m} / \mathrm{cm}$ & $3.079 \mathrm{E}-06$ & $\mathrm{~m} / \mathrm{s}$ \\
\hline 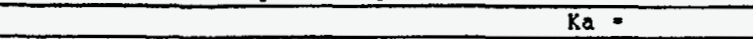 & $8.68 \mathrm{E}-06$ & $9.872 \mathrm{E}-13 \mathrm{~m}^{\wedge} 2 / \mathrm{d}$ & $8.77 \mathrm{E}-18$ & $m^{2} 2$ \\
\hline $\mathrm{Ka}=$ & $8.88 E-03$ & & $8.77 \mathrm{E}-14$ & $\mathrm{~cm}^{\star 2} 2$ \\
\hline $\mathrm{Ka}=$ & $8.88 \mathrm{E}+00$ & & & \\
\hline
\end{tabular}




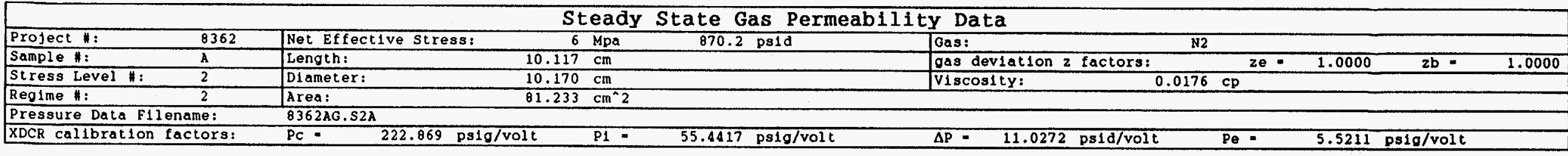

\begin{tabular}{|c|c|c|c|c|c|c|c|c|c|c|c|c|}
\hline Date & $\begin{array}{l}\text { T1me } \\
\text { of } \\
\text { Day }\end{array}$ & $\begin{array}{l}\text { File } \\
\text { Time } \\
\text { (min) }\end{array}$ & $\underset{\#}{\text { Regime }}$ & \begin{tabular}{|l}
$\mathrm{Pb}$ \\
Barometric \\
Pressure
\end{tabular} & $\begin{array}{l}\text { Pc } \\
\text { Confining } \\
\text { Pressure }\end{array}$ & $\begin{array}{l}\text { P1 } \\
\text { Inlet } \\
\text { Pressure }\end{array}$ & $\begin{array}{l}\Delta P \\
\text { D1fferential } \\
\text { Pressure }\end{array}$ & $\begin{array}{l}\text { Pm } \\
\text { Mean Pore } \\
\text { Pressure } \\
\text { Pe }+\Delta P / 2\end{array}$ & $\begin{array}{l}\text { Pe } \\
\text { Ex1t } \\
\text { Pressure }\end{array}$ & $\begin{array}{l}\text { Te } \\
\text { Flow } \\
\text { Temp } \\
\left({ }^{\circ} \mathrm{C}\right)\end{array}$ & $\begin{array}{c}\text { Tb } \\
\text { Amblent } \\
\text { Temp } \\
\left({ }^{\circ} \mathrm{C}\right)\end{array}$ & $\begin{array}{c}Q b \\
\text { Flow Rate } \\
\text { OPb\&Tb } \\
\text { (ml/sec) }\end{array}$ \\
\hline 28 May 93 & $14: 34$ & 407 & AS2R2a & 12.30 ps1a & 4.071 & volts & 3.829 volts & & 2.871 & $\overline{23}$ & 23 & 0.04414 \\
\hline 28 May 93 & $14: 37$ & 412 & AS2R2b & $12.30 \mathrm{ps} 1 \mathrm{a}$ & volts & volts & 3.829 volts & & volts & 23 & 23 & 0.04410 \\
\hline 28 May 93 & $14: 39$ & 412 & AS2R2C & $12.30 \mathrm{ps} 1 \mathrm{a}$ & 4.071 volts & volts & 3.829 volts & & volts & 23 & 23 & 0.04411 \\
\hline 28 May 93 & $14: 47$ & 422 & AS2R2d & $12.30 \mathrm{ps} 1 \mathrm{a}$ & 4.071 volts & volts & $3.830 \mathrm{volts}$ & & volts & 23 & 23 & 0.04403 \\
\hline \multirow{9}{*}{ AVERAGES } & & & \multirow{9}{*}{ AS2R2 } & & छUACE & GUAOE & DTGFERENTIA & GUXGE & OUNOE & क्त) & 8वृ: & \\
\hline & & & & & 4.071 volts & 1.040 volts & 3.829 volts & & 2.871 & \multirow{4}{*}{23} & \multirow{4}{*}{23} & \\
\hline & & & & & 907.3 & 57.66 & $42.23 \mathrm{ps} 1 \mathrm{~d}$ & $36.96 \mathrm{psig}$ & 15.850 & & & \\
\hline & & & & & 61.74 & 3.923 & 2.873 & $2.515 \mathrm{~atm}$ & 1.0785 & & & \\
\hline & & & & & $6.256 \quad \mathrm{Mpa}$ & 0.3975 & $0.2911 \mathrm{Mpa}$ & $0.2548 \mathrm{Mpa}$ & 0.10928 & & & \\
\hline & & & & AQSOLUTTE & ABSOLUTE & ABSOLUTE & DIFFERENTIAL & ABSOLTRE & ABSOtUTE & $(0 x)$ & (OX) & $(\mathrm{m}) \mathrm{sec})$ \\
\hline & & & & $12.3 \mathrm{psia}$ & $919.6 \quad \mathrm{ps} 1 \mathrm{a}$ & $69.96 \quad$ psia & 42.23 ps1d & $49.26 \mathrm{psla}$ & $28.15 \quad$ psia & \multirow{3}{*}{296} & \multirow{3}{*}{296} & \multirow{3}{*}{0.04409} \\
\hline & & & & $0.8370 \mathrm{~atm}$ & 62.57 & 4.760 & 2.873 & $3.352 \mathrm{~atm}$ & 1.915 & & & \\
\hline & & & & 0.08481 & 6.340 & 0.4824 & 0.2911 & 0.3397 & 0.1941 & & & \\
\hline
\end{tabular}

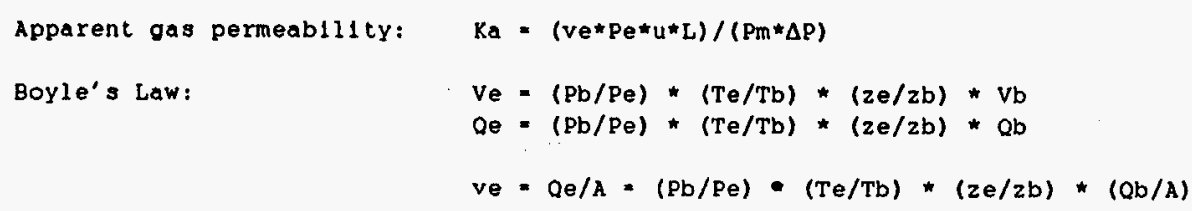

\begin{tabular}{|c|c|c|c|c|}
\hline Parameter & $\begin{array}{l}\text { Traditional } \\
\text { Units }\end{array}$ & & $\begin{array}{l}\text { SI } \\
\text { Units }\end{array}$ & \\
\hline$\mu=$ gas viscosity & 0.0176 & $1.0 \mathrm{E}-3 \mathrm{~Pa} * \mathrm{sec} / \mathrm{Cp}$ & $1.760 \mathrm{E}-05$ & Pawsec \\
\hline $\mathrm{L}=$ sample length & 10.117 & $1.0 \mathrm{E}-2 \mathrm{~m} / \mathrm{cm}$ & $1.012 \mathrm{E}-01$ & $\mathrm{~m}$ \\
\hline$A=$ sample clrcular cross sectional area & 81.233 & $1.0 \mathrm{E}-4 \mathrm{~m}^{\wedge} 2 / \mathrm{cm}^{\wedge} 2$ & $8.123 \mathrm{E}-03$ & $m^{\wedge} 2$ \\
\hline $\mathrm{Pb}=$ flow measurement basls pressure (absolute) & 0.8370 & $* 1.013 \mathrm{E}+5 \mathrm{~Pa} / \mathrm{atm}$ & $8.478 E+04$ & $\mathrm{~Pa}$ \\
\hline$\Delta \mathrm{P}=$ pressure drop across sample length & 2.873 & $\star 1.013 \mathrm{E}+5 \mathrm{~Pa} / \mathrm{atm}$ & $2.911 E+05$ & $\mathrm{~Pa}$ \\
\hline $\mathrm{Pm}=$ mean pore pressure (absolute) & 3.352 & $\star 1.013 \mathrm{E}+5 \mathrm{~Pa} / \mathrm{atm}$ & $3.396 \mathrm{E}+05$ & $\mathbf{P a}$ \\
\hline Pe - exit pressure (absolute) & 1.915 & $\star 1.013 \mathrm{E}+5 \mathrm{~Pa} / \mathrm{atm}$ & $1.940 E+05$ & $\mathrm{~Pa}$ \\
\hline Te - sample temperature (absolute) & 296 & & 296 & ${ }^{\circ} \mathrm{K}$ \\
\hline $\mathrm{Tb}=$ flow measurement basls temperature (absolute) & 296 & & 296 & ${ }^{\circ} \mathrm{K}$ \\
\hline$z e=$ gas deviation factor at $\mathrm{Pe}$ and $\mathrm{Te}$ & 1.0000 & & 1.0000 & \\
\hline $2 \mathrm{~b}=$ gas devlation factor $\mathrm{at} \mathrm{Pb}$ and $\mathrm{Te}$ & 1.0000 & & 1.0000 & \\
\hline$Q b$ - flow rate at base conditions & $0.04409 \mathrm{~cm}^{\wedge} 3 / \mathrm{s}$ & $\star 1.0 \mathrm{E}-6 \mathrm{~m}^{\wedge} 3 / \mathrm{cm}^{\wedge} 3$ & $4.409 \mathrm{E}-08$ & $m^{\wedge} 3 / 3$ \\
\hline ve = flow velocity at sample exit end & $2.372 \mathrm{E}-04 \mathrm{~cm} / \mathrm{s}$ & $\star 1.0 \mathrm{E}-2 \mathrm{~m} / \mathrm{cm}$ & $2.372 E-06$ & $\mathrm{~m} / \mathrm{s}$ \\
\hline $\mathrm{Ka}_{\mathrm{a}}=$ & $8.40 \mathrm{E}-06$ & $9.872 \mathrm{E}-13 \mathrm{~m}^{\wedge} 2 / \mathrm{d}$ & $8.29 \mathrm{E}-18$ & $m^{2} 2$ \\
\hline $\mathrm{Ka}=$ & $8.40 \mathrm{E}-03$ & & $8.29 \mathrm{E}-14$ & $\mathrm{~cm}^{\star} 2$ \\
\hline $\mathrm{Ka}=$ & $8.40 E+00$ & & & \\
\hline
\end{tabular}


Steady State Gas Permeability Data

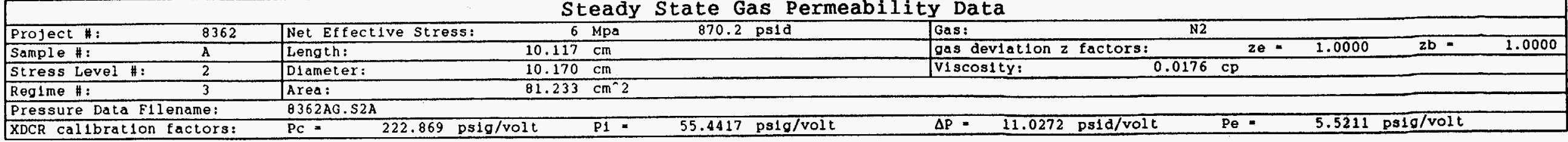

\begin{tabular}{|c|c|c|c|c|c|c|c|c|c|c|c|c|}
\hline Date & $\begin{array}{l}\text { Time } \\
\text { of } \\
\text { Day }\end{array}$ & $\begin{array}{l}\text { File } \\
\text { Time } \\
(m i n)\end{array}$ & Regime & $\begin{array}{l}\mathrm{Pb} \\
\text { Barometric } \\
\text { Pressure }\end{array}$ & $\begin{array}{l}\text { Pe } \\
\text { Confining } \\
\text { Pressure }\end{array}$ & $\begin{array}{l}\text { P1 } \\
\text { Inlet } \\
\text { Pressure }\end{array}$ & $\begin{array}{l}\Delta P \\
\text { Differential } \\
\text { Pressure }\end{array}$ & $\begin{array}{l}\text { Pm } \\
\text { Mean pore } \\
\text { Pressure } \\
\text { Pe } \Delta \mathrm{P} / 2\end{array}$ & $\begin{array}{l}\text { Pe } \\
\text { Ex1t } \\
\text { Pressure }\end{array}$ & $\begin{array}{l}\text { Te } \\
\text { Flow } \\
\text { Temp } \\
\left({ }^{\circ} \mathrm{C}\right)\end{array}$ & $\begin{array}{c}\text { Tb } \\
\text { Ambient } \\
\text { Temp } \\
\left({ }^{\circ} \mathrm{C}\right)\end{array}$ & $\begin{array}{c}\text { Qb } \\
\text { Flow Rate } \\
\text { OPb\&Tb } \\
(\mathrm{ml} / \mathrm{sec})\end{array}$ \\
\hline 01 Jun 93 & $13: 27$ & 6102 & AS2R3a & 12.32 ps1a & volts & volts & 3.041 volts & & volts & 23 & 22 & 0.04952 \\
\hline 01 Jun 93 & $13: 32$ & 6107 & $A S 2 R 3 b$ & $12.32 \mathrm{ps} 1 \mathrm{a}$ & volls & 1.225 & 3.841 volts & & volts & 23 & 22 & 0.04948 \\
\hline 01 Jun 93 & $13: 34$ & 6107 & AS2R3C & $12.32 \mathrm{ps} 1 \mathrm{a}$ & volts & 1.225 & 3.841 volts & & volts & 23 & 22 & 0.04962 \\
\hline 01 Jun 93 & $13: 37$ & 6112 & AS2R3d & 12.32 ps1a & 4.116 & 1.225 & 3.841 volts & & 4.715 & 23 & 22 & 0.04954 \\
\hline \multirow{9}{*}{ AVERAGES } & & & \multirow{9}{*}{ AS 2 R 3} & & GUAGE & OUAQE & DTEFERENT TAL & GUAGE: & OUDOE & \multirow[t]{5}{*}{ (90) } & \multirow[t]{5}{*}{ (rol } & \\
\hline & & & & & 4.116 volts & 1.225 volts & 3.841 volts & & 4.716 & & & \\
\hline & & & & & 917.3 & 67.92 & 42.36 psid & $47.21 \mathrm{psig}$ & 26.035 & & & \\
\hline & & & & & 62.42 & 4.621 & 2.882 & $3.213 \mathrm{~atm}$ & 1.7716 & & & \\
\hline & & & & & $6.325 \quad \mathrm{Mpa}$ & 0.4683 & $0.2920 \quad \mathrm{Mpa}$ & $0.3255 \quad \mathrm{Mpa}$ & 0.17950 & & & \\
\hline & & & & ABSOLUTE: & ABSOLUTE & ABSOLUTE & DIFFERENTLAL & \multicolumn{2}{|l|}{ AQSOLUTE } & \multirow{4}{*}{296} & (5) & (milsoc) \\
\hline & & & & $12.32 \mathrm{ps} 1 \mathrm{a}$ & 929.6 psia & 80.24 psia & 42.36 ps1d & 59.53 psia & 38.35 & & \multirow{3}{*}{295} & \multirow{3}{*}{0.04954} \\
\hline & & & & 0.8383 atm & 63.26 & 5.460 & 2.882 & $4.051 \mathrm{acm}$ & 2.610 & & & \\
\hline & & & & $0.08494 \mathrm{Mpa}$ & 6.410 & 0.5532 & 0.2920 & 0.4105 & 0.2644 & & & \\
\hline
\end{tabular}

Apparent gas permeability: Ka $=\left(v e^{*} \mathrm{Pe}^{*} \mathrm{U}^{*} \mathrm{~L}\right) /(\mathrm{Pm} * \Delta \mathrm{P})$

Boyle's Law:

$\mathrm{Ve}=(\mathrm{Pb} / \mathrm{Pe}) *(\mathrm{Te} / \mathrm{Tb}) *(\mathrm{ze} / \mathrm{zb}) * \mathrm{Vb}$

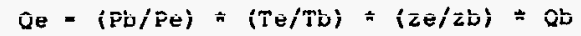

$v_{e}=Q_{e} / A=(\mathrm{Pb} / \mathrm{Pe}) *(\mathrm{Te} / \mathrm{Tb}) *(z e / z b) *(\mathrm{Qb} / \mathrm{A})$

\begin{tabular}{|c|c|c|c|c|}
\hline Parameter & $\begin{array}{l}\text { Traditional } \\
\text { Undts }\end{array}$ & & $\begin{array}{l}\text { SI } \\
\text { Units }\end{array}$ & \\
\hline$\mu=$ gas $v 1 \operatorname{scos} 1 t y$ & 0.0176 & $* 1.0 \mathrm{E}-3 \mathrm{~Pa} * \mathrm{sec} / \mathrm{cp}$ & $1.760 \mathrm{E}-05$ & Pa*sec \\
\hline$L=$ sample length & 10.117 & $\hbar 1.0 \mathrm{E}-2 \mathrm{~m} / \mathrm{cm}$ & $1.012 \mathrm{E}-01$ & $m$ \\
\hline$A=$ sample clrcular cross sectional area & 81.233 & $\star 1.0 \mathrm{E}-4 \mathrm{~m}^{2} 2 / \mathrm{cm}^{2} 2$ & $8.123 \mathrm{E}-03$ & $m^{n} 2$ \\
\hline $\mathrm{pb}=$ flow measurement basis pressure (absolute) & 0.8383 & $1.013 E+5 \mathrm{~Pa} / \mathrm{atm}$ & $8.492 E+04$ & $\mathrm{~Pa}$ \\
\hline$\Delta \mathrm{P}=$ pressure drop across sample length & 2.882 & $* 1.013 \mathrm{E}+5 \mathrm{~Pa} / \mathrm{atm}$ & $2.920 E+05$ & Po \\
\hline $\mathrm{P}_{\mathrm{m}}$ - mean pore pressure (absolute) & 4.051 & $\% 1.013 \mathrm{E}+5 \mathrm{~Pa} / \mathrm{atm}$ & $4.104 E+05$ & Po \\
\hline $\mathrm{Pe}=$ exit pressure (absolute) & 2.610 & $* 1.013 \mathrm{E}+5 \mathrm{~Pa} / \mathrm{atm}$ & $2.644 E+05$ & $\mathbf{P a}$ \\
\hline Te - sample temperature (absolute) & 296 & & 296 & ${ }^{\circ} \mathrm{K}$ \\
\hline $\mathrm{Tb}=$ flow measurement basis temperature (absolute) & 295 & & 295 & ${ }^{\circ} \mathrm{K}$ \\
\hline $2 e=$ gas deviation factor at $\mathrm{Pe}$ and $\mathrm{Te}$ & 1.0000 & & 1.0000 & \\
\hline $2 b=$ gas deviation factor at $\mathrm{Pb}$ and $\mathrm{Te}$ & 1.0000 & & 1.0000 & \\
\hline$Q b=$ flow rate at base conditions & 0.04954 & $1.0 \mathrm{E}-6 \mathrm{~m}^{\wedge} 3 / \mathrm{cm}^{\wedge} 3$ & $4.954 \mathrm{E}-08$ & $m^{\wedge} 3 / s$ \\
\hline ve - flow velocity at sample exit end & $1.966 \mathrm{E}-04$ & $\$ 1.0 \mathrm{E}-2 \mathrm{~m} / \mathrm{cm}$ & $1.966 \mathrm{E}-06$ & $\mathrm{~m} / \mathrm{s}$ \\
\hline Ka $=$ & $7.82 \mathrm{E}-06$ & $9.872 \mathrm{E}-13 \mathrm{~m}^{\wedge} 2 / \mathrm{d}$ & $7.72 \mathrm{E}-18$ & $m^{*} 2$ \\
\hline $\mathrm{Ka}=$ & $7.82 E-03$ & & $7.72 \mathrm{E}-14$ & $\mathrm{~cm}^{\wedge} 2$ \\
\hline $\mathrm{Ka}=$ & $7.82 \mathrm{E}+00$ & & & \\
\hline
\end{tabular}




\begin{tabular}{|c|c|c|c|c|c|c|c|c|c|c|c|c|c|}
\hline \multicolumn{14}{|c|}{ Steady State Gas Permeability Data } \\
\hline Project \#: & 8362 & Net Effective stress: & 6 & Mpa & 870.2 psid & Gas: & & $\sqrt{2}$ & & & & & \\
\hline Sample \#: & $\bar{A}$ & Length: & 10.117 & $\mathrm{~cm}$ & & gas deviation $z$ & factors: & & & $2 \theta=$ & 1.0000 & $2 \mathrm{~b}=$ & 1.0000 \\
\hline Stress Level \#: & 2 & Diameter: & 10.170 & $\mathrm{~cm}$ & & V1scosity: & & 0.0176 & $\mathrm{cp}$ & & & & \\
\hline Reglme \#: & 4 & Area: & 81.233 & $\mathrm{~cm}^{2} 2$ & & & & & & & & & \\
\hline Pressure Data F & me: & $8362 \mathrm{AG} . \mathrm{S} 2 \mathrm{~A}$ & & & & & & & & & & & \\
\hline XDCR callbrat10 & tors: & $P C=\quad 222.869$ & $01 \mathrm{t}$ & $P_{1}=$ & $55.4417 \mathrm{ps} 1 \mathrm{~g} / \mathrm{volt}$ & 11.0272 & ps1d/volt & & $\mathrm{Pe}$ & & 5.5211 & g/volt & \\
\hline
\end{tabular}

\begin{tabular}{|c|c|c|c|c|c|c|c|c|c|c|c|c|}
\hline Date & $\begin{array}{l}\text { Time } \\
\text { of } \\
\text { Day }\end{array}$ & $\begin{array}{l}\text { File } \\
\text { Time } \\
\text { (m1n) }\end{array}$ & $\underset{\#}{\text { Reglme }}$ & $\begin{array}{l}\mathrm{Pb} \\
\text { Barometrlc } \\
\text { Pressure }\end{array}$ & $\begin{array}{l}\text { Pc } \\
\text { Confining } \\
\text { Pressure }\end{array}$ & $\begin{array}{l}\text { P1 } \\
\text { Inlet } \\
\text { Pressure }\end{array}$ & $\begin{array}{l}\Delta P \\
\text { D1fferential } \\
\text { Pressure }\end{array}$ & $\begin{array}{l}\text { Pm } \\
\text { Mean pore } \\
\text { Pressure } \\
\text { Pe }+\Delta P / 2\end{array}$ & $\begin{array}{l}\text { Pe } \\
\text { Exit } \\
\text { Pressure }\end{array}$ & $\begin{array}{l}\text { Te } \\
\text { Flow } \\
\text { Temp } \\
\left({ }^{\circ} \mathrm{C}\right)\end{array}$ & $\begin{array}{c}\text { Tb } \\
\text { Amblent } \\
\text { Temp } \\
\left({ }^{\circ} \mathrm{C}\right)\end{array}$ & $\begin{array}{c}\text { Qb } \\
\text { Flow Rate } \\
\text { PbdTb } \\
\text { (m1/sec) }\end{array}$ \\
\hline 02 Jun 93 & $13: 14$ & 7527 & AS2R4a & 12.21 psia & volts & 1.407 & 3.874 volts & & volts & 23 & 22 & 0.05676 \\
\hline 02 Jun 93 & $13: 17$ & 7532 & AS2R4b & $12.21 \mathrm{ps10}$ & volts & 1.407 & 3.874 volts & & volts & 23 & 22 & 0.05679 \\
\hline 02 Jun 93 & $13: 19$ & 7532 & AS2R4C & 12.21 psia & volts & 1.407 & 3.874 volts & & volts & 23 & 22 & 0.05683 \\
\hline 02 Jun 9.3 & $13: 21$ & 7537 & AS2R4d & $12.21 \mathrm{ps} 1 \mathrm{a}$ & volts & 1.407 & 3.875 volts & & volts & 23 & 22 & 0.05682 \\
\hline \multirow{9}{*}{ AVERAGES } & & & \multirow{9}{*}{ AS2R4 } & & GUAGE & OUAOE & DIEGEREMTIAL & OUAGE & GUAOE & $90 \mathrm{C}$ & (क) & \\
\hline & & & & & 4.160 volts & 1.407 voles & 3.874 volts & & 6.496 & \multirow{4}{*}{23} & \multirow{4}{*}{22} & \\
\hline & & & & & 927.1 & 78.01 & 42.72 ps1d & $57.23 \mathrm{ps} 1 \mathrm{~g}$ & 35.865 & & & \\
\hline & & & & & 63.09 & 5.308 & 2.907 & 3.894 atm & 2.4405 & & & \\
\hline & & & & & 6.392 & 0.5378 & $0.2946 \quad \mathrm{Mpa}$ & $0.3946 \quad \mathrm{Mpa}$ & 0.24728 & & & \\
\hline & & & & ABSOLUTE & ABSOLUTE & ABSOLUTE: & DIFFERENTIAL & ABSOUTTE & AESOLOTE & $(0 \mathrm{k})$ & $(0 k)$ & $(\mathrm{ml} / \mathrm{sec})$ \\
\hline & & & & $12.21 \mathrm{ps} 1 \mathrm{a}$ & $939.3 \quad \mathrm{ps} 1 \mathrm{a}$ & $90.22 \quad$ ps1a & $42.72 \quad \mathrm{ps} 1 \mathrm{~d}$ & $69.44 \mathrm{pg} 1 \mathrm{a}$ & 48.08 & \multirow{3}{*}{296} & \multirow{3}{*}{295} & \multirow{3}{*}{0.05680} \\
\hline & & & & $0.8308 \quad \mathrm{~atm}$ & 63.92 & 6.139 & 2.907 & $0.725 \mathrm{~atm}$ & 3.271 & & & \\
\hline & & & & $0.08419 \quad \mathrm{Mpa}$ & 6.477 & 0.6220 & 0.2946 & 0.4787 & 0.3315 & & & \\
\hline
\end{tabular}

Apparent gas permeabtlity:

$K_{a}=\left(v e^{\star P} e^{\star} u^{\star} L\right) /\left(P{ }^{\star} \Delta P\right)$

Boyle's Law:

$\mathrm{Ve}=(\mathrm{Pb} / \mathrm{Pe}) *(\mathrm{Te} / \mathrm{Tb}) *(\mathrm{ze} / \mathrm{zb}) * \mathrm{Vb}$

$Q e=(\mathrm{Pb} / \mathrm{Pe}) *(\mathrm{Te} / \mathrm{Tb}) *(z e / 2 \mathrm{~b}) * \mathrm{Qb}$

$v_{e}=Q_{e} / \mathrm{A}=(\mathrm{Pb} / \mathrm{Pe}) *(\mathrm{Te} / \mathrm{Tb}) \cdot(\mathrm{ze} / \mathrm{zb}) *(\mathrm{Qb} / \mathrm{A})$

\begin{tabular}{|c|c|c|c|c|}
\hline Parameter & $\begin{array}{l}\text { Traditional } \\
\text { Units }\end{array}$ & & $\begin{array}{l}\text { SI } \\
\text { Undes }\end{array}$ & \\
\hline$\mu=g a s v 1 s \cos 1 t y$ & 0.0176 & $\star 1.0 \mathrm{E}-3 \mathrm{~Pa}$ * sec/cp & $1.760 \mathrm{E}-05$ & Pa*sec \\
\hline$L=$ sample length & 10.117 & $\star 1.0 \mathrm{E}-2 \mathrm{~m} / \mathrm{cm}$ & $1.012 \mathrm{E}-01$ & $\mathrm{~m}$ \\
\hline A - sample clrcular cross sectlonal area & 81.233 & $\star 1.0 \mathrm{E}-4 \mathrm{~m}^{\wedge} 2 / \mathrm{cm}^{\wedge} 2$ & $8.123 \mathrm{E}-03$ & $\mathrm{~m}^{\wedge} 2$ \\
\hline$\overline{p b}=$ flow measurement basis pressure (absolute) & 0.8308 & $\star 1.013 \mathrm{E}+5 \mathrm{~Pa} / \mathrm{atm}$ & $8.416 \mathrm{E}+04$ & Pa \\
\hline$\Delta \mathrm{P}=$ pressure drop across sample length & 2.907 & $1.013 \mathrm{E}+5 \mathrm{~Pa} / \mathrm{atm}$ & $2.945 \mathrm{E}+05$ & $\mathrm{~Pa}$ \\
\hline $\mathrm{Pm}=$ mean pore pressure (absolute) & 4.725 & $1.013 \mathrm{E}+5 \mathrm{Po} / \mathrm{atm}$ & $4.786 \mathrm{E}+05$ & Pa \\
\hline $\mathrm{Pe}=$ exlt pressure (absolute) & 3.271 & $\star 1.013 \mathrm{E}+5 \mathrm{~Pa} / \mathrm{atm}$ & $3.314 E+05$ & $\mathrm{~Pa}$ \\
\hline $\mathrm{Te}$ = sample temperature (absolute) & 296 & & 296 & ${ }^{\circ} \mathrm{K}$ \\
\hline$T b=$ flow measurement basis temperature (absolute) & 295 & & 295 & ${ }^{\circ} \mathrm{K}$ \\
\hline $2 e$ - gas deviation factor at $\mathrm{Pe}$ and Te & 1.0000 & & 1.0000 & \\
\hline $\mathrm{zb}=$ gas deviation factor $\mathrm{at} \mathrm{Pb}$ and $\mathrm{Te}$ & 1.0000 & & 1.0000 & \\
\hline$Q b=$ flow rate at base conditions & $0.05580 \mathrm{~cm}^{\wedge} 3 / \mathrm{s}$ & $1.0 \mathrm{E}-6 \mathrm{~m}^{\wedge} 3 / \mathrm{cm}^{\wedge} 3$ & $5.680 E-08$ & $\mathrm{~m}^{2} 3 / \mathrm{s}$ \\
\hline ve $=$ flow velocity at sample exit end & $1.782 \mathrm{E}-0.4 \mathrm{~cm} / \mathrm{s}$ & $\$ 1.0 \mathrm{E}-2 \mathrm{~m} / \mathrm{cm}$ & $1.782 \mathrm{E}-06$ & $\mathrm{~m} / \mathrm{s}$ \\
\hline $\mathrm{Ka} *$ & $7.56 \mathrm{E}-06$ & $9.872 \mathrm{E}-13 \mathrm{~m}^{\wedge} 2 / \mathrm{d}$ & $7.46 \mathrm{E}-18$ & $m^{\wedge} 2$ \\
\hline $\mathrm{Ka}=$ & $7.56 \mathrm{E}-03$ & & $7.46 \mathrm{E}-14$ & $\mathrm{~cm}^{2} 2$ \\
\hline $\mathrm{Ka}=$ & $7.56 \mathrm{E}+00$ & & & \\
\hline
\end{tabular}




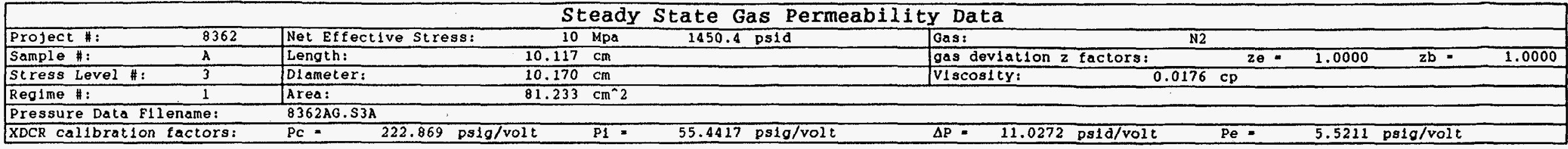

\begin{tabular}{|c|c|c|c|c|c|c|c|c|c|c|c|c|}
\hline Date & $\begin{array}{l}\text { Time } \\
\text { of } \\
\text { Day }\end{array}$ & $\begin{array}{l}\text { File } \\
\text { Time } \\
\text { (min) }\end{array}$ & $\underset{\#}{\text { Reglme }}$ & $\begin{array}{l}\text { Pb } \\
\text { Barometric } \\
\text { Pressure }\end{array}$ & $\begin{array}{l}\text { Pc } \\
\text { Confining } \\
\text { Pressure }\end{array}$ & $\begin{array}{l}\text { P1 } \\
\text { Inlet } \\
\text { Pressure }\end{array}$ & $\begin{array}{l}\Delta P \\
D \text { lf ferential } \\
\text { Pressure }\end{array}$ & $\begin{array}{l}\text { Pm } \\
\text { Mean Pore } \\
\text { Pressure } \\
\text { Pe }+\Delta P / 2\end{array}$ & $\begin{array}{l}\text { Pe } \\
\text { Exit } \\
\text { Pressure }\end{array}$ & $\begin{array}{l}\text { Te } \\
\text { Flow } \\
\text { Temp } \\
\left({ }^{\circ} \mathrm{C}\right)\end{array}$ & $\begin{array}{c}\mathrm{Tb} \\
\text { Amblent } \\
\text { Temp } \\
\left({ }^{\circ} \mathrm{C}\right)\end{array}$ & $\begin{array}{c}\text { Qb } \\
\text { Flow Rate } \\
\text { PbsTb } \\
\text { (ml/sec) }\end{array}$ \\
\hline 03 Jun 93 & $09: 28$ & $10 \overline{85}$ & AS3R1a & $12.30 \mathrm{psia}$ & yolts & volts & 3.853 volts & & volts & 23 & 21 & 0.03065 \\
\hline 03 Jun 93 & $09: 33$ & 1090 & AS3R1b & 12.30 ps1a & 6.629 & volts & 3.853 volts & & volts & 23 & 21 & 0.03056 \\
\hline 03 Jun 93 & $09: 35$ & 1090 & AS3R1C & $12.30 \mathrm{psia}$ & volts & volts & 3.853 volts & & volts & 23 & 21 & 0.03057 \\
\hline 03 Jun 93 & $09: 38$ & 1095 & AS3R1d & $12.30 \mathrm{psia}$ & volts & volts & 3.853 volts & & volts & 23 & 21 & 0.03062 \\
\hline \multirow{9}{*}{ AVERAGES } & & & \multirow{9}{*}{ AS3R 1} & & OUUAGE & OUAGE & DIFFERENTAR & GUAGE & GUAOE & 10 & $1 \%$ & \\
\hline & & & & & 6.629 volts & 0.866 volts & 3.853 volts & & 1.058 & \multirow{4}{*}{23} & \multirow{4}{*}{21} & \\
\hline & & & & & 1477.4 & 48.01 & 42.49 ps1d & $27.09 \mathrm{ps} 1 \mathrm{~g}$ & 5.843 & & & \\
\hline & & & & & 100.53 & 3.267 & 2.891 & $1.843 \mathrm{~atm}$ & 0.3976 & & & \\
\hline & & & & & 10.186 & 0.3310 & 0.2929 & 0.1868 & 0.04028 & & & \\
\hline & & & & ABSOLUTE & ABSOLUTE & ABSOLUTE & DIFEERENTIAL & \multicolumn{2}{|l|}{ ABSOLYTE } & (बK) & (OK) & (thI $/ \mathrm{sacl}$ \\
\hline & & & & $12.3 \mathrm{ps} 1 \mathrm{a}$ & $1489.7 \quad \mathrm{ps} 1 \mathrm{a}$ & 60.31 psia & 42.49 ps1d & 39.39 ps1a & 18.14 & \multirow{3}{*}{296} & \multirow{3}{*}{294} & \multirow{3}{*}{0.03060} \\
\hline & & & & $0.8370 \mathrm{~atm}$ & 101.37 & 4.104 & 2.891 & $2.680 \mathrm{~atm}$ & 1.235 & & & \\
\hline & & & & 0.08481 & 10.271 & 0.4158 & 0.2929 & 0.2716 & 0.1251 & & & \\
\hline
\end{tabular}

至

Apparent gas permeability:

Boyle's Law:

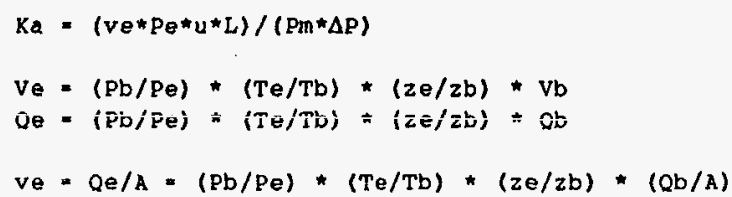

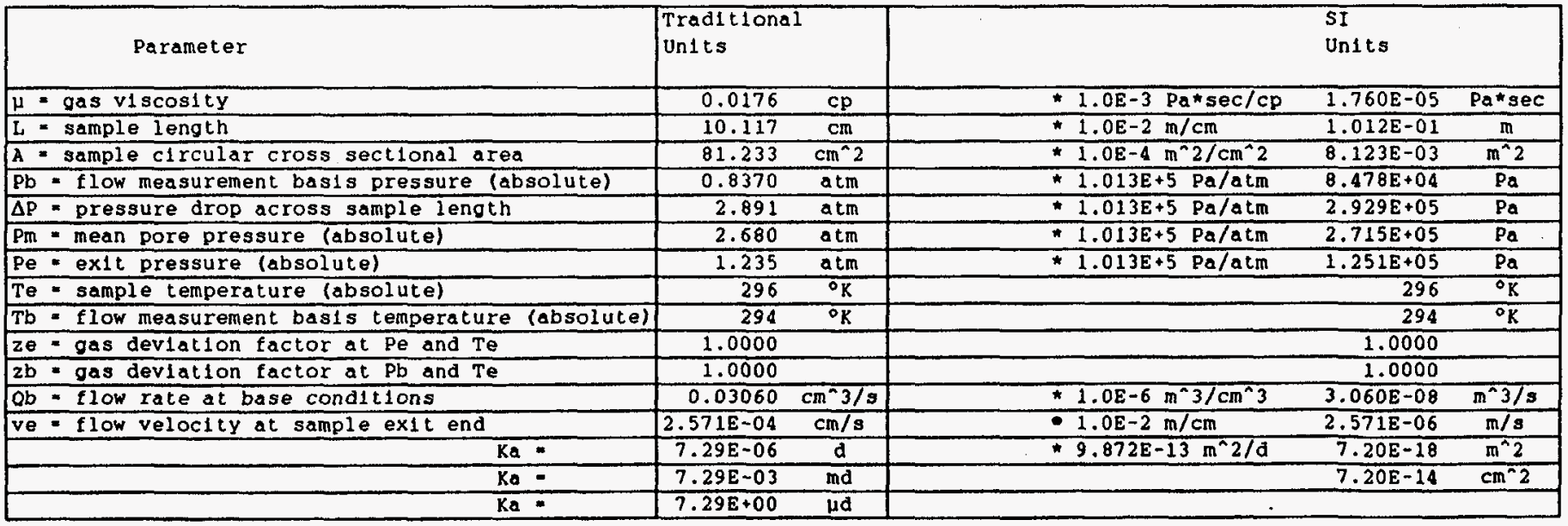


Steady State Gas Permeability Data

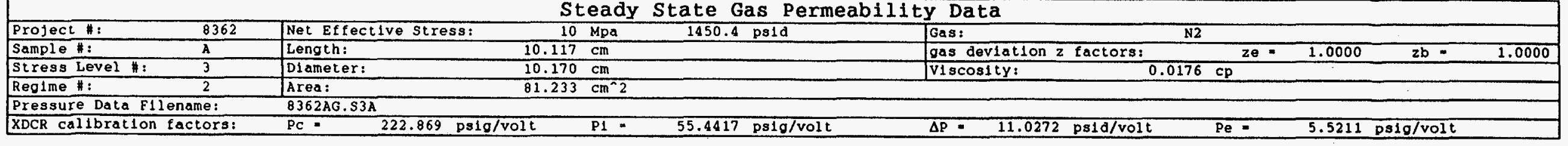

\begin{tabular}{|c|c|c|c|c|c|c|c|c|c|c|c|c|}
\hline Date & $\begin{array}{l}\text { Time } \\
\text { of } \\
\text { Day }\end{array}$ & $\begin{array}{l}\text { File } \\
\text { Time } \\
\text { (min) }\end{array}$ & $\underset{*}{\text { Reglme }}$ & $\begin{array}{l}\mathrm{pb} \\
\text { Barometric } \\
\text { Pressure }\end{array}$ & $\begin{array}{l}\text { Pc } \\
\text { Contining } \\
\text { Pressure }\end{array}$ & $\begin{array}{l}\text { P1 } \\
\text { Inlet } \\
\text { Pressure }\end{array}$ & $\begin{array}{l}\Delta \mathrm{P} \\
\text { D1fferent1al } \\
\text { Pressure }\end{array}$ & $\begin{array}{l}\mathrm{Pm} \\
\text { Mean pore } \\
\mathrm{Pressure} \\
\mathrm{Pe}+\Delta \mathrm{P} / 2\end{array}$ & $\begin{array}{l}\text { Pe } \\
\text { Exit } \\
\text { Pressure }\end{array}$ & $\begin{array}{l}\text { Te } \\
\text { Flow } \\
\text { Temp } \\
\left({ }^{\circ} \mathrm{C}\right)\end{array}$ & $\begin{array}{c}\text { Tb } \\
\text { Amblent } \\
\text { Temp } \\
\left({ }^{\circ} \mathrm{C}\right)\end{array}$ & $\begin{array}{c}\text { Qb } \\
\text { Flow Rate } \\
\text { Pbstb } \\
\text { (ml/sec) }\end{array}$ \\
\hline 03 Jun 93 & $14: 15$ & 1372 & AS3R2a & $12.32 \mathrm{psia}$ & volts & volts & 3.820 volts & & 2.939 & 23 & 22 & 0.03577 \\
\hline 03 Jun 93 & $14: 19$ & 1372 & AS3R2b & $12.32 \mathrm{ps} 1 \mathrm{a}$ & volts & volts & $3.821 \mathrm{volts}$ & & volts & 23 & 22 & 0.03584 \\
\hline 03 Jun 93 & $14: 21$ & 1377 & AS3R2C & $12.32 \mathrm{ps} 1 \mathrm{a}$ & volts & volts & 3.821 volts & & volts & 23 & $\frac{22}{22}$ & 0.03595 \\
\hline 03 Jun 93 & $14: 24$ & 1377 & AS3R2d & 12.32 ps1a & volts & volts & 3.821 volts & & volts & 23 & 22 & 0.03599 \\
\hline 03 Jun 93 & $14: 26$ & 1382 & AS3R2e & $12.32 \mathrm{ps} 1 \mathrm{a}$ & 6.674 volts & volts & 3.821 volts & & volts & 23 & 22 & 0.03601 \\
\hline \multirow{9}{*}{ AVERAGES } & & & \multirow{9}{*}{ AS3R 2} & & OUAGE & OUACE & DTFEERENTIA & GUNGE & OUAOE & 90 & 601 & \\
\hline & & & & & 6.674 volts & 1.047 volts & 3.821 volts & & 2.939 & \multirow{4}{*}{23} & \multirow{4}{*}{22} & \\
\hline & & & & & 1487.4 & 58.05 & 42.13 psid & $37.29 \mathrm{psig}$ & 16.225 & & & \\
\hline & & & & & 101.21 & 3.950 & 2.867 & $2.538 \mathrm{~atm}$ & 1.1041 & & & \\
\hline & & & & & 10.255 & 0.4002 & 0.2905 & $0.2571 \mathrm{Mpa}$ & 0.11187 & & & \\
\hline & & & & ABSOLUTTE & ABSOLUTE & ABSOLUTE & DTEFERENTIAL & Ras SOLUTE & ABSOTUTE & (OK) & (OK) & 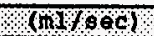 \\
\hline & & & & 12.32 ps1a & $1499.7 \quad$ ps1a & 70.37 ps1a & 42.13 psid & $49.61 \mathrm{ps} 1 \mathrm{a}$ & 28.55 & \multirow{3}{*}{296} & \multirow{3}{*}{295} & \multirow{3}{*}{0.03589} \\
\hline & & & & $0.8383 \mathrm{~atm}$ & 102.05 & 4.788 & 2.867 & $3.376 \mathrm{~atm}$ & 1.942 & & & \\
\hline & & & & 0.08494 & 10.340 & 0.4852 & 0.2905 & 0.3421 & 0.1968 & & & \\
\hline
\end{tabular}

E

Apparent gas permeability: $K a=\left(v e * P e^{*} u^{*} L\right) /(P m * \Delta P)$

Boyle's Law:

$\mathrm{Ve}=(\mathrm{Pb} / \mathrm{Pe}) \cdot(\mathrm{Te} / \mathrm{Tb}) *(\mathrm{ze} / \mathrm{zb}) * \mathrm{Vb}$

$\mathrm{Qe}=(\mathrm{Pb} / \mathrm{Pe}) *(\mathrm{Te} / \mathrm{Tb}) *(2 e / 2 \mathrm{~b}) * \mathrm{Qb}$

$v e=Q e / A=(\mathrm{Pb} / \mathrm{Pe}) *(\mathrm{Te} / \mathrm{Tb}) *(\mathrm{ze} / \mathrm{zb}) *(\mathrm{Qb} / \mathrm{A})$

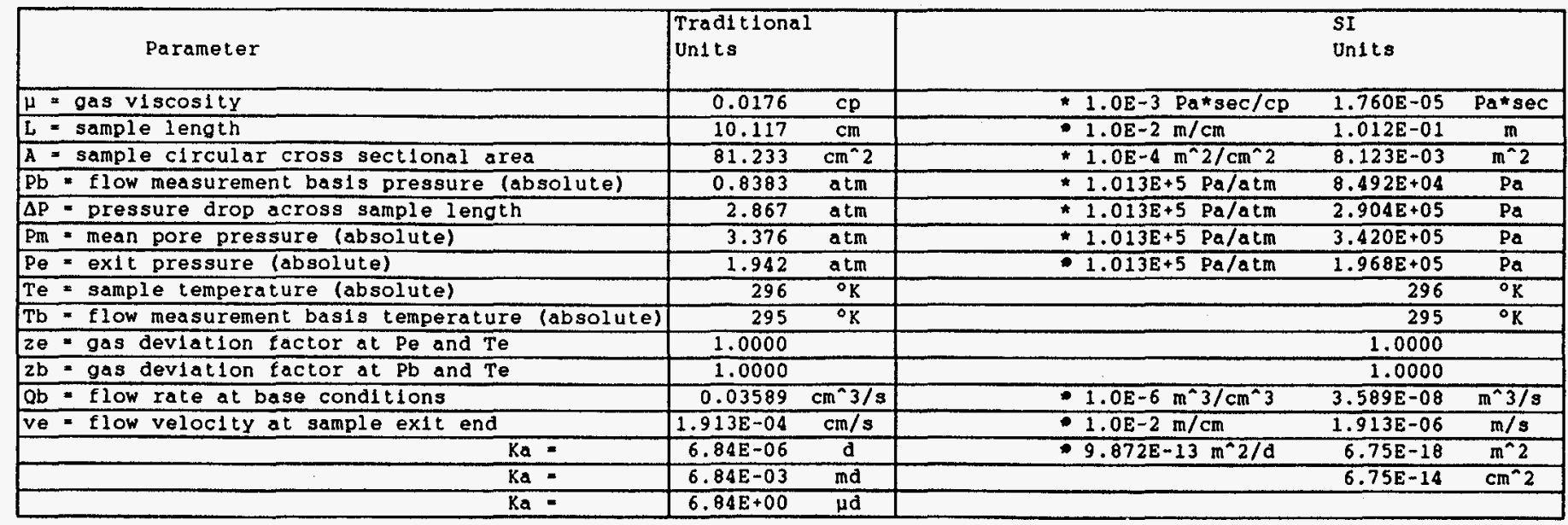


Steady State Gas Permeability Data

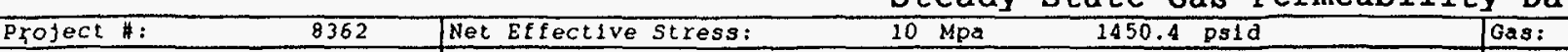

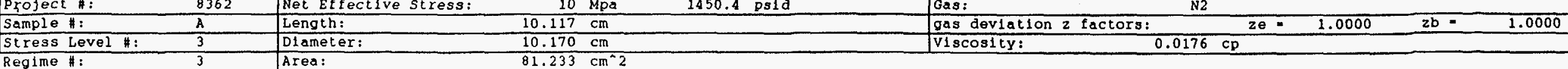

$81.233 \mathrm{~cm}^{\wedge} 2$ V1scosity:

$0.0176 \mathrm{cp}$

Pressure Data Fllename: $8362 \mathrm{AG}$ S3A

\begin{tabular}{|llllll}
\hline XDCR Callbration factors: & PC & 222.869 & pslg/volt & PI -
\end{tabular}

\begin{tabular}{|c|c|c|c|c|c|c|c|c|c|c|c|c|}
\hline Date & $\begin{array}{l}\text { T1me } \\
\text { of } \\
\text { Day }\end{array}$ & $\begin{array}{l}\text { File } \\
\text { Time } \\
\text { (min) }\end{array}$ & $\begin{array}{l}\text { Regime } \\
\#\end{array}$ & $\begin{array}{l}\text { Pb } \\
\text { Barometric } \\
\text { Pressure }\end{array}$ & $\begin{array}{l}\text { PC } \\
\text { Confining } \\
\text { Pressure }\end{array}$ & $\begin{array}{l}\text { P1 } \\
\text { Inlet } \\
\text { Pressure }\end{array}$ & $\begin{array}{l}\Delta P \\
\text { Differential } \\
\text { Pressure }\end{array}$ & $\begin{array}{l}\text { Pm } \\
\text { Mean Pore } \\
\text { Pressure } \\
\text { Pe }+\Delta P / 2\end{array}$ & $\begin{array}{l}\text { Pe } \\
\text { Exit } \\
\text { Pressure }\end{array}$ & $\begin{array}{l}\text { Te } \\
\text { Flow } \\
\text { Temp } \\
\left({ }^{\circ} \mathrm{C}\right)\end{array}$ & $\begin{array}{c}\text { Tb } \\
\text { Ambient } \\
\text { Temp } \\
\left({ }^{\circ} \mathrm{C}\right)\end{array}$ & $\begin{array}{c}\text { Qb } \\
\text { Flow Rate } \\
\text { Pb\&Tb } \\
\text { (ml/sec) }\end{array}$ \\
\hline 04 Jun 93 & $09: 31$ & 2527 & AS3R3a & $12.29 \mathrm{ps} 1 \mathrm{a}$ & 6.719 & volts & 3.879 volts & & 4.669 & 23 & 21 & 0.04180 \\
\hline 04 Jun 93 & $09: 39$ & 2532 & AS 3R3B & $12.29 \mathrm{ps} 1 \mathrm{a}$ & 6.719 & volts & $3.880 \mathrm{volts}$ & & volts & 23 & 21 & 0.04169 \\
\hline 04 Jun 93 & $09: 43$ & 2537 & AS3R3C & $12.29 \mathrm{psia}$ & 6.719 & volts & 3.879 volts & & volts & 23 & 21 & 0.04179 \\
\hline 04 Jun 93 & $09: 46$ & 2542 & AS3R3d & 12.29 ps1a & 6.719 & volts & 3.879 volts & & volts & 23 & 21 & 0.04180 \\
\hline \multirow{9}{*}{ AVERAGES } & & & \multirow{9}{*}{ AS3R3 } & & GUAGE & ROUAOE & DTFEERENTIAL & DUAGE & GUACE & 100 & $\mathrm{CoO}$ & \\
\hline & & & & & 6.719 volts & 1.229 volts & 3.879 volts & & 4.670 & \multirow{4}{*}{23} & \multirow{4}{*}{21} & \\
\hline & & & & & 1497.5 & 68.14 & 42.78 psid & $47.17 \mathrm{ps1g}$ & 25.781 & & & \\
\hline & & & & & 101.90 & 4.636 & 2.911 & $3.210 \mathrm{~atm}$ & 1.7543 & & & \\
\hline & & & & & 10.325 & 0.4698 & 0.2949 & $0.3252 \mathrm{Mpa}$ & 0.17775 & & & \\
\hline & & & & ABSOLUTE & ABSOLUTE & ABSOLUTE & DIFFERENTIAL & ABSOLUTE & ABSOLUTE & (OK) & $8 \mathrm{~kJ}$ & $(\mathrm{~m} Y \mathrm{sec})$ \\
\hline & & & & $12.29 \mathrm{ps} 1 \mathrm{a}$ & $1509.7 \quad \mathrm{ps} 1 \mathrm{a}$ & $80.43 \quad \mathrm{ps} 1 \mathrm{a}$ & $\begin{array}{rr}42.78 \quad \text { psid } \\
\end{array}$ & 59.46 ps1a & 38.07 & \multirow{3}{*}{296} & \multirow{3}{*}{294} & \multirow{3}{*}{0.04177} \\
\hline & & & & $0.8363 \quad \mathrm{~atm}$ & 102.73 & 5.473 & 2.911 & $4.046 \mathrm{~atm}$ & 2.591 & & & \\
\hline & & & & 0.08474 & 10.409 & 0.5545 & 0.2949 & 0.4100 & 0.2625 & & & \\
\hline
\end{tabular}

穴
Apparent gas permeab1lity:
$K a=\left(v e^{\star} P e^{\star} u^{\star} L\right) /\left(P m^{*} \Delta P\right)$

Boyle's Law:

$\mathrm{Ve}=(\mathrm{Pb} / \mathrm{Pe}) *(\mathrm{Te} / \mathrm{Tb}) \cdot(\mathrm{ze} / \mathrm{zb}) * \mathrm{Vb}$

$\mathrm{ne}=(\mathrm{Pb} / \mathrm{Pe}) \cdot(\mathrm{Te} / \mathrm{Tb}) *(z e / 2 b) * \mathrm{Qb}$

$v e=Q e / A=(\mathrm{Pb} / \mathrm{Pe}) *(\mathrm{Te} / \mathrm{Tb}) *(z e / z \mathrm{~b}) *(\mathrm{Qb} / \mathrm{A})$

\begin{tabular}{|c|c|c|c|c|}
\hline Parameter & $\begin{array}{l}\text { Traditional } \\
\text { Undts }\end{array}$ & . & $\begin{array}{l}\text { SI } \\
\text { Units }\end{array}$ & \\
\hline$\mu=g a s v 1 s \cos i t y$ & 0.0176 & $\star 1.0 \mathrm{E}-3 \mathrm{~Pa} \mathrm{Pec}^{\circ} \mathrm{Cp}$ & $1.760 \mathrm{E}-05$ & Pa*sec \\
\hline L = sample length & 10.117 & $\$ 1.0 \mathrm{E}-2 \mathrm{~m} / \mathrm{cm}$ & $1.012 \mathrm{E}-01$ & $\mathrm{~m}$ \\
\hline A - sample clrcular cross sectlonal area & 81.233 & $\star 1.0 \mathrm{E}-4 \mathrm{~m}^{\wedge} 2 / \mathrm{cm}^{\wedge} 2$ & $8.123 \mathrm{E}-03$ & $m^{\wedge} 2$ \\
\hline Pb $=$ flow measurement basls pressure (absolute) & 0.8363 & $\approx 1.013 E+5 \mathrm{~Pa} / \mathrm{atm}$ & $8.472 E+04$ & $\mathrm{pa}$ \\
\hline$\Delta \mathrm{P}=$ pressure drop across sample length & 2.911 & $\star 1.013 \mathrm{E}+5 \mathrm{~Pa} / \mathrm{atm}$ & $2.949 \mathrm{E}+05$ & Pa \\
\hline $\mathrm{p}_{\mathrm{m}}=$ mean pore pressure (absolute) & 4.046 & $1.013 \mathrm{E}+5 \mathrm{~Pa} / \mathrm{atm}$ & $4.099 \mathrm{E}+05$ & $\mathrm{~Pa}$ \\
\hline Pe $=$ exlt pressure (absolute) & 2.591 & $\star 1.013 \mathrm{E}+5 \mathrm{~Pa} / \mathrm{atm}$ & $2.624 E+05$ & $\mathrm{~Pa}$ \\
\hline $\mathrm{Te}$ sample temperature (absolute) & 296 & & 296 & ${ }^{\circ} \mathrm{K}$ \\
\hline Tb - flow measurement basis temperature (absolute) & 294 & & 294 & ${ }^{\circ} \mathrm{K}$ \\
\hline $2 \mathrm{e}=$ gas devlation factor at $\mathrm{Pe}$ and $\mathrm{Te}$ & 1.0000 & & 1.0000 & \\
\hline $2 \mathrm{~b}$ - gas deviation factor $\mathrm{at} \mathrm{Pb}$ and $\mathrm{Te}$ & 1.0000 & & 1.0000 & \\
\hline$Q b=$ flow rate at base conditions & $0.04177 \mathrm{~cm} \wedge 3 / \mathrm{s}$ & $1.0 \mathrm{E}-6 \mathrm{~m}^{\wedge} 3 / \mathrm{cm}^{\wedge} 3$ & $4.177 \mathrm{E}-08$ & $\mathrm{~m}^{2} 3 / \mathrm{s}$ \\
\hline ve $=$ flow velocity at sample exit end & $1.671 \mathrm{E}-04 \mathrm{~cm} / \mathrm{s}$ & $1.0 \mathrm{E}-2 \mathrm{~m} / \mathrm{cm}$ & $1.671 \mathrm{E}-06$ & $\mathrm{~m} / \mathrm{s}$ \\
\hline $\mathrm{Ka}=$ & $6.55 E-06$ & $9.872 E-13 \mathrm{~m}^{2} 2 / \mathrm{d}$ & $6.46 \mathrm{E}-18$ & $m^{\wedge} 2$ \\
\hline $\mathrm{Ka} \cdot$ & $6.55 \mathrm{E}-03$ & & $6.46 E-14$ & $\mathrm{Cm}^{\wedge} 2$ \\
\hline $\mathrm{Ka}=$ & $6.55 \mathrm{E}+00$ & & & \\
\hline
\end{tabular}




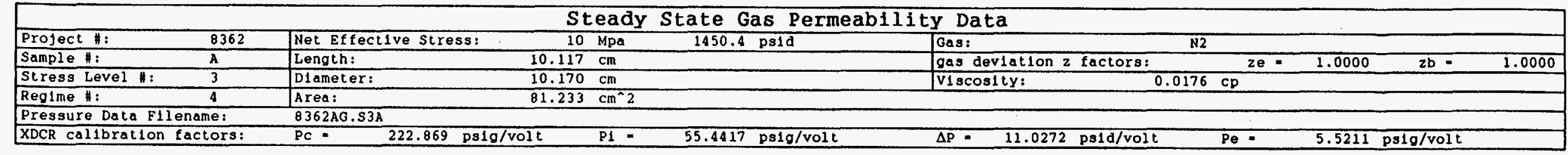

\begin{tabular}{|c|c|c|c|c|c|c|c|c|c|c|c|c|}
\hline Date & $\begin{array}{l}\text { Time } \\
\text { of } \\
\text { Day }\end{array}$ & $\begin{array}{l}\text { File } \\
\text { Time } \\
(m i n)\end{array}$ & $\begin{array}{c}\text { Reg1me } \\
\#\end{array}$ & $\begin{array}{l}\text { Pb } \\
\text { Barometr1c } \\
\text { Pressure }\end{array}$ & $\begin{array}{l}\text { Pc } \\
\text { Confining } \\
\text { Pressure }\end{array}$ & $\begin{array}{l}\text { P1 } \\
\text { Inlet } \\
\text { Pressure }\end{array}$ & $\begin{array}{l}\Delta P \\
\text { D1fferential } \\
\text { Pressure }\end{array}$ & $\begin{array}{l}\text { Pm } \\
\text { Mean Pore } \\
\text { Pressure } \\
\text { Pe }+\Delta P / 2\end{array}$ & $\begin{array}{l}\text { Pe } \\
\text { Exit } \\
\text { Pressure }\end{array}$ & $\begin{array}{l}\text { Te } \\
\text { Flow } \\
\text { Temp } \\
\left({ }^{\circ} \mathrm{C}\right)\end{array}$ & $\begin{array}{c}\text { Tb } \\
\text { Amblent } \\
\text { Temp } \\
\left({ }^{\circ} \mathrm{C}\right\rangle\end{array}$ & $\begin{array}{c}\text { Qb } \\
\text { Flow Rate } \\
\text { OPb\&Tb } \\
\text { (ml/sec) }\end{array}$ \\
\hline 04 Jun 93 & $16: 35$ & 2952 & AS3R4a & $12.23 \mathrm{pS} 1 \mathrm{a}$ & volts & 1.407 & 3.857 volts & & 6.530 & 23 & $\overline{22}$ & 0.04725 \\
\hline 04 Jun 93 & $16: 37$ & 2952 & AS3R4b & 12.23 pq1a & volts & volts & 3.857 vol ts & & volts & 23 & 22 & 0.04723 \\
\hline 04 Jun 93 & $16: 39$ & 2957 & AS3R4C & $12.23 \mathrm{psia}$ & volts & volts & 3.057 volts & & volts & 23 & 22 & 0.04720 \\
\hline 04 Jun 93 & $16: 42$ & 2957 & AS3R4d & 12.23 ps1a & volts & volts & $3.857 \mathrm{vo1ts}$ & & volts & 23 & 22 & 0.04728 \\
\hline \multirow{9}{*}{ AVERAGES } & & & \multirow{9}{*}{ AS 3R4 } & & OUAGE: & OOUAE: & DIESERENTIAI & OUAGE & GDXOE & कटा & बक्वश: & \\
\hline & & & & & 6.764 volts & 1.407 volts & 3.857 volts & & 6.530 & \multirow{4}{*}{23} & \multirow{4}{*}{22} & \\
\hline & & & & & 1507.5 & 78.01 & 42.53 ps1d & $57.32 \mathrm{psig}$ & 36.053 & & & \\
\hline & & & & & 102.58 & 5.308 & $2.894 \quad \mathrm{~atm}$ & $3.900 \mathrm{~atm}$ & 2.4532 & & & \\
\hline & & & & & $10.394 \quad \mathrm{Mpa}$ & 0.5378 & $0.2932 \mathrm{Mpa}$ & $0.3952 \mathrm{Mpa}$ & 0.24858 & & & \\
\hline & & & & ABSOLUTE & ABSOLUTE & ABSOLUTE & DHFEERENT TAL & ARSOLUTE & ABSOLUTE & (OK) & (0.88 & (DIf $/ S A C)$ \\
\hline & & & & $12.23 \mathrm{psia}$ & 1519.7 psia & 90.24 ps1a & 42.53 psid & 69.55 Ds1a & 48.28 & \multirow{3}{*}{296} & \multirow{3}{*}{295} & \multirow{3}{*}{0.04724} \\
\hline & & & & $0.8322 \mathrm{~atm}$ & 103.41 & 6.140 & 2.894 & $4.732 \quad \mathrm{~atm}$ & 3.285 & & & \\
\hline & & & & $0.08432 \mathrm{Mpa}$ & 10.478 & 0.6222 & 0.2932 & 0.4795 & 0.3329 & & & \\
\hline
\end{tabular}

Apparent gas permeablilty:

Boyle's Law:

$K a=(v e * P e * u * L) /(P m * \Delta P)$

$\mathrm{Ve}=(\mathrm{Pb} / \mathrm{Pe}) *(\mathrm{Te} / \mathrm{Tb}) *(\mathrm{ze} / \mathrm{zb}) * \mathrm{Vb}$

$\mathrm{Qe}_{\mathrm{e}}=(\mathrm{Pb} / \mathrm{Pe}) *(\mathrm{Te} / \mathrm{Tb}) \cdot(\mathrm{ze} / \mathrm{zb}) * \mathrm{Qb}$

$v e=Q e / A=(P b / P e) *(T e / T b) *(z e / Z b) *(Q b / A)$

\begin{tabular}{|c|c|c|c|c|}
\hline Parameter & $\begin{array}{l}\text { Trad1tional } \\
\text { Units }\end{array}$ & & $\begin{array}{l}\text { SI } \\
\text { Units }\end{array}$ & \\
\hline$\mu=g a s v 1 s \cos 1 t y$ & 0.0176 & $\star 1.0 \mathrm{E}-3 \mathrm{~Pa} * \mathrm{sec} / \mathrm{CP}$ & $1.760 \mathrm{E}-05$ & $\mathrm{~Pa} a_{\mathrm{sec}}$ \\
\hline$L=$ sample length & 10.117 & $\star 1.0 \mathrm{E}-2 \mathrm{~m} / \mathrm{cm}$ & $1.012 \mathrm{E}-01$ & m \\
\hline$A=$ sample clrcular cross sectional area & 81.233 & $* 1.0 \mathrm{E}-4 \mathrm{~m}^{2} 2 / \mathrm{cm}^{2} 2$ & $8.123 \mathrm{E}-03$ & $m^{\wedge} 2$ \\
\hline $\mathrm{Pb}=$ flow measurement basis pressure (absolute) & 0.8322 & $\star 1.013 \mathrm{E}+5 \mathrm{~Pa} / \mathrm{atm}$ & $8.430 E+04$ & Pa \\
\hline$\Delta \mathrm{P}$ - pressure drop across sample length & 2.894 & $\star 1.013 \mathrm{E}+5 \mathrm{~Pa} / \mathrm{atm}$ & $2.932 E+05$ & $\mathrm{~Pa}$ \\
\hline $\mathrm{Pm}=$ mean pore pressure (absolute) & 4.732 & $\star 1.013 \mathrm{E}+5 \mathrm{~Pa} / \mathrm{atm}$ & $4.794 E+05$ & $\mathrm{~Pa}$ \\
\hline $\mathrm{Pe}=$ exit pressure (absolute) & 3.285 & $\star 1.013 \mathrm{E}+5 \mathrm{~Pa} / \mathrm{atm}$ & $3.328 E+05$ & $\mathrm{~Pa}$ \\
\hline $\mathrm{Te}=$ sample temperature (absolute) & 296 & & 296 & ${ }^{\circ} \mathrm{K}$ \\
\hline $\mathrm{Tb}$ - flow measurement basis temperature (absolute) & 295 & & 295 & ${ }^{\circ} \mathrm{K}$ \\
\hline $\mathrm{ze}=\mathrm{gas}$ deviation factor at $\mathrm{Pe}$ and $\mathrm{Te}$ & 1.0000 & & 1.0000 & \\
\hline $\mathrm{zb}=$ gas devlation factor $\mathrm{at} \mathrm{Pb}$ and $\mathrm{Te}$ & 1.0000 & & 1.0000 & \\
\hline$Q b=$ flow rate at base conditions & $0.04724 \mathrm{~cm}^{\wedge} 3 / \mathrm{s}$ & $1.0 \mathrm{E}-6 \mathrm{~m}^{\wedge} 3 / \mathrm{cm}^{\wedge} 3$ & $4.726 \mathrm{E}-08$ & $\mathrm{~m}^{\wedge} 3 / \mathrm{s}$ \\
\hline$v e=$ tlow velocity at sample exit end & $1.478 \mathrm{E}-04$ & $1.0 \mathrm{E}-2 \mathrm{~m} / \mathrm{cm}$ & $1.478 \mathrm{E}-06$ & $\mathrm{~m} / \mathrm{s}$ \\
\hline $\mathrm{Ka}=$ & $6.31 \mathrm{E}-06$ & $\star 9.872 \mathrm{E}-13 \mathrm{~m}^{\wedge} 2 / \mathrm{d}$ & $6.23 \mathrm{E}-18$ & $m^{\wedge} 2$ \\
\hline $\mathrm{Ka}=$ & $6.31 \mathrm{E}-03$ & & $6.23 \mathrm{E}-14$ & $c m^{2} 2$ \\
\hline $\mathrm{Ka}=$ & $6.31 \mathrm{E}+00$ & & & \\
\hline
\end{tabular}




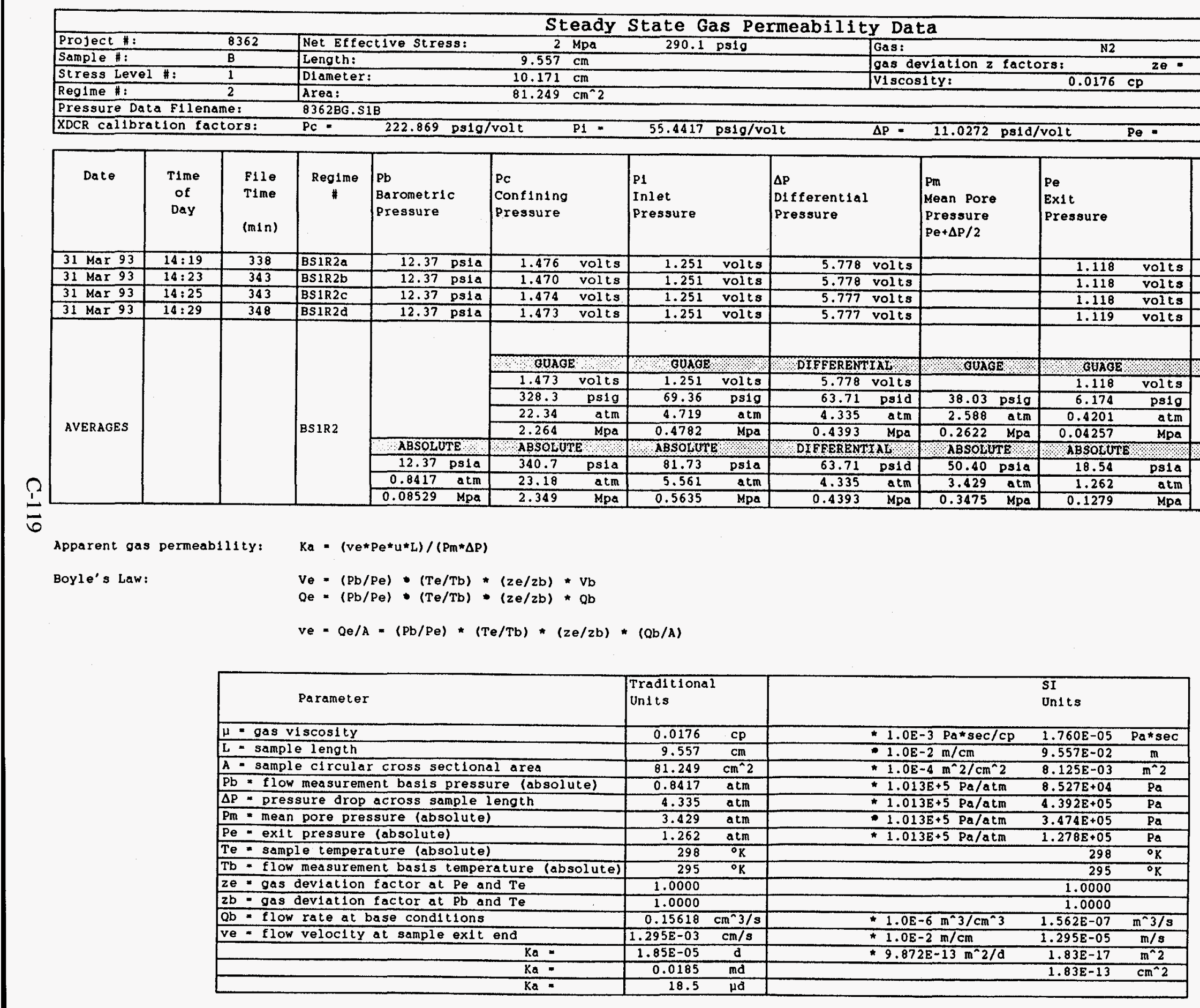


Steady State Gas Permeability Data

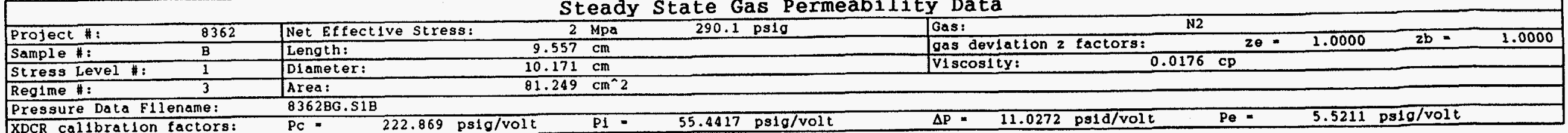

\begin{tabular}{l} 
Pressure Data Fllename: \\
\hline XDCR callbration factors:
\end{tabular} $\mathrm{PC}=222.869 \mathrm{ps} / \mathrm{g} / \mathrm{volt} \quad \mathrm{PI}$

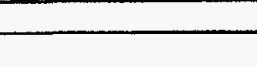

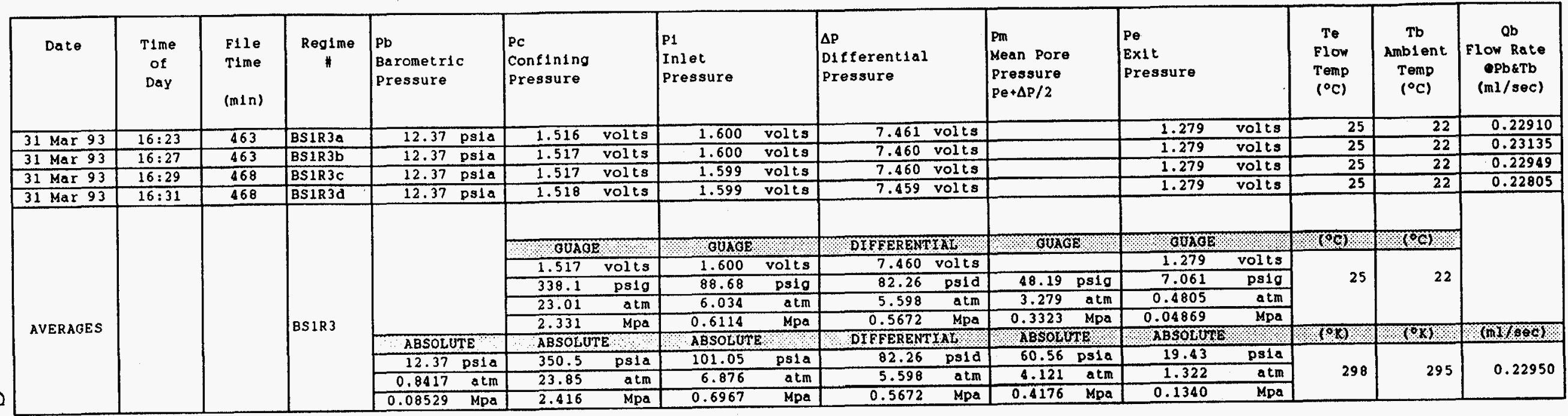

$\stackrel{1}{0}$

Apparent gas permeab1l1ty:

Boyle's Law:
$K a=\left(v e * P^{*} u * L\right) /(P m * \Delta P)$

$\mathrm{Ve}=(\mathrm{Pb} / \mathrm{Pe}) *(\mathrm{Te} / \mathrm{Tb}) *(\mathrm{ze} / \mathrm{Zb}) * \mathrm{Vb}$

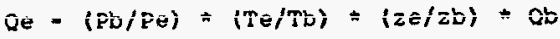

$v e * Q e / A=(\mathrm{Pb} / \mathrm{Pe}) *(\mathrm{Te} / \mathrm{Tb}) *(2 \mathrm{e} / \mathrm{Zb}) *(\mathrm{Qb} / \mathrm{A})$

\begin{tabular}{|c|c|c|c|c|}
\hline Parameter & $\begin{array}{l}\text { Traditional } \\
\text { Units }\end{array}$ & & $\begin{array}{l}\text { SI } \\
\text { Units }\end{array}$ & \\
\hline$\mu=\operatorname{gas} v 1 s \cos 1 t y$ & 0.0176 & $\star 1.0 \mathrm{E}-3 \mathrm{~Pa} * \mathrm{sec} / \mathrm{CP}$ & $1.760 \mathrm{E}-05$ & Pa*sec \\
\hline $\mathrm{L}=$ sample length & 9.557 & $1.0 \mathrm{E}-2 \mathrm{~m} / \mathrm{cm}$ & $9.557 \mathrm{E}-02$ & m \\
\hline$A=$ sample clrcular cross sectlonal area & 81.249 & $\pi 1.0 \mathrm{E}-4 \mathrm{~m}^{\wedge} 2 / \mathrm{cm}^{\wedge} 2$ & $8.125 \mathrm{E}-03$ & $m^{\wedge} 2$ \\
\hline $\mathrm{Pb}=$ flow measurement basls pressure (absolute) & 0.8417 & $1.013 \mathrm{E}+5 \mathrm{~Pa} / \mathrm{atm}$ & $8.527 \mathrm{E}+04$ & $\mathrm{~Pa}$ \\
\hline$\Delta p=$ pressure drop across sample length & 5.598 & $1.013 \mathrm{E}+5 \mathrm{~Pa} / \mathrm{atm}$ & $5.670 \mathrm{E}+05$ & $\mathrm{~Pa}_{\mathrm{a}}$ \\
\hline $\mathrm{Pm}=$ mean pore pressure (absolute) & 4.121 & $1.013 \mathrm{E}+5 \mathrm{~Pa} / \mathrm{atm}$ & $4.175 E+05$ & Pa \\
\hline Pe = exit pressure (absolute) & 1.322 & * $1.013 \mathrm{E}+5 \mathrm{~Pa} / \mathrm{atm}$ & $1.339 E+05$ & $\mathrm{~Pa}$ \\
\hline Te - sample temperature (absolute) & 298 & & 298 & ${ }^{\circ} \mathrm{K}$ \\
\hline$T b=$ flow measurement basls temperature (absolute) & 295 & & 295 & ${ }^{\circ} \mathrm{K}$ \\
\hline$z e=$ gas deviation factor at $P e$ and $T \theta$ & 1.0000 & & 1.0000 & \\
\hline $2 \mathrm{~b}$ - gas deviation factor at $\mathrm{Pb}$ and $\mathrm{Te}$ & 1.0000 & & $\frac{1.0000}{2.205 E-07}$ & \\
\hline$Q b$ - flow rate at base condltions & $0.22950 \mathrm{~cm}^{\wedge} 3 / \mathrm{s}$ & $1.0 \mathrm{E}-6 \mathrm{~m}^{2} 3 / \mathrm{cm}^{3} 3$ & $2.295 E-07$ & $\frac{m^{2} 3 / s}{m / s}$ \\
\hline ve - flow velocity at sample exit end & $1.816 \mathrm{E}-03$ & $1.0 \mathrm{E}-2 \mathrm{~m} / \mathrm{cm}$ & $\frac{1.816 E-05}{1.73 E-17}$ & $\mathrm{~m} / \mathrm{s}$ \\
\hline $\begin{array}{ll}\mathrm{Ka}_{\mathrm{a}}= \\
\end{array}$ & $1.75 \mathrm{E}-05$ & $9.872 \mathrm{E}-13 \mathrm{~m}^{\wedge} 2 / \mathrm{d}$ & $\frac{1.73 \mathrm{E}-17}{1.73 \mathrm{E}-13}$ & $\frac{m^{2} 2}{c m^{\wedge} 2}$ \\
\hline $\begin{array}{l}\mathrm{Ka}- \\
\mathrm{Ka}=\end{array}$ & 0.0175 & & $1.73 \mathrm{E}-13$ & $\mathrm{~cm}^{2} 2$ \\
\hline $\mathrm{Ka}=$ & 17.5 & & & \\
\hline
\end{tabular}


Steady State Gas Permeability

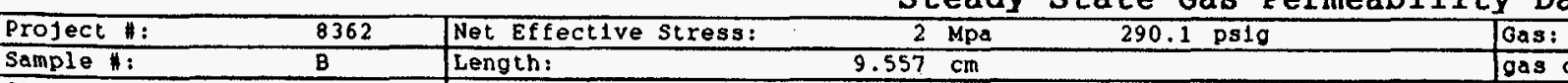

\begin{tabular}{l|l|lr}
\hline Sample \#: & B & Length: & $9.557 \mathrm{~cm}$ \\
\hline Stress Level \#: & 1 & Dlameter: & $10.171 \mathrm{~cm}$ \\
\hline
\end{tabular}

\begin{tabular}{l|l} 
Regime : & Drea:
\end{tabular}

$81.249 \mathrm{~cm}^{\wedge}$

N2

$\begin{array}{lll}\text { Pressure Data Filename: } & \text { B362BG.S1日 }\end{array}$

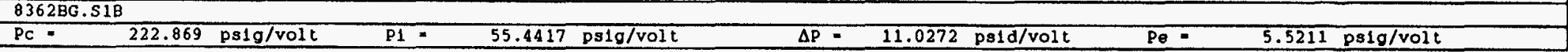

XDCR callbration factors:

\begin{tabular}{|c|c|c|c|c|c|c|c|c|c|c|c|c|}
\hline Date & $\begin{array}{l}\text { Time } \\
\text { of } \\
\text { Day }\end{array}$ & $\begin{array}{l}\text { File } \\
\text { Time } \\
(m 1 n)\end{array}$ & $\begin{array}{c}\text { Regime } \\
\#\end{array}$ & $\begin{array}{l}\mathrm{pb} \\
\text { Barometric } \\
\text { Pressure }\end{array}$ & $\begin{array}{l}\text { Pc } \\
\text { Confining } \\
\text { Pressure }\end{array}$ & $\begin{array}{l}\text { P1 } \\
\text { Inlet } \\
\text { Pressure }\end{array}$ & $\begin{array}{l}\Delta P \\
D 1 \text { fferential } \\
\text { Pressure }\end{array}$ & $\begin{array}{l}\text { Pm } \\
\text { Mean Pore } \\
\text { Pressure } \\
\text { Pe }+\Delta \mathrm{P} / 2\end{array}$ & $\mid \begin{array}{l}\text { Pe } \\
\text { Exit } \\
\text { Pressure }\end{array}$ & $\begin{array}{l}\text { Te } \\
\text { Flow } \\
\text { Temp } \\
\left({ }^{\circ} \mathrm{C}\right)\end{array}$ & $\begin{array}{c}\text { Tb } \\
\text { Amblent } \\
\text { Temp } \\
\left({ }^{\circ} \mathrm{C}\right)\end{array}$ & $\begin{array}{c}\text { Qb } \\
\text { Flow Rate } \\
\text { Pbetb } \\
\text { (ml } / \text { sec) }\end{array}$ \\
\hline 01 Apr 93 & $09: 20$ & 1480 & BS1R4a & $12.28 \mathrm{ps} 1 \mathrm{a}$ & 1.569 & 1.947 & 9.099 volts & & volts & 25 & 21 & 0.31721 \\
\hline 01 Apr 93 & $09: 23$ & 1480 & BS1RAb & 12.28 ps1a & 1.570 volts & volts & 9.097 volts & & volts & 25 & 22 & 0.31422 \\
\hline 01 ADI 93 & $09: 25$ & 1485 & BS1R4C & $12.28 \mathrm{psia}$ & 1.570 & volts & 9.097 volts & & volts & 25 & 22 & 0.31496 \\
\hline 01 Apr 93 & $09: 27$ & 1485 & BS1R4d & 12.28 psia & 1.568 & volts & 9.096 volts & & volts & 25 & 22 & 0.31671 \\
\hline \multirow{9}{*}{ AVERAGES } & & & \multirow{9}{*}{ BS1R4 } & & OUAGE & GUAOE & DIGEERENTIAL & GUXGE & OUAOE & क्षा & क्टा & \\
\hline & & & & & 1.569 volts & 1.947 volts & 9.097 volts & & 1.516 & \multirow{4}{*}{25} & \multirow{4}{*}{22} & \\
\hline & & & & & 349.7 & 107.93 & 100.32 ps1d & $58.52 \mathrm{psig}$ & 8.359 & & & \\
\hline & & & & & 23.80 & 7.344 & 6.826 & $3.982 \mathrm{~atm}$ & 0.5688 & & & \\
\hline & & & & & $2.411 \quad \mathrm{Mpa}$ & $0.7442 \quad \mathrm{Mpa}$ & $0.6917 \quad \mathrm{Mpa}$ & $0.4035 \mathrm{Mpa}$ & 0.05763 & & & \\
\hline & & & & ABSOLUTE & ABSOLUTE & ABSOLUTE & DIFFERENTIAL & A 8 OLUTE & ABSOLOTE: & $(\mathrm{OK})$ & $(0 \mathrm{~K})$ & $(\mathrm{m}) / \mathrm{s} \theta \mathrm{c})$ \\
\hline & & & & $12.28 \mathrm{ps} 1 \mathrm{a}$ & $362.0 \quad \mathrm{ps} 1 \mathrm{a}$ & 120.21 psia & $100.32 \mathrm{ps} 1 \mathrm{~d}$ & $70.80 \mathrm{ps} 1 \mathrm{a}$ & 20.64 & \multirow{3}{*}{298} & \multirow{3}{*}{295} & \multirow{3}{*}{0.31577} \\
\hline & & & & $0.8356 \quad \mathrm{~atm}$ & 24.63 & 8.180 & 6.826 & 4.817 atm & 1.404 & & & \\
\hline & & & & 0.08467 & 2.496 & 0.8288 & 0.6917 & 0.4881 & 0.1423 & & & \\
\hline
\end{tabular}

N

Apparent gas permeab111ty:

$K a=\left(v e * P e^{\star} u * L\right) /(P m \star \Delta P)$

Boyle's Law:

$v_{e}=(\mathrm{Pb} / \mathrm{Pe}) *(\mathrm{Te} / \mathrm{Tb}) *(2 e / 2 \mathrm{~b}) * \mathrm{Vb}$

$\mathrm{Q}_{e}=(\mathrm{Pb} / \mathrm{Pe}) \cdot(\mathrm{Te} / \mathrm{Tb}) \cdot(\mathrm{ze} / \mathrm{zb}) * \mathrm{Qb}$

ve $=\mathrm{Qe} / \mathrm{A}=(\mathrm{Pb} / \mathrm{Pe}) *(\mathrm{Te} / \mathrm{Tb}) *(\mathrm{ze} / \mathrm{zb}) *(\mathrm{Qb} / \mathrm{A})$

\begin{tabular}{|c|c|c|c|c|}
\hline Parameter & $\begin{array}{l}\text { Traditional } \\
\text { Units }\end{array}$ & & $\begin{array}{l}\text { SI } \\
\text { Unt ts }\end{array}$ & \\
\hline$\mu=$ gas viscosity & 0.0176 & $\pitchfork 1.0 \mathrm{E}-3 \mathrm{~Pa} \# \mathrm{sec} / \mathrm{cp}$ & $1.760 \mathrm{E}-05$ & Pa*sec \\
\hline$L=$ sample length & 9.557 & $1.0 \mathrm{E}-2 \mathrm{~m} / \mathrm{cm}$ & $9.557 E-02$ & $\mathbf{m}$ \\
\hline$A$ - sample clrcular cross sectional area & 81.249 & $\star 1.0 \mathrm{E}-4 \mathrm{~m}^{\wedge} 2 / \mathrm{cm}^{\wedge} 2$ & $8.125 E-03$ & $m^{\wedge} 2$ \\
\hline $\mathrm{Pb}=$ flow measurement basis pressure (absolute) & 0.8356 & $\star 1.013 \mathrm{E}+5 \mathrm{~Pa} / \mathrm{atm}$ & $8.465 E+04$ & $\mathrm{~Pa}$ \\
\hline$\Delta \mathrm{P}=$ pressure drop across sample length & 6.826 & $\approx 1.013 \mathrm{E}+5 \mathrm{~Pa} / \mathrm{atm}$ & $6.915 E+05$ & $\mathbf{P a}$ \\
\hline $\mathrm{Pm}=$ mean pore pressure (absolute) & 4.817 & $\star 1.013 \mathrm{E}+5 \mathrm{~Pa} / \mathrm{atm}$ & $4.880 E+05$ & $\mathrm{~Pa}$ \\
\hline $\mathrm{pe}_{\mathrm{e}}=$ exit pressure (absolute) & 1.404 & $\star 1.013 \mathrm{E}+5 \mathrm{~Pa} / \mathrm{atm}$ & $1.423 E+05$ & $\mathrm{~Pa}$ \\
\hline $\mathrm{Te}=$ sample temperature (absolute) & 298 & & 298 & ${ }^{\circ} \mathrm{K}$ \\
\hline $\mathrm{Tb}$ - flow measurement basls temperature (absolute) & 295 & & 295 & ${ }^{\circ} \mathrm{K}$ \\
\hline $2 e=$ gas deviation factor at $\mathrm{Pe}$ and $\mathrm{Te}$ & 1.0000 & & 1.0000 & \\
\hline $\mathrm{zb}=$ gas deviation factor at $\mathrm{pb}$ and $\mathrm{Te}$ & 1.0000 & & 1.0000 & \\
\hline $\mathrm{Qb}=$ flow rate at base conditions & $0.31577 \mathrm{~cm}^{\wedge} 3 / \mathrm{s}$ & $\star 1.0 \mathrm{E}-6 \mathrm{~m}^{\wedge} 3 / \mathrm{cm}^{\wedge} 3$ & $3.158 \mathrm{E}-07$ & $\mathrm{~m}^{\wedge} 3 / \mathrm{s}$ \\
\hline ve $=$ flow velocity at sample exit end & $2.338 \mathrm{E}-03 \mathrm{~cm} / \mathrm{s}$ & $1.0 \mathrm{E}-2 \mathrm{~m} / \mathrm{cm}$ & $2.338 \mathrm{E}-05$ & $\mathrm{~m} / \mathrm{s}$ \\
\hline $\mathrm{Ka}=$ & $1.68 \mathrm{E}-05$ & $9.872 \mathrm{E}-13 \mathrm{~m}^{\wedge} 2 / \mathrm{d}$ & $1.66 \mathrm{E}-17$ & $m^{\wedge} 2$ \\
\hline Ka $=$ & 0.0168 & & $1.66 \mathrm{E}-13$ & $\mathrm{~cm} \wedge^{\wedge} 2$ \\
\hline $\mathrm{Ka}=$ & 16.8 & & & \\
\hline
\end{tabular}




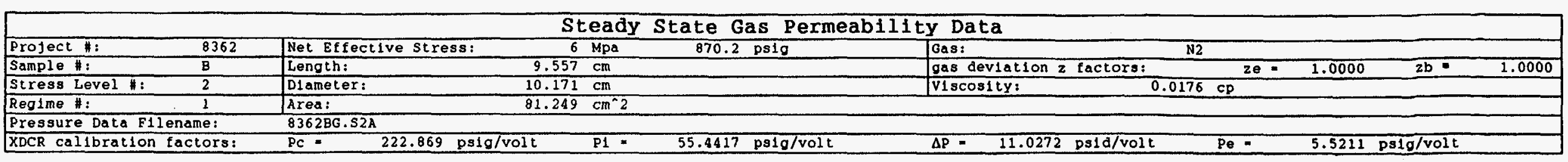

\begin{tabular}{|c|c|c|c|c|c|c|c|c|c|c|c|c|}
\hline Date & $\begin{array}{l}\text { Time } \\
\text { of } \\
\text { Day }\end{array}$ & $\begin{array}{l}\text { File } \\
\text { Time } \\
(m 1 n)\end{array}$ & $\begin{array}{c}\text { Reglme } \\
\#\end{array}$ & $\begin{array}{l}\text { Pb } \\
\text { Barometric } \\
\text { Pressure }\end{array}$ & $\begin{array}{l}\text { PC } \\
\text { Confining } \\
\text { Pressure }\end{array}$ & $\begin{array}{l}\text { p1 } \\
\text { Inlet } \\
\text { Pressure }\end{array}$ & $\begin{array}{l}\Delta \mathrm{P} \\
\text { Differential } \\
\text { Pressure }\end{array}$ & \begin{tabular}{|l} 
Pm \\
Mean Pore \\
Pressure \\
Pe $+\Delta P / 2$
\end{tabular} & $\begin{array}{l}\text { Pe } \\
\text { Ex1t } \\
\text { Pressure }\end{array}$ & $\begin{array}{l}\text { Te } \\
\text { Flow } \\
\text { Temp } \\
\left({ }^{\circ} \mathrm{C}\right)\end{array}$ & $\begin{array}{c}\text { Tb } \\
\text { Amblent } \\
\text { Temp } \\
\left({ }^{\circ} \mathrm{C}\right)\end{array}$ & $\begin{array}{c}\text { ab } \\
\text { Flow Rate } \\
\text { RPbETb } \\
(\mathrm{ml} / \mathrm{sec})\end{array}$ \\
\hline 01 Apr 93 & $14: 46$ & 177 & BS2R1a & $12.23 \mathrm{ps} 1 \mathrm{a}$ & 4.030 & 0.903 & 4.135 volts & & volts & 25 & 22 & 0.06385 \\
\hline 01 Apr 93 & $14: 50$ & 182 & BS2R1b & 12.23 psia & 4.030 & 0.902 & 4.135 volts & & volts & 25 & 22 & 0.06384 \\
\hline 01 Apr 93 & $14: 53$ & 182 & BS2R1C & $12.23 \mathrm{ps} 1 \mathrm{a}$ & 4.036 & 0.902 & 4.135 volts & & volts & 25 & 22 & 0.06393 \\
\hline 01 Apr 93 & $14: 56$ & 187 & BS2R1d & 12.23 Dsia & 4.039 & 0.902 volts & 4.135 volts & & volts & 25 & 22 & 0.06401 \\
\hline \multirow{9}{*}{ AVERAGES } & & & \multirow{9}{*}{ BS2R1 } & & OUAOE & GUAGE & DIEFERENTIKL & OUAGE & GUABE & (बC) & (क) & \\
\hline & & & & & 4.034 volts & 0.902 volts & 4.135 volts & & 0.900 & \multirow{4}{*}{25} & \multirow{4}{*}{22} & \\
\hline & & & & & 899.0 & 50.02 & 45.60 ps1d & $27.76 \mathrm{psig}$ & 4.966 & & & \\
\hline & & & & & 61.17 & 3.404 & 3.103 & $1.889 \mathrm{~atm}$ & 0.3379 & & & \\
\hline & & & & & $6.198 \quad \mathrm{Mpa}$ & 0.3449 & $0.3144 \quad \mathrm{Mpa}$ & 0.1914 & 0.03424 & & & \\
\hline & & & & ABSOLUTE & ABSOLUTSE & ABSOLUTE & DTEFERENTEX & \multicolumn{2}{|l|}{ ABSOLUTE } & (8) & (OX) & $(\mathrm{n}) / \mathrm{sec})$ \\
\hline & & & & 12.23 psta & 911.2 ps1a & 62.25 ps1a & 45.60 psid & $39.99 \mathrm{ps1a}$ & 17.20 & \multirow{3}{*}{298} & \multirow{3}{*}{295} & \multirow{3}{*}{0.06391} \\
\hline & & & & 0.8322 atm & 62.01 & 4.236 & 3.103 & $2.721 \quad \mathrm{~atm}$ & 1.170 & & & \\
\hline & & & & 0.08432 & 6.283 & 0.4292 & 0.3144 & 0.2758 & 0.1186 & & & \\
\hline
\end{tabular}

Apparent gas permeab111ty:

$K a=\left(v e * P e^{*} U * L\right) /(P m * \Delta P)$

Boyle's Law:

$\mathrm{Ve}=(\mathrm{Pb} / \mathrm{Pe}) \cdot(\mathrm{Te} / \mathrm{Tb}) *(\mathrm{ze} / \mathrm{zb}) \cdot \mathrm{Vb}$

$\mathrm{Qe}=(\mathrm{Pb} / \mathrm{Pe}) *(\mathrm{Te} / \mathrm{Tb}) \cdot(\mathrm{ze} / \mathrm{zb}) \cdot \mathrm{Qb}$

ve $=Q_{e} / \mathrm{A} *(\mathrm{~Pb} / \mathrm{Pe}) *(\mathrm{Te} / \mathrm{Tb}) *(2 e / 2 \mathrm{~b}) *(\mathrm{Ob} / \mathrm{A})$

\begin{tabular}{|c|c|c|c|c|}
\hline Parameter & $\begin{array}{l}\text { Traditional } \\
\text { Units }\end{array}$ & & $\begin{array}{l}\text { SI } \\
\text { Unt ts }\end{array}$ & \\
\hline$\mu=$ gas v1scosity & 0.0176 & $\star 1.0 \mathrm{E}-3 \mathrm{~Pa} * \mathrm{sec} / \mathrm{CP}$ & $1.760 \mathrm{E}-05$ & Pa*sec \\
\hline L. sample length & 9.557 & * $1.0 \mathrm{E}-2 \mathrm{~m} / \mathrm{cm}$ & $9.557 \mathrm{E}-02$ & $\mathrm{~m}$ \\
\hline A = sample clrcular cross sectlonal area & 81.249 & * $1.0 \mathrm{E}-4 \mathrm{~m}^{\wedge} 2 / \mathrm{cm}^{\wedge} 2$ & $8.125 E-03$ & $m^{2} 2$ \\
\hline $\mathrm{Pb}=$ flow measurement basis pressure (absolute) & 0.8322 & $1.013 \mathrm{E}+5 \mathrm{~Pa} / \mathrm{atm}$ & $8.430 \mathrm{E}+04$ & Pa \\
\hline$\Delta \mathrm{P}=$ pressure drop across sample length & 3.103 & $1.013 \mathrm{E}+5 \mathrm{~Pa} / \mathrm{atm}$ & $3.143 E+05$ & $\mathrm{~Pa}$ \\
\hline $\mathrm{Pm}=$ mean pore pressure (absolute) & 2.721 & $\approx 1.013 \mathrm{E}+5 \mathrm{~Pa} / \mathrm{atm}$ & $2.757 E+05$ & $\mathrm{~Pa}$ \\
\hline Pe $=$ ex1t pressure (absolute) & 1.170 & $\approx 1.013 \mathrm{E}+5 \mathrm{~Pa} / \mathrm{atm}$ & $1.185 \mathrm{E}+05$ & $\mathrm{~Pa}$ \\
\hline $\mathrm{Te}$ - sample temperature (absolute) & 298 & & 298 & ${ }^{\circ} \mathrm{K}$ \\
\hline$T b=$ flow messurement basls temperature (absolute) & 295 & & 295 & ${ }^{\circ} \mathrm{K}$ \\
\hline $2 \mathrm{e}=$ gas deviatlon factor at $\mathrm{Pe}$ and $\mathrm{Te}$ & 1.0000 & & 1.0000 & \\
\hline $\mathrm{zb}=$ gas devlation factor $\mathrm{at} \mathrm{Pb}$ and $\mathrm{Te}$ & 1.0000 & & 1.0000 & \\
\hline$Q b=$ flow rate at base conditions & $0.06391 \mathrm{~cm}^{2} 3 / \mathrm{s}$ & $1.0 \mathrm{E}-6 \mathrm{~m}^{\wedge} 3 / \mathrm{cm}^{\wedge} 3$ & $6.391 \mathrm{E}-08$ & $m^{n} 3 / 3$ \\
\hline ve $=$ flow velocity at sample exit end & $5.651 \mathrm{E}-04$ & $1.0 \mathrm{E}-2 \mathrm{~m} / \mathrm{cm}$ & $5.651 E-06$ & $\mathrm{~m} / \mathrm{s}$ \\
\hline $\mathrm{Ka}_{\mathrm{a}}=$ & $1.32 \mathrm{E}-05$ & $\$ 9.872 \mathrm{E}-13 \mathrm{~m}^{\wedge} 2 / \mathrm{d}$ & $1.308-17$ & $m^{\wedge} 2$ \\
\hline $\mathrm{Ka}=$ & 0.0132 & & $1.30 \mathrm{E}-13$ & $\mathrm{~cm}^{\wedge} 2$ \\
\hline $\mathrm{Ka}=$ & 13.2 & & & \\
\hline
\end{tabular}




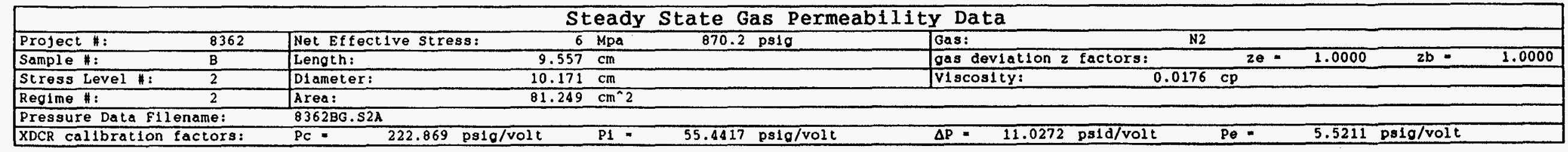

\begin{tabular}{|c|c|c|c|c|c|c|c|c|c|c|c|c|}
\hline Date & $\begin{array}{l}\text { Time } \\
\text { of } \\
\text { Day }\end{array}$ & $\begin{array}{l}\text { File } \\
\text { Time } \\
\text { (min) }\end{array}$ & $\underset{*}{\text { Reglme }}$ & $\begin{array}{l}\text { Pb } \\
\text { Barometric } \\
\text { Pressure }\end{array}$ & $\begin{array}{l}\text { Pc } \\
\text { Confining } \\
\text { Pressure }\end{array}$ & $\begin{array}{l}\text { P1 } \\
\text { Inlet } \\
\text { Pressure }\end{array}$ & $\begin{array}{l}\Delta P \\
\text { Dif ferential } \\
\text { Pressure }\end{array}$ & $\begin{array}{l}\mathrm{Pm} \\
\text { Mean pore } \\
\text { Pressure } \\
\mathrm{Pe}+\Delta \mathrm{P} / 2\end{array}$ & $\begin{array}{l}\text { Pe } \\
\text { Ex1t } \\
\text { Pressure }\end{array}$ & $\begin{array}{l}\text { Te } \\
\text { Elaw } \\
\text { Temp } \\
\left({ }^{\circ} \mathrm{C}\right)\end{array}$ & $\begin{array}{c}\text { Tb } \\
\text { Ambient } \\
\text { Temp } \\
\left({ }^{\circ} \mathrm{C}\right)\end{array}$ & $\begin{array}{c}\text { Qb } \\
\text { Flow Rate } \\
\text { Pb\&Tb } \\
\text { (ml /sec) }\end{array}$ \\
\hline 01 Apr 93 & $16: 24$ & 272 & BS2R2a & $12.21 \mathrm{ps1a}$ & volts & 1.251 volts & 5.800 volts & & 1.097 & 25 & 22 & 0.10411 \\
\hline 01 Apr 93 & $16: 28$ & 277 & BS2R2b & $12.21 \mathrm{ps} 1 \mathrm{a}$ & volts & 1.251 volts & 5.800 volts & & volts & 25 & 22 & 0.10400 \\
\hline 01 Apr 93 & $16: 30$ & 277 & $B S 2 R 2 C$ & $12.21 \mathrm{ps} 1 \mathrm{a}$ & volts & 1.251 volts & 5.799 volts & & volts & 25 & 22 & 0.10349 \\
\hline 01 Apr 93 & $16: 31$ & 282 & BS2R2d & $12.21 \mathrm{ps} 1 \mathrm{a}$ & 4.061 volts & 1.251 volts & 5.799 volts & & volts & 25 & 22 & 0.10360 \\
\hline \multirow{9}{*}{ AVERAGES } & & & \multirow{9}{*}{ BS2R2 } & & OUAGE & OUAGE & DYFFERENTKAL & OUAGE & GOXOE & एवस & (G) & \\
\hline & & & & & 4.065 volts & 1.251 volts & 5.800 volts & & 1.098 & \multirow{4}{*}{25} & \multirow{4}{*}{22} & \\
\hline & & & & & 906.0 & 69.36 & 63.95 ps1d & $38.04 \mathrm{ps} 1 \mathrm{~g}$ & 6.061 & & & \\
\hline & & & & & 61.65 & 4.719 & 4.352 & $2.588 \mathrm{~atm}$ & 0.4124 & & & \\
\hline & & & & & 6.246 & 0.4782 & $0.4409 \quad$ Mpa & $0.2623 \mathrm{Mpa}$ & 0.04179 & & & \\
\hline & & & & ABSOLUTE & ABSOLUTE & ABSOLUTE & DIFEERENTIAL & \multicolumn{2}{|l|}{ ABSOLUTE } & (०k) & 10X & (nI $7 \mathrm{sec}$ \\
\hline & & & & 12.21 psia & $918.2 \quad$ ps1a & $81.57 \quad$ psia & 63.95 ps1d & 50.25 psia & 18.27 & \multirow{3}{*}{298} & \multirow{3}{*}{295} & \multirow{3}{*}{0.10380} \\
\hline & & & & 0.8308 atm & 62.48 & 5.550 & 4.352 & $3.419 \quad \mathrm{~atm}$ & 1.243 & & & \\
\hline & & & & 0.08419 & 6.331 & 0.5624 & 0.4409 & 0.3464 & 0.1260 & & & \\
\hline
\end{tabular}

$\bar{\omega}$

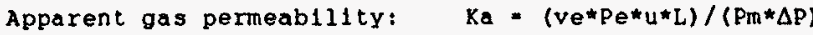

Boyle's Law:

$V_{e}=(\mathrm{Pb} / \mathrm{Pe}) *(\mathrm{Te} / \mathrm{Tb}) *(\mathrm{ze} / \mathrm{zb}) * \mathrm{Vb}$

$\mathrm{Q}_{e}=(\mathrm{Pb} / \mathrm{Pe}) *(\mathrm{Te} / \mathrm{Tb}) *(\mathrm{ze} / \mathrm{zb}) * \mathrm{ab}$

$v_{e}=Q_{e} / A=(\mathrm{Pb} / \mathrm{Pe}) \cdot(\mathrm{Te} / \mathrm{Tb}) *(2 e / 2 \mathrm{~b}) *(\mathrm{Qb} / \mathrm{A})$

\begin{tabular}{|c|c|c|c|c|}
\hline Parameter & $\begin{array}{l}\text { Traditional } \\
\text { Un1ts }\end{array}$ & & $\begin{array}{l}\text { SI } \\
\text { Un1ts }\end{array}$ & \\
\hline$\mu=$ gas $v i s \cos i t y$ & 0.0176 & $1.0 \mathrm{E}-3 \mathrm{~Pa} * \mathrm{sec} / \mathrm{CP}$ & $1.760 \mathrm{E}-05$ & $\mathrm{~Pa} * \mathrm{sec}$ \\
\hline $\mathrm{L}=$ sample length & 9.557 & $1.0 \mathrm{E}-2 \mathrm{~m} / \mathrm{cm}$ & $9.557 E-02$ & $\mathrm{~m}$ \\
\hline$A=$ sample clrcular cross sectlonal area & 81.249 & $1.0 \mathrm{E}-4 \mathrm{~m}^{\wedge} 2 / \mathrm{cm}^{\wedge} 2$ & $8.125 \mathrm{E}-03$ & $m^{\wedge} 2$ \\
\hline $\mathrm{pb}=$ flow measurement bas ls pressure (absolute) & 0.8308 & $\star 1.013 \mathrm{E}+5 \mathrm{~Pa} / \mathrm{atm}$ & $8.416 E+04$ & $\mathrm{~Pa}$ \\
\hline$\Delta \mathrm{P}$ - pressure drop across sample length & 4.352 & $\approx 1.013 \mathrm{E}+5 \mathrm{~Pa} / \mathrm{atm}$ & $4.408 E+05$ & $\mathrm{~Pa}$ \\
\hline $\mathrm{Pm}=$ mean pore pressure (absolute) & 3.419 & $1.013 \mathrm{E}+5 \mathrm{~Pa} / \mathrm{atm}$ & $3.464 E+05$ & Pa \\
\hline Pe $=$ exit pressure (absolute) & 1.243 & $1.013 \mathrm{E}+5 \mathrm{~Pa} / \mathrm{atm}$ & $1.259 \mathrm{E}+05$ & $\mathrm{~Pa}$ \\
\hline Te - sample temperature (absolute) & 298 & & 298 & ${ }^{\circ} \mathrm{K}$ \\
\hline $\mathrm{Tb}$ - flow measurement bas1s temperature (absolute) & 295 & & 295 & ${ }^{\circ} \mathrm{K}$ \\
\hline$z e=$ gas deviation factor at $\mathrm{Pe}$ and $\mathrm{Te}$ & 1.0000 & & 1.0000 & \\
\hline $2 \mathrm{~b}=$ gas deviation factor $\mathrm{at} \mathrm{Pb}$ and $\mathrm{Te}$ & 1.0000 & & 1.0000 & \\
\hline $\mathrm{Qb}=$ flow rate at base condltions & 0.10380 & $1.0 \mathrm{E}-6 \mathrm{~m}^{\wedge} 3 / \mathrm{cm}^{\wedge} 3$ & $1.038 \mathrm{E}-07$ & $\mathrm{~m}^{n} 3 / \mathrm{s}$ \\
\hline$v e=$ flow veloclty at sample extt end & $8.625 \mathrm{E}-04$ & $1.0 \mathrm{E}-2 \mathrm{~m} / \mathrm{cm}$ & $8.625 E-06$ & $\mathrm{~m} / \mathrm{s}$ \\
\hline $\mathrm{Ka}-$ & $1.21 \mathrm{E}-05$ & $9.872 \mathrm{E}-13 \mathrm{~m}^{\wedge} 2 / \mathrm{d}$ & $1.20 \mathrm{E}-17$ & $m^{2} 2$ \\
\hline $\mathrm{Ka}=$ & 0.0121 & & $1.20 \mathrm{E}-13$ & $\mathrm{~cm}^{2} 2$ \\
\hline $\mathrm{Ka}=$ & 12.1 & & & \\
\hline
\end{tabular}




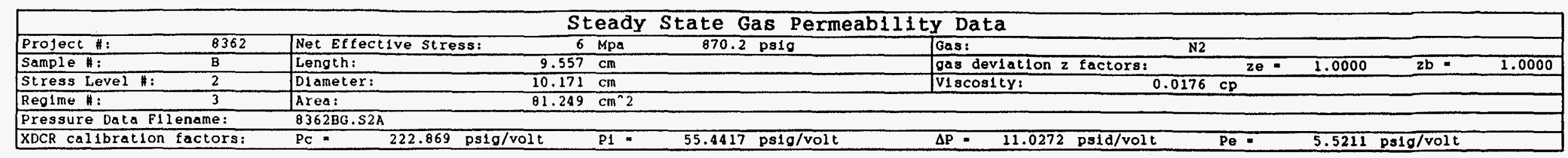

\begin{tabular}{|c|c|c|c|c|c|c|c|c|c|c|c|c|}
\hline Date & $\begin{array}{l}\text { Time } \\
\text { of } \\
\text { Day }\end{array}$ & $\begin{array}{l}\text { File } \\
\text { Time } \\
\text { (min) }\end{array}$ & $\underset{\#}{\text { Reg1me }}$ & \begin{tabular}{|l} 
Pb \\
Barometric \\
Pressure
\end{tabular} & $\begin{array}{l}\text { Pc } \\
\text { Confining } \\
\text { Pressure }\end{array}$ & $\begin{array}{l}\text { PI } \\
\text { Inlet } \\
\text { Pressure }\end{array}$ & $\begin{array}{l}\Delta \mathrm{P} \\
\text { Differential } \\
\text { Pressure }\end{array}$ & $\begin{array}{l}\mathrm{Pm} \\
\text { Mean Pore } \\
\text { Pressure } \\
\mathrm{Pe}+\Delta \mathrm{P} / 2\end{array}$ & $\begin{array}{l}\text { Pe } \\
\text { Exit } \\
\text { Pressure }\end{array}$ & $\begin{array}{l}\text { Te } \\
\text { Flow } \\
\text { Temp } \\
\left({ }^{\circ} \mathrm{C}\right)\end{array}$ & $\begin{array}{c}\text { Tb } \\
\text { Amb1ent } \\
\text { Temp } \\
\left({ }^{\circ} \mathrm{C}\right)\end{array}$ & $\begin{array}{c}\text { Ob } \\
\text { Flow Rate } \\
\text { OPb\&Tb } \\
\text { (m1/sec) }\end{array}$ \\
\hline 02 Apr 93 & $08: 40$ & 1253 & BS2R3a & $12.31 \mathrm{ps} 1 \mathrm{a}$ & 4.120 & volts & 7.475 volts & & volts & 25 & 21 & 0.14744 \\
\hline 02 Apr 93 & $08: 42$ & 1253 & BS2R3b & $12.31 \mathrm{ps} 1 \mathrm{a}$ & 4.113 & volts & 7.475 volts & & volts & 25 & 21 & 0.14782 \\
\hline 02 Apr 93 & $08: 44$ & 1253 & $B S 2 R 3 C$ & $12.31 \mathrm{ps} 1 \mathrm{a}$ & 4.115 & volts & 7.474 volts & & volts & 25 & 21 & 0.14782 \\
\hline 02 Apr 93 & $08: 46$ & 1258 & BS2R3d & $12.31 \mathrm{psia}$ & 4.117 & valts & 7.474 volts & & volts & 25 & 21 & 0.14806 \\
\hline \multirow{9}{*}{ AVERAGES } & & & \multirow{9}{*}{ BS2R3 } & & GOAOE & GUAGE & DYFEERENTIAL & OUXGE & GUaGE & $\mathrm{OCl}$ & (re) & \\
\hline & & & & & 4.116 voltg & 1.601 volts & 7.475 volts & & 1.250 & \multirow{4}{*}{25} & \multirow{4}{*}{21} & \\
\hline & & & & & 917.4 ps1g & 88.76 & $82.42 \mathrm{ps} 1 \mathrm{~d}$ & 48.11 psig & 6.900 & & & \\
\hline & & & & & 62.42 & 6.040 & 5.609 & $3,274 \mathrm{~atm}$ & 0.4695 & & & \\
\hline & & & & & 6.325 & 0.6120 & 0.5683 & 0.3317 & 0.04757 & & & \\
\hline & & & & ABSOLUTE & ABSOLUTE & ABSOLUTE & DIFEERENTIAT & KBSOLUTE & ABSOLOTE & \%) & (OK) & (ill $100 \mathrm{cl}$ \\
\hline & & & & $12.31 \mathrm{ps} 1 \mathrm{a}$ & $929.7 \quad$ psia & 101.07 psia & $82.42 \quad \mathrm{ps} 1 \mathrm{~d}$ & 60.42 psia & 19.21 & \multirow{3}{*}{298} & \multirow{3}{*}{294} & \multirow{3}{*}{0.14778} \\
\hline & & & & $0.8376 \mathrm{~atm}$ & 63.26 & 6.878 & 5.609 & $4.111 \mathrm{~atm}$ & 1.307 & & & \\
\hline & & & & $0.08487 \mathrm{MPa}$ & 6.410 & 0.6969 & 0.5683 & $0.4166 \mathrm{Mpa}$ & 0.1324 & & & \\
\hline
\end{tabular}

$\stackrel{\leftrightarrow}{\oplus}$

Apparent gas permeability:

Boyle's Law:

$K a=(v e * P e * u * L) /(P m * \Delta P)$

$V_{e}=(\mathrm{Pb} / \mathrm{Pe}) *(\mathrm{Te} / \mathrm{Tb}) *(\mathrm{ze} / \mathrm{zb}) * \mathrm{Vb}$

$\mathrm{Qe}=(\mathrm{Pb} / \mathrm{Pe}) *(\mathrm{Te} / \mathrm{Tb}) *(2 \mathrm{ze} / \mathrm{zb}) \cdot \mathrm{Qb}$

$v_{e}=Q_{e} / A=(P b / P e) *(T e / T b) *(2 e / 2 b) *(Q b / A)$

\begin{tabular}{|c|c|c|c|c|}
\hline Parameter & $\begin{array}{l}\text { Traditional } \\
\text { Unlts }\end{array}$ & & $\begin{array}{l}\text { SI } \\
\text { Units }\end{array}$ & \\
\hline$\mu=$ gas $v i s c o s i t y$ & 0.0176 & $\star 1.0 \mathrm{E}-3 \mathrm{~Pa} \mathrm{sec}_{\mathrm{Sep}}$ & $1.760 \mathrm{E}-05$ & $\mathrm{~Pa} * \sec$ \\
\hline$L=$ sample length & 9.557 & $1.0 \mathrm{E}-2 \mathrm{~m} / \mathrm{cm}$ & $9.557 \mathrm{E}-02$ & $\mathbf{m}$ \\
\hline$A$ - sample clrcular cross sectlonal area & 81.249 & $\star 1.0 \mathrm{E}-4 \mathrm{~m}^{\wedge} 2 / \mathrm{cm}^{\wedge} 2$ & $8.125 E-03$ & $m^{2} 2$ \\
\hline $\mathrm{Pb}=$ flow measurement basis pressure (absolute) & 0.8376 & $* 1.013 \mathrm{E}+5 \mathrm{~Pa} / \mathrm{atm}$ & $8.485 E+04$ & $\mathrm{~Pa}$ \\
\hline$\Delta \mathrm{P}=$ pressure drop across sample length & 5.609 & $\star 1.013 \mathrm{E}+5 \mathrm{~Pa} / \mathrm{atm}$ & $5.681 E+05$ & $\mathrm{~Pa}$ \\
\hline $\mathrm{Pm}=$ mean pore pressure (absolute) & 4.111 & $* 1.013 \mathrm{E}+5 \mathrm{~Pa} / \mathrm{atm}$ & $4.165 E+05$ & $\mathrm{~Pa}$ \\
\hline $\mathrm{Pe}=$ exit pressure (absolute) & 1.307 & $\approx 1.013 \mathrm{E}+5 \mathrm{~Pa} / \mathrm{atm}$ & $1.324 \mathrm{E}+05$ & $\mathrm{~Pa}$ \\
\hline $\mathrm{Te}$ : sample temperature (absolute) & 298 & & 298 & ${ }^{\circ} \mathrm{K}$ \\
\hline Tb - flow measurement basis temperature (absolute) & 294 & & 294 & ${ }^{\circ} \mathrm{K}$ \\
\hline$z e-$ gas devlation factor at $\mathrm{Pe}$ and $\mathrm{Te}$ & 1.0000 & & 1.0000 & \\
\hline $2 \mathrm{~b}$ : gas devlation factor $\mathrm{at} \mathrm{Pb}$ and $\mathrm{Te}$ & 1.0000 & & 1.0000 & \\
\hline $\mathrm{Qb}=$ flow rate at base conditions & $0.14778 \mathrm{~cm}^{2} 3 / \mathrm{s}$ & $\$ 1.0 \mathrm{E}-6 \mathrm{~m}^{4} 3 / \mathrm{cm}^{\wedge} 3$ & $1.478 \mathrm{E}-07$ & $m^{\wedge} 3 / s$ \\
\hline ve $=$ flow velocity at sample exit end & $1.181 \mathrm{E}-03$ & $1.0 \mathrm{E}-2 \mathrm{~m} / \mathrm{cm}$ & $1.181 \mathrm{E}-05$ & $\mathrm{~m} / \mathrm{s}$ \\
\hline $\mathrm{Ka}=$ & $1.13 \mathrm{E}-05$ & $* 9.872 \mathrm{E}-13 \mathrm{~m}^{\wedge} 2 / \mathrm{d}$ & $1.11 \mathrm{E}-17$ & $m^{2} 2$ \\
\hline $\mathrm{Ka}=$ & 0.0113 & & $1.11 \mathrm{E}-13$ & $c m^{\wedge} 2$ \\
\hline $\mathrm{Ka}=$ & 11.3 & & & \\
\hline
\end{tabular}




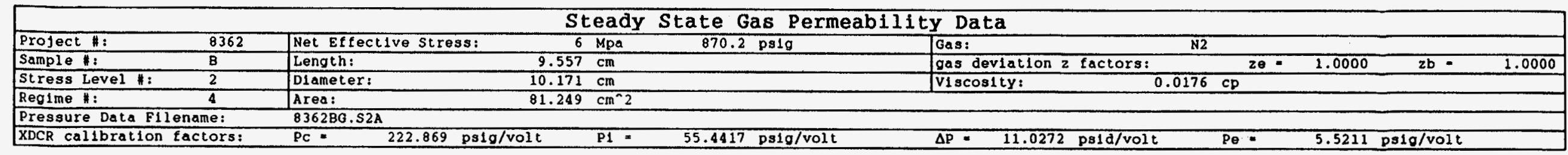

\begin{tabular}{|c|c|c|c|c|c|c|c|c|c|c|c|c|}
\hline Date & $\begin{array}{l}\text { Time } \\
\text { of } \\
\text { Day }\end{array}$ & $\begin{array}{l}\text { File } \\
\text { Time } \\
\text { (min) }\end{array}$ & $\underset{\#}{\operatorname{Reg} 1 \mathrm{me}}$ & $\begin{array}{l}\text { pb } \\
\text { Barometrlc } \\
\text { Pressure }\end{array}$ & $\begin{array}{l}\text { Pc } \\
\text { Confining } \\
\text { Pressure }\end{array}$ & $\begin{array}{l}\text { P1 } \\
\text { Inlet } \\
\text { Pressure }\end{array}$ & $\begin{array}{l}\Delta P \\
D 1 \text { fferential } \\
\text { Pressure }\end{array}$ & $\begin{array}{l}\text { Pm } \\
\text { Mean Pore } \\
\text { Pressure } \\
\text { Pe }+\Delta P / 2\end{array}$ & $\begin{array}{l}\text { Pe } \\
\text { Ex1t } \\
\text { Pressure }\end{array}$ & $\begin{array}{l}\text { Te } \\
\text { Flow } \\
\text { Temp } \\
\left({ }^{\circ} \mathrm{C}\right)\end{array}$ & $\begin{array}{c}\text { Tb } \\
\text { Amblent } \\
\text { Temp } \\
\left({ }^{\circ} \mathrm{C}\right)\end{array}$ & $\begin{array}{c}\text { Ob } \\
\text { Flow Rate } \\
\text { OPb\&Tb } \\
\text { (m1/sec) }\end{array}$ \\
\hline 02 Apr 93 & $14: 31$ & 1602 & BS2R4a & $12.32 \mathrm{ps} 1 \mathrm{a}$ & volts & 1.951 & 9.144 volts & & volts & 25 & 22 & 0.20243 \\
\hline 02 Apr 93 & $14: 33$ & 1602 & BS2R4b & $12.32 \mathrm{ps} 1 \mathrm{a}$ & volts & 1.950 & 9.143 volts & & volts & 25 & 22 & 0.20222 \\
\hline 02 Apr 93 & $14: 35$ & 1607 & BS2R4C & 12.32 psia & $\begin{array}{ll}4.168 & \text { volts }\end{array}$ & 1.950 & 9.143 volts & & volts & 25 & 22 & 0.20121 \\
\hline 02 Apr 93 & $14: 37$ & 1607 & BS2R4a & 12.32 psia & 4.168 volts & 1.950 & 9.143 volts & & volts & 25 & 22 & 0.20161 \\
\hline \multirow{9}{*}{ AVERAGES } & & & \multirow{9}{*}{ BS2RA } & & GUAGE & ODACE & DIFEERENT III & \multirow[t]{2}{*}{ GUAGE: } & & & क्टा & \\
\hline & & & & & 4.168 volts & 1.950 volts & 9.143 volts & & $\frac{\text { GUAGE }}{1.451}$ & \multirow{4}{*}{25} & \multirow{4}{*}{22} & \\
\hline & & & & & $929.0 \quad$ psig & 108.13 & 100.82 psid & $58.42 \mathrm{ps} 1 \mathrm{~g}$ & 8.011 & & & \\
\hline & & & & & 63.21 & 7.357 & 6.861 & $3.975 \mathrm{~atm}$ & 0.5451 & & & \\
\hline & & & & & 6.405 & 0.7455 & 0.6952 & 0.4028 & 0.05523 & & & \\
\hline & & & & ABSOLUTE & ABSOLUTE & ABSOLUTE & DYFEERENTIAL & ABSOLUTE & Agsottutes & $8 \mathrm{x})$ & (OK) & (nin $7 s Q C)$ \\
\hline & & & & 12.32 psia & 941.3 ps1a & 120.45 ps1a & 100.82 ps1d & $70.74 \mathrm{psia}$ & 20.33 & \multirow{3}{*}{298} & \multirow{3}{*}{295} & \multirow{3}{*}{0.20187} \\
\hline & & & & $0.8383 \mathrm{~atm}$ & 64.05 & 8.196 & 6.861 & $4.814 \mathrm{~atm}$ & 1.383 & & & \\
\hline & & & & 0.08494 & 6.490 & 0.8304 & 0.6952 & 0.4878 & 0.1402 & & & \\
\hline
\end{tabular}

Apparent gas permeab111ty:

Boyle's Law:

$K a=\left(v e * P_{e} * u * L\right) /(P m * \Delta P)$

$\mathrm{Ve} \cdot(\mathrm{Pb} / \mathrm{Pe}) \cdot(\mathrm{Te} / \mathrm{Tb}) \cdot(\mathrm{ze} / \mathrm{zb}) \cdot \mathrm{Vb}$

$Q e *(\mathrm{~Pb} / \mathrm{Pe}) *(\mathrm{~T} \theta / \mathrm{Tb}) *(\mathrm{ze} / \mathrm{zb}) * \mathrm{Qb}$

$v e=Q_{e} / \mathrm{A}=(\mathrm{Pb} / \mathrm{Pe}) *(\mathrm{Te} / \mathrm{Tb}) *(2 \mathrm{e} / \mathrm{zb}) *(\mathrm{Qb} / \mathrm{A})$

\begin{tabular}{|c|c|c|c|c|}
\hline Parameter & $\begin{array}{l}\text { Traditional } \\
\text { Undts }\end{array}$ & & $\begin{array}{l}\text { SI } \\
\text { Units }\end{array}$ & \\
\hline$\mu=$ gas viscosity & 0.0176 & $-1.0 \mathrm{E}-3 \mathrm{~Pa} * \mathrm{sec} / \mathrm{CP}$ & $1.760 \mathrm{E}-05$ & Pa*sec \\
\hline$L=$ sample length & 9.557 & $1.0 \mathrm{E}-2 \mathrm{~m} / \mathrm{cm}$ & $9.557 \mathrm{E}-02$ & $m$ \\
\hline$A$ - sample clrcular cross sectional area & 81.249 & $1.0 \mathrm{E}-4 \mathrm{~m}^{\wedge} 2 / \mathrm{cm}^{\wedge} 2$ & $8.125 \mathrm{E}-03$ & $m^{n} 2$ \\
\hline $\mathrm{Pb}=$ flow measurement basis pressure (absolute) & 0.8383 & $\approx 1.013 \mathrm{E}+5 \mathrm{~Pa} / \mathrm{atm}$ & $8.492 E+04$ & $\mathrm{~Pa}$ \\
\hline$\Delta \mathrm{P}=$ pressure drop across sample length & 6.861 & $1.013 \mathrm{E}+5 \mathrm{~Pa} / \mathrm{atm}$ & $6.950 \varepsilon+05$ & Pa \\
\hline Pm = mean pore pressure (absolute) & 4.814 & - $1.013 \mathrm{E}+5 \mathrm{~Pa} / \mathrm{atm}$ & $4.876 \mathrm{E}+05$ & $\mathrm{~Pa}$ \\
\hline Pe $=$ exit pressure (absolute) & 1.383 & $1.013 \mathrm{E}+5 \mathrm{~Pa} / \mathrm{atm}$ & $1.401 \mathrm{E}+05$ & Po \\
\hline $\mathrm{Te}$ - sample temperature (absolute) & 298 & & 298 & ${ }^{\circ} \mathrm{K}$ \\
\hline $\mathrm{Tb}=$ flow measurement basis temperature (absolute) & 295 & & 295 & ${ }^{\circ} \mathrm{K}$ \\
\hline$z e=$ gas deviation factor at $\mathrm{Pe}$ and $\mathrm{Te}$ & 1.0000 & & 1.0000 & \\
\hline $2 b=$ gas deviation factor at $\mathrm{Pb}$ and $\mathrm{Te}$ & 1.0000 & & 1.0000 & \\
\hline$Q b$ - flow rate at base conditions & $0.20187 \mathrm{~cm}^{2} 3 / \mathrm{s}$ & $\star 1.0 \mathrm{E}-6 \mathrm{~m}^{\wedge} 3 / \mathrm{cm}^{\wedge} 3$ & $2.019 \mathrm{E}-07$ & $m^{-3 / 3}$ \\
\hline ve = flow velocity at sample exit end & $1.521 \mathrm{E}-03 \mathrm{~cm} / \mathrm{s}$ & $\hbar 1.0 \mathrm{E}-2 \mathrm{~m} / \mathrm{cm}$ & $1.521 \mathrm{E}-05$ & $\mathrm{~m} / \mathrm{s}$ \\
\hline $\mathrm{Ka}=$ & $1.07 E-05$ & $9.872 \mathrm{E}-13 \mathrm{~m}^{\wedge} 2 / \mathrm{d}$ & $1.06 \mathrm{E}-17$ & $m^{\star} 2$ \\
\hline $\mathrm{Ka}=$ & 0.0107 & & $1.06 \mathrm{E}-13$ & $\mathrm{~cm}^{\wedge} 2$ \\
\hline $\mathrm{Ka}=$ & 10.7 & & & \\
\hline
\end{tabular}




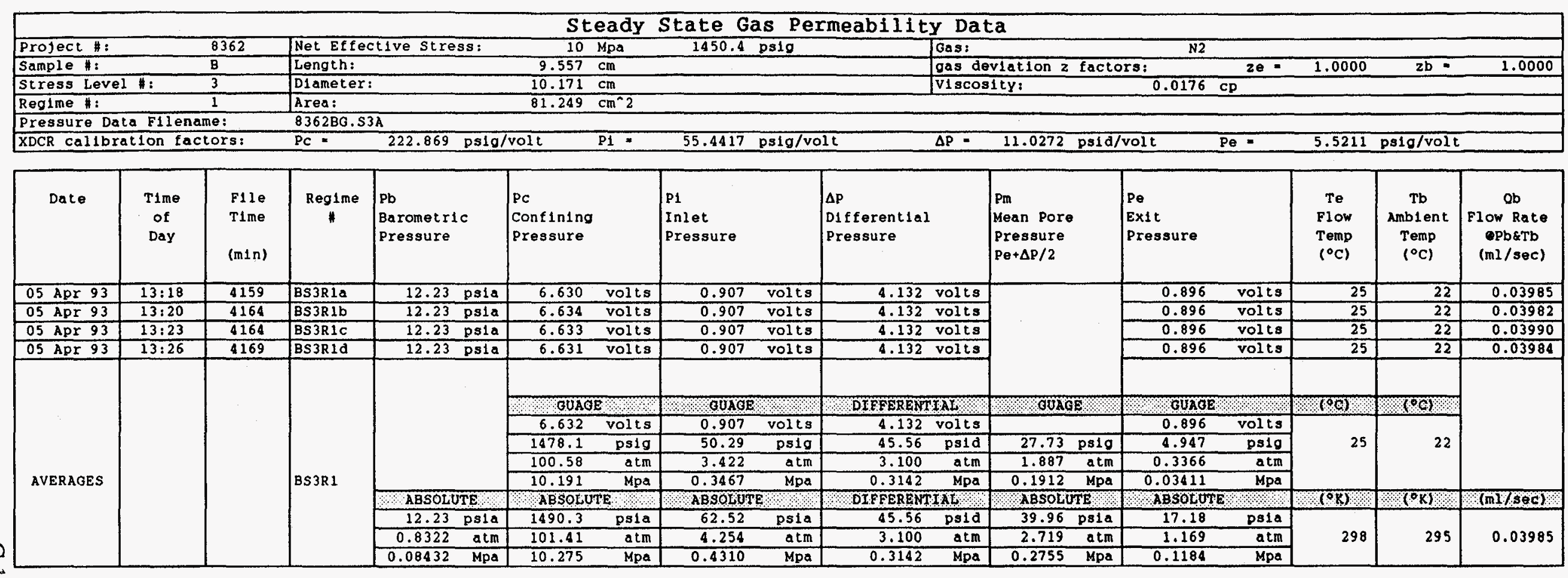

N
Apparent gas permeabillty:
Boyle's Law:
$K a=(v e \star P e \star u \star L) /(P m \star \Delta P)$
$V_{e}=(\mathrm{Pb} / \mathrm{Pe}) *(\mathrm{Te} / \mathrm{Tb}) *(z e / 2 b) * \mathrm{Vb}$
$\mathrm{Qe}=(\mathrm{Pb} / \mathrm{Pe}) *(\mathrm{Te} / \mathrm{Tb}) *(z e / 2 \mathrm{~b}) * \mathrm{Qb}$
$v_{e}=Q_{\theta} / A=(\mathrm{Pb} / \mathrm{Pe}) *(\mathrm{Te} / \mathrm{Tb}) *(z e / z b) *(\mathrm{Qb} / \mathrm{A})$

\begin{tabular}{|c|c|c|c|c|}
\hline Parameter & $\begin{array}{l}\text { Traditional } \\
\text { Units }\end{array}$ & & $\begin{array}{l}\text { SI } \\
\text { Units }\end{array}$ & \\
\hline$\mu=g a s v 1 s \cos 1 t y$ & 0.0176 & $\star 1.0 \mathrm{E}-3 \mathrm{~Pa} * \mathrm{sec} / \mathrm{cp}$ & $1.760 \mathrm{E}-05$ & $P a * \sec$ \\
\hline $\mathrm{L}=$ sample length & 9.557 & $1.0 \mathrm{E}-2 \mathrm{~m} / \mathrm{cm}$ & $9.557 E-02$ & $\mathrm{~m}$ \\
\hline$A$ - sample cl rcular cross sectional area & 81.249 & $\star 1.0 \mathrm{E}-4 \mathrm{~m}^{\wedge} 2 / \mathrm{cm}^{\wedge} 2$ & $8.125 E-03$ & $\mathrm{~m}^{\wedge} 2$ \\
\hline $\mathrm{Pb}$ - flow measurement basis pressure (absolute) & 0.8322 & $\star 1.013 \mathrm{E}+5 \mathrm{~Pa} / \mathrm{atm}$ & $8.430 E+04$ & $\mathrm{~Pa}$ \\
\hline$\Delta \mathrm{P}=$ pressure drop across sample length & 3.100 & $\pitchfork 1.013 \mathrm{E}+5 \mathrm{~Pa} / \mathrm{atm}$ & $3.141 E+05$ & Pa \\
\hline $\mathrm{Pm}_{\mathrm{m}}=$ mean pore pressure (absolute) & 2.719 & $\pi 1.013 \mathrm{E}+5 \mathrm{~Pa} / \mathrm{atm}$ & $2.754 E+05$ & $\mathrm{~Pa}$ \\
\hline $\mathrm{Pe}=$ ex1t pressure (absolute) & 1.169 & $\approx 1.013 \mathrm{E}+5 \mathrm{~Pa} / \mathrm{atm}$ & $1.184 E+05$ & Pa \\
\hline Te = sample temperature (absolute) & 298 & & 298 & ${ }^{\circ} \mathrm{K}$ \\
\hline $\mathrm{Tb}=$ flow measurement basis temperature (absolute) & 295 & & 295 & ${ }^{\circ} \mathrm{K}$ \\
\hline$z e=$ gas deviation factor at $P e$ and $T e$ & 1.0000 & & 1.0000 & \\
\hline $2 b=$ gas devlation factor $a t p b$ and $T e$ & 1.0000 & & 1.0000 & \\
\hline $\mathrm{Qb}$ - flow rate at base conditlons & $0.03985 \mathrm{~cm}^{-3} 3 / \mathrm{s}$ & $\star 1.0 \mathrm{E}-6 \mathrm{~m}^{2} 3 / \mathrm{cm}^{2} 3$ & $3.985 \mathrm{E}-08$ & $m^{n} 3 / s$ \\
\hline ve $=$ flow velocity at sample exit end & $3.528 E-04$ & $1.0 \mathrm{E}-2 \mathrm{~m} / \mathrm{cm}$ & $3.528 \mathrm{E}-06$ & $\mathrm{~m} / \mathrm{s}$ \\
\hline $\mathrm{ka}=$ & $0.23 \mathrm{E}-06$ & $\$ 9.872 \mathrm{E}-13 \mathrm{~m}^{2} 2 / \mathrm{d}$ & $8.12 \mathrm{E}-18$ & $m^{2} 2$ \\
\hline $\mathrm{Ka}=$ & 0.0082 & & $8,12 \mathrm{E}-14$ & $\mathrm{~cm}^{2} 2$ \\
\hline Ka $=$ & 8.23 & & & \\
\hline
\end{tabular}




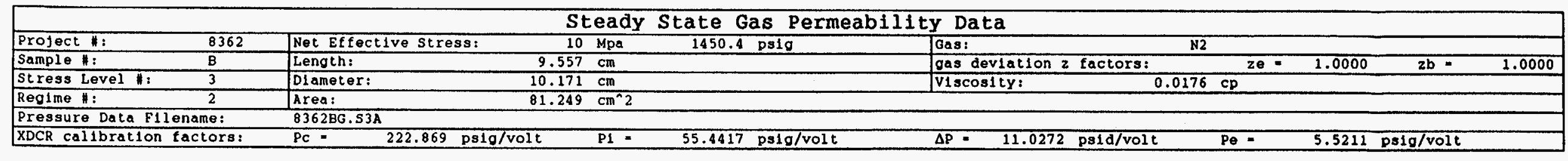

\begin{tabular}{|c|c|c|c|c|c|c|c|c|c|c|c|c|}
\hline Date & $\begin{array}{l}\text { Time } \\
\text { of } \\
\text { Day }\end{array}$ & $\begin{array}{l}\text { File } \\
\text { Time } \\
\text { (min) }\end{array}$ & $\begin{array}{c}\text { Reglme } \\
\#\end{array}$ & $\begin{array}{l}\text { Pb } \\
\text { Barometr1c } \\
\text { Pressure }\end{array}$ & $\begin{array}{l}\text { Pc } \\
\text { Confining } \\
\text { Pressure }\end{array}$ & $\begin{array}{l}\text { Pi } \\
\text { Inlet } \\
\text { Pressure }\end{array}$ & $\begin{array}{l}\Delta P \\
D 1 \text { f ferential } \\
\text { Pressure }\end{array}$ & $\begin{array}{l}\text { Pra } \\
\text { Mean Pore } \\
\text { Pressure } \\
\mathrm{Pe}+\Delta \mathrm{P} / 2\end{array}$ & $\begin{array}{l}\text { Pe } \\
\text { Exit } \\
\text { Pressure }\end{array}$ & $\begin{array}{l}\text { Te } \\
\text { Flow } \\
\text { Temp } \\
\left\langle{ }^{\circ} \mathrm{C}\right\rangle\end{array}$ & $\begin{array}{c}\text { Tb } \\
\text { Amblent } \\
\text { Temp } \\
\left({ }^{\circ} \mathrm{C}\right)\end{array}$ & $\begin{array}{c}\text { Qb } \\
\text { Flow Rate } \\
\text { OPbeTb } \\
\text { (ml / sec) }\end{array}$ \\
\hline 05 ApI 93 & $16: 04$ & 4324 & $B S 3 R 2 a$ & 12.21 psla & 6.661 & volts & 5.791 volts & & volts & 25 & 22 & 0.06445 \\
\hline 05 Apr 93 & $16: 07$ & 4329 & BS3R2b & 12.21 psia & 6.682 & volts & 5.790 volts & & volts & 25 & 22 & 0.06401 \\
\hline 05 Apr 93 & $16: 10$ & 4334 & BS3R2C & $12.21 \mathrm{ps} 1 \mathrm{a}$ & 6.690 & volts & 5.790 volts & & volts & 25 & 22 & 0.06430 \\
\hline 05 Apr 93 & $16: 15$ & 4339 & BS3R2d & $12.21 \mathrm{ps} 10$ & 6.695 volts & 1.252 volts & 5.791 volts & & volts & 25 & 22 & 0.06430 \\
\hline \multirow{9}{*}{ AVERAGES } & & & \multirow{9}{*}{ BS3R2 } & & OUAGE & OUAOE & DIFEERENTIXL & OONGE & GUXGe & $\mathrm{re}$ & $10 \mathrm{Cl}$ & \\
\hline & & & & & 6.682 volts & 1.252 volts & 5.791 volts & & 1.073 & \multirow{4}{*}{25} & \multirow{4}{*}{22} & \\
\hline & & & & & 1489.2 & 69.41 & $63.85 \mathrm{ps} 1 \mathrm{~d}$ & $37.85 \mathrm{pslg}$ & 5.926 & & & \\
\hline & & & & & 101.33 & 4.723 & 4.345 & $2.576 \mathrm{~atm}$ & 0.4032 & & & \\
\hline & & & & & $10.268 \quad \mathrm{Mpa}$ & 0.4786 & $0.4403 \quad \mathrm{Mpa}$ & $0.2610 \mathrm{Mpa}$ & 0.04086 & & & \\
\hline & & & & ABSOLUTE & ABSOLUTE & ABSOLUTE & DIFFEREMTIAL & ABSOLUTE & ABSOLUTE: & 8x) & Tax: & $\mathrm{ml} / \mathrm{sec}$ \\
\hline & & & & $12.21 \mathrm{ps} 1 \mathrm{a}$ & 1501.4 ps1a & 81.62 ps1a & $63.85 \mathrm{ps} 1 \mathrm{~d}$ & $50.06 \mathrm{ps} 1 \mathrm{a}$ & 18.14 & \multirow{3}{*}{298} & \multirow{3}{*}{295} & \multirow{3}{*}{0.06427} \\
\hline & & & & $0.8308 \mathrm{~atm}$ & 102.17 & 5.554 & 4.345 & $3.407 \mathrm{~atm}$ & 1.234 & & & \\
\hline & & & & 0.08419 & 10.352 & 0.5628 & 0.4403 & 0.3452 & 0.1250 & & & \\
\hline
\end{tabular}

Apparent gas permeability:

$K a=(v e * P e * u * L) /(P m * \Delta P)$

Boyle's Law:

$V_{e}=(\mathrm{Pb} / \mathrm{Pe}) *(\mathrm{Te} / \mathrm{Tb}) *(\mathrm{ze} / \mathrm{zb}) * \mathrm{Vb}$

$\mathrm{Qe}=(\mathrm{Pb} / \mathrm{Pe}) *(\mathrm{Te} / \mathrm{Tb}) *(\mathrm{ze} / \mathrm{zb}) * \mathrm{Qb}$

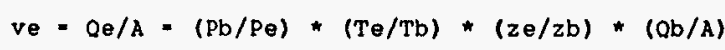

\begin{tabular}{|c|c|c|c|c|}
\hline Parameter & $\begin{array}{l}\text { Traditional } \\
\text { Units }\end{array}$ & & $\begin{array}{l}\text { SI } \\
\text { Und ts }\end{array}$ & \\
\hline$\mu=\operatorname{gas} v 1 \operatorname{scos} 1 t y$ & 0.0176 & $* 1.0 \mathrm{E}-3 \mathrm{~Pa} \mathrm{sec}^{\mathrm{c}} \mathrm{cp}$ & $1.760 \mathrm{E}-05$ & Pa*sec \\
\hline$L=$ sample length & 9.557 & * $1.0 \mathrm{E}-2 \mathrm{~m} / \mathrm{cm}$ & $9.557 \mathrm{E}-02$ & $\mathrm{~m}$ \\
\hline$A=$ sample c1rcular cross sectional area & 81.249 & $\star 1.0 \mathrm{E}-4 \mathrm{~m}^{\wedge} 2 / \mathrm{cm}^{\wedge} 2$ & $8.125 \mathrm{E}-03$ & $m^{\wedge} 2$ \\
\hline $\mathrm{pb}=$ flow measurement basis pressure (absolute) & 0.8308 & $\star 1.013 \mathrm{E}+5 \mathrm{~Pa} / \mathrm{atm}$ & $8.416 \mathrm{E}+04$ & $\mathrm{~Pa}$ \\
\hline$\Delta \mathrm{Q}=$ pressure drop across sample length & 4.345 & $\pitchfork 1.013 \mathrm{E}+5 \mathrm{~Pa} / \mathrm{atm}$ & $4.401 E+05$ & $\mathrm{~Pa}$ \\
\hline $\mathrm{Pm}=$ mean pore pressure (absolute) & 3.407 & $\star 1.013 \mathrm{E}+5 \mathrm{~Pa} / \mathrm{atm}$ & $3.451 E+05$ & $\mathrm{~Pa}$ \\
\hline Fe exit pressure (absolute) & 1.234 & $\star 1.013 \mathrm{E}+5 \mathrm{~Pa} / \mathrm{atm}$ & $1.250 \mathrm{E}+05$ & Pa \\
\hline$T_{e}=$ sample temperature (absolute) & 298 & & 298 & ${ }^{\circ} \mathrm{K}$ \\
\hline $\mathrm{Tb}=$ flow measurement basis temperature (absolute) & 295 & & 295 & ${ }^{\circ} \mathrm{K}$ \\
\hline$z e=$ gas deviation factor at $P e$ and $T e$ & 1.0000 & & 1.0000 & \\
\hline $\mathrm{zb}=$ gas deviation factor $\mathrm{at} \mathrm{Pb}$ and $\mathrm{Te}$ & 1.0000 & & 1.0000 & \\
\hline$Q b=$ flow rate at base conditions & $0.06427 \mathrm{~cm}^{2} 3 / \mathrm{s}$ & $1.0 \mathrm{E}-6 \mathrm{~m}^{\wedge} 3 / \mathrm{cm}^{\wedge} 3$ & $6.427 \mathrm{E}-08$ & $\mathrm{~m}^{*} 3 / \mathrm{s}$ \\
\hline ve $=$ flow velocity at sample exit end & $5.379 \mathrm{E}-04 \mathrm{~cm} / \mathrm{s}$ & $1.0 \mathrm{E}-2 \mathrm{~m} / \mathrm{cm}$ & $5.379 \mathrm{E}-06$ & $\mathrm{~m} / \mathrm{s}$ \\
\hline $\mathrm{Ka}=$ & $7.54 \mathrm{E}-06$ & $* 9.872 \mathrm{E}-13 \mathrm{~m}^{2} 2 / \mathrm{d}$ & $7.45 E-18$ & $m^{\wedge} 2$ \\
\hline Ka. & 0.00754 & & $7.45 E-14$ & $\mathrm{~cm}^{\wedge} 2$ \\
\hline Ka & 7.54 & & & \\
\hline
\end{tabular}




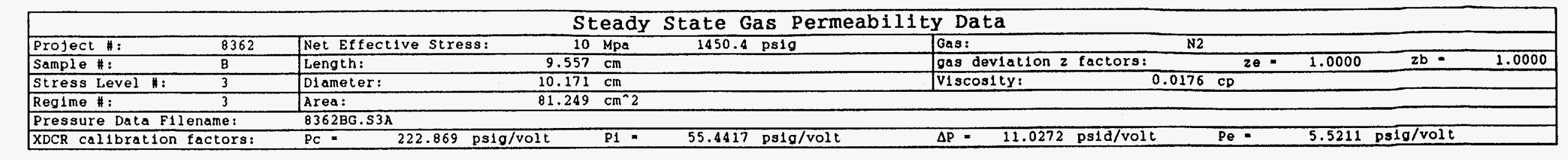

\begin{tabular}{|c|c|c|c|c|c|c|c|c|c|c|c|c|}
\hline Date & $\begin{array}{l}\text { Time } \\
\text { of } \\
\text { Day }\end{array}$ & $\begin{array}{l}\text { File } \\
\text { Time } \\
(m \mid n)\end{array}$ & $\begin{array}{c}\text { Reg1me } \\
\#\end{array}$ & $\begin{array}{l}\mathrm{Pb} \\
\text { Barometr1c } \\
\text { Pressure }\end{array}$ & $\begin{array}{l}\text { Pc } \\
\text { Confining } \\
\text { Pressure }\end{array}$ & $\begin{array}{l}\text { Pi } \\
\text { Inlet } \\
\text { Pressure }\end{array}$ & $\begin{array}{l}\Delta \mathrm{P} \\
\text { D1fferent1al } \\
\text { Pressure }\end{array}$ & $\mid \begin{array}{l}\text { Pm } \\
\text { Mean Pore } \\
\text { Pressure } \\
\text { Pe }+\Delta P / 2\end{array}$ & \begin{tabular}{|l} 
Pe \\
Exit \\
Pressure
\end{tabular} & $\begin{array}{l}\text { Te } \\
\text { Elow } \\
\text { Temp } \\
\left({ }^{\circ} \mathrm{C}\right)\end{array}$ & $\begin{array}{c}\text { Tb } \\
\text { Amb 1ent } \\
\text { Temp } \\
\left({ }^{\circ} \mathrm{C}\right)\end{array}$ & $\begin{array}{c}\text { Qb } \\
\text { Flow Rate } \\
\text { apb\&Tb } \\
\text { (m1/sec) }\end{array}$ \\
\hline 06 Apr 93 & $10: 17$ & 5419 & BS3R3a & $12.27 \mathrm{pg} 1 \mathrm{a}$ & volts & volts & 7.460 volts & & volts & 25 & 22 & 0.09236 \\
\hline 06 Apr 93 & $10: 21$ & 5424 & BS3R 3b & 12.27 ps1a & volts & volts & 7.460 volts & & volts & 25 & 22 & 0.09234 \\
\hline 06 Apr 93 & $10: 24$ & 5424 & $\mathrm{BS} 3 \mathrm{R} 3 \mathrm{C}$ & $12.27 \mathrm{psia}$ & volts & volts & 7.460 volts & & volis & 25 & 22 & 0.09236 \\
\hline $06 \mathrm{Apr} 93$ & $10: 26$ & 5429 & BS3R3d & $12.27 \quad \mathrm{ps} 1 \mathrm{a}$ & volts & volts & 7.460 volts & & volts & 25 & 22 & 0.09227 \\
\hline \multirow{9}{*}{ AVERAGES } & & & \multirow{9}{*}{ BS 3R 3} & & GUAGE & BOAGE & DIREERENTIAL & GUAGE & GWAGE & (ब्) & (9व) & \\
\hline & & & & & 6.736 volts & 1.602 volts & 7.460 vol ts & & 1.253 & \multirow{4}{*}{25} & \multirow{4}{*}{22} & \\
\hline & & & & & 1501.2 & 88.82 & 82.26 ps1d & $48.05 \mathrm{psig}$ & 6.918 & & & \\
\hline & & & & & 102.15 & 6.044 & 5.598 & $3.270 \mathrm{~atm}$ & 0.4707 & & & \\
\hline & & & & & $10.350 \quad \mathrm{Mpa}$ & 0.6124 & $0.5672 \quad \mathrm{Mpa}$ & $0.3313 \mathrm{Mpa}$ & 0.04770 & & & \\
\hline & & & & ABSOLUTE & ABSOLUTE & ABSOLUTE & DIFFERENTIAL & ABSOLUTE & ABSOLUTE & (0x) & एKI & (nimI.sec) \\
\hline & & & & $12.27 \mathrm{ps} 1 \mathrm{a}$ & 1513.5 ps1a & $101.09 \quad \mathrm{ps} 1 \mathrm{a}$ & 82.26 ps1d & 60.32 ps1a & 19.19 & \multirow{3}{*}{298} & \multirow{3}{*}{295} & \multirow{3}{*}{0.09233} \\
\hline & & & & $0.8349 \mathrm{~atm}$ & 102.98 & 6.879 & 5.598 & $4.104 \mathrm{~atm}$ & 1.306 & & & \\
\hline & & & & $0.08460 \quad \mathrm{Mpa}$ & 10.435 & 0.6970 & 0.5672 & 0.4159 & 0.1323 & & & \\
\hline
\end{tabular}

$\vec{N}$

Apparent gas permeability:

$K a=(v e \star P e \star u \star L) /(P m \star \Delta P)$

Boyle's Law

$\mathrm{Ve}=(\mathrm{Pb} / \mathrm{Pe}) *(\mathrm{Te} / \mathrm{Tb}) *(z e / z \mathrm{~b}) * \mathrm{Vb}$

$\mathrm{Qe}=(\mathrm{Pb} / \mathrm{Pe}) *(\mathrm{Te} / \mathrm{Tb}) *(2 \mathrm{ze} / 2 \mathrm{~b}) * \mathrm{Qb}$

$v_{e}=Q_{e} / A=(P b / P e) *(T e / T b) *(2 e / 2 b) *\left(\mathrm{Cb}^{2} / \mathrm{A}\right)$

\begin{tabular}{|c|c|c|c|c|}
\hline Parameter & $\begin{array}{l}\text { Traditional } \\
\text { Undts }\end{array}$ & & $\begin{array}{l}\text { SI } \\
\text { Un1ts }\end{array}$ & \\
\hline$\mu=$ gas viscosity & 0.0176 & $\approx 1.0 \mathrm{E}-3 \mathrm{~Pa} * \mathrm{sec} / \mathrm{Cp}$ & $1.760 \mathrm{E}-05$ & Pa*sec \\
\hline$L=$ sample length & 9.557 & $1.0 \mathrm{E}-2 \mathrm{~m} / \mathrm{cm}$ & $9.557 \mathrm{E}-02$ & $\mathrm{~m}$ \\
\hline A = sample circular cross sectional area & 81.249 & $1.0 \mathrm{E}-4 \mathrm{~m}^{\wedge} 2 / \mathrm{cm}^{\wedge} 2$ & $8.125 \mathrm{E}-03$ & $m^{2} 2$ \\
\hline $\mathrm{Pb}=$ flow measurement basls pressure (absolute) & 0.8349 & $1.013 \mathrm{E}+5 \mathrm{~Pa} / \mathrm{atm}$ & $8.458 \mathrm{E}+04$ & $\mathrm{~Pa}$ \\
\hline$\Delta \mathrm{P}=$ pressure drop across sample length & 5.598 & $\star 1.013 E+5 \mathrm{~Pa} / \mathrm{atm}$ & $5.670 \mathrm{E}+05$ & $\mathrm{~Pa}$ \\
\hline $\mathrm{Pm}_{\mathrm{m}}=$ mean pore pressure (absolute) & 4.104 & $1.013 \mathrm{E}+5 \mathrm{~Pa} / \mathrm{atm}$ & $4.158 \mathrm{BE}+05$ & Pa \\
\hline Pe = exit pressure (absolute) & 1.306 & $\star 1.013 \mathrm{E}+5 \mathrm{~Pa} / \mathrm{atm}$ & $1.323 E+05$ & $\overline{\mathrm{Pa}}$ \\
\hline $\mathrm{Te}=$ sample temperature (absolute) & 298 & & 298 & ${ }^{\circ} \mathrm{K}$ \\
\hline $\mathrm{Tb}$ - flow measurement basis temperature (absolute) & 295 & & 295 & ${ }^{\circ} \mathrm{K}$ \\
\hline $2 e=$ gas devlation factor at pe and Te & 1.0000 & & 1.0000 & \\
\hline $\mathrm{zb}=$ gas deviation factor $\mathrm{at} \mathrm{Pb}$ and $\mathrm{Te}$ & 1.0000 & & 1.0000 & \\
\hline $\mathrm{ab}=$ flow rate at base conditions & 0.09233 & $\approx 1.0 \mathrm{E}-6 \mathrm{~m}^{\wedge} 3 / \mathrm{cm}^{\wedge} 3$ & $9.233 \mathrm{E}-08$ & $\mathrm{~m}^{\wedge} 3 / \mathrm{s}$ \\
\hline ve $=$ flow velocity at sample exit end & $7.341 \mathrm{E}-04$ & $1.0 \mathrm{E}-2 \mathrm{~m} / \mathrm{cm}$ & $7.341 \mathrm{E}-06$ & $\mathrm{~m} / \mathrm{s}$ \\
\hline $\mathrm{Ka}=$ & $7.02 E-06$ & $\$ 9.872 \varepsilon-13 \mathrm{~m}^{*} 2 / \mathrm{d}$ & $6.93 \mathrm{E}-18$ & $m^{*} 2$ \\
\hline Ka $=$ & 0.00702 & & $6.93 \mathrm{E}-14$ & $\mathrm{~cm}^{2} 2$ \\
\hline $\mathrm{Ka}=$ & 7.02 & & & \\
\hline
\end{tabular}


Steady State Gas Permeability Data

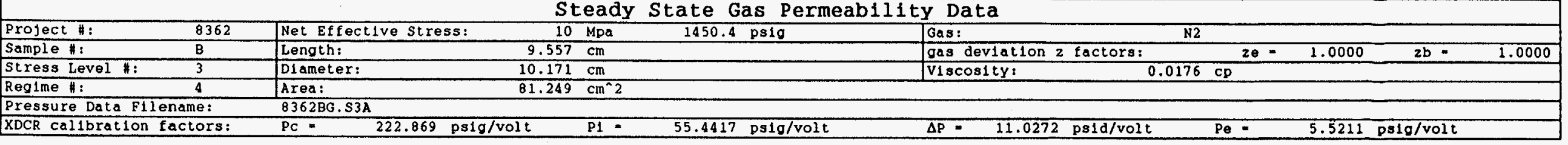

\begin{tabular}{|c|c|c|c|c|c|c|c|c|c|c|c|c|}
\hline Date & $\begin{array}{l}\text { Time } \\
\text { of } \\
\text { Day }\end{array}$ & $\begin{array}{l}\text { File } \\
\text { T1me } \\
(m 1 n)\end{array}$ & $\begin{array}{l}\text { Reglme } \\
\#\end{array}$ & $\begin{array}{l}\mathrm{Pb} \\
\text { Barometric } \\
\text { Pressure }\end{array}$ & $\begin{array}{l}\text { Pc } \\
\text { Confining } \\
\text { Pressure }\end{array}$ & $\begin{array}{l}\text { P1 } \\
\text { Inlet } \\
\text { Pressure }\end{array}$ & $\begin{array}{l}\Delta \mathrm{P} \\
\text { D1fferential } \\
\text { Pressure }\end{array}$ & $\begin{array}{l}\text { Pm } \\
\text { Mean Pore } \\
\text { Pressure } \\
\text { Pe }+\Delta P / 2\end{array}$ & $\begin{array}{l}\text { Pe } \\
\text { Ex1t } \\
\text { Pressure }\end{array}$ & $\begin{array}{l}\text { Te } \\
\text { Flow } \\
\text { Temp } \\
\left({ }^{\circ} \mathrm{C}\right)\end{array}$ & $\begin{array}{c}\text { Tb } \\
\text { Amblent } \\
\text { Temp } \\
\left({ }^{\circ} \mathrm{C}\right)\end{array}$ & $\begin{array}{c}\text { Qb } \\
\text { Flow Rate } \\
\text { Ppbstb } \\
\text { (m1/sec) }\end{array}$ \\
\hline 06 Apr 93 & $13: 39$ & 5619 & BS3R4a & $12.28 \mathrm{psia}$ & volts & volts & 9.122 volts & & 1.451 & 25 & 22 & 0.12571 \\
\hline 06 Apr 93 & $13: 42$ & 5624 & BS3R4b & $12.28 \mathrm{ps} 1 \mathrm{a}$ & volts & 1.950 & 9.122 volts & & volts & 25 & 22 & 0.12590 \\
\hline 06 Apr 93 & $13: 44$ & 5624 & BS3R4C & $12.28 \mathrm{ps} 1 \mathrm{a}$ & volts & volts & 9.122 volts & & volts & 25 & 22 & 0.12598 \\
\hline 06 Apr 93 & $13: 46$ & 5629 & BS3R4d & $12.28 \mathrm{ps} 1 \mathrm{a}$ & volts & volts & 9.122 volts & & volts & 25 & 22 & 0.12571 \\
\hline \multirow{9}{*}{ AVERAGES } & & & \multirow{9}{*}{ BS3R4 } & & OUAOE & GOUAE & DIFEERENTIAL & OUAGE & BUAOE & (ए) & (ब्रा & \\
\hline & & & & & 6.777 volts & 1.950 volis & 9.122 volts & & 1.451 & \multirow{4}{*}{25} & \multirow{4}{*}{22} & \\
\hline & & & & & 1510.4 & 108.11 & 100.59 ps1d & $58.31 \mathrm{ps} 1 \mathrm{~g}$ & 8.011 & & & \\
\hline & & & & & 102.78 & 7.357 & 6.845 & 3.967 atm & 0.5451 & & & \\
\hline & & & & & $10.414 \quad \mathrm{Mpa}$ & 0.7454 & $0.6935 \quad \mathrm{Mpa}$ & $0.4020 \mathrm{Mpa}$ & 0.05523 & & & \\
\hline & & & & ABSOLUTE & ABSOLUTE & ABSOLUTE & DIFFERENTIAL & \multicolumn{2}{|l|}{ ABSOLUTE } & $(0 x)$ & $\mathrm{OKJ}$ & $(\operatorname{mI} / \mathrm{SQC})$ \\
\hline & & & & 12.28 psla & $1522.7 \quad$ ps1a & $120.39 \quad \mathrm{ps} 1 \mathrm{a}$ & $100.59 \mathrm{ps} 1 \mathrm{~d}$ & 70.59 psia & 20.29 & \multirow{3}{*}{298} & \multirow{3}{*}{295} & \multirow{3}{*}{0.12583} \\
\hline & & & & $0.8356 \quad \mathrm{~atm}$ & 103.61 & 8.192 & 6.845 & $4.803 \mathrm{~atm}$ & 1.381 & & & \\
\hline & & & & $0.08467 \quad \mathrm{Mpa}$ & 10.499 & 0.8301 & 0.6935 & 0.4867 & 0.1399 & & & \\
\hline
\end{tabular}

Apparent gas permeablltty: $K a=($ ve* $P e * u * L) /(P m * \Delta P)$

Boyle's Law:

$V_{e}=(\mathrm{Pb} / \mathrm{Pe}) *(\mathrm{Te} / \mathrm{Tb}) *(\mathrm{ze} / \mathrm{zb}) * \mathrm{Vb}$

$\mathrm{Qe}=(\mathrm{Pb} / \mathrm{Pe}) *(\mathrm{Te} / \mathrm{Tb}) *(z e / z b) * \mathrm{Qb}$

$\mathrm{ve}=\mathrm{Qe} / \mathrm{A}=(\mathrm{Pb} / \mathrm{Pe}) *(\mathrm{Te} / \mathrm{Tb}) *(\mathrm{ze} / \mathrm{Zb}) *(\mathrm{Qb} / \mathrm{A})$

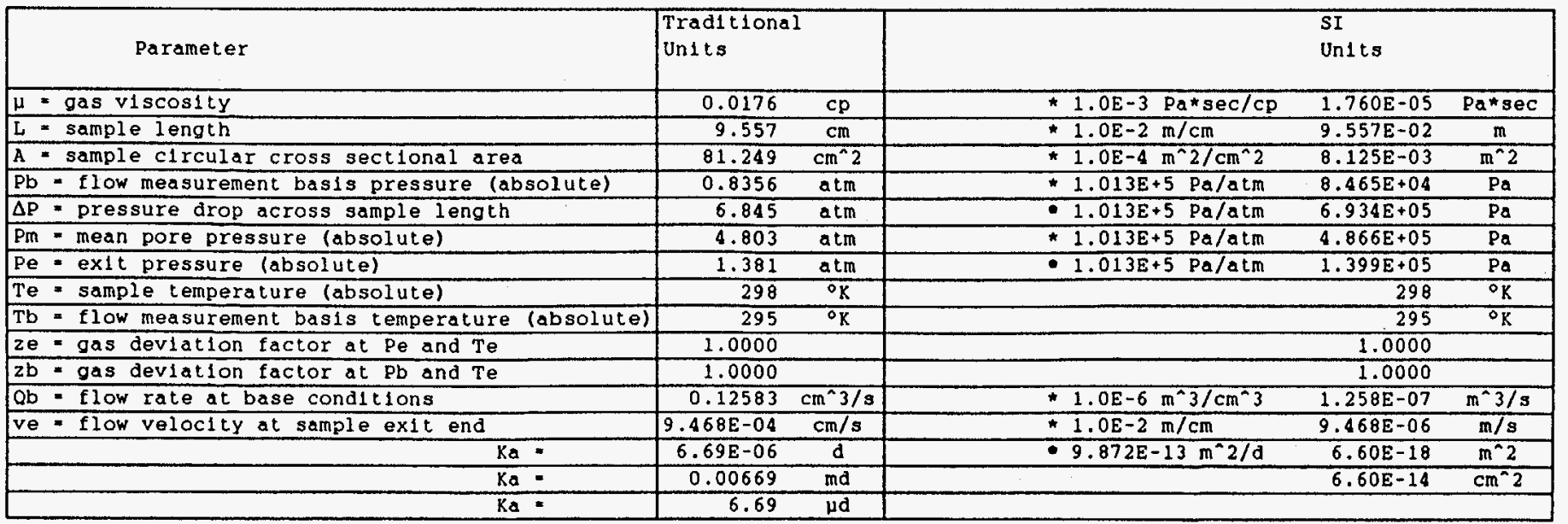




\begin{tabular}{|c|c|c|c|c|c|c|c|c|c|c|c|c|c|}
\hline \multicolumn{14}{|c|}{ Steady State Gas Permeability Data } \\
\hline Project & $t \#:$ & 8362 & Net Effective Stress: & 2 & Mpa & 290.1 psid & Gas: & & N2 & & & & \\
\hline Sample & H: & $c$ & Length: & 10.043 & $\mathrm{~cm}$ & & gas deviation 2 & factors: & & $2 e=$ & 1.0000 & $2 b=$ & 1.0000 \\
\hline Stress & Level \#: & 1 & Diameter: & 10.168 & $\mathrm{~cm}$ & & Vtscosit $y:$ & & 0.0176 & $\mathrm{cp}$ & & & \\
\hline Regime & \#: & 1 & Area: & 81.201 & $\mathrm{~cm}^{\wedge} 2$ & & & & & & & & \\
\hline \multicolumn{3}{|c|}{ Pressure Data Fllename: } & $8362 \mathrm{CG} .51 \mathrm{~B}$ & & & & & & & & & & \\
\hline XDCR CO & al1bratior & tors: & PC $=222.869$ & sit & P1 $=$ & $55.4417 \mathrm{psig} / \mathrm{volt}$ & 11.0272 & pgld/volt & & $\mathrm{Pe}_{\mathrm{e}}=$ & 5.5211 & psig/volt & \\
\hline
\end{tabular}

\begin{tabular}{|c|c|c|c|c|c|c|c|c|c|c|c|c|}
\hline Date & $\begin{array}{l}\text { Time } \\
\text { of } \\
\text { Day }\end{array}$ & $\begin{array}{l}\text { File } \\
\text { Time } \\
\text { (min) }\end{array}$ & $\begin{array}{c}\text { Regime } \\
\#\end{array}$ & $\begin{array}{l}\text { Pb } \\
\text { Barometric } \\
\text { Pressure }\end{array}$ & $\begin{array}{l}\text { Pc } \\
\text { Confining } \\
\text { Pressure }\end{array}$ & $\begin{array}{l}\text { P1 } \\
\text { Inlet } \\
\text { Pressure }\end{array}$ & $\begin{array}{l}\Delta \mathrm{P} \\
\mathrm{D} 1 \mathrm{f} \text { ferential } \\
\text { Pressure }\end{array}$ & $\begin{array}{l}\mathrm{Pm} \\
\text { Mean Pore } \\
\mathrm{Pressure} \\
\mathrm{Pe}+\Delta \mathrm{P} / 2\end{array}$ & $\begin{array}{l}\text { Pe } \\
\text { Exit } \\
\text { Pressure }\end{array}$ & $\begin{array}{l}\text { Te } \\
\text { Flow } \\
\text { Temp } \\
\left({ }^{\circ} \mathrm{C}\right)\end{array}$ & $\begin{array}{c}\text { Tb } \\
\text { Amb1ent } \\
\text { Temp } \\
\left({ }^{\circ} \mathrm{C}\right)\end{array}$ & $\begin{array}{c}\text { Ob } \\
\text { Flow Rate } \\
\text { OPb\&Tb } \\
\text { (m1/sec) }\end{array}$ \\
\hline 27 Apr 93 & $13: 40$ & 266 & CS1R1a & $12.36 \mathrm{psia}$ & volts & 0.962 & 4.416 volts & & volts & 23 & 23 & 0.04023 \\
\hline 27 Apr 93 & $13: 45$ & 271 & CS1RIb & 12.36 psia & 1.434 volts & 0.963 & 4.415 volts & & volts & 23 & 23 & 0.04023 \\
\hline 27 Apr 93 & $23: 49$ & 271 & $\operatorname{Cs} 1 \mathrm{R} 1 \mathrm{C}$ & $12.36 \mathrm{ps} 1 \mathrm{a}$ & 1.434 vo1ts & 0.963 & 4.415 volts & & volts & 23 & 23 & 0.04007 \\
\hline 27 Apr 93 & $13: 52$ & 276 & CSIR1d & $12.36 \mathrm{ps} 1 \mathrm{a}$ & volts & 0.963 & 4.415 volts & & volts & 23 & 23 & 0.04025 \\
\hline \multirow{9}{*}{ AVERAGES } & & & \multirow{9}{*}{ CSIRI } & & OOAAE & OOAGE & OTFEERENTIXI & OUNGE & OWAOE: & काल) & $(90)$ & \\
\hline & & & & & 1.434 vo1ts & 0.963 volts & 4.415 volts & & 0.895 & \multirow{4}{*}{23} & \multirow{4}{*}{23} & \\
\hline & & & & & 319.6 & 53.38 & 48.69 psid & $29.29 \mathrm{ps} 1 \mathrm{~g}$ & 4.941 & & & \\
\hline & & & & & 21.75 & 3.632 & 3.313 & 1.993 atm & 0.3362 & & & \\
\hline & & & & & 2.204 & 0.3680 & 0.3357 & 0.2019 & 0.03407 & & & \\
\hline & & & & ABSOLUTE & ABSOLUTE & ABSOLUTE & DIFFERENTIAL & ABSOUUTE & ABSOLUTE: & (बर) & $(\% x)$ & (sitsoc) \\
\hline & & & & $12.36 \mathrm{psia}$ & 332.0 ps1a & 65.74 psta & 48.69 ps1d & 41.65 psia & 17.30 & \multirow{3}{*}{296} & \multirow{3}{*}{296} & \multirow{3}{*}{0.04020} \\
\hline & & & & $0.8410 \mathrm{~atm}$ & 22.59 & 4.473 & 3.313 & $2.834 \mathrm{~atm}$ & 1.177 & & & \\
\hline & & & & $0.08522 \quad \mathrm{Mpa}$ & 2.289 & 0.4532 & 0.3357 & 0.2871 & 0.1193 & & & \\
\hline
\end{tabular}

Apparent gas permeability:

$K_{a}=\left(v e^{\star P e} e^{\star} u^{\star} L\right) /(P m \star \Delta P)$

Boyle's Law:

$\mathrm{V}_{e}=(\mathrm{Pb} / \mathrm{Pe}) \cdot(\mathrm{Te} / \mathrm{Tb}) *(\mathrm{ze} / \mathrm{zb}) * \mathrm{Vb}$

$\mathrm{Qe}^{\mathrm{C}}=(\mathrm{Pb} / \mathrm{Pe}) *(\mathrm{Te} / \mathrm{Tb}) *(\mathrm{ze} / \mathrm{Zb}) * \mathrm{Qb}$

$v_{e}=Q_{e} / A=\left(\mathrm{Pb} / \mathrm{Pe}_{\mathrm{e}}\right)(\mathrm{Te} / \mathrm{Tb}) *(2 e / 2 \mathrm{~b}) *(\mathrm{Qb} / \mathrm{A})$

\begin{tabular}{|c|c|c|c|c|}
\hline Parameter & $\begin{array}{l}\text { Traditional } \\
\text { Units }\end{array}$ & & $\begin{array}{l}\text { SI } \\
\text { Un1ts }\end{array}$ & \\
\hline$\mu=$ gas viscosity & 0.0176 & $\approx 1.0 \mathrm{E}-3 \mathrm{~Pa}$ * $\mathrm{sec} / \mathrm{cp}$ & $1.760 \mathrm{E}-05$ & Pa*sec \\
\hline$L=$ sample length & 10.043 & $\star 1.0 \mathrm{E}-2 \mathrm{~m} / \mathrm{cm}$ & $1.004 E-01$ & $\mathrm{~m}$ \\
\hline A - sample clrcular cross sectlonal area & 81.201 & $1.0 E^{-4} \mathrm{~m}^{n} 2 / \mathrm{cm}^{\wedge} 2$ & $8.120 \mathrm{E}-03$ & $m^{2} 2$ \\
\hline $\mathrm{Pb}=$ flow measurement bas1s pressure (absolute) & 0.8410 & $\$ 1.013 \mathrm{E}+5 \mathrm{~Pa} / \mathrm{atm}$ & $8.520 E+04$ & $\mathrm{~Pa}$ \\
\hline$\Delta \mathrm{P}=$ pressure drop across sample length & 3.313 & $\$ 1.013 E+5 \mathrm{~Pa} / \mathrm{atm}$ & $3.356 \mathrm{E}+05$ & $\mathrm{~Pa}$ \\
\hline $\mathrm{Pm}_{\mathrm{m}}=$ mean pore pressure (absolule) & 2.834 & $\approx 1.013 \mathrm{E}+5 \mathrm{~Pa} / \mathrm{atm}$ & $2.871 E+05$ & $\overline{P a}$ \\
\hline $\mathrm{Pe}=$ exit pressure (absolute) & 1.177 & $1.013 \mathrm{E}+5 \cdot \mathrm{Pa} / \mathrm{atm}$ & $1.1938+05$ & $\mathrm{~Pa}$ \\
\hline Te = sample temperature (absolute) & 296 & & 296 & ${ }^{\circ} \mathrm{K}$ \\
\hline $\mathrm{Tb}=$ flow measurement basis temperature (absolute) & 296 & & 296 & ${ }^{\circ} \mathrm{K}$ \\
\hline$z e=$ gas deviation factor at $\mathrm{Pe}$ and $\mathrm{Te}$ & 1.0000 & & 1.0000 & \\
\hline $\mathrm{zb}=$ gas deviation factor $\mathrm{at} \mathrm{Pb}$ and $\mathrm{Te}$ & 1.0000 & & 1.0000 & \\
\hline$Q b$ - flow rate at base conditions & 0.04020 & $1.0 \mathrm{E}-6 \mathrm{~m}^{\wedge} 3 / \mathrm{cm}^{2} 3$ & $4.020 \mathrm{E}-08$ & $m^{n} 3 / s$ \\
\hline ve = flow velocity at sample exit end & $3.536 \mathrm{E}-04$ & $1.0 \mathrm{E}-2 \mathrm{~m} / \mathrm{cm}$ & $3.536 \mathrm{E}-06$ & $\mathrm{~m} / \mathrm{s}$ \\
\hline $\mathrm{Ka}_{\mathrm{a}}$ & $7.84 \mathrm{E}-06$ & $9.872 E-13 m^{\prime \prime} 2 / d$ & $7.74 E-18$ & $m^{2} 2$ \\
\hline $\mathrm{Ka}=$ & $7.84 \mathrm{E}-03$ & & $7.74 E-14$ & $\mathrm{~cm}^{\star} 2$ \\
\hline $\mathrm{Ka}=$ & $7.84 \mathrm{E}+00$ & & & \\
\hline
\end{tabular}




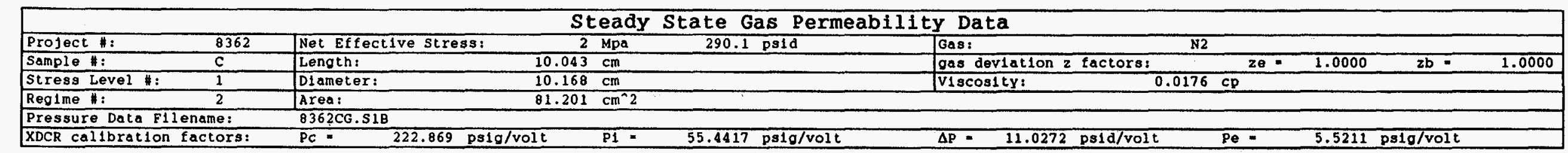

\begin{tabular}{|c|c|c|c|c|c|c|c|c|c|c|c|c|}
\hline Date & $\begin{array}{l}\text { Time } \\
\text { of } \\
\text { Day }\end{array}$ & $\begin{array}{l}\text { File } \\
\text { Time } \\
(m 1 n)\end{array}$ & $\begin{array}{c}\text { Regime } \\
\#\end{array}$ & $\begin{array}{l}\text { Pb } \\
\text { Barometric } \\
\text { Pressure }\end{array}$ & $\begin{array}{l}\text { Pc } \\
\text { Confining } \\
\text { Pressure }\end{array}$ & $\begin{array}{l}\text { P1 } \\
\text { Inlet } \\
\text { Pressure }\end{array}$ & $\begin{array}{l}\Delta \mathrm{P} \\
\text { D1fferent1al } \\
\text { Pressure }\end{array}$ & $\begin{array}{l}\text { Pm } \\
\text { Mean Pore } \\
\text { Pressure } \\
\mathrm{Pe}+\Delta \mathrm{P} / 2\end{array}$ & $\begin{array}{l}\text { Pe } \\
\text { Ex1t } \\
\text { Pressure }\end{array}$ & $\begin{array}{l}\text { Te } \\
\text { Flow } \\
\text { Temp } \\
\left({ }^{\circ} \mathrm{C}\right)\end{array}$ & $\begin{array}{c}\mathrm{Tb} \\
\text { Amblent } \\
\text { Temp } \\
\left({ }^{\circ} \mathrm{C}\right)\end{array}$ & $\begin{array}{c}\text { ab } \\
\text { Flow Rate } \\
\text { opb\&tb } \\
\text { (m1/sec) }\end{array}$ \\
\hline 27 Apr 93 & $16: 45$ & 451 & CSIR2a & $12.34 \mathrm{ps} 1 \mathrm{a}$ & volts & volts & 4.375 volts & & volts & 23 & 23 & 0.04577 \\
\hline 27 Apr 93 & $16: 47$ & 451 & CS1R2b & 12.34 ps1a & volts & volts & 4.375 volts & & volts & 23 & $\overline{23}$ & 0.04583 \\
\hline $27 \mathrm{Apr} 93$ & $16: 50$ & 456 & CS1R2c & $12.34 \mathrm{ps} 1 \mathrm{a}$ & volts & volts & 4.375 volts & & volts & 23 & 23 & 0.04571 \\
\hline 27 Apr 93 & $16: 58$ & 461 & CS1R2d & 12.34 ps1a & volts & volts & 4.375 volts & & volts & 23 & 23 & 0.04579 \\
\hline 27 Apr 93 & $17: 01$ & 466 & CS1R2d & $12.34 \mathrm{ps} 1 \mathrm{a}$ & volts & volts & 4.375 volts & & 2.743 & 23 & 23 & 0.04568 \\
\hline \multirow{9}{*}{ AVERAGES } & & & \multirow{9}{*}{$\operatorname{Cs} 1 \operatorname{R}^{2}$} & & GUACE & GUAOE & DIMFEREMTI II & OUXaE: & OUnOD: & $60)$ & $190 \%$ & \\
\hline & & & & & 1.478 volts & 1.138 volts & 4.375 volts & & 2.744 & \multirow{4}{*}{23} & \multirow{4}{*}{23} & \\
\hline & & & & & 329.4 & 63.09 & 48.24 psid & $39.27 \mathrm{psig}$ & 15.151 & & & \\
\hline & & & & & 22.41 & 4.293 & 3.283 & $2.672 \mathrm{~atm}$ & 1.0310 & & & \\
\hline & & & & & 2.271 & 0.4350 & $0.3326 \quad \mathrm{Mpa}$ & $0.2708 \mathrm{Mpa}$ & 0.10446 & & & \\
\hline & & & & ABSOLUTE & ABSOLUTE & ABSOLUME & DIFFERENT IL & \multicolumn{2}{|l|}{ RaSOLUTE } & बर & $6 \times 1$ & (nTHec) \\
\hline & & & & $12.34 \mathrm{pg} 1 \mathrm{a}$ & 341.7 ps1a & 75.43 ps1a & $4 \theta .24 \mathrm{pg} 1 \mathrm{~d}$ & 51.61 ps1o & 27.49 & \multirow{3}{*}{296} & \multirow{3}{*}{296} & \multirow{3}{*}{0.04578} \\
\hline & & & & $0.8397 \quad \mathrm{~atm}$ & 23.25 & 5.133 & 3.283 & $3.512 \mathrm{~atm}$ & 1.871 & & & \\
\hline & & & & $0.08508 \mathrm{Mpa}$ & 2.356 & 0.5201 & 0.3326 & 0.3559 & 0.1895 & & & \\
\hline
\end{tabular}

Apparent gas permeab1lity:

Boyle's Law:

$K a=\left(v e * P e^{\star} u \star L\right) /(P m \star \Delta P)$

$\mathrm{Ve}=(\mathrm{Pb} / \mathrm{Pe}) *(\mathrm{Te} / \mathrm{Tb}) *(\mathrm{ze} / \mathrm{zb}) * \mathrm{Vb}$

$\mathrm{Q}$ - $(\mathrm{Pb} / \mathrm{Pe}) *(\mathrm{Te} / \mathrm{Tb}) \cdot(2 e / 2 \mathrm{~b}) * \mathrm{Qb}$

$v_{e}=\mathrm{Qe} / \mathrm{A}=(\mathrm{Pb} / \mathrm{Pe}) *(\mathrm{Te} / \mathrm{Tb}) \cdot(2 \mathrm{e} / \mathrm{zb}) *(\mathrm{Qb} / \mathrm{A})$

\begin{tabular}{|c|c|c|c|c|}
\hline Parameter & $\begin{array}{l}\text { Traditional } \\
\text { Units }\end{array}$ & & $\begin{array}{l}\text { SI } \\
\text { Un1ts }\end{array}$ & \\
\hline$\mu=$ gas viscosity & 0.0176 & $\star 1.0 \mathrm{E}-3 \mathrm{~Pa} \star \sec / \mathrm{cp}$ & $1.760 \mathrm{E}-05$ & $\mathrm{~Pa} \star \mathrm{sec}$ \\
\hline $\mathrm{L}$ - sample length & 10.043 & * $1.0 \mathrm{E}-2 \mathrm{~m} / \mathrm{cm}$ & $1.004 \mathrm{E}-01$ & $\mathbf{m}$ \\
\hline A - sample circular cross sectlonal area & 81.201 & $\star 1.0 \mathrm{E}-4 \mathrm{~m}^{\wedge} 2 / \mathrm{cm}^{\wedge} 2$ & $8.120 \mathrm{E}-03$ & $m^{\wedge} 2$ \\
\hline $\mathrm{Pb}=$ flow measurement basis pressure (absolute) & 0.8397 & $\star 1.013 \mathrm{E}+5 \mathrm{~Pa} / \mathrm{atm}$ & $8.506 \mathrm{E}+04$ & $\mathrm{~Pa}$ \\
\hline$\Delta \mathrm{P}=$ pressure arop across sample length & 3.283 & $\pi 1.013 \mathrm{E}+5 \mathrm{~Pa} / \mathrm{atm}$ & $3.325 E+05$ & $\mathbf{P a}$ \\
\hline $\mathrm{Pm}=$ mean pore pressure (absolute) & 3.512 & $1.013 \mathrm{E}+5 \mathrm{~Pa} / \mathrm{atm}$ & $3.558 \mathrm{E}+05$ & $\mathrm{~Pa}$ \\
\hline $\mathrm{Pe}=$ exit pressure (absolute) & 1.871 & $1.013 \mathrm{E}+5 \mathrm{~Pa} / \mathrm{atm}$ & $1.895 \mathrm{E}+05$ & $\mathrm{~Pa}$ \\
\hline Te - sample temperature (absolute) & 296 & & 296 & ${ }^{\circ} \mathrm{K}$ \\
\hline $\mathrm{Tb}=$ flow measurement basis temperature (absolute) & 296 & & 296 & ${ }^{\circ} \mathrm{K}$ \\
\hline $2 e=$ gas devlation factor at $\mathrm{Pe}$ and $\mathrm{Te}$ & 1.0000 & & 1.0000 & \\
\hline $2 \mathrm{~b}=$ gas deviation factor $\mathrm{at} \mathrm{Pb}$ and $\mathrm{Te}$ & 1.0000 & & 1.0000 & \\
\hline$a b=$ flow rale at base conditions & 0.04578 & $1.0 \mathrm{E}-6 \mathrm{~m}^{\wedge} 3 / \mathrm{cm}^{4} 3$ & $4.578 \mathrm{E}-08$ & $m^{2} 3 / s$ \\
\hline ve $=$ flow velocity at sample exit end & $2.531 \mathrm{E}-04$ & $* 1.0 \mathrm{E}-2 \mathrm{~m} / \mathrm{cm}$ & $2.531 \mathrm{E}-06$ & $\mathrm{~m} / \mathrm{s}$ \\
\hline $\mathrm{Ka}=$ & $7.26 \mathrm{E}-06$ & $\$ 9.872 \mathrm{E}-13 \mathrm{~m}^{\wedge} 2 / \mathrm{d}$ & $7.16 \mathrm{E}-18$ & $m^{\wedge} 2$ \\
\hline$R_{a}=$ & $7.26 \varepsilon-03$ & & $7.16 \mathrm{E}-14$ & $\mathrm{~cm}^{\wedge} 2$ \\
\hline $\mathbf{K a}=$ & $7.26 \mathrm{E}+00$ & & & \\
\hline
\end{tabular}




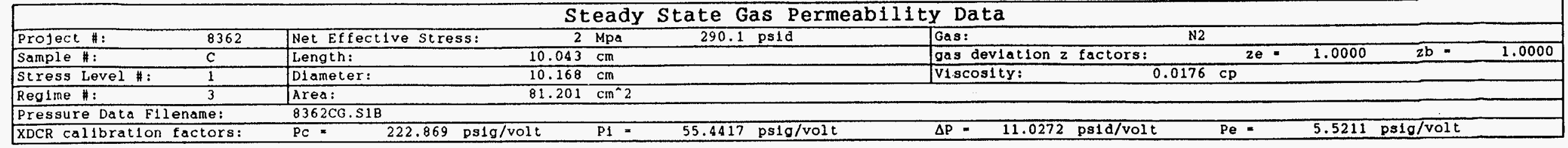

\begin{tabular}{|c|c|c|c|c|c|c|c|c|c|c|c|c|}
\hline Date & $\begin{array}{l}\text { Time } \\
\text { of } \\
\text { Day }\end{array}$ & $\begin{array}{l}\text { File } \\
\text { Time } \\
\text { (min) }\end{array}$ & $\begin{array}{c}\text { Reglme } \\
\#\end{array}$ & $\begin{array}{l}\mathrm{Pb} \\
\text { Barometric } \\
\text { Pressure }\end{array}$ & $\begin{array}{l}\text { Pc } \\
\text { Confining } \\
\text { Pressure }\end{array}$ & $\begin{array}{l}\text { P1 } \\
\text { Inlet } \\
\text { Pressure }\end{array}$ & $\begin{array}{l}\Delta \mathrm{P} \\
\mathrm{D} 1 \mathrm{f} \text { ferential } \\
\text { Pressure }\end{array}$ & $\begin{array}{l}\text { Pm } \\
\text { Mean Pore } \\
\text { Pressure } \\
\text { Pe }+\Delta P / 2\end{array}$ & $\begin{array}{l}\text { Pe } \\
\text { Exit } \\
\text { Pressure }\end{array}$ & $\begin{array}{l}\text { Te } \\
\text { Flow } \\
\text { Temp } \\
\left({ }^{\circ} \mathrm{C}\right)\end{array}$ & $\begin{array}{c}\text { Tb } \\
\text { Amb1ent } \\
\text { Temp } \\
\left({ }^{\circ} \mathrm{C}\right)\end{array}$ & $\begin{array}{c}\text { Qb } \\
\text { Flow Rate } \\
\text { OPbsTb } \\
\text { (ml/sec) }\end{array}$ \\
\hline 28 Apr 93 & $10: 37$ & 1523 & $\operatorname{Cs} 1 \mathrm{R} 3 a$ & 12.42 psia & 1.522 volts & 1.312 volts & 4.393 volts & & 4.459 & 23 & 22 & 0.05075 \\
\hline 28 Apr 93 & $10: 40$ & 1523 & CSIR $3 b$ & 12.42 psia & volts & volts & 4.393 volts & & volts & 23 & 22 & 0.05068 \\
\hline $28 \mathrm{Apr} 93$ & $10: 43$ & 1528 & $\operatorname{CS1R} 3 \mathrm{C}$ & 12.42 psta & volts & volts & 4.394 volts & & volts & 23 & 22 & 0.05072 \\
\hline 28 Apr 93 & $10: 45$ & 1528 & $\operatorname{CS1R3d}$ & $12.42 \mathrm{psia}$ & volts & volts & 4.395 volts & & 4.456 & 23 & 22 & 0.05076 \\
\hline \multirow{9}{*}{ AVERAGES } & & & \multirow{9}{*}{$\operatorname{cs} 1 \mathrm{R} 3$} & & GUAGE & GUAGE & DIFEEREATTIAL & GOKGE & GUAGE & $(96)$ & (ए) & \\
\hline & & & & & 1.523 volts & $1.312 \mathrm{volts}$ & 4.394 volts & & 4.458 & \multirow{4}{*}{23} & \multirow{4}{*}{22} & \\
\hline & & & & & 339.3 & 72.74 & 48.45 psid & $48.84 \mathrm{ps} 1 \mathrm{~g}$ & 24.612 & & & \\
\hline & & & & & 23.09 & 4.950 & 3.297 & $3.323 \mathrm{~atm}$ & 1.6747 & & & \\
\hline & & & & & 2.340 & 0.5015 & $0.3341 \quad \mathrm{Mpo}$ & $0.3367 \mathrm{Mpa}$ & 0.16969 & & & \\
\hline & & & & ABSOLUTE & ABSOLUTE & ABSOLUTE & DIFFERENTIAL & \multicolumn{2}{|l|}{ AESOLUTE } & OKM & TKX & (StI $\mathrm{sec}$ \\
\hline & & & & $12.42 \mathrm{ps} 1 \mathrm{a}$ & $351.7 \quad$ ps10 & $85.16 \quad$ ps1a & 48.45 psid & $61.26 \mathrm{ps} 1 \mathrm{a}$ & 37.03 & \multirow{3}{*}{296} & \multirow{3}{*}{295} & \multirow{3}{*}{0.05073} \\
\hline & & & & $0.8451 \mathrm{~atm}$ & 23.93 & 5.795 & 3.297 & $4.168 \mathrm{~atm}$ & 2.520 & & & \\
\hline & & & & 0.08563 & 2.425 & 0.5872 & 0.3341 & 0.4224 & 0.2553 & & & \\
\hline
\end{tabular}

心

Apparent gas permeablilty: Ka $=\left(\right.$ ve $\left.\mathrm{P}^{\star} \mathrm{u}^{\star} \mathrm{L}\right) /\left(\mathrm{Pm}^{\star} \Delta \mathrm{P}\right)$

Boyle's Law:

$\mathrm{Ve}=(\mathrm{Pb} / \mathrm{Pe}) *(\mathrm{Te} / \mathrm{Tb}) *(\mathrm{ze} / \mathrm{zb}) * \mathrm{Vb}$

Qe $=(\mathrm{Db} / \mathrm{PO}) *(\mathrm{Te} / \mathrm{Tb}) *(z e / 2 b) * Q b$

$v e * Q e / A=(\mathrm{Pb} / \mathrm{Pe}) *(\mathrm{Te} / \mathrm{Tb}) *(z e / 2 \mathrm{~b}) *(\mathrm{Qb} / \mathrm{A})$

\begin{tabular}{|c|c|c|c|c|}
\hline Parameter & $\begin{array}{l}\text { Traditional } \\
\text { Units }\end{array}$ & & $\begin{array}{l}\text { SI } \\
\text { Units }\end{array}$ & \\
\hline$\mu=\operatorname{gas} v 1 \mathrm{scos} 1 \mathrm{ty}$ & 0.0176 & $\star 1.0 \mathrm{E}-3 \mathrm{~Pa} * \mathrm{sec} / \mathrm{CP}$ & $1.760 \mathrm{E}-05$ & $\mathrm{~Pa} * \mathrm{sec}$ \\
\hline $\mathrm{L}=$ sample length & 10.043 & $1.0 \mathrm{E}-2 \mathrm{~m} / \mathrm{cm}$ & $1.004 \mathrm{E}-01$ & $\mathbf{m}$ \\
\hline A = sample clrcular cross sectlonal area & 81.201 & $\star 1.0 \mathrm{E}-4 \mathrm{~m}^{\wedge} 2 / \mathrm{cm}^{\wedge} 2$ & $8.120 \mathrm{E}-03$ & $m^{\wedge} 2$ \\
\hline $\mathrm{Pb}$ - flow measurement bas1s pressure (absolute) & 0.8451 & $\star 1.013 \mathrm{E}+5 \mathrm{~Pa} / \mathrm{atm}$ & $8.561 \mathrm{E}+04$ & Pa \\
\hline$\Delta \mathrm{P}=$ pressure drop across sample length & 3.297 & $\star 1.013 \mathrm{E}+5 \mathrm{~Pa} / \mathrm{atm}$ & $3.340 \mathrm{E}+05$ & $\mathrm{~Pa}$ \\
\hline Pm - mean pore pressure (absolute) & 4.168 & $1.013 \mathrm{E}+5 \mathrm{~Pa} / \mathrm{atm}$ & $4.222 E+05$ & $\mathrm{~Pa}$ \\
\hline Pe = exit pressure (absolute) & 2.520 & $1.013 \mathrm{E}+5 \mathrm{~Pa} / \mathrm{atm}$ & $2.553 E+05$ & $\mathrm{~Pa}$ \\
\hline Te = sample temperature (absolute) & 296 & & 296 & ${ }^{\circ} \mathrm{K}$ \\
\hline$T b=$ flow measurement basis temperature (absolute) & 295 & & 295 & ${ }^{\circ} \mathrm{K}$ \\
\hline $2 e=$ gas deviation factor at Pe and $\mathrm{Te}$ & 1.0000 & & 1.0000 & \\
\hline $2 \mathrm{~b}=$ gas deviation factor at $\mathrm{pb}$ and $\mathrm{Te}$ & 1.0000 & & 1.0000 & \\
\hline $\mathrm{Qb}=$ flow rate at base conditions & 0.05073 & $1.0 \mathrm{E}-6 \mathrm{~m}^{\wedge} 3 / \mathrm{cm}^{\wedge} 3$ & $5.073 E-08$ & $m^{\wedge} 3 / 3$ \\
\hline ve = flow velocity at sample exit end & $2.102 \mathrm{E}-04$ & $* 1.0 \overline{\mathrm{E}}-2 \mathrm{~m} / \mathrm{cm}$ & $2.102 \mathrm{E}-06$ & $\mathrm{~m} / \mathrm{s}$ \\
\hline $\mathrm{Ka}=$ & $6.81 \mathrm{E}-06$ & $9.872 \mathrm{E}-13 \mathrm{~m}^{\wedge} 2 / \mathrm{d}$ & $6.73 E-18$ & $m^{\wedge} 2$ \\
\hline $\mathrm{Ka}=$ & $6.81 E-03$ & & $6.73 \mathrm{E}-14$ & $\mathrm{~cm}^{2} 2$ \\
\hline $\mathrm{Ka}=$ & $6.81 \mathrm{E}+00$ & & & \\
\hline
\end{tabular}


Steady State Gas Permeability Data

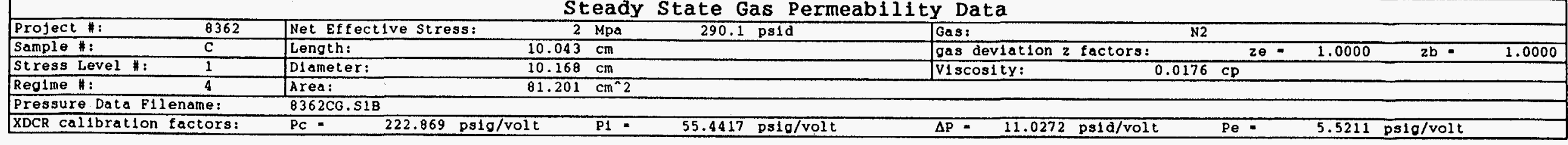

\begin{tabular}{|c|c|c|c|c|c|c|c|c|c|c|c|c|}
\hline Date & $\begin{array}{l}\text { Time } \\
\text { of } \\
\text { Day }\end{array}$ & $\begin{array}{l}\text { File } \\
\text { Time } \\
\text { (min) }\end{array}$ & $\begin{array}{c}\text { Reg1me } \\
\#\end{array}$ & $\begin{array}{l}\mathrm{pb} \\
\text { Barometric } \\
\text { Pressure }\end{array}$ & $\begin{array}{l}\text { Pc } \\
\text { Contining } \\
\text { Pressure }\end{array}$ & $\begin{array}{l}\text { P1 } \\
\text { Inlet } \\
\text { Pressure }\end{array}$ & $\begin{array}{l}\Delta \mathrm{P} \\
\mathrm{D} 1 \mathrm{f} \text { ferent1al } \\
\text { Pressure }\end{array}$ & $\begin{array}{l}\mathrm{Pm} \\
\text { Mean Pore } \\
\text { Pressure } \\
\text { P } \theta+\Delta P / 2\end{array}$ & $\begin{array}{l}\text { Pe } \\
\text { Ex1t } \\
\text { Pressure }\end{array}$ & $\begin{array}{l}\text { Te } \\
\text { Flow } \\
\text { Temp } \\
\left({ }^{\circ} \mathrm{C}\right)\end{array}$ & $\begin{array}{c}\text { Tb } \\
\text { Amblent } \\
\text { Temp } \\
\left({ }^{\circ} \mathrm{C}\right)\end{array}$ & $\begin{array}{c}\text { Ob } \\
\text { Elow Rate } \\
\text { OPbLTb } \\
\text { (ml/sec) }\end{array}$ \\
\hline 28 Apr 93 & $14: 15$ & 1741 & CS1R4a & 12.42 psla & volts & volts & 4.477 volts & & volts & 23 & 23 & 0.05789 \\
\hline 28 Apr 93 & $14: 17$ & 1741 & CS1R4b & 12.42 psia & volts & volts & 4.477 volts & & volts & 23 & 23 & 0.05798 \\
\hline 28 Apr 93 & $14: 20$ & 1746 & CS1R4C & $12.42 \mathrm{psia}$ & volts & volts & 4.478 volts & & volts & 23 & 23 & 0.05784 \\
\hline 28 Apr 93 & $14: 23$ & 1746 & CS1R4d & 12.42 ps1a & 1.566 volts & 1.506 volts & 4.478 volt s & & volts & 23 & 23 & 0.05785 \\
\hline \multirow{9}{*}{ AVERAGES } & & & \multirow{9}{*}{$\operatorname{Cs} 1 \mathrm{R} 4$} & & OUAGE & TOUAGE & DHEREREMTIAL & OOAGE & ownOE: & क्टा: & क्टा & \\
\hline & & & & & 1.566 volts & 1.506 volts & 4.478 volts & & 6.258 & \multirow{4}{*}{23} & \multirow{4}{*}{23} & \\
\hline & & & & & 349.0 & 83.50 & 49.37 ps1d & $59.24 \mathrm{psig}$ & 34.548 & & & \\
\hline & & & & & 23.75 & 5.681 & 3.360 & $4.031 \mathrm{~atm}$ & 2.3509 & & & \\
\hline & & & & & 2.406 & 0.5757 & 0.3404 & 0.4084 & 0.23820 & & & \\
\hline & & & & ABSOLUTE & ABSOLUTE & ABSOLUTE & DIFFERENTIA 6 & ABSOLUTE & ABSOLUTE & (बK) & (क्य) & $(\mathrm{m} / \mathrm{s} \theta \mathrm{C})$ \\
\hline & & & & 12.42 psla & 361.4 ps1a & $95.92 \quad$ ps1a & 49.37 ps1d & 71.66 psia & 46.97 psia & \multirow{3}{*}{296} & \multirow{3}{*}{296} & \multirow{3}{*}{0.05789} \\
\hline & & & & $0.8451 \quad \Delta \mathrm{cm}$ & 24.59 & 6.527 & 3.360 & $4.876 \mathrm{~atm}$ & 3.196 & & & \\
\hline & & & & $0.08563 \mathrm{Mpa}$ & 2.492 & 0.6613 & 0.3404 & 0.4940 & 0.3238 & & & \\
\hline
\end{tabular}

Apparent gas permeability:

Boyle's Law:

$K a=\left(V e^{\star P e * u * L)} /(P m * \Delta P)\right.$

$\mathrm{Ve}=(\mathrm{Pb} / \mathrm{Pe}) *(\mathrm{Te} / \mathrm{Tb}) *(\mathrm{ze} / \mathrm{zb}) * \mathrm{Vb}$

$\mathrm{Qe}=(\mathrm{Pb} / \mathrm{Pe}) *(\mathrm{Te} / \mathrm{Tb}) *(\mathrm{ze} / \mathrm{zb}) * \mathrm{Qb}$

$v e=Q e / A=(\mathrm{Pb} / \mathrm{Pe}) *(\mathrm{Te} / \mathrm{Tb}) *(\mathrm{ze} / \mathrm{Zb}) *(\mathrm{Qb} / \mathrm{A})$

\begin{tabular}{|c|c|c|c|c|}
\hline Parameter & $\begin{array}{l}\text { Traditional } \\
\text { Units }\end{array}$ & & $\begin{array}{l}\text { SI } \\
\text { Untts }\end{array}$ & \\
\hline$\mu=g a s v 1 \operatorname{scos} 1 t y$ & 0.0176 & $\star 1.0 \mathrm{E}-3 \mathrm{~Pa} \mathrm{a}^{\star} \mathrm{sec} / \mathrm{Cp}$ & $1.760 \mathrm{E}-05$ & $\mathrm{~Pa}^{*} \sec$ \\
\hline$L=$ sample length & 10.043 & $\star 1.0 \mathrm{E}-2 \mathrm{~m} / \mathrm{cm}$ & $1.004 \mathrm{E}-01$ & $\mathrm{~m}$ \\
\hline$A$ = sample clrcular cross sectional area & 81.201 & $\star 1.0 \mathrm{E}-4 \mathrm{~m}^{\wedge} 2 / \mathrm{cm}^{\wedge} 2$ & $8.120 \mathrm{E}-03$ & $m^{\wedge} 2$ \\
\hline $\mathrm{Pb}=$ flow measurement basis pressure (absolute) & 0.8451 & $\star 1.013 \mathrm{E}+5 \mathrm{~Pa} / \mathrm{atm}$ & $8.561 E+04$ & $\mathrm{~Pa}$ \\
\hline$\Delta \mathrm{P}=$ pressure drop across sample length & 3.360 & $\# 1.013 \mathrm{E}+5 \mathrm{~Pa} / \mathrm{atm}$ & $3.403 E+05$ & $\mathrm{~Pa}$ \\
\hline $\mathrm{Pm}=$ mean pore pressure (absolute) & 4.876 & $\$ 1.013 \mathrm{E}+5 \mathrm{~Pa} / \mathrm{atm}$ & $4.939 E+05$ & Pa \\
\hline $\mathrm{Pe}=$ exit pressure (absolute) & 3.196 & $\star 1.013 \mathrm{E}+5 \mathrm{~Pa} / \mathrm{atm}$ & $3.230 E+05$ & $\mathrm{~Pa}$ \\
\hline $\mathrm{Te}=$ sample temperature (absolute) & 296 & & 296 & ${ }^{\circ} \mathrm{K}$ \\
\hline $\mathrm{Tb}=$ flow measurement basis temperature (absolute) & 296 & & 296 & ${ }^{\circ} \mathrm{K}$ \\
\hline$z e=$ gas deviation factor at $\mathrm{Pe}$ and $\mathrm{Te}$ & 1.0000 & & 1.0000 & \\
\hline $\mathrm{zb}=$ gas deviation factor $\mathrm{at} \mathrm{Pb}$ and $\mathrm{Te}$ & 1.0000 & & 1.0000 & \\
\hline $\mathrm{Qb}=$ flow rate at base conditions & $0.05789 \mathrm{~cm}^{\wedge} 3 / \mathrm{s}$ & $\star 1.0 \mathrm{E}-6 \mathrm{~m}^{\wedge} 3 / \mathrm{cm}^{\wedge} 3$ & $5.789 \mathrm{E}-08$ & $\mathrm{~m}^{n} 3 / \mathrm{s}$ \\
\hline ve $=$ flow velocity at sample exit end & $1.885 \mathrm{E}-04 \mathrm{~cm} / \mathrm{s}$ & * $1.0 \mathrm{E}-2 \mathrm{~m} / \mathrm{cm}$ & $1.885 \mathrm{E}-06$ & $\mathrm{~m} / \mathrm{s}$ \\
\hline $\mathrm{K}-\mathrm{K}-\mathrm{Ka}-$ & $6.50 \mathrm{E}-06$ & $\$ 9.872 \mathrm{E}-13 \mathrm{~m}-2 / \mathrm{d}$ & $6.42 E-18$ & $m^{2} 2$ \\
\hline Ka $=$ & $6.50 \mathrm{E}-03$ & & $6.42 \mathrm{E}-14$ & $c m^{\wedge} 2$ \\
\hline $\mathrm{Ka}=$ & $6.50 E+00$ & & & \\
\hline
\end{tabular}




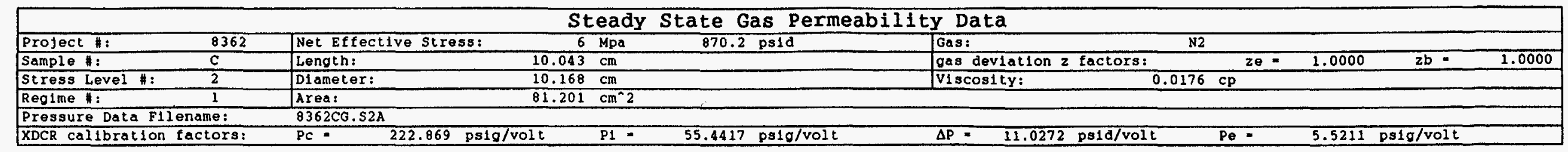

\begin{tabular}{|c|c|c|c|c|c|c|c|c|c|c|c|c|}
\hline Date & $\begin{array}{l}\text { Time } \\
\text { of } \\
\text { Day }\end{array}$ & $\begin{array}{l}\text { File } \\
\text { Time } \\
\text { (min) }\end{array}$ & Reglme & $\begin{array}{l}\mathrm{Pb} \\
\text { Barometric } \\
\text { Pressure }\end{array}$ & $\begin{array}{l}\text { Pc } \\
\text { Confining } \\
\text { Pressure }\end{array}$ & \begin{tabular}{|l} 
P1 \\
Inlet \\
Pressure
\end{tabular} & $\begin{array}{l}\Delta P \\
D 1 f \text { ferential } \\
\text { Pressure }\end{array}$ & $\begin{array}{l}\text { Pm } \\
\text { Mean Pore } \\
\text { Pressure } \\
\text { Pe }+\Delta P / 2\end{array}$ & $\begin{array}{l}\text { Pe } \\
\text { Exlt } \\
\text { Pressure }\end{array}$ & $\begin{array}{l}\text { Te } \\
\text { Flow } \\
\text { Temp } \\
\left({ }^{\circ} \mathrm{C}\right)\end{array}$ & $\begin{array}{c}\text { Tb } \\
\text { Amblent } \\
\text { Temp } \\
\left({ }^{\circ} \mathrm{C}\right)\end{array}$ & $\begin{array}{c}\text { Ob } \\
\text { Flow Rate } \\
\text { Pb\&Tb } \\
\text { (ml/sec) }\end{array}$ \\
\hline $29 \mathrm{Apr} 93$ & $16: 26$ & 255 & CS2R1a & $12.34 \mathrm{ps} 1 \mathrm{a}$ & volts & volts & 4.388 volts & & 0.897 & 23 & 23 & 0.02748 \\
\hline 29 Apr 93 & $16: 30$ & 260 & CS2R1b & $12.34 \mathrm{ps} 1 \mathrm{a}$ & volts & volts & 4.389 volts & & volts & 23 & 23 & 0.02747 \\
\hline $29 \mathrm{Apr} 93$ & $16: 34$ & 265 & $\operatorname{CS} 2 \mathrm{R} 1 \mathrm{C}$ & $12.34 \mathrm{ps} 1 \mathrm{a}$ & volts & volts & 4.389 volts & & volts & 23 & 23 & 0.02755 \\
\hline 29 Apr 93 & $16: 38$ & 265 & $\operatorname{cs} 2 \mathrm{R} 1 \mathrm{~d}$ & $12.34 \mathrm{ps} 1 \mathrm{a}$ & volts & volts & $4.389 \mathrm{voltg}$ & & volts & 23 & 23 & 0.02750 \\
\hline \multirow{9}{*}{ AVERAGES } & & & \multirow{9}{*}{$\operatorname{CS} 2 \mathrm{R} 1$} & & GUAGE & GUAGE & DIEGERENTLAI & OUXGE & GUABE & (ब) & (O) & \\
\hline & & & & & 4.036 volts & 0.956 volts & $4.389 \mathrm{volts}$ & & 0.897 & \multirow{4}{*}{23} & \multirow{4}{*}{23} & \\
\hline & & & & & $899.5 \quad$ psig & 53.00 & $48.40 \mathrm{ps} 1 \mathrm{~d}$ & $29.15 \mathrm{ps} 1 \mathrm{~g}$ & 4.950 & & & \\
\hline & & & & & 61.21 & 3.607 & 3.293 & $1.983 \mathrm{~atm}$ & 0.3368 & & & \\
\hline & & & & & 6.202 & 0.3654 & 0.3337 & $0.2010 \mathrm{Mpa}$ & 0.03413 & & & \\
\hline & & & & ABSOLUTE & ABSOLUTE & ABSOLUTE & DIFFERENTIAL & \multicolumn{2}{|l|}{ ABSOEUTE } & $(9 x)$ & (OK) & (nim/sac) \\
\hline & & & & $12.34 \mathrm{ps} 1 \mathrm{a}$ & 911.8 ps1a & $65.34 \quad$ psla & 48.40 psid & $41.49 \mathrm{psia}$ & 17.29 & \multirow{3}{*}{296} & \multirow{3}{*}{296} & \multirow{3}{*}{0.02750} \\
\hline & & & & $0.8397 \quad \mathrm{~atm}$ & 62.05 & 4.446 & 3.293 & 2.823 atm & 1.176 & & & \\
\hline & & & & 0.08508 Mpo & 6.287 & 0.4505 & 0.3337 & 0.2860 & 0.1192 & & & \\
\hline
\end{tabular}

$\underset{\omega}{\oplus}$

Apparent gas permeabll1ty:

Boyle's Law:

$K a *($ Ve*Pe*u*L) $/(P m * \Delta P)$

$\mathrm{Ve}=(\mathrm{Pb} / \mathrm{Pe}) *(\mathrm{Te} / \mathrm{Tb}) *(2 \mathrm{e} / \mathrm{zb}) * \mathrm{Vb}$

$Q c=(\mathrm{Pb} / \mathrm{Pe}) *(\mathrm{Te} / \mathrm{Tb}) *(z e / z b) * \mathrm{Qb}$

$v e=Q_{e} / \mathrm{A}=(\mathrm{Pb} / \mathrm{Pe}) *(\mathrm{Te} / \mathrm{Tb}) *(2 e / 2 \mathrm{~b}) *(\mathrm{Qb} / \mathrm{A})$

\begin{tabular}{|c|c|c|c|c|}
\hline Parameter & $\begin{array}{l}\text { Traditional } \\
\text { Units }\end{array}$ & & $\begin{array}{l}\text { SI } \\
\text { Un1ts }\end{array}$ & \\
\hline$\mu=$ gas viscosity & 0.0176 & $* 1.0 \mathrm{E}-3 \mathrm{~Pa} * \mathrm{sec} / \mathrm{Cp}$ & $1.760 \mathrm{E}-05$ & $\mathrm{~Pa} * \mathrm{sec}$ \\
\hline$L=$ sample length & 10.043 & $\star 1.0 \mathrm{E}-2 \mathrm{~m} / \mathrm{cm}$ & $1.004 \mathrm{E}-01$ & $m$ \\
\hline A sample clrcular cross sectional area & 81.201 & $1.0 \mathrm{E}-4 \mathrm{~m}^{\wedge} 2 / \mathrm{cm}^{\wedge} 2$ & $8.120 \mathrm{E}-03$ & $m^{n} 2$ \\
\hline $\mathrm{Pb}=$ flow measurement basis pressure (absolute) & 0.8397 & $1.013 \mathrm{E}+5 \mathrm{~Pa} / \mathrm{atm}$ & $8.506 \mathrm{E}+04$ & $\mathrm{~Pa}$ \\
\hline$\Delta \mathrm{P}=$ pressure drop across sample length & 3.293 & $1.013 E+5 \mathrm{~Pa} / \mathrm{atm}$ & $3.336 \mathrm{E}+05$ & $\overline{P_{a}}$ \\
\hline Pm - mean pore pressure (absolute) & 2.823 & $\star 1.013 \mathrm{E}+5 \mathrm{~Pa} / \mathrm{atm}$ & $2.860 \mathrm{E}+05$ & $\mathrm{~Pa}$ \\
\hline Pe - ex1t pressure (absolute) & 1.176 & $\star 1.013 \mathrm{E}+5 \mathrm{~Pa} / \mathrm{atm}$ & $1.192 \mathrm{E}+05$ & $\mathrm{~Pa}$ \\
\hline Te = sample temperature (absolute) & 296 & & 296 & ${ }^{\circ} \mathrm{K}$ \\
\hline Tb = flow measurement basis temperature (absolute) & 296 & & 296 & ${ }^{\circ} \mathrm{K}$ \\
\hline $2 e=$ gas devlation factor at $P e$ and $T e$ & 1.0000 & & 1.0000 & \\
\hline $2 b-$ gas deviation factor $a t \mathrm{pb}$ and $\mathrm{Te}$ & 1.0000 & & 1.0000 & \\
\hline $\mathrm{ab}$ - flow rate at base conditions & 0.02750 & $1.0 \mathrm{E}-6 \mathrm{~m}^{\wedge} 3 / \mathrm{cm}^{\wedge} 3$ & $2.750 \mathrm{E}-08$ & $m^{n} 3 / s$ \\
\hline ve - flow veloclty at sample exit end & $2.417 \mathrm{E}-04$ & $1.0 \mathrm{E}-2 \mathrm{~m} / \mathrm{cm}$ & $2.417 E-06$ & $\mathrm{~m} / \mathrm{s}$ \\
\hline $\mathrm{Ka}=$ & $5.818-06$ & $9.872 \mathrm{E}-13 \mathrm{~m}^{\wedge} 2 / \mathrm{d}$ & $5.34 \mathrm{E}-18$ & $m^{\wedge} 2$ \\
\hline $\mathrm{Ka}=$ & $5.41 \mathrm{E}-03$ & & $5.34 \mathrm{E}-14$ & $\mathrm{~cm}^{\wedge} 2$ \\
\hline $\mathrm{ka}=$ & $5.41 \mathrm{E}+00$ & & & \\
\hline
\end{tabular}


Steady State Gas Permeability Data

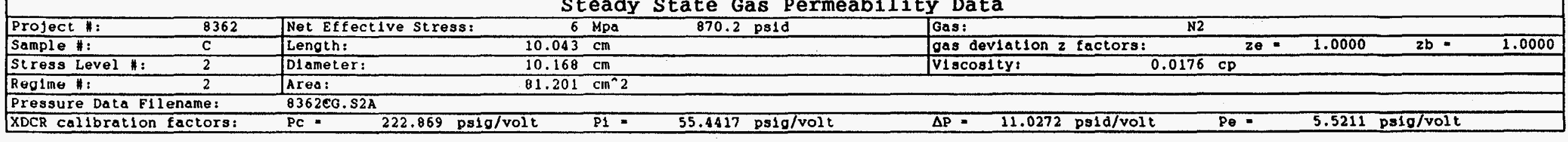

\begin{tabular}{|c|c|c|c|c|c|c|c|c|c|c|c|c|}
\hline Date & $\begin{array}{l}\text { Time } \\
\text { of } \\
\text { Day }\end{array}$ & $\begin{array}{l}\text { File } \\
\text { Time } \\
(m i n)\end{array}$ & $\underset{*}{\text { Reglme }}$ & \begin{tabular}{|l} 
Pb \\
Barometrlc \\
Pressure
\end{tabular} & $\begin{array}{l}\text { Pc } \\
\text { Confining } \\
\text { Pressure }\end{array}$ & $\begin{array}{l}\text { P1 } \\
\text { Inlet } \\
\text { Pressure }\end{array}$ & $\begin{array}{l}\Delta P \\
D 1 \text { fferential } \\
\text { Pressure }\end{array}$ & $\begin{array}{l}\text { Pm } \\
\text { Mean Pore } \\
\text { Pressure } \\
\text { Pe }+\Delta \mathrm{P} / 2\end{array}$ & \begin{tabular}{|l} 
Pe \\
Ex1t \\
Pressure
\end{tabular} & $\begin{array}{l}\text { Te } \\
\text { Flow } \\
\text { Temp } \\
\left({ }^{\circ} \mathrm{C}\right)\end{array}$ & $\begin{array}{c}\text { Tb } \\
\text { Amblent } \\
\text { Temp } \\
\left({ }^{\circ} \mathrm{C}\right)\end{array}$ & $\begin{array}{c}Q b \\
\text { Flow Rate } \\
\text { OPb\&Tb } \\
\text { (ml/sec) }\end{array}$ \\
\hline 30 Apr 93 & $12: 42$ & 1470 & $\operatorname{CS} 2 \mathrm{R} 2 \mathrm{a}$ & $12.33 \mathrm{ps} 1 \mathrm{a}$ & volts & volts & 4.367 volts & & volts & 23 & 22 & 0.03043 \\
\hline $30 \mathrm{Apr} 93$ & $12: 45$ & 1475 & CS2R2b & $12.33 \mathrm{ps} 1 \mathrm{a}$ & 4.080 volts & 1.134 volts & 4.366 vol ts & & volts & 23 & 22 & 0.03040 \\
\hline 30 Apr 93 & $12: 48$ & 1475 & CS2R2C & 12.33 psia & volts & volts & 4.365 volts & & volts & 23 & 22 & 0.03040 \\
\hline 30 Apr 93 & $12: 52$ & 1480 & CS2R2d & $12.33 \mathrm{ps} 1 \mathrm{a}$ & 4.080 volts & 1.134 volts & 4.365 volts & & volts & 23 & 22 & 0.03046 \\
\hline \multirow{9}{*}{ AVERAGES } & & & \multirow{9}{*}{$\operatorname{Cs} 2 R 2$} & & BOUAOE & OOKOE & DYGFEREMTIX & ONONGE & Gona & 60 & क्ष & \\
\hline & & & & & 4.080 volts & 1.134 volts & 4.366 volts & & 2.737 & \multirow{4}{*}{23} & \multirow{4}{*}{22} & \\
\hline & & & & & 909.3 & 62.86 & 48.14 psid & $39.18 \mathrm{psig}$ & 15.110 & & & \\
\hline & & & & & 61.87 & 4.277 & 3.276 & $2.666 \mathrm{~atm}$ & 1.0282 & & & \\
\hline & & & & & $6.269 \quad \mathrm{Mpa}$ & 0.4334 & $0.3319 \quad \mathrm{Mpa}$ & $0.2701 \mathrm{Mpa}$ & 0.10418 & & & \\
\hline & & & & ABSOLUTE & ABSOLUTE & ABSOLUTE & DIFFERENT XL & AESOLUTE & ABSOLUTE & \% $)$ & Pok! & (mifsec) \\
\hline & & & & 12.33 psia & 921.6 ps1a & 75.19 psia & 48.14 psid & $51.51 \mathrm{ps} 1 \mathrm{a}$ & 27.44 ps1a & \multirow{3}{*}{296} & \multirow{3}{*}{295} & \multirow{3}{*}{0.03042} \\
\hline & & & & $0.8390 \mathrm{~atm}$ & 62.71 & 5.116 & 3.276 & 3.505 atm & 1.867 & & & \\
\hline & & & & $0.08501 \mathrm{Mpa}$ & 6.354 & 0.5184 & 0.3319 & 0.3552 & 0.1892 & & & \\
\hline
\end{tabular}

Apparent gas permeability:

Boyle's Law:
$K a=\left(v e * P e^{\star} u * L\right) /\left(P_{m} * \Delta P\right)$

$v_{e}=(\mathrm{Pb} / \mathrm{Pe}) *(\mathrm{Te} / \mathrm{Tb}) *(2 e / z b) * \mathrm{Vb}$

$\mathrm{Qe}^{-}(\mathrm{Pb} / \mathrm{Pe}) *(\mathrm{Te} / \mathrm{Tb}) \cdot(\mathrm{ze} / \mathrm{Zb}) \cdot \mathrm{Qb}$

ve $=\mathrm{Qe} / \mathrm{A}=(\mathrm{Pb} / \mathrm{Pe}) \cdot(\mathrm{Te} / \mathrm{Tb}) *(\mathrm{ze} / 2 \mathrm{~b}) *(\mathrm{Qb} / \mathrm{A})$

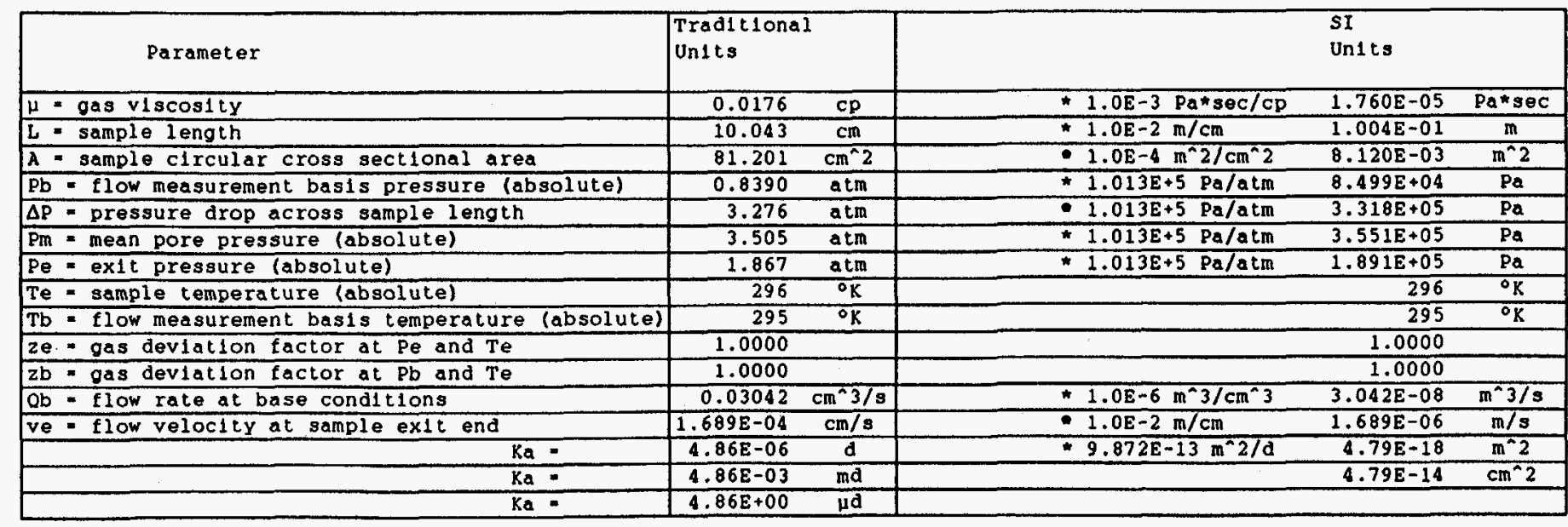




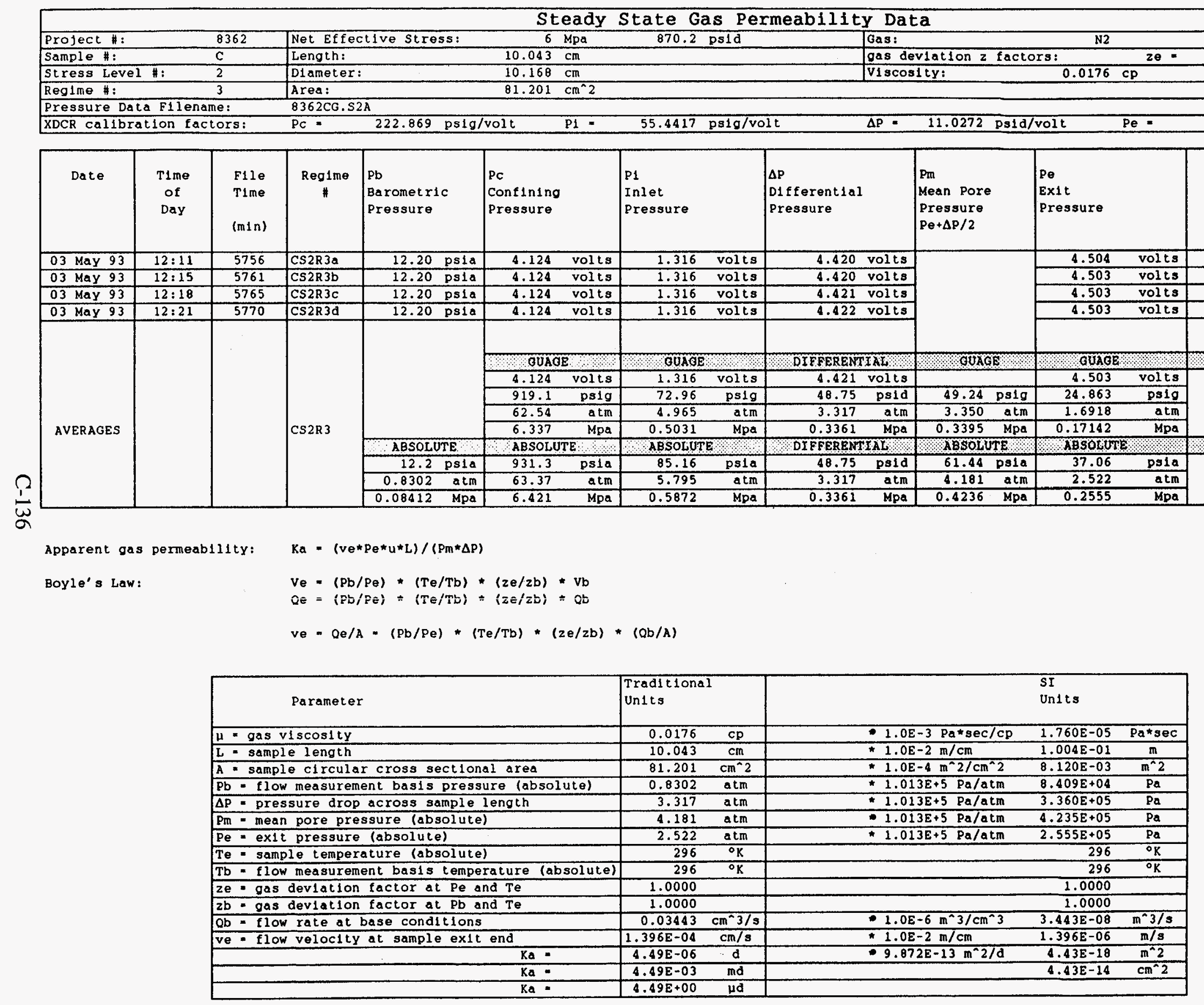


Steady State Gas Permeability Data

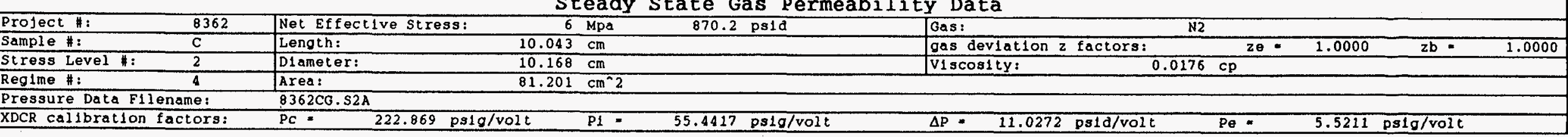

\begin{tabular}{|c|c|c|c|c|c|c|c|c|c|c|c|c|}
\hline Date & $\begin{array}{l}\text { T1me } \\
\text { of } \\
\text { Day }\end{array}$ & $\begin{array}{l}\text { File } \\
\text { Time } \\
\text { (min) }\end{array}$ & Reg1me & $\begin{array}{l}\text { Pb } \\
\text { Barometric } \\
\text { Pressure }\end{array}$ & $\begin{array}{l}\text { Pc } \\
\text { Confining } \\
\text { Pressure }\end{array}$ & $\begin{array}{l}\text { P1 } \\
\text { Inlet } \\
\text { Pressure }\end{array}$ & $\begin{array}{l}\Delta \mathrm{P} \\
\text { D1fferent1al } \\
\text { Pressure }\end{array}$ & $\begin{array}{l}\text { Pm } \\
\text { Mean Pore } \\
\text { Pressure } \\
\mathrm{Pe}+\Delta \mathrm{P} / 2\end{array}$ & $\begin{array}{l}\text { Pe } \\
\text { Ex1t } \\
\text { Pressure }\end{array}$ & $\begin{array}{l}\text { Te } \\
\text { Flow } \\
\text { Temp } \\
\left({ }^{\circ} \mathrm{C}\right)\end{array}$ & $\begin{array}{c}\text { Tb } \\
\text { Ambient } \\
\text { Temp } \\
\left({ }^{\circ} \mathrm{C}\right)\end{array}$ & $\begin{array}{c}\text { Qb } \\
\text { Flow Rate } \\
\text { Pb\&Tb } \\
\text { (ml/sec) }\end{array}$ \\
\hline 03 May 93 & $16: 29$ & 6015 & CS2R4a & $12.12 \mathrm{ps} 1 \mathrm{a}$ & 4.169 & volts & 4.431 volts & & volts & $\overline{23}$ & $\overline{23}$ & 0.03828 \\
\hline 03 May 93 & $16: 32$ & 6020 & CS2R4b & 12.12 ps1a & volts & volts & 4.432 volts & & volts & 23 & 23 & 0.03846 \\
\hline 03 May 93 & $16: 35$ & 6025 & $\operatorname{CS} 2 \mathrm{R} 4 \mathrm{C}$ & $12.12 \mathrm{ps12}$ & volts & volts & 4.432 volts & & volts & 23 & 23 & 0.03843 \\
\hline $03 \mathrm{MaY} 93$ & $16: 41$ & 6030 & $\operatorname{Cs} 2 R 4 d$ & 12.12 ps1a & volts & volts & 4.433 volts & & volts & 23 & 23 & 0.03827 \\
\hline $03 \mathrm{MaY} 93$ & $16: 44$ & 6035 & Cs2R4e & $12.12 \mathrm{psia}$ & volts & volts & 4.434 volts & & volts & 23 & 23 & 0.03842 \\
\hline \multirow{9}{*}{ AVERAGES } & & & \multirow{9}{*}{ CS2R4 } & & OUAAE & OUNGE & DHAFEREATIAL & DOUAGE: & GUAOE & क्र & (ए) & \\
\hline & & & & & 4.169 volts & 1.498 volts & 4.432 volts & & 6.337 & \multirow{4}{*}{23} & \multirow{4}{*}{23} & \\
\hline & & & & & 929.1 & 83.05 & $48.88 \quad \mathrm{ps} 1 \mathrm{~d}$ & $59.43 \mathrm{ps} 1 \mathrm{~g}$ & 34.989 & & & \\
\hline & & & & & 63.22 & 5.651 & 3.326 & $4.044 \mathrm{~atm}$ & 2.3809 & & & \\
\hline & & & & & 6.406 & 0.5726 & $0.3370 \mathrm{Mpa}$ & $0.4097 \mathrm{Mpa}$ & 0.24124 & & & \\
\hline & & & & ABSOLUTE & ABSOLUTE & ABSOLUTE & DYFFERENTIAL & ABSOLUTE & ABSOLOTE & (ox) & $(0 k)$ & $(\mathrm{ml} / \mathrm{sec})$ \\
\hline & & & & 12.12 psia & $941.3 \quad$ ps1a & $95.17 \quad \mathrm{ps} 1 \mathrm{a}$ & $48.88 \quad p s 1 d$ & $71.55 \mathrm{ps} 1 \mathrm{a}$ & 47.11 psia & \multirow{3}{*}{296} & \multirow{3}{*}{296} & \multirow{3}{*}{0.03836} \\
\hline & & & & 0.8247 atm & 64.05 & 6.476 & 3.326 & $4.869 \mathrm{~atm}$ & 3.206 & & & \\
\hline & & & & $0.08356 \mathrm{Mpa}$ & 6.490 & 0.6562 & 0.3370 & 0.4933 & 0.3248 & & & \\
\hline
\end{tabular}

Apparent gas permeab111ty:

Boyle's Law:

$K a *\left(v e * P e^{\star} u * L\right) /(P m * \Delta P)$

$\mathrm{Ve}=(\mathrm{Pb} / \mathrm{Pe}) *(\mathrm{Te} / \mathrm{Tb}) \cdot(\mathrm{ze} / \mathrm{zb}) * \mathrm{Vb}$

$\mathrm{Qe}=(\mathrm{Pb} / \mathrm{Pe}) *(\mathrm{Te} / \mathrm{Tb}) *(z e / 2 \mathrm{~b}) * \mathrm{Qb}$

$v e=Q_{e} / \mathrm{A} *\left(\mathrm{~Pb} / \mathrm{Pe}_{\theta}\right) *(\mathrm{Te} / \mathrm{Tb}) *(\mathrm{ze} / \mathrm{Zb}) *\left(\mathrm{Qb}^{\mathrm{A}} / \mathrm{A}\right)$

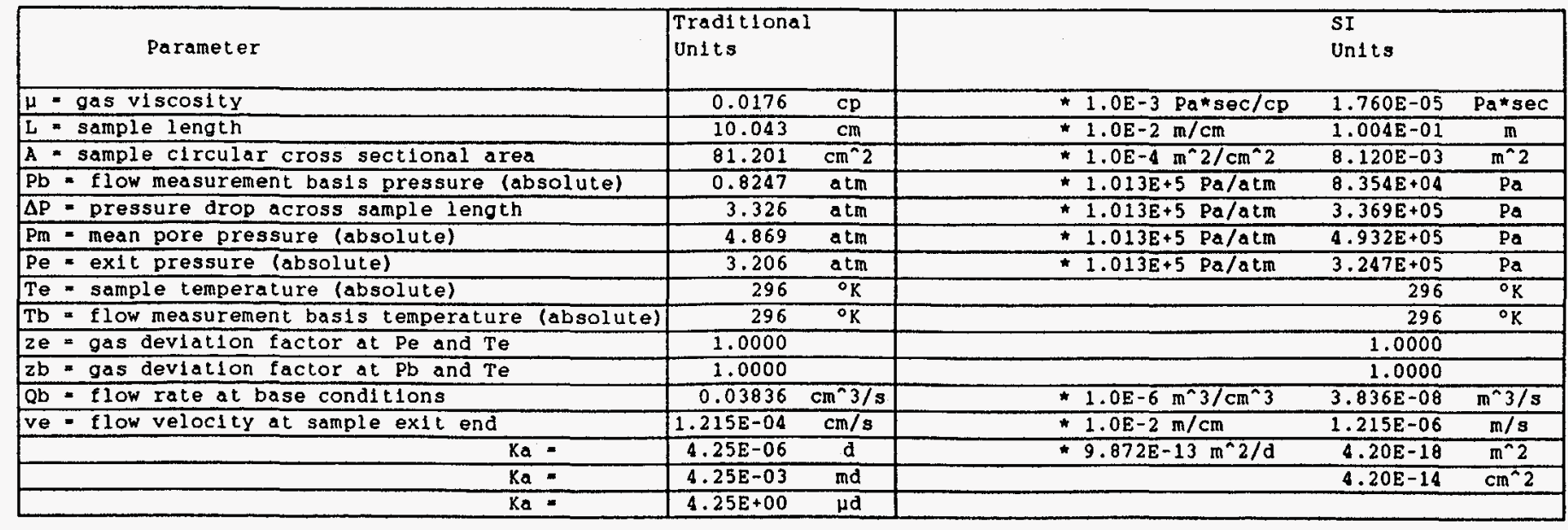


Steady State Gas Permeability Data

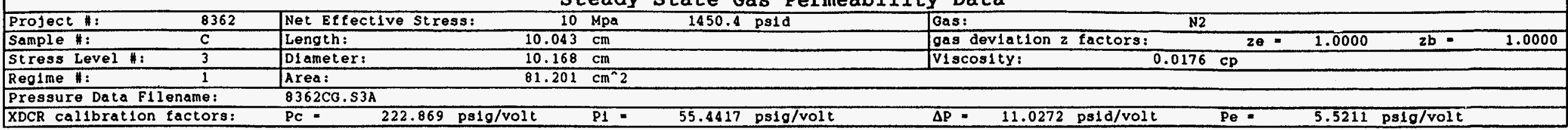

\begin{tabular}{|c|c|c|c|c|c|c|c|c|c|c|c|c|}
\hline Date & $\begin{array}{l}\text { Time } \\
\text { of } \\
\text { Day }\end{array}$ & $\begin{array}{l}\text { File } \\
\text { Time } \\
\text { (min) }\end{array}$ & Reglme & $\begin{array}{l}\text { Pb } \\
\text { Barometrlc } \\
\text { Pressure }\end{array}$ & $\begin{array}{l}\text { Pc } \\
\text { Confining } \\
\text { Pressure }\end{array}$ & $\begin{array}{l}\text { P1 } \\
\text { Inlet } \\
\text { Pressure }\end{array}$ & $\begin{array}{l}\Delta P \\
D 1 \text { ifferent1al } \\
\text { Pressure }\end{array}$ & $\begin{array}{l}\text { Pm } \\
\text { Mean pore } \\
\text { Pressure } \\
\text { Pe+ }+\Delta \mathrm{P} / 2\end{array}$ & \begin{tabular}{|l} 
Pe \\
Ex1t \\
Pressure
\end{tabular} & $\begin{array}{l}\text { Te } \\
\text { Flow } \\
\text { Temp } \\
\left({ }^{\circ} \mathrm{C}\right)\end{array}$ & $\begin{array}{c}\text { Tb } \\
\text { Amblent } \\
\text { Temp } \\
\left({ }^{\circ} \mathrm{C}\right)\end{array}$ & $\begin{array}{c}\text { Qb } \\
\text { Flow Rate } \\
\text { OPbstb } \\
\text { (ml/sec) }\end{array}$ \\
\hline 04 May 93 & $17: 19$ & 350 & CS3R1a & $12.20 \mathrm{psia}$ & volts & volts & 4.420 volts & & volts & 23 & 22 & 0.02110 \\
\hline 04 May 93 & $17: 25$ & 355 & CS3R1b & 12.20 psla & volts & volts & 4.420 volts & & volts & 23 & 22 & 0.02108 \\
\hline 04 May 93 & $17: 29$ & 355 & $\operatorname{CS3R} 1 \mathrm{C}$ & 12.20 psia & volts & volts & 4.420 volts & & 0.875 & 23 & 22 & 0.02104 \\
\hline 04 May 93 & $17: 34$ & 360 & CS3R1d & 12.20 psia & volts & volts & 4.420 volts & & 0.875 & 23 & 22 & 0.02105 \\
\hline \multirow{9}{*}{ AVERAGES } & & & \multirow{9}{*}{$\operatorname{Cs} 3 R 1$} & & ROUAGE & OUAOE & DHEFEREMYXY & OOXOE & 3unoe & क्ला & 801 & \\
\hline & & & & & 6.638 volts & 0.957 volts & 4.420 volts & & 0.875 & \multirow{4}{*}{23} & \multirow{4}{*}{22} & \\
\hline & & & & & 1479.4 & 53.06 & 48.74 psid & $29.20 \mathrm{ps} 1 \mathrm{~g}$ & 4.832 & & & \\
\hline & & & & & 100.67 & 3.610 & 3.317 & $1.987 \mathrm{~atm}$ & 0.3288 & & & \\
\hline & & & & & $10.200 \quad \mathrm{Mpa}$ & 0.3658 & $0.3361 \quad \mathrm{Mpa}$ & $0.2013 \mathrm{Mpa}$ & 0.03332 & & & \\
\hline & & & & ABSOLUTE & ABSOLUTE & ABSOLUTE & DEFGERENTIAL & ABSOLUTE & XBSOLOTE: & बK & $6 \mathrm{k} /$ & $(S \mathrm{~S} / \mathrm{SOC})$ \\
\hline & & & & $12.2 \mathrm{psla}$ & 1491.6 ps1a & 65.26 ps1a & 48.74 ps1d & $41.40 \mathrm{ps} 1 \mathrm{a}$ & 17.03 & \multirow{3}{*}{296} & \multirow{3}{*}{295} & \multirow{3}{*}{0.02107} \\
\hline & & & & $0.8302 \mathrm{~atm}$ & 101.50 & 4.441 & 3.317 & $2.817 \mathrm{~atm}$ & 1.159 & & & \\
\hline & & & & $0.08412 \mathrm{Mpa}$ & 10.284 & 0.4499 & 0.3361 & 0.2855 & 0.1174 & & & \\
\hline
\end{tabular}

$\ddot{\omega}$

Apparent gas permeab111ty:

Boyle's Law:

$K a=\left(v e e^{\star P e} e^{\star} u L\right) /(P m \star \Delta P)$

$v_{e}=(P b / P e) *(T e / T b) *(z e / z b) * v b$

$Q E=\{P S / P E) *(T \in / T b) *\{z \in / z b) * Q b$

ve $=Q e / A=(\mathrm{Pb} / \mathrm{Pe}) *(\mathrm{Te} / \mathrm{Tb}) *(\mathrm{ze} / \mathrm{zb}) *(\mathrm{Qb} / \mathrm{A})$

\begin{tabular}{|c|c|c|c|c|}
\hline Parameter & $\begin{array}{l}\text { Trad1tional } \\
\text { Units }\end{array}$ & & $\begin{array}{l}\text { SI } \\
\text { Units }\end{array}$ & \\
\hline$\mu=$ gas viscosity & 0.0176 & $-1.0 \mathrm{E}-3 \mathrm{~Pa} \times \mathrm{sec} / \mathrm{cp}$ & $1.760 \mathrm{E}-05$ & Pa*sec \\
\hline L = sample length & 10.043 & $\star 1.0 \mathrm{E}-2 \mathrm{~m} / \mathrm{cm}$ & $1.004 \mathrm{E}-01$ & $m$ \\
\hline A = sample clrcular cross sectlonal area & 81.201 & $1.0 \mathrm{E}-4 \mathrm{~m}^{\wedge} 2 / \mathrm{cm}^{\wedge} 2$ & $8.120 \mathrm{E}-03$ & $m^{\star 2} 2$ \\
\hline $\mathrm{Pb}=$ flow measurement basls pressure (absolute) & 0.8302 & $\$ 1.013 \mathrm{E}+5 \mathrm{~Pa} / \mathrm{atm}$ & $8.409 E+04$ & $\mathrm{~Pa}$ \\
\hline$\Delta \mathrm{p}=$ pressure drop across sample length & 3.317 & $\star 1.013 \mathrm{E}+5 \mathrm{~Pa} / \mathrm{atm}$ & $3.360 E+05$ & Pa \\
\hline Pm - mean pore pressure (absolute) & 2.817 & $\star 1.013 \mathrm{E}+5 \mathrm{~Pa} / \mathrm{atm}$ & $2.854 E+05$ & $\mathrm{~Pa}$ \\
\hline $\mathrm{Pe}=$ exit pressure (absolute) & 1.159 & $1.013 \mathrm{E}+5 \mathrm{~Pa} / \mathrm{atm}$ & $1.174 \mathrm{E}+05$ & Pa \\
\hline Te = sample temperature (absolute) & 296 & & 296 & ${ }^{\circ} \mathrm{K}$ \\
\hline $\mathrm{Tb}=$ flow measurement bas1s temperature (absolute) & 295 & & 295 & ${ }^{\circ} \mathrm{K}$ \\
\hline$z e=$ gas deviation factor at $\mathrm{Pe}$ and $\mathrm{Te}$ & 1.0000 & & 1.0000 & \\
\hline $\mathrm{zb}=$ gas deviation factor $\mathrm{at} \mathrm{Pb}$ and $\mathrm{Te}$ & 1.0000 & & 1.0000 & \\
\hline$Q b=$ flow rate at base conditlons & 0.02107 & $1.0 \mathrm{E}-6 \mathrm{~m}^{\wedge} 3 / \mathrm{cm}^{\wedge} 3$ & $2.107 E-08$ & $m^{\wedge} 3 / s$ \\
\hline ve - flow velocity at sample exit end & $1.865 \mathrm{E}-04$ & $1.0 \mathrm{E}-2 \mathrm{~m} / \mathrm{cm}$ & $1.865 \mathrm{E}-06$ & $\mathrm{~m} / \mathrm{s}$ \\
\hline $\mathrm{Ka}=$ & $4.09 \mathrm{E}-06$ & $9.872 \mathrm{E}-13 \mathrm{~m}^{\wedge} 2 / \mathrm{d}$ & $4.04 E-18$ & $m^{\star 2} 2$ \\
\hline $\mathrm{Ka}=$ & $4.09 E-03$ & & $4.04 \mathrm{E}-14$ & $\mathrm{~cm}^{\wedge} 2$ \\
\hline $\mathrm{Ka}=$ & $4.09 \mathrm{E}+00$ & & & \\
\hline
\end{tabular}


Steady State Gas Permeability Data

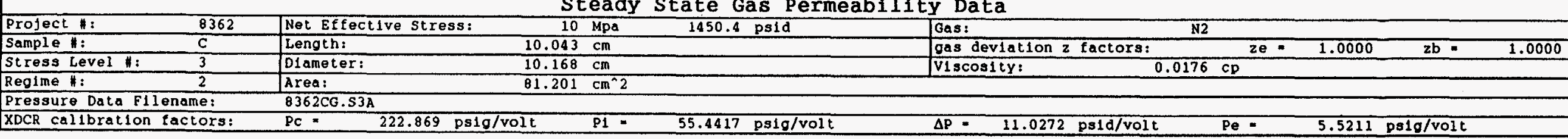

\begin{tabular}{|c|c|c|c|c|c|c|c|c|c|c|c|c|}
\hline Date & $\begin{array}{l}\text { Time } \\
\text { of } \\
\text { Day }\end{array}$ & $\begin{array}{l}\text { File } \\
\text { Time } \\
\text { (min) }\end{array}$ & Regime & \begin{tabular}{|l}
$\mathrm{Pb}$ \\
Barometrlc \\
Pressure
\end{tabular} & $\begin{array}{l}\text { Pc } \\
\text { Confining } \\
\text { Pressure }\end{array}$ & $\begin{array}{l}\text { P1 } \\
\text { Inlet } \\
\text { Pressure }\end{array}$ & $\begin{array}{l}\Delta \mathrm{P} \\
\text { D1f ferential } \\
\text { Pressure }\end{array}$ & $\begin{array}{l}\text { Pm } \\
\text { Mean Pore } \\
\text { Pressure } \\
\text { Pe }+\Delta P / 2\end{array}$ & $\begin{array}{l}\text { Pe } \\
\text { Exit } \\
\text { Pressure }\end{array}$ & $\begin{array}{l}\text { Te } \\
\text { Flow } \\
\text { Temp } \\
\left({ }^{\circ} \mathrm{C}\right)\end{array}$ & $\begin{array}{c}\text { Tb } \\
\text { Amb 1ent } \\
\text { Temp } \\
\left({ }^{\circ} \mathrm{C}\right)\end{array}$ & $\begin{array}{c}\text { Qb } \\
\text { Flow Rate } \\
\text { OPbeTb } \\
\text { (ml/sec) }\end{array}$ \\
\hline 05 May 93 & $13: 04$ & 1535 & CS3R2a & $12.28 \mathrm{ps} 1 \mathrm{a}$ & 6.683 & volts & 4.434 volts & & volts & 23 & 21 & 0.02341 \\
\hline 05 May 93 & $13: 10$ & 1540 & $\operatorname{Cs} 3 R 2 b$ & $12.28 \quad \mathrm{ps} 1 \mathrm{a}$ & 6.683 & volts & 4.434 volts & & volts & 23 & 21 & 0.02325 \\
\hline 05 May 93 & $13: 15$ & 1545 & $\operatorname{cs} 3 R 2 c$ & $12.28 \mathrm{ps} 1 \mathrm{a}$ & 6.683 & volts & 4.434 volts & & volts & 23 & 21 & 0.02329 \\
\hline 05 May 93 & $13: 20$ & 1550 & $\operatorname{cs3} 322$ & $12.28 \mathrm{ps} 1 \mathrm{a}$ & 6.683 & volts & 4.434 volts & & volts & 23 & $\frac{21}{21}$ & 0.02340 \\
\hline 05 May 93 & $13: 25$ & 1555 & $\operatorname{cs3R2e}$ & 12.28 psia & 6.683 & volts & 4.434 vo1ts & & volts & 23 & 21 & 0.02330 \\
\hline \multirow{9}{*}{ AVERAGES } & & & \multirow{9}{*}{$\operatorname{Cs} 3 \mathrm{R} 2$} & & OUACE & OUAOE: & DYFEEREMTIAL & GUKGE & Gunge: & $\mathrm{COC}$ & rogr & \\
\hline & & & & & 6.683 volts & 1.138 volts & $\frac{4.434 \text { volts }}{4.45}$ & & $\frac{2000}{2.660}$ & \multirow{4}{*}{23} & \multirow{4}{*}{21} & \\
\hline & & & & & 1489.4 & 63.09 & 48.89 ps1d & $39.13 \mathrm{psig}$ & 14.686 & & & \\
\hline & & & & & 101.35 & 4.293 & 3.327 & $2.663 \mathrm{~atm}$ & 0.9993 & & & \\
\hline & & & & & $10.269 \quad \mathrm{Mpa}$ & 0.4350 & $0.3371 \mathrm{Mpa}$ & $0.2698 \mathrm{Mpa}$ & 0.10126 & & & \\
\hline & & & & ABSOLUTE & ABSOLUTE & ABSOLUTE & DHF TEREMTI I & Xesorut: & ABSOLUTE & (1) & \%र & $(\operatorname{mi} 1 / 66 \mathrm{c})$ \\
\hline & & & & 12.28 psia & 1501.7 psia & 75.37 gs1a & $\frac{48.89 \mathrm{ps} 1 \mathrm{~d}}{4}$ & $51.41 \mathrm{ps} 1 \mathrm{a}$ & 26.97 & \multirow{3}{*}{296} & \multirow{3}{*}{294} & \multirow{3}{*}{0.02334} \\
\hline & & & & 0.8356 atm & 102.19 & 5.129 & 3.327 & 3.498 atm & 1.835 & & & \\
\hline & & & & $0.08467 \mathrm{Mpa}$ & 10.354 & 0.5197 & 0.3371 & 0.3545 & 0.1859 & & & \\
\hline
\end{tabular}

Apparent gas permeab1lity:

$K a *\left(v e \star P e^{\star} u * L\right) /(P m \star \Delta P)$

Boyle's Law:

$\mathrm{Ve}=(\mathrm{Pb} / \mathrm{Pe}) \cdot(\mathrm{Te} / \mathrm{Tb}) *(\mathrm{ze} / \mathrm{zb}) \bullet \mathrm{Vb}$

$\mathrm{Qe}=(\mathrm{Pb} / \mathrm{Pe}) *(\mathrm{Te} / \mathrm{Tb}) *(\mathrm{ze} / \mathrm{zb}) * \mathrm{Qb}$

ve $* \mathrm{Qe}_{\mathrm{A}} / \mathrm{A}=(\mathrm{Pb} / \mathrm{Pe}) *(\mathrm{Te} / \mathrm{Tb}) *\left(\mathrm{ze}_{\mathrm{C}} / \mathrm{zb}\right) *(\mathrm{Qb} / \mathrm{A})$

\begin{tabular}{|c|c|c|c|c|}
\hline Parameter & $\begin{array}{l}\text { Traditional } \\
\text { Units }\end{array}$ & & $\begin{array}{l}\text { SI } \\
\text { Un1ts }\end{array}$ & \\
\hline$\mu=g a s v 1 s \cos 1 t y$ & 0.0176 & $\star 1.0 \mathrm{E}-3 \mathrm{~Pa} * \mathrm{Sec} / \mathrm{CP}$ & $1.760 \mathrm{E}-05$ & Pa*sec \\
\hline $\mathrm{L}=$ sample length & 10.043 & $\$ 1.0 \mathrm{E}-2 \mathrm{~m} / \mathrm{cm}$ & $1.004 E-01$ & $\mathrm{~m}$ \\
\hline$A=$ sample clrcular cross sectional area & 81.201 & * $1.0 \mathrm{E}-4 \mathrm{~m}^{\wedge} 2 / \mathrm{cm}^{\wedge} 2$ & $8.120 \mathrm{E}-03$ & $m^{\wedge} 2$ \\
\hline $\mathrm{Pb}=$ flow measurement basis pressure (absolute) & 0.8356 & * $1.013 \mathrm{E}+5 \mathrm{~Pa} / \mathrm{atm}$ & $8.465 E+04$ & $\mathrm{~Pa}$ \\
\hline$\Delta \mathrm{P}=$ pressure drop across sample length & 3.327 & $\star 1.013 \mathrm{E}+5 \mathrm{~Pa} / \mathrm{atm}$ & $3.370 E+05$ & $\mathbf{P a}$ \\
\hline $\mathrm{Pm}$ = mean pore pressure (absolute) & 3.498 & $\star 1.013 \mathrm{E}+5 \mathrm{~Pa} / \mathrm{atm}$ & $3.544 \mathrm{E}+05$ & $\mathbf{P a}$ \\
\hline$P_{e}=$ exit pressure (absolute) & 1.835 & $1.013 \mathrm{E}+5 \mathrm{~Pa} / \mathrm{atm}$ & $1.859 \mathrm{E}+05$ & Pa \\
\hline $\mathrm{Te}=$ sample temperature (absolute) & 296 & & 296 & ${ }^{\circ} \mathbf{K}$ \\
\hline $\mathrm{Tb}=$ flow measurement basis temperature (absolute) & 294 & & 294 & ${ }^{\circ} \mathbf{K}$ \\
\hline$z e=$ gas deviation factor at $P e$ and $T_{e}$ & 1.0000 & & 1.0000 & \\
\hline $2 \mathrm{~b}=$ gas deviation factor at $\mathrm{pb}$ and $\mathrm{Te}$ & 1.0000 & & 1.0000 & \\
\hline $\mathrm{Qb}=$ flow rate at base conditions & $0.02334 \mathrm{~cm}^{\wedge} 3 / \mathrm{s}$ & $1.0 \mathrm{E}-6 \mathrm{~m}^{\wedge} 3 / \mathrm{cm}^{\wedge} 3$ & $2.334 E-08$ & $m^{*} 3 / s$ \\
\hline ve = flow veloclty at sample exit end & $1.318 \mathrm{E}-04 \mathrm{~cm} / \mathrm{s}$ & $1.0 \mathrm{E}-2 \mathrm{~m} / \mathrm{cm}$ & $1.318 \mathrm{E}-06$ & $\mathrm{~m} / \mathrm{s}$ \\
\hline $\mathrm{Ka}_{\mathrm{a}}=$ & $3.67 \mathrm{E}-06$ & $9.872 \mathrm{E}-13 \mathrm{~m}^{\wedge} 2 / \mathrm{d}$ & $3.62 \mathrm{E}-18$ & $m^{-2}$ \\
\hline $\mathrm{Ka}=$ & $3.67 \mathrm{E}-03$ & & $3.62 \mathrm{E}-14$ & $\mathrm{~cm}^{2} 2$ \\
\hline $\mathrm{Ka}=$ & $3.67 \mathrm{E}+00$ & & & \\
\hline
\end{tabular}




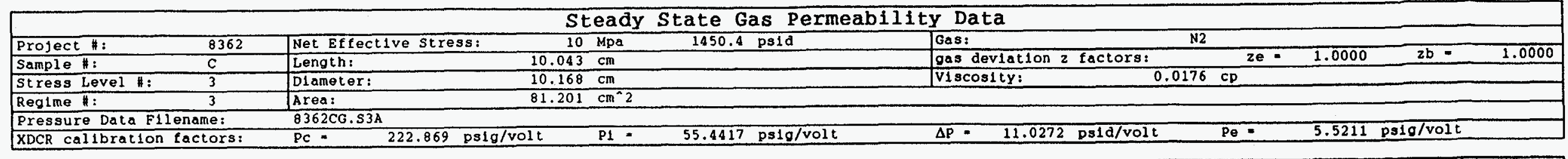

\begin{tabular}{|c|c|c|c|c|c|c|c|c|c|c|c|c|}
\hline Date & $\begin{array}{l}\text { Time } \\
\text { of } \\
\text { Day }\end{array}$ & $\begin{array}{l}\text { File } \\
\text { Time } \\
(m 1 n)\end{array}$ & $\underset{\#}{\text { Reglme }}$ & $\begin{array}{l}\mathrm{Pb} \\
\text { Barometrlc } \\
\text { Pressure }\end{array}$ & $\begin{array}{l}\text { Pc } \\
\text { Confining } \\
\text { Pressure }\end{array}$ & $\begin{array}{l}\text { p1 } \\
\text { Inlet } \\
\text { Pressure }\end{array}$ & $\begin{array}{l}\Delta \mathrm{P} \\
\text { D1fferential } \\
\text { Pressure }\end{array}$ & $\begin{array}{l}\mathrm{Pm} \\
\text { Mean Pore } \\
\text { Pressure } \\
\mathrm{Po}+\Delta \mathrm{P} / 2\end{array}$ & $\begin{array}{l}\text { Pe } \\
\text { Exit } \\
\text { Pressure }\end{array}$ & $\begin{array}{l}\text { Te } \\
\text { Flow } \\
\text { Temp } \\
\left({ }^{\circ} \mathrm{C}\right)\end{array}$ & $\begin{array}{c}\text { Tb } \\
\text { Amb1ent } \\
\text { Temp } \\
\left({ }^{\circ} \mathrm{C}\right)\end{array}$ & $\begin{array}{c}\text { Qb } \\
\text { Flow Rate } \\
\text { Pb\&Tb } \\
(\mathrm{ml} / \mathrm{sec})\end{array}$ \\
\hline 05 May 93 & $16: 56$ & 1765 & $\operatorname{cs} 3 R 3 a$ & $12.29 \mathrm{ps} 1 \mathrm{a}$ & volts & volts & 4.390 volts & & volts & 23 & 22 & 0.02584 \\
\hline 05 May 93 & $17: 00$ & 1770 & $C S 3 R 3 b$ & 12.29 ps1a & volts & volts & 4.390 volts & & volts & 23 & 22 & 0.02579 \\
\hline 05 May 93 & $17: 04$ & 1770 & $\operatorname{CS} 3 \mathrm{R} 3 \mathrm{C}$ & $12.29 \mathrm{ps} 1 \mathrm{a}$ & volts & volts & 4.390 volts & & volts & 23 & 22 & 0.02574 \\
\hline 05 May 93 & $17: 04$ & 1775 & $\operatorname{cs} 3 \mathrm{R} 3 \mathrm{~d}$ & 12.29 ps1a & volts & volts & 4.390 volts & & volts & 23 & 22 & 0.02571 \\
\hline 05 May 93 & $17: 13$ & 1780 & $\operatorname{cs} 3 \mathrm{R} 3 \mathrm{e}$ & 12.29 pg1a & $6.728 \mathrm{volts}$ & volts & 4.390 volts & & volts & 23 & 22 & 0.02579 \\
\hline \multirow{9}{*}{ AVERAGES } & & & \multirow{9}{*}{$\operatorname{cs} 3 R^{2} 3$} & & OUAGE & OUAGE & DIFEERENTIAL & OONGE & OUAGE: & 601 & $\mathrm{OCO}$ & \\
\hline & & & & & 6.728 volts & 1.316 volts & 4.390 volts & & 4.550 & \multirow{4}{*}{23} & \multirow{4}{*}{22} & \\
\hline & & & & & 1499.5 & 72.96 & 48.41 ps1d & $49.32 \mathrm{pslg}$ & 25.120 & & & \\
\hline & & & & & 102.03 & 4.965 & 3.294 & $3.356 \mathrm{~atm}$ & 1.7093 & & & \\
\hline & & & & & $10.338 \quad \mathrm{Mpa}$ & 0.5031 & $0.3338 \quad \mathrm{Mpa}$ & $0.3401 \mathrm{Mpa}$ & 0.17320 & & & \\
\hline & & & & ABSOLUTE & ABSOLUTE & ABSOLUTE $\$$ & DTFFERENTIAL & \multicolumn{2}{|l|}{ ABSOLUTE } & OK) & $(0 \mathrm{~K})$ & (BIT:SoC) \\
\hline & & & & $12.29 \mathrm{ps} 1 \mathrm{a}$ & $1511.8 \quad$ ps1a & $85.25 \quad$ ps1a & 48.41 psid & $61.61 \mathrm{ps1a}$ & 37.41 & \multirow{3}{*}{296} & \multirow{3}{*}{295} & \multirow{3}{*}{0.02577} \\
\hline & & & & 0.8363 atm & 102.87 & 5.801 & 3.294 & $4.193 \mathrm{~atm}$ & 2.546 & & & \\
\hline & & & & $0.08474 \quad$ Mpa & 10.423 & 0.5878 & 0.3338 & 0.4248 & 0.2579 & & & \\
\hline
\end{tabular}

$\frac{1}{b}$

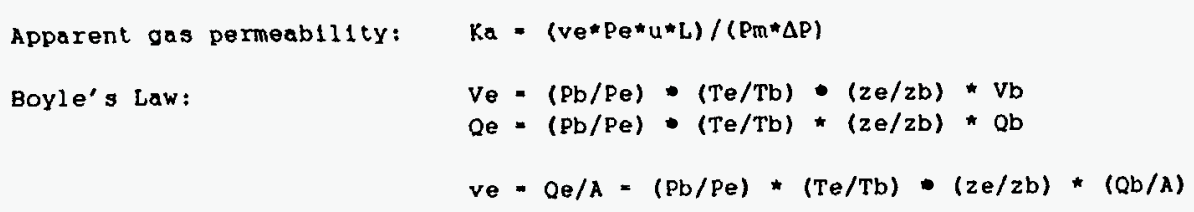

\begin{tabular}{|c|c|c|c|c|}
\hline - Parameter & $\begin{array}{l}\text { Traditional } \\
\text { Units }\end{array}$ & & $\begin{array}{l}\text { SI } \\
\text { Un1 ts }\end{array}$ & \\
\hline$\mu=$ gas v1 scosity & 0.0176 & $* 1.0 \mathrm{E}-3 \mathrm{~Pa}$ *sec/cp & $1.760 \mathrm{E}-05$ & Pa*sec \\
\hline $\mathrm{L}=$ sample length & 10.043 & $\$ 1.0 \mathrm{E}-2 \mathrm{~m} / \mathrm{cm}$ & $1.004 \mathrm{E}-01$ & $m$ \\
\hline A - sample clrcular cross sectlonal area & 81.201 & $\star 1.0 \mathrm{E}-4 \mathrm{~m}^{\wedge} 2 / \mathrm{cm}^{\wedge} 2$ & $8.120 \mathrm{E}-03$ & $m^{\wedge} 2$ \\
\hline $\mathrm{pb}=$ flow measurement basis pressure (absolute) & 0.8363 & $* 1.013 \mathrm{E}+5 \mathrm{~Pa} / \mathrm{atm}$ & $8.472 \mathrm{E}+04$ & $\mathrm{~Pa}$ \\
\hline$\Delta \vec{P}=$ pressure drop across sample length & 3.294 & $1.013 \mathrm{E}+5 \mathrm{~Pa} / \mathrm{atm}$ & $3.337 E+05$ & $\mathrm{~Pa}$ \\
\hline$P_{m}=$ mean pore pressure (absolute) & 4.193 & $1.013 \mathrm{E}+5 \mathrm{~Pa} / \mathrm{atm}$ & $4.247 E+05$ & $\mathrm{~Pa}$ \\
\hline $\mathrm{Pe}$ : exit pressure (absolute) & 2.546 & $1.013 \mathrm{E}+5 \mathrm{~Pa} / \mathrm{atm}$ & $2.579 E+05$ & $\mathrm{~Pa}$ \\
\hline Te = sample temperature (absolute) & 296 & & 296 & ${ }^{\circ} \mathrm{K}$ \\
\hline$T b=$ flow measurement basis temperature (absolute) & 295 & & 295 & ${ }^{\circ} \mathrm{K}$ \\
\hline$z e=$ gas deviation factor at $\mathrm{Pe}$ and $\mathrm{Te}$ & 1.0000 & & 1.0000 & \\
\hline $\mathrm{zb}=$ gas deviation factor at $\mathrm{Pb}$ and $\mathrm{Te}$ & 1.0000 & & 1.0000 & \\
\hline$Q b=$ flow rate at base conditions & $0.02577 \mathrm{~cm}^{n} 3 / \mathrm{s}$ & $\star 1.0 \mathrm{E}-6 \mathrm{~m}^{\wedge} 3 / \mathrm{cm}^{\wedge} 3$ & $2.577 \mathrm{E}-08$ & $m^{\wedge} 3 / s$ \\
\hline$v e=$ flow velocity at sample exit end & $1.046 \mathrm{E}-04 \mathrm{~cm} / \mathrm{s}$ & $1.0 \mathrm{E}-2 \mathrm{~m} / \mathrm{cm}$ & $1.046 \mathrm{E}-06$ & $\mathrm{~m} / \mathrm{s}$ \\
\hline $\mathrm{Ka}=$ & $3.41 \mathrm{E}-06$ & $* 9.872 \mathrm{E}-13 \mathrm{~m}^{\wedge} 2 / \mathrm{d}$ & $3.36 \mathrm{E}-18$ & $m^{\wedge} 2$ \\
\hline $\mathrm{ka}=$ & $3.41 \mathrm{E}-03$ & & $3.36 \mathrm{E}-14$ & $\mathrm{~cm}^{\wedge} 2$ \\
\hline $\mathrm{Ka}=$ & $3.41 E+00$ & & & \\
\hline
\end{tabular}




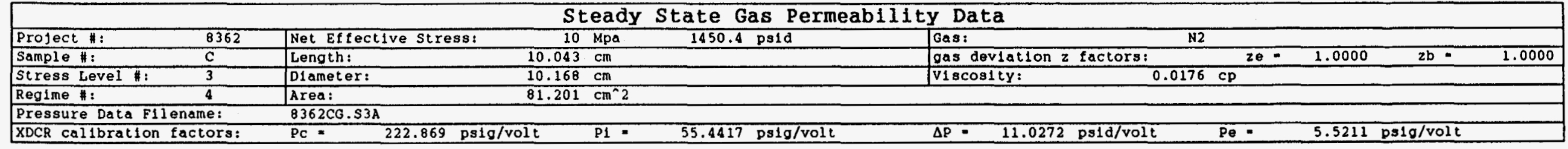

\begin{tabular}{|c|c|c|c|c|c|c|c|c|c|c|c|c|}
\hline Date & $\begin{array}{l}\text { Time } \\
\text { of } \\
\text { Day }\end{array}$ & $\begin{array}{l}\text { File } \\
\text { Time } \\
(m 1 n)\end{array}$ & Regime & $\begin{array}{l}\text { Pb } \\
\text { Barometric } \\
\text { Pressure }\end{array}$ & $\begin{array}{l}\text { Pe } \\
\text { Confining } \\
\text { Pressure }\end{array}$ & $\begin{array}{l}\text { Pi } \\
\text { Inlet } \\
\text { Pressure }\end{array}$ & $\begin{array}{l}\Delta \mathrm{P} \\
\text { Differential } \\
\text { Pressure }\end{array}$ & $\begin{array}{l}\text { Pm } \\
\text { Mean Pore } \\
\text { Pressure } \\
\text { Pe }+\Delta P / 2\end{array}$ & \begin{tabular}{|l} 
Pe \\
Exit \\
Pressure
\end{tabular} & $\begin{array}{l}\text { Te } \\
\text { Elow } \\
\text { Temp } \\
\left({ }^{\circ} \mathrm{C}\right)\end{array}$ & $\begin{array}{c}\text { Tb } \\
\text { Ambient } \\
\text { Temp } \\
\left({ }^{\circ} \mathrm{C}\right)\end{array}$ & $\begin{array}{c}\text { Qb } \\
\text { Flow Rate } \\
\text { PPb\&Tb } \\
\text { (ml/sec) }\end{array}$ \\
\hline $06 \mathrm{MaY} 93$ & $14: 34$ & 3060 & $\operatorname{cs} 3 R 4 a$ & 12.29 ps1a & volts & volts & $4.422 \mathrm{volts}$ & & volts & 23 & 22 & 0.02838 \\
\hline 06 MaY 93 & $14: 38$ & 3065 & CS3R4b & $12.29 \mathrm{ps} 1 \mathrm{a}$ & 6.772 volts & volts & 4.422 volts & & volts & 23 & 22 & 0.02854 \\
\hline 06 MaY 93 & $14: 43$ & 3070 & $\operatorname{CS} 3 R 4 C$ & $12.29 \mathrm{ps} 1 \mathrm{a}$ & 6.772 & volts & 4.422 volts & & volts & 23 & 22 & 0.02851 \\
\hline 06 May 93 & $14: 48$ & 3075 & CS3R4d & $12.29 \mathrm{ps} 1 \mathrm{a}$ & vol ts & volts & 4.422 volts & & volts & 23 & 22 & 0.02842 \\
\hline 06 MaY 93 & $14: 53$ & 3080 & $\operatorname{cs3R} 4 \theta$ & 12.29 ps1a & 6.772 & volts & 4.422 vol ts & & volts & 23 & 22 & 0.02848 \\
\hline \multirow{9}{*}{ AVERAGES } & & & \multirow{9}{*}{$\operatorname{cs} 3 \mathrm{R} 4$} & & OUAGE & OUAOE & DIFFEREATIX & OUAGE & QGOXGE & क्ला & $60)$ & \\
\hline & & & & & 6.772 volts & 1.499 volts & 4.422 volts & & 6.335 & \multirow{4}{*}{23} & \multirow{4}{*}{22} & \\
\hline & & & & & 1509.3 & 83.11 & 48.76 psid & $59.36 \mathrm{ps} 1 \mathrm{~g}$ & 34.978 & & & \\
\hline & & & & & 102.70 & 5.655 & 3.318 & $4.039 \mathrm{~atm}$ & 2.3801 & & & \\
\hline & & & & & 10.406 & 0.5730 & $0.3362 \mathrm{Mpa}$ & 0.4093 & 0.24117 & & & \\
\hline & & & & ABSOLUTE & ABSOLUTE & ABSOLUTE & DHFFERENTAAL & \multicolumn{2}{|l|}{ AESOLWTE } & (9x) & (कर) & $(\mathrm{kL} / \mathrm{sec}$ \\
\hline & & & & $12.29 \mathrm{ps} 1 \mathrm{a}$ & 1521.6 ps1a & 95.40 psta & 48.76 psid & $71.65 \mathrm{ps1a}$ & 47.27 & \multirow{3}{*}{296} & \multirow{3}{*}{295} & \multirow{3}{*}{0.02846} \\
\hline & & & & $0.8363 \mathrm{~atm}$ & 103.54 & 6.491 & 3.318 & $4.875 \mathrm{~atm}$ & 3.216 & & & \\
\hline & & & & 0.08474 & 10.491 & 0.6577 & 0.3362 & 0.4940 & 0.3259 & & & \\
\hline
\end{tabular}

Apparent gas permeability: Ka $=\left(v^{\left.\star P^{*}{ }^{*}{ }^{*} \mathrm{~L}\right) /\left(\mathrm{Pm}^{\star} \Delta \mathrm{P}\right)}\right.$

Boyle's Law:

$V_{e}=(\mathrm{Pb} / \mathrm{Pe}) *(\mathrm{Te} / \mathrm{Tb}) *(\mathrm{ze} / \mathrm{zb}) * \mathrm{Vb}$

$\mathrm{Qe}=(\mathrm{Pb} / \mathrm{Pe}) *(\mathrm{Te} / \mathrm{Tb}) *(\mathrm{ze} / \mathrm{zb}) * \mathrm{Qb}$

$v_{e}=Q e / A=(\mathrm{Pb} / \mathrm{Pe}) *(\mathrm{Te} / \mathrm{Tb}) *(z e / z \mathrm{~b}) *(\mathrm{Ob} / \mathrm{A})$

\begin{tabular}{|c|c|c|c|c|}
\hline Parameter & $\begin{array}{l}\text { Traditional } \\
\text { Units }\end{array}$ & & $\begin{array}{l}\text { SI } \\
\text { Un1ts }\end{array}$ & \\
\hline$\mu=$ gas viscosity & 0.0176 & $-1.0 \mathrm{E}-3 \mathrm{~Pa}$ * SeC/CP & $1.760 \mathrm{E}-05$ & Pa*sec \\
\hline$L$ - sample length & 10.043 & $1.0 \mathrm{E}-2 \mathrm{~m} / \mathrm{cm}$ & $1.004 \mathrm{E}-01$ & $m$ \\
\hline A = sample clrcular cross sectlonal area & 81.201 & - $1.0 \mathrm{E}-4 \mathrm{~m}^{2} 2 / \mathrm{cm}^{2} 2$ & $8.120 E-03$ & $m^{2} 2$ \\
\hline $\mathrm{Pb}=$ flow measurement basis pressure (absolute) & 0.8363 & $\star 1.013 \mathrm{E}+5 \mathrm{~Pa} / \mathrm{atm}$ & $8.472 E+04$ & $\mathrm{pa}_{\mathrm{a}}$ \\
\hline$\Delta \mathrm{P}$ - pressure drop across sample length & 3.318 & $1.013 \mathrm{E}+5 \mathrm{~Pa} / \mathrm{atm}$ & $3.361 E+05$ & $\overline{\mathrm{Pa}}$ \\
\hline $\mathrm{Pm}=$ mean pore pressure (absolute) & 4.875 & $1.013 \mathrm{E}+5 \mathrm{~Pa} / \mathrm{atm}$ & $4.939 E+05$ & $\mathrm{~Pa}$ \\
\hline Pe $=$ exit pressure (absolute) & 3.216 & $\hbar 1.013 \mathrm{E}+5 \mathrm{~Pa} / \mathrm{atm}$ & $3.258 \mathrm{E}+05$ & $\mathrm{~Pa}$ \\
\hline $\mathrm{Te}$ = sample temperature (absolute) & 296 & & 296 & ${ }^{\circ} \mathrm{K}$ \\
\hline$T b=$ flow measurement basis temperature (absolute) & 295 & & 295 & ${ }^{\circ} \mathrm{K}$ \\
\hline$z e=g a s$ dev1ation factor at $\mathrm{Pe}$ and $\mathrm{Te}$ & 1.0000 & & 1.0000 & \\
\hline $2 b=$ gas deviation factor $a t \mathrm{pb}$ and $\mathrm{Te}$ & 1.0000 & & 1.0000 & \\
\hline $\mathrm{ab}=$ flow rate at base conditions & 0.02846 & $\star 1.0 \mathrm{E}-6 \mathrm{~m}^{\wedge} 3 / \mathrm{cm}^{\wedge} 3$ & $2.846 \mathrm{E}-08$ & $m^{2} 3 / s$ \\
\hline $\mathrm{ve}=$ flow veloc1ty at sample exit end & $9.144 \mathrm{E}-05$ & $1.0 \mathrm{E}-2 \mathrm{~m} / \mathrm{cm}$ & $9.144 E-07$ & $\mathrm{~m} / \mathrm{s}$ \\
\hline $\mathrm{Ka}=$ & $3.21 \mathrm{E}-06$ & $\% 9.872 \mathrm{E}-13 \mathrm{~m}^{\wedge} 2 / \mathrm{d}$ & $3.17 \mathrm{E}-18$ & $m^{2} 2$ \\
\hline $\mathrm{Ka}=$ & $3.21 E-03$ & & $3.17 \mathrm{E}-14$ & $\mathrm{~cm}^{\wedge} 2$ \\
\hline & $3.21 \mathrm{E}+00$ & & & \\
\hline
\end{tabular}




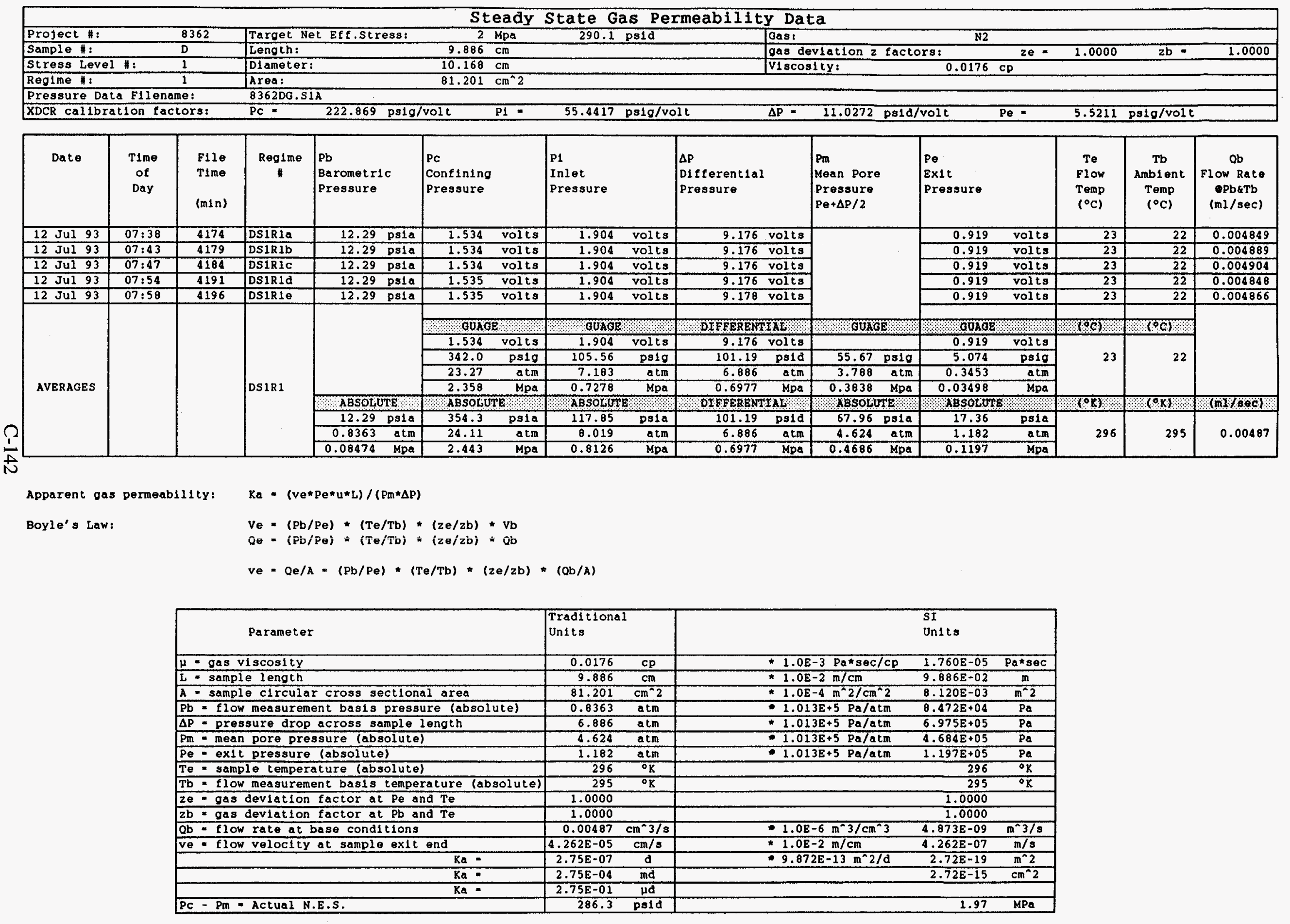




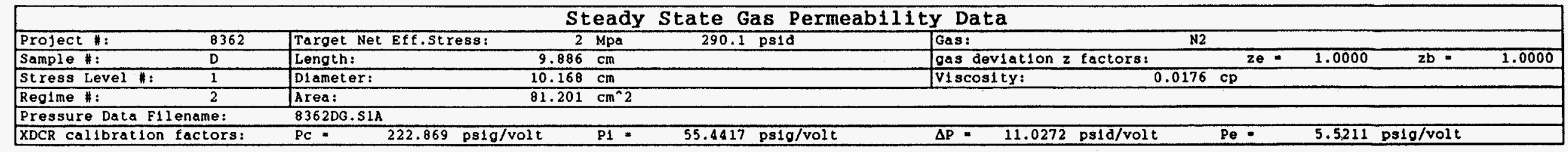

\begin{tabular}{|c|c|c|c|c|c|c|c|c|c|c|c|c|}
\hline Date & $\begin{array}{l}\text { Time } \\
\text { of } \\
\text { Day }\end{array}$ & $\begin{array}{l}\text { File } \\
\text { Time } \\
(m 1 n)\end{array}$ & $\begin{array}{c}\text { Regime } \\
*\end{array}$ & $\begin{array}{l}\mathrm{Pb} \\
\text { Barometrlc } \\
\text { Pressure }\end{array}$ & $\begin{array}{l}\text { Pc } \\
\text { Confining } \\
\text { Pressure }\end{array}$ & $\begin{array}{l}\text { P1 } \\
\text { Inlet } \\
\text { Pressure }\end{array}$ & $\begin{array}{l}\Delta P \\
D 1 \text { fferential } \\
\text { Pressure }\end{array}$ & $\begin{array}{l}\mathrm{Pm} \\
\text { Mean Pore } \\
\text { Pressure } \\
\mathrm{Pe}+\Delta \mathrm{P} / 2\end{array}$ & $\begin{array}{l}\text { Pe } \\
\text { Ex1t } \\
\text { Pressure }\end{array}$ & $\begin{array}{l}\text { Te } \\
\text { Flow } \\
\text { Temp } \\
\left({ }^{\circ} \mathrm{C}\right)\end{array}$ & $\begin{array}{c}\mathrm{Tb} \\
\text { Amblent } \\
\text { Temp } \\
\left({ }^{\circ} \mathrm{C}\right)\end{array}$ & $\begin{array}{c}\text { Qb } \\
\text { Flow Rate } \\
\text { PbETb } \\
\text { (m1/sec) }\end{array}$ \\
\hline $12 \mathrm{Jul} 93$ & $12: 44$ & 4476 & DS1R2a & $12.32 \mathrm{ps} 1 \mathrm{a}$ & volts & 2.102 & 9.155 volts & & volts & 23 & 23 & 0.005378 \\
\hline $12 \mathrm{Jul} 93$ & $12: 47$ & 4481 & DS1R2b & $12.32 \mathrm{ps} 1 \mathrm{a}$ & volts & 2.102 & 9.155 volts & & volts & 23 & 23 & 0.005387 \\
\hline 12 Jul 93 & $12: 51$ & 4486 & DS1R2C & 12.32 psia & volts & 2.102 & 9.155 volts & & volts & 23 & 23 & 0.005362 \\
\hline 12 Jul 93 & $12: 55$ & 4491 & DS1R2d & $12.32 \mathrm{ps} 1 \mathrm{a}$ & volts & 2.102 & 9.156 volts & & volts & 23 & 23 & 0.005371 \\
\hline 12 Jul 93 & $13: 01$ & 4496 & DS1R2e & 12.32 psia & 1.598 vol ts & 2.102 volts & 9.156 volts & & volts & 23 & 23 & 0.005358 \\
\hline \multirow{9}{*}{ AVERAGES } & & & \multirow{9}{*}{ DS1R2 } & & GOAGE & GOAGE & DIFEERENTIXI & OUAGE & GUXXE: & (9) & 10ला\% & \\
\hline & & & & & 1.598 volts & 2.102 volts & $9: 155$ volts & & 2.950 & \multirow{4}{*}{23} & \multirow{4}{*}{23} & \\
\hline & & & & & 356.1 & 116.54 & 100.96 ps1d & $66.77 \mathrm{psig}$ & 16.287 & & & \\
\hline & & & & & 24.23 & 7.930 & 6.870 & $4.543 \mathrm{~atm}$ & 1.1083 & & & \\
\hline & & & & & 2.456 & 0.8035 & $0,6961 \quad \mathrm{Mpa}$ & $0.4603 \mathrm{Mpa}$ & 0.11230 & & & \\
\hline & & & & ABSOLUTE & ABSOLUTE & ABSOLURE & DTFFERENT AL & \multicolumn{2}{|l|}{ MaSOLCTE } & (1) & (OK) & $(\mathrm{mI} / \mathrm{s \theta c)}$ \\
\hline & & & & 12.32 psia & 368.5 ps1a & $128.86 \quad \mathrm{ps} 1 \mathrm{a}$ & 100.96 psid & 79.09 psia & 28.61 & \multirow{3}{*}{296} & \multirow{3}{*}{296} & \multirow{3}{*}{0.00537} \\
\hline & & & & 0.8383 atm & 25.07 & 8.768 & 6.870 & $5.381 \mathrm{~atm}$ & 1.947 & & & \\
\hline & & & & $0.08494 \mathrm{Mpa}$ & 2.540 & 0.8884 & 0.6961 & 0.5453 & 0.1972 & & & \\
\hline
\end{tabular}

Apparent gas permeability:

$K a=(v e * P e * u * L) /(P m * \Delta P)$

Boyle's Law:

$\mathrm{Ve}=(\mathrm{Pb} / \mathrm{Pe}) *(\mathrm{Te} / \mathrm{Tb}) *(\mathrm{ze} / \mathrm{2b}) * \mathrm{Vb}$

$\mathrm{Qe}_{e}=(\mathrm{Pb} / \mathrm{Pe}) *(\mathrm{Te} / \mathrm{Tb}) *(\mathrm{ze} / \mathrm{Zb}) * \mathrm{Qb}$

$v_{e}=Q_{e} / \mathrm{A}=(\mathrm{Pb} / \mathrm{Pe}) \cdot(\mathrm{Te} / \mathrm{Tb}) *(z e / 2 \mathrm{~b}) \cdot(\mathrm{Qb} / \mathrm{A})$

\begin{tabular}{|c|c|c|c|c|}
\hline Parameter & $\begin{array}{l}\text { Traditional } \\
\text { Units }\end{array}$ & & $\begin{array}{l}\text { SI } \\
\text { Un1ts }\end{array}$ & \\
\hline$\mu=$ gas $v 1 s \cos 1 t y$ & 0.0176 & $\star 1.0 \mathrm{E}-3 \mathrm{~Pa} \mathrm{~Pa}^{\star \mathrm{sec} / \mathrm{cp}}$ & $1.760 \mathrm{E}-05$ & $\mathrm{~Pa}^{\star} \mathrm{sec}$ \\
\hline $\mathrm{L}=$ sample length & 9.886 & $1.0 \mathrm{E}-2 \mathrm{~m} / \mathrm{cm}$ & $9.886 \mathrm{E}-02$ & $\mathrm{~m}$ \\
\hline$A=$ sample clrcular cross sectional area & 81.201 & $* 1.0 \mathrm{E}-4 \mathrm{~m}^{\wedge} 2 / \mathrm{cm}^{\wedge} 2$ & $8.120 \mathrm{E}-03$ & $m^{\wedge} 2$ \\
\hline $\mathrm{Pb}=$ flow measurement basis pressure (absolute) & 0.8383 & $-1.013 \mathrm{E}+5 \mathrm{~Pa} / \mathrm{atm}$ & $8.492 E+04$ & $\mathrm{~Pa}$ \\
\hline$\Delta p=$ pressure drop across sample length & 6.870 & $-1.013 \mathrm{E}+5 \mathrm{~Pa} / \mathrm{atm}$ & $6.959 E+05$ & $\mathrm{~Pa}$ \\
\hline $\mathrm{pm}_{\mathrm{m}}=$ mean pore pressure (absolute) & 5.381 & $-1.013 \mathrm{E}+5 \mathrm{~Pa} / \mathrm{atm}$ & $5.451 E+05$ & $\mathrm{~Pa}$ \\
\hline $\mathrm{Pe}=$ exit pressure (absolute) & 1.947 & $* 1.013 \mathrm{E}+5 \quad \mathrm{~Pa} / \mathrm{atm}$ & $1.972 \mathrm{E}+05$ & Pa \\
\hline Te = sample temperature (absolute) & 296 & & 296 & ${ }^{\circ} \mathrm{K}$ \\
\hline $\mathrm{Tb}=$ flow measurement basis temperature (absolute) & 296 & & 296 & ${ }^{\circ} \mathrm{K}$ \\
\hline$z e=$ gas deviation factor at $\mathrm{Pe}$ and $\mathrm{Te}$ & 1.0000 & & 1.0000 & \\
\hline $2 b=$ gas devlation factor at $\mathrm{Pb}$ and $\mathrm{Te}$ & 1.0000 & & 1.0000 & \\
\hline$Q b=$ flow rate at base conditions & 0.00537 & $1.0 \mathrm{E}-6 \mathrm{~m}^{\wedge} 3 / \mathrm{cm}^{\wedge} 3$ & $5.374 E-09$ & $m^{n} 3 / s$ \\
\hline ve = flow velocity at sample exit end & $2.850 \mathrm{E}-05$ & $\star 1.0 \mathrm{E}-2 \mathrm{~m} / \mathrm{cm}$ & $2.850 E-07$ & $\mathrm{~m} / \mathrm{s}$ \\
\hline $\mathrm{Ka}^{-}=$ & $2.61 \mathrm{E}-07$ & $9.872 \mathrm{E}-13 \mathrm{~m}^{\wedge} 2 / \mathrm{d}$ & $2.58 \mathrm{E}-19$ & $m^{2} 2$ \\
\hline$K_{a}=$ & $2.61 \mathrm{E}-04$ & & $2.58 \mathrm{E}-15$ & $\mathrm{~cm}^{\wedge} 2$ \\
\hline $\mathrm{K}_{0}=$ & $2.61 \mathrm{E}-01$ & & & \\
\hline$P c-P m=$ Actuel N.E.S. & 289.4 & & 2.00 & MPO \\
\hline
\end{tabular}




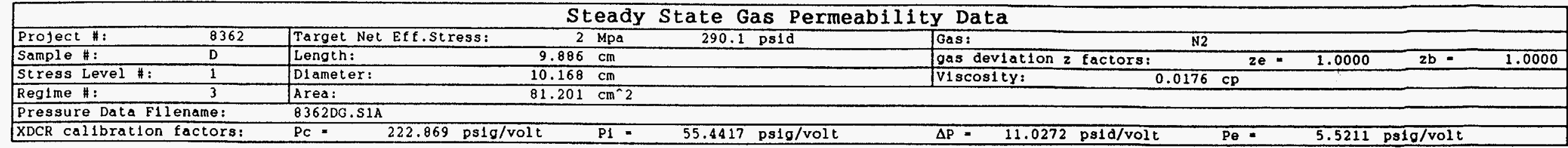

\begin{tabular}{|c|c|c|c|c|c|c|c|c|c|c|c|c|}
\hline Date & $\begin{array}{l}\text { Time } \\
\text { of } \\
\text { Day }\end{array}$ & $\begin{array}{l}\text { File } \\
\text { Time } \\
\text { (min) }\end{array}$ & $\begin{array}{c}\text { Reglme } \\
\#\end{array}$ & $\begin{array}{l}\mathrm{Pb} \\
\text { Barometric } \\
\text { Pressure }\end{array}$ & $\begin{array}{l}\text { Pc } \\
\text { Confining } \\
\text { Pressure }\end{array}$ & $\begin{array}{l}\text { Pi } \\
\text { Inlet } \\
\text { Pressure }\end{array}$ & $\begin{array}{l}\Delta P \\
D 1 \text { fferential } \\
\text { Pressure }\end{array}$ & $\begin{array}{l}\text { Pm } \\
\text { Mean Pore } \\
\text { Pressure } \\
\text { Pe }+\Delta P / 2\end{array}$ & \begin{tabular}{|l} 
Pe \\
Exlt \\
Pressure
\end{tabular} & $\begin{array}{l}\text { Te } \\
\text { Flow } \\
\text { Temp } \\
\left({ }^{\circ} \mathrm{C}\right)\end{array}$ & $\begin{array}{c}\text { Tb } \\
\text { Amblent } \\
\text { Temp } \\
\left({ }^{\circ} \mathrm{C}\right)\end{array}$ & $\begin{array}{c}Q b \\
\text { Flow Rate } \\
\text { Pb\&Tb } \\
\text { (ml/sec) }\end{array}$ \\
\hline 12 Jul 93 & $17: 05$ & 4738 & DS1R3a & $12.29 \mathrm{ps} 1 \mathrm{a}$ & volts & volts & 9.160 volts & & 4.720 & 23 & 23 & 0.005750 \\
\hline 12 Jul 93 & $17: 09$ & 4743 & DS1R3b & $12.29 \mathrm{ps} 1 \mathrm{a}$ & volts & volts & 9.160 volts & & volts & 23 & 23 & 0.005742 \\
\hline $12 \mathrm{JuI} 93$ & $17: 13$ & 4748 & DS1R3C & $12.29 \mathrm{ps} 1 \mathrm{a}$ & volts & volts & 9.590 volts & & volts & 23 & 23 & 0.005760 \\
\hline 12 Ju1 93 & $17: 16$ & 4753 & DS1R3d & 12.29 psla & volts & volts & 9.590 volts & & volts & 23 & 23 & 0.005767 \\
\hline 12 Jul 93 & $17: 19$ & 4758 & DS1R3e & $12.29 \mathrm{psia}$ & volts & volts & 9.590 volts & & volts & 23 & 23 & 0.005764 \\
\hline \multirow{9}{*}{ AVERAGES } & & & \multirow{9}{*}{ DS1R3 } & & OUAGE & OOAGE & DIFEERENTIALI & OUAGE & GUAOE & $\mathrm{Te})$ & $\mathrm{Pel}$ & \\
\hline & & & & & 1.645 volts & 2.277 volts & 9.418 volts & & 4.720 & \multirow{4}{*}{23} & \multirow{4}{*}{23} & \\
\hline & & & & & 366.7 & 126.24 & 103.85 ps1d & 77.99 psig & 26.060 & & & \\
\hline & & & & & 24.95 & 8.590 & 7.067 & $5.307 \mathrm{~atm}$ & 1.7732 & & & \\
\hline & & & & & $2.528 \quad \mathrm{Mpa}$ & 0.8704 & 0.7160 & $0.5377 \mathrm{Mpa}$ & 0.17967 & & & \\
\hline & & & & ABSOLUTE & ABSOLUTE & ABSOLUTE & DIFFERENTIAL & AESOLUTE & ABSOLUTE & (OK) & $(0 \times)$ & $\mathrm{mm} / \mathrm{sec})$ \\
\hline & & & & 12.29 ps1a & $379.0 \quad$ ps10 & $138.53 \quad$ psla & 103.85 ps1d & 90.28 ps1a & 38.35 & \multirow{3}{*}{296} & \multirow{3}{*}{296} & \multirow{3}{*}{0.00575} \\
\hline & & & & $0.8363 \quad \mathrm{~atm}$ & 25.79 & 9.426 & 7.067 & $6.143 \mathrm{~atm}$ & 2.610 & & & \\
\hline & & & & $0.08474 \quad \mathrm{Mpa}$ & 2.613 & 0.9551 & 0.7160 & 0.6224 & 0.2644 & & & \\
\hline
\end{tabular}

Apparent gas permeab111ty:

Boyle's Law:

$K a=\left(v e^{\star P e} e^{\star} u L\right) /(P m \star \Delta P)$

$\mathrm{Ve}=(\mathrm{Pb} / \mathrm{Pe}) *(\mathrm{Te} / \mathrm{Tb}) *(2 e / \mathrm{zb}) * \mathrm{Vb}$

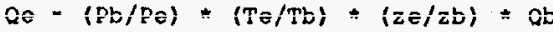

$v_{e}=Q_{e} / \mathrm{A}=(\mathrm{Pb} / \mathrm{Pe}) *(\mathrm{Te} / \mathrm{Tb}) *(\mathrm{ze} / \mathrm{zb}) *(\mathrm{Qb} / \mathrm{A})$

\begin{tabular}{|c|c|c|c|c|}
\hline Parameter & $\begin{array}{l}\text { Traditional } \\
\text { Units }\end{array}$ & & $\begin{array}{l}\text { SI } \\
\text { Un1ts }\end{array}$ & \\
\hline$\mu=\operatorname{gas} v 1 s \cos 1 t y$ & 0.0176 & $\star 1.0 \mathrm{E}-3 \mathrm{~Pa} * \mathrm{sec} / \mathrm{cp}$ & $1.760 \mathrm{E}-05$ & $\mathrm{~Pa}^{\star} \mathrm{sec}$ \\
\hline $\mathrm{L}$ - sample length & 9.886 & $\star 1.0 \mathrm{E}-2 \mathrm{~m} / \mathrm{cm}$ & $9.886 \mathrm{E}-02$ & $\mathbf{m}$ \\
\hline$A=$ sample clrcular cross sectional area & 81.201 & $21.0 \mathrm{E}-4 \mathrm{~m}^{\wedge} 2 / \mathrm{cm}^{\wedge} 2$ & $8.120 \mathrm{E}-03$ & $m^{\wedge} 2$ \\
\hline $\mathrm{Pb}=$ flow measurement basls pressure (absolute) & 0.8363 & $\star 1.013 \mathrm{E}+5 \mathrm{~Pa} / \mathrm{atm}$ & $8.472 E+04$ & $\mathrm{~Pa}$ \\
\hline$\Delta \mathrm{P}=$ pressure drop across sample length & 7.067 & $1.013 \mathrm{E}+5 \mathrm{~Pa} / \mathrm{atm}$ & $7.159 \mathrm{E}+05$ & Pa \\
\hline Pm $=$ mean pore pressure (absolute) & 6.143 & $1.013 \mathrm{E}+5 \mathrm{~Pa} / \mathrm{acm}$ & $6.223 \mathrm{E}+05$ & $\mathrm{~Pa}$ \\
\hline Pe $=$ exit pressure (absolute) & 2.610 & $1.013 \mathrm{E}+5 \mathrm{~Pa} / \mathrm{atm}$ & $2.643 \mathrm{E}+05$ & $\mathrm{~Pa}$ \\
\hline $\mathrm{Te}$ - sample temperature (absolute) & 296 & & 296 & ${ }^{\circ} \mathrm{K}$ \\
\hline $\mathrm{Tb}=$ flow measurement basis temperature (absolute) & 296 & & 296 & ${ }^{\circ} \mathrm{K}$ \\
\hline $2 e=$ gas deviation factor at $\mathrm{Pe}$ and $\mathrm{Te}$ & 1.0000 & & 1.0000 & \\
\hline $2 \mathrm{~b}$ - gas deviation factor at $\mathrm{pb}$ and $\mathrm{Te}$ & 1.0000 & & 1.0000 & \\
\hline$Q b=$ flow rate at base conditions & $0.00575 \mathrm{~cm}^{\wedge} 3 / \mathrm{s}$ & $1.0 \mathrm{E}-6 \mathrm{~m}^{\wedge} 3 / \mathrm{cm}^{\wedge} 3$ & $5.755 \mathrm{E}-09$ & $m^{n} 3 / s$ \\
\hline ve = flow velocity at sample exit end & $2.271 \mathrm{E}-05 \mathrm{~cm} / \mathrm{s}$ & $\star 1.0 \mathrm{E}-2 \mathrm{~m} / \mathrm{cm}$ & $2.271 \mathrm{E}-07$ & $\mathrm{~m} / \mathrm{s}$ \\
\hline $\mathrm{Ka}=$ & $2.38 \mathrm{E}-07$ & $9.872 \mathrm{E}-13 \mathrm{~m}^{2} 2 / \mathrm{d}$ & $2.34 E-19$ & $\mathrm{~m}^{\wedge} 2$ \\
\hline $\mathrm{Ka}=$ & $2.38 \mathrm{E}-04$ & & $2.34 \mathrm{E}-15$ & $\mathrm{~cm}^{n} 2$ \\
\hline $\mathrm{Ka}=$ & $2.38 \mathrm{E}-01$ & & & \\
\hline$P C-P m=$ ACtual N.E.S. & 288.7 & & 1.99 & MPa \\
\hline
\end{tabular}




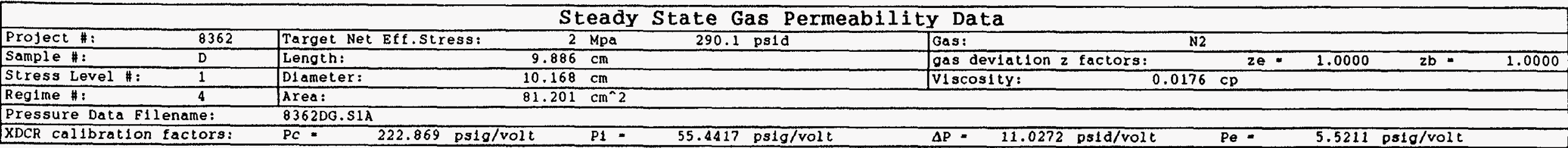

\begin{tabular}{|c|c|c|c|c|c|c|c|c|c|c|c|c|}
\hline Date & $\begin{array}{l}\text { Time } \\
\text { of } \\
\text { Day }\end{array}$ & $\begin{array}{l}\text { File } \\
\text { Time } \\
\text { (min) }\end{array}$ & $\underset{\#}{\text { Regime }}$ & $\begin{array}{l}\text { Pb } \\
\text { Barometric } \\
\text { Pressure }\end{array}$ & $\begin{array}{l}\text { Pc } \\
\text { Confining } \\
\text { Pressure }\end{array}$ & $\begin{array}{l}\text { P1 } \\
\text { Inlet } \\
\text { Pressure }\end{array}$ & $\begin{array}{l}\Delta \mathrm{P} \\
\text { Differential } \\
\text { Pressure }\end{array}$ & $\begin{array}{l}\text { Pm } \\
\text { Mean Pore } \\
\text { Pressure } \\
\mathrm{Pe}+\Delta \mathrm{P} / 2\end{array}$ & $\begin{array}{l}\text { Pe } \\
\text { Exit } \\
\text { Pressure }\end{array}$ & $\begin{array}{l}\text { Te } \\
\text { Flow } \\
\text { Temp } \\
\left({ }^{\circ} \mathrm{C}\right)\end{array}$ & $\begin{array}{c}\text { Tb } \\
\text { Amblent } \\
\text { Temp } \\
\left({ }^{\circ} \mathrm{C}\right)\end{array}$ & $\begin{array}{c}\text { Qb } \\
\text { Flow Rate } \\
\text { Pbstb } \\
\text { (ml/sec) }\end{array}$ \\
\hline 13 Jul 93 & $07: 41$ & 5613 & DS1R4a & $12.33 \mathrm{ps} 1 \mathrm{a}$ & volts & volts & 9.158 volts & & volts & 23 & 21 & 0.006108 \\
\hline 13 JuL 93 & $07: 44$ & 5618 & DS1R4b & $12.33 \mathrm{ps} 1 \mathrm{a}$ & volts & volts & 9.158 volts & & volts & 23 & 21 & 0.006070 \\
\hline 13 JuI 93 & $07: 47$ & 5623 & DSIR4C & $12.33 \mathrm{ps} 1 \mathrm{a}$ & volts & volts & 9.159 volts & & volts & 23 & 21 & 0.006066 \\
\hline 13 Ju1 93 & $07: 50$ & 5628 & DS1R4a & $12.33 \mathrm{ps} 1 \mathrm{a}$ & volts & volts & 9.159 volts & & volts & 23 & 21 & 0.006107 \\
\hline 13 Jul 93 & $07: 53$ & 5633 & DS1R4e & $12.33 \mathrm{ps} 1 \mathrm{a}$ & volts & volts & 9.158 volts & & volts & 23 & 21 & 0.006111 \\
\hline \multirow{9}{*}{ AVERAGES } & & & \multirow{9}{*}{ DS1R4 } & & GUAGE & OUAGE & DIFFERENTIAI & OOAGE & GUXOE: & का & क्व) & \\
\hline & & & & & 1.678 volts & 2.449 volts & 9.158 volts & & 6.450 & \multirow{4}{*}{23} & \multirow{4}{*}{21} & \\
\hline & & & & & 374.0 & 135.78 & 100.99 ps1d & $86.11 \mathrm{ps} 1 \mathrm{~g}$ & 35.613 & & & \\
\hline & & & & & 25.45 & 9.239 & 6.872 & $5.859 \mathrm{~atm}$ & 2.4233 & & & \\
\hline & & & & & 2.578 & 0.9361 & 0.6963 & $0.5937 \mathrm{Mpa}$ & 0.24555 & & & \\
\hline & & & & ABSOLUTE & ABSOLUTE & ABSOLUTE & DIFFERENTIAL & \multicolumn{2}{|l|}{ ABSOLUTE } & (OK) & ok & $(\mathrm{m} I / \mathrm{sec})$ \\
\hline & & & & $12.33 \mathrm{ps} 1 \mathrm{a}$ & 386.3 psia & 148.11 psia & 100.99 ps1d & $98.44 \mathrm{ps} 1 \mathrm{a}$ & 47.94 & \multirow{3}{*}{296} & \multirow{3}{*}{294} & \multirow{3}{*}{0.00609} \\
\hline & & & & $0.8390 \mathrm{~atm}$ & 26.29 & 10.078 & 6.872 & $6.698 \mathrm{~atm}$ & 3.262 & & & \\
\hline & & & & $0.08501 \mathrm{Mpa}$ & 2.663 & 1.0212 & 0.6963 & $0.6787 \mathrm{Mpa}$ & 0.3306 & & & \\
\hline
\end{tabular}

Apparent gas permeab111ty: Ka $=\left(v e^{\left.* P e^{*} u^{* L} L\right) /(P m * \Delta P)}\right.$

Boyle's Law:

$\mathrm{Ve}_{e}=(\mathrm{Pb} / \mathrm{Pe}) *(\mathrm{Te} / \mathrm{Tb}) *(\mathrm{ze} / \mathrm{zb}) * \mathrm{Vb}$

$\mathrm{Qe}=(\mathrm{Pb} / \mathrm{Pe}) \cdot(\mathrm{Te} / \mathrm{Tb}) *(\mathrm{ze} / \mathrm{zb}) * \mathrm{Qb}$

$v e=Q e / A=(\mathrm{Pb} / \mathrm{Pe}) \cdot(\mathrm{Te} / \mathrm{Tb}) *(2 \mathrm{e} / \mathrm{zb}) *(\mathrm{Qb} / \mathrm{A})$

\begin{tabular}{|c|c|c|c|c|}
\hline Parameter & $\begin{array}{l}\text { Traditional } \\
\text { Un1ts }\end{array}$ & & $\begin{array}{l}\text { SI } \\
\text { Undts }\end{array}$ & \\
\hline$\mu=\operatorname{gas} v i s \cos i t y$ & 0.0176 & $\star 1.0 E-3 \mathrm{~Pa}^{*} \mathrm{sec} / \mathrm{cp}$ & $1.760 E-05$ & Pa*sec \\
\hline $\mathrm{L}=$ sample length & 9.886 & $* 1.0 \mathrm{E}-2 \mathrm{~m} / \mathrm{cm}$ & $9.886 \mathrm{E}-02$ & $\mathrm{~m}$ \\
\hline$A=$ sample circular cross sectional area & 81.201 & $\star 1.0 \mathrm{E}-4 \mathrm{~m}^{\star} 2 / \mathrm{cm}^{\star} 2$ & $8.120 \mathrm{E}-03$ & $m^{\wedge} 2$ \\
\hline $\mathrm{Pb}=$ flow measurement basis pressure (absolute) & 0.8390 & $\star 1.013 \mathrm{E}+5 \mathrm{~Pa} / \mathrm{atm}$ & $8.499 \mathrm{E}+04$ & $\mathrm{~Pa}$ \\
\hline$\Delta \mathrm{P}=$ pressure drop across sample length & $6 . \overline{872}$ & $\star 1.013 \mathrm{E}+5 \mathrm{~Pa} / \mathrm{atm}$ & $6.961 \mathrm{E}+05$ & Pa \\
\hline $\mathrm{P} m_{\mathrm{m}}=$ mean pore pressure (absolute) & 6.698 & $1.013 \mathrm{E}+5 \mathrm{~Pa} / \mathrm{atm}$ & $6.785 \mathrm{E}+05$ & Pa \\
\hline $\mathrm{Pe}=$ exit pressure (absolute) & 3.262 & $\star 1.013 \mathrm{E}+5 \mathrm{~Pa} / \mathrm{atm}$ & $3.305 \mathrm{E}+05$ & $\mathrm{~Pa}$ \\
\hline$T e=$ sample temperature (absolute) & 296 & & 296 & ${ }^{\circ} \mathrm{K}$ \\
\hline$T b=$ flow measurement bas1s temperature (absolute) & 294 & & 294 & ${ }^{\circ} \mathrm{K}$ \\
\hline $2 \mathrm{e}=$ gas deviation factor at $\mathrm{Pe}$ and $\mathrm{Te}$ & 1.0000 & & 1.0000 & \\
\hline$z b=$ gas deviation factor at $\mathrm{Pb}$ and $\mathrm{Te}$ & 1.0000 & & 1.0000 & \\
\hline$Q b$ flow rate at base conditions & 0.00609 & $\pi 1.0 \mathrm{E}-6 \mathrm{~m}^{\wedge} 3 / \mathrm{cm}^{\wedge} 3$ & $6.088 \mathrm{E}-09$ & $m^{2} 3 / s$ \\
\hline ve = flow velocity at sample exit end & $1.941 E-05$ & $\star 1.0 \mathrm{E}-2 \mathrm{~m} / \mathrm{cm}$ & $1.941 \mathrm{E}-07$ & $\mathrm{~m} / \mathrm{s}$ \\
\hline $\mathrm{Ka}=$ & $2.39 \mathrm{E}-07$ & $\star 9.872 \mathrm{E}-13 \mathrm{~m}^{\wedge} 2 / \mathrm{d}$ & $2.36 \mathrm{E}-19$ & $m^{2} 2$ \\
\hline $\mathrm{Ka}=$ & $2.39 \mathrm{E}-04$ & & $2.36 \mathrm{E}-15$ & $\mathrm{~cm}^{\wedge} 2$ \\
\hline $\mathrm{Ka}=$ & $2.39 \mathrm{E}-01$ & & & \\
\hline $\mathrm{PC}-\mathrm{Pm}_{\mathrm{P}}=$ Actual N.E.S. & 287.9 & & 1.98 & $\mathrm{MPa}$ \\
\hline
\end{tabular}




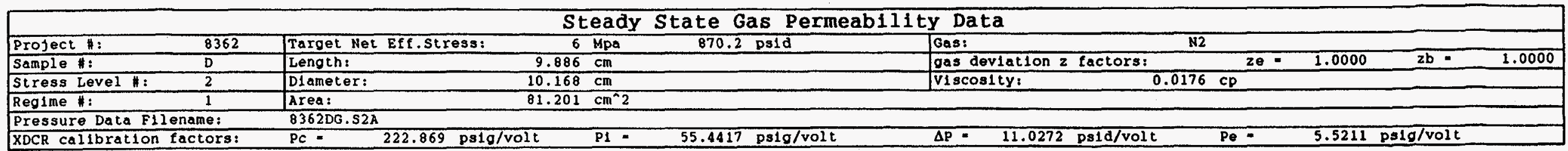

\begin{tabular}{|c|c|c|c|c|c|c|c|c|c|c|c|c|}
\hline Date & $\begin{array}{l}\text { Time } \\
\text { of } \\
\text { Day }\end{array}$ & $\begin{array}{l}\text { File } \\
\text { Time } \\
\text { (min) }\end{array}$ & $\underset{\#}{\text { Reg1me }}$ & $\begin{array}{l}\text { Pb } \\
\text { Barometric } \\
\text { Pressure }\end{array}$ & $\begin{array}{l}\text { Pc } \\
\text { Confining } \\
\text { Pressure }\end{array}$ & $\begin{array}{l}\text { P1 } \\
\text { Inlet } \\
\text { Pressure }\end{array}$ & $\begin{array}{l}\Delta \mathrm{P} \\
\text { D1fferentlal } \\
\text { Pressure }\end{array}$ & $\begin{array}{l}\text { Pm } \\
\text { Mean Pore } \\
\text { Pressure } \\
\text { Pe }+\Delta P / 2\end{array}$ & $\begin{array}{l}\text { Pe } \\
\text { Exit } \\
\text { Pressure }\end{array}$ & $\begin{array}{l}\text { To } \\
\text { Flow } \\
\text { Temp } \\
\left({ }^{\circ} \mathrm{C}\right\rangle\end{array}$ & $\begin{array}{c}\text { Tb } \\
\text { Amb1ent } \\
\text { Temp } \\
\left({ }^{\circ} \mathrm{C}\right)\end{array}$ & $\begin{array}{c}\text { Qb } \\
\text { Flow Rate } \\
\text { PPbETb } \\
\text { (ml/sec) }\end{array}$ \\
\hline 15 Jul 93 & $14: 47$ & 1353 & DS2R1a & 12.30 psla & volts & volts & 9.165 volts & & volts & 23 & 23 & 0.002292 \\
\hline 15 Jul 93 & $14: 51$ & 1358 & DS2R1b & $12.30 \mathrm{psia}$ & 4.158 volts & volts & 9.165 volts & & volts & 23 & 23 & 0.002289 \\
\hline 15 Jul 93 & $14: 56$ & 1363 & DS2R1C & 12.30 psla & volts & volts & 9.164 volts & & volts & 23 & 23 & 0.002281 \\
\hline 15 Jul 93 & $15: 01$ & 1368 & DS2R1d & $12.30 \mathrm{psla}$ & 4.158 volts & volts & 9.164 volts & & volts & 23 & 23 & 0.002275 \\
\hline 15 Jul 93 & $15: 06$ & 1373 & DS2R1e & $12.30 \quad \mathrm{ps} 1 \mathrm{a}$ & 4.158 volts & volts & 9.164 volts & & 1.081 & 23 & 23 & 0.002287 \\
\hline \multirow{9}{*}{ AVERAGES } & & & \multirow{9}{*}{ DS2R1 } & & OUAOE & G0XOE & DIFFEREATIAI & OUAGE: & OUXXOE: & (क) & Tदा? & \\
\hline & & & & & 4.158 volts & 1.918 volts & 9.164 volts & & 1.079 & \multirow{4}{*}{23} & \multirow{4}{*}{23} & \\
\hline & & & & & 926.7 & 106.34 & 101.06 psid & $56.49 \mathrm{ps} 1 \mathrm{~g}$ & 5.958 & & & \\
\hline & & & & & 63.06 & 7.236 & 6.877 & $3.844 \mathrm{~atm}$ & 0.4054 & & & \\
\hline & & & & & $6.389 \quad \mathrm{Mpa}$ & 0.7332 & $0.6968 \quad \mathrm{Mpa}$ & $0.3895 \quad$ Mpa & 0.04108 & & & \\
\hline & & & & ABSOLUTE & ABSOLUTE & ABSOLURE & DYFFEREMTIAL & \multicolumn{2}{|l|}{ RESOLLTE } & $P^{\circ} \mathbf{x}$ & $(0 \mathrm{k})$ & $\sin 7 \mathrm{sec})$ \\
\hline & & & & $12.3 \mathrm{ps} 1 \mathrm{a}$ & $939.0 \quad$ psla & 118.64 ps1a & 101.06 ps1d & $68.79 \mathrm{psia}$ & 18.26 & \multirow{3}{*}{296} & \multirow{3}{*}{296} & \multirow{3}{*}{0.00228} \\
\hline & & & & $0.8370 \mathrm{~atm}$ & 63.89 & 8.073 & 6.877 & $4.681 \mathrm{~atm}$ & 1.242 & & & \\
\hline & & & & $0.08481 \mathrm{Mpa}$ & 6.474 & 0.8180 & 0.6968 & 0.4743 & 0.1259 & & & \\
\hline
\end{tabular}

南

Apparent gas permeabllity:

$K a=\left(v e * P e^{\star} u^{\star L} L\right) /(P m * \Delta P)$

Boyle's Law:

$V_{e}=(\mathrm{Pb} / \mathrm{Pe}) *(\mathrm{Te} / \mathrm{Tb}) *(2 \mathrm{e} / \mathrm{zb}) * \mathrm{Vb}$

$\mathrm{Qe}=(\mathrm{Pb} / \mathrm{Pe}) *(\mathrm{Te} / \mathrm{Tb}) *(\mathrm{ze} / \mathrm{zb}) * \mathrm{Qb}$

$v_{e}=Q_{e} / \mathrm{A}=(\mathrm{Pb} / \mathrm{Pe}) *(\mathrm{Te} / \mathrm{Tb}) *(\mathrm{ze} / \mathrm{Zb}) *(\mathrm{Qb} / \mathrm{A})$

\begin{tabular}{|c|c|c|c|c|}
\hline Parameter & $\begin{array}{l}\text { Traditional } \\
\text { Units }\end{array}$ & & $\begin{array}{l}\text { SI } \\
\text { Un1ts }\end{array}$ & \\
\hline$\mu=g a s v 1 s \cos 1 t y$ & 0.0176 & $\star 1.0 \varepsilon-3 \mathrm{~Pa} * \mathrm{sec} / \mathrm{cp}$ & $1.760 \mathrm{E}-05$ & $\mathrm{~Pa}^{*} \mathrm{sec}$ \\
\hline$L=$ sample length & 9.886 & $1.0 \mathrm{E}-2 \mathrm{~m} / \mathrm{cm}$ & $9.886 \mathrm{E}-02$ & $m$ \\
\hline A : sample clrcular cross sectional area & 81.201 & $\star 1.0 \mathrm{E}-4 \mathrm{~m}^{\wedge} 2 / \mathrm{cm}^{2} 2$ & $8.120 E-03$ & $m^{*} 2$ \\
\hline $\mathrm{Pb}=$ flow measurement basls pressure (absolute) & 0.8370 & $\star 1.013 E+5 \mathrm{~Pa} / \mathrm{atm}$ & $8.478 \mathrm{E}+04$ & $\mathrm{~Pa}$ \\
\hline$\Delta \mathrm{P}$ - pressure drop across sample length & 6.877 & $1.013 \mathrm{E}+5 \mathrm{~Pa} / \mathrm{atm}$ & $6.966 E+05$ & Pa \\
\hline$P_{m}=$ mean pore pressure (absolute) & 4.681 & $\$ 1.013 \mathrm{E}+5 \mathrm{~Pa} / \mathrm{atm}$ & $4.742 E+05$ & $\mathrm{~Pa}$ \\
\hline $\mathrm{Pe}=$ exit pressure (absolute) & 1.242 & $\$ 1.013 \mathrm{E}+5 \mathrm{~Pa} / \mathrm{atm}$ & $1.259 \mathrm{E}+05$ & Pa \\
\hline$T e=$ sample temperature (absolute) & 296 & & 296 & ${ }^{\circ} \mathrm{K}$ \\
\hline $\mathrm{Tb}=$ flow measurement bas1s temperature (absolute) & 296 & & 296 & ${ }^{\circ} \mathrm{K}$ \\
\hline$z e$ - gas devlation factor at $P e$ and $T e$ & 1.0000 & & 1.0000 & \\
\hline $2 \mathrm{~b}$ - gas devlation factor at $\mathrm{Pb}$ and $\mathrm{Te}$ & 1.0000 & & 1.0000 & \\
\hline $\mathrm{Qb}=$ flow rate at base conditions & 0.00228 & $1.0 \mathrm{E}-6 \mathrm{~m}^{2} 3 / \mathrm{cm}^{-3}$ & $2.284 \mathrm{E}-09$ & $\mathrm{~m}^{\wedge} 3 / \mathrm{s}$ \\
\hline ve $=$ flow velocity at sample exit end & $1.895 \mathrm{E}-05$ & $* 1.0 \mathrm{E}-2 \mathrm{~m} / \mathrm{cm}$ & $1.095 \mathrm{E}-07$ & $\mathrm{~m} / \mathrm{s}$ \\
\hline $\mathrm{Ka}_{\mathrm{a}}=$ & $1.27 E-07$ & * $9.872 \mathrm{E}-13 \mathrm{~m} 2 / \mathrm{d}$ & $1.26 \mathrm{E}-19$ & $m^{2} 2$ \\
\hline Ka * & $1.27 \varepsilon-04$ & & $1.26 \mathrm{E}-15$ & $\mathrm{~cm}^{\wedge} 2$ \\
\hline $\mathrm{Ka}=$ & $1.27 \mathrm{E}-01$ & & & \\
\hline$\overline{P C}-P_{m}$ & 870.2 & & 6.00 & MPa \\
\hline
\end{tabular}




\begin{tabular}{|c|c|c|c|c|c|c|c|c|c|c|c|c|c|c|c|}
\hline \multicolumn{16}{|c|}{ Steady State Gas Permeability Data } \\
\hline Projec & $t:$ & 8362 & Target Ne & Eff.stress: & 6 & Mpa & 870.2 psld & Gas: & & N2 & & & & & \\
\hline Sample & $\#:$ & D & Length: & & 9.886 & $\mathrm{~cm}$ & & gas deviation $z$ & factors: & & & $\mathrm{ze}=$ & 1.0000 & $2 b=$ & 1.0000 \\
\hline Stress & Level i: & 2 & D1ameter: & & 10.168 & $\mathrm{~cm}$ & & V1scosity: & & 176 & $\mathrm{cp}$ & & & & \\
\hline Reglme & H: & 2 & Area: & & 81.201 & $\mathrm{~cm}^{\wedge} 2$ & & & & & & & & & \\
\hline \multicolumn{3}{|c|}{ Pressure Data Fll ename: } & $8362 D G .52$ & & & & & & & & & & & & \\
\hline XDCR C & al1bratior & tors: & $P C=$ & $222.869 \mathrm{ps} 1 \mathrm{~g}$ & & $\mathrm{P1}=$ & psig/volt & 11.0272 & psid/volt & & Pe & & 5.5211 & psig/volt & \\
\hline
\end{tabular}

\begin{tabular}{|c|c|c|c|c|c|c|c|c|c|c|c|c|}
\hline Date & $\begin{array}{l}\text { Time } \\
\text { of } \\
\text { Day }\end{array}$ & $\begin{array}{l}\text { File } \\
\text { Time } \\
\text { (mIn) }\end{array}$ & Reglme & $\begin{array}{l}\mathrm{Pb} \\
\text { Barometric } \\
\text { Pressure }\end{array}$ & $\begin{array}{l}\text { Pc } \\
\text { Confining } \\
\text { Pressure }\end{array}$ & $\begin{array}{l}\text { P1 } \\
\text { Inlet } \\
\text { Pressure }\end{array}$ & $\begin{array}{l}\Delta \mathrm{P} \\
\text { D1fferential } \\
\text { Pressure }\end{array}$ & $\begin{array}{l}\mathrm{Pm} \\
\text { Mean Pore } \\
\text { Pressure } \\
P_{\theta}+\Delta \mathrm{P} / 2\end{array}$ & $\begin{array}{l}\text { Pe } \\
\text { ExLt } \\
\text { Pressure }\end{array}$ & $\begin{array}{l}\text { Te } \\
\text { Flow } \\
\text { Temp } \\
\left({ }^{\circ} \mathrm{C}\right)\end{array}$ & $\begin{array}{c}\text { Tb } \\
\text { Amblent } \\
\text { Temp } \\
\left({ }^{\circ} \mathrm{C}\right\rangle\end{array}$ & $\begin{array}{c}\text { Ob } \\
\text { Flow Rate } \\
\text { OPbtrb } \\
(\mathrm{ml} / \mathrm{sec})\end{array}$ \\
\hline 16 Ju1 93 & $13: 47$ & 2735 & DS2R2a & $12.36 \mathrm{ps} 1 \mathrm{a}$ & volts & volts & 9.129 volts & & volts & 23 & 23 & 0.002449 \\
\hline 16 Jul 93 & $13: 52$ & 2740 & DS2R2b & $12.36 \mathrm{psia}$ & volts & volts & 9.131 volts & & voltg & 23 & 23 & 0.002424 \\
\hline 16 Jul 93 & $13: 57$ & 2745 & DS2R2C & $12.36 \mathrm{ps} 1 \mathrm{a}$ & volts & volts & 9.132 volts & & volts & 23 & 23 & 0.002444 \\
\hline 16 Jul 93 & $14: 01$ & 2750 & DS2R2d & $12.36 \mathrm{psia}$ & volts & volts & 9.132 volts & & volts & 23 & 23 & 0.002460 \\
\hline 16 Jul 93 & $14: 06$ & 2755 & DS2R2e & $12.36 \mathrm{ps} 1 \mathrm{a}$ & volts & volts & 9.133 volts & & volts & 23 & 23 & 0.002442 \\
\hline \multirow{9}{*}{ AVERAGES } & & & \multirow{9}{*}{ DS2R2 } & & OONOE & OUACE & DHEFERENTALI & OOXGE & GUAG & (6) & का & \\
\hline & & & & & 4.203 volts & 2.095 volts & 9.131 volts & & 2.931 & \multirow{4}{*}{23} & \multirow{4}{*}{23} & \\
\hline & & & & & 936.7 & 116.17 & 100.69 psid & $66.53 \mathrm{ps} 1 \mathrm{~g}$ & 16.183 & & & \\
\hline & & & & & 63.74 & 7.905 & 6.852 & $6.527 \mathrm{~atm}$ & 1.1012 & & & \\
\hline & & & & & 6.458 & 0.8010 & 0.6943 & 0.4587 & 0.11158 & & & \\
\hline & & & & ABSOLUTE & ABSOLUTE & ABSOLOTE & DRFFERENTIA & AESOLUTE & AaSOLOTE & $8 \%$ & (\%K) & $(\mathrm{m} 1 / \mathrm{sec})$ \\
\hline & & & & 12.36 ps1a & $949.1 \quad$ ps1a & $128.53 \quad$ ps1a & 100.69 ps1d & $78.89 \mathrm{ps1a}$ & 28.54 & \multirow{3}{*}{296} & \multirow{3}{*}{296} & \multirow{3}{*}{0.00244} \\
\hline & & & & $0.8410 \quad \mathrm{~atm}$ & 64.58 & 8.746 & 6.852 & $5.368 \mathrm{~atm}$ & 1.942 & & & \\
\hline & & & & $0.08522 \mathrm{Mpa}$ & 6.544 & 0.8862 & 0.6943 & 0.5439 & 0.1968 & & & \\
\hline
\end{tabular}

I

Apparent gas permeab1lity:

$K_{a}=\left(v e^{\star P e} e^{\star} u^{\star L}\right) /\left(P m^{\star} \Delta P\right)$

Boyle's Law:

$V_{e}=(P b / P e) \cdot(T e / T b) *(2 e / 2 b) \cdot V b$

$\mathrm{Qe}^{-}=(\mathrm{Pb} / \mathrm{Pe}) *(\mathrm{Te} / \mathrm{Tb}) \cdot(2 e / 2 \mathrm{~b}) * \mathrm{Qb}$

$v e=Q_{e} / \mathrm{A}=(\mathrm{Pb} / \mathrm{Pe}) *(\mathrm{Te} / \mathrm{Tb}) *(2 \mathrm{e} / \mathrm{Zb}) *(\mathrm{Ob} / \mathrm{A})$

\begin{tabular}{|c|c|c|c|c|}
\hline Parameter & $\begin{array}{l}\text { Traditlonal } \\
\text { Un1ts }\end{array}$ & & $\begin{array}{l}\text { SI } \\
\text { Units }\end{array}$ & \\
\hline$\mu=$ gas viscosity & 0.0176 & $\approx 1.0 \mathrm{E}-3 \mathrm{~Pa} \mathrm{~B}_{\mathrm{Bec} / \mathrm{CP}}$ & $1.760 \mathrm{E}-05$ & $\mathrm{~Pa} \star \mathrm{sec}$ \\
\hline L a sample length & 9.886 & $1.0 \mathrm{E}-2 \mathrm{~m} / \mathrm{cm}$ & $9.886 \mathrm{E}-02$ & $\mathrm{~m}$ \\
\hline A - sample clrcular cross sectional area & 81.201 & $1.0 \mathrm{E}-4 \mathrm{~m}^{\wedge} 2 / \mathrm{cm}^{\wedge} 2$ & $8.120 \mathrm{E}-03$ & $m^{2} 2$ \\
\hline $\mathrm{Pb}$ - flow measurement basis pressure (absolute) & 0.8410 & $\star 1.013 \mathrm{E}+5 \mathrm{~Pa} / \mathrm{atm}$ & $8.520 E+04$ & Po \\
\hline$\Delta \mathrm{P}=$ pressure drop across sample length & 6.852 & $\star 1.013 \mathrm{E}+5 \mathrm{~Pa} / \mathrm{atm}$ & $6.941 E+05$ & $\mathbf{P a}$ \\
\hline $\mathrm{Pm}=$ mean pore pressure (absolute) & 5.368 & $\star 1.013 \mathrm{E}+5 \mathrm{~Pa} / \mathrm{atm}$ & $5.438 E+05$ & $\overline{\mathrm{Pa}}$ \\
\hline$P_{\theta}=$ exit pressure (absolute) & 1.942 & $.1 .013 \mathrm{E}+5 \mathrm{~Pa} / \mathrm{atm}$ & $1.968 \varepsilon+05$ & Pa \\
\hline Te - sample temperature (absolute) & 296 & & 296 & OK \\
\hline $\mathrm{Tb}=$ flow measurement basis temperature (absolute) & 296 & & 296 & ${ }^{\circ} \mathrm{K}$ \\
\hline$z e=$ gas devlation factor at Pe and Te & 1.0000 & & 1.0000 & \\
\hline $\mathrm{zb}=$ gas devlacion factor at $\mathrm{Pb}$ and $\mathrm{Te}$ & 1.0000 & & 1.0000 & \\
\hline $\mathrm{Qb}=$ flow rate at base conditions & $0.00244 \mathrm{~cm}^{2} 3 / \mathrm{s}$ & $\pi 1.0 \mathrm{E}-6 \mathrm{~m}^{\wedge} 3 / \mathrm{cm}^{2} 3$ & $2.444 E-09$ & $m^{2} 3 / 5$ \\
\hline ve $=$ flow veloclty at sample exit end & $1.303 \mathrm{E}-05 \mathrm{~cm} / \mathrm{s}$ & $\star 1.0 \mathrm{E}-2 \mathrm{~m} / \mathrm{cm}$ & $1.303 \mathrm{E}-07$ & $\mathrm{~m} / \mathrm{s}$ \\
\hline $\mathrm{Ka}=$ & $1.20 \mathrm{E}-07$ & $\$ 9.872 \mathrm{E}-13 \mathrm{~m}^{4} 2 / \mathrm{d}$ & $1.18 \mathrm{E}-19$ & $m^{\wedge} 2$ \\
\hline $\mathrm{Ka}-$ & $1,20 \mathrm{E}-04$ & & $1.18 \mathrm{E}-15$ & $c m^{\wedge} 2$ \\
\hline $\mathrm{Ka}=$ & $1.20 \mathrm{E}-01$ & & & \\
\hline Pc - Pm $\rightarrow$ Actual N.E.S. & 870.2 & & 6.00 & $\mathrm{MPa}$ \\
\hline
\end{tabular}


Steady State Gas Permeability Data

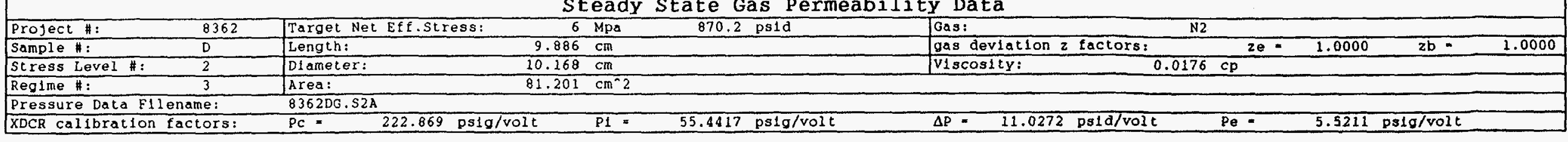

\begin{tabular}{|c|c|c|c|c|c|c|c|c|c|c|c|c|}
\hline Date & $\begin{array}{l}\text { Time } \\
\text { of } \\
\text { Day }\end{array}$ & $\begin{array}{l}\text { File } \\
\text { T1me } \\
\text { (m1n) }\end{array}$ & $\underset{\#}{\text { Regime }}$ & $\begin{array}{l}\mathrm{Pb} \\
\text { Barometric } \\
\text { Pressure }\end{array}$ & $\begin{array}{l}\text { Pc } \\
\text { Confining } \\
\text { Pressùre }\end{array}$ & $\begin{array}{l}\text { pi } \\
\text { Inlet } \\
\text { Pressure }\end{array}$ & $\begin{array}{l}\Delta P \\
D 1 \text { f ferentlal } \\
\text { Pressure }\end{array}$ & $\begin{array}{l}\text { Pm } \\
\text { Mean pore } \\
\text { Pressure } \\
\text { Pe+ }+\Delta P / 2\end{array}$ & $\begin{array}{l}\text { Pe } \\
\text { Exit } \\
\text { Pressure }\end{array}$ & $\begin{array}{l}\text { Te } \\
\text { Flow } \\
\text { Temp } \\
\left({ }^{\circ} \mathrm{C}\right)\end{array}$ & $\begin{array}{c}\text { Tb } \\
\text { Amblent } \\
\text { Temp } \\
\left({ }^{\circ} \mathrm{C}\right)\end{array}$ & $\begin{array}{c}Q b \\
\text { Flow Rate } \\
\text { Pb\&Tb } \\
\text { (ml/sec) }\end{array}$ \\
\hline 17 Ju1 93 & $14: 20$ & 4208 & DS2R3a & $12.35 \mathrm{ps} 1 \mathrm{a}$ & volts & volts & 9.050 volts & & 4.859 & 23 & 23 & 0.002590 \\
\hline 17 Jul 93 & $14: 25$ & 4213 & DS2R3b & $12.35 \mathrm{ps} 1 \mathrm{a}$ & volts & 2.271 & 9.051 volts & & volts & 23 & 23 & 0.002603 \\
\hline 17 Jul 93 & $14: 29$ & 4213 & DS2R3C & $12.35 \mathrm{psia}$ & volts & 2.271 & 9.052 volts & & volts & 23 & 23 & 0.002565 \\
\hline $17 \mathrm{Jul} 93$ & $14: 33$ & 4218 & $D S 2 R 3 d$ & $12.35 \mathrm{ps} 1 \mathrm{a}$ & volts & volts & 9.054 volts & & volts & 23 & 23 & 0.002562 \\
\hline 17 Jul 93 & $14: 37$ & 4223 & DS2R3e & 12.35 ps1a & volts & 2.271 & 9.054 volts & & volts & 23 & 23 & 0.002574 \\
\hline \multirow{9}{*}{ AVERAGES } & & & \multirow{9}{*}{ DS2R3 } & & OUAGE & OUAGE & DTFFERENTIAL & OOWGE & GUAOE & (9) & (1) & \\
\hline & & & & & 4.249 volts & 2.271 volts & 9.052 volts & & 4.859 & \multirow{4}{*}{23} & \multirow{4}{*}{23} & \\
\hline & & & & & 946.9 & 125.90 & 99.82 ps1d & $76.74 \mathrm{psig}$ & 26.827 & & & \\
\hline & & & & & 64.43 & 8.567 & 6.792 & $5.222 \mathrm{~atm}$ & 1.8255 & & & \\
\hline & & & & & $6.529 \quad \mathrm{Mpa}$ & 0.8680 & 0.6882 & $0.5291 \mathrm{Mpa}$ & 0.18497 & & & \\
\hline & & & & ABSOLUTE & ABSOLUTE & ABSOLUTE & DTFEERENTIAL & \multicolumn{2}{|l|}{ ABSOLUTE } & $(\circ)$ & $(0 \bar{x}\rangle$ & $\mathrm{m} / \mathrm{sec}$ \\
\hline & & & & $12.35 \mathrm{ps} 1 \mathrm{a}$ & 959.3 ps1a & 138.25 psla & 99.82 ps1d & 89.09 psia & 39.18 & \multirow{3}{*}{296} & \multirow{3}{*}{296} & \multirow{3}{*}{0.00258} \\
\hline & & & & $0.8404 \mathrm{~atm}$ & 65.27 & 9.407 & 6.792 & $6.052 \mathrm{~atm}$ & 2.666 & & & \\
\hline & & & & $0.08515 \mathrm{Mpa}$ & 6.614 & 0.9532 & 0.6882 & 0.6142 & 0.2701 & & & \\
\hline
\end{tabular}

$\stackrel{+}{\infty}$

Apparent gas permeability:

$K a=\left(v e * P^{*} u * L\right) /\left(P_{m} * \Delta P\right)$

Boyle's Law:

$\mathrm{Ve}=(\mathrm{Pb} / \mathrm{Pe}) *(\mathrm{Te} / \mathrm{Tb}) *(\mathrm{ze} / \mathrm{zb}) * \mathrm{Vb}$

Qe - $(\mathrm{Pb} / \mathrm{Pe}) *(\mathrm{Te} / \mathrm{Tb}) *(z e / z b) * \mathrm{Qb}$

$v e=Q_{e} / A=(P b / P e) *(T e / T b) *(z e / z b) *(Q b / A)$

\begin{tabular}{|c|c|c|c|c|}
\hline Parameter & $\begin{array}{l}\text { Traditional } \\
\text { Units }\end{array}$ & & $\begin{array}{l}\text { SI } \\
\text { Un1ts }\end{array}$ & \\
\hline$\mu=g a s$ viscosity & 0.0176 & $1.0 \mathrm{E}-3 \mathrm{~Pa}$ Sec/cp & $1.7608-05$ & $\mathrm{~Pa}^{\star} \mathrm{sec}$ \\
\hline$L=$ sample length & 9.886 & $* 1.0 \mathrm{E}-2 \mathrm{~m} / \mathrm{cm}$ & $9.886 \mathrm{E}-02$ & $m$ \\
\hline$A$ - sample clrcular cross sectional area & 81.201 & $\star 1.0 \mathrm{E}-4 \mathrm{~m}^{\wedge} 2 / \mathrm{cm}^{\wedge} 2$ & $8.120 E-03$ & $m^{2} 2$ \\
\hline $\mathrm{pb}=$ flow measurement basis pressure (absolute) & 0.8404 & $\$ 1.013 \mathrm{E}+5 \mathrm{~Pa} / \mathrm{atm}$ & $8.513 E+04$ & $\mathrm{~Pa}$ \\
\hline$\Delta P=$ pressure drop across sample length & 6.792 & $\$ 1.013 \mathrm{E}+5 \mathrm{~Pa} / \mathrm{atm}$ & $6.881 E+05$ & Pa \\
\hline $\mathrm{Pm}=$ mean pore pressure (absolute) & 6.062 & $\star 1.013 \mathrm{E}+5 \mathrm{~Pa} / \mathrm{atm}$ & $6.141 E+05$ & $\overline{P_{a}}$ \\
\hline $\mathrm{Pe}=$ exit pressure (absolute) & 2.666 & $\star 1.013 \mathrm{E}+5 \mathrm{~Pa} / \mathrm{atm}$ & $2.700 E+05$ & $\mathrm{~Pa}$ \\
\hline$T e=$ sample temperature (absolute) & 296 & & 296 & ${ }^{\circ} \mathrm{K}$ \\
\hline$T b=$ tlow measurement basis temperature (absolule) & 296 & & 296 & ${ }^{\circ} \mathrm{K}$ \\
\hline$z e=$ gas deviation factor at $P e$ and $T e$ & 1.0000 & & 1.0000 & \\
\hline $2 b=$ gas deviation factor $a t \mathrm{~Pb}$ and $\mathrm{Te}$ & 1.0000 & & 1.0000 & \\
\hline $\mathrm{Qb}=$ flow rate at base conditions & 0.00258 & $1.0 \mathrm{E}-6 \mathrm{~m}^{\wedge} 3 / \mathrm{cm}^{\wedge} 3$ & $2.580 \mathrm{E}-09$ & $m^{-3 / s}$ \\
\hline ve $=$ flow velocity at sample exit end & $1.002 \mathrm{E}-05$ & $\div 1.0 \mathrm{E}-2 \mathrm{~m} / \mathrm{cm}$ & $1.002 \mathrm{E}-07$ & $\mathrm{~m} / \mathrm{s}$ \\
\hline $\mathrm{K}_{\mathrm{a}}=$ & $1.13 \varepsilon-07$ & $\$ 9.872 \mathrm{E}-13 \mathrm{~m}^{\wedge} 2 / \mathrm{d}$ & $1.11 E-19$ & $m^{\wedge} 2$ \\
\hline $\mathrm{Ka}=$ & $1.13 E-04$ & & $1.11 \mathrm{E}-15$ & $\mathrm{~cm}^{\wedge} 2$ \\
\hline$K_{a}=$ & $1.13 \mathrm{E}-01$ & & & \\
\hline Pc $-\mathrm{Pm}=$ Actual N.E.S. & 870.2 & & 6.00 & $\mathrm{MPa}$ \\
\hline
\end{tabular}


Steady State Gas Permeability Data

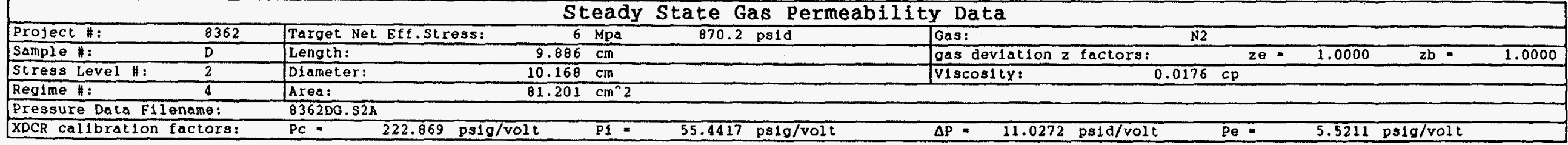

\begin{tabular}{|c|c|c|c|c|c|c|c|c|c|c|c|c|}
\hline Date & $\begin{array}{l}\text { Time } \\
\text { of } \\
\text { Day }\end{array}$ & $\begin{array}{l}\text { Elle } \\
\text { Time } \\
\text { (min) }\end{array}$ & $\underset{\#}{\text { Regime }}$ & $\begin{array}{l}\text { Pb } \\
\text { Barometric } \\
\text { Pressure }\end{array}$ & $\begin{array}{l}\text { Pc } \\
\text { Conflning } \\
\text { Pressure }\end{array}$ & $\begin{array}{l}\text { P1 } \\
\text { Inlet } \\
\text { Pressure }\end{array}$ & $\begin{array}{l}\Delta \mathrm{P} \\
\text { Differential } \\
\text { Pressure }\end{array}$ & $\begin{array}{l}\text { Pm } \\
\text { Mean Pore } \\
\text { Pressure } \\
\text { Pe+ }+\Delta \mathrm{P} / 2\end{array}$ & $\begin{array}{l}\text { Pe } \\
\text { Exit } \\
\text { Pressure }\end{array}$ & $\begin{array}{l}\text { Te } \\
\text { Flow } \\
\text { Temp } \\
\left({ }^{\circ} \mathrm{C}\right)\end{array}$ & $\begin{array}{c}\text { Tb } \\
\text { Amblent } \\
\text { Temp } \\
\left({ }^{\circ} \mathrm{C}\right)\end{array}$ & $\begin{array}{c}\text { Qb } \\
\text { Flow Rate } \\
\text { OPbstb } \\
\text { (m1/sec) }\end{array}$ \\
\hline 19 Jul 93 & $17: 37$ & 7285 & DS2R4a & 12.32 psia & volts & volts & 8.982 volts & & volts & 23 & 23 & 0.002709 \\
\hline 19 Jul 93 & $17: 41$ & 7290 & DS2R4b & 12.32 psla & volts & volts & 8.983 volts & & volts & 23 & 23 & 0.002700 \\
\hline 19 JuI 93 & $17: 45$ & 7295 & DS2R4C & 12.32 psia & 4.297 & vol ts & 8.982 volts & & volks & 23 & 23 & 0.002684 \\
\hline 19 Jul 93 & $17: 49$ & 7295 & DS2R4d & 12.32 ps1a & volts & volts & 8.983 volts & & volts & 23 & 23 & 0.002684 \\
\hline 19 Ju1 93 & $17: 53$ & 7300 & DS2R4e & $12.32 \mathrm{psia}$ & volts & volts & 8.983 volts & & volts & 23 & 23 & 0.002677 \\
\hline \multirow{9}{*}{ AVERAGES } & & & \multirow{9}{*}{ DS2R4 } & & OUAGE & OOAOE & DTFEERENTIAL & OOAGE & GOAOE & (ब) & एक्ल) & \\
\hline & & & & & 4.297 volts & 2.454 volts & 8.983 volts & & 6.858 & \multirow{4}{*}{23} & \multirow{4}{*}{23} & \\
\hline & & & & & 957.7 & 136.05 & 99.05 psid & $87.39 \mathrm{ps} 1 \mathrm{~g}$ & 37.865 & & & \\
\hline & & & & & 65.17 & 9.258 & 6.740 & $5.947 \mathrm{~atm}$ & 2.5765 & & & \\
\hline & & & & & 6.603 & 0.9381 & 0.6829 & $0.6025 \mathrm{Mpa}$ & 0.26107 & & & \\
\hline & & & & ABSOLUTE & ABSOLUTE & ABSOLUTE & DIFFERENTIAL & ABSOLUTE & ABSOLUTE & $(\circ \mathrm{K})$ & (OK) & $($ (nI/sec) \\
\hline & & & & $12.32 \mathrm{ps} 1 \mathrm{a}$ & 970.0 ps10 & $148.37 \quad \mathrm{ps} 1 \mathrm{a}$ & 99.05 ps1d & $99.71 \mathrm{ps} 1 \mathrm{a}$ & 50.18 & \multirow{3}{*}{296} & \multirow{3}{*}{296} & \multirow{3}{*}{0.00269} \\
\hline & & & & $0.8383 \mathrm{~atm}$ & 66.00 & 10.096 & 6.740 & 6.785 atm & 3.415 & & & \\
\hline & . & & & 0.08494 & 6.688 & 1.0230 & 0.6829 & 0.6875 & 0.3450 & & & \\
\hline
\end{tabular}

Apparent gas permeab111ty: $K a=(v e * P e * u * L) /(P m * \Delta P)$

Boyle's Law:

$V_{e}=(P b / P e) *(T e / T b) *(z e / z b) * V b$

$\mathrm{Qe}=(\mathrm{Pb} / \mathrm{Pe}) *(\mathrm{Te} / \mathrm{Tb}) *(2 \theta / 2 \mathrm{~b}) * \mathrm{Qb}$

$\mathrm{ve}=\mathrm{Qe} / \mathrm{A}=(\mathrm{Pb} / \mathrm{Pe}) \cdot(\mathrm{Te} / \mathrm{Tb}) *(\mathrm{ze} / \mathrm{zb}) *(\mathrm{Qb} / \mathrm{A})$

\begin{tabular}{|c|c|c|c|c|}
\hline Parameter & $\begin{array}{l}\text { Traditlonal } \\
\text { Und ts }\end{array}$ & & $\begin{array}{l}\text { SI } \\
\text { Undts }\end{array}$ & \\
\hline$\mu=g a s v 1 s \cos 1 t y$ & 0.0176 & $\star 1.0 \mathrm{E}-3 \mathrm{~Pa} * \mathrm{sec} / \mathrm{cp}$ & $1.760 \mathrm{E}-05$ & $p_{a} \star s e c$ \\
\hline$L=$ sample length & 9.886 & $1.0 \mathrm{E}-2 \mathrm{~m} / \mathrm{cm}$ & $9.886 \mathrm{E}-02$ & $\mathrm{~m}$ \\
\hline$A=$ sample circular cross sectlonal area & 81.201 & $-1.0 \mathrm{E}-4 \mathrm{~m}^{n} 2 / \mathrm{cm}^{2} 2$ & $8.120 E-03$ & $\mathrm{~m}^{\circ} 2$ \\
\hline $\mathrm{Pb}=$ flow measurement basis pressure (absolute) & 0.8383 & $* 1.013 \mathrm{E}+5 \mathrm{~Pa} / \mathrm{atm}$ & $8.492 \mathrm{E}+04$ & $\mathrm{~Pa}$ \\
\hline$\Delta \mathrm{P}$ - pressure drop across sample length & 6.740 & $=1.013 \mathrm{E}+5 \mathrm{~Pa} / \mathrm{atm}$ & $6.828 \mathrm{E}+05$ & $\mathrm{~Pa}$ \\
\hline $\mathrm{Pm}$ - mean pore pressure (absolute) & 6.785 & $\star 1.013 \mathrm{E}+5 \mathrm{~Pa} / \mathrm{atm}$ & $6.873 \mathrm{E}+05$ & $P_{Q}$ \\
\hline Pe $=$ exit pressure (absolute) & 3.415 & $\$ 1.013 \mathrm{E}+5 \mathrm{~Pa} / \mathrm{atm}$ & $3.459 \mathrm{E}+05$ & $\mathrm{~Pa}$ \\
\hline Te = sample temperature (absolute) & 296 & & 296 & ${ }^{\circ} \mathrm{K}$ \\
\hline $\mathrm{Tb}=$ flow measurement basis temperature (absolute) & 296 & & 296 & ${ }^{\circ} \mathrm{K}$ \\
\hline$z e=$ gas deviation factor at $P_{e}$ and $T e$ & 1.0000 & & 1.0000 & \\
\hline $2 b=$ gas deviation factor $a t \quad \mathrm{~Pb}$ and $\mathrm{Te}$ & 1.0000 & & 1.0000 & \\
\hline$a b=$ flow rate at base conditions & 0.00269 & $\pi 1.0 \mathrm{E}-6 \mathrm{~m}^{2} 3 / \mathrm{cm}^{2} 3$ & $2.694 \mathrm{E}-09$ & $m^{2} 3 / s$ \\
\hline ve = flow velocity at sample exit end & $8.146 \mathrm{E}-06$ & $1.0 \mathrm{E}-2 \mathrm{~m} / \mathrm{cm}$ & $8.146 \mathrm{E}-08$ & $\mathrm{~m} / \mathrm{s}$ \\
\hline $\mathrm{Ka}=$ & $1.06 \mathrm{E}-07$ & $\pi 9.872 \mathrm{E}-13 \mathrm{~m}^{\wedge} 2 / \mathrm{d}$ & $1.04 \mathrm{E}-19$ & $m^{2} 2$ \\
\hline $\mathrm{Ka}=$ & $1.06 \mathrm{E}-04$ & & $1.04 \mathrm{E}-15$ & $\mathrm{~cm}^{2} 2$ \\
\hline $\mathrm{Ka}=$ & $1.06 \mathrm{E}-01$ & & & \\
\hline$P_{c}-P_{m}=$ Actual N.E.S. & 870.3 & & 6.00 & MPa \\
\hline
\end{tabular}




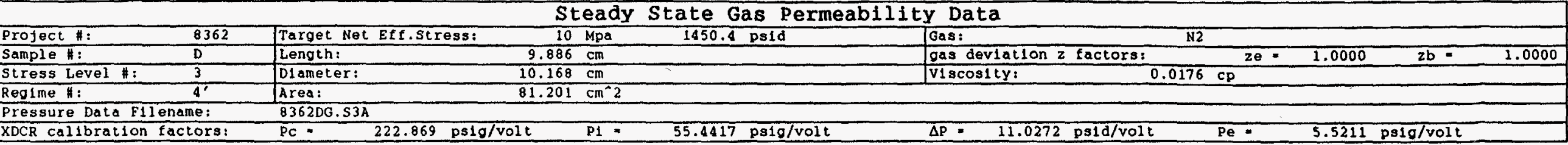

\begin{tabular}{|c|c|c|c|c|c|c|c|c|c|c|c|c|}
\hline Date & $\begin{array}{l}\text { Time } \\
\text { of } \\
\text { Day }\end{array}$ & $\begin{array}{l}\text { File } \\
\text { Time } \\
\text { (min) }\end{array}$ & $\begin{array}{c}\text { Regime } \\
\#\end{array}$ & $\begin{array}{l}\text { Pb } \\
\text { Barometric } \\
\text { Pressure }\end{array}$ & $\begin{array}{l}\text { Pc } \\
\text { Confining } \\
\text { Pressure }\end{array}$ & $\begin{array}{l}\text { PI } \\
\text { Inlet } \\
\text { Pressure }\end{array}$ & $\begin{array}{l}\Delta \mathrm{P} \\
\text { D1fferential } \\
\text { Pressure }\end{array}$ & 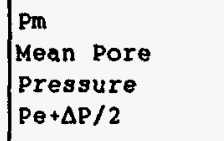 & $\begin{array}{l}\text { Pe } \\
\text { Exit } \\
\text { Pressure }\end{array}$ & $\begin{array}{l}\text { Te } \\
\text { Elow } \\
\text { Temp } \\
\left({ }^{\circ} \mathrm{C}\right)\end{array}$ & $\begin{array}{c}\text { Tb } \\
\text { Amblent } \\
\text { Temp } \\
\left({ }^{\circ} \mathrm{C}\right)\end{array}$ & $\begin{array}{c}\text { Ob } \\
\text { Flow Rate } \\
\text { OPb\&Tb } \\
\text { (ml/sec) }\end{array}$ \\
\hline 27 JuI 93 & $14: 36$ & 10262 & $D S 3 R 4^{\prime} a$ & $12.40 \mathrm{ps} 1 \mathrm{a}$ & 6.898 volts & 2.460 & 9.088 volts & & volts & 23 & 23 & 0.002014 \\
\hline $27 \mathrm{JuI} 93$ & $14: 41$ & 10267 & DS3R4'b & $12.40 \mathrm{ps} 1 \mathrm{a}$ & volis & volts & 9.087 volts & & volts & 23 & 23 & 0.001987 \\
\hline 27 Jul 93 & $14: 46$ & 10272 & $D S 3 R 4^{\prime} \mathrm{C}$ & 12.40 psia & volts & volts & 9.088 volts & & volts & 23 & 23 & 0.001970 \\
\hline 27 Jul 93 & $14: 52$ & 10277 & DS3R4'd & $12.40 \mathrm{ps} 1 \mathrm{a}$ & volts & volts & 9.089 volts & & voles & 23 & 23 & 0.001979 \\
\hline 27 Jul 93 & $14: 57$ & 10282 & DS3R4'e & $12.40 \mathrm{ps} 1 \mathrm{a}$ & 6.898 volts & volts & 9.089 volts & & volts. & 23 & 23 & 0.001993 \\
\hline \multirow{9}{*}{ AVERAGES } & & & \multirow{9}{*}{ DS3R4' } & & OUAGE & GUAGE & DCEFEREHTYII & coXGE: & OUAOE & (oc) & $\mathrm{res}$ & \\
\hline & & & & & 6.898 volts & 2.460 volts & 9.088 volts & & 6.688 & \multirow{4}{*}{23} & \multirow{4}{*}{23} & \\
\hline & & & & & 1537.4 & 136.39 & 100.22 ps1d & $87.03 \mathrm{psig}$ & 36.925 & & & \\
\hline & & & & & 104.61 & 9.281 & 6.819 & $5.922 \quad \mathrm{~atm}$ & 2.5126 & & & \\
\hline & & & & & 10.600 & 0.9404 & $0.6910 \quad \mathrm{Mpo}$ & $0.6001 \mathrm{Mpa}$ & 0.25459 & & & \\
\hline & & & & ABSOTUTE & ABSOLUTE & ABSOLUTE & DIFFERENTXX & ABSOLUTE & ABSOLOTE & (0x) & $(0 x)$ & $(\mathrm{nI} / \mathrm{sec})$ \\
\hline & & & & $12.4 \mathrm{ps} 1 \mathrm{a}$ & 1549.8 psia & 148.79 psla & 100.22 psld & 99.43 ps1a & 49.33 & \multirow{3}{*}{296} & \multirow{3}{*}{296} & \multirow{3}{*}{0.00199} \\
\hline & & & & $0.8438 \mathrm{~atm}$ & 105.45 & 10.124 & 6.819 & 6.766 atm & 3.356 & & & \\
\hline & & & & 0.08550 & 10.685 & 1.0258 & 0.6910 & 0.6856 & 0.3401 & & & \\
\hline
\end{tabular}

Apparent gas permeab111ty:

$R a=\left(v e * P e^{\star} u^{\star} L\right) /\left(P_{m} * \Delta P\right)$

Boyle's Law:

$\mathrm{Ve}=(\mathrm{Pb} / \mathrm{Pe}) \cdot(\mathrm{Te} / \mathrm{Tb}) \cdot\langle z e / \mathrm{zb}) \cdot \mathrm{Vb}$

$\mathrm{Qe}=(\mathrm{Pb} / \mathrm{Pe}) *(\mathrm{Te} / \mathrm{Tb}) *(z e / \mathrm{zb}) * \hat{\mathrm{Q}}$

$v_{e}=Q_{e} / A=\left(P b / P_{Q}\right) *(T e / T b) *(z e / z b) *(O b / A)$

\begin{tabular}{|c|c|c|c|c|}
\hline Parameter & $\begin{array}{l}\text { Traditional } \\
\text { Unlts }\end{array}$ & & $\begin{array}{l}\text { SI } \\
\text { Units }\end{array}$ & \\
\hline$\mu=\operatorname{gas} v 1 \operatorname{scos} 1 t y$ & 0.0176 & $\star 1.0 \mathrm{E}-3 \mathrm{~Pa} \mathrm{PaC}_{\mathrm{sep}}$ & $1.760 \mathrm{E}-05$ & $p_{a} * \sec$ \\
\hline $\mathrm{L}=$ sample length & 9.886 & $1.0 \mathrm{E}-2 \mathrm{~m} / \mathrm{cm}$ & $9.886 \mathrm{E}-02$ & m \\
\hline A - sample circular cross sectional area & 81.201 & $1.0 \mathrm{E}-4 \mathrm{~m}^{\wedge} 2 / \mathrm{cm}^{\wedge} 2$ & $8.120 \mathrm{E}-03$ & $m^{2} 2$ \\
\hline $\mathrm{Pb}=$ flow measurement basis pressure (absolute) & 0.8438 & $\star 1.013 \mathrm{E}+5 \mathrm{~Pa} / \mathrm{atm}$ & $8.547 \mathrm{E}+04$ & Pa \\
\hline$\Delta \mathrm{P}=$ pressure drop across sample length & 6.819 & $\star 1.013 \mathrm{E}+5 \mathrm{~Pa} / \mathrm{atm}$ & $6.908 \mathrm{E}+05$ & $\mathrm{~Pa}$ \\
\hline $\mathrm{Pm}_{\mathrm{m}}=$ mean pore pressure (absolute) & 6.766 & $\star 1.013 \mathrm{E}+5 \mathrm{~Pa} / \mathrm{atm}$ & $6.854 E+05$ & $\mathrm{~Pa}$ \\
\hline Pe $=$ exit pressure (absolute) & 3.356 & $\pi 1.013 \mathrm{E}+5 \mathrm{~Pa} / \mathrm{atm}$ & $3.400 E+05$ & $\mathrm{~Pa}$ \\
\hline$T e=$ sample temperature (absolute) & 296 & & 296 & ${ }^{\circ} \mathbf{K}$ \\
\hline $\mathrm{Tb}=$ flow measurement basis temperature (absolute) & 296 & & 296 & ${ }^{\circ} \mathrm{K}$ \\
\hline $2 e=$ gas deviation factor at $\mathrm{Pe}$ and $\mathrm{Te}$ & 1.0000 & & 1.0000 & \\
\hline $2 \mathrm{~b}=$ gas devlation factor $\mathrm{at} \mathrm{Pb}$ and $\mathrm{Te}$ & 1.0000 & & 1.0000 & \\
\hline$Q b=$ flow rate at base conditions & 0.00199 & $\star 1.0 \mathrm{E}-6 \mathrm{~m}^{-3} / \mathrm{cm}^{-3}$ & $1.987 \mathrm{E}-09$ & $\mathrm{~m}^{\wedge} 3 / \mathrm{s}$ \\
\hline ve = flow velocity at sample exit end & $6.152 E-06$ & * $1.0 \mathrm{E}-2 \mathrm{~m} / \mathrm{cm}$ & $6.152 \mathrm{E}-08$ & $\mathrm{~m} / \mathrm{s}$ \\
\hline $\mathrm{ar}-\mathrm{Ka}=$ & $7.79 E-08$ & $\star 9.872 E-13 m^{\wedge} 2 / d$ & $7.69 \mathrm{E}-20$ & $m^{n} 2$ \\
\hline $\mathrm{Ka}=$ & $7.79 E-05$ & & $7.69 \mathrm{E}-16$ & $\mathrm{~cm}^{n} 2$ \\
\hline $\mathrm{Ka}=$ & $7.79 \mathrm{E}-02$ & & & \\
\hline Pc $-P_{m}=$ Actual N.E.S. & 1450.3 & & 10.00 & $\mathrm{MPa}$ \\
\hline
\end{tabular}




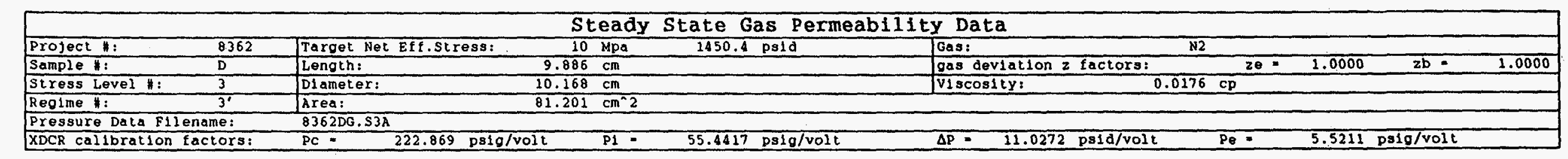

\begin{tabular}{|c|c|c|c|c|c|c|c|c|c|c|c|c|}
\hline Date & $\begin{array}{l}\text { Time } \\
\text { of } \\
\text { Day }\end{array}$ & $\begin{array}{l}\text { File } \\
\text { Time } \\
\text { (min) }\end{array}$ & $\underset{\#}{\text { Reglme }}$ & $\begin{array}{l}\mathrm{Pb} \\
\text { Barometr1c } \\
\text { Pressure }\end{array}$ & $\begin{array}{l}\text { Pc } \\
\text { Confining } \\
\text { Pressure }\end{array}$ & $\begin{array}{l}\text { P1 } \\
\text { Inlet } \\
\text { Pressure }\end{array}$ & $\left\{\begin{array}{l}\Delta P \\
D 1 \text { f ferential } \\
\text { Pressure }\end{array}\right.$ & $\begin{array}{l}\text { Pm } \\
\text { Mean Pore } \\
\text { Pressure } \\
\mathrm{Pe}+\Delta \mathrm{P} / 2\end{array}$ & $\mid \begin{array}{l}P \theta \\
\text { Exit } \\
\text { Pressure }\end{array}$ & $\begin{array}{l}\text { Te } \\
\text { Flow } \\
\text { Temp } \\
\left({ }^{\circ} \mathrm{C}\right)\end{array}$ & $\begin{array}{c}\text { Tb } \\
\text { Amblent } \\
\text { Temp } \\
\left({ }^{\circ} \mathrm{C}\right)\end{array}$ & $\begin{array}{c}Q b \\
\text { Flow Rate } \\
\text { Ppbtb } \\
(\mathrm{ml} / \mathrm{sec})\end{array}$ \\
\hline 28 Jul 93 & $08: 47$ & 11353 & DS3R3'a & $12.38 \mathrm{ps} 1 \mathrm{a}$ & volts & volts & 9.136 volts & & volts & 23 & 22 & 0.001837 \\
\hline 28 Jul 93 & $08: 53$ & 11358 & $D S 3 R 3^{\prime} \mathrm{b}$ & 12.38 ps1a & volts & volts & 9.137 volts & & volts & 23 & 22 & 0.001822 \\
\hline 28 Ju1 93 & $08: 58$ & 11363 & $D S 3 R 3^{\prime} \mathrm{C}$ & $12.38 \mathrm{ps} 1 \mathrm{Q}$ & volts & 2.270 & 9.137 volts & & volts & 23 & 22 & 0.001828 \\
\hline 28 Jul 93 & $09: 04$ & 11368 & $D S 33^{\prime} d$ & $12.38 \mathrm{ps} 1 \mathrm{~s}$ & volts & 2.270 & 9.138 volts & & volts & 23 & 22 & 0.001847 \\
\hline 28 Jul 93 & $09: 10$ & 11373 & DS3R3'e & $12.38 \quad \mathrm{ps} 1 \mathrm{a}$ & volts & 2.270 & 9.139 yolts & & volts & 23 & 22 & 0.001831 \\
\hline \multirow{9}{*}{ AVERAGES } & & & \multirow{9}{*}{ DS3R3' } & & BUAGE & BUAGE & TOYFFERENT WL & OUXGE & 6UWhOB & खा & \%०1 & \\
\hline & & & & & 6.849 volts & 2.270 volts & 9.137 volts & & 4.687 & \multirow{4}{*}{23} & \multirow{4}{*}{22} & \\
\hline & & & & & 1526.4 & 125.85 & 100.76 psid & $76.26 \mathrm{psig}$ & 25.877 & & & \\
\hline & & & & & 103.87 & 8.564 & 6.856 & 5.189 atm & 1.7608 & & & \\
\hline & & & & & 10.524 & 0.8677 & $0.6947 \mathrm{Mpa}$ & $0.5258 \mathrm{Mpa}$ & 0.17842 & & & \\
\hline & & & & ABSOLUTE & ABSOLUTE & ABSOLUTE & ORFEERENT TAL & ABSOLUTE & ABSOLTTR & (OK) & $(0 \times)$ & (nin $/ 506)$ \\
\hline & & & & $12.38 \mathrm{ps} 1 \mathrm{a}$ & 1538.8 ps1a & 138.23 psia & 100.76 psid & 88.64 psia & 38.26 & \multirow{3}{*}{296} & \multirow{3}{*}{295} & \multirow{3}{*}{0.00183} \\
\hline & & & & 0.8424 atm & 104.71 & 9.406 & 6.856 & $6.031 \mathrm{~atm}$ & 2.603 & & & \\
\hline & & & & $0.08536 \mathrm{Mpa}$ & 20.610 & 0.9531 & 0.6947 & 0.6111 & 0.2638 & & & \\
\hline
\end{tabular}

Apparent gas permeability:

$K a=(v e * P e * u * L) /\left(P m^{\star} \Delta P\right)$

Boyle's Law:

$\mathrm{Ve}=(\mathrm{Pb} / \mathrm{Pe}) *(\mathrm{Te} / \mathrm{Tb}) \cdot(\mathrm{ze} / \mathrm{zb}) * \mathrm{Vb}$

$Q e=(\mathrm{Pb} / \mathrm{Pe}) *(\mathrm{Te} / \mathrm{Tb}) *(2 \mathrm{e} / \mathrm{zb}) * \mathrm{Qb}$

ve $=\mathrm{Qe} / \mathrm{A}=(\mathrm{Pb} / \mathrm{Pe}) *(\mathrm{Te} / \mathrm{Tb}) *(2 e / 2 \mathrm{~b}) \cdot(\mathrm{Qb} / \mathrm{A})$

\begin{tabular}{|c|c|c|c|c|}
\hline Parameter & $\begin{array}{l}\text { Traditional } \\
\text { Un1ts }\end{array}$ & & $\begin{array}{l}\text { SI } \\
\text { Unt ts }\end{array}$ & \\
\hline$\mu=$ gas viscosity & 0.0176 & $\approx 1.0 \mathrm{E}-3 \mathrm{pa} * \mathrm{sec} / \mathrm{CD}$ & $1.760 \mathrm{E}-05$ & Pa\#sec \\
\hline$L=$ sample length & 9.886 & $\star 1.0 \mathrm{E}-2 \mathrm{~m} / \mathrm{cm}$ & $9.886 \mathrm{E}-02$ & $m$ \\
\hline A = sample clrcular cross sectlonal area & 81.201 & $1.0 \mathrm{E}-4 \mathrm{~m}^{\wedge} 2 / \mathrm{cm}^{\wedge} 2$ & $0.120 \mathrm{E}-03$ & $m^{* 2} 2$ \\
\hline $\mathrm{Pb}=$ flow measurement basis pressure (absolute) & 0.8424 & * $1.013 \mathrm{E}+5 \mathrm{~Pa} / \mathrm{atm}$ & $8.534 E+04$ & $\mathrm{~Pa}$ \\
\hline$\Delta \mathrm{p}=$ pressure drop across sample length & 6.856 & $\star 1.013 \mathrm{E}+5 \mathrm{~Pa} / \mathrm{atm}$ & $6.945 E+05$ & Pa \\
\hline $\mathrm{Pm}_{\mathrm{m}}=$ mean pore pressure (absolute) & 6.031 & $\pi 1.013 \mathrm{E}+5 \mathrm{~Pa} / \mathrm{atm}$ & $6.110 \mathrm{E}+05$ & Pa \\
\hline$p_{e}=$ exit pressure (absolute) & 2.603 & $\star 1.013 \mathrm{E}+5 \mathrm{~Pa} / \mathrm{atm}$ & $2.637 E+05$ & pa \\
\hline Te = sample temperature (absolute) & 296 & & 296 & ${ }^{\circ} \mathrm{K}$ \\
\hline $\mathrm{Tb}=$ flow measurement basls temperature (absolute) & 295 & & 295 & $\sigma_{\mathrm{K}}$ \\
\hline$z e=$ gas deviation factor at pe and Te & 1.0000 & & 1.0000 & \\
\hline $2 b=$ gas deviation tactor $a t$ pb and Te & 1.0000 & & 1.0000 & \\
\hline$\overline{Q b}=$ flow rate at base conditlons & $0.00183 \mathrm{~cm}^{\wedge} 3 / \mathrm{s}$ & $1.0 \mathrm{E}-6 \mathrm{~m}^{\wedge} 3 / \mathrm{cm}^{\wedge} 3$ & $1.833 \mathrm{E}-09$ & $m^{2} 3 / s$ \\
\hline ve $=$ flow velocity at samplo exit end & $7.330 \mathrm{E}-06 \mathrm{~cm} / \mathrm{s}$ & * $1.0 \mathrm{E}-2 \mathrm{~m} / \mathrm{cm}$ & $7.330 E-08$ & $\mathrm{~m} / \mathrm{s}$ \\
\hline $\mathrm{Ka}=$ & $8.03 E-08$ & $\star 9.872 \mathrm{E}-13 \mathrm{~m}^{\wedge} 2 / \mathrm{d}$ & $7.93 \mathrm{E}-20$ & $m^{2} 2$ \\
\hline $\mathrm{Ka}=$ & $8.03 E-05$ & & $7.93 E-16$ & $\mathrm{~cm}^{2} 2$ \\
\hline $\mathrm{Ka}=$ & $8.03 E-02$ & & & \\
\hline $\mathrm{PC}-\mathrm{Pm}=$ Actual N.E.S. & 1450.2 & & 10.00 & $\mathrm{MPa}$ \\
\hline
\end{tabular}


Steady State Gas Permeability Data

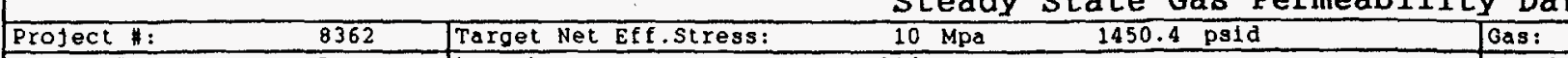

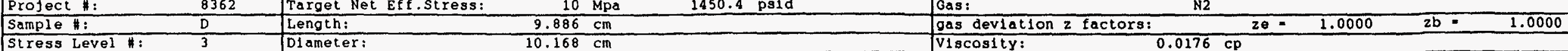

Stress Level \#: 3 - 3 Diameter:

2. Area:

$81.201 \mathrm{~cm}^{2} 2$

V1scosity: $0.0176 \mathrm{cP}$

$83620 \mathrm{DG} .53 \mathrm{~A}$

XDCR callbration factors: $\quad \mathrm{PC}=222.869 \mathrm{ps}=2 \mathrm{~g} / \mathrm{volt} \quad \mathrm{PI}=55.4417 \mathrm{ps} 1 \mathrm{~g} / \mathrm{volt}$ $\Delta \mathrm{P}=\quad 11.0272 \mathrm{ps} 1 \mathrm{~d} / \mathrm{volt}$

$\mathrm{Pe}^{-}=$

$5.5211 \mathrm{ps} 1 \mathrm{~g} / \mathrm{volt}$

\begin{tabular}{|c|c|c|c|c|c|c|c|c|c|c|c|c|}
\hline Date & $\begin{array}{l}\text { TIme } \\
\text { of } \\
\text { Day }\end{array}$ & $\begin{array}{l}\text { File } \\
\text { Time } \\
(m 1 n)\end{array}$ & $\underset{\#}{R \in g 1 m e}$ & $\begin{array}{l}\text { Pb } \\
\text { Barometric } \\
\text { Pressure }\end{array}$ & $\begin{array}{l}\text { Pc } \\
\text { Confining } \\
\text { Pressure }\end{array}$ & $\begin{array}{l}\text { P1 } \\
\text { Inlet } \\
\text { Pressure }\end{array}$ & $\begin{array}{l}\Delta P \\
\text { Differential } \\
\text { Pressure }\end{array}$ & $\begin{array}{l}\text { Pm } \\
\text { Mean Pore } \\
\text { Pressure } \\
\text { Pe }+\Delta P / 2\end{array}$ & $\begin{array}{l}\text { Pe } \\
\text { Ex1t } \\
\text { Pressure }\end{array}$ & $\begin{array}{l}\text { Te } \\
\text { Flow } \\
\text { Temp } \\
\left({ }^{\circ} \mathrm{C}\right)\end{array}$ & $\begin{array}{c}\text { Tb } \\
\text { Amblent } \\
\text { Temp } \\
\left({ }^{\circ} \mathrm{C}\right)\end{array}$ & $\begin{array}{c}\text { Ob } \\
\text { Flow Rate } \\
\text { OPbsTb } \\
(\mathrm{ml} / \mathrm{sec})\end{array}$ \\
\hline $29 \mathrm{Ju} 193$ & $10: 54$ & 12917 & DS 3R2'a & $12.38 \quad \mathrm{ps} 1 \mathrm{a}$ & 6.805 volts & 2.097 volts & 9.176 volts & & volts & 23 & 23 & 0.001712 \\
\hline 2934193 & $11: 00$ & 12922 & $\mathrm{DS} 3 \mathrm{R} 2^{\prime} \mathrm{b}$ & 12.38 ps1a & 6.805 & volts & 9.175 vol ts & & volts & 23 & 23 & 0.001704 \\
\hline 29 Jul 93 & $11: 05$ & 12932 & DS3R2' C & $12.38 \mathrm{psta}$ & 6.805 & volts & 9.176 volts & & volts & 23 & 23 & 0.001699 \\
\hline 29 Jul 93 & $11: 11$ & 12937 & DS3R2'd & 12.38 psia & 6.805 & volts & 9.177 volts & & volts & 23 & 23 & 0.001699 \\
\hline 29 Ju1 93 & $12: 21$ & 12947 & DS3R2'e & $12.38 \mathrm{psia}$ & 6.805 & volts & 9.177 volts & & volts & 23 & 23 & 0.001704 \\
\hline \multirow{9}{*}{ AVERAGES } & & & \multirow{9}{*}{ DS3R2' } & & OUAGE & OUACE & DYFFERENTIAL & GUXGE & OUAOE & (ब) & (oc) & \\
\hline & & & & & 6.805 volts & 2.097 volts & 9.176 volts & & 2.860 & \multirow{4}{*}{23} & \multirow{4}{*}{23} & \\
\hline & & & & & 1516.6 & 116.27 & 101.19 psid & $66.39 \mathrm{ps1g}$ & 15.791 & & & \\
\hline & & & & & 103.20 & 7.912 & 6.885 & $4.517 \mathrm{~atm}$ & 1.0745 & & & \\
\hline & & & & & 10.457 & 0.8017 & 0.6977 & 0.4577 Mpa & 0.10888 & & & \\
\hline & & & & ABSOLUTE & ABSOLUTE & ABSOLUTE & DIFFERENTIAL & ABSOEUTE & ABSOLUTE & (0x) & $(0 \mathrm{~K})$ & $\mathrm{(ml} / \mathrm{sec})$ \\
\hline & & & & $12.38 \mathrm{p3} 1 \mathrm{a}$ & 1529.0 ps1a & 128.65 ps10 & 101.19 psid & 78.77 psia & 28.17 & \multirow{3}{*}{296} & \multirow{3}{*}{296} & \multirow{3}{*}{0.001704} \\
\hline & & & & $0.8424 \quad a \mathrm{tm}$ & 104.04 & 8.754 & 6.885 & 5.360 atm & 1.917 & & & \\
\hline & & & & 0.08536 & 10.542 & 0.8870 & 0.6977 & 0.5431 & 0.1942 & & & \\
\hline
\end{tabular}

岕

Apparent gas permeab1l1ty:

Boyle's Law:

$K a=\left(v e^{*} P^{*} u * L\right) /(P m * \Delta P)$

$v_{e}=(\mathrm{Pb} / \mathrm{Pe}) *(\mathrm{Te} / \mathrm{Tb}) *(2 \mathrm{Ce} / \mathrm{zb}) * \mathrm{Vb}$

$\mathrm{Qe}=(\mathrm{Pb} / \mathrm{Pe}) *(\mathrm{Te} / \mathrm{Tb})=\{\mathrm{za} / z \mathrm{zb}) * Q b$

$v e=Q_{e} / A=(\mathrm{Pb} / \mathrm{Pe}) *(\mathrm{Te} / \mathrm{Tb}) *(2 \mathrm{e} / \mathrm{Zb}) \cdot(\mathrm{Ob} / \mathrm{A})$

\begin{tabular}{|c|c|c|c|c|}
\hline parameter & $\begin{array}{l}\text { Traditional } \\
\text { Units }\end{array}$ & & $\begin{array}{l}\text { SI } \\
\text { Undts }\end{array}$ & \\
\hline$\mu=\operatorname{gas} v 1 \mathrm{scos} 1 t y$ & 0.0176 & $\star 1.0 \mathrm{E}-3 \mathrm{~Pa} \mathrm{Pec}_{\mathrm{sep}}$ & $1.760 \mathrm{E}-05$ & $\mathrm{~Pa}$ sec \\
\hline$L_{1}=$ sample length & 9.886 & $\star 1.0 \mathrm{E}-2 \mathrm{~m} / \mathrm{cm}$ & $9.886 \mathrm{E}-02$ & $\mathrm{~m}$ \\
\hline$A$ = sample clrcular cross sectional area & 81.201 & $1.0 E-4 \mathrm{~m}^{2} 2 / \mathrm{cm}^{2} 2$ & $8.120 E-03$ & $m^{2} 2$ \\
\hline $\mathrm{Pb}=$ flow measurement basls pressure (absolute) & 0.8424 & $\$ 1.013 \mathrm{E}+5 \mathrm{~Pa} / \mathrm{atm}$ & $8.534 E+04$ & Pa \\
\hline$\Delta \mathrm{P}=$ pressure drop across sample length & 6.885 & $\star 1.013 \mathrm{E}+5 \mathrm{~Pa} / \mathrm{atm}$ & $6.975 E+05$ & Pa \\
\hline $\mathrm{Pm}_{\mathrm{m}}=$ mean pore pressure (absolute) & 5.360 & $\$ 1.013 \mathrm{E}+5 \mathrm{~Pa} / \mathrm{atm}$ & $5.429 E+05$ & $\mathrm{~Pa}$ \\
\hline $\mathrm{Pe}=$ exit pressure (absolute) & 1.917 & $\star 1.013 \mathrm{E}+5 \mathrm{~Pa} / \mathrm{atm}$ & $1.942 \mathrm{E}+05$ & Pa \\
\hline $\mathrm{Te}$ - sample temperature (absolute) & 296 & & 296 & ${ }^{\circ} \mathrm{K}$ \\
\hline $\mathrm{Tb}=$ flow measurement basls temperature (absolute) & 296 & & 296 & ${ }^{\circ} \mathrm{K}$ \\
\hline $\mathrm{Ze}=$ gas devlation factor at $\mathrm{Pe}$ and $\mathrm{Te}$ & 1.0000 & & 1.0000 & \\
\hline $2 b=$ gas devtation factor $a t \quad P b$ and $\mathrm{Te}$ & 1.0000 & & 1.0000 & \\
\hline$Q b=$ flow rate at base conditions & 0.00170 & $1.0 \mathrm{E}-6 \mathrm{~m}^{\wedge} 3 / \mathrm{cm}^{\wedge} 3$ & $1.704 E-09$ & $m^{\wedge} 3 / s$ \\
\hline ve = flow velocity at sample exit end & $9.220 \mathrm{E}-06$ & $\pi 1.0 \mathrm{E}-2 \mathrm{~m} / \mathrm{cm}$ & $9.220 \mathrm{E}-08$ & $\mathrm{~m} / \mathrm{s}$ \\
\hline $\mathrm{Ka}=$ & $8.33 \mathrm{E}-08$ & $9.872 \mathrm{E}-13 \mathrm{~m}^{\wedge} 2 / \mathrm{d}$ & $8.23 \mathrm{E}-20$ & $m^{2} 2$ \\
\hline$K_{a}=$ & $8.33 E-05$ & & $8.23 E-16$ & $\mathrm{~cm}^{\wedge} 2$ \\
\hline $\mathrm{Ka}=$ & $8.33 \mathrm{E}-02$ & & & \\
\hline$P C-P m$ & 1450.2 & & 10.00 & MPa \\
\hline
\end{tabular}




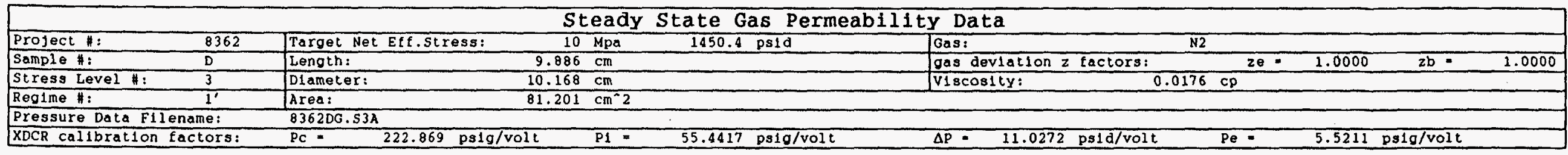

\begin{tabular}{|c|c|c|c|c|c|c|c|c|c|c|c|c|}
\hline Date & $\begin{array}{l}\text { Time } \\
\text { of } \\
\text { Day }\end{array}$ & $\begin{array}{l}\text { Flle } \\
\text { Time } \\
\text { (min) }\end{array}$ & $\begin{array}{c}\text { Regime } \\
\#\end{array}$ & $\begin{array}{l}\text { pb } \\
\text { Barometric } \\
\text { Pressure }\end{array}$ & $\begin{array}{l}\text { Pc } \\
\text { Confining } \\
\text { Pressure }\end{array}$ & $\begin{array}{l}\text { P1 } \\
\text { Inlet } \\
\text { Pressure }\end{array}$ & $\begin{array}{l}\Delta P \\
\text { D1fferential } \\
\text { Pressure }\end{array}$ & $\begin{array}{l}\text { Pm } \\
\text { Mean Pore } \\
\text { Pressure } \\
\text { Pe }+\Delta P / 2\end{array}$ & $\begin{array}{l}\text { Pe } \\
\text { Ex1t } \\
\text { Pressure }\end{array}$ & $\begin{array}{l}\text { Te } \\
\text { Flow } \\
\text { Temp } \\
\left({ }^{\circ} \mathrm{C}\right)\end{array}$ & $\begin{array}{c}\text { Tb } \\
\text { Amblent } \\
\text { Temp } \\
\left({ }^{\circ} \mathrm{C}\right)\end{array}$ & $\begin{array}{c}\text { Qb } \\
\text { Flow Rate } \\
\text { PbsTb } \\
\text { (ml/sec) }\end{array}$ \\
\hline 30 Jul 93 & $15: 31$ & 14637 & DS3R1'a & $12.42 \mathrm{psta}$ & volts & volts & 9.174 volts & & volts & 23 & 23 & 0.001578 \\
\hline 30 Jul 93 & $15: 37$ & 14642 & $D S 3 R 1^{\prime} b$ & 12.42 psia & volts & volts & 9.174 volts & & volts & 23 & 23 & 0.001568 \\
\hline $30 \mathrm{JuI} 93$ & $15: 56$ & 14662 & $\mathrm{DS} 3 \mathrm{R} 1^{\prime} \mathrm{C}$ & 12.42 ps $1 \mathrm{a}$ & volts & volts & 9.175 volts & & volts & 23 & 23 & 0.001553 \\
\hline 30 Jul 93 & $16: 10$ & 14677 & DS3R1'd & 12.42 ps1a & volts & volts & 9.175 volts & & volts & 23 & 23 & 0.001544 \\
\hline 30 Ju1 93 & $16: 16$ & 14682 & DS3R1'e & 12.42 ps10 & volts & volts & 9.175 volts & & volts & 23 & 23 & 0.001548 \\
\hline \multirow{9}{*}{ AVERAOES } & & & \multirow{9}{*}{ DS3R1' } & & GUACE & GOXGE & DTFEERENTIAL & GUAGE & GUROE & का & (9) & \\
\hline & & & & & 6.758 volts & $\begin{array}{ll}1.918 & \text { volts }\end{array}$ & 9.175 volts & & 1.049 & \multirow{4}{*}{23} & \multirow{4}{*}{23} & \\
\hline & & & & & 1506.1 & 106.34 & 101.17 psid & $56.38 \mathrm{psig}$ & 5.792 & & & \\
\hline & & & & & 102.49 & 7.236 & 6.884 & $3.836 \mathrm{~atm}$ & 0.3941 & & & \\
\hline & & & & & 10.385 & 0.7332 & 0.6975 & 0.3887 & 0.03993 & & & \\
\hline & & & & ABSOLUTE: & ABSOLLUTE & ABSOLUTE & DTEEERENTT AL & AESOLUTE & ABSOLOTE & (क) & (OK) & $(\mathrm{m} / \mathrm{sec})$ \\
\hline & & & & $12.42 \mathrm{ps} 1 \mathrm{a}$ & 1518.6 ps1a & 118.76 psla & 101.17 ps1d & 68.80 ps1a & 18.21 & \multirow{3}{*}{296} & \multirow{3}{*}{296} & \multirow{3}{*}{0.001561} \\
\hline & & & & $0.8451 \quad \mathrm{~atm}$ & 103.33 & 8.081 & 6.884 & $4.681 \mathrm{~atm}$ & 1.239 & & & \\
\hline & & & & $0.08563 \mathrm{Mpa}$ & 10.470 & 0.8188 & 0.6975 & 0.4743 & 0.1256 & & & \\
\hline
\end{tabular}

\begin{tabular}{|c|c|c|c|c|}
\hline Parameter & $\begin{array}{l}\text { Traditional } \\
\text { Un1ts }\end{array}$ & & $\begin{array}{l}\text { SI } \\
\text { Units }\end{array}$ & \\
\hline$\mu=g a s v 1 s \cos 1 t y$ & 0.0176 & $* 1.0 \mathrm{E}-3 \mathrm{~Pa} * \mathrm{sec} / \mathrm{cp}$ & $1.760 \mathrm{E}-05$ & $\mathrm{~Pa} \star \mathrm{sec}$ \\
\hline$L=$ sample length & 9.886 & $\star 1.0 \mathrm{E}-2 \mathrm{~m} / \mathrm{cm}$ & $9.886 \mathrm{E}-02$ & $m$ \\
\hline A - sample clrcular cross sectlonal area & 81.201 & $1.0 \mathrm{E}-4 \mathrm{~m}^{\wedge} 2 / \mathrm{cm}^{2} 2$ & $8.120 E-03$ & $m^{n} 2$ \\
\hline $\mathrm{pb}=$ flow measurement basis pressure (absolute) & 0.8451 & $\approx 1.013 \mathrm{E}+5 \mathrm{~Pa} / \mathrm{atm}$ & $8.561 E+04$ & $\mathrm{~Pa}$ \\
\hline$\Delta \mathrm{P}=$ pressure drop across sample length & 6.884 & $1.013 \mathrm{E}+5 \mathrm{~Pa} / \mathrm{atm}$ & $6.974 E+05$ & $\overline{P a}$ \\
\hline Pm = mean pore pressure (absolute) & 4.681 & $1.013 \mathrm{E}+5 \mathrm{~Pa} / \mathrm{atm}$ & $4.742 E+05$ & Pa \\
\hline$P e=$ exlt pressure (absolute) & 1.239 & $\star 1.013 \mathrm{E}+5 \mathrm{~Pa} / \mathrm{atm}$ & $1.255 \mathrm{E}+05$ & Pa \\
\hline Te = sample temperature (absolute) & 296 & & 296 & ${ }^{\circ} \mathrm{K}$ \\
\hline $\mathrm{Tb}=$ flow measurement bas1s temperature (absolute) & 296 & & 296 & ${ }^{\circ} \mathrm{K}$ \\
\hline$z e=$ gas deviation factor at $\mathrm{Pe}$ and $\mathrm{Te}$ & 1.0000 & & 1.0000 & \\
\hline $2 \mathrm{D}=$ gas deviation factor at $\mathrm{Pb}$ and $\mathrm{Te}$ & 1.0000 & & 1.0000 & \\
\hline$Q b=$ flow rate at base conditions & $0.00156 \mathrm{~cm}^{2} 3 / \mathrm{s}$ & * $1.0 \mathrm{E}-6 \mathrm{~m}^{\wedge} 3 / \mathrm{cm}^{\wedge} 3$ & $1.561 \mathrm{E}-09$ & $m^{4} 3 / 3$ \\
\hline ve $=$ flow velocity at sample exit end & $1.311 \mathrm{E}-05 \mathrm{~cm} / \mathrm{s}$ & $1.0 \mathrm{E}-2 \mathrm{~m} / \mathrm{cm}$ & $1.311 \mathrm{E}-07$ & $\mathrm{~m} / \mathrm{s}$ \\
\hline $\mathrm{Ka}-\mathrm{Ka}-$ & $8.77 E-08$ & $\star 9.872 \mathrm{E}-13 \mathrm{~m}^{\wedge} 2 / \mathrm{d}$ & $8.66 \mathrm{E}-20$ & $m^{\wedge} 2$ \\
\hline $\mathrm{Ka}-$ & $8.77 E-05$ & & $8.66 \mathrm{E}-16$ & $\mathrm{~cm}^{\wedge} 2$ \\
\hline $\mathrm{Ka}=$ & $8.77 \varepsilon-02$ & & & \\
\hline Pc $-P_{m}=$ ACtual N.E.S. & 1449.8 & & 10.00 & $\mathrm{MPa}$ \\
\hline
\end{tabular}




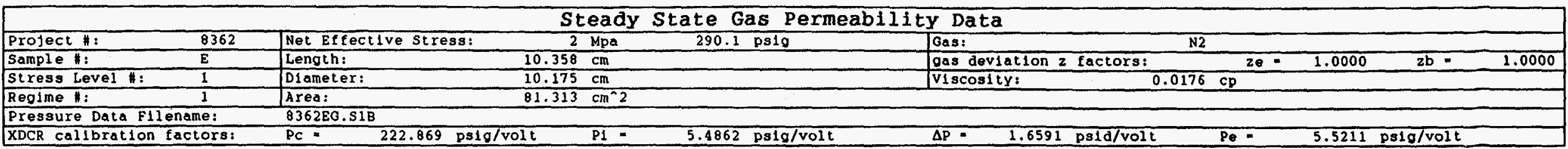

\begin{tabular}{|c|c|c|c|c|c|c|c|c|c|c|c|c|}
\hline Date & $\begin{array}{l}\text { Time } \\
\text { of } \\
\text { DaY }\end{array}$ & $\begin{array}{l}\text { F1le } \\
\text { Time } \\
\text { (min) }\end{array}$ & $\begin{array}{c}\text { Reg1me } \\
\end{array}$ & \begin{tabular}{|l} 
Pb \\
Barometrle \\
Pressure
\end{tabular} & $\begin{array}{l}\text { Pc } \\
\text { Confining } \\
\text { Pressure }\end{array}$ & $\begin{array}{l}\text { PI } \\
\text { Inlet } \\
\text { Pressure }\end{array}$ & $\mid \begin{array}{l}\Delta P \\
\text { DLfferential } \\
\text { Pressure }\end{array}$ & $\begin{array}{l}\mathrm{Pm} \\
\text { Mean Pore } \\
\text { Pressure } \\
\mathrm{Pe}+\Delta \mathrm{P} / 2\end{array}$ & $\begin{array}{l}\text { Pe } \\
\text { Exit } \\
\text { Pressure }\end{array}$ & $\begin{array}{l}\text { Te } \\
\text { Flow } \\
\text { Temp } \\
\left({ }^{\circ} \mathrm{C}\right)\end{array}$ & $\begin{array}{c}\text { Tb } \\
\text { Amblent } \\
\text { Temp } \\
\left({ }^{\circ} \mathrm{C}\right)\end{array}$ & $\begin{array}{c}\text { Ob } \\
\text { Flow Rate } \\
\text { epbeTb } \\
\text { (ml/sec) }\end{array}$ \\
\hline 16 Apr 93 & $11: 32$ & 1454 & ES1R1a & $12.32 \mathrm{ps} 1 \mathrm{a}$ & volts & volts & 1.857 volts & & volts & 23 & 22 & 0.13136 \\
\hline 16 Apr 93 & $21: 36$ & 1459 & ES1R1b & $12.32 \mathrm{ps} 1 \mathrm{a}$ & volts & volts & 1.857 volts & & volts & 23 & 22 & 0.13098 \\
\hline $16 \mathrm{Apr} 93$ & $11: 39$ & 1459 & ESIR1C & $12.32 \mathrm{ps} 1 \mathrm{a}$ & volts & volts & 1.856 volts & & volts & 23 & 22 & 0.13119 \\
\hline $16 \mathrm{Apr} 93$ & $11: 42$ & 1464 & ES1R1d & $12.32 \mathrm{ps} 1 \mathrm{a}$ & volts & 0.729 & 1.856 volts & & volts & 23 & 22 & 0.13106 \\
\hline \multirow{9}{*}{ AVERAGES } & & & \multirow{9}{*}{ ESIRI } & & GOACE & OUAOE & DrFERREMTII & GUAGE: & Gunce & 60 & Tol & \\
\hline & & & & & 1.313 volts & 0.729 volts & 1.857 volts & & 0.178 & \multirow{4}{*}{23} & \multirow{4}{*}{22} & \\
\hline & & & & & 292.5 & psig & $3.080 \quad \mathrm{ps} 1 \mathrm{~d}$ & $2.523 \mathrm{psig}$ & 0.9828 & & & \\
\hline & & & & & 19.90 & 0.272 & 0.2096 & 0.1717 atm & 0.06687 & & & \\
\hline & & & & & 2.017 & 0.0276 & $0.02124 \mathrm{Mpa}$ & 0.01739 Мpa & 0.006776 & & & \\
\hline & & & & ABSOLUTE & ABSOLUTE & ABSOLUTE & DIFFERENTIXL & A BSOLUTE & ABSOLOTE: & $(6 \mathrm{x})$ & rox & (nITsec) \\
\hline & & & & 12.32 pgla & 304.8 ps1a & $16.32 \quad \mathrm{ps} 1 \mathrm{a}$ & 3.080 ps1d & 14.84 ps1a & 13.30 & \multirow{3}{*}{296} & \multirow{3}{*}{295} & \multirow{3}{*}{0.13115} \\
\hline & & & & $0.8383 \mathrm{~atm}$ & 20.74 & 1.110 & 0.2096 & $1.010 \quad \mathrm{tm}$ & 0.9052 & & & \\
\hline & & & & $0.08494 \mathrm{Mpa}$ & 2.102 & 0.1125 & 0.02128 & 0.1023 & 0.09172 & & & \\
\hline
\end{tabular}

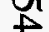

Apparent gas permeabillty:

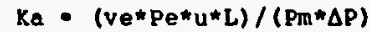

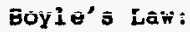

$\mathrm{V}_{\theta}=(\mathrm{Pb} / \mathrm{Pe}) *(\mathrm{Te} / \mathrm{Tb}) *(2 \mathrm{ze} / \mathrm{zb}) * \mathrm{Vb}$

$\mathrm{Qe} \cdot(\mathrm{Pb} / \mathrm{Pe}) \cdot(\mathrm{Te} / \mathrm{Tb}) *(\mathrm{ze} / \mathrm{Zb}) \cdot \mathrm{Qb}$

$v_{e}=Q_{e} / A \cdot(\mathrm{Pb} / \mathrm{Pe}) *(T e / T b) *(2 e / 2 b) *(Q b / A)$

\begin{tabular}{|c|c|c|c|c|}
\hline Parameter & $\begin{array}{l}\text { Traditional } \\
\text { Units }\end{array}$ & & $\begin{array}{l}\text { SI } \\
\text { Units }\end{array}$ & \\
\hline$\mu=$ gas v1scosity & 0.0176 & $1.0 \mathrm{E}-3 \mathrm{~Pa} \star \mathrm{SeC} / \mathrm{cp}$ & $1.760 \mathrm{E}-05$ & $\mathrm{~Pa} \star \mathrm{sec}$ \\
\hline$L=$ sample length & 10.358 & $1.0 \mathrm{E}-2 \mathrm{~m} / \mathrm{cm}$ & $1.036 \mathrm{E}-01$ & $m$ \\
\hline A - sample circular cross sectional area & 81.313 & $1.0 \mathrm{E}-4 \mathrm{~m}^{2} 2 / \mathrm{cm}^{2} 2$ & $8.131 E-03$ & $m^{2} 2$ \\
\hline $\mathrm{pb}=$ flow measurement basis pressure (absolute) & 0.8383 & $" 1.013 \mathrm{E}+5 \mathrm{~Pa} / \mathrm{atm}$ & $8.492 \mathrm{E}+04$ & $\mathrm{~Pa}$ \\
\hline$\Delta \mathrm{P}=$ pressure drop across sample length & 0.210 & $1.013 \mathrm{E}+5 \mathrm{~Pa} / \mathrm{atm}$ & $2.123 E+04$ & $\mathrm{~Pa}$ \\
\hline $\mathrm{P}_{\mathrm{m}}=$ mean pore pressure (absolute) & 1.010 & $=1.013 \mathrm{E}+5 \mathrm{~Pa} / \mathrm{atm}$ & $1.023 E+0 S$ & $\mathrm{~Pa}$ \\
\hline Pe - exit pressure (absolute) & 0.905 & $1.013 \mathrm{E}+5 \mathrm{~Pa} / \mathrm{atm}$ & $9.270 E+04$ & Pa \\
\hline Te - sample temperature (absolute) & 296 & & 296 & ${ }^{\circ} \mathrm{K}$ \\
\hline $\mathrm{Tb}=$ flow measurement basls temperature (absolute) & 295 & & 295 & ${ }^{\circ} \mathrm{K}$ \\
\hline$z e=$ gas deviation factor at $\mathrm{Pe}$ and $\mathrm{Te}$ & 1.0000 & & 1.0000 & \\
\hline $\mathrm{zb}=$ gas deviation factor $\mathrm{at} \mathrm{Pb}$ and $\mathrm{Te}$ & 1.0000 & & 1.0000 & \\
\hline $\mathrm{Qb}=$ flow rate at base conditions & $0.13115 \mathrm{~cm}^{\wedge} 3 / \mathrm{s}$ & $1.0 \mathrm{E}-6 \mathrm{~m}^{2} 3 / \mathrm{cm}^{2} 3$ & $1.311 E-07$ & $m^{n} 3 / 3$ \\
\hline ve = flow velocity at sample exit end & $1.499 \mathrm{E}-03 \mathrm{~cm} / \mathrm{s}$ & $1.0 \mathrm{E}-2 \mathrm{~m} / \mathrm{cm}$ & $1.499 \mathrm{E}-05$ & $\mathrm{~m} / \mathrm{s}$ \\
\hline $\mathrm{K}_{\mathrm{a}}=$ & $1.17 E-03$ & $9.872 \mathrm{E}-13 \mathrm{~m}^{\wedge} 2 / \mathrm{d}$ & $1.15 E-15$ & $m^{2} 2$ \\
\hline $\mathrm{Ka}_{\mathbf{a}} \cdot$ & 1.17 & & $1.15 E-11$ & $\mathrm{~cm}^{2} 2$ \\
\hline $\mathrm{Ka}=$ & 1168 & & & \\
\hline
\end{tabular}




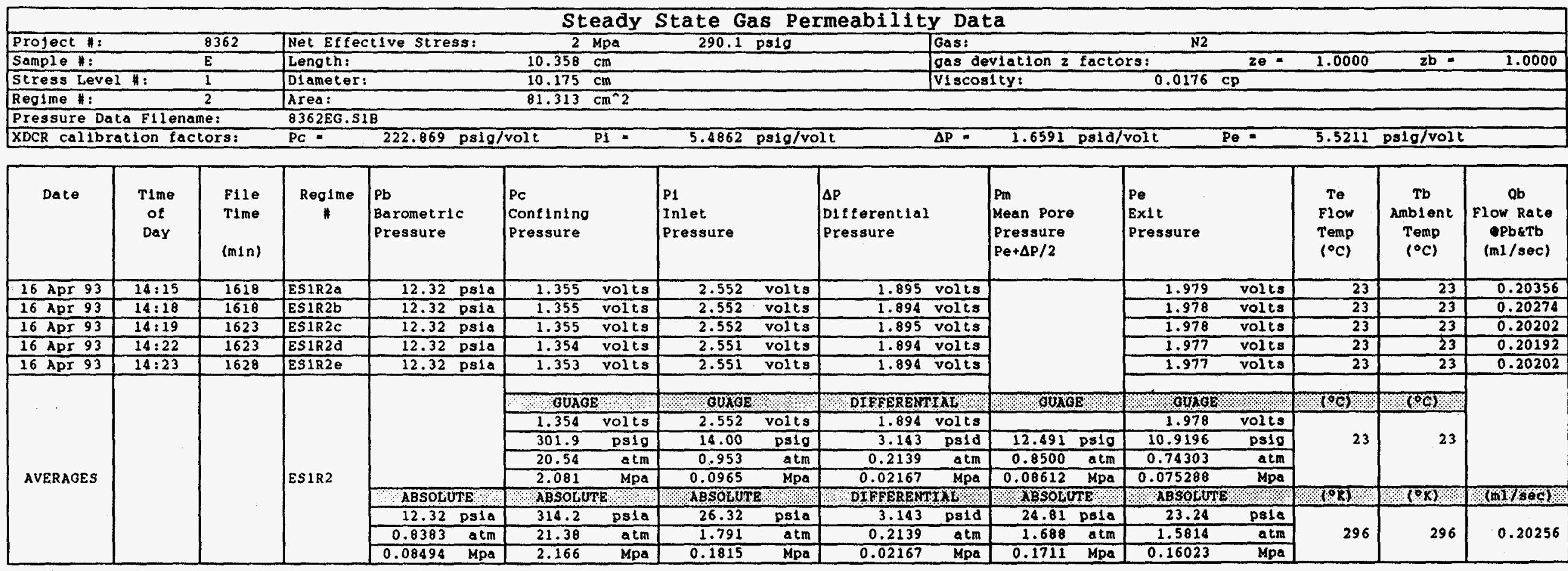

un

Apparent gas permeab1l1ty:

$K a=\left(v e * P e^{\star} u * L\right) /(P m \star \Delta P)$

Boyle's Law:

$\mathrm{Ve}=(\mathrm{Pb} / \mathrm{Pe}) \cdot(\mathrm{Te} / \mathrm{Tb}) *(\mathrm{ze} / \mathrm{zb}) * \mathrm{Vb}$

$\mathrm{Qe}=(\mathrm{Pb} / \mathrm{Pe}) *(\mathrm{Te} / \mathrm{Tb}) *(z \mathrm{ze} / \mathrm{zb}) * \mathrm{Qb}$

ve $=\mathrm{Qe} / \mathrm{A}-(\mathrm{Pb} / \mathrm{Pe}) \cdot(\mathrm{Te} / \mathrm{Tb}) *(\mathrm{ze} / \mathrm{zb}) *(\mathrm{Qb} / \mathrm{A})$

\begin{tabular}{|c|c|c|c|c|}
\hline Parameter & $\begin{array}{l}\text { Traditlonal } \\
\text { Untts }\end{array}$ & & $\begin{array}{l}\text { SI } \\
\text { Un1ts }\end{array}$ & \\
\hline$\mu \cdot$ gas viscosity & 0.0176 & $\star 1.0 \mathrm{E}-3 \mathrm{~Pa} * \mathrm{sec} / \mathrm{cp}$ & $1.760 \mathrm{E}-05$ & Pa*sec \\
\hline$L=$ sample length & 10.358 & $\star 1.0 \mathrm{E}-2 \mathrm{~m} / \mathrm{cm}$ & $1.036 \mathrm{E}-01$ & $\mathrm{~m}$ \\
\hline A - sample clrcular cross sectlonal area & 81.313 & $1.0 \mathrm{E}-4 \mathrm{~m}^{\wedge} 2 / \mathrm{cm}^{\wedge} 2$ & $8.131 \mathrm{E}-03$ & $m^{\star 2} 2$ \\
\hline $\mathrm{pb}=$ flow measurement bas1s pressure (absolute) & 0.8383 & $\approx 1.013 \mathrm{E}+5 \mathrm{~Pa} / \mathrm{atm}$ & $8.492 \mathrm{E}+04$ & $\mathrm{~Pa}$ \\
\hline$\Delta \mathrm{P}=$ pressure drop across sample length & 0.2139 & $* 1.013 E+5 \mathrm{~Pa} / \mathrm{atm}$ & $2.166 \mathrm{E}+04$ & $\overline{P a}$ \\
\hline $\mathrm{P}_{m}=$ mean pore pressure (absolute) & 1.688 & $\star 1.013 \mathrm{E}+5 \mathrm{~Pa} / \mathrm{atm}$ & $1.710 \mathrm{E}+05$ & $\mathbf{P a}$ \\
\hline $\mathrm{Pe}=$ exit pressure (absolute) & 1.581 & $\star 1.013 \mathrm{E}+5 \mathrm{~Pa} / \mathrm{atm}$ & $1.602 \mathrm{E}+05$ & $\mathrm{~Pa}$ \\
\hline Te = sample temperature (absolute) & 296 & & 296 & ${ }^{\circ} \mathrm{K}$ \\
\hline $\mathrm{Tb}=$ flow measurement basis temperature (absolute) & 296 & & 296 & ${ }^{\circ} \mathrm{K}$ \\
\hline$z e=$ gas deviation factor at $P e$ and $T e$ & 1.0000 & & 1.0000 & \\
\hline$z b=$ gas deviation factor $a t \quad \mathrm{~Pb}$ and $\mathrm{Te}$ & 1.0000 & & 1.0000 & \\
\hline $\mathrm{Qb}=$ flow rate at base conditions & $0.20256 \mathrm{~cm}^{2} 3 / \mathrm{s}$ & $1.0 \mathrm{E}-6 \mathrm{~m}^{\wedge} 3 / \mathrm{cm}^{\wedge} 3$ & $2.026 \mathrm{E}-07$ & $m^{\wedge} 3 / s$ \\
\hline ve = flow veloclty at sample exit end & $1.321 \mathrm{E}-03 \mathrm{~cm} / \mathrm{s}$ & $1.0 \mathrm{E}-2 \mathrm{~m} / \mathrm{cm}$ & $1.321 E-05$ & $\mathrm{~m} / \mathrm{s}$ \\
\hline $\mathrm{Ka}=$ & $1.05 E-03$ & $0.872 \mathrm{E}-13 \mathrm{~m} \mathrm{~m}^{\wedge} 2 / \mathrm{d}$ & $1.04 \mathrm{E}-15$ & $m^{\wedge} 2$ \\
\hline $\mathrm{Ka}=$ & 1.05 & & $1.04 E-11$ & $\mathrm{~cm}^{2} 2$ \\
\hline $\mathrm{Ka}=$ & 1054 & & & \\
\hline
\end{tabular}


Steady State Gas Permeability Data

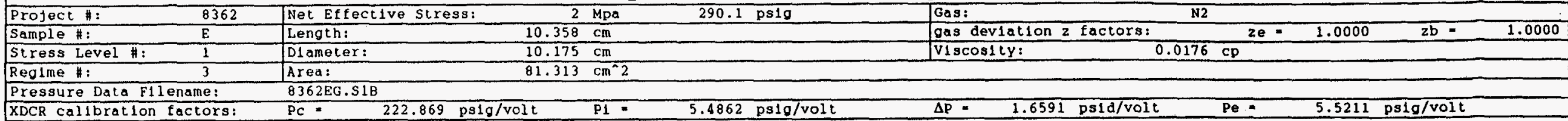

\begin{tabular}{|c|c|c|c|c|c|c|c|c|c|c|c|c|}
\hline Date & $\begin{array}{l}\text { Time } \\
\text { of } \\
\text { Day }\end{array}$ & $\begin{array}{l}\text { File } \\
\text { Time } \\
(m 1 n)\end{array}$ & $\underset{\#}{\text { Reglme }}$ & $\begin{array}{l}\mathrm{Pb} \\
\text { Barometric } \\
\text { Pressure }\end{array}$ & $\begin{array}{l}\text { Pc } \\
\text { Confining } \\
\text { Pressure }\end{array}$ & $\begin{array}{l}\text { PI } \\
\text { Inlet } \\
\text { Pressure }\end{array}$ & $\begin{array}{l}\Delta P \\
D 1 f \text { ferential } \\
\text { Pressure }\end{array}$ & $\begin{array}{l}\mathrm{Pm} \\
\text { Mean pore } \\
\text { Pressure } \\
\mathrm{Pe}+\Delta \mathrm{P} / 2\end{array}$ & $\begin{array}{l}\text { Pe } \\
\text { Ex1t } \\
\text { Pressure }\end{array}$ & $\begin{array}{l}\text { Te } \\
\text { Flow } \\
\text { Temp } \\
\left({ }^{\circ} \mathrm{C}\right)\end{array}$ & $\begin{array}{c}\text { Tb } \\
\text { Amblent } \\
\text { Temp } \\
\left({ }^{\circ} \mathrm{C}\right)\end{array}$ & $\begin{array}{c}\text { Ob } \\
\text { Flow Rate } \\
\text { Pb\&Tb } \\
\text { (ml/sec) }\end{array}$ \\
\hline 16 Apr 93 & $16: 30$ & 1753 & ES1R3a & $12.32 \mathrm{ps} 1 \mathrm{a}$ & volts & volts & 1.867 volts & & volts & 23 & 23 & 0.26508 \\
\hline $16 \mathrm{Apr} 93$ & $16: 34$ & 1753 & ES1R3b & 12.32 psia & 1.405 volts & 4.376 volts & 1.866 volts & & volts & 23 & 23 & 0.26438 \\
\hline 16 Apr 93 & $16: 36$ & 1758 & ES1R3C & $12.32 \mathrm{psla}$ & volts & volts & 1.869 volts & & volts & 23 & 23 & 0.26438 \\
\hline 16 Apr 93 & $16: 37$ & 1758 & ES1R3d & $12.32 \mathrm{ps} 1 \mathrm{a}$ & volts & volts & 1.868 volts & & volts & 23 & 23 & 0.26560 \\
\hline \multirow{9}{*}{ AVERAGES } & & & \multirow{9}{*}{ ESIR3 } & & COUACE & OUAOE & DTFEEREMTIAL & OOAGE & GUরG & rel & $\mathrm{PQT}$ & \\
\hline & & & & & 1.405 volts & 4.377 volts & 1.868 volts & & 3.795 & \multirow{4}{*}{23} & \multirow{4}{*}{23} & \\
\hline & & & & & 313.1 & 24.01 & 3.098 ps1d & $22.500 \mathrm{psig}$ & 20.9512 & & & \\
\hline & & & & & 21.30 & 1.634 & 0.2108 & $1.5311 \mathrm{~atm}$ & 1.42564 & & & \\
\hline & & & & & $2.159 \quad \mathrm{Mpa}$ & 0.1656 & $0.02136 \quad \mathrm{MpA}$ & $0.15513 \mathrm{Mpa}$ & 0.144453 & & & \\
\hline & & & & ABSOLUTE & ABSOLUTE & ABSOLUTE & DTFFERENTIXL & ABSOLUTE & ABSOLUTE & (6) & $(9)$ & $(\mathrm{ml} / \mathrm{sec})$ \\
\hline & & & & $12.32 \mathrm{psia}$ & $325.4 \quad \mathrm{ps} 1 \mathrm{a}$ & $36.33 \quad$ ps1a & $3.098 \quad$ ps1d & $34.82 \mathrm{ps} 1 \mathrm{a}$ & 33.27 & \multirow{3}{*}{296} & \multirow{3}{*}{296} & \multirow{3}{*}{0.26486} \\
\hline & & & & $0.8383 \mathrm{~atm}$ & 22.14 & 2.472 & 0.2108 & $2.369 \quad \mathrm{~atm}$ & 2.2640 & & & \\
\hline & & & & $0.08494 \quad \mathrm{Mpa}$ & 2.244 & 0.2505 & 0.02136 & 0.2401 & 0.22940 & & & \\
\hline
\end{tabular}

ă

Apparent gas permeability:

$K_{a}=\left(V e^{*} P e^{*} u * L\right) /\left(P m^{*} \Delta P\right)$

Boyle's Law:

$v_{e}=(\mathrm{Pb} / \mathrm{Pe}) *(\mathrm{Te} / \mathrm{Tb}) \cdot(\mathrm{ze} / \mathrm{zb}) \cdot \mathrm{Vb}$

$\mathrm{Qe}=(\mathrm{Pb} / \mathrm{Pe}) *(\mathrm{Te} / \mathrm{TD}) *(z e / z \mathrm{D}) * \mathrm{QD}$

$v e=Q e / A=(P b / P e) \cdot(T e / T b) *(Z e / Z b) *(Q b / A)$

\begin{tabular}{|c|c|c|c|c|}
\hline Parameter & $\begin{array}{l}\text { Traditional } \\
\text { Units }\end{array}$ & & $\begin{array}{l}\text { SI } \\
\text { Undts }\end{array}$ & \\
\hline$\mu=g a s v i s \cos 1 t y$ & 0.0176 & $-1.0 \mathrm{E}-3 \mathrm{~Pa} * \mathrm{sec} / \mathrm{CP}$ & $1.760 \mathrm{E}-05$ & pa*sec \\
\hline$L=$ sample length & 10.358 & $\star 1.0 \mathrm{E}-2 \mathrm{~m} / \mathrm{cm}$ & $1.036 \mathrm{E}-01$ & $\mathrm{~m}$ \\
\hline A sample clrcular cross sectlonal area & 81.313 & $2.0 \mathrm{E}-4 \mathrm{~m}^{2} 2 / \mathrm{cm}^{2} 2$ & $8.131 E-03$ & $m^{\wedge} 2$ \\
\hline $\mathrm{Pb}$ - flow measurement basis pressure (absolute) & 0.8383 & $1.013 \mathrm{E}+5 \mathrm{~Pa} / \mathrm{atm}$ & $8.492 E+04$ & $\mathrm{~Pa}$ \\
\hline$\overline{\Delta P}$ - pressure drop across sample length & 0.2108 & $\star 1.013 E+5 \quad \mathrm{~Pa} / \mathrm{atm}$ & $2.136 \mathrm{E}+04$ & Pa \\
\hline Pm - mean pore pressure (absolute) & 2.369 & $1.013 \mathrm{E}+5 \mathrm{~Pa} / \mathrm{atm}$ & $2.400 E+05$ & $\mathrm{~Pa}$ \\
\hline Pe $=$ exit pressure (absolute) & 2.264 & $1.013 \mathrm{E}+5 \mathrm{~Pa} / \mathrm{atm}$ & $2.293 \mathrm{E}+05$ & Pa \\
\hline Te = sample temperature (absolute) & 296 & & 296 & ${ }^{\circ} \mathrm{K}$ \\
\hline$T b=$ flow measurement basls temperature (absolute) & 296 & & 296 & ${ }^{\circ} \mathrm{K}$ \\
\hline $2 e$ - gas deviation factor at $\mathrm{Pe}$ and $\mathrm{Te}$ & 1.0000 & & 1.0000 & \\
\hline $\mathrm{zb}=$ gas devlation tactor at $\mathrm{pb}$ and $\mathrm{Te}$ & 1.0000 & & 1.0000 & \\
\hline$a b=$ flow rate at base conditions & $0.26486 \mathrm{~cm}^{\wedge} 3 / \mathrm{s}$ & $1.0 \mathrm{E}-6 \mathrm{~m}^{\wedge} 3 / \mathrm{cm}^{\wedge} 3$ & $2.649 \mathrm{E}-07$ & $m^{2} 3 / s$ \\
\hline ve = flow velocity at sample exit end & $1.206 \mathrm{E}-03 \mathrm{~cm} / \mathrm{s}$ & $1.0 \mathrm{E}-2 \mathrm{~m} / \mathrm{cm}$ & $1.206 \mathrm{E}-05$ & $\mathrm{~m} / \mathrm{s}$ \\
\hline $\mathrm{Ka}=$ & $9.97 \mathrm{E}-04$ & $.9 .872 \mathrm{E}-13 \mathrm{~m}^{\wedge} 2 / \mathrm{d}$ & $9.84 \mathrm{E}-16$ & $m^{2} 2$ \\
\hline$K_{a}=$ & 1.00 & & $9.84 E-12$ & $\mathrm{~cm}^{\wedge} 2$ \\
\hline $\mathrm{Ka}=$ & 997 & & & \\
\hline
\end{tabular}


Steady State Gas Permeability Data

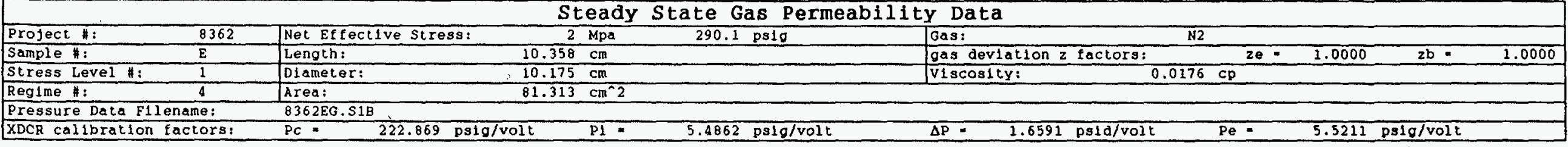

\begin{tabular}{|c|c|c|c|c|c|c|c|c|c|c|c|c|}
\hline Date & $\begin{array}{l}\text { Time } \\
\text { of } \\
\text { Day }\end{array}$ & $\begin{array}{l}\text { File } \\
\text { Time } \\
(m 1 n)\end{array}$ & $\underset{*}{\text { Reglme }}$ & $\begin{array}{l}\text { pb } \\
\text { Barometric } \\
\text { pressure }\end{array}$ & $\begin{array}{l}\text { Pc } \\
\text { Confining } \\
\text { Pressure }\end{array}$ & $\begin{array}{l}\text { P1 } \\
\text { Inlet } \\
\text { Pressure }\end{array}$ & $\begin{array}{l}\Delta P \\
D 1 \text { fferential } \\
\text { Pressure }\end{array}$ & $\begin{array}{l}\text { Pm } \\
\text { Mean pore } \\
\text { Pressure } \\
\text { Pe }+\Delta P / 2\end{array}$ & $\begin{array}{l}\text { Pe } \\
\text { Exit } \\
\text { Pressure }\end{array}$ & $\begin{array}{l}\text { Te } \\
\text { Flow } \\
\text { Temp } \\
\left({ }^{\circ} \mathrm{C}\right)\end{array}$ & $\begin{array}{c}\text { Tb } \\
\text { Amb 1ent } \\
\text { Temp } \\
\left({ }^{\circ} \mathrm{C}\right)\end{array}$ & $\begin{array}{c}\text { Qb } \\
\text { Flow Rate } \\
\text { pabtb } \\
(\mathrm{ml} / \mathrm{sec})\end{array}$ \\
\hline 19 Apr 93 & $14: 45$ & 5968 & ES1R4a & $12.38 \mathrm{ps1a}$ & volts & volts & 1.792 volts & & volts & 23 & 23 & 0.30303 \\
\hline 19 Apr 93 & $14: 48$ & 5968 & ES1RAb & $12.38 \mathrm{psla}$ & volts & volts & $1.794 \mathrm{volts}$ & & volts & 23 & 23 & 0.30326 \\
\hline 19 Apr 93 & $14: 51$ & 5973 & ES1R4C & $12.38 \mathrm{ps} 1 \mathrm{a}$ & volts & volts & 1.794 volts & & volts & 23 & 23 & 0.30211 \\
\hline $19 \mathrm{Apr} 93$ & $14: 53$ & 5973 & ES1R4d & $12.38 \mathrm{psla}$ & volts & volts & 1.794 volts & & volts & 23 & 23 & 0.30234 \\
\hline \multirow{9}{*}{ AVERAGES } & & & \multirow{9}{*}{ ES1R4 } & & GUAGE & GUAGE & DIFFERENTIAL & OORGE & Guinoz & $\mathrm{PCl}$ & (०द) & \\
\hline & & & & & 1.446 volts & 6.197 vo1ts & 1.794 volts & & 5.595 & \multirow{4}{*}{23} & \multirow{4}{*}{23} & \\
\hline & & & & & 322.2 psig & 34.00 & 2.976 psid & $32.380 \mathrm{ps} 1 \mathrm{~g}$ & 30.8919 & & & \\
\hline & & & & & 21.93 & 2.313 & 0.2025 & $2.2033 \mathrm{~atm}$ & 2.10206 & & & \\
\hline & & & & & $2.222 \quad \mathrm{Mpa}$ & 0.2344 & $0.02052 \quad \mathrm{Mpa}$ & $0.22325 \mathrm{Mpa}$ & 0.212992 & & & \\
\hline & & & & ABSOLUTE & ABSOLUTE & ABSOLUTE & DIFFEREMT IAL & ABSOLUTE & ABSOLUTE & (Ox) & $0 \times 1)$ & $(\mathrm{mI} / \mathrm{sec})$ \\
\hline & & & & $12.38 \mathrm{ps} 1 \mathrm{a}$ & $334.6 \quad \mathrm{ps} 1 \mathrm{a}$ & $46.38 \quad \mathrm{ps} 1 \mathrm{a}$ & 2.976 ps1d & $44.76 \mathrm{ps1a}$ & 43.27 & \multirow{3}{*}{296} & \multirow{3}{*}{296} & \multirow{3}{*}{0.30269} \\
\hline & & & & $0.8424 \quad \mathrm{~atm}$ & 22.77 & 3.156 & 0.2025 & $3.046 \quad \mathrm{~atm}$ & 2.9445 & & & \\
\hline & & & & $0.08536 \mathrm{Mpa}$ & 2.307 & 0.3198 & 0.02052 & 0.3086 & 0.29835 & & & \\
\hline
\end{tabular}

U

Apparent gas permeabllity:

$K a=\left(v \theta^{*} P e^{*} u^{*} L\right) /\left(P m^{*} \Delta P\right)$

Boyle's Law:

$v_{e}=(\mathrm{Pb} / \mathrm{Pe}) *(\mathrm{Te} / \mathrm{Tb}) *(\mathrm{ze} / \mathrm{zb}) * \mathrm{Vb}$

$Q_{e}=(\mathrm{Pb} / \mathrm{Pe}) *(\mathrm{Te} / \mathrm{Tb}) \cdot(\mathrm{ze} / \mathrm{zb}) * \mathrm{Ob}$

$v e=Q_{e} / A=(P b / P e) *(T e / T b) *(z e / z b) *(Q b / A)$

\begin{tabular}{|c|c|c|c|c|}
\hline Parameter & $\begin{array}{l}\text { Traditional } \\
\text { Untes }\end{array}$ & & $\begin{array}{l}\text { SI } \\
\text { Units }\end{array}$ & \\
\hline$\mu=$ gas v1scosity & 0.0176 & $\star 1.0 \mathrm{E}-3 \mathrm{~Pa} * \mathrm{sec} / \mathrm{CD}$ & $1.760 \mathrm{E}-05$ & $\mathrm{~Pa}^{\star} \mathrm{sec}$ \\
\hline$L=$ sample length & 10.358 & $1.0 \mathrm{E}-2 \mathrm{~m} / \mathrm{cm}$ & $1.036 \mathrm{E}-01$ & $\mathrm{~m}$ \\
\hline$A$ - sample clrcular cross sectional area & 81.313 & $\star 1.0 \mathrm{E}-4 \mathrm{~m}^{\wedge} 2 / \mathrm{cm}^{\wedge} 2$ & $8.131 E-03$ & $m^{2} 2$ \\
\hline $\mathrm{Pb}$ - flow measurement basls pressure (absolute) & 0.8424 & $\star 1.013 \mathrm{E}+5 \mathrm{~Pa} / \mathrm{atm}$ & $8.534 \mathrm{E}+04$ & $\mathrm{~Pa}$ \\
\hline$\Delta \mathrm{P}=$ pressure drop across sample length & 0.2025 & $\star 1.013 \mathrm{E}+5 \mathrm{~Pa} / \mathrm{atm}$ & $2.051 \mathrm{E}+04$ & $\mathrm{~Pa}$ \\
\hline $\mathrm{Pm}=$ mean pore pressure (absolute) & 3.046 & $1.013 \mathrm{E}+5 \mathrm{~Pa} / \mathrm{atm}$ & $3.085 E+05$ & $\mathrm{~Pa}$ \\
\hline $\mathrm{Pe}=$ exit pressure (absolute) & 2.944 & $\pi 1.013 \mathrm{E}+5 \mathrm{~Pa} / \mathrm{atm}$ & $2.983 E+05$ & $\mathrm{~Pa}$ \\
\hline $\mathrm{Te}=$ sample temperature (absolute) & 296 & & 296 & ${ }^{\circ} \mathrm{K}$ \\
\hline $\mathrm{Tb}=$ flow measurement basis temperature (absolute) & 296 & & 296 & ${ }^{\circ} \mathrm{K}$ \\
\hline$z e=$ gas deviation factor at $P e$ and $T e$ & 1.0000 & & 1.0000 & \\
\hline $\mathrm{zb}=$ gas devlation factor $\mathrm{at} \mathrm{Pb}$ and $\mathrm{Te}$ & 1.0000 & & 1.0000 & \\
\hline $\mathrm{Qb}=$ flow rate at base conditlons & 0.30269 & $\star 1.0 \mathrm{E}-6 \mathrm{~m}^{\wedge} 3 / \mathrm{cm}^{\wedge} 3$ & $3.027 E-07$ & $m^{2} 3 / s$ \\
\hline ve - flow velocity at sample exit end & $1.065 \mathrm{E}-03$ & $1.0 \mathrm{E}-2 \mathrm{~m} / \mathrm{cm}$ & $1.065 \mathrm{E}-05$ & $\mathrm{~m} / \mathrm{s}$ \\
\hline $\mathrm{Ka}=$ & $9.27 E-04$ & $9.872 \mathrm{E}-13 \mathrm{~m}^{\wedge} 2 / \mathrm{d}$ & $9.15 \mathrm{E}-16$ & $m^{\wedge} 2$ \\
\hline $\mathrm{Ka}=$ & 0.927 & & $9.15 \mathrm{E}-12$ & $\mathrm{~cm}^{\wedge} 2$ \\
\hline $\mathrm{Ka}=$ & 927 & & & \\
\hline
\end{tabular}




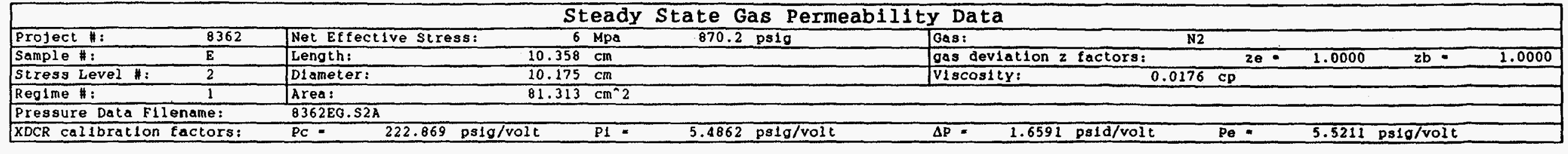

\begin{tabular}{|c|c|c|c|c|c|c|c|c|c|c|c|c|}
\hline Date & $\begin{array}{l}\text { Time } \\
\text { of } \\
\text { Day }\end{array}$ & $\begin{array}{l}\text { Eile } \\
\text { Time } \\
\text { (min) }\end{array}$ & $\begin{array}{c}\text { Regime } \\
*\end{array}$ & $\begin{array}{l}\mathrm{Pb} \\
\text { Barometric } \\
\text { Pressure }\end{array}$ & $\begin{array}{l}\text { Pc } \\
\text { Confining } \\
\text { Pressure }\end{array}$ & $\begin{array}{l}\text { P1 } \\
\text { Inlet } \\
\text { Pressure }\end{array}$ & \begin{tabular}{|l}
$\Delta P$ \\
D1fferent1al \\
Pressure
\end{tabular} & $\begin{array}{l}\text { Pm } \\
\text { Mean Pore } \\
\text { Pressure } \\
\text { Pe+ }+\Delta / 2\end{array}$ & $\begin{array}{l}\text { Pe } \\
\text { Ex1t } \\
\text { Pressure }\end{array}$ & $\begin{array}{l}\text { Te } \\
\text { Flow } \\
\text { Temp } \\
\left({ }^{\circ} \mathrm{C}\right)\end{array}$ & $\begin{array}{c}\text { Tb } \\
\text { Amblent } \\
\text { Temp } \\
\left({ }^{\circ} \mathrm{C}\right)\end{array}$ & $\begin{array}{c}\text { Ob } \\
\text { Flow Rate } \\
\text { OPbETb } \\
(\mathrm{ml} / \mathrm{sec})\end{array}$ \\
\hline 20 Apr 93 & $17: 05$ & 421 & ES2R1a & 12.39 psia & volts & volts & 1.669 volts & & volts & 23 & 23 & 0.06384 \\
\hline $20 \mathrm{Apr} 93$ & $17: 14$ & 426 & ES2R1b & $12.39 \mathrm{ps} 1 \mathrm{a}$ & 3.931 volts & volts & 1.680 volts & & volts & 23 & 23 & 0.06322 \\
\hline 20 Apr 93 & $17: 15$ & 431 & ES2R1C & 12.39 psio & volts & voits & 1.676 volts & & volts & 23 & 23 & 0.06337 \\
\hline $20 \mathrm{Apr} 93$ & $17: 20$ & 436 & ES2R1d & 12.39 ps1a & volts & volts & 1.676 volts & & volts & 23 & 23 & 0.06324 \\
\hline 20 Apr 93 & $17: 22$ & 436 & ES2R10 & 12.39 psia & 3.938 volts & volts & 1.675 volts & & volts & 23 & 23 & 0.06324 \\
\hline \multirow{9}{*}{ AVERAGES } & & & \multirow{9}{*}{ ES2R1 } & & OUAOE & OUAGE & DTFFERENTIAL & OUUAE: & OUhOE & कळ & (क्ट) & \\
\hline & & & & & 3.934 volts & 1.402 volts & 1.679 volts & & 0.887 & \multirow{4}{*}{23} & \multirow{4}{*}{23} & \\
\hline & & & & & 876.9 & 7.69 & 2.79 psld & $6.29 \mathrm{ps} 1 \mathrm{~g}$ & 4.899 & & & \\
\hline & & & & & 59.67 & 0.524 & 0.190 & 0.428 atm & 0.3334 & & & \\
\hline & & & & & 6.046 & 0.0530 & 0.0192 & 0.0434 & 0.03378 & & & \\
\hline & & & & ABSOLUTE: & ABSOLUTE & ABSOLUTE & DIFFERENT TAL & XBSOLUTL & ABSOLUT & (\%) & (कK) & (mYToc) \\
\hline & & & & 12.39 psia & 889.2 ps1a & $20.08 \quad$ ps1a & 2.79 ps1d & $18.68 \mathrm{ps} 1 \mathrm{a}$ & 17.29 & \multirow{3}{*}{296} & \multirow{3}{*}{296} & \multirow{3}{*}{0.06342} \\
\hline & & & & 0.8431 atm & 60.51 & 1.367 & 0.190 & $1.271 \mathrm{~atm}$ & 1.176 & & & \\
\hline & & & & $0.08543 \mathrm{Mpa}$ & 6.131 & 0.1385 & 0.0192 & 0.1288 & 0.1192 & & & \\
\hline
\end{tabular}

$\stackrel{1}{\dot{L}}$

Apparent gas permeabillty:

Boyle's Law:

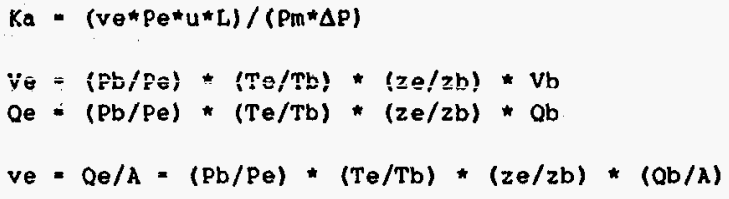

\begin{tabular}{|c|c|c|c|c|}
\hline Parameter & $\begin{array}{l}\text { Traditional } \\
\text { Units }\end{array}$ & & $\begin{array}{l}\text { SI } \\
\text { Units }\end{array}$ & \\
\hline$\mu=$ gas viscosity & 0.0176 & $* 1.0 \mathrm{E}-3 \mathrm{~Pa} * \mathrm{sec} / \mathrm{CD}$ & $1.760 \mathrm{E}-05$ & Patsec \\
\hline$L=$ sample length & 10.358 & $\$ 1.0 \mathrm{E}-2 \mathrm{~m} / \mathrm{cm}$ & $1.036 \mathrm{E}-01$ & $m$ \\
\hline A sample clrcular cross sectlonal area & 81.313 & $1.0 \mathrm{E}-4 \mathrm{~m}^{2} 2 / \mathrm{cm}^{2} 2$ & $8.131 E-03$ & $\mathrm{~m}^{2} 2$ \\
\hline $\mathrm{Pb}=$ flow measurement basis pressure (absolute) & 0.8431 & $\$ 1.013 \mathrm{E}+5 \mathrm{~Pa} / \mathrm{atm}$ & $8.540 \mathrm{E}+04$ & Pa \\
\hline$\Delta \mathrm{P}=$ pressure drop across sample length & 0.190 & $\$ 1.013 \mathrm{E}+5 \mathrm{~Pa} / \mathrm{atm}$ & $1.920 E+04$ & $\mathrm{~Pa}$ \\
\hline $\mathrm{Pm}$ - mean pore pressure (absolute) & 1.271 & $1.013 \mathrm{E}+5 \mathrm{~Pa} / \mathrm{atm}$ & $1.288 E+05$ & Pa \\
\hline Pe = exit pressure (absolute) & 1.176 & $71.013 \mathrm{E}+5 \mathrm{~Pa} / \mathrm{atm}$ & $1.192 \mathrm{E}+05$ & $\mathrm{~Pa}$ \\
\hline $\mathrm{Te}=$ sample temperature (absolute) & 296 & & 296 & ${ }^{\circ} \mathrm{K}$ \\
\hline $\mathrm{Tb}=$ flow measurement basis temperature (absolute) & 296 & & 296 & ${ }^{\circ} \mathrm{K}$ \\
\hline$z e=$ gas devlation factor at $\mathrm{Pe}$ and $\mathrm{Te}$ & 1.0000 & & 1.0000 & \\
\hline $\mathrm{zb}=$ gas deviation factor at $\mathrm{Pb}$ and $\mathrm{Te}$ & 1.0000 & & 1.0000 & \\
\hline$a b=$ flow rate at base conditions & 0.06342 & $\star 1.0 \mathrm{E}-6 \mathrm{~m}^{n} 3 / \mathrm{cm}^{\wedge} 3$ & $6.342 \mathrm{E}-08$ & $m \wedge 3 / s$ \\
\hline ve $=$ flow velocity at sample exit end & $5.589 \mathrm{E}-04$ & $1.0 \mathrm{E}-2 \mathrm{~m} / \mathrm{cm}$ & $5.589 \mathrm{E}-06$ & $\mathrm{~m} / \mathrm{s}$ \\
\hline $\mathrm{Ka}=$ & $4.97 \mathrm{E}-04$ & $9.872 \mathrm{E}-13 \mathrm{~m} \mathrm{~m}^{*} 2 / \mathrm{d}$ & $4.91 \mathrm{E}-16$ & $m^{n} 2$ \\
\hline $\mathrm{Ka}=$ & $4.97 \mathrm{E}-01$ & & $4.918-12$ & $\mathrm{~cm}^{\star} 2$ \\
\hline$K_{a} \cdot$ & $4.97 \mathrm{E}+02$ & & & \\
\hline
\end{tabular}




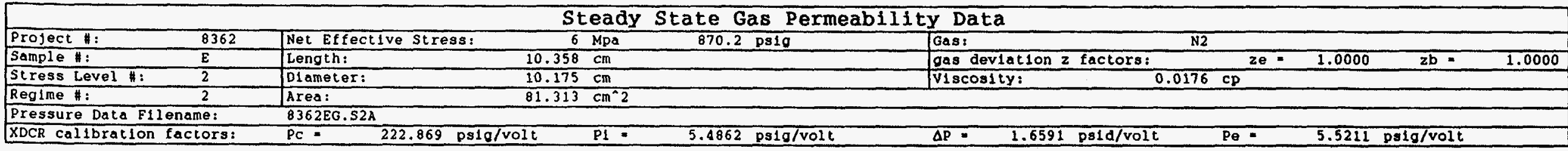

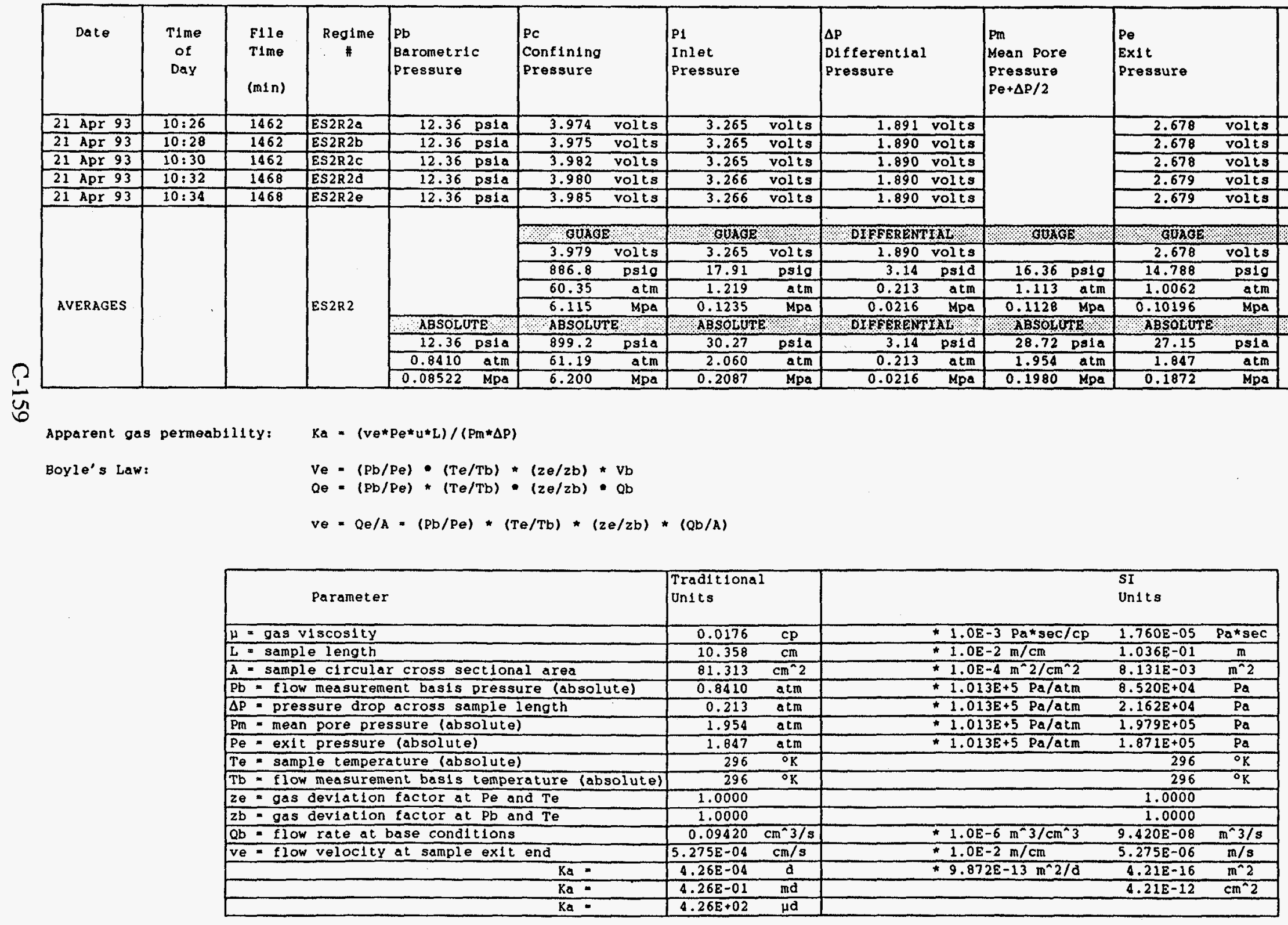




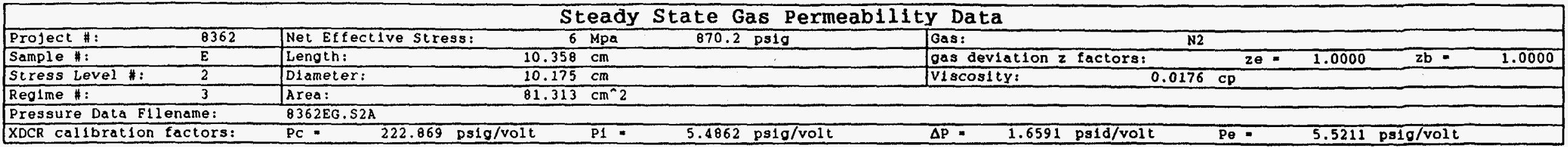

\begin{tabular}{|c|c|c|c|c|c|c|c|c|c|c|c|c|}
\hline Date & $\begin{array}{l}\text { Time } \\
\text { of } \\
\text { Day }\end{array}$ & $\begin{array}{l}\text { Flle } \\
\text { Time } \\
\text { (min) }\end{array}$ & $\begin{array}{c}\text { Regime } \\
\|\end{array}$ & $\begin{array}{l}\mathrm{Pb} \\
\text { Barometric } \\
\text { Pressure }\end{array}$ & $\begin{array}{l}\text { Pc } \\
\text { Confining } \\
\text { Pressure }\end{array}$ & $\begin{array}{l}\text { Pi } \\
\text { Inlet } \\
\text { Pressure }\end{array}$ & $\begin{array}{l}\Delta P \\
\text { Differential } \\
\text { Pressure }\end{array}$ & \begin{tabular}{|l} 
Pm \\
Mean pore \\
pressure \\
Pe+ $+\Delta \mathrm{P} / 2$
\end{tabular} & $\left\{\begin{array}{l}\mathrm{Pe} \\
\text { Exit } \\
\text { Pressure }\end{array}\right.$ & $\begin{array}{l}\text { Te } \\
\text { Flow } \\
\text { Temp } \\
\left({ }^{\circ} \mathrm{C}\right)\end{array}$ & $\begin{array}{c}\text { Tb } \\
\text { Amblent } \\
\text { Temp } \\
\left({ }^{\circ} \mathrm{C}\right\rangle\end{array}$ & $\begin{array}{c}\text { Qb } \\
\text { Flow Rate } \\
\text { PbtTb } \\
\text { (m1/sec) }\end{array}$ \\
\hline 21 Apr 93 & $14: 42$ & 1716 & ES2R3a & 12.35 psia & 4.022 & 5.078 & 1.911 volts & & 4.463 & 23 & 23 & 0.11980 \\
\hline 21 Apr 93 & $14: 44$ & 1716 & ES2R3b & 12.35 ps1a & volts & 5.078 & 1.912 volts & & volts & 23 & 23 & 0.11958 \\
\hline 21 Apr 93 & $14: 46$ & 1721 & ES2R3C & 12.35 psia & volts & 5.078 & 1.912 volts & & volts & 23 & 23 & 0.11930 \\
\hline 21 ApY 93 & $14: 47$ & 1721 & ES2R3d & $12.35 \mathrm{psia}$ & volts & 5.077 & 1.912 volts & & volts & 23 & 23 & 0.11965 \\
\hline 21 Apr 93 & $14: 49$ & 1726 & ES2R3e & 12.35 ps1a & 4.023 volts & 5.077 volts & 1.911 volts & & 4.462 & 23 & 23 & 0.11947 \\
\hline \multirow{9}{*}{ AVERAGES } & & & \multirow{9}{*}{ ES2R3 3} & & GUACE & OUAOE & DTEEEREKTIAL & OUAGE & Guno: & 100 & क्व & \\
\hline & & & & & 4.023 volts & 5.078 volts & 1.912 volts & & 4.462 & \multirow{4}{*}{23} & \multirow{4}{*}{23} & \\
\hline & & & & & 896.6 & 27.86 & 3.17 psid & $26.22 \mathrm{ps1g}$ & 24.637 & & & \\
\hline & & & & & 61.01 & 1.896 & 0.216 & 1.784 atm & 1.6765 & & & \\
\hline & & & & & 6.182 & 0.1921 & $0.0219 \quad \mathrm{Mpa}$ & $0.1808 \quad \mathrm{Mpa}$ & 0.16987 & & & \\
\hline & & & & ABSOLUTE & ABSOLUTE & ABSOLUTE & DFFEEREMTYA & ABSOLUTE & ABSOLUTE & $(9 x)$ & एवरा & (mI Toc) \\
\hline & & & & $12.35 \mathrm{ps} 1 \mathrm{a}$ & 908.9 psla & 40.21 & 3.17 ps1d & $38.57 \mathrm{ps} 1 \mathrm{a}$ & 36.99 & \multirow{3}{*}{296} & \multirow{3}{*}{296} & \multirow{3}{*}{0.11958} \\
\hline & & & & $0.8404 \quad \mathrm{~atm}$ & 61.85 & 2.736 & 0.216 & $2.625 \mathrm{~atm}$ & 2.517 & & & \\
\hline & & & & 0.08515 & 6.267 & 0.2772 & 0.0219 & 0.2660 & 0.2550 & & & \\
\hline
\end{tabular}

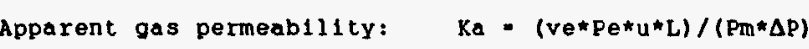

Boyle's Law:

$V_{e}=(P b / P e) \cdot(T e / T b) *(z e / z b) * V b$

$Q_{e}=(\mathrm{Pb} / \mathrm{Pe}) \cdot(\mathrm{Te} / \mathrm{Tb}) *(z e / 2 \mathrm{~b}) * \mathrm{Q}$

$v e=Q e / A=(\mathrm{Pb} / \mathrm{Pe}) \cdot(\mathrm{Te} / \mathrm{Tb}) *(2 e / 2 \mathrm{~b}) *(\mathrm{Qb} / \mathrm{A})$

\begin{tabular}{|c|c|c|c|c|}
\hline Parameter & $\begin{array}{l}\text { Troditional } \\
\text { Unlts }\end{array}$ & & $\begin{array}{l}\text { SI } \\
\text { Units }\end{array}$ & \\
\hline$\mu=g a s v 1 s \cos 1 t y$ & 0.0176 & $\star 1.0 \mathrm{E}-3 \mathrm{~Pa} * \mathrm{sec} / \mathrm{Cp}$ & $1.760 \mathrm{E}-05$ & Pa*sec \\
\hline$L=$ sample length & 10.358 & $\hbar 1.0 \mathrm{E}-2 \mathrm{~m} / \mathrm{cm}$ & $1.036 \mathrm{E}-01$ & $\mathrm{~m}$ \\
\hline$A=$ sample clrcular cross sectlonal area & 81.313 & $\$ 1.0 \mathrm{E}-4 \mathrm{~m}^{\wedge} 2 / \mathrm{cm}^{\wedge} 2$ & $8.131 \mathrm{E}-03$ & $m^{\wedge} 2$ \\
\hline$\overline{\mathrm{pb}}=$ flow measurement basis pressure (absolute) & 0.8404 & $\pitchfork 1.013 \mathrm{E}+5 \mathrm{~Pa} / \mathrm{atm}$ & $8.513 E+04$ & $\mathrm{~Pa}$ \\
\hline$\overline{\Delta P}$ : pressure drop across sample length & 0.216 & $\pi 1.013 \mathrm{E}+5 \mathrm{~Pa} / \mathrm{atm}$ & $2.186 \mathrm{E}+08$ & $\mathbf{P a}$ \\
\hline$P_{m}=$ mean pore pressure (absolute) & 2.625 & $\star 1.013 E+5 \mathrm{~Pa} / \mathrm{atm}$ & $2.659 E+05$ & $\overline{P a}$ \\
\hline Pe = exit pressure (absolute) & 2.517 & $1.013 \mathrm{E}+5 \mathrm{~Pa} / \mathrm{atm}$ & $2.550 E+05$ & $\mathbf{P a}$ \\
\hline Te = sample temperature (absolute) & 296 & & 296 & ${ }^{\circ} \mathbf{K}$ \\
\hline $\mathrm{Tb}=$ flow measurement basis temperature (absolute) & 296 & & 296 & ${ }^{\circ} \mathrm{K}$ \\
\hline$z e=$ gas deviation factor at $\mathrm{Pe}$ and $\mathrm{Te}$ & 1.0000 & & 1.0000 & \\
\hline $\mathrm{zb}=$ gas deviation factor at $\mathrm{pb}$ and $\mathrm{Te}$ & 1.0000 & & 1.0000 & \\
\hline$\overline{Q b}=$ flow rate at base conditions & 0.11958 & $1.0 \mathrm{E}-6 \mathrm{~m}^{\wedge} 3 / \mathrm{cm}^{\wedge} 3$ & $1.196 \mathrm{E}-07$ & $m^{2} 3 / s$ \\
\hline ve flow velocity at sample exit end & $4.910 \mathrm{E}-04$ & $-1.0 \mathrm{E}-2 \mathrm{~m} / \mathrm{cm}$ & $4.910 E-06$ & $\mathrm{~m} / \mathrm{s}$ \\
\hline $\mathrm{K}_{\mathrm{a}}=$ & $3.98 \mathrm{E}-04$ & $0.872 \mathrm{E}-13 \mathrm{~m}^{\circ} 2 / \mathrm{d}$ & $3.93 \mathrm{E}-16$ & $m^{2} 2$ \\
\hline $\mathrm{Ka}=$ & $3.98 \mathrm{E}-01$ & & $3.93 E-12$ & $c m^{n} 2$ \\
\hline $\mathrm{Ka}=$ & $3.98 \mathrm{E}+02$ & & & \\
\hline
\end{tabular}




\begin{tabular}{|c|c|c|c|c|c|c|c|c|c|c|c|c|c|}
\hline \multicolumn{14}{|c|}{ Steady State Gas Permeability Data } \\
\hline Project \#: & 8362 & Net Effective stress: & 6 & $\mathrm{Mpa}$ & $870.2 \mathrm{ps} 1 \mathrm{~g}$ & Gas: & & N2 & & & & & \\
\hline Sample : : & E & Length: & 10.358 & $\mathrm{~cm}$ & & gas deviation 2 & factors: & & & $2 e=$ & 1.0000 & $\mathrm{zb}-$ & 1.0000 \\
\hline Stress Level \#: & $\overline{2}$ & Dlameter: & 10.175 & $\mathrm{~cm}$ & & VIscosity: & & 0.0176 & $\mathrm{CP}$ & & & & \\
\hline Regime $\|:$ & 4 & Area: & 81.313 & $\mathrm{~cm}^{2} 2$ & & & & & & & & & \\
\hline Pressure Data F1 & ne: & $8362 \mathrm{EG} .52 \mathrm{~A}$ & & & & & & & & & & & \\
\hline XDCR calibration & Eors: & $\mathrm{Pc}=\quad 222.869 \mathrm{ps1}$ & & $\mathrm{P1}=$ & $5.4862 \mathrm{ps} 1 \mathrm{~g} / \mathrm{volt}$ & 1.6591 & psid/volt & & $\mathrm{Pe}$ & & 5.5211 & psig/volt & \\
\hline
\end{tabular}

\begin{tabular}{|c|c|c|c|c|c|c|c|c|c|c|c|c|}
\hline Date & $\begin{array}{l}\text { Time } \\
\text { of } \\
\text { Day }\end{array}$ & $\begin{array}{l}\text { File } \\
\text { Time } \\
\text { (min) }\end{array}$ & $\begin{array}{c}\text { Regime } \\
*\end{array}$ & $\begin{array}{l}\mathrm{Pb} \\
\text { Barometric } \\
\text { Pressure }\end{array}$ & $\begin{array}{l}\text { Pc } \\
\text { Confining } \\
\text { Pressure }\end{array}$ & $\begin{array}{l}\text { P1 } \\
\text { Inlet } \\
\text { Pressure }\end{array}$ & $\begin{array}{l}\Delta \mathrm{P} \\
\text { D1fferentlal } \\
\text { Pressure }\end{array}$ & $\begin{array}{l}\text { Pm } \\
\text { Mean pore } \\
\text { Pressure } \\
\text { Pe }+\Delta \mathrm{P} / 2\end{array}$ & $\begin{array}{l}\text { Pe } \\
\text { Exit } \\
\text { Pressure }\end{array}$ & $\begin{array}{l}\text { Te } \\
\text { Flow } \\
\text { Temp } \\
\left({ }^{\circ} \mathrm{C}\right)\end{array}$ & $\begin{array}{c}\text { Tb } \\
\text { Amb1 ent } \\
\text { Temp } \\
\left({ }^{\circ} \mathrm{C}\right)\end{array}$ & $\begin{array}{c}Q b \\
\text { Flow Rate } \\
\text { OPb\&Tb } \\
\text { (ml/sec) }\end{array}$ \\
\hline 21 ApI 93 & $17: 01$ & 1716 & ES2R4a & $12.34 \mathrm{ps} 1 \mathrm{a}$ & 4.068 volts & 6.918 volts & 1.982 volts & & 6.255 & 23 & 23 & 0.14981 \\
\hline 21 ApI 93 & $17: 03$ & 1716 & ES2R4b & $12.34 \mathrm{ps} 1 \mathrm{a}$ & 4.068 & volts & 1.985 volts & & volts & 23 & 23 & 0.15021 \\
\hline 21 Apr 93 & $17: 05$ & 1721 & ES2R4C & $12.34 \mathrm{ps} 1 \mathrm{a}$ & volts & volts & 1.985 vol ts & & volts & 23 & 23 & 0.15032 \\
\hline 21 ApI 93 & $17: 07$ & 1721 & ES2R4d & $12.34 \mathrm{psia}$ & 4.068 volts & volts & 1.985 volts & & volts & 23 & 23 & 0.15026 \\
\hline \multirow{9}{*}{ AVERAGES } & & & \multirow{9}{*}{ ES2R4 } & & ऽOUAGE & GOACE & DTFEEREMTXI & OUXOE: & GUAOE & (ब) & (rc) & \\
\hline & & & & & 4.068 volts & 6.917 volts & 1.984 volts & & 6.254 & \multirow{4}{*}{23} & \multirow{4}{*}{23} & \\
\hline & & & & & 906.6 & 37.95 & 3.29 psid & $36.17 \mathrm{psig}$ & 34.528 & & & \\
\hline & & & & & 61.69 & 2.582 & 0.224 & $2.461 \mathrm{~atm}$ & 2.3495 & & & \\
\hline & & & & & 6.251 & 0.2616 & 0.0227 & 0.2494 & 0.23806 & & & \\
\hline & & & & ABSOLUTE & ABSOLUTE & ABSOLUTE & DIFFERENTIAL & AESOLUTE & ABSOLLTE & (स) & $(0 x)$ & $(\mathrm{m} / \mathrm{sec})$ \\
\hline & & & & 12.34 psia & 919.0 psia & 50.29 psia & $3.29 \quad$ psid & $48.51 \mathrm{psla}$ & 46.87 & \multirow{3}{*}{296} & \multirow{3}{*}{296} & \multirow{3}{*}{0.15015} \\
\hline & & & & 0.8397 atm & 62.53 & 3.422 & 0.224 & $3.301 \mathrm{~atm}$ & 3.189 & & & \\
\hline & & & & 0.08508 & 6.336 & 0.3467 & 0.0227 & 0.3345 & 0.3231 & & & \\
\hline
\end{tabular}

o

Apparent gas permeabillty: $\quad K a=\left(v e^{*} P^{*} u^{*} L\right) /\left(P m^{*} \Delta P\right)$

Boyle's Law:

$\mathrm{Ve}=(\mathrm{Pb} / \mathrm{Pe}) \cdot(\mathrm{Te} / \mathrm{Tb}) *(\mathrm{ze} / \mathrm{zb}) * \mathrm{Vb}$

$\mathrm{Qe}=(\mathrm{Pb} / \mathrm{Pe}) *(\mathrm{Te} / \mathrm{Tb}) *(\mathrm{ze} / \mathrm{zb}) * \mathrm{Qb}$

$v_{e}=Q_{e} / A=(\mathrm{Pb} / \mathrm{Pe}) \cdot(\mathrm{Te} / \mathrm{Tb}) *(z e / z b) *(\mathrm{Qb} / \mathrm{A})$

\begin{tabular}{|c|c|c|c|c|}
\hline Parameter & $\begin{array}{l}\text { Traditional } \\
\text { Un1ts }\end{array}$ & & $\begin{array}{l}\text { SI } \\
\text { Units }\end{array}$ & \\
\hline$\mu=\operatorname{gas} v i s \cos 1 t y$ & 0.0176 & $* 1.0 \mathrm{E}-3 \mathrm{~Pa}$ (sec/cp & $1.760 \mathrm{E}-05$ & $\mathrm{~Pa} * \sec$ \\
\hline $\mathrm{L}=$ sample length & 10.358 & $\star 1.0 \mathrm{E}-2 \mathrm{~m} / \mathrm{cm}$ & $1.036 \mathrm{E}-01$ & $\mathrm{~m}$ \\
\hline$A=$ sample circular cross sectional area & 81.313 & $\star 1.0 \mathrm{E}-4 \mathrm{~m}^{\wedge} 2 / \mathrm{cm}^{\wedge} 2$ & $8.131 E-03$ & $m^{2} 2$ \\
\hline $\mathrm{pb}=$ flow measurement basis pressure (absolute) & 0.8397 & $\pi 1.013 \mathrm{E}+5 \mathrm{~Pa} / \mathrm{atm}$ & $8.506 \mathrm{E}+04$ & $\mathrm{~Pa}$ \\
\hline$\Delta \mathrm{P}=$ pressure drop across sample length & 0.224 & $\approx 1.013 \mathrm{E}+5 \mathrm{~Pa} / \mathrm{atm}$ & $2.269 \mathrm{E}+04$ & $\mathrm{~Pa}$ \\
\hline $\mathrm{Pm}=$ mean pore pressure (absolute) & 3.301 & $\star 1.013 \mathrm{E}+5 \mathrm{~Pa} / \mathrm{atm}$ & $3.344 \mathrm{E}+05$ & $\mathrm{~Pa}_{\mathrm{a}}$ \\
\hline $\mathrm{Pe}=$ exlt pressure (absoluce) & 3.189 & $\star 1.013 \mathrm{E}+5 \mathrm{~Pa} / \mathrm{atm}$ & $3.231 \mathrm{E}+05$ & $\mathrm{~Pa}$ \\
\hline Te = sample temperature (absolute) & 296 & & 296 & ${ }^{\circ} \mathrm{K}$ \\
\hline $\mathrm{Tb}=$ flow measurement basis temperature (absolute) & 296 & & 296 & ${ }^{\circ} \mathrm{K}$ \\
\hline$z e=$ gas deviation factor at $\mathrm{Pe}$ and $\mathrm{Te}$ & 1.0000 & & 1.0000 & \\
\hline $\mathrm{zb}=$ gas deviation factor at $\mathrm{Pb}$ and $\mathrm{Te}$ & 1.0000 & & 1.0000 & \\
\hline $\mathrm{Qb}=$ flow rate at base conditlons & $0.15015 \mathrm{~cm}^{\wedge} 3 / \mathrm{s}$ & $\pm 1.0 \mathrm{E}-6 \mathrm{~m}^{\wedge} 3 / \mathrm{cm}^{\wedge} 3$ & $1.502 E-07$ & $\mathrm{~m}^{2} 3 / \mathrm{s}$ \\
\hline ve = flow velocity at sample exit end & $4.862 E-04$ & * $1.0 \mathrm{E}-2 \mathrm{~m} / \mathrm{cm}$ & $4.862 \mathrm{E}-06$ & $\mathrm{~m} / \mathrm{s}$ \\
\hline$x_{a}=$ & $3.82 \mathrm{E}-04$ & $\star 9.872 \mathrm{E}-13 \mathrm{~m}^{\wedge} 2 / \mathrm{d}$ & $3.77 \mathrm{E}-16$ & $m^{n} 2$ \\
\hline $\mathrm{Ka}=$ & $3.82 \mathrm{E}-01$ & & $3.77 \mathrm{E}-12$ & $\mathrm{~cm}^{\wedge} 2$ \\
\hline $\mathrm{Ka}=$ & $3.82 \mathrm{E}+02$ & & & \\
\hline
\end{tabular}




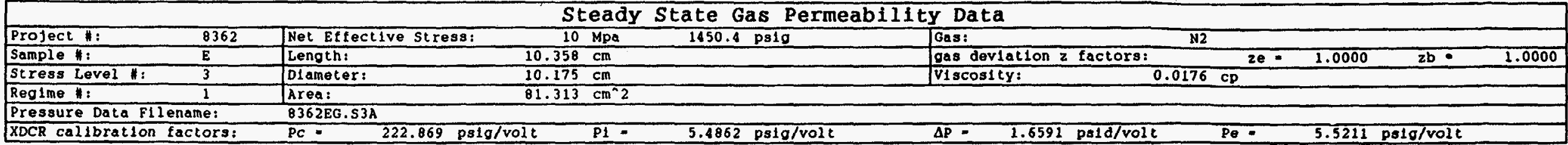

\begin{tabular}{|c|c|c|c|c|c|c|c|c|c|c|c|c|}
\hline Date & $\begin{array}{l}\text { Time } \\
\text { of } \\
\text { Day }\end{array}$ & $\begin{array}{l}\text { Flle } \\
\text { Time } \\
\text { (min) }\end{array}$ & $\begin{array}{c}\text { Reglme } \\
k\end{array}$ & $\begin{array}{l}\mathrm{Pb} \\
\text { Barometrlc } \\
\text { Pressure }\end{array}$ & $\begin{array}{l}\text { PC } \\
\text { Confining } \\
\text { Pressure }\end{array}$ & $\begin{array}{l}\text { P1 } \\
\text { Inlet } \\
\text { Pressure }\end{array}$ & $\begin{array}{l}\Delta \mathrm{P} \\
\text { D1fferential } \\
\text { Pressure }\end{array}$ & \begin{tabular}{|l} 
Pm \\
Mean Pore \\
Pressure \\
Pe+ $\Delta P / 2$
\end{tabular} & $\begin{array}{l}\text { Pe } \\
\text { Exit } \\
\text { Pressure }\end{array}$ & $\begin{array}{l}\text { Te } \\
\text { Flow } \\
\text { Temp } \\
\left({ }^{\circ} \mathrm{C}\right)\end{array}$ & $\begin{array}{c}\text { Tb } \\
\text { Amblent } \\
\text { Temp } \\
\left({ }^{\circ} \mathrm{C}\right)\end{array}$ & $\begin{array}{c}\text { Qb } \\
\text { Flow Rate } \\
\text { OPb\&Tb } \\
\text { (ml/sec) }\end{array}$ \\
\hline 22 Apr 93 & $14: 17$ & 308 & ES3R1a & 12.20 psia & 6.537 & volts & 2.038 voltg & & 0.872 & 23 & 23 & 0.04611 \\
\hline $22 \operatorname{Apr} 93$ & $14: 21$ & 313 & ES3R1D & $12.20 \mathrm{ps} 1 \mathrm{a}$ & volts & volts & 2.042 volts & & 0.871 & 23 & $\overline{23}$ & 0.04595 \\
\hline 22 Apr 93 & $14: 26$ & 318 & ES3RIC & $12.20 \mathrm{psia}$ & volts & volts & 2.043 volts & & volts & 23 & 23 & 0.04615 \\
\hline $22 \mathrm{Apr} 93$ & $14: 30$ & 323 & ES3R1d & 12.20 psia & 6.537 & volts & 2.044 volts & & volts & 23 & 23 & 0.04579 \\
\hline 22 Apr 93 & $14: 33$ & 323 & ES3R1e & $12.20 \mathrm{psia}$ & 6.536 volts & volts & 2.045 volts & & 0.871 & 23 & 23 & 0.04575 \\
\hline \multirow{9}{*}{ AVERAGES } & & & \multirow{9}{*}{ ES3R 1} & & OONOE & OOUXOE & DIFFERENTIXL & OVXGE & 00000 & (क्) & \%ला। & \\
\hline & & & & & 6.537 volts & 1.498 volts & 2.042 volts & & 0.871 & \multirow{4}{*}{23} & \multirow{4}{*}{23} & \\
\hline & & & & & 1456.8 & psig & $3.39 \mathrm{psid}$ & $6.50 \mathrm{ps} 10$ & 4.809 & & & \\
\hline & & & & & 99.13 & 0.559 & 0.231 & 0.943 atm & 0.3272 & & & \\
\hline & & & & & 10.044 & 0.0567 & 0.0234 & 0.0448 & 0.03316 & & & \\
\hline & & & & ABSOLUTE & ABSOLUTE & ABSOLUTE & DIFFERENTIAL & \multicolumn{2}{|l|}{ A8SOLLTEP } & (OR: & (\%) & (nTlogr \\
\hline & & & & $12.2 \mathrm{ps} 1 \mathrm{a}$ & $1469.0 \quad$ psla & 20.42 ps1a & 3.39 psid & $18.70 \mathrm{psia}$ & 17.01 & \multirow{3}{*}{296} & \multirow{3}{*}{296} & \multirow{3}{*}{0.04600} \\
\hline & & & & 0.8302 atm & 99.96 & 1.389 & 0.231 & $1.273 \mathrm{~atm}$ & 1.157 & & & \\
\hline & & & & $0.08412 \mathrm{Mpa}$ & 10.128 & 0.1408 & 0.0234 & 0.1290 & 0.1173 & & & \\
\hline
\end{tabular}

ลิ

Apparent gas permeability:

Boyle's Law:

$K a=\left(v e * P e^{*} u * L\right) /(P m * \Delta P)$

$V_{e}=(P b / P e) \cdot(T e / T b) \cdot(z e / z b) \cdot V b$

$Q \theta=(P b / P e) *(T e / T b) *(2 \theta / 2 D) * 0 \dot{O b}$

ve $\cdot Q e / A=(\mathrm{Pb} / \mathrm{Pe}) \cdot(\mathrm{Te} / \mathrm{Tb}) \cdot(\mathrm{ze} / \mathrm{zb}) \cdot(\mathrm{Ob} / \mathrm{A})$

\begin{tabular}{|c|c|c|c|c|}
\hline Parameter & $\begin{array}{l}\text { Tradltional } \\
\text { Units }\end{array}$ & & $\begin{array}{l}\text { SI } \\
\text { Units }\end{array}$ & \\
\hline$\mu=$ gas v1scosity & 0.0176 & $* 1.0 \mathrm{E}-3$ Pa*sec/ep & $1.760 \mathrm{E}-05$ & Pa"sec \\
\hline $\mathrm{L}=$ sample length & 10.358 & $1.0 \mathrm{E}-2 \mathrm{~m} / \mathrm{cm}$ & $1.036 \mathrm{E}-01$ & $m$ \\
\hline$A=$ sample clrcular cross sectlonal area & 81.313 & $1.0 \mathrm{E}-4 \mathrm{~m}^{\wedge} 2 / \mathrm{cm}^{\wedge} 2$ & $8.131 \mathrm{E}-03$ & $m^{\wedge} 2$ \\
\hline $\mathrm{Pb}=$ tlow measurement basls pressure (absolute) & 0.8302 & $1.013 \mathrm{E}+5 \mathrm{~Pa} / \mathrm{atm}$ & $8.409 E+04$ & Pa \\
\hline$\Delta P=$ pressure drop across sample length & 0.231 & $1.013 \mathrm{E}+5 \mathrm{~Pa} / \mathrm{arm}$ & $2.336 E+04$ & $\mathbf{P a}$ \\
\hline Pm - mean pore pressure (absolute) & 1.273 & $1.013 \mathrm{E}+5 \mathrm{~Pa} / \mathrm{atm}$ & $1.289 E+05$ & $\mathrm{~Pa}$ \\
\hline Pe: exit pressure (absolute) & 1.157 & $1.013 \mathrm{E}+5 \mathrm{~Pa} / \mathrm{atm}$ & $1.172 \mathrm{E}+05$ & $\mathbf{P a}$ \\
\hline Te = sample temperature (absolute) & 296 & & 296 & ${ }^{\circ} \mathrm{K}$ \\
\hline$T b=$ flow measurement basis temperature (absolute) & 296 & & 296 & ${ }^{\circ} \mathrm{K}$ \\
\hline$z e=$ gas devlation factor at $\mathrm{Pe}$ and $\mathrm{Te}$ & 1.0000 & & 1.0000 & \\
\hline $\mathrm{zb}=$ gas deviation factor at $\mathrm{Pb}$ and $\mathrm{Te}$ & 1.0000 & & 1.0000 & \\
\hline$a b$ - flow rate at base condltions & $0.04600 \mathrm{~cm}^{2} 3 / \mathrm{s}$ & $21.0 \mathrm{E}-6 \mathrm{~m}^{\wedge} 3 / \mathrm{cm}^{\wedge} 3$ & $4.600 \mathrm{E}-08$ & $m^{n} 3 / 3$ \\
\hline ve - flow velocity at sample exit end & $4.058 \mathrm{E}-04 \mathrm{~cm} / \mathrm{s}$ & $1.0 \mathrm{E}-2 \mathrm{~m} / \mathrm{cm}$ & $4.058 \mathrm{E}-06$ & $\mathrm{~m} / \mathrm{s}$ \\
\hline$x_{a}=$ & $2.92 \mathrm{E}-04$ & $9.872 \mathrm{E}-13 \mathrm{~m}^{2} 2 / \mathrm{d}$ & $2.88 \mathrm{E}-16$ & $m^{\wedge} 2$ \\
\hline$K_{\mathbf{A}}=$ & $2.92 \mathrm{E}-01$ & & $2.8 B E-12$ & $\mathrm{~cm}^{-2} \mathrm{2}$ \\
\hline$R_{a}=$ & $2.92 E+02$ & & & \\
\hline
\end{tabular}




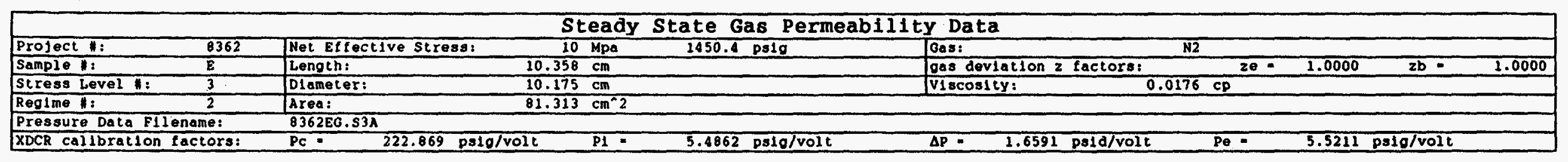

\begin{tabular}{|c|c|c|c|c|c|c|c|c|c|c|c|c|}
\hline Date & $\begin{array}{c}\text { Time } \\
\text { of } \\
\text { Day }\end{array}$ & $\begin{array}{l}\text { Flle } \\
\text { Time } \\
\text { (min) }\end{array}$ & Regime & $\begin{array}{l}\text { Pb } \\
\text { Barometrle } \\
\text { Pressure }\end{array}$ & $\begin{array}{l}\text { Pc } \\
\text { Contining } \\
\text { Pressure }\end{array}$ & $\begin{array}{l}\text { PI } \\
\text { Inlet } \\
\text { Pressure }\end{array}$ & $\begin{array}{l}\Delta \mathrm{P} \\
\text { Differential } \\
\text { Pressure }\end{array}$ & 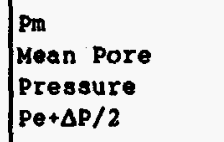 & $\begin{array}{l}\text { Pe } \\
\text { ExIt } \\
\text { Pressure }\end{array}$ & $\begin{array}{l}\text { To } \\
\text { Fiow } \\
\text { Temp } \\
\left({ }^{\circ} \mathrm{C}\right)\end{array}$ & $\begin{array}{l}\text { Tb } \\
\text { Amblent } \\
\text { Temp } \\
\left({ }^{\circ} \mathrm{C}\right)\end{array}$ & $\begin{array}{c}\text { Qb } \\
\text { Flow Race } \\
\text { OPbstb } \\
\text { (ml/sec) }\end{array}$ \\
\hline 22 Apr 93 & $16: 57$ & 468 & ES3R2a & $12.21 \mathrm{pg} 1 \mathrm{a}$ & volts & volts & 1.920 volts & & 2.671 & 23 & 23 & 0.05722 \\
\hline $22 \mathrm{Aps} 93$ & $17: 00$ & 473 & ES3R2b & $12.21 \mathrm{ps} 1 \mathrm{a}$ & volts & volts & 1.921 volts & & volts & 23 & 23 & 0.05741 \\
\hline 22 Apr 93 & $17: 03$ & 873 & ES3R2C & $12.21 \mathrm{psia}$ & volts & volts & 1.922 volts & & volks & 23 & 23 & 0.05727 \\
\hline $22 \mathrm{Apr} 93$ & $17: 06$ & 478 & ES3R2d & $12.21 \mathrm{ps} 1 \mathrm{a}$ & volts & volts & 1.924 volts & & volts & 23 & 23 & 0.05731 \\
\hline \multirow{9}{*}{ AVERAGES } & & & \multirow{9}{*}{ ES3R2 } & & POUAGE & POONOE & DTHEERENTHK & OUXXG: & auno: & बक्ष & करतः & \\
\hline & & & & & 6.581 volts & 3.274 volts & 1.922 volts & & 2.670 & \multirow{4}{*}{23} & \multirow{4}{*}{23} & \\
\hline & & & & & 1466.8 & 17.96 & $3.19 \mathrm{psid}$ & $16.34 \mathrm{pg10}$ & $14.741^{\circ}$ & & & \\
\hline & & & & & 99.81 & 1.222 & 0.217 & $1.112 \mathrm{~atm}$ & 1.0031 & & & \\
\hline & & & & & $10.113 \quad \mathrm{Mpa}$ & 0.1238 & $0.0220 \mathrm{Mpa}$ & $0.1126 \quad \mathrm{Mpa}$ & 0.10164 & & & \\
\hline & & & & ABSOLUTE & ABSOLUTE & ABSOCOTE & DrFFEREMTIX L & \multicolumn{2}{|l|}{ TESOLUTE } & (9x) & YOXY & (n⿴囗十 $60 \mathrm{~s}$ \\
\hline & & & & $12.21 \mathrm{ps} 1 \mathrm{a}$ & 1479.0 DS1a & 30.17 ps1a & $3.19 \mathrm{ps} 1 \mathrm{~d}$ & $28.55 \mathrm{ps} 1 \mathrm{a}$ & 26.95 & \multirow{3}{*}{296} & \multirow{3}{*}{296} & \multirow{3}{*}{0.05730} \\
\hline & & & & $0.8308 \quad \mathrm{~atm}$ & 100.64 & 2.053 & 0.217 & 1.942 atm & 1.834 & & & \\
\hline & & & & $0.08419 \mathrm{Mpa}$ & 10.197 & 0.2080 & 0.0220 & 0.1968 & 0.1858 & & & \\
\hline
\end{tabular}

$\overrightarrow{\mathrm{s}}$

Apparent gas permeab111ty:

Boyle's Law:

$K a \cdot(v e * P e * u * L) /(P m * \Delta P)$

$v_{e}=(P b / P e) *(T e / T b) *(z e / z b) * V b$

$a e=(\mathrm{Pb} / \mathrm{Pe}) *(\mathrm{Te} / \mathrm{Tb}) \cdot(z e / 2 \mathrm{~b}) \cdot \mathrm{Ob}$

$v_{e}=Q_{e} / A=\left(P b / P_{e}\right) \cdot(T e / T b) *(z e / 2 b) \cdot(Q b / A)$

\begin{tabular}{|c|c|c|c|c|}
\hline Parameter & $\begin{array}{l}\text { Traditlonal } \\
\text { Units }\end{array}$ & & $\begin{array}{l}\text { SI } \\
\text { Un1ts }\end{array}$ & \\
\hline$\mu=$ gas viscosity & 0.0176 & $1.0 \mathrm{E}-3 \mathrm{~Pa}+\mathrm{sec} / \mathrm{Cp}$ & $1.760 \mathrm{E}-05$ & Pa*sec \\
\hline$L=$ sample length & 10.358 & $1.0 \mathrm{E}-2 \mathrm{~m} / \mathrm{cm}$ & $1.036 \mathrm{E}-01$ & $m$ \\
\hline$A$ - sample clrcular cross sectlonal area & 81.313 & $1.0 \mathrm{E}-4 \mathrm{~m}^{n} 2 / \mathrm{cm}^{n} 2$ & $8.131 E-03$ & $m^{-2} 2$ \\
\hline $\mathrm{Pb}=$ flow measurement basis pressure (absolute) & 0.8308 & $1.013 \mathrm{E}+5 \mathrm{~Pa} / \mathrm{atm}$ & $8.116 \mathrm{E}+04$ & Po \\
\hline$\Delta \mathrm{p}=$ pressure drop across sample length & 0.217 & $1.013 \mathrm{E}+5 \mathrm{~Pa} / \mathrm{atm}$ & $2.198 \mathrm{E}+04$ & Pa \\
\hline $\mathrm{Pm}=$ mean pore pressure (absolute) & 1.942 & $1.013 \mathrm{E}+5 \mathrm{~Pa} / \mathrm{atm}$ & $1.968 E+05$ & $\mathbf{P a}$ \\
\hline $\mathrm{Pe}_{\mathrm{e}}=$ exit pressure (absolute) & 1.834 & $1.013 \mathrm{E}+5 \mathrm{~Pa} / \mathrm{atm}$ & $1.858 \mathrm{E}+05$ & $\mathrm{~Pa}$ \\
\hline $\mathrm{Te} \cdot$ sample temperature (absolute) & 296 & & 296 & \% $\mathrm{K}$ \\
\hline $\mathrm{Tb}=$ flow measurement basis temperalure (absolute) & 296 & & 296 & ${ }^{\circ} \mathrm{K}$ \\
\hline $2 e=$ gas devlation factor at Pe and Te & 1.0000 & & 1.0000 & \\
\hline $2 \mathrm{~b}$ - gas devlation factor $\mathrm{at} \mathrm{Pb}$ and $\mathrm{Te}$ & 1.0000 & & 1.0000 & \\
\hline Qb - llow rate at base conditlons & $0.05730 \mathrm{~cm}^{\wedge} 3 / \mathrm{s}$ & $1.0 \mathrm{E}-6 \mathrm{~m}^{\wedge} 3 / \mathrm{cm}^{\wedge} 3$ & $5.730 \varepsilon-08$ & $m^{2} 3 / 3$ \\
\hline ve $=$ flow velocity at sample exit end & $3.193 \mathrm{E}-04$ & $1.0 \mathrm{E}-2 \mathrm{~m} / \mathrm{cm}$ & $3.193 \mathrm{E}-06$ & $\mathrm{~m} / \mathrm{s}$ \\
\hline $\mathrm{Ka}_{\mathrm{a}}=$ & $2.53 \mathrm{E}-04$ & $9.872 \mathrm{E}-13 \mathrm{~m}^{\wedge} 2 / \mathrm{d}$ & $2.50 \mathrm{E}-16$ & $m^{\wedge} 2$ \\
\hline $\mathrm{Ka}=$ & $2.53 \mathrm{E}-01$ & & $2.50 \mathrm{E}-12$ & $c m^{\wedge} 2$ \\
\hline $\mathrm{Ka}=$ & $2.53 \mathrm{E}+02$ & & & \\
\hline
\end{tabular}




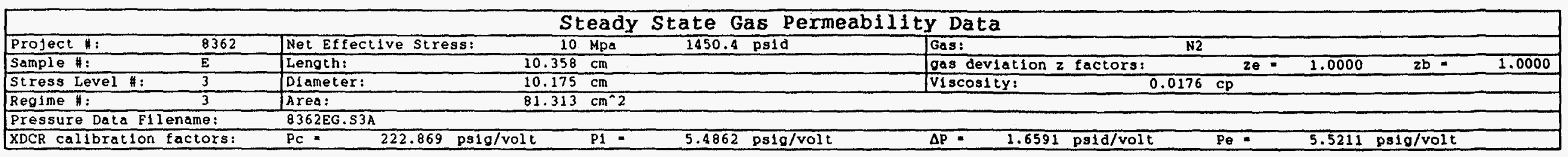

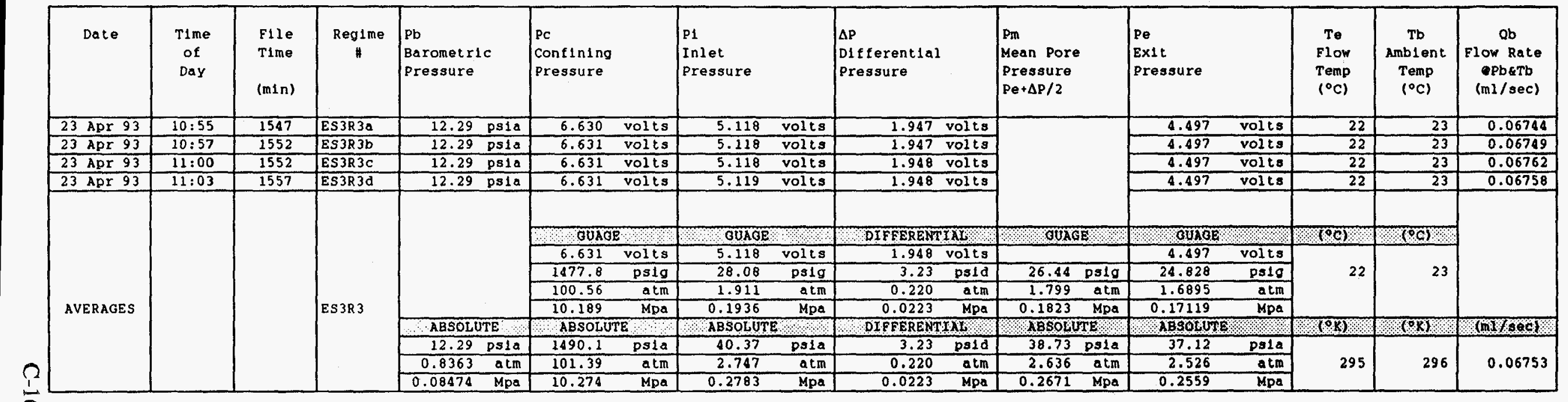

क

Apparent gas permeablifty:

$K a=\left(v e^{*} e^{*} U * L\right) /\left(P m^{*} \Delta P\right)$

Bợit's LaW:

$V_{e}=(P b / P e) *(T e / T b) *(z e / z b) * V b$

$Q e=(\mathrm{Pb} / \mathrm{Pe}) \cdot(\mathrm{Te} / \mathrm{Tb}) \cdot(z e / 2 \mathrm{~b}) * \mathrm{Qb}$

$v_{e}=Q_{e} / A=(\mathrm{Pb} / \mathrm{Pe}) *(\mathrm{Te} / \mathrm{Tb}) *(z e / 2 b) *(\mathrm{Ob} / \mathrm{A})$

\begin{tabular}{|c|c|c|c|c|}
\hline Parameter & $\begin{array}{l}\text { Trad1tional } \\
\text { Un1ts }\end{array}$ & & $\begin{array}{l}\text { SI } \\
\text { Units }\end{array}$ & \\
\hline$\mu=$ gas viscosity & 0.0176 & $01.0 \mathrm{E}-3 \mathrm{~Pa} * \mathrm{sec} / \mathrm{cp}$ & $1.760 \varepsilon-05$ & Pa*sec \\
\hline$L=$ sample length & 10.358 & $1.0 \mathrm{E}-2 \mathrm{~m} / \mathrm{cm}$ & $1.036 \mathrm{E}-01$ & $\mathbf{m}$ \\
\hline$A=$ sample clrcular cross sectional area & 81.313 & $\$ 1.0 \mathrm{E}-4 \mathrm{~m}^{\wedge} 2 / \mathrm{cm}^{\wedge} 2$ & $8.131 E-03$ & $m^{\wedge} 2$ \\
\hline $\mathrm{pb}=$ flow measurement basis pressure (absolute) & 0.8363 & $* 1.013 \mathrm{E}+5 \mathrm{~Pa} / \mathrm{atm}$ & $8.472 E+04$ & $\mathrm{~Pa}$ \\
\hline$\Delta \mathrm{P}$ - pressure drop across sample length & 0.220 & $1.013 \mathrm{E}+5 \mathrm{~Pa} / \mathrm{atm}$ & $2.227 E+04$ & $\mathrm{~Pa}$ \\
\hline Pm $=$ mean pore pressure (absolute) & 2.636 & $1.013 \mathrm{E}+5 \mathrm{~Pa} / \mathrm{atm}$ & $2.670 E+05$ & Pa \\
\hline Pe $=$ exlt pressure (absolute) & 2.526 & $1.013 E+5 \mathrm{~Pa} / \mathrm{atm}$ & $2.559 E+05$ & Pa \\
\hline Te = sample temperature (absolute) & 295 & & 295 & ${ }^{\circ} \mathrm{K}$ \\
\hline $\mathrm{Tb}=$ flow measurement basis temperature (absolute) & 296 & & 296 & ${ }^{\circ} \mathrm{K}$ \\
\hline$z e=$ gas deviation factor at $\mathrm{Pe}$ and $\mathrm{Te}$ & 1.0000 & & 1.0000 & \\
\hline $\mathrm{zb}=$ gas deviation factor $\mathrm{at} \mathrm{Pb}$ and $\mathrm{Te}$ & 1.0000 & & 1.0000 & \\
\hline$\overline{Q b}=$ flow rate at base conditions & $0.06753 \mathrm{~cm}^{\wedge} 3 / \mathrm{s}$ & $-1.0 \mathrm{E}-6 \mathrm{~m}^{2} 3 / \mathrm{cm}^{2} 3$ & $6.753 \mathrm{E}-08$ & $m^{2} 3 / 3$ \\
\hline ve $=$ flow velocity at sample exit end & $2.741 \mathrm{E}-04 \mathrm{~cm} / \mathrm{s}$ & $1.0 \mathrm{E}-2 \mathrm{~m} / \mathrm{cm}$ & $2.741 E-06$ & $\mathrm{~m} / \mathrm{s}$ \\
\hline - Ka $=$ & $2.18 \mathrm{E}-04$ & $9.872 \mathrm{E}-13 \mathrm{~m}^{\wedge} 2 / \mathrm{d}$ & $2.15 E-16$ & $m^{\wedge} 2$ \\
\hline $\mathrm{Ka}=$ & $2.18 \mathrm{E}-01$ & & $2.15 E-12$ & $\mathrm{~cm}^{2} 2$ \\
\hline $\mathrm{Ka}=$ & $2.18 E+02$ & & & \\
\hline
\end{tabular}




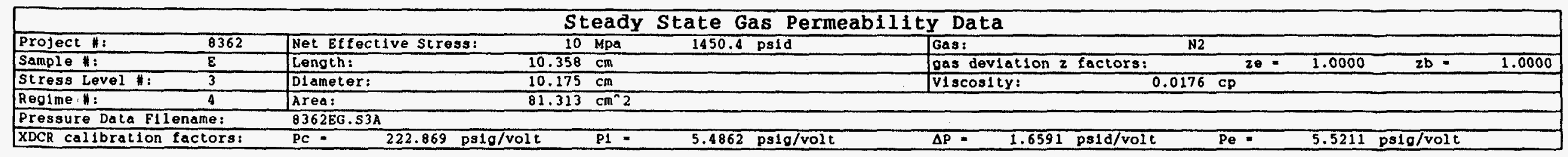






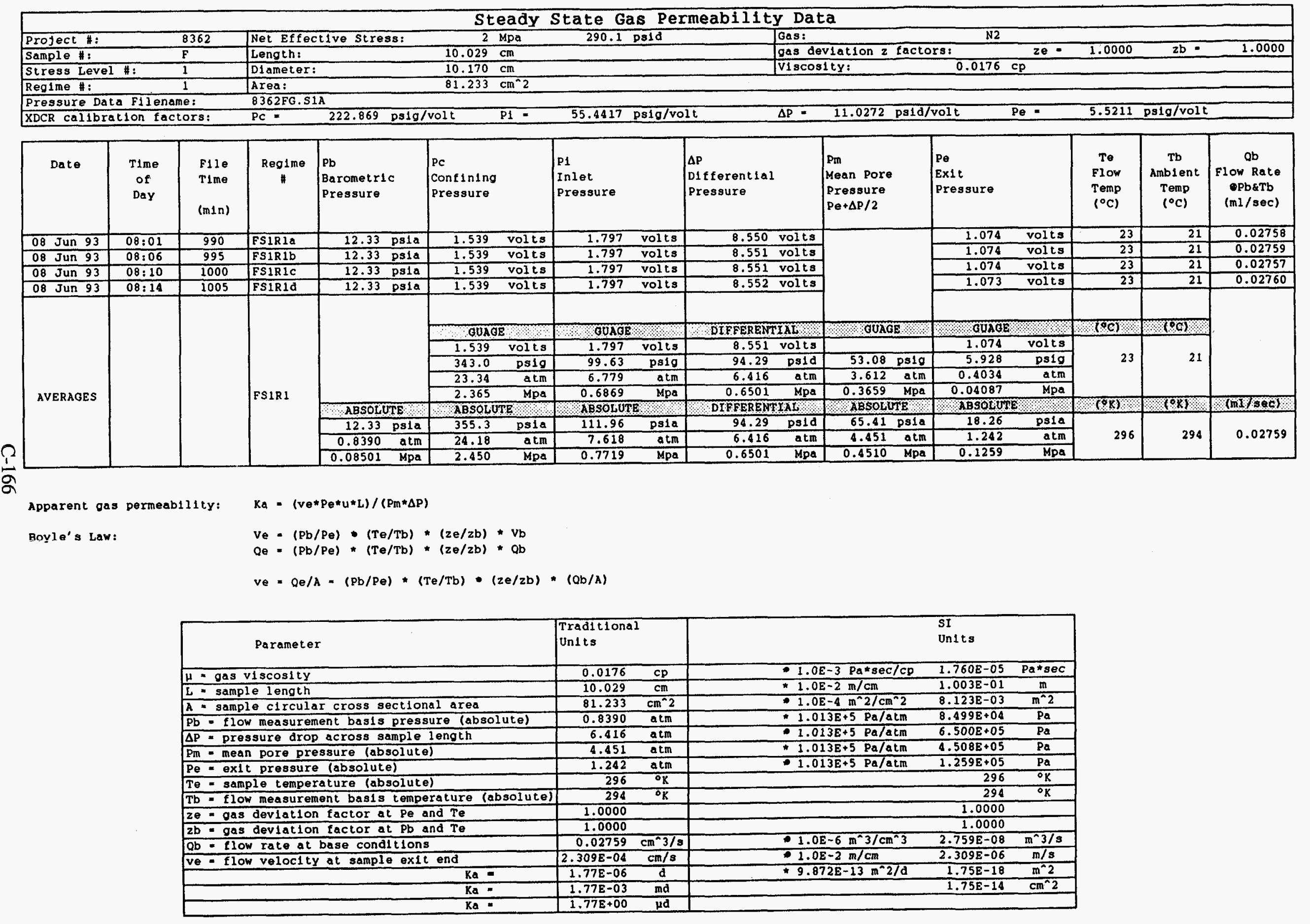




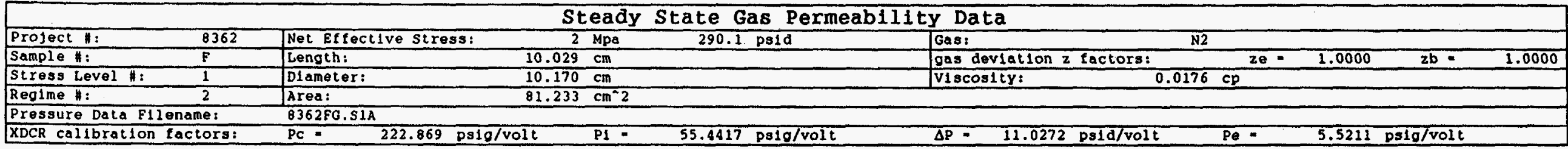

\begin{tabular}{|c|c|c|c|c|c|c|c|c|c|c|c|c|}
\hline Date & $\begin{array}{l}\text { Time } \\
\text { of } \\
\text { Day }\end{array}$ & $\begin{array}{l}\text { Flle } \\
\text { Time } \\
(m i n)\end{array}$ & Regime & $\begin{array}{l}\text { Pb } \\
\text { Barometric } \\
\text { Pressure }\end{array}$ & $\begin{array}{l}\text { Pc } \\
\text { Confining } \\
\text { Pressure }\end{array}$ & $\begin{array}{l}\text { P1 } \\
\text { Inlet } \\
\text { Pressure }\end{array}$ & $\begin{array}{l}\Delta \mathrm{P} \\
\text { DIfferential } \\
\text { Pressure }\end{array}$ & $\begin{array}{l}\mathrm{Pm} \\
\text { Mean Pore } \\
\text { Pressure } \\
\mathrm{Pe}+\Delta \mathrm{P} / 2\end{array}$ & $\begin{array}{l}\text { Pe } \\
\text { Ex1t } \\
\text { Pressure }\end{array}$ & $\begin{array}{l}\text { Te } \\
\text { Flow } \\
\text { Temp } \\
\left({ }^{\circ} \mathrm{C}\right)\end{array}$ & $\begin{array}{c}\text { Tb } \\
\text { Amb1ent } \\
\text { Temp } \\
\left({ }^{\circ} \mathrm{C}\right)\end{array}$ & $\begin{array}{c}\text { Ob } \\
\text { Flow Rate } \\
\text { opbstb } \\
\text { (m1/sec) }\end{array}$ \\
\hline 08 Jun 93 & $11: 52$ & 1226 & FS1R2a & $12.35 \mathrm{ps} 1 \mathrm{a}$ & volts & volts & 8.549 volts & & volts & 23 & 21 & 0.03051 \\
\hline 08 Jun 93 & $11: 56$ & 1226 & FS1R2b & $12.35 \mathrm{psia}$ & 1.584 & volts & 8.550 volts & & volts & 23 & 21 & 0.03048 \\
\hline 08 Jun 93 & $12: 00$ & 1231 & FS1R2C & $12.35 \mathrm{ps} 1 \mathrm{a}$ & volts & volts & 8.550 volts & & volts & 23 & 21 & 0.03040 \\
\hline 08 Jun 93 & $22: 04$ & 1231 & ES1R2d & 12.35 ps1a & volts & 1.985 volts & 8.550 volts & & volts & 23 & 21 & 0.03041 \\
\hline \multirow{9}{*}{ AVERAGES } & & & \multirow{9}{*}{ FS1R2 } & & WOUACE & 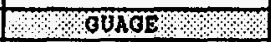 & DIFEERENTIAL & PैOOXGE & ouroe & \%) & roc) & \\
\hline & & & & & 1.584 volts & 1.985 volts & 8.550 volts & & 2.971 & \multirow{4}{*}{23} & \multirow{4}{*}{21} & \\
\hline & & & & & 353.0 & 110.05 & 94.28 psid & $63.54 \mathrm{psig}$ & 16.403 & & & \\
\hline & & & & & 24.02 & 7.489 & 6.415 & 4.324 atm & 1.1162 & & & \\
\hline & & & & & 2.434 & 0.7588 & $0.6500 \quad \mathrm{Mpa}$ & $0.4381 \mathrm{Mpa}$ & 0.11310 & & & \\
\hline & & & & ABSOLUTE & ABSOLUTE & ABSOLUTE & DIFFERENTIAL & ABSOLUTY & AsSOCOTC & \%) & \%) & (WW $60 \mathrm{C}$ \\
\hline & & & & $12.35 \mathrm{ps} 1 \mathrm{a}$ & $365.4 \quad$ ps1a & 122.40 ps1a & 94.28 psid & $75.89 \mathrm{pS1a}$ & 28.75 & \multirow{3}{*}{296} & \multirow{3}{*}{294} & \multirow{3}{*}{0.03045} \\
\hline & & & & 0.8404 atm & 24.86 & 8.329 & 6.415 & $5.164 \mathrm{~atm}$ & 1.957 & & & \\
\hline & & & & $0.08515 \mathrm{Mpa}$ & 2.519 & 0.8439 & 0.6500 & 0.5233 & 0.1982 & & & \\
\hline
\end{tabular}

Apparent gas permeab111ty: Ka $=\left(v e^{\star P e * u * L)} /(P m \star \Delta P)\right.$

Boyle's Law:

$V_{e}=(\mathrm{Pb} / \mathrm{Pe}) *(\mathrm{Te} / \mathrm{Tb}) *(z e / 2 b) * \mathrm{Vb}$

$\mathrm{ge} \cdot(\mathrm{Pb} / \mathrm{Pe}) \cdot(\mathrm{T} \theta / \mathrm{Tb}) \cdot(2 e / z \mathrm{~b}) \cdot \mathrm{Qb}$

ve $=Q_{e} / \mathrm{A}=(\mathrm{Pb} / \mathrm{Pe}) *(\mathrm{Te} / \mathrm{Tb}) *(\mathrm{ze} / \mathrm{Zb}) *(\mathrm{Ob} / \mathrm{A})$

\begin{tabular}{|c|c|c|c|c|}
\hline Parameter & $\begin{array}{l}\text { Traditlonal } \\
\text { Untts }\end{array}$ & & $\begin{array}{l}\text { SI } \\
\text { Units }\end{array}$ & \\
\hline$\mu=$ gas viscosity & 0.0176 & $1.0 \mathrm{E}-3 \mathrm{~Pa} * \mathrm{seC} / \mathrm{CP}$ & $1.760 \mathrm{E}-05$ & Pa*sec \\
\hline$L=$ sample length & 10.029 & $\star 1.0 \mathrm{E}-2 \mathrm{~m} / \mathrm{cm}$ & $1.003 \mathrm{E}-01$ & $m$ \\
\hline A = sample clrcular cross sectlonal area & 81.233 & $* 1.0 E-4 \mathrm{~m}^{2} 2 / \mathrm{cm}^{2} 2$ & $8.123 E-03$ & $\overline{m^{2} 2}$ \\
\hline $\mathrm{Pb}=$ flow measurement basis pressure (absolute) & 0.8404 & $\star 1.013 \mathrm{E}+5 \mathrm{~Pa} / \mathrm{atm}$ & $8.513 E+04$ & $\mathrm{~Pa}$ \\
\hline$\Delta \mathrm{P}=$ pressure drop across sample length & 6.415 & $\$ 1.013 \mathrm{E}+5 \mathrm{~Pa} / \mathrm{atm}$ & $6.499 E+05$ & $\overline{\mathbf{P a}}$ \\
\hline $\mathrm{Pm}=$ mean pore pressure (absolute) & 5.164 & $\star 1.013 \mathrm{E}+5 \mathrm{~Pa} / \mathrm{atm}$ & $5.231 E+05$ & $\mathbf{P a}$ \\
\hline$P_{e}=$ exit pressure (absolute) & 1.957 & $1.013 \mathrm{E}+5 \mathrm{~Pa} / \mathrm{atm}$ & $1.982 \mathrm{E}+05$ & $\mathbf{P a}$ \\
\hline Te = sample temperature (absolute) & 296 & & 296 & ${ }^{\circ} \mathrm{K}$ \\
\hline $\mathrm{Tb}=$ flow measurement bas1s temperature (absolute) & 294 & & 294 & ${ }^{\circ} \mathrm{K}$ \\
\hline$z e=$ gas deviation factor at $\mathrm{Pe}_{\mathrm{e}}$ and $\mathrm{Te}$ & 1.0000 & & 1.0000 & \\
\hline $\mathrm{zb}=$ gas deviatlon factor at $\mathrm{Pb}$ and $\mathrm{Te}$ & 1.0000 & & 1.0000 & \\
\hline $\mathrm{ab}=$ flow rate at base conditions & $0.03045 \mathrm{~cm}^{\wedge} 3 / \mathrm{s}$ & $\star 1.0 \mathrm{E}-6 \mathrm{~m}^{\wedge} 3 / \mathrm{cm}^{\wedge} 3$ & $3.045 E-08$ & $m^{2} 3 / s$ \\
\hline ve $=$ flow velocity at sample exit end & $1.621 E-04$ & $\star 1.0 \mathrm{E}-2 \mathrm{~m} / \mathrm{cm}$ & $1.621 \mathrm{E}-06$ & $\mathrm{~m} / \mathrm{s}$ \\
\hline $\mathrm{Ka}=$ & $1.69 E-06$ & $\star 9.872 \mathrm{E}-13 \mathrm{~m}^{\wedge} 2 / \mathrm{d}$ & $1.67 \mathrm{E}-18$ & $m^{\wedge} 2$ \\
\hline $\mathrm{Ka}=$ & $1.69 \mathrm{E}-03$ & & $1.67 E-14$ & $\mathrm{~cm}^{2} 2$ \\
\hline Ka- & $1.69 E+00$ & & & \\
\hline
\end{tabular}




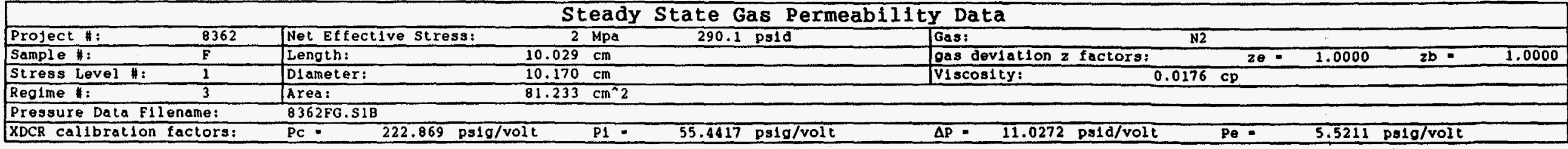

\begin{tabular}{|c|c|c|c|c|c|c|c|c|c|c|c|c|}
\hline Date & $\begin{array}{l}\text { Time } \\
\text { of } \\
\text { Day }\end{array}$ & $\begin{array}{l}\text { Flle } \\
\text { Time } \\
\text { (min) }\end{array}$ & Reglme & \begin{tabular}{|l} 
Pb \\
Barometrlc \\
Pressure
\end{tabular} & $\begin{array}{l}\text { Pc } \\
\text { Confining } \\
\text { Pressure }\end{array}$ & $\begin{array}{l}\text { Pl } \\
\text { Inlet } \\
\text { Pressure }\end{array}$ & \begin{tabular}{|l}
$\Delta \mathrm{P}$ \\
Differential \\
Pressure
\end{tabular} & $\begin{array}{l}\mathrm{Pm} \\
\text { Mean Pore } \\
\text { Pressure } \\
\mathrm{Pe}+\Delta \mathrm{P} / 2\end{array}$ & $\begin{array}{l}\text { Pe } \\
\text { Exit } \\
\text { Pressure }\end{array}$ & $\begin{array}{l}\text { Te } \\
\text { Flow } \\
\text { Temp } \\
\text { (oc) }\end{array}$ & $\begin{array}{c}\text { Tb } \\
\text { Amblent } \\
\text { Temp } \\
\left({ }^{\circ} \mathrm{C}\right)\end{array}$ & $\begin{array}{c}\text { Qb } \\
\text { Flow Race } \\
\text { Pps\&Tb } \\
\text { (m1/sec) }\end{array}$ \\
\hline 08 Jun 93 & $16: 36$ & 140 & FS1R3a & $12.35 \mathrm{psla}$ & 1.629 volts & volts & 8.601 volts & & 4.695 & 23 & 22 & 0.03333 \\
\hline 08 Jun 93 & $16: 42$ & 145 & ESIR3b & 12.35 ps1a & volts & volts & 8.601 volts & & volts & 23 & 22 & 0.03327 \\
\hline 08 Jun 93 & $16: 46$ & 150 & FS1R3c & $12.35 \mathrm{pg} 1 \mathrm{~A}$ & volts & volts & 8.601 volts & & volts & 23 & 22 & 0.03329 \\
\hline 08 Jun 93 & $16: 49$ & 155 & FSIR3d & $12.35 \mathrm{ps} 1 \mathrm{a}$ & 1.629 volts & volts & 8.601 volts & & volts & 23 & 22 & 0.03332 \\
\hline \multirow{9}{*}{ AVERAOES } & & & \multirow{9}{*}{ FSIR3 } & & GUXGE & OOOKGE: & DTFFERAKXXX & OOOAG: & oonor & (6) & $8 \mathrm{~d}$ & \\
\hline & & & & & 1.629 volts & 2.166 volts & 8.601 volts & & 4.695 & \multirow{4}{*}{23} & \multirow{4}{*}{22} & \\
\hline & & & & & 363.1 & 120.09 & 94.84 psid & $73.34 \mathrm{ps} 1 \mathrm{~g}$ & 25.920 & & & \\
\hline & & & & & 24.70 & 8.171 & 6.454 & $4.991 \mathrm{~atm}$ & 1.7638 & & & \\
\hline & & & & & $2.503 \quad \mathrm{Mpa}$ & 0.8280 & 0.6539 & $0.5057 \mathrm{Mpa}$ & 0.17871 & & & \\
\hline & & & & ABSOLUTE & ABSOLUTE & ABSOLOTE & DTEFERENT IX L & KaSOLUTE & XB30turs: & (6) & 6x: & (hisecr \\
\hline & & & & $12.35 \mathrm{psia}$ & 375.4 ps1a & 132.44 psia & 94.84 psid & $85.69 \mathrm{ps} 1 \mathrm{a}$ & 38.27 & \multirow{3}{*}{296} & \multirow{3}{*}{295} & \multirow{3}{*}{0.03330} \\
\hline & & & & $0.8404 \mathrm{~atm}$ & 25.54 & 9.012 & 6.454 & 5.831 atm & 2.604 & & & \\
\hline & & & & 0.08515 & 2.588 & 0.9131 & 0.6539 & 0.5908 & 0.2639 & & & \\
\hline
\end{tabular}

$\frac{1}{\infty}$

Apporent gas permeabllity: $\quad K a=($ ve*Pe*u*L)/(PntaP)

Boyle's Law

Ve $=(F b / F e) \neq(T e / T b)+\{z e / z b) \cdot v b$

$\mathrm{Qe} \cdot(\mathrm{Pb} / \mathrm{Pe}) *(\mathrm{Te} / \mathrm{Tb}) \cdot(\mathrm{ze} / \mathrm{zb}) * \mathrm{Qb}$

$v_{e}=Q_{e} / \mathrm{A}=(\mathrm{Pb} / \mathrm{Pe}) *(\mathrm{Te} / \mathrm{Tb}) *(\mathrm{ze} / \mathrm{zb}) \cdot(\mathrm{Qb} / \mathrm{A})$

\begin{tabular}{|c|c|c|c|c|}
\hline Parameter & $\begin{array}{l}\text { Trad1tional } \\
\text { Un1ts }\end{array}$ & & $\begin{array}{l}\text { SI } \\
\text { Un1ts }\end{array}$ & \\
\hline$\mu=$ gas viscosity & 0.0176 & $-1.0 \mathrm{E}-3 \mathrm{~Pa} * \mathrm{SeC} / \mathrm{CP}$ & $1.760 \mathrm{E}-05$ & Patsec \\
\hline $\mathrm{L}=$ sample length & 10.029 & $1.0 \mathrm{E}-2 \mathrm{~m} / \mathrm{cm}$ & $1.003 \mathrm{E}-01$ & $\mathrm{~m}$ \\
\hline A - sample clrcular cross sectlonal area & 81.233 & $1.0 \mathrm{E}-4 \mathrm{~m}^{\wedge} 2 / \mathrm{cm}^{\wedge} 2$ & $8.123 \mathrm{E}-03$ & $m^{\wedge} 2$ \\
\hline $\mathrm{Pb}$ - flow measurement basls pressure (absolute) & 0.8404 & $-1.013 \mathrm{E}+5 \mathrm{~Pa} / \mathrm{alm}$ & $8.513 E+04$ & $\mathrm{~Pa}_{\mathrm{a}}$ \\
\hline$\Delta \mathrm{P}=$ pressure drop across sample length & 6.454 & $01.013 \mathrm{E}+5 \mathrm{~Pa} / \mathrm{atm}$ & $6.538 E+05$ & $\mathrm{~Pa}$ \\
\hline $\mathrm{Pm}_{\mathrm{m}}=$ mean pore pressure (absolute) & 5.831 & $\$ 1.013 \mathrm{E}+5 \mathrm{~Pa} / \mathrm{atm}$ & $5.907 E+05$ & Pa \\
\hline$P_{e}=$ exit pressure (absolute) & 2.604 & $1.013 \mathrm{E}+5 \mathrm{~Pa} / \mathrm{atm}$ & $2.638 E+05$ & $\mathbf{P a}$ \\
\hline Te - sample temperature (absolute) & 296 & & 296 & ${ }^{\circ} \mathrm{K}$ \\
\hline $\mathrm{Tb}=$ flow measurement basls temperature (absolute) & 295 & & 295 & ${ }^{\circ} \mathrm{K}$ \\
\hline $2 e=$ gas deviatlon factor at $\mathrm{Pe}$ and $\mathrm{Te}$ & 1.0000 & & 1.0000 & \\
\hline $2 \mathrm{~b}=$ gas deviallon factor at $\mathrm{Pb}$ and $\mathrm{Te}$ & 1.0000 & & 1.0000 & \\
\hline $\mathrm{Qb}=$ flow rate at base conditions & $0.03330 \mathrm{~cm}^{1} 3 / \mathrm{s}$ & $1.0 \mathrm{E}-6 \mathrm{~m}^{\wedge} 3 / \mathrm{cm}^{\wedge} 3$ & $3.330 \mathrm{E}-08$ & $m^{2} 3 / 3$ \\
\hline ve = flow veloclty at sample exit end & $1.327 \mathrm{E}-04 \mathrm{~cm} / \mathrm{s}$ & $1.0 \mathrm{E}-2 \mathrm{~m} / \mathrm{cm}$ & $1.327 \mathrm{E}-06$ & $\mathrm{~m} / \mathrm{s}$ \\
\hline $\mathrm{Ka}_{a}=$ & $1.62 \mathrm{E}-06$ & $9.872 \mathrm{E}-13 \mathrm{~m}^{\wedge} 2 / \mathrm{d}$ & $1.60 \mathrm{E}-18$ & $m^{\wedge} 2$ \\
\hline $\mathrm{Ka} \cdot$ & $1.62 \mathrm{E}-03$ & & $1.60 E-14$ & $\mathrm{~cm}^{\wedge} 2$ \\
\hline $\mathbf{R a}_{\mathbf{a}}=$ & $1.62 E+00$ & & & \\
\hline
\end{tabular}




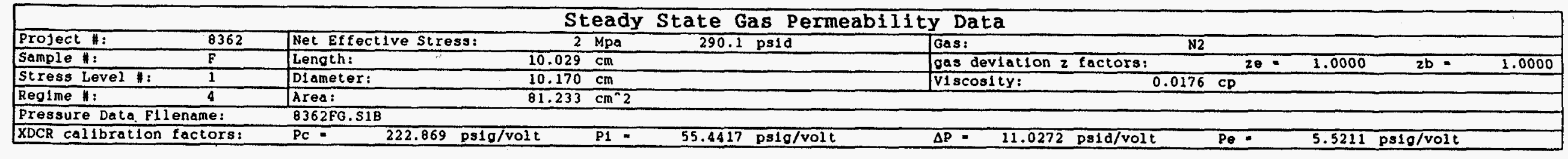

\begin{tabular}{|c|c|c|c|c|c|c|c|c|c|c|c|c|}
\hline Date & $\begin{array}{l}\text { TIme } \\
\text { of } \\
\text { Day }\end{array}$ & $\begin{array}{l}\text { File } \\
\text { Time } \\
(m 1 n)\end{array}$ & Regime & $\begin{array}{l}\text { Pb } \\
\text { Barometric } \\
\text { Pressure }\end{array}$ & $\begin{array}{l}\text { Pc } \\
\text { Confining } \\
\text { Pressure }\end{array}$ & $\begin{array}{l}\text { P1 } \\
\text { Inlet } \\
\text { Pressure }\end{array}$ & $\begin{array}{l}\Delta P \\
D 1 \text { fferential } \\
\text { Pressure }\end{array}$ & $\begin{array}{l}\mathrm{Pm} \\
\text { Mean Pore } \\
\text { Pressure } \\
\mathrm{Pe}+\Delta \mathrm{P} / 2\end{array}$ & $\begin{array}{l}\text { Pe } \\
\text { Exit } \\
\text { Pressure }\end{array}$ & $\begin{array}{l}\text { Te } \\
\text { Flow } \\
\text { Temp } \\
\left({ }^{\circ} \mathrm{C}\right)\end{array}$ & $\begin{array}{c}\text { Tb } \\
\text { Amblent } \\
\text { Temp } \\
\left({ }^{\circ} \mathrm{C}\right)\end{array}$ & $\begin{array}{c}\text { Ob } \\
\text { Flow Race } \\
\text { PPbTb } \\
\text { (m1/sec) }\end{array}$ \\
\hline 09 Jun 93 & $09: 09$ & 1132 & FS1R4a & $12.39 \mathrm{ps} 1 \mathrm{a}$ & volts & volts & 8.509 volts & & volts & 23 & 21 & 0.03535 \\
\hline 09 Jun 93 & $09: 11$ & 1137 & FS1R4b & $12.39 \mathrm{ps1a}$ & volts & volts & 8,509 volts & & volls & 23 & 21 & 0.03532 \\
\hline 09 Jun 93 & $09: 14$ & 1132 & FS1R4C & $12.39 \quad \mathrm{ps} 1 \mathrm{a}$ & volts & volts & 8.510 volts & & volts & 23 & 21 & 0.03532 \\
\hline 09 Jun 93 & $09: 18$ & 1142 & FS1R4d & $12.39 \mathrm{ps} 1 \mathrm{a}$ & volts & volus & 0.511 volis & & volts & 23 & 21 & 0.03539 \\
\hline \multirow{9}{*}{ AVERAGES } & & & \multirow{9}{*}{ FS1R4 } & & GOAGE & OUNGE & DYFFERETH IA L & OUXGE & GUAGE & (6) & (क्ल) & \\
\hline & & & & & 1.674 volts & 2.333 volts & 8.510 volts & & 6.546 & \multirow{4}{*}{23} & \multirow{4}{*}{21} & \\
\hline & & & & & 373.1 & 129.35 & 93.84 ps1d & $83.06 \mathrm{ps} 1 \mathrm{~g}$ & 36.141 & & & \\
\hline & & & & & 25.39 & 8.801 & 6.385 & $5.652 \mathrm{~atm}$ & 2.4592 & & & \\
\hline & & & & & $2.572 \quad \mathrm{Mpa}$ & 0.8918 & 0.6470 & 0.5727 & 0.24918 & & & \\
\hline & & & & ABSOLUTE & ABSOLUTE & ABSOIUTE & DTFEEREATIAL & XESOLUTE & ABSOLUTE & (Ox) & $\mathrm{OK})$ & (nil/sec) \\
\hline & & & & 12.39 psia & $385.5 \quad$ ps1a & $141.74 \quad \mathrm{ps10}$ & 93.04 psid & 95.45 ps1a & 48.53 & \multirow{3}{*}{296} & \multirow{3}{*}{294} & \multirow{3}{*}{0.03535} \\
\hline & & & & $0.0431 \mathrm{~atm}$ & 26.23 & 9.644 & 6.385 & $6.495 \quad \mathrm{~atm}$ & 3.302 & & & \\
\hline & & & & 0.08543 & 2.658 & 0.9772 & 0.6470 & 0.6581 & 0.3346 & & & \\
\hline
\end{tabular}

8

Apparent gas permeability:

Boyle's Law:

$K a=\left(V e * P e^{*} u * L\right) /\left(P P^{*} \Delta P\right)$

$\mathrm{Ve}=(\mathrm{Pb} / \mathrm{Pe}) \star(\mathrm{Te} / \mathrm{Tb}) \cdot(\mathrm{ze} / \mathrm{zb}) * \mathrm{Vb}$

$\mathrm{Qe}^{-}(\mathrm{Pb} / \mathrm{Pe}) *(\mathrm{Te} / \mathrm{Tb}) \cdot(\mathrm{ze} / \mathrm{zb}) * \mathrm{Qb}$

$v e=Q e / A=(\mathrm{Pb} / \mathrm{Pe}) *(\mathrm{Te} / \mathrm{Tb}) *(z e / z b) *(\mathrm{Qb} / \mathrm{A})$

\begin{tabular}{|l|l|l|}
\hline Parameter & $\begin{array}{l}\text { Traditlonal } \\
\text { Un1ts }\end{array}$ & SI \\
Un1ts
\end{tabular}




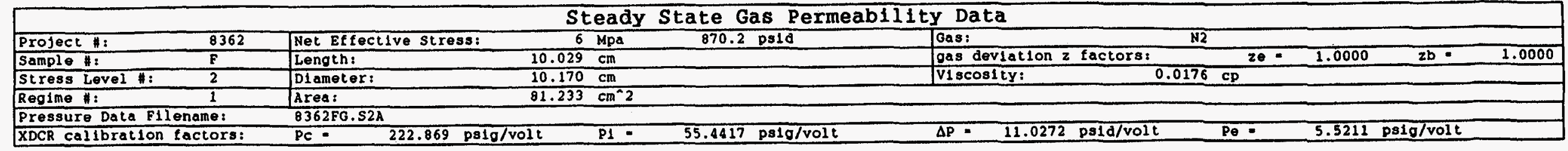

\begin{tabular}{|c|c|c|c|c|c|c|c|c|c|c|c|c|}
\hline Date & $\begin{array}{l}\text { Time } \\
\text { of } \\
\text { Day }\end{array}$ & $\begin{array}{l}\text { File } \\
\text { Time } \\
(m 1 n)\end{array}$ & Regime & \begin{tabular}{|l} 
Pb \\
Barometric \\
Pressure
\end{tabular} & $\begin{array}{l}\text { Pc } \\
\text { Confining } \\
\text { Pressure }\end{array}$ & $\begin{array}{l}\text { P1 } \\
\text { Inlet } \\
\text { Pressure }\end{array}$ & $\begin{array}{l}\Delta \mathrm{P} \\
\text { D1fferential } \\
\text { Prossuro }\end{array}$ & $\begin{array}{l}\mathrm{Pm} \\
\text { Mean Pore } \\
\text { Pressure } \\
\mathrm{Pe}+\Delta \mathrm{P} / 2\end{array}$ & \begin{tabular}{|l} 
Pe \\
Exit \\
Pressure
\end{tabular} & $\begin{array}{l}\text { Te } \\
\text { Flow } \\
\text { Temp } \\
\left({ }^{\circ} \mathrm{C}\right)\end{array}$ & $\begin{array}{c}\text { Tb } \\
\text { Amblent } \\
\text { Temp } \\
\left({ }^{\circ} \mathrm{C}\right)\end{array}$ & $\begin{array}{c}\text { Ob } \\
\text { Flow Rate } \\
\text { OPb\&Tb } \\
\text { (m1/sec) }\end{array}$ \\
\hline 09 Jun 93 & $15: 20$ & 230 & FS2R1a & $12.37 \quad \mathrm{ps1a}$ & volts & volts & 8.612 volts & & volts & 23 & 22 & 0.01939 \\
\hline 09 Jun 93 & $15: 25$ & 235 & FS2R1b & 12.37 psla & volts & volts & 8.613 volts & & volts & 23 & 22 & 0.01935 \\
\hline 09 Jun 93 & $15: 33$ & 240 & FS2R1C & $12.37 \mathrm{psia}$ & volts & volts & 8.613 volts & & volts & 23 & 22 & 0.01939 \\
\hline 09 Jun 93 & $15: 38$ & 245 & FS2R1d & 12.37 psla & 4.142 volts & volts & 0.613 volts & & volts & 23 & 22 & 0.01934 \\
\hline \multirow{9}{*}{ AVERAGES } & & & \multirow{9}{*}{ FS2RI } & & OOUKE & GOAAD & DTFEERENT IAI I & GOXGE: & OUAOE & rod & (oc) & \\
\hline & & & & & 4.142 vo1ts & \begin{tabular}{|ll}
1.806 & volts
\end{tabular} & 8.613 volts & & 1.044 & \multirow{4}{*}{23} & \multirow{4}{*}{22} & \\
\hline & & & & & 923.1 & 100.13 & 94.97 psid & 53.25 ps1g & 5.764 & & & \\
\hline & & & & & 62.81 & 6.813 & 6.463 & 3.624 atm & 0.3922 & & & \\
\hline & & & & & $6.365 \quad \mathrm{Mpa}$ & 0.6904 & $0.6548 \quad \mathrm{Mpa}$ & $0.3672 \mathrm{Mpa}$ & 0.03974 & & & \\
\hline & & & & ABSDLUTE & ABSOLUTE & ABsaLTIS & OIFFERENTIX & \multirow{2}{*}{\multicolumn{2}{|c|}{$\frac{\text { ABSOLTTE }}{65.62 \text { ps1a }}$}} & (OX) & (ox) & (m1/Bec) \\
\hline & & & & 12.37 psla & 935.5 ps1a & 112.50 psia & 94.97 ps1d & & & \multirow{3}{*}{296} & \multirow{3}{*}{295} & \multirow{3}{*}{0.01937} \\
\hline & & & & $0.8417 \mathrm{~atm}$ & 63.66 & 7.655 & 6.463 & $4.465 \mathrm{~atm}$ & 1.234 & & & \\
\hline & & & & 0.08529 & 6.450 & 0.7756 & 0.6548 & 0.4524 & 0.1250 & & & \\
\hline
\end{tabular}

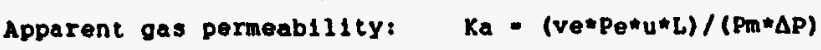

Dựiéc Lä̀i:

$\mathrm{Ye}_{e} \cdot\left(\mathrm{ph} / \mathrm{Pe}_{e}\right) \cdot(\mathrm{Te} / \mathrm{Tb}) \cdot(\mathrm{ze} / \mathrm{zb}) \cdot \mathrm{Vb}$

$\mathrm{Qe}-(\mathrm{Pb} / \mathrm{Pe}) \cdot(\mathrm{Te} / \mathrm{Tb}) \cdot(\mathrm{ze} / \mathrm{zb}) \cdot \mathrm{Qb}$

ve $=Q e / A=(P b / P Q) *(T e / T b) *(2 e / z b) *(Q b / A)$

\begin{tabular}{|c|c|c|c|c|}
\hline Parameter & \begin{tabular}{|l|} 
Trad1tional \\
Units
\end{tabular} & & $\begin{array}{l}\text { SI } \\
\text { Un1ts }\end{array}$ & \\
\hline$\mu=$ gas viscosity & 0.0176 & $1.0 \mathrm{E}-3 \mathrm{~Pa} * \mathrm{sec} / \mathrm{CP}$ & $1.760 \mathrm{E}-05$ & Pa*sec \\
\hline $\mathrm{L}=$ sample length & 10.029 & $1.0 \mathrm{E}-2 \mathrm{~m} / \mathrm{cm}$ & $1.003 \mathrm{E}-01$ & $\mathbf{m}$ \\
\hline A - sample clrcular cross sectlonal area & 81.233 & $1.0 \mathrm{E}-4 \mathrm{~m}^{\wedge} 2 / \mathrm{cm}^{\wedge} 2$ & $8.123 E-03$ & $m^{2} 2$ \\
\hline $\mathrm{Pb}$ - flow measurement basls pressure (absolute) & 0.8417 & $1.013 \mathrm{E}+5 \mathrm{~Pa} / \mathrm{atm}$ & $8.527 E+04$ & $\mathrm{~Pa}$ \\
\hline$\Delta \mathrm{P}=$ pressure arop across sample length & 6.463 & $1.013 \mathrm{E}+5 \mathrm{~Pa} / \mathrm{atm}$ & $6.547 E+05$ & Pa \\
\hline $\mathrm{P}_{m}=$ mean pore pressure (absolute) & 4.465 & $1.013 \mathrm{E}+5 \mathrm{~Pa} / \mathrm{atm}$ & $4.523 E+05$ & $\mathrm{~Pa}$ \\
\hline Pe = exlt pressure (absolute) & 1.234 & $1.013 \mathrm{E}+5 \mathrm{~Pa} / \mathrm{atm}$ & $1.250 \mathrm{E}+05$ & Pa \\
\hline Te - sample temperature (absolute) & 296 & & 296 & ${ }^{\circ} \mathbf{K}$ \\
\hline Tb - tlow measurement basls temperature (absolute) & 295 & & 295 & ${ }^{\circ} \mathbf{K}$ \\
\hline $2 e$ - gas deviation factor at $\mathrm{Pe}$ and $\mathrm{Te}$ & 1.0000 & & 1.0000 & \\
\hline $2 b$ - gas devlation tactor at pb and Te & 1.0000 & & 1.0000 & \\
\hline $\mathrm{ab}=$ flow rate at base conditions & $0.01937 \mathrm{~cm}^{\wedge} 3 / \mathrm{s}$ & $1.0 \mathrm{E}-6 \mathrm{~m}^{2} 3 / \mathrm{cm}^{4} 3$ & $1.937 E-08$ & $\mathrm{~m}^{\wedge} 3 / \mathrm{s}$ \\
\hline ve - flow velocity at sample exit end & $1.632 \mathrm{E}-04$ & $1.0 \mathrm{E}-2 \mathrm{~m} / \mathrm{cm}$ & $1.632 \mathrm{E}-06$ & $\mathrm{~m} / \mathrm{s}$ \\
\hline Ka & $1.23 \mathrm{E}-05$ & $0.872 \mathrm{E}-13 \mathrm{~m}^{\wedge} 2 / \mathrm{d}$ & $1.22 \mathrm{E}-18$ & $m^{\wedge} 2$ \\
\hline $\mathrm{K}_{a}=$ & $1.23 E-03$ & & $1.22 \mathrm{E}-14$ & $\mathrm{~cm}^{\wedge} 2$ \\
\hline $\mathrm{Ka}=$ & $1.23 E+00$ & & & \\
\hline
\end{tabular}




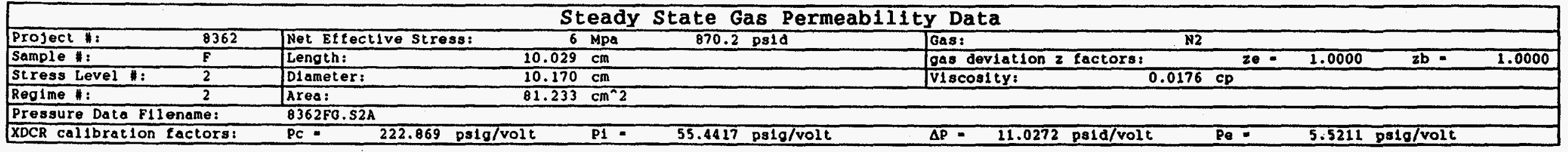

\begin{tabular}{|c|c|c|c|c|c|c|c|c|c|c|c|c|}
\hline Date & $\begin{array}{l}\text { T1me } \\
\text { of } \\
\text { Day }\end{array}$ & $\begin{array}{l}\text { File } \\
\text { Time } \\
\text { (min) }\end{array}$ & $\underset{n}{\text { Regime }}$ & $\begin{array}{l}\text { Pb } \\
\text { Barometrlc } \\
\text { Pressure }\end{array}$ & $\begin{array}{l}\text { Pc } \\
\text { Contining } \\
\text { Pressure }\end{array}$ & $\begin{array}{l}\text { P1 } \\
\text { Inlet } \\
\text { Pressure }\end{array}$ & $\begin{array}{l}\Delta \mathrm{P} \\
\text { Differential } \\
\text { Pressure }\end{array}$ & $\mid \begin{array}{l}\mathrm{Pm} \\
\text { Mean pore } \\
\mathrm{Pressure} \\
\mathrm{Pe}+\Delta \mathrm{P} / 2\end{array}$ & $\begin{array}{l}\text { Pe } \\
\text { ExIt } \\
\text { Pressure }\end{array}$ & $\begin{array}{l}\text { Te } \\
\text { Flow } \\
\text { Temp } \\
\left({ }^{\circ} \mathrm{C}\right)\end{array}$ & $\begin{array}{c}\text { Tb } \\
\text { Amblent } \\
\text { Temp } \\
\left({ }^{\circ} \mathrm{C}\right)\end{array}$ & $\begin{array}{c}\text { Ob } \\
\text { Flow Rate } \\
\text { OPbETb } \\
(\mathrm{ml} / \mathrm{sec})\end{array}$ \\
\hline 10 Jun 93 & $09: 38$ & 1325 & FS2R2a & 12.37 psia & volts & volts & 8.664 volts & & volts & 23 & 22 & 0.02084 \\
\hline 10 Jun 93 & $09: 45$ & 1335 & FS2R2b & 12.37 psla & 4.187 & volts & 8.666 volts & & volts & 23 & 22 & 0.02094 \\
\hline $10 \operatorname{Jun} 93$ & $09: 49$ & 1340 & FS2R2C & $12.37 \mathrm{ps} 1 \mathrm{a}$ & 4.187 & 1.987 & 8.666 volts & & volts & 23 & 22 & 0.02098 \\
\hline 10 Jun 93 & $09: 54$ & 1340 & FS2R2d & 12.37 psia & volts & 1.987 & 8.666 volts & & volts & 23 & 22 & 0.02091 \\
\hline 10 Jun 93 & $10: 00$ & 1345 & FS2R2e & $12.37 \mathrm{psia}$ & 4.187 volts & volts & 8.667 volts & & volts & 23 & 22 & 0.02092 \\
\hline \multirow{9}{*}{ AVERAGES } & & & \multirow{9}{*}{ FS2R 2} & & OONAOE & OOACE & OTHFEREMTXXY & GOXOE & $60 \times 08$ & बख्य & $10 \mathrm{cl}$ & \\
\hline & & & & & 4.187 volts & 1.987 volts & 8.666 volts & & 2.749 & \multirow{4}{*}{23} & \multirow{4}{*}{22} & \\
\hline & & & & & 933.2 ps1g & 110.15 & $95.56 \mathrm{ps} 1 \mathrm{~d}$ & $62.96 \mathrm{ps} 1 \mathrm{~g}$ & 15.175 & & & \\
\hline & & & & & 63.50 & 7.495 & 6.502 & $4.284 \mathrm{~atm}$ & 1.0326 & & & \\
\hline & & & & & $6.434 \mathrm{Mpa}$ & 0.7595 & $0.6589 \mathrm{Mpa}$ & $0.4341 \mathrm{Mpa}$ & 0.10463 & & & \\
\hline & & & & ABSOLUTE & ABSOLUTE & ABSOLUTE & DIFEEREMTIXI & MasouUT $\mathrm{A}$ & ×ASOCONA $/$ ২ & (बK) & 6x) & (n) $/ \mathrm{sec}$ \\
\hline & & & & $12.37 \mathrm{ps} 1 \mathrm{a}$ & 945.5 ps1a & 122.52 ps1a & $95.56 \mathrm{ps1d}$ & $75.33 \mathrm{psia}$ & 27.55 ps10 & \multirow{3}{*}{296} & \multirow{3}{*}{295} & \multirow{3}{*}{0.02092} \\
\hline & & & & $0.8417 \quad \mathrm{~atm}$ & 64.34 & 8.337 & 6.502 & $5.126 \quad \mathrm{~atm}$ & 1.074 & & & \\
\hline & & & & 0.08529 & 6.519 & 0.8448 & 0.6589 & 0.5193 & 0.1899 & & & \\
\hline
\end{tabular}

$\exists$

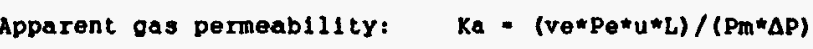

Boyle's Law:

$v_{e}=(\mathrm{Pb} / \mathrm{Pe}) *(\mathrm{Te} / \mathrm{Tb}) \cdot(\mathrm{ze} / \mathrm{zb}) * \mathrm{Vb}$

$Q_{e}=(\mathrm{Pb} / \mathrm{Pe}) *(\mathrm{Te} / \mathrm{Tb}) *(2 e / 2 b) * Q b$

$v e \cdot Q_{\theta} / \mathrm{A}=(\mathrm{Pb} / \mathrm{Pe}) \cdot(\mathrm{Te} / \mathrm{Tb}) \cdot\left(\mathrm{ze}_{e} / \mathrm{zb}\right) \cdot(\mathrm{Ob} / \mathrm{A})$

\begin{tabular}{|c|c|c|c|c|}
\hline Parameter & $\begin{array}{l}\text { Traditional } \\
\text { Units }\end{array}$ & & $\begin{array}{l}\text { SI } \\
\text { Units }\end{array}$ & \\
\hline$\mu=$ gas viscosity & 0.0176 & $\star 1.0 \mathrm{E}-3 \mathrm{~Pa} * \mathrm{seC} / \mathrm{Cp}$ & $1.760 \mathrm{E}-05$ & Pa*sec \\
\hline$L=$ sample length & 10.029 & $\star 1.0 \mathrm{E}-2 \mathrm{~m} / \mathrm{cm}$ & $1.003 \mathrm{E}-01$ & $\mathbf{m}$ \\
\hline A - sample clrcular cross sectlonal area & 81.233 & $1.08-4 \mathrm{~m}^{\wedge} 2 / \mathrm{cm}^{\wedge} 2$ & $8.123 \mathrm{E}-03$ & $m^{2} 2$ \\
\hline $\mathrm{pb}=$ flow measurement basis pressure (absolute) & 0.8417 & \# $1.013 \mathrm{E}+5 \mathrm{~Pa} / \mathrm{atm}$ & $8.527 \mathrm{E}+04$ & $\mathrm{~Pa}$ \\
\hline$\Delta \mathrm{P}=$ pressure drop across sample length & 6.502 & $01.013 \mathrm{E}+5 \mathrm{~Pa} / \mathrm{atm}$ & $6.587 E+05$ & $\mathrm{~Pa}$ \\
\hline$P_{m}=$ mean pore pressure (absolute) & 5.126 & $\star 1.013 E+5 \mathrm{~Pa} / \mathrm{atm}$ & $5.192 E+05$ & $\mathbf{P a}$ \\
\hline $\mathrm{Pe}=$ exit pressure (absolute) & 1.874 & $-1.013 \mathrm{E}+5 \mathrm{~Pa} / \mathrm{atm}$ & $1.899 \mathrm{E}+05$ & $\mathbf{P a}$ \\
\hline $\mathrm{Te}$ - sample temperature (absolute) & 296 & & 296 & 。र \\
\hline Tb - flow measurement basls temperature (absolute) & 295 & & 295 & ${ }^{\circ} \mathrm{K}$ \\
\hline $2 e$ - gas deviation factor at $\mathrm{Pe}$ and $\mathrm{Te}$ & 1.0000 & & 1.0000 & \\
\hline $2 \mathrm{~b}$ - gas deviation factor at $\mathrm{pb}$ and $\mathrm{Te}$ & 1.0000 & & 1.0000 & \\
\hline$Q b=$ flow rate at base conditions & 0.02092 & $1.0 \mathrm{E}-6 \mathrm{~m}^{\wedge} 3 / \mathrm{cm}^{\wedge} 3$ & $2.092 \mathrm{E}-08$ & $m^{\wedge} 3 / s$ \\
\hline$v e=$ flow velocity at sample exit end & $1.160 \mathrm{E}-04$ & $1.0 \mathrm{E}-2 \mathrm{~m} / \mathrm{cm}$ & $1.160 \mathrm{E}-06$ & $\mathrm{~m} / \mathrm{s}$ \\
\hline$-K_{a}=$ & $1.15 E-06$ & $9.872 \mathrm{E}-13 \mathrm{~m}^{\wedge} 2 / \mathrm{a}$ & $1.14 E-18$ & $m^{\wedge} 2$ \\
\hline $\mathrm{Ka}=$ & $1.15 \mathrm{E}-03$ & & $1.14 E-14$ & $\mathrm{~cm}^{2} 2$ \\
\hline $\mathrm{Ka} \cdot$ & $1.15 \mathrm{E}+00$ & & & \\
\hline
\end{tabular}




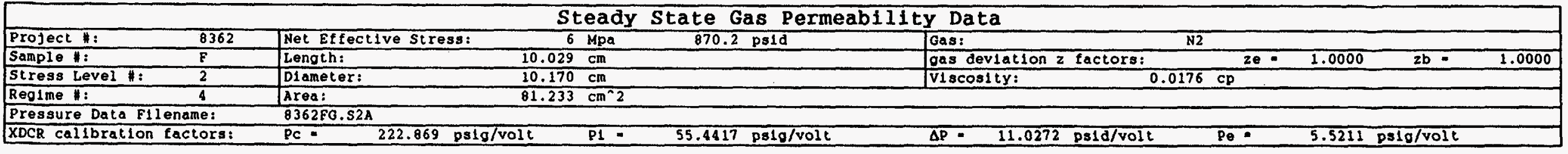

\begin{tabular}{|c|c|c|c|c|c|c|c|c|c|c|c|c|}
\hline Date & $\begin{array}{l}\text { Time } \\
\text { of } \\
\text { Day }\end{array}$ & $\begin{array}{l}\text { File } \\
\text { Time } \\
\text { (min) }\end{array}$ & $\begin{array}{c}\text { Regime } \\
*\end{array}$ & $\begin{array}{l}\mathrm{Pb} \\
\text { Barometric } \\
\text { Pressure }\end{array}$ & $\begin{array}{l}\text { Pc } \\
\text { Confining } \\
\text { Pressure }\end{array}$ & $\begin{array}{l}\text { P1 } \\
\text { Inlet } \\
\text { Pressure }\end{array}$ & $\begin{array}{l}\Delta \mathrm{P} \\
\text { Differential } \\
\text { Pressure }\end{array}$ & $\begin{array}{l}\mathrm{Pm} \\
\text { Mean pore } \\
\text { Pressure } \\
\mathrm{Pe}+\Delta \mathrm{P} / 2\end{array}$ & \begin{tabular}{|l} 
Pe \\
Exit \\
Pressure
\end{tabular} & $\begin{array}{l}\text { Te } \\
\text { Flow } \\
\text { Temp } \\
\left({ }^{\circ} \mathrm{C}\right)\end{array}$ & $\begin{array}{c}\text { Tb } \\
\text { Amblent } \\
\text { Temp } \\
\left({ }^{\circ} \mathrm{C}\right)\end{array}$ & $\begin{array}{c}\text { Ob } \\
\text { Flow Rate } \\
\text { OPbETb } \\
\text { (ml/sec) }\end{array}$ \\
\hline 11 Jun 93 & $08: 57$ & 2725 & FS2R4a & 12.31 psia & volts & 2.338 & 8.530 volts & & volts & 23 & 22 & 0.02422 \\
\hline 11 Jun 93 & $09: 01$ & 2730 & FS2R4b & $12.31 \mathrm{psia}$ & volts & 2.338 & 8.530 volts & & volts & 23 & 22 & 0.02425 \\
\hline 11 Jun 93 & $09: 05$ & 2735 & FS2R4C & 12.31 psia & volts & 2.338 & 8.530 volts & & volts & 23 & 22 & 0.02422 \\
\hline 11 Jun 93 & $09: 09$ & 2740 & FS2R4d & $12.31 \mathrm{ps1a}$ & volts & 2.338 & 0.530 volts & & 6.582 & 23 & 22 & 0.02423 \\
\hline \multirow{9}{*}{ AVERAGES } & & & \multirow{9}{*}{ FS2R4 } & & GUXOE & ODANE & DTFFEREMTIXIF & OOXAB & 60UROE & (खC) & rocts & \\
\hline & & & & & 4.277 volts & 2.338 volts & 8.530 volts & & 6.581 & \multirow{4}{*}{23} & \multirow{4}{*}{22} & \\
\hline & & & & & 953.2 & 129.62 & 94.06 psid & 83.37 psig & 36.336 & & & \\
\hline & & & & & 64.86 & 8.820 & 6.401 & $5.673 \mathrm{~atm}$ & 2.4725 & & & \\
\hline & & & & & 6.572 & 0.8937 & $0.6485 \mathrm{Mpa}$ & $0.5748 \quad \mathrm{Mpa}$ & 0.25053 & & & \\
\hline & & & & ABSOLUTE & ABSOLOTE & ABSOLUTE & DTHFERENTIAZ & HaSOLUTE & TBSOLOTE & (6) & $6 \times)$ & (niffor) \\
\hline & & & & $12.31 \mathrm{ps} 1 \mathrm{a}$ & 965.5 psia & $141.93 \quad$ psla & 94.06 ps1d & 95.68 ps1a & 48.65 & \multirow{3}{*}{296} & \multirow{3}{*}{295} & \multirow{3}{*}{0.02423} \\
\hline & & & & 0.8376 atm & 65.70 & 9.658 & $6.401 \quad \mathrm{~atm}$ & $6.510 \mathrm{~atm}$ & 3.310 & & & \\
\hline & & & & $0.08487 \mathrm{Mpa}$ & 6.657 & 0.9786 & 0.6485 & 0.6597 & 0.3354 & & & \\
\hline
\end{tabular}

$\vec{\omega}$

Apparent gas permeability: $\quad \mathrm{Ka}=\left(\mathrm{Ve}^{*} \mathrm{Pe}^{*} \mathrm{U}^{*} \mathrm{~L}\right) /\left(\mathrm{Pm}_{\mathrm{m}}^{*} \Delta \mathrm{P}\right)$

Boyle's Law:

$\mathrm{Ve}=(\mathrm{Pb} / \mathrm{Pe}) *(\mathrm{Te} / \mathrm{Tb}) *(\mathrm{ze} / \mathrm{2b}) * \mathrm{Vb}$

$\mathrm{Qe}_{e}=(\mathrm{Pb} / \mathrm{Pe}) *(\mathrm{Te} / \mathrm{Tb}) *(\mathrm{ze} / \mathrm{zb}) * \mathrm{Qb}$

$v_{e}=\mathrm{Qe}_{\mathrm{A}}=(\mathrm{Pb} / \mathrm{Pe}) \cdot(\mathrm{Te} / \mathrm{Tb}) \cdot(\mathrm{ze} / \mathrm{Zb}) \star(\mathrm{Qb} / \mathrm{A})$

\begin{tabular}{|c|c|c|c|c|}
\hline parameter & $\begin{array}{l}\text { Tradltional } \\
\text { Un1ts }\end{array}$ & & $\begin{array}{l}\text { SI } \\
\text { Un1ts }\end{array}$ & \\
\hline$\mu=\operatorname{gas} v 1 \mathrm{scos} 1 \mathrm{ty}$ & 0.0176 & $\star 1.0 \mathrm{E}-3 \mathrm{~Pa} * \mathrm{sec} / \mathrm{Cp}$ & $1.760 E-05$ & Pa*sec \\
\hline $\mathrm{L}=$ sample length & 10.029 & $* 1.0 \mathrm{E}-2 \mathrm{~m} / \mathrm{cm}$ & $1.003 \mathrm{E}-01$ & $\mathbf{m}$ \\
\hline$A$ - sample clrcular cross sectlonal area & 81.233 & $1.0 \mathrm{E}-\mathrm{A} \mathrm{m}^{\wedge} 2 / \mathrm{cm}^{\wedge} 2$ & $8.123 E-03$ & $m^{2} 2$ \\
\hline $\mathrm{Pb}=$ flow measurement bas1s pressure (absolute) & 0.8376 & $\star 1.013 \mathrm{E}+5 \mathrm{~Pa} / \mathrm{atm}$ & $8.485 E+04$ & $\mathrm{~Pa}$ \\
\hline$\Delta \mathrm{p}$ - pressure drop across sample length & 6.401 & $\approx 1.013 \mathrm{E}+5 \mathrm{~Pa} / \mathrm{atm}$ & $6.484 E+05$ & $\mathbf{P a}$ \\
\hline Pm - mean pore pressure (absolute) & 6.510 & $1.013 \mathrm{E}+5 \mathrm{~Pa} / \mathrm{atm}$ & $6.595 E+05$ & Pa \\
\hline$P_{e}=$ exit pressure (absolute) & 3.310 & $\star 1.013 \mathrm{E}+5 \mathrm{~Pa} / \mathrm{atm}$ & $3.353 \mathrm{E}+05$ & $\mathbf{P a}$ \\
\hline Te - sample temperature (absolute) & 296 & & 296 & ${ }^{\circ} \mathrm{K}$ \\
\hline $\mathrm{Tb}=$ flow measurement bas1s temperature (absolute) & 295 & & 295 & ${ }^{\circ} \mathbf{K}$ \\
\hline $2 e$ - gas deviation factor at $\mathrm{Pe}$ and $\mathrm{Te}$ & 1.0000 & & 1.0000 & \\
\hline $2 \mathrm{~b}$ - gas deviation factor at $\mathrm{Pb}$ and $\mathrm{Te}$ & 1.0000 & & 1.0000 & \\
\hline$Q b$ - flow rate at base conditions & $0.02423 \cdot \mathrm{cm}^{\wedge} 3 / \mathrm{s}$ & $.1 .0 \mathrm{E}-6 \mathrm{~m}^{\wedge} 3 / \mathrm{cm}^{\wedge} 3$ & $2.423 E-08$ & $m^{\wedge} 3 / 3$ \\
\hline ve = flow velocity at sample exit end & $7.573 \mathrm{E}-05 \mathrm{~cm} / \mathrm{s}$ & $\star 1.0 \mathrm{E}-2 \mathrm{~m} / \mathrm{cm}$ & $7.573 \mathrm{E}-07$ & $\mathrm{~m} / \mathrm{s}$ \\
\hline $\mathrm{Ka}=$ & $1.06 \mathrm{E}-06$ & $* 9.872 \mathrm{E}-13 \mathrm{~m}^{\wedge} 2 / \mathrm{d}$ & $1.05 E-18$ & $m^{\wedge} 2$ \\
\hline $\mathrm{Ka}=$ & $1.06 \mathrm{E}-03$ & & $1.05 E-14$ & $\mathrm{~cm}^{\wedge} 2$ \\
\hline $\mathrm{Ka}=$ & $1.06 \mathrm{E}+00$ & & & \\
\hline
\end{tabular}




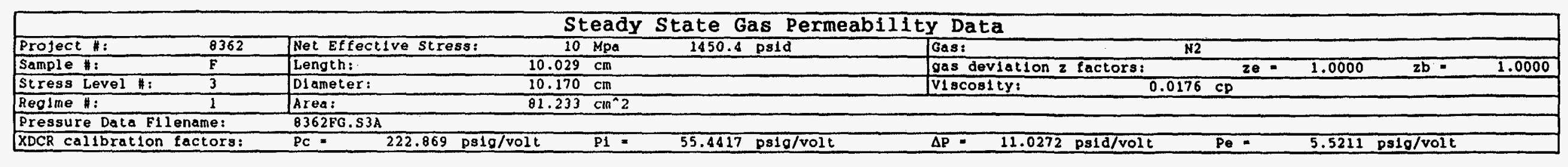

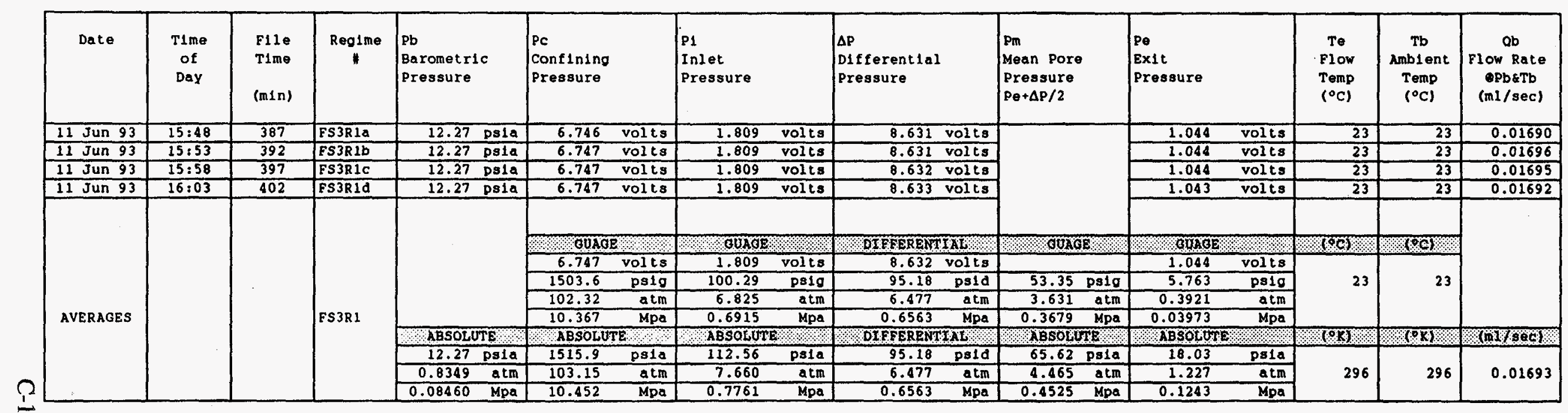

$\frac{\pi}{7}$

Apparent gas permeabllity:

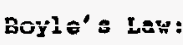

$K a=\left(v e * p^{*} u^{*} L\right) /(P m * \Delta P)$

$v_{e}=(\mathrm{Pb} / \mathrm{Pe}) *(\mathrm{Te} / \mathrm{Tb}) *(2 e / 2 \mathrm{~b}) \bullet \mathrm{Vb}$

$\mathrm{Qe}=(\mathrm{Pb} / \mathrm{Pe}) *(\mathrm{Te} / \mathrm{Tb}) *(\mathrm{ze} / \mathrm{Zb}) * \mathrm{Qb}$

$v e=Q_{e} / \mathrm{A}=(\mathrm{Pb} / \mathrm{Pe}) \cdot(\mathrm{Te} / \mathrm{Tb}) *(\mathrm{ze} / \mathrm{Zb}) \cdot(\mathrm{Qb} / \mathrm{A})$

\begin{tabular}{|c|c|c|c|c|}
\hline Parameter & $\begin{array}{l}\text { Traditional } \\
\text { Unlts }\end{array}$ & & $\begin{array}{l}\text { SI } \\
\text { Units }\end{array}$ & \\
\hline$\mu=g a s v 13 \cos 1 t y$ & 0.0176 & $* 1.0 \mathrm{E}-3 \mathrm{~Pa} * \mathrm{sec} / \mathrm{Cp}$ & $1.760 \mathrm{E}-05$ & Pa*sec \\
\hline $\mathrm{L}=$ sample length & 10.029 & $1.0 \mathrm{E}-2 \mathrm{~m} / \mathrm{cm}$ & $1.003 \mathrm{E}-01$ & $\mathrm{~m}$ \\
\hline$A$ sample clrcular cross sectional area & 81.233 & $1.0 \mathrm{E}-4 \mathrm{~m}^{*} 2 / \mathrm{cm}^{\wedge} 2$ & $8.123 \mathrm{E}-03$ & $\mathrm{~m}^{\wedge} \mathbf{2}$ \\
\hline $\mathrm{Pb}=$ tlow measurement basis pressure (absolute) & 0.8349 & * $1.013 \mathrm{E}+5 \mathrm{~Pa} / \mathrm{atm}$ & $8.458 E+04$ & Pa \\
\hline$\Delta \mathrm{P}=$ pressure drop across sample length & 6.477 & $\$ 1.013 \mathrm{E}+5 \mathrm{~Pa} / \mathrm{atm}$ & $6.561 \mathrm{E}+05$ & $\mathbf{P a}$ \\
\hline$P_{m}=$ mean pore pressure (absolute) & 4.465 & $1.013 \mathrm{E}+5 \mathrm{~Pa} / \mathrm{atm}$ & $4.524 E+05$ & $\mathrm{~Pa}$ \\
\hline$P e=$ exlt pressure (absolute) & 1.227 & $1.013 \mathrm{E}+5 \mathrm{~Pa} / \mathrm{atm}$ & $1.243 E+05$ & Pa \\
\hline Te - sample temperature (absolute) & 296 & & 296 & ${ }^{\circ} \mathrm{K}$ \\
\hline $\mathrm{Tb}=$ flow meesurement basis temperature (absolute) & 296 & & 296 & ${ }^{\circ} \mathrm{K}$ \\
\hline$z e$ - gas devlation factor at $p_{\theta}$ and $T e$ & 1.0000 & & 1.0000 & \\
\hline $2 \mathrm{~b}=$ gas deviation factor $\mathrm{at} \mathrm{Pb}$ and $\mathrm{Te}$ & 1.0000 & & 1.0000 & \\
\hline$a b=$ llow rate at base conditlons & 0.01693 & $01.0 \mathrm{E}-6 \mathrm{~m}^{\wedge} 3 / \mathrm{cm}^{\wedge} 3$ & $1.693 E-08$ & $m^{\wedge} 3 / s$ \\
\hline ve - flow veloclty at sample exit end & $1.418 \mathrm{E}-04$ & $\star 1.0 \mathrm{E}-2 \mathrm{~m} / \mathrm{cm}$ & $1.418 \mathrm{E}-06$ & $\mathrm{~m} / \mathrm{s}$ \\
\hline $\mathrm{K}_{\mathrm{a}}-\mathrm{Ka}=$ & $1.06 \mathrm{E}-06$ & $+9.872 \mathrm{E}-13 \mathrm{~m}^{2} 2 / \mathrm{d}$ & $1.05 E-18$ & $m^{\wedge} 2$ \\
\hline $\mathrm{Ka}=$ & $1.06 \mathrm{E}-03$ & & $1.05 \mathrm{E}-14$ & $\mathrm{~cm}^{2} 2$ \\
\hline $\mathrm{Ka}=$ & $1.06 \mathrm{E}+00$ & & & \\
\hline
\end{tabular}


Steady State Gas Permeability Data

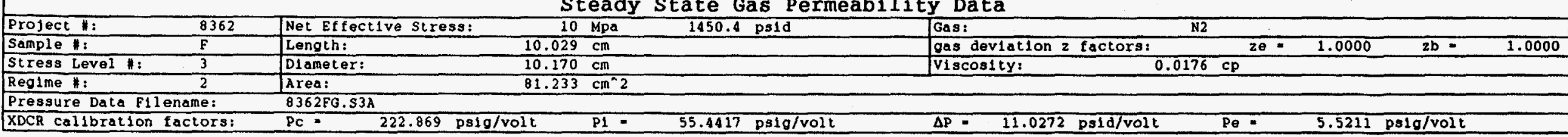

\begin{tabular}{|c|c|c|c|c|c|c|c|c|c|c|c|c|}
\hline Dace & $\begin{array}{l}\text { Time } \\
\text { of } \\
\text { Day }\end{array}$ & $\begin{array}{l}\text { File } \\
\text { Time } \\
\text { (min) }\end{array}$ & Reg1me & $\begin{array}{l}\mathrm{Pb} \\
\text { Barometric } \\
\text { Pressure }\end{array}$ & $\begin{array}{l}\text { Pc } \\
\text { Confining } \\
\text { Pressure }\end{array}$ & \begin{tabular}{|l} 
P1 \\
Inlet \\
Pressure
\end{tabular} & \begin{tabular}{|l}
$\Delta \mathrm{P}$ \\
Differential \\
Pressure
\end{tabular} & \begin{tabular}{|l} 
Pm \\
Mean Pore \\
Pressure \\
Pe+ $+P$ P /2
\end{tabular} & \begin{tabular}{|l} 
Pe \\
Exit \\
Pressure
\end{tabular} & $\begin{array}{l}\text { Te } \\
\text { Flow } \\
\text { Temp } \\
\left({ }^{\circ} \mathrm{C}\right)\end{array}$ & $\begin{array}{c}\text { Tb } \\
\text { Amblent } \\
\text { Temp } \\
\left({ }^{\circ} \mathrm{C}\right)\end{array}$ & $\begin{array}{c}\text { Qb } \\
\text { Flow Rate } \\
\text { Opb\&Tb } \\
\text { (ml/sec) }\end{array}$ \\
\hline 14 Jun 93 & $09: 29$ & 4330 & FS3R2a & $12.40 \mathrm{psia}$ & volts & volts & 8.670 volts & & volts & 23 & 22 & 0.01790 \\
\hline 14 Jun 93 & $09: 33$ & 4335 & FS3R2b & 12.40 psla & 6.790 volts & 1.986 & 8.670 volts & & volts & 23 & 22 & 0.01791 \\
\hline 14 Jun 93 & $09: 39$ & 4340 & FS3R2C & $12.40 \mathrm{ps} 1 \mathrm{a}$ & 6.790 volts & $\begin{array}{ll}1.986 & \mathrm{v} 01 \mathrm{ts}\end{array}$ & 8.610 volts & & volts & $\frac{25}{23}$ & $\frac{24}{22}$ & 0.01785 \\
\hline 14 Jun 93 & $09: 44$ & 4345 & ES3R2d & $12.40 \mathrm{ps} / \mathrm{a}$ & 6.790 volts & 1.986 volts & 8.620 volts & & 2.721 & 23 & 22 & 0.01792 \\
\hline \multirow{9}{*}{ AVERAGES } & & & \multirow{9}{*}{ FS3R2 } & & GOAGE & কেতOXOE & DFEFERATXI & \% GOXGE & 600 & Tops: & Trec: & \\
\hline & & & & & 6.790 volts & $\frac{1.986}{1.01 t s}$ & $\frac{1.643 \text { Volt3 }}{8.63}$ & & $\frac{.0402}{2.720}$ & \multirow{4}{*}{23} & \multirow{4}{*}{22} & \\
\hline & & & & & 1513.3 psig & 110.11 & 95.30 ps1d & $62.67 \mathrm{ps} 1 \mathrm{~g}$ & 15.019 & & & \\
\hline & & & & & $\frac{131.3}{102.98}$ & $\frac{11.11}{7.492}$ & $\begin{array}{lll}6.085 & \mathrm{stm}\end{array}$ & $4.264 \mathrm{~atm}$ & $\frac{\mathrm{atm}}{1.0220}$ & & & \\
\hline & & & & & $10.434 \quad \mathrm{Mpa}$ & 0.7592 & $0.6571 \mathrm{Mpa}$ & $0.4321 \mathrm{Mpa}$ & $0.10355 \quad$ Mpa & & & \\
\hline & & & & ABSOLUTE & ABSOLUTE & ABSOLUTE & DIEFERENTIAS & ABSOLUTE & $\frac{0.10355}{\text { ABSOLtore }}$ & (6x) & (बx) & (mI/sac) \\
\hline & & & & 12.4 psia & 1525.7 psia & 122.51 ps1a & 95.30 p91d & $75.07 \mathrm{psla}$ & 27.42 ps1a & & & \\
\hline & & & & $0.8438 \quad \mathrm{~atm}$ & 103.82 & 8.336 & $6.485 \quad \mathrm{acm}$ & $5.108 \mathrm{~atm}$ & 1.866 & 296 & 295 & 0.01789 \\
\hline & & & & $0.08550 \quad \mathrm{Mpa}$ & 10.520 & 0.8447 & $0.6571 \quad \mathrm{Mpa}$ & $0.5176 \mathrm{Mpa}$ & 0.1890 & & & \\
\hline
\end{tabular}

ù

Apparent gas permeab111ty: Ka $=\left(v^{\star} \mathrm{Pe}^{\star} \mu^{\star} L\right) /\left(P_{m} \star \Delta P\right)$

Boyle's Law:

$V_{e}=(P b / P e) *(T e / T b) *(z e / z b) * V b$

$Q_{e}=(\mathrm{Pb} / \mathrm{Pe}) *(T e / T b) *(z e / z b) * Q b$

ve $\cdot Q e / A=(P b / P e) *(T e / T b) *(2 e / 2 b) *(Q b / A)$

\begin{tabular}{|c|c|c|c|c|}
\hline Parameter & $\begin{array}{l}\text { Traditional } \\
\text { Units }\end{array}$ & & $\begin{array}{l}\text { SI } \\
\text { Units }\end{array}$ & \\
\hline$\mu=\operatorname{gas} v i s \cos 1 \mathrm{ty}$ & 0.0176 & $* 1.0 \mathrm{E}-3 \mathrm{~Pa} * \mathrm{SeC} / \mathrm{CP}$ & $1.760 \mathrm{E}-05$ & $\mathrm{~Pa}$ * $\mathrm{BeC}$ \\
\hline $\mathrm{L}=$ sample length & 10.029 & $\pi 1.0 \mathrm{E}-2 \mathrm{~m} / \mathrm{cm}$ & $1.003 \mathrm{E}-01$ & $\mathbf{m}$ \\
\hline $\mathrm{A}=$ sample circular cross sectional area & 81.233 & $1.0 \mathrm{E}-4 \mathrm{~m}^{\wedge} 2 / \mathrm{cm}^{\wedge} 2$ & $8.123 \mathrm{E}-03$ & $m^{2} 2$ \\
\hline $\mathrm{Pb}=$ flow measurement basis pressure (absolute) & 0.8438 & $1.013 \mathrm{E}+5 \mathrm{~Pa} / \mathrm{atm}$ & $8.547 E+04$ & $\mathrm{~Pa}$ \\
\hline$\Delta \mathrm{P}=$ pressure drop across sample length & 6.485 & $\approx 1.013 \mathrm{E}+5 \mathrm{~Pa} / \mathrm{atm}$ & $6.569 E+05$ & $\mathrm{~Pa}$ \\
\hline Pm - mean pore pressure (absolute) & 5.108 & $* 1.013 \mathrm{E}+5 \mathrm{~Pa} / \mathrm{atm}$ & $5.1758+05$ & $\mathrm{~Pa}$ \\
\hline Pe = exit pressure (absolute) & 1.866 & $\approx 1.013 \mathrm{E}+5 \mathrm{~Pa} / \mathrm{atm}$ & $1.890 \mathrm{E}+05$ & Pa \\
\hline Te sample temperature (absolute) & 296 & & 296 & ${ }^{\circ} \mathrm{K}$ \\
\hline Tb - flow measurement bas1s temperature (absolute) & 295 & & 295 & ${ }^{\circ} \mathrm{K}$ \\
\hline$z e=$ gas deviation factor at Pe and $T e$ & 1.0000 & & 1.0000 & \\
\hline $\mathrm{zb}=$ gas devlation factor at $\mathrm{Pb}$ and $\mathrm{Te}$ & 1.0000 & & 1.0000 & \\
\hline $\mathrm{Qb}=$ flow rate at base conditions & $0.01789 \mathrm{~cm}^{\wedge} 3 / \mathrm{s}$ & * $1.0 \mathrm{E}-6 \mathrm{~m}^{\wedge} 3 / \mathrm{cm}^{\wedge} 3$ & $1.789 \mathrm{E}-08$ & $m^{n} 3 / s$ \\
\hline$v_{e}=$ flow velocity at sample exit end & $9.996 \mathrm{E}-05 \mathrm{~cm} / \mathrm{s}$ & $* 1.0 \mathrm{E}-2 \mathrm{~m} / \mathrm{cm}$ & $9.996 \mathrm{E}-07$ & $\mathrm{~m} / \mathrm{s}$ \\
\hline $\mathrm{Ka}=$ & $9.94 \mathrm{E}-07$ & $9.872 \mathrm{E}-13 \mathrm{~m}^{\mathrm{A}} 2 / \mathrm{d}$ & $9.81 \mathrm{E}-19$ & $m^{\wedge} 2$ \\
\hline $\mathrm{Ka}=$ & $9.94 \mathrm{E}-04$ & & $9.81 \mathrm{E}-15$ & $\mathrm{~cm}^{\wedge} 2$ \\
\hline $\mathrm{Ka}=$ & $9.94 \mathrm{E}-01$ & & & \\
\hline
\end{tabular}




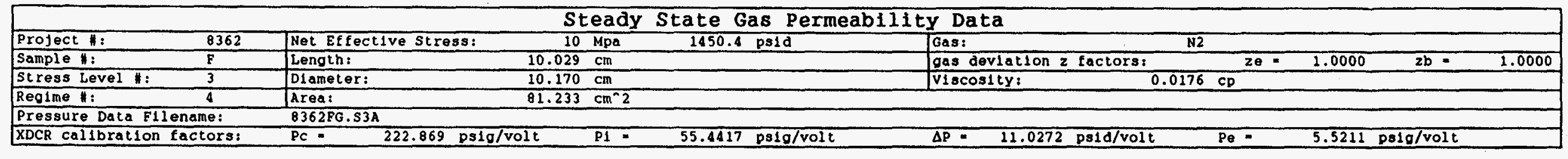

\begin{tabular}{|c|c|c|c|c|c|c|c|c|c|c|c|c|}
\hline Date & $\begin{array}{l}\text { Time } \\
\text { of } \\
\text { Day }\end{array}$ & $\begin{array}{l}\text { File } \\
\text { Time } \\
\text { (min) }\end{array}$ & Regime & $\begin{array}{l}\mathrm{Pb} \\
\text { Barometric } \\
\text { Pressure }\end{array}$ & $\begin{array}{l}\text { Pc } \\
\text { Confining } \\
\text { Pressure }\end{array}$ & $\begin{array}{l}\text { Pi } \\
\text { Inlet } \\
\text { Pressure }\end{array}$ & $\begin{array}{l}\Delta P \\
D i f f e r e n t i a l \\
\text { Pressure }\end{array}$ & $\begin{array}{l}\text { Pm } \\
\text { Mean Pore } \\
\text { Pressure } \\
\text { Pe }+\Delta P / 2\end{array}$ & $\begin{array}{l}\text { Pe } \\
\text { Exit } \\
\text { Pressure }\end{array}$ & $\begin{array}{l}\text { Te } \\
\text { Flow } \\
\text { Temp } \\
\left({ }^{\circ} \mathrm{C}\right)\end{array}$ & $\begin{array}{c}\text { Tb } \\
\text { Amb1ent } \\
\text { Temp } \\
\left({ }^{\circ} \mathrm{C}\right)\end{array}$ & $\begin{array}{c}\text { Qb } \\
\text { Flow Rate } \\
\text { opbeTb } \\
(\mathrm{ml} / \mathrm{sec})\end{array}$ \\
\hline 15 Jun 93 & $12: 47$ & 5967 & FS3R4a & $12.30 \mathrm{psia}$ & 6.883 & 2.337 & 8.406 volts & & 6.808 & 23 & 23 & 0.02060 \\
\hline 15 Jun 93 & $12: 51$ & 5972 & FS3R4b & 12.30 ps1a & volts & 2.337 & 8.407 volts & & volts & 23 & 23 & 0.02062 \\
\hline 15 Jun 93 & $12: 56$ & 5977 & FS3R4C & 12.30 psla & volts & 2.338 & 8.408 volts & & volts & 23 & 23 & 0.02062 \\
\hline 15 Jun 93 & $13: 01$ & 5982 & FS3R4d & 12.30 psia & volts & 2.338 & 8.408 volts & & volts & 23 & 23 & 0.02060 \\
\hline \multirow{9}{*}{ AVERAGES } & & & \multirow{9}{*}{ FS3R4 } & & GONGE & SEOAGE & DYFFERGKT XI & OONGE & ouno6: & (ब) & (ब) & \\
\hline & & & & & 6.884 volts & 2.338 volts & 8.407 volts & & 6.809 & \multirow{4}{*}{23} & \multirow{4}{*}{23} & \\
\hline & & & & & 1534.1 & 129.59 & 92.71 psid & $83.94 \mathrm{ps} 1 \mathrm{~g}$ & 37.590 & & & \\
\hline & & & & & 104.39 & 8.818 & 6.308 & $5.712 \quad \mathrm{~atm}$ & 2.5579 & & & \\
\hline & & & & & $10.577 \quad \mathrm{Mpa}$ & 0.8935 & $0.6392 \mathrm{Mpa}$ & 0.5788 & 0.25918 & & & \\
\hline & & & & ABSOLUTE & ABSOLUTE & ABSOLUTE & DIFFERGATIAI & \multicolumn{2}{|l|}{ AssouUr: } & (\%) & $8 \times 1$ & (nIfobe) \\
\hline & & & & $12.3 \mathrm{psia}$ & 1546.4 psia & $141.89 \mathrm{ps} 1 \mathrm{a}$ & 92.71 psid & $96.24 \mathrm{psia}$ & 49.89 & \multirow{3}{*}{296} & \multirow{3}{*}{296} & \multirow{3}{*}{0.02061} \\
\hline & & & & 0.8370 atm & 105.23 & 9.655 & 6.308 & $6.549 \mathrm{~atm}$ & 3.395 & & & \\
\hline & & & & $0.08481 \mathrm{Mpq}$ & 10.662 & 0.9783 & 0.6392 & 0.6636 & 0.3440 & & & \\
\hline
\end{tabular}

Apparent gas permeab111ty:

Boyle's Law:

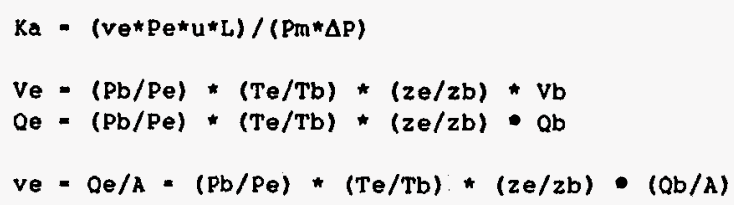

\begin{tabular}{|c|c|c|c|c|}
\hline Parameter & $\begin{array}{l}\text { Traditional } \\
\text { Units }\end{array}$ & & $\begin{array}{l}\text { SI } \\
\text { Units }\end{array}$ & \\
\hline$\mu=$ gas $v 1 s \cos 1 t y$ & 0.0176 & $\approx 1.0 \mathrm{E}-3 \mathrm{~Pa}$ sec/cp & $1.760 \mathrm{E}-05$ & Pansec \\
\hline$L=$ sample length & 10.029 & $1.0 \mathrm{E}-2 \mathrm{~m} / \mathrm{cm}$ & $1.003 \mathrm{E}-01$ & $\mathrm{~m}$ \\
\hline$A=$ sample clrcular cross sectional area & 81.233 & $\$ 1.0 \mathrm{E}-4 \mathrm{~m}^{\wedge} 2 / \mathrm{cm}^{\wedge} 2$ & $8.123 \mathrm{E}-03$ & $m^{\wedge} 2$ \\
\hline $\mathrm{pb}=$ flow measurement basis pressure (absolute) & 0.8370 & $\star 1.013 \mathrm{E}+5 \mathrm{~Pa} / \mathrm{atm}$ & $8.478 \mathrm{E}+04$ & Pa \\
\hline$\Delta \mathrm{P}=$ pressure drop across sample length & 6.308 & $\$ 1.013 \mathrm{E}+5 \mathrm{~Pa} / \mathrm{atm}$ & $6.390 \mathrm{E}+05$ & Pa \\
\hline $\mathrm{Pm}=$ mean pore pressure (absolute) & 6.549 & $\approx 1.013 \mathrm{E}+5 \mathrm{~Pa} / \mathrm{atm}$ & $6.634 E+05$ & $\mathrm{~Pa}$ \\
\hline $\mathrm{Pe}_{\mathrm{e}}=$ exit pressure (absolute) & 3.395 & $1.013 \mathrm{E}+5 \mathrm{~Pa} / \mathrm{atm}$ & $3.439 E+05$ & Pa \\
\hline $\mathrm{Te}$ - sample temperature (absolute) & 296 & & 296 & ${ }^{\circ} \mathrm{K}$ \\
\hline $\mathrm{Tb}=$ flow measurement basis temperature (absolute) & 296 & & 296 & ${ }^{\circ} \mathrm{K}$ \\
\hline$z e=$ gas deviation factor at Pe and Te & 1.0000 & & 1.0000 & \\
\hline $\mathrm{zb}=$ gas deviation factor at $\mathrm{pb}$ and $\mathrm{Te}$ & 1.0000 & & 1.0000 & \\
\hline $\mathrm{Qb}=$ flow rate at base conditions & $0.02061 \mathrm{~cm}^{\wedge} 3 / \mathrm{s}$ & $1.0 \mathrm{E}-6 \mathrm{~m}^{2} 3 / \mathrm{cm}^{2} 3$ & $2.061 \mathrm{E}-08$ & $\mathrm{~m}^{*} 3 / \mathrm{s}$ \\
\hline$v_{\theta}=$ flow velocity at sample exit end & $6.255 \mathrm{E}-05$ & $1.0 \mathrm{E}-2 \mathrm{~m} / \mathrm{cm}$ & $6.255 \mathrm{E}-07$ & $\mathrm{~m} / \mathrm{s}$ \\
\hline $\mathrm{K}_{\mathrm{a}}=$ & $9.07 E-07$ & $9.872 \mathrm{E}-13 \mathrm{~m}^{2} 2 / \mathrm{d}$ & $8.96 \mathrm{E}-19$ & $m^{*} 2$ \\
\hline $\mathrm{Ka}=$ & $9.07 E-04$ & & $8.96 \mathrm{E}-15$ & $\mathrm{~cm}^{\wedge} 2$ \\
\hline $\mathrm{Ka}=$ & $9.07 E-01$ & & & \\
\hline
\end{tabular}


C-178 


\section{APPENDIX C-E: Liquid Permeability Spreadsheets}




\begin{tabular}{|c|c|c|c|c|c|c|c|c|}
\hline \multicolumn{9}{|c|}{ Steady State Liquid Permeability Data } \\
\hline Project \#: & 8362 & Target Eff.Stress: & 2 & Mpa & 290.1 psid & & Flu1d: & Odorless Mineral spirit \\
\hline Sample \#: & A & Length: & 10.117 & $\mathrm{~cm}$ & & & & \\
\hline Stress Level \#: & 1 & Diameter: & 10.170 & $\mathrm{~cm}$ & & & Viscosity & $1.29 \mathrm{Cp} \cdot 23^{\circ} \mathrm{C}$ \\
\hline & & Area: & 81.233 & $\mathrm{~cm}^{\wedge} 2$ & & & & \\
\hline Pressure Data F & lename: & $8362 \mathrm{AL} . \mathrm{S} 1 \mathrm{~B}$ & & & & & & \\
\hline XDCR calibratior & factors: & $\mathrm{PC}=\quad 222.869$ & $1 \mathrm{~g} / \mathrm{volt}$ & & & & $\Delta \mathrm{P}=$ & $11.0272 \mathrm{ps} 1 \mathrm{~d} / \mathrm{volt}$ \\
\hline
\end{tabular}

All measurements made at room temperature of $\sim 23^{\circ} \mathrm{C}$ and with 0 psig back pressure

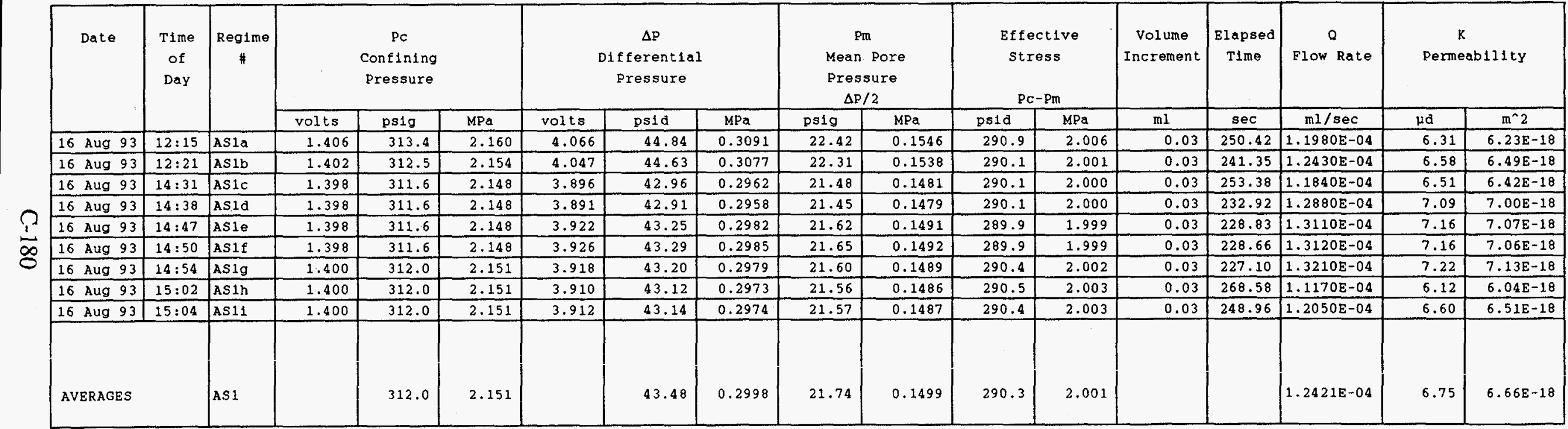

Darcy's law: $K=\left(Q^{*} \mu^{*} L\right) /\left(\Delta P^{*} A\right)$

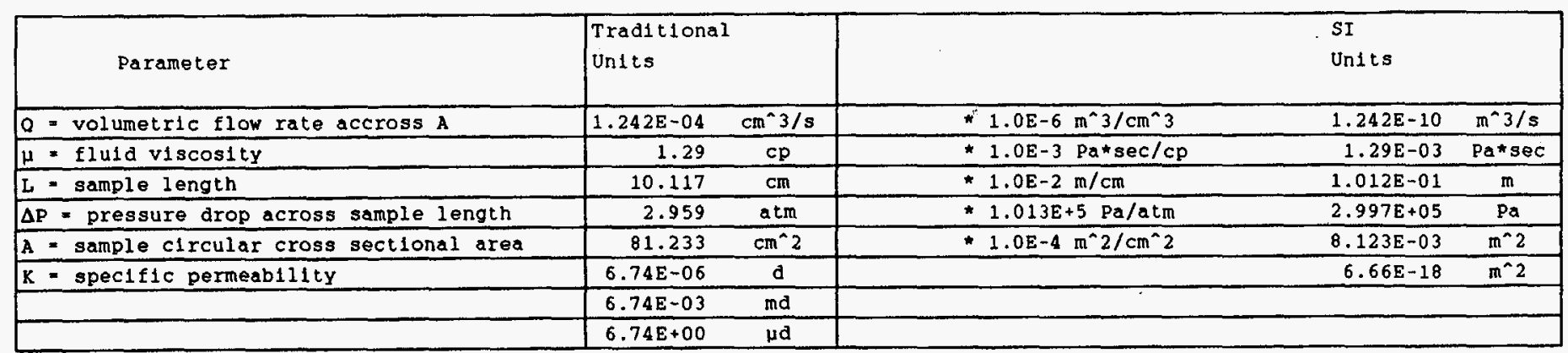




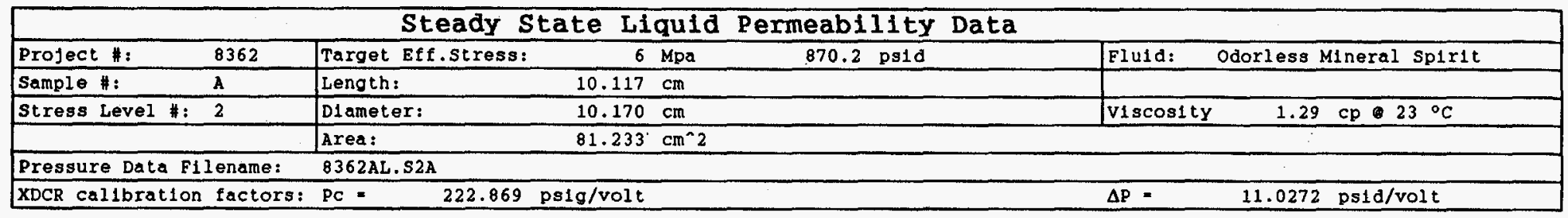

All measurements made at room temperature of $-23^{\circ} \mathrm{C}$ and with 0 psig back pressure

\begin{tabular}{|c|c|c|c|c|c|c|c|c|c|c|c|c|c|c|c|c|c|}
\hline \multirow[t]{2}{*}{ Date } & \multirow[t]{2}{*}{$\begin{array}{c}\text { Time } \\
\text { of } \\
\text { Day }\end{array}$} & \multirow[t]{2}{*}{$\begin{array}{c}\text { Reglme } \\
\#\end{array}$} & \multicolumn{3}{|c|}{$\begin{array}{c}\text { PC } \\
\text { Confining } \\
\text { Pressure }\end{array}$} & \multicolumn{3}{|c|}{$\begin{array}{c}\Delta P \\
\text { Differential } \\
\text { Pressure }\end{array}$} & \multicolumn{2}{|c|}{\begin{tabular}{l}
\multicolumn{1}{c}{ Pm } \\
Mean Pore \\
Pressure \\
$\Delta \mathrm{P} / 2$
\end{tabular}} & \multicolumn{2}{|c|}{$\begin{array}{c}\text { Effective } \\
\text { Stress } \\
\text { Pc-Pm }\end{array}$} & \multirow[t]{2}{*}{$\begin{array}{c}\begin{array}{c}\text { Volume } \\
\text { Increment }\end{array} \\
\text { ml }\end{array}$} & \multirow[t]{2}{*}{$\begin{array}{c}\begin{array}{c}\text { Elapsed } \\
\text { Time }\end{array} \\
\text { sec }\end{array}$} & \multirow[t]{2}{*}{$\frac{0}{\text { Flow Rate }}$} & \multicolumn{2}{|c|}{$\frac{K}{\text { Permeab1lity }}$} \\
\hline & & & volts & psig & $\mathrm{MPa}$ & volts & psid & $\mathrm{MPa}$ & ps1g & $\mathrm{MPa}$ & psid & $\mathrm{MPa}$ & & & & $\mu \mathrm{d}$ & $m^{\wedge} 2$ \\
\hline 19 Aug 93 & $13: 24$ & AS2a & 4.007 & 893.0 & 6.157 & 4.094 & 45.15 & 0.3113 & 22.57 & 0.1556 & 870.5 & 6.002 & 0.10 & 951.27 & $1.0512 \mathrm{E}-04$ & 5.50 & $5.43 E-18$ \\
\hline 19 Aug 93 & $13: 46$ & AS2b & 4.007 & 893.0 & 6.157 & 4.114 & 45.37 & 0.3128 & 22.68 & 0.1564 & 870.4 & 6.001 & 0.06 & 553.56 & $1.0839 \mathrm{E}-04$ & 5.64 & $5.57 \varepsilon-18$ \\
\hline 19 Aug 93 & $14: 19$ & AS2C & 4.007 & 893.0 & 6.157 & 4.087 & 45.07 & 0.3107 & 22.53 & 0.1554 & 870.5 & 6.002 & 0.07 & 671.94 & $1.0418 \mathrm{E}-04$ & 5.46 & $5.39 \mathrm{E}-18$ \\
\hline 19 Aug 93 & $14: 51$ & As2d & 4.007 & 893.0 & 6.157 & 4.047 & 44.63 & 0.3077 & 22.31 & 0.1538 & 870.7 & 6.003 & 0.10 & 854.49 & $1.1703 \mathrm{E}-04$ & 6.19 & $6.11 E-18$ \\
\hline 19 Aug 93 & $15: 19$ & AS2e & 4.007 & 893.0 & 6.157 & 4.016 & 44.29 & 0.3053 & 22.14 & 0.1527 & 870.9 & 6.005 & 0.10 & 890.20 & $1.1233 \mathrm{E}-04$ & 5.99 & $5.91 \mathrm{E}-18$ \\
\hline & & & & & & & & & & & & & & & & & \\
\hline AVERAGES & & AS2 & & 893.0 & 6.157 & & 44.90 & 0.3096 & 22.45 & 0.1548 & 870.6 & 6.002 & & & $1.0941 \mathrm{E}-04$ & 5.76 & $5.68 E-18$ \\
\hline
\end{tabular}

Darcy's law: $K=\left(Q^{*} \mu^{*} L\right) /\left(\Delta P^{*} A\right)$

\begin{tabular}{|c|c|c|c|c|}
\hline Parameter & $\begin{array}{l}\text { Traditional } \\
\text { Un1ts }\end{array}$ & & $\begin{array}{l}\text { SI } \\
\text { Un1ts }\end{array}$ & \\
\hline$Q=$ volumetric flow rate accross $A$ & $1.094 \mathrm{E}-04 \quad \mathrm{~cm}^{n} 3 / \mathrm{s}$ & $\therefore 1.0 \mathrm{E}-6 \mathrm{~m}^{2} 3 / \mathrm{cm}^{\wedge} 3$ & $1.094 \mathrm{E}-10$ & $m^{n} 3 / 5$ \\
\hline$\mu=$ flu1d viscosity & 1.29 & $* 1.0 \mathrm{E}-3 \quad \mathrm{pa}_{\mathrm{a}} * \mathrm{sec} / \mathrm{cp}$ & $1.29 \mathrm{E}-03$ & $\mathrm{~Pa} \star \sec$ \\
\hline$L=$ sample length & 10.117 & $\star 1.0 \mathrm{E}-2 \mathrm{~m} / \mathrm{cm}$ & $1.012 \mathrm{E}-01$ & m \\
\hline$\Delta \mathrm{P}=$ pressure drop across sample length & 3.055 & $\star 1.013 \mathrm{E}+5 \mathrm{~Pa} / \mathrm{atm}$ & $3.095 \mathrm{E}+05$ & $\mathrm{~Pa}$ \\
\hline$A=$ sample c1rcular cross sectional area & 81.233 & $1.0 \mathrm{E}-4 \mathrm{~m}^{\wedge} 2 / \mathrm{cm}^{\wedge} 2$ & $8.123 E-03$ & $m^{n} 2$ \\
\hline \multirow[t]{3}{*}{$K=$ speciflc permeability } & $5.75 E-06$ & & $5.68 \mathrm{E}-18$ & $\overline{m^{\wedge} 2}$ \\
\hline & $5.75 E-03$ & & & \\
\hline & $5.75 E+00$ & & & \\
\hline
\end{tabular}




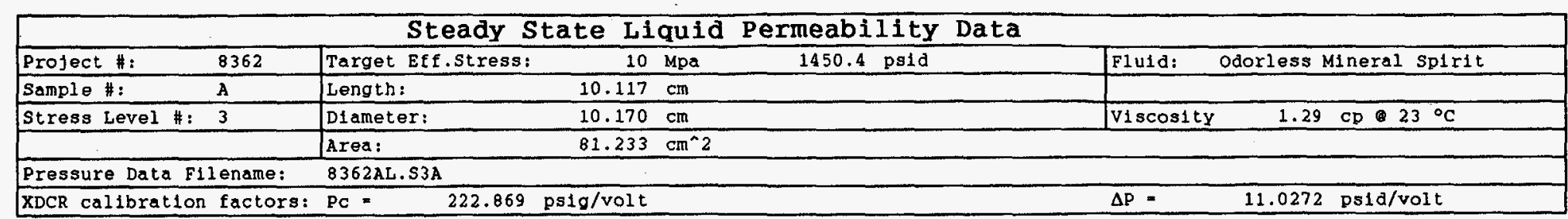

All measurements made at room temperature of $\sim_{23}^{\circ} \mathrm{C}$ and with 0 psig back pressure

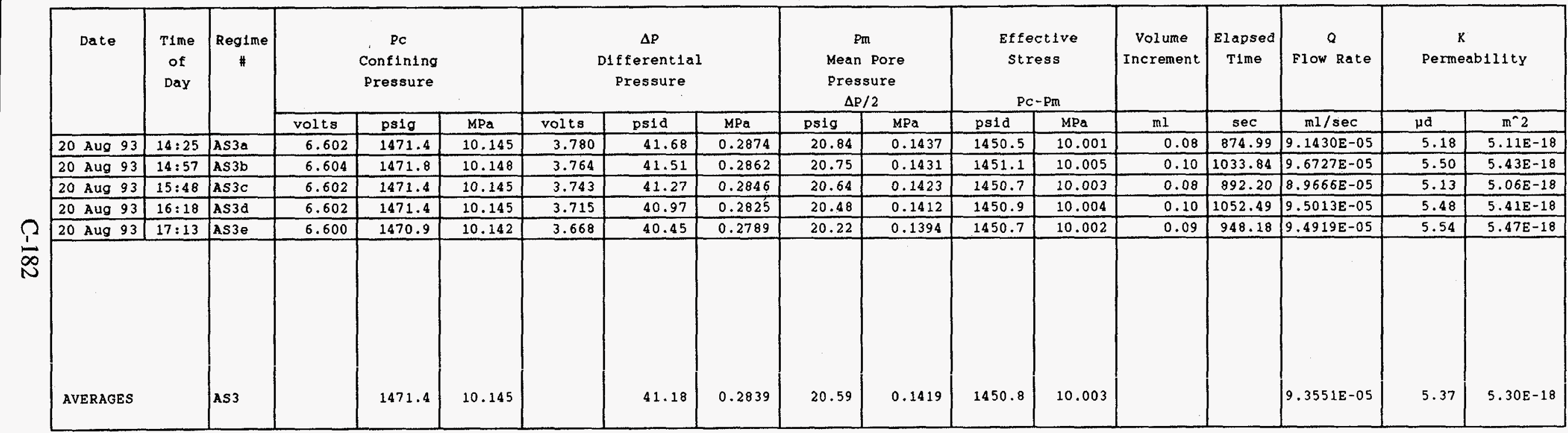

Darcy's law: $K=\left(Q^{\star} \mu \star L\right) /(\Delta \mathrm{D} * A)$

\begin{tabular}{|c|c|c|c|c|c|}
\hline Parameter & \multicolumn{2}{|c|}{$\begin{array}{l}\text { Traditional } \\
\text { Units }\end{array}$} & \multicolumn{3}{|c|}{$\begin{array}{l}\text { SI } \\
\text { Units }\end{array}$} \\
\hline$Q$ - volumetric flow rate accross $A$ & $9.355 \mathrm{E}-0$ & $\mathrm{~cm}^{\wedge} 3 / \mathrm{s}$ & $* 1.0 \mathrm{E}-6 \mathrm{~m}^{\wedge} 3 / \mathrm{cm}^{\wedge} 3$ & $9.355 \mathrm{E}-11$ & $\mathrm{~m}^{n} 3 / \mathrm{s}$ \\
\hline$\mu=f l u i d v i s \cos 1 t y$ & 1.25 & $\mathrm{Cp}$ & $\pi 1.0 \mathrm{E}-3 \mathrm{~Pa} * \mathrm{sec} / \mathrm{Cp}$ & $1.29 \mathrm{E}-03$ & $\mathrm{~Pa}^{\star} \mathrm{sec}$ \\
\hline $\mathrm{L}=$ sample length & 10.11 & $\mathrm{~cm}$ & $-1.0 \mathrm{E}-2 \mathrm{~m} / \mathrm{cm}$ & $1.012 \mathrm{E}-01$ & $\mathrm{~m}$ \\
\hline$\Delta P=$ pressure drop across sample length & 2.802 & atm & $* 1.013 \mathrm{E}+5 \mathrm{~Pa} / \mathrm{atm}$ & $2.838 \mathrm{E}+05$ & $\mathrm{~Pa}$ \\
\hline A - sample circular cross sectional area & 81.23 & $\mathrm{~cm}^{\wedge} 2$ & $1.0 \mathrm{E}-4 \mathrm{~m}^{\wedge} 2 / \mathrm{cm}^{\wedge} 2$ & $8.123 E-03$ & $\mathrm{~m}^{\wedge} 2$ \\
\hline \multirow[t]{3}{*}{$K=$ specif1c permeab1lity } & $5.36 \mathrm{E}-0$ & $\mathrm{~d}$ & & $5.30 \mathrm{E}-18$ & $\mathrm{~m}^{\wedge} 2$ \\
\hline & $5.36 E-03$ & ma & & & \\
\hline & $5.36 \mathrm{E}+0 \mathrm{C}$ & $\mu \mathrm{d}$ & & & \\
\hline
\end{tabular}




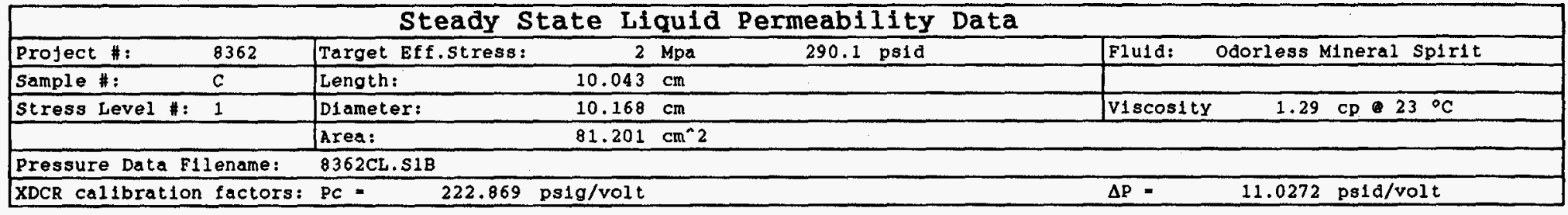

All measurements made at room temperature of $-23^{\circ} \mathrm{C}$ and with 0 psig back pressure

\begin{tabular}{|c|c|c|c|c|c|c|c|c|c|c|c|c|c|c|c|c|c|}
\hline \multirow[t]{2}{*}{ Date } & \multirow[t]{2}{*}{$\begin{array}{c}\text { Time } \\
\text { of } \\
\text { Day }\end{array}$} & \multirow[t]{2}{*}{$\begin{array}{c}\text { Regime } \\
\#\end{array}$} & \multicolumn{3}{|c|}{$\begin{array}{c}\text { PC } \\
\text { Confining } \\
\text { Pressure }\end{array}$} & \multicolumn{3}{|c|}{$\begin{array}{c}\Delta P \\
\text { D1fferential } \\
\text { Pressure }\end{array}$} & \multicolumn{2}{|c|}{$\begin{array}{c}\quad \mathrm{Pm} \\
\text { Mean Pore } \\
\text { Pressure } \\
\Delta \mathrm{P} / 2\end{array}$} & \multicolumn{2}{|c|}{$\begin{array}{c}\text { Effective } \\
\text { stress } \\
\text { Pe-Pm }\end{array}$} & \multirow{2}{*}{$\begin{array}{c}\text { volume } \\
\text { Increment }\end{array}$} & \multirow[t]{2}{*}{\begin{tabular}{|c}
$\begin{array}{c}\text { Elapsed } \\
\text { Time }\end{array}$ \\
sec
\end{tabular}} & \multirow[t]{2}{*}{\begin{tabular}{|c} 
Flow Rate \\
$\frac{\mathrm{ml} / \mathrm{sec}}{}$
\end{tabular}} & \multicolumn{2}{|c|}{$\stackrel{K}{\text { Permeab1lity }}$} \\
\hline & & & volts & psig & $\mathrm{MPa}$ & volts & psid & $\mathrm{MPa}$ & psig & $\mathrm{MPa}$ & ps1d & $\mathrm{MPa}$ & & & & $\mu d$ & $\mathrm{~m}^{\wedge} 2$ \\
\hline 28 Aug 93 & $14: 22$ & $\operatorname{cs} 1 a$ & 1.430 & 318.7 & 2.197 & 5.303 & 58.48 & 0.4032 & 29.24 & 0.2016 & 289.5 & 1.996 & 0.09 & 968.22 & $9.2954 \mathrm{E}-05$ & 3.73 & $3.68 \mathrm{E}-18$ \\
\hline 28 Aug 93 & $14: 49$ & $\operatorname{cs} 1 b$ & 1.431 & 318.9 & 2.199 & 5.311 & 58.57 & 0.4038 & 29.28 & 0.2019 & 289.6 & 1.997 & 0.10 & 1090.49 & $9.1702 \mathrm{E}-05$ & 3.67 & $3.62 E-18$ \\
\hline 28 Aug 93 & $15: 24$ & csic & 1.437 & 320.3 & 2.208 & 5.191 & 57.24 & 0.3947 & 28.62 & 0.1973 & 291.6 & 2.011 & 0.09 & 1007.17 & $8.9359 \mathrm{E}-05$ & 3.66 & $3.61 \mathrm{E}-18$ \\
\hline 28 Aug 93 & $15: 58$ & $\operatorname{cs} 1 d$ & 1.432 & 319.1 & 2.200 & 5.033 & 55.50 & 0.3827 & 27.75 & 0.1913 & 291.4 & 2.009 & 0.09 & 1065.45 & $8.4471 \mathrm{E}-05$ & 3.57 & $3.52 \mathrm{E}-18$ \\
\hline 28 Aug 93 & $16: 36$ & csie & 1.429 & 318.5 & 2.196 & 4.792 & 52.84 & 0.3643 & 26.42 & 0.1822 & 292.1 & 2.014 & 0.09 & 1095.34 & $8.2166 \mathrm{E}-05$ & 3.65 & $3.60 E-18$ \\
\hline & & & & & & & & & & & & & & & & & \\
\hline AVERAGES & & $\operatorname{cs} 1$ & & 319.1 & 2.200 & & 56.53 & 0.3897 & 28.26 & 0.1949 & 290.8 & 2.005 & & & $8.8131 \mathrm{E}-05$ & 3.65 & $3.61 \mathrm{E}-18$ \\
\hline
\end{tabular}

Darcy 's law: $K=\left(Q^{*} \mu^{\star} \mathrm{L}\right) /(\Delta \mathrm{P} * \mathrm{~A})$

\begin{tabular}{|c|c|c|c|c|}
\hline Parameter & $\begin{array}{l}\text { Traditlonal } \\
\text { Units }\end{array}$ & \multicolumn{3}{|c|}{$\begin{array}{l}\text { SI } \\
\text { Un1ts }\end{array}$} \\
\hline$Q=$ volumetric flow rate accross $A$ & $8.813 \mathrm{E}-05 \quad \mathrm{~cm}^{\wedge} 3 / \mathrm{s}$ & $\star 1.0 \mathrm{E}-6 \mathrm{~m}^{\wedge} 3 / \mathrm{cm}^{\wedge} 3$ & $8.813 E-11$ & $m^{\wedge} 3 / s$ \\
\hline$\mu=$ flu1d v1scosity & 1.29 & - $1.0 \mathrm{E}-3 \mathrm{~Pa}$ *sec/Cp & $1.29 \mathrm{E}-03$ & $\mathrm{~Pa}$ sec \\
\hline$L=$ sample length & 10.043 & $* 1.0 \mathrm{E}-2 \mathrm{~m} / \mathrm{cm}$ & $1.004 \mathrm{E}-01$ & $\mathrm{~m}$ \\
\hline$\Delta \mathbf{P}=$ pressure drop across sample length & 3.846 & *1.013E+5 Pa/atm & $3.896 E+05$ & $\mathrm{~Pa}$ \\
\hline$A=$ sample c1rcular cross sectional area & 81.201 & $* 1.0 \mathrm{E}-4 \mathrm{~m}^{n} 2 / \mathrm{cm}^{n} 2$ & $8.120 \mathrm{E}-03$ & $m^{\wedge} 2$ \\
\hline$x=$ specific permeabllity & $3.66 \mathrm{E}-06$ & & $3.61 \mathrm{E}-18$ & $m^{\wedge} 2$ \\
\hline & $3.66 \mathrm{E}-03$ & & & \\
\hline & $3.66 \mathrm{E}+00$ & & & \\
\hline
\end{tabular}




\begin{tabular}{|c|c|c|c|c|c|c|c|}
\hline \multicolumn{8}{|c|}{ Steady state Liquid Permeability Data } \\
\hline Project \#: & 8362 & Target Eff.Stress: & 6 & Mpa & 870.2 ps1d & Fluld: & Odorless Mineral spirit \\
\hline Sample \#: & c & Length: & 10.043 & $\mathrm{~cm}$ & & & \\
\hline \multirow[t]{2}{*}{ Stress Level \#: } & 2 & D1ameter: & 10.168 & $\mathrm{~cm}$ & & V1scosity & $1.29 \mathrm{cp} 23^{\circ} \mathrm{C}$ \\
\hline & & Area: & 81.201 & $\mathrm{~cm}^{\wedge} 2$ & & & \\
\hline \multicolumn{2}{|c|}{ Pressure Data Filename: } & $8362 \mathrm{CL} . \mathrm{S} 2 \mathrm{~A}$ & & & & & \\
\hline XDCR callbrat10 & factors: & Pc $=\quad 222.869$ & slg/volt & & & $\Delta \mathrm{P}=$ & $11.0272 \mathrm{ps} 1 \mathrm{~d} / \mathrm{volt}$ \\
\hline
\end{tabular}

All measurements made at room temperature of $\sim_{23}^{\circ} \mathrm{C}$ and with 0 psig back pressure

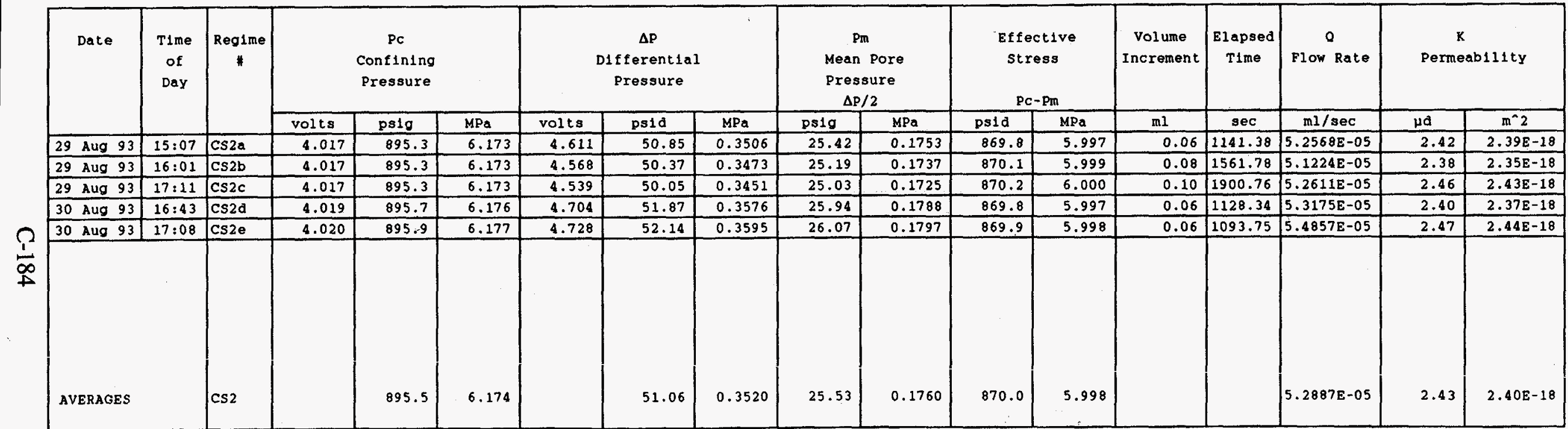

Darcy's law: $K=(Q * \mu * L) /(\Delta P * A)$

\begin{tabular}{|c|c|c|c|c|}
\hline Parameter & $\begin{array}{l}\text { Trad1 tlonal } \\
\text { Undts }\end{array}$ & \multicolumn{3}{|c|}{$\begin{array}{l}\text { SI } \\
\text { Undts }\end{array}$} \\
\hline Q - volumetric flow rate accross $A$ & $5.289 \mathrm{E}-05$ & $1.0 \mathrm{E}-6 \mathrm{~m}^{\wedge} 3 / \mathrm{cm}^{\wedge} 3$ & $5.289 \mathrm{E}-11$ & $m^{\wedge} 3 / s$ \\
\hline$\mu=$ fluld viscosity & 1.29 & $* 1.0 \mathrm{E}-3 \mathrm{~Pa} * \mathrm{sec} / \mathrm{Cp}$ & $1.29 \mathrm{E}-03$ & $\mathrm{~Pa}$ *sec \\
\hline$L$ - sample length & 10.043 & $\star 1.0 \mathrm{E}-2 \mathrm{~m} / \mathrm{cm}$ & $1.004 \mathrm{E}-01$ & $\mathrm{~m}$ \\
\hline$\Delta P=$ pressure drop across sample length & 3.474 & $* 1.013 \mathrm{E}+5 \mathrm{~Pa} / \mathrm{atm}$ & $3.519 E+05$ & $\mathrm{~Pa}$ \\
\hline$A=$ sample clrcular cross sectional area & 81.201 & $\star 1.0 \mathrm{E}-4 \mathrm{~m}^{\wedge} 2 / \mathrm{cm}^{\wedge} 2$ & $8.120 E-03$ & $m^{\wedge} 2$ \\
\hline$K$ - spec1fic permeability & $2.43 \mathrm{E}-06$ & & $2.40 \mathrm{E}-18$ & $m^{\wedge} 2$ \\
\hline & $2.43 E-03$ & & & \\
\hline & $2.43 E+00$ & & & \\
\hline
\end{tabular}




\begin{tabular}{|c|c|c|c|c|c|c|c|c|}
\hline \multicolumn{9}{|c|}{ Steady state Liquid Permeability Data } \\
\hline Project \#: & 8362 & Target Eff.stress: & 10 & Mpa & 1450.4 psid & & Fluid: & Odorless Mineral spirit \\
\hline Sample \#: & c & Length: & 10.043 & $\mathrm{~cm}$ & & & & \\
\hline \multirow[t]{2}{*}{ Stress Level \#: } & 3 & Dlameter: & 10.168 & $\mathrm{~cm}$ & & & viscosity & $1.29 \mathrm{cp} \cdot 23^{\circ} \mathrm{C}$ \\
\hline & & Area: & 81.201 & $\overline{c m^{\wedge} 2}$ & & & & \\
\hline \multicolumn{2}{|c|}{ Pressure Data Filename: } & 8362 CL.S3A & & & & & & \\
\hline XOCR callbrat1or & factors: & Pc $=\quad 222.869$ & psig/volt & & & & $\Delta \mathrm{P}=$ & $11.0272 \mathrm{ps} 1 \mathrm{~d} / \mathrm{volt}$ \\
\hline
\end{tabular}

All measurements made at room temperature of $\sim 23^{\circ} \mathrm{C}$ and w1th 0 psig back pressure

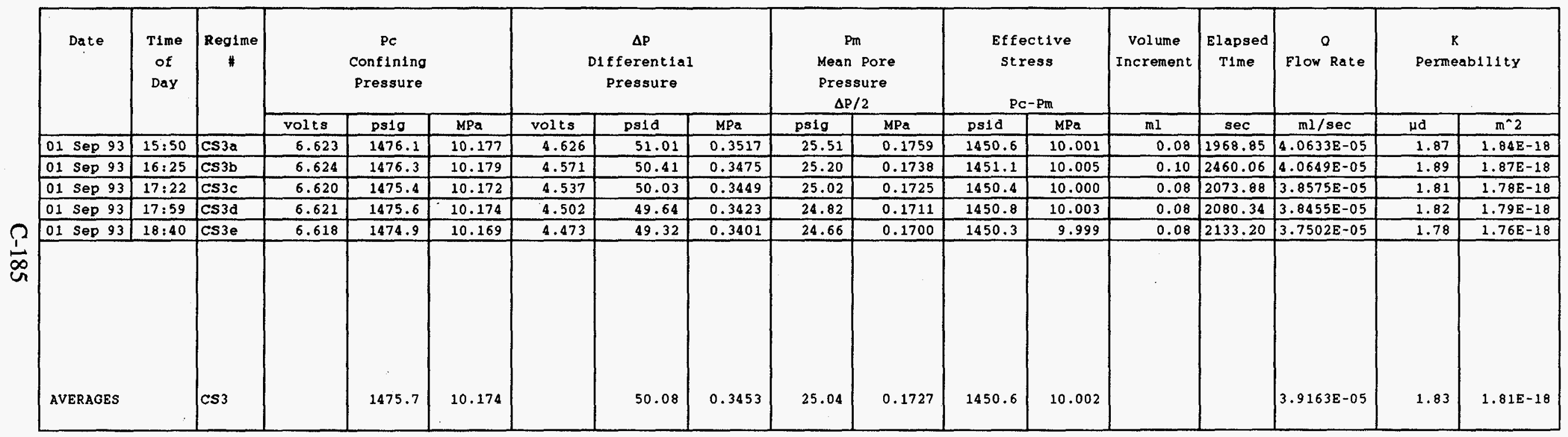

Darcy's law: $K=\left(Q^{\star} \mu^{\star} L\right) /\left(\Delta P^{\star} A\right)$

\begin{tabular}{|c|c|c|c|c|c|}
\hline Parameter & \multicolumn{2}{|c|}{$\begin{array}{l}\text { Traditional } \\
\text { Un1ts }\end{array}$} & \multicolumn{3}{|c|}{$\begin{array}{l}\text { SI } \\
\text { Units }\end{array}$} \\
\hline$Q$ - volumetric flow rate accross $A$ & $3.916 \mathrm{E}-05$ & $\mathrm{~cm}^{\wedge} 3 / \mathrm{s}$ & $\star 1.0 \mathrm{E}-6 \mathrm{~m}^{\wedge} 3 / \mathrm{cm}^{\wedge} 3$ & $3.916 \mathrm{E}-11$ & $m^{\wedge} 3 / s$ \\
\hline$\mu=$ fluid viscosity & 1.29 & $\mathrm{cp}$ & $* 1.0 \mathrm{E}-3 \mathrm{~Pa} * \sec / \mathrm{CP}$ & $1.29 E-03$ & $\mathrm{~Pa}^{*}$ sec \\
\hline L - sample length & 10.043 & $\mathrm{~cm}$ & $1.0 \mathrm{E}-2 \mathrm{~m} / \mathrm{cm}$ & $1.004 \mathrm{E}-01$ & $\mathbf{m}$ \\
\hline$\Delta P=$ pressure drop across sample length & 3.408 & atm & $\because 1.013 \mathrm{E}+5 \mathrm{~Pa} / \mathrm{atm}$ & $3.452 E+05$ & $\mathrm{~Pa}$ \\
\hline $\mathrm{A}$ - sample clrcular cross sectional area & 81.201 & $\mathrm{~cm}^{\wedge} 2$ & $* 1.0 \mathrm{E}-4 \mathrm{~m}^{\wedge} 2 / \mathrm{cm}^{\wedge} 2$ & $8.120 \mathrm{E}-03$ & $\mathrm{~m}^{\wedge} 2$ \\
\hline \multirow[t]{3}{*}{$K$ - specific permeab1l1ty } & $1.83 \mathrm{E}-06$ & d & & $1.81 E-18$ & $m^{\wedge} 2$ \\
\hline & $1.83 \mathrm{E}-03$ & md & & & \\
\hline & $1.83 E+00$ & $\mu \mathrm{d}$ & & & \\
\hline
\end{tabular}




\begin{tabular}{|c|c|c|c|c|c|c|c|c|}
\hline \multicolumn{9}{|c|}{ Steady state Iiquid Permeability Data } \\
\hline Project & \#: & 8362 & Target Eff.Stress: & 2 & Mpa & 290.1 psid & Fluld: & odorless Mineral splrit \\
\hline Sample & \#: & $F$ & Length: & 10.029 & $\mathrm{~cm}$ & & & \\
\hline \multirow[t]{2}{*}{ Stress } & Level \# & $1: 1$ & D1ameter: & 10.170 & $\mathrm{~cm}$ & & $V 1 s \cos 1 t y$ & $1.29 \mathrm{cp} 23^{\circ} \mathrm{C}$ \\
\hline & & & Area: & 81.233 & $\mathrm{~cm}^{\wedge} 2$ & & & \\
\hline \multicolumn{3}{|c|}{ Pressure Data Fllename: } & $8362 \mathrm{FL} . \mathrm{S} 1 \mathrm{~B}$ & & & & & \\
\hline XDCR Ca & alibrat1 & Ion factors: & Pc $=\quad 222.663$ & psig/volt & & & $\Delta \mathrm{P}=$ & $11.0303 \mathrm{psid} / \mathrm{volt}$ \\
\hline
\end{tabular}

All measurements made at room temperature of ${ }^{\circ} 23^{\circ} \mathrm{C}$ and with 0 psig back pressure

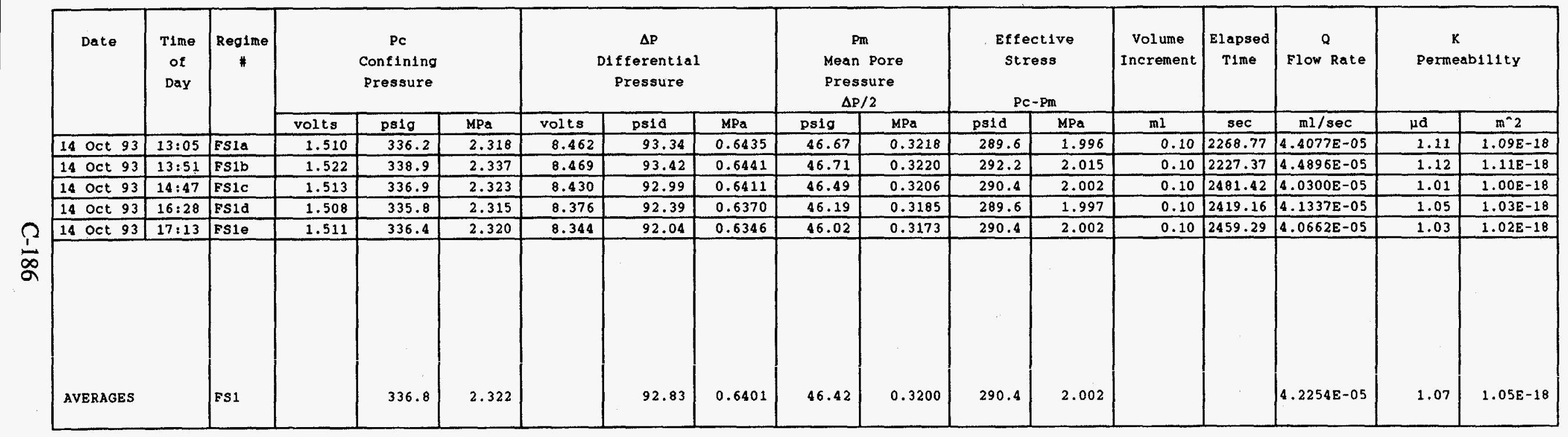

Darcy's law: $K=(Q * \mu * L) /(\Delta P * A)$

\begin{tabular}{|c|c|c|c|c|}
\hline Parameter & $\begin{array}{l}\text { Traditional } \\
\text { Units }\end{array}$ & \multicolumn{3}{|c|}{$\begin{array}{l}\text { SI } \\
\text { Units }\end{array}$} \\
\hline$Q=$ volumetrlc flow rate accross $A$ & $4.225 \mathrm{E}-05 \quad \mathrm{~cm}^{\wedge} 3 / \mathrm{s}$ & $1.0 \mathrm{E}-6 \mathrm{~m}^{\wedge} 3 / \mathrm{cm}^{-} 3$ & $4.225 \mathrm{E}-11$ & $m^{n} 3 / s$ \\
\hline$\mu=$ fluid viscosity & 1.29 & $* 1.0 \mathrm{E}-3 \mathrm{~Pa} * \mathrm{sec} / \mathrm{cp}$ & $1.29 E-03$ & $\mathrm{~Pa} * \mathrm{sec}$ \\
\hline $\mathrm{L}=$ sample length & 10.029 & $1.0 \mathrm{E}-2 \mathrm{~m} / \mathrm{cm}$ & $1.003 \mathrm{E}-01$ & $\mathrm{~m}$ \\
\hline$\Delta P$ - pressure drop across sample length & 6.317 & $* 1.013 \mathrm{E}+5 \mathrm{~Pa} / \mathrm{atm}$ & $6.399 E+05$ & $\mathrm{~Pa}$ \\
\hline$\lambda=$ sample clrcular cross sectlonal area & 81.233 & $-1.0 \mathrm{E}-4 \mathrm{~m}^{\wedge} 2 / \mathrm{cm}^{\wedge} 2$ & $8.123 E-03$ & $m^{\wedge} 2$ \\
\hline $\mathrm{K}=$ spec1fic permeability & $1.07 E-06$ & & $1.05 \mathrm{E}-18$ & $m^{\wedge} 2$ \\
\hline & $1.07 \mathrm{E}-03$ & & & \\
\hline & $1.07 E+00$ & & & \\
\hline
\end{tabular}




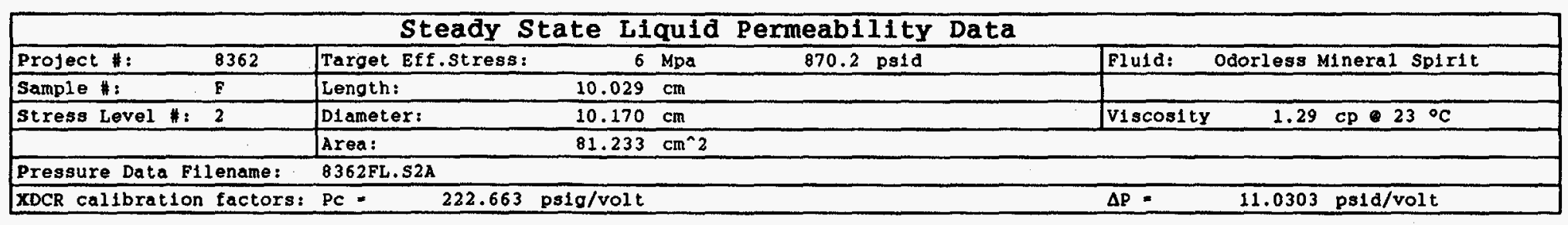

All measurements made at room temperature of ${ }^{\circ} 23^{\circ} \mathrm{C}$ and with 0 psig back pressure

\begin{tabular}{|c|c|c|c|c|c|c|c|c|c|c|c|c|c|c|c|c|c|}
\hline \multirow[t]{2}{*}{ Date } & \multirow[t]{2}{*}{$\begin{array}{c}\text { Time } \\
\text { of } \\
\text { Day }\end{array}$} & \multirow[t]{2}{*}{$\begin{array}{c}\text { Regime } \\
*\end{array}$} & \multicolumn{3}{|c|}{$\begin{array}{c}\text { PC } \\
\text { Confining } \\
\text { Pressure }\end{array}$} & \multicolumn{3}{|c|}{$\begin{array}{c}\Delta P \\
\text { Differential } \\
\text { Pressure }\end{array}$} & \multicolumn{2}{|c|}{$\begin{array}{c}\text { Pm } \\
\text { Mean Pore } \\
\text { Pressure } \\
\Delta \mathrm{P} / 2 \\
\end{array}$} & \multicolumn{2}{|c|}{$\begin{array}{c}\text { Effective } \\
\text { Stress } \\
\text { PC-Pm }\end{array}$} & \multirow[t]{2}{*}{$\begin{array}{c}\begin{array}{c}\text { volume } \\
\text { Increment }\end{array} \\
\mathrm{ml} \\
\end{array}$} & \multirow[t]{2}{*}{\begin{tabular}{|c}
$\begin{array}{c}\text { Elapsed } \\
\text { T1me }\end{array}$ \\
sec
\end{tabular}} & \multirow[t]{2}{*}{\begin{tabular}{|c} 
\\
Flow Rate \\
$\mathrm{ml} / \mathrm{sec}$ \\
\end{tabular}} & \multicolumn{2}{|c|}{$\stackrel{\text { K }}{\text { Permeab111ty }}$} \\
\hline & & & volts & psig & $\mathrm{MPa}$ & vol ts & psid & $\mathrm{MPa}$ & psig & $\mathrm{MPa}$ & psid & $\mathrm{MPa}$ & & & & $\mu \mathrm{d}$ & $\mathrm{m}^{\wedge} 2$ \\
\hline 16 oct 93 & $11: 59$ & FS2a & 4.119 & 917.1 & 6.324 & 8.367 & 92.29 & 0.6363 & 46.15 & 0.3182 & 871.0 & 6.005 & $\begin{array}{l}0.09 \\
\end{array}$ & 3705.41 & $2.4289 \mathrm{E}-05$ & 0.616 & $6.08 \mathrm{E}-19$ \\
\hline 16 oct 93 & $13: 05$ & FS2b & 4.121 & 917.6 & 6.327 & 8.551 & 94.32 & 0.6503 & 47.16 & 0.3252 & 870.4 & 6.001 & 0.08 & 3230.25 & $2.4766 \mathrm{E}-05$ & 0.615 & $6.07 E-19$ \\
\hline 16 oct 93 & $14: 02$ & ES2C & 4.123 & 918.0 & 6.330 & 8.564 & 94.46 & 0.6513 & 47.23 & 0.3257 & 870.8 & 6.004 & 0.09 & 3557.91 & $2.5296 \mathrm{E}-05$ & 0.627 & $6.19 \mathrm{E}-19$ \\
\hline 16 oct 93 & $15: 05$ & ES2d & 4.124 & 918.3 & 6.331 & 8.560 & 94.42 & 0.6510 & 47.21 & 0.3255 & 871.1 & 6.006 & 0.07 & 2719.19 & $2.5743 \mathrm{E}-05$ & 0.638 & $6.30 \mathrm{E}-19$ \\
\hline 16 oct 93 & $15: 55$ & Fs2e & 4.123 & 918.0 & 6.330 & 8.552 & 94.33 & 0.6504 & 47.17 & 0.3252 & 870.9 & 6.004 & 0.06 & 2392.26 & $2.5081 \mathrm{E}-05$ & 0.622 & $6.14 E-19$ \\
\hline 16 Oct 93 & $16: 39$ & FS2f & 4.121 & 917.6 & 6.327 & 8.549 & 94.30 & 0.6502 & 47.15 & 0.3251 & 870.4 & 6.002 & 0.06 & 2406.01 & $2.4938 \mathrm{E}-05$ & 0.619 & $6.11 \mathrm{E}-19$ \\
\hline & & & & & & & & & & & & & & & & & \\
\hline AVERAGES & & FS2 & & 917.8 & 6.328 & & 94.02 & 0.6482 & 47.01 & 0.3241 & 870.8 & 6.004 & & & $2.5019 E-05$ & 0.623 & $6.15 E-19$ \\
\hline
\end{tabular}

Darcy's law: $K=\left(Q^{*} \mu * L\right) /(\Delta P * A)$

\begin{tabular}{|c|c|c|c|c|}
\hline Parameter & $\begin{array}{l}\text { Traditional } \\
\text { Units }\end{array}$ & & $\begin{array}{l}\text { SI } \\
\text { Units }\end{array}$ & \\
\hline$Q=$ volumetric flow rate accross A & $2.502 \mathrm{E}-05 \quad \mathrm{~cm}^{\wedge} 3 / \mathrm{s}$ & $* 1.0 \mathrm{E}-6 \mathrm{~m}^{\wedge} 3 / \mathrm{cm}^{\wedge} 3$ & $2.502 \mathrm{E}-11$ & $m^{n} 3 / s$ \\
\hline$\mu=$ fluid viscosity & 1.29 & $1.0 \mathrm{E}-3 \mathrm{~Pa}{ }^{*} \mathrm{sec} / \mathrm{cp}$ & $1.29 \mathrm{E}-03$ & Pa*sec \\
\hline$L=$ sample length & 10.029 & $\star 1.0 \mathrm{E}-2 \mathrm{~m} / \mathrm{cm}$ & $1.003 \mathrm{E}-01$ & $\mathrm{~m}$ \\
\hline$\Delta \mathrm{p}=$ pressure drop across sample length & 6.398 & * $1.013 \mathrm{E}+5 \mathrm{~Pa} / \mathrm{atm}$ & $6.481 E+05$ & $\mathrm{~Pa}$ \\
\hline A - sample c1rcular cross sectional area & 81.233 & $\star 1.0 \mathrm{E}-4 \mathrm{~m}^{2} 2 / \mathrm{cm}^{-2}$ & $8.123 E-03$ & $m^{\wedge} 2$ \\
\hline $\mathrm{K}=$ specific permeab111ty & $6.23 E-07$ & & $6.15 \mathrm{E}-19$ & $m^{\wedge} 2$ \\
\hline & $6.23 E-04$ & & & \\
\hline & $6.23 \mathrm{E}-01$ & & & \\
\hline
\end{tabular}




\begin{tabular}{|c|c|c|c|c|c|c|c|c|}
\hline \multicolumn{9}{|c|}{ Steady State Liquid Permeability Data } \\
\hline Project \#: & 8362 & Target Eff.Stress: & 10 & $\mathrm{Mpa}$ & $1450.4 \mathrm{ps} 1 \mathrm{~d}$ & & Flu1d: & Odorless Mineral Spirit \\
\hline Sample \#: & F & Length: & 10.029 & $\mathrm{~cm}$ & & & & \\
\hline Stress Level \#: & 3 & Diameter: & 10.170 & $\mathrm{Cm}$ & & & Viscosity & $1.29 \mathrm{cp} 23^{\circ} \mathrm{C}$ \\
\hline & & Area: & 81.233 & $\mathrm{~cm}^{\wedge} 2$ & & & & \\
\hline Pressure Data F1 & lename: & 8362FL.S3A & & & & & & \\
\hline XDCR calibration & factors: & $\mathrm{Pc}=\quad 222.663$ & $1 g /$ volt & & & & $\Delta P=$ & $11.0303 \mathrm{ps} 1 \mathrm{~d} / \mathrm{volt}$ \\
\hline
\end{tabular}

All measurements made at room temperature of ${ }^{\circ} 23^{\circ} \mathrm{C}$ and with 0 psig back pressure

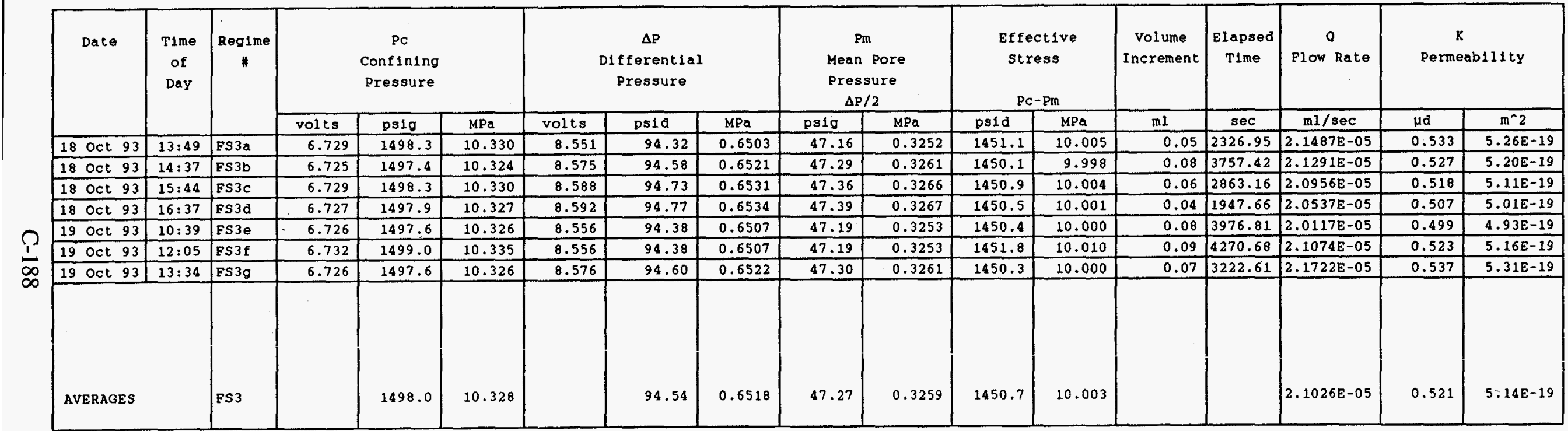

Darcy's law: $K=\left(Q^{*} \mu * L\right) /(\Delta P * A)$

\begin{tabular}{|c|c|c|c|c|}
\hline Parameter & $\begin{array}{l}\text { Traditlonal } \\
\text { Units }\end{array}$ & \multicolumn{3}{|c|}{$\begin{array}{l}\text { SI } \\
\text { Units }\end{array}$} \\
\hline$Q=$ volumetric flow rate accross $A$ & $2.103 \mathrm{E}-05 \quad \mathrm{~cm}^{\wedge} 3 / \mathrm{s}$ & $\star 1.0 \mathrm{E}-6 \mathrm{~m}^{\wedge} 3 / \mathrm{cm}^{\wedge} 3$ & $2.103 E-11$ & $\mathrm{~m}^{4} 3 / \mathrm{s}$ \\
\hline$\mu=$ fluid viscosity & 1.29 & $1.0 \mathrm{E}-3 \quad \mathrm{~Pa} * \mathrm{sec} / \mathrm{cp}$ & $1.29 E-03$ & $\mathrm{~Pa} * \mathrm{sec}$ \\
\hline $\mathrm{L}$ = sample length & 10.029 & $1.0 \mathrm{E}-2 \mathrm{~m} / \mathrm{cm}$ & $1.003 \mathrm{E}-01$ & $\mathrm{~m}$ \\
\hline$\Delta P=$ pressure drop across sample length & 6.433 & $\star 1.013 E+5 \mathrm{~Pa} / \mathrm{atm}$ & $6.516 E+05$ & Pa \\
\hline A - sample circular cross sectlonal area & 81.233 & $1.0 \mathrm{E}-4 \mathrm{~m}^{\wedge} 2 / \mathrm{cm}^{\wedge} 2$ & $8.123 E-03$ & $\mathrm{~m}^{\wedge} 2$ \\
\hline$K=$ specific permeability & $5.21 E-07$ & & $5.14 E-19$ & $m^{n} 2$ \\
\hline & $5.21 E-04$ & & & \\
\hline & $5.21 E-01$ & & & \\
\hline
\end{tabular}


Appendix D.

Marker Bed 139 Brine Recipe Documentation

The following information is provided as Appendix D of this document.

D-1 


\section{Appendix D}

Marker Bed 139 Brine Recipe Documentation

\section{Errata Sheet}

The following modifications should be made to the reference citations in Appendix D.

\begin{tabular}{|l|l|}
\hline Page No. & \multicolumn{1}{|c|}{ Change } \\
\hline D-3 & the existence of Lab Notebook No. WIPP 04 could not be verified \\
\hline D-4 & the existence of Lab Notebook No. WIPP 02 could not be verified \\
\hline D-10 & the existence of Lab Notebook No. WIPP 04 could not be verified \\
\hline D-10 & the existence of Lab Notebook No. WIPP 02 could not be verified \\
\hline D-14 & the existence of Lab Notebook No. WIPP 04 could not be verified \\
\hline D-14 & the existence of Lab Notebook No. WIPP 02 could not be verified \\
\hline D-29 & the existence of the Chem-Nuclear Geotech reports could not be verified \\
\hline D-29 & Felmy and Weare, 1986 is in Vol. 50, no. 12; copy on file in SWCF as WPO\#30421 \\
\hline D-29 & copy of Finley et al., 1992 on file in SWCF as WPO\#26222 \\
\hline D-30 & Harvie and Weare, 1980 is in Vol. 44, no. 7; copy on file in SWCF as WPO\#30423 \\
\hline D-30 & in Harvie et al, 1984 "Strengths" is plural in the title; paper is in Vol. 48, no. 4; copy on \\
file in SWCF as WPO\#30422 \\
\hline D-30 & copy of Krumhansl et al., 1991 on file in SWCF as WPO\#27786 \\
\hline D-30 & copy of McCaffrey et al., 1987 on file in SWCF as WPO\#42577 \\
\hline D-30 & the existence of the UNC Geotech Analytical Laboratory report could not be verified \\
\hline
\end{tabular}


Date: 27 March 1993

To: Craig Novak, 6119; and Susan Howarth, 6119

$$
\text { Karen Rolinaon. }
$$

From: Karen Robinson, 6119

Subject: Status Report on the Preparation of Standard Brines SB-139-A and SB-139-95A

\section{SUMMARY}

In February, I prepared 100-mL batches of two synthetic brines. SB-139-A has a composition close to that of an "average" QPB brine and is expected to be saturated with respect to the minerals in Marker Bed 139. SB-139-95A is expected to be slightiy undersaturated because the element concentrations are about $95 \%$ of those in the first brine. I have calculated the probable compositions of those brines based on the masses of salts used.

I have not yet measured the $\mathrm{pH}$ of either brine, but expect to do so by the first week of April. I have not yet confirmed the compositions of the brines by chemical analyses; I hope this will be done in early April.

In the following paragraphs I give some detaits about the recipes, my procedure, probiem areas and recommendations, and what remains to be done.

\section{RECIPES}

Craig Novak supplied a recipe for an average QPB brine. Table la shows his recipe (amounts of salts needed for 1 liter of brine) as well as the amounts needed for $100 \mathrm{~mL}$ of brine (saturated) and the amounts needed for $100 \mathrm{~mL}$ of "95\%" brine. Table 1b shows the average QPB composition (target composition), the calculated composition based on the "saturated" recipe, and the calcuiated composition based on the " $95 \%$ " recipe.

Table $2 a$ shows the masses of salts used (weighed out) for the two brines. Table $2 b$ shows the calculated compositions of those two brines based on the masses of salts used.

\section{PROCEDURE}

Detailed notes about my procedure are in my lab notebook (Lab Notebook No. WIPP 04, pp.6-12).

\section{Reagents}

Reagent grade salts were used. Some of these (magnesium sulfate, sodium chloride, sodium bromide, and sodium tetraborate) had been dried in the $7 a b$ oven at $-110^{\circ} \mathrm{C}$ and stored in a desiccator (by S. Yeh or J. Kelly). Others (calcium chloride dihydrate, magnesium chloride hexahydrate, and potassium chloride) were used "as is" from the bottle.

Deionized water from the Barnstead Nanopure A deionizer was used. 
Equipment

Reagents were weighed out using the Mettler AEI63 balance. The balance was calibrated before use with the internal calibration weight. The calibration was checked with selected standard weights. Details can be found in the balance $\log$ book. (Lab Notebook No. WIPP 02, p. 24).

Glassware included a $100-\mathrm{mL}$ class-A volumetric flask, glass beakers, and watchglasses.

Plasticware included weighing boats, $125-\mathrm{mL}$ polyethylene bottles, a small plastic funnel, and a teflon stirring rod (which also served as a boiling stick when solutions were heated).

Other equipment included a Thermolyne Nuova 7 stir plate; a Bransonic Ultrasonic bath; a Nalgene hand-operated vacuum pump; a Nalgene filter holder with receiver (Nalge Cat. No. 300-4000); Whatman filter paper (grade 4l, size $4.7 \mathrm{~cm})$.

\section{Preparation}

In brief, the required amounts of salts were dissolved in deionized water, the volume was adjusted to $100 \mathrm{~mL}$ in the volumetric flask, and the solution was filtered and transferred to a polyethylene bottle for storage. The step-bystep details for each solution are in Attachment 1.

In practice, preparing saturated and near-saturated solutions is somewhat challenging. Problem areas are discussed below.

\section{PROBLEM AREAS/RECOMMENDATIONS}

To prepare a standard solution, one usually dissolves the salt (or salts) in deionized water in a beaker and keeps the volume less than (perhaps half or three-quarters of) the final desired volume. This concentrated solution is then allowed to cool (if necessary) and is transferred quantitatively to the appropriate size volumetric flask. This won't work with a saturated solution. One ends up trying to quantitatively transfer the solution plus the undissolved stuff (a kind of wet slush). Under these conditions it is difficult to be certain that everything was rinsed out of the beaker.

Alternatively, one can put the dry salts directly into the volumetic, add water (a little less than the final desired amount), shake the flask occasionally (to speed mixing), and wait patiently for the salts to dassolve. The problem encountered with the $100-\mathrm{mL}$ volumetric flask was that because of the narrow neck, the salts wouldn't flow freely into the flask; more than $100 \mathrm{~mL}$ of water were needed to get all the salts into the flask!

Another problem I noted was that after the volumetric flask was inverted to mix the contents, the brine didn't drain cleanly from the stopper or the neck of the flask. Droplets clung to the neck and eventually salt precipitated out. (The amounts were small and apparently immediately redissolved when the flask was shaken or inverted to mix the contents.) Also, droplets clung to the ground glass stopper, which had to be left ajar to avoid being "cemented" in place. 
Recommendation

Attachment 2 gives the procedure that I would try next, if 1 were asked to prepare another batch.

\section{WORK REMAINING}

I still need to measure the $\mathrm{pH}$ of the solutions and adjust them to -6.1 if necessary. I al so need to confirm the brine compositions by chemical analysis.

\section{Measurement/Adjustment of $\mathrm{pH}$}

I plan to use the Sentron Model $2001 \mathrm{pH}$ System to check the pH of the samples. With this system I can use as little as one drop of solution. This will both conserve the solution and reduce the chance of contamination. If necessary, I' 11 use reagent grade $\mathrm{HCl}$ and $\mathrm{NaOH}$ to adjust the $\mathrm{pH}$ to -6.1 .

\section{Chemical Analyses}

Cations ( $\mathrm{B}, \mathrm{Ca}, \mathrm{K}, \mathrm{Mg}$, and $\mathrm{Na}$ ) will be determined by ICP-MS by Jeff Reich (1824). Anions ( $\mathrm{Br}, \mathrm{Cl}, \mathrm{SO4}$, and perhaps HCO3) will be determined by ion chromatography by John Kelly $(6119)$. Fred will pay for the ICP-MS analyses. I will need to dilute the samples to the appropriate concentration ranges for these analyses.

With both of these analytical techniques, the time-consuming (and therefore expensive) part of the procedure is the instrument set-up and calibration. For efficiency, therefore, these brines will be run along with a number of Fred Gelbard's Culebra brines.

I need to do the following: 1) calculate dilutions factors for the SB-139 brines and for Fred's Culebra brines, 2) meet with John to select some of Fred's brines based on the $\mathrm{Cl}$ and $\mathrm{SO} 4$ results (John has both the brines and the data), 3) check with Fred to see if he agrees with our selections, 4) check with Jeff Reich to find out when he can do the cation analyses, 5) dilute the samples for cation analyses the morning they will be run, 6) find out when John will be running the IC again, 7) dilute the SB-139 samples for anion analyses.

|karen\misc|sb-139-a.l

copy to: $6119 \mathrm{~K}$. L. Robinson 
TABLE 1a: Recipes -- Amounts of Salts Needed

$\begin{array}{cccc}\text { Amount needed } & \text { Amount needed } & \text { Amount needed } \\ \text { for 1 liter of } & \text { for } 100 \mathrm{ml} \text { of } & \text { for } 100 \mathrm{~mL} \text { of } \\ \text { "saturated" soln } & \text { "saturated" soln } & \text { "95\%" solution } \\ \text { Salt } & \text { (grams) } & \text { (grams) } & \text { (grams) }\end{array}$

$\mathrm{NaHCO} 3$
$\mathrm{CaCl} 2 \cdot 2 \mathrm{H} 2 \mathrm{O}$
$\mathrm{MgSO} 4$
$\mathrm{MgCl} \cdot 6 \mathrm{H} 2 \mathrm{O}$
$\mathrm{KCl}$
$\mathrm{NaCl}$
$\mathrm{Na} 2 \mathrm{~B} 407$
$\mathrm{NaBr}$

0.00133547
1.27954
19.92105
130.606
32.395
204.105
7.0024
1.87565

0.000134

0.12795

1.99210

13.0606

3.2395

20.4105

0.70024

0.18756

0.000127

0.12156

1.89250

12.4076

3.0775

19.3900

0.66523

0.17819

Table 1b: Brine Compositions -- Target and Calculated

$\begin{array}{lccc}\text { Species } & \begin{array}{c}\text { Target Comp. } \\ \text { Average QPB } \\ (\mathrm{mg} / \mathrm{L})\end{array} & \begin{array}{c}\text { Calc'd Comp. } \\ \text { "Sat'd"Recipe } \\ (\mathrm{mg} / \mathrm{L})\end{array} & \begin{array}{c}\text { Calc'd Comp. } \\ \text { "95\%" Recipe } \\ \text { (mg/L) }\end{array} \\ \mathrm{HCO3} & 0.970 & 0.970 & 0.922 \\ \mathrm{Cl} & 192171 & 185391 & 176121 \\ \mathrm{SO4} & 15898 & 15898 & 15103 \\ \mathrm{Na} & 82315 & 82315 & 78199 \\ \mathrm{~K} & 16990 & 16990 & 16141 \\ \mathrm{Ca} & 348.8 & 348.8 & 331.4 \\ \mathrm{Mg} & 19641 & 19641 & 18659 \\ \mathrm{~B} & 1505 & 1505 & 1430 \\ \mathrm{Br} & 1457 & 1457 & 1384\end{array}$


TABLE 2a: Actual Amounts of Salts Used (Weighed Out)

\begin{tabular}{|c|c|c|}
\hline Salt & $\begin{array}{l}\text { Amount used } \\
\text { for } 100 \mathrm{~mL} \text { of } \\
\text { "saturated" soln } \\
\text { (grams) }\end{array}$ & $\begin{array}{l}\text { Amount used } \\
\text { for } 100 \mathrm{~mL} \\
\text { "95\%" soluti } \\
\text { (grams) }\end{array}$ \\
\hline $\begin{array}{l}\mathrm{NaHCO3} \\
\mathrm{CaCl2} \cdot 2 \mathrm{H} 2 \mathrm{O} \\
\mathrm{MgSO} 4 \\
\mathrm{MgCl} 2 \cdot 6 \mathrm{H} 2 \mathrm{O} \\
\mathrm{KCl} \\
\mathrm{NaCl} \\
\mathrm{Na} 2 \mathrm{~B} 407 \\
\mathrm{NaBr}\end{array}$ & $\begin{array}{r}0^{*} \\
0.1284 \\
1.9921 \\
13.0605 \\
3.2412 \\
20.4109 \\
0.7027 \\
0.1875\end{array}$ & $\begin{array}{c}0 \star \\
0.1218 \\
1.8907 \\
12.4072 \\
3.0783 \\
19.3903 \\
0.66574 \\
0.1789\end{array}$ \\
\hline
\end{tabular}

* don't have appropriate equipment to accurately measure $0.00013 \mathrm{~g}$ of a salt.

Table 2b: Calculated Brine Compositions*

\begin{tabular}{|c|c|c|}
\hline Species & $\begin{array}{l}\text { SB-139-A } \\
\text { ("Sat'd" Recipe) } \\
\text { (mg/L) }\end{array}$ & $\begin{array}{l}\text { SB-139-95A } \\
\text { ("95\%" Recipe) } \\
\text { (mg/L) }\end{array}$ \\
\hline $\begin{array}{l}\mathrm{HCO} \\
\mathrm{Cl} \\
\mathrm{SO} 4 \\
\mathrm{Na} \\
\mathrm{K} \\
\mathrm{Ca} \\
\mathrm{Mg} \\
\mathrm{B} \\
\mathrm{Br}\end{array}$ & $\begin{array}{c}185390^{-* *} \\
15890 \\
82320 \\
17000 \\
350 \\
19640 \\
1510 \\
1460\end{array}$ & $\begin{array}{c}176120^{-\star \star} \\
15080 \\
78200 \\
16140 \\
330 \\
18660 \\
1430 \\
1390\end{array}$ \\
\hline
\end{tabular}


ATTACHMENT 1: Preparation of SB-139-A and SB-139-95A (aka "Dear Diary")

Preparation of SB-139-A

02/04/93: Weighed Na2B407. Transferred quantitatively to $150 \mathrm{~mL}$ beaker with DI water; adjusted volume to $-50 \mathrm{~mL}$; placed in ultrasonic bath -30 min.

02/05/93: Na2B407 dissolved overnight. Transferred solution to 100-mL vol. flask. Weighed other saits (CaCl2 $2 \mathrm{H}_{2} \mathrm{O}, \mathrm{MgCl} 2 \cdot 6 \mathrm{H} 2 \mathrm{O}, \mathrm{KCl}, \mathrm{NaBr}$, $\mathrm{MgSO}, \mathrm{NaCl}$ ) and transferred to vol. flask. Salts wouldn't dissolve (flask too full to mix and volume $>100 \mathrm{~mL}$ ), so transferred contents of flask to $250-\mathrm{mL}$ beaker and adjusted volume to $-200 \mathrm{~mL}$. Covered with evaporating watchglass and heated gently to dissolve salts. Salts dissolved in -30 min. Removed watchglass and continued heating to reduce volume. Left covered overnight (not on hot plate).

02/08/93: Continued evaporating, uncovered, on hot plate. Reduced volume to $-100 \mathrm{~mL}$. Removed from heat; rinsed walls of beaker with a few $\mathrm{mLs}$ of DI water. Left covered overnight.

02/09/93: Continued evaporating, uncovered, on hot plate. Reduced volume to $-75 \mathrm{~mL}$ (solution plus precipitates). Removed from heat; rinsed walls of beaker with a few mLs of DI water. Left to cool, covered. Transferred contents of beaker to $100-\mathrm{ml}$ vol. flask. A thin cloudy residue remained in the bottom of the beaker. (It wouldn't rinse out, but scrubbed out easily with Alconox and a bottle brush.) Diluted contents of vol. flask to $-99 \mathrm{~mL}$. Left stoppered overnight.

02/10/93: Significant quantity of undissolved salt in flask. Inverted several times to mix. Added DI water to within $-2 \mathrm{~mm}$ of mark on flask.

02/16/93: Salts appeared dissolved but solution was cloudy. Diluted to mark. Inverted to mix. Cleaned 125-mL poly. bottle by soaking in DI water for -3 hrs. Left to air-dry overnight.

02/17/93: Filtered solution through Whatman 41 filter paper. Transferred to clean, dry poly. bottle. Labelled "SB-139-A"; dated 2/17/93.

Preparation of SB-139-95A

02/17/93: Weighed Na2B407. Transferred quantitatively to $150 \mathrm{~mL}$ beaker with DI water; adjusted volume to $-50 \mathrm{~mL}$. Left covered with watch glass.

02/18/93: Na2B407 dissolved overnight. Weighed other salts ( $\mathrm{CaCl} 2 \cdot 2 \mathrm{H} 20$, $\mathrm{MgCl} 2 \cdot 6 \mathrm{H} 2 \mathrm{O}, \mathrm{KCl}, \mathrm{NaBr}$, $\mathrm{MgSO} 4, \mathrm{NaCl}$ ) and transferred to beaker with Na2B407 solution. Put on hot plate to reduce volume to $-80 \mathrm{~mL}$. Rinsed beaker walls with a few $\mathrm{mL}$ of DI water. Left coveres overnight (not on hot plate).

02/19/93: Transferred contents of beaker to 100-mL vol. flask. (Spilled a few $\mathrm{mL}$ of the final rinse water - shouldn't measurably affect final concentrations.) Diluted almost to mark ( $-5 \mathrm{~mm}$ below line). Shook to mix.

02/22/93: Small amount of salt remained undissolved. Added a little more DI water.

02/23/93: Everything dissolved. Diluted to mark. Mixed. Filtered through Whatman 41 filter paper. Transferred to clean, dry poly. bottle. Labelled "SB-139-95A"; dated 2/23/93. 


\section{ATTACHMENT 2: Recommendation}

If I were to prepare another batch I would try the following:

- Prepare a larger batch $(500 \mathrm{~mL}$ or $1 \mathrm{~L})$. This allows the use of a flask with a wider neck.

- Be sure the volumetric flask is clean and dry.

- Transfer the salts to the flask. Use a powder funnel (wide stem) and, if needed, a tefion stirring rod. Save the weighing containers (boats or whatever the salts were in) but don't rinse them yet.

- After all the salts have been transferred (except for the small amounts of residue in the weighing containers), rinse the stirring rod, then the weighing containers, and finally the funnel with deionized water. All rinse water should go into the flask. This ensures that the salts were transferred quantitatively.

- Add deionized water to the flask to just below the neck. Mix the contents by gently swirling the flask (don't invert to mix). Wait patiently for salts to dissolve, swirling occasionally (every half-hour or so during the day).

- When no more salts appear to dissolve (probably after a day or two), add deionized water to within 1 or $2 \mathrm{~cm}$ of the mark on the neck of the flask. Mix by tipping the flask. Avoid allowing the solution to touch the stopper. The stopper will not drain completely and is likely to get "cemented" in place by tiny salt crystals.

- Eventually, everything should dissolve (this could take several days). At this point, adjust to the final volume with deionized water, mix the solution thoroughly by inverting the flask, and transfer the solution immediately to a clean, dry plastic bottle.

If this doesn't work, I have some other ideas to try. 
Date: 07 June 1993

To: $\quad$ Susan Howarth, 6119

Karen Rolinoon

From: Karen Robinson, 6119

Subject: Preparation of Standard Brine SB-139-95B

\section{SUMMARY}

This memo describes the preparation of the standard brine SB-139-95B. I am giving quite a bit of detail in case you want to use this to generate a brinepreparation procedure for future use. In brief, I prepared 1 1 iter of brine, adjusted the $\mathrm{pH}$ to -6.1 with $\mathrm{HCl}$, and split the brine into two $500-\mathrm{mL}$ lots. You sent one bottle to Chem Nuclear Geotech for analysis; the other bottle is being stored in $823 / 2079$.

\section{RECIPE}

Craig Novak supplied a recipe for an average QPB brine, a brine expected to be saturated with respect to the minerals in Marker Bed 139. The brine described here is slightly undersaturated and contains $95 \%$ of the salts recommended by Craig.

Table la shows the "95\%" recipe and the amounts of salts actually weighed out. Table $1 b$ shows the calculated composition based on the "95\%" recipe and the calculated composition based on the amounts of salts actually weighed out.

\section{PROCEDURE}

Detailed notes about the preparation are in my 1 ab notebook (Lab Notebook No. WIPP 04, pp.21-23); those notes are summarized in Attachment 1.

\section{Reagents}

Reagent grade salts were used. All salts were used "as is" from the bottle (that is, they were not dried in the lab oven).

Deionized water from the Barnstead Nanopure A deionizer was used.

Standard $\mathrm{pH}$ buffer solutions were prepared from pHydrion buffer capsules.

Trace-metal grade hydrochioric acid was used to adjust the $\mathrm{pH}$.

\section{Equipment}

Reagents were weighed out using the Mettler AEl63 balance. The balance was calibrated before use with the internal calibration weight. The calibration was checked with selected standard weights. Details can be found in the balance $\log$ book (Lab Notebook No. WIPP 02, p. 25).

Glassware included a $1000-\mathrm{mL}$ class-A volumetric flask and a powder funnel. 
Plasticware included weighing boats, $500-\mathrm{mL}$ polyethylene bottles, various plastic beakers, and a teflon stirring rod.

Other equipment included a Thermolyne Nuova 7 stir plate; a magnetic stir bar and stir-bar retriever; and a Sentron model $2001 \mathrm{pH}$ system (meter and probe).

\section{Preparation}

In brief, the required amounts of salts were dissolved in deionized water in the volumetric flask; dissolution was speeded by using the magnetic stirrer. The volume was adjusted to $1000 \mathrm{~mL}$ in the volumetric flask. The $\mathrm{pH}$ was then adjusted by adding $-4 \mathrm{~mL}$ of $\mathrm{HCl}$. The solution was then transferred to two 500-mL polyethylene bottles. The step-by-step details are in Attachment 1.

Note that although the final volume of the solution was $~ 1004 \mathrm{~mL}$ (after the $\mathrm{pH}$ was adjusted), I used a volume of $1000 \mathrm{~mL}$ to calculated the concentrations of the solutes.

\section{HORK REMAINING}

As we discussed, I will also prepare one liter of the "saturated" recipe. This work has been delayed somewhat because there wasn't enough $\mathrm{NaCl}$ in the 1ab. More was ordered and has recently arrived. I expect to have the brine prepared and the memo documenting its preparation written by Friday, June 18 .

|karen \misc \sb-139-b. 1

copy to: 6119 C. F. Novak

6119 K. L. Robinson 
TABLE 1a: Recipes -- Amounts of Salts Needed and Weighed Out

$\begin{array}{lcr} & \begin{array}{c}\text { Amount needed } \\ \text { for 1 liter of } \\ \text { "95\%" soln } \\ \text { (grams) }\end{array} & \begin{array}{r}\text { Amount we } \\ \text { out for } \\ \text { SB-139-9 } \\ \text { (grams) }\end{array} \\ \text { Salt } & & \\ & & * \\ \mathrm{NaHC03} & 0.00127 & 1.2144 \\ \mathrm{CaCl2} \cdot 2 \mathrm{H} 20 & 1.2156 & 18.9238 \\ \mathrm{MgSO} 4 & 18.9250 & 124.0775 \\ \mathrm{MgC12} \cdot 6 \mathrm{H} 20 & 124.076 & 30.7727 \\ \mathrm{KCl} & 30.7753 & 193.8973 \\ \mathrm{NaCl} & 193.8998 & 6.6519 \\ \mathrm{Na2B407} & 6.6523 & 1.7837 \\ \mathrm{NaBr} & 1.7819 & \end{array}$

* Don't have appropriate equipment to accurately measure $0.00127 \mathrm{~g}$ of a salt.

Table 1b: Brine Compositions -- Target and Calculated

\begin{tabular}{|c|c|c|}
\hline Species & $\begin{array}{l}\text { Calc'd Comp. } \\
\text { "95\%" Recipe } \\
\text { (mg/L) }\end{array}$ & $\begin{array}{c}\text { Calc'd Comp } \\
\text { SB-139-95B* } \\
(\mathrm{mg} / \mathrm{L})\end{array}$ \\
\hline $\begin{array}{l}\mathrm{HCO} 3 \\
\mathrm{Cl} \\
\mathrm{SO} 4 \\
\mathrm{Na} \\
\mathrm{K} \\
\mathrm{Ca} \\
\mathrm{Mg} \\
\mathrm{B} \\
\mathrm{Br}\end{array}$ & $\begin{array}{c}0.922 \\
176106 \\
15103 \\
78198 \\
16141 \\
331 \\
18657 \\
1430 \\
1384\end{array}$ & $\begin{array}{r}\star \star \\
176100 \\
15100 \\
78200 \\
16140 \\
330 \\
18660 \\
1430 \\
1390\end{array}$ \\
\hline
\end{tabular}


ATTACHMENT 1: Preparation of SB-139-95B

04/30/93: Put $-200 \mathrm{~mL}$ deionized water and small magnetic stir bar into 1000-mL volumetric flask.

Weighed Na2B407; transferred quantitatively to vol. flask.

Began stirring. Stirred for $-3 \mathrm{hrs}$. Left standing over weekend. Weighed other salts (CaCl2.2H20, $\mathrm{MgCl} 2 \cdot 6 \mathrm{H} 20, \mathrm{KCl}, \mathrm{NaCl}, \mathrm{MgSO} 4, \mathrm{NaBr}$ ) into plastic beakers. Covered with parafilm.

05/03/93: Resumed stirring.

Quantitatively transferred chloride salts $(\mathrm{CaCl} 2 \cdot 2 \mathrm{H} 2 \mathrm{O}, \mathrm{MgCl} 2 \cdot 2 \mathrm{H} 2 \mathrm{O}$, $\mathrm{KCl}, \mathrm{NaCl}$ ) to vol. flask.

Added deionized water to fill flask *two-thirds.

Stirred $\sim 2$ hrs.

Quantitatively transferred remaining salts (MgSO4, $\mathrm{NaBr}$ ) to

vol. flask.

Continued stirring. At end of work day turned off stirrer and left to stand overnight.

05/04/93: Removed stir bar with magnetic stir-bar retriever. Rinsed with deionized water, adding all rinse water to flask.

Diluted with deionized water to volume and inverted to mix thoroughty.

Calitrated $\mathrm{pH}$ system with standard buffers 7 and 4 . Checked calibration with standard buffer 6.4 .

Measured initial $\mathrm{pH}$ of solution as 7.0 .

Alternately added aliquots of $\mathrm{HCl}$, mixed the solution by inverting the vol. flask, and checked the $\mathrm{pH}$ of the solution. After $-4 \mathrm{~mL}$ of $\mathrm{HCl}$ were added (in 6 unequal increments) the $\mathrm{pH}$ of the soiution was 6.14 .

The final volume of the solution was $-1004 \mathrm{~mL}$. Note that concentrations of solutes were calculated using a volume of $1000 \mathrm{~mL}$. The solution was transferred to two $500-\mathrm{mL}$ polyethylene bottles. One was given to $S$. Howarth for shipping to ChemNuclear Geotech for chemical analysis. The other is currently stored in $823 / 2079$. 
Date: 18 June 1993

To: $\quad$ Susan Howarth, 6119

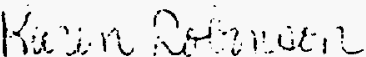

From: Karen Robinson, 6119

Subject: Preparation of Standard Brine SB-139-B

\section{SUMMARY}

This memo describes the preparation of the standard brine SB-139-B. I am giving quite a bit of detail in case you want to use this to generate a brinepreparation procedure for future use. In brief, I prepared l liter of brine, adjusted the $\mathrm{pH}$ to $\approx 6.1$ with $\mathrm{HCl}$, and split the brine into two 500-mL lots. I am giving one bottle to you for possible shipment to Chem Nuclear Geotech for analysis; the other bottle is being stored in $823 / 2079$.

\section{RECIPE}

Craig Novak supplied a recipe for an average QPB brine, a brine expected to be saturated with respect to the minerals in Marker Bed 139.

Table la shows his recipe ("saturated solution") and the amounts of salts actually weighed out. Table lb shows the calculated composition based on the recipe and the calculated composition based on the amounts of salts actually weighed out.

\section{PROCEDURE}

Detailed notes about the preparation are in my 1 ab notebook (Lab Notebook No. WIPP 04, pp.26-27 \& 34); those notes are summarized in Attachment 1.

\section{Reagents}

Reagent grade salts were used. All salts were used "as is" from the bottle (that is, they were not dried in the lab oven).

Deionized water from the Barnstead Nanopure A deionizer was used.

Standard $\mathrm{pH}$ buffer solutions were prepared from pHydrion buffer capsules.

Trace-metal grade hydrochioric acid was used to adjust the $\mathrm{pH}$.

\section{Equipment}

Reagents were weighed out using the Mettler AE163 balance. The balance was calibrated before use with the internal calibration weight. The calibration was checked with selected standard weights. Details can be found in the balance log book (Lab Notebook No. WIPP 02, p. 25).

Glassware included a $1000-\mathrm{mL}$ class-A volumetric flask and a powder funnel.

Plasticware included weighing boats, $500-\mathrm{mL}$ polyethylene bottles, various plastic beakers, and a teflon stirring rod. 
Other equipment included a Thermolyne Nuova 7 stir plate; a magnetic stir bar and stir-bar retriever; an adjustable Finnpipette $(1-5 \mathrm{~mL})$, and a Sentron model $2001 \mathrm{pH}$ system (meter and probe).

\section{Preparation}

In brief, the required amounts of salts were dissolved in deionized water in the volumetric flask; dissolution was speeded by using the magnetic stirrer. The volume was adjusted to $1000 \mathrm{~mL}$ in the volumetric flask. The $\mathrm{pH}$ was then adjusted by adding $-4 \mathrm{~mL}$ of $\mathrm{HCl}$. The solution was then transferred to two 500-mL polyethylene bottles. The step-by-step details are in Attachment 1 .

Note that although the final volume of the solution was $\sim 1004 \mathrm{~mL}$ (after the pH was adjusted), I used a volume of $1000 \mathrm{~mL}$ to calculated the concentrations of the solutes. Also, I ignored the $\mathrm{HCl}$ in calculating the chloride concentration.

$\mid k a r e n \backslash m i s c \backslash s b-139-b .2$

copy to: 6119 C. F. Novak

6119 K. L. Robinson 
TABLE 1a: Recipes -- Amounts of Salts Needed and Weighed Out

Salt

$\mathrm{NaHCO} 3$

$\mathrm{CaCl} 2 \cdot 2 \mathrm{H} 2 \mathrm{O}$

$\mathrm{MgSO} 4$

$\mathrm{MgCl} \cdot 6 \mathrm{H} 2 \mathrm{O}$

$\mathrm{KCl}$

$\mathrm{NaCl}$

$\mathrm{Na2B} 407$

$\mathrm{NaBr}$

$$
\begin{aligned}
& \text { Amount needed } \\
& \text { for } 1 \text { liter of } \\
& \text { "sat'd" soln }
\end{aligned}
$$$$
\text { (grams) }
$$

$$
0.00134
$$

1.27954

19.92105

130.606

32.395

204.105

7.0024

1.87565
Amount weighed

out for

SB-139-B

(grams)

* Don't have appropriate equipment to accurately measure $0.00013 \mathrm{~g}$ of a salt.

Table 1b: Brine Compositions -- Target and Calculated

$\begin{array}{lcc}\text { Calc'd Comp. } & \begin{array}{c}\text { Calc'd Comp } \\ \text { "Sat'd" Recipe } \\ (\mathrm{mg} / \mathrm{L})\end{array} & \begin{array}{c}\text { SB-139-B* } \\ (\mathrm{mg} / \mathrm{L})\end{array} \\ \text { Species } & 0.970 & \star \\ \mathrm{HCO3} & 185391 & 184770 \\ \mathrm{Cl} & 15898 & 15900 \\ \mathrm{SO} 4 & 82315 & 81920 \\ \mathrm{Na} & 16990 & 16990 \\ \mathrm{~K} & 349 & 350 \\ \mathrm{Ca} & 19641 & 19640 \\ \mathrm{Mg} & 1505 & 1500 \\ \mathrm{~B} & 1457 & 1460 \\ \mathrm{Br} & & \\ & & \\ & & \end{array}$


ATTACHMENT 1: Preparation of SB-139-B

05/21/93: Put $200 \mathrm{~mL}$ deionized water and smal1 magnetic stir bar into 1000-mL volumetric flask.

Discovered shortage of salts ( $\mathrm{NaCl}$, possibly KCl) in lab. Postponed further work until they arrive. Covered vol. flask.

06/10/93: Weighed Na2B407; transferred quantitatively to vol. flask.

Began stirring. Stirred for -3 hrs. Left standing over night.

Weighed other salts $(\mathrm{CaCl} 2 \cdot 2 \mathrm{H} 20, \mathrm{MgCl} 2 \cdot 6 \mathrm{H} 2 \mathrm{O}, \mathrm{KCl}, \mathrm{NaCl}, \mathrm{MgSO} 4, \mathrm{NaBr})$ into plastic beakers. Covered with parafilm.

06/11/93: Resumed stirring.

Quantitatively transferred chloride salts ( $\mathrm{CaCl} 2 \cdot 2 \mathrm{H} 2 \mathrm{O}, \mathrm{MgCl} 2 \cdot 2 \mathrm{H} 2 \mathrm{O}$, $\mathrm{KCl}, \mathrm{NaCl}$ ) to vol. flask.

Added deionized water to fill flask *two-thirds.

Stirred $-1 \mathrm{hr}$. Let sit over weekend.

06/14/93: Resumed stirring.

Quantitatively transferred remaining salts (MgSO4, $\mathrm{NaBr}$ ) to vol.

flask. Added deionized water until base of flask was almost full. Continued stirring. At end of work day turned off stirrer and left to stand overnight.

06/15/93: Continued stirring. Let sit overnight.

06/16/93: Continued stirring. Left stirring overnight.

06/17/93: Stopped stirring.

Removed stir bar with magnetic stir-bar retriever. Rinsed with deionized water, adding all rinse water to flask.

Diluted with deionized water to volume and inverted to mix thoroughly.

00/18/93: Calibrated $\mathrm{pH}$ system with standard buffers 7 and 4 . Checked

calibration with standard buffer 6.4.

Measured initial pH of solution as 7.04 .

Added $4.0 \mathrm{~mL}$ of trace-metal grade $\mathrm{HCl}$, mixed the solution by inverting the vol. flask several times, and checked the $\mathrm{pH}$ of the solution. The final pH of the solution was 6.15 .

The final volume of the solution was $~ 1004 \mathrm{~mL}$. Note that concentrations of solutes were calculated using a volume of $1000 \mathrm{~mL}$. The solution was transferred to two $500-\mathrm{mL}$ polyethylene bottles. One was given to $\mathrm{S}$. Howarth for possible shipment to ChemNuclear Geotech for chemical analysis. The other is currently stored in $823 / 2079$. 


\section{闻 Sandia National Laboratories}

MANAGED BY MARTIN MARIETTA CORPORATION FOR THE U. S. DEPARTMENT OF ENERGY

date: 26 April 1994

to: Susan M. Howarth, Dopt. 6115, MS 1324, 848-0676

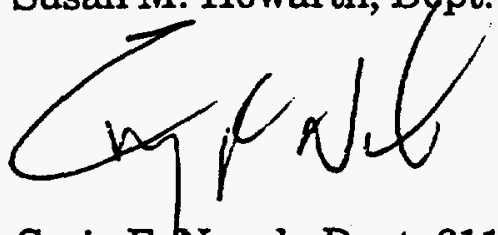

from: Craig F. Novak, Dept. 6119, MS 1320, 848-0619

subject: Formulation and Recipe for a "Standard" Marker Bed 139 Brine

A standard brine with little or no potential to dissolve rock from Marker Bed 139 was needed for flow experiments. This memo documents the process used to develop a composition for this standard brine, which will be given the name SB139-B.

The brines collected in the QPB boreholes in the Q-access drift (Finley et al., 1992) are believed to represent brines from Marker Bed 139. If this is indeed the case, these brine compositions should be in equilibrium with the solids in the marker bed, and thus should not dissolve marker bed material. Chemical analyses of 20 brine samples from these boreholes are available, collected between October 1989 and July 1992. Discussion of these and other Salado brine samples can be found in the memo "Evaluation of Chemical Analysis Data of Brine Samples from the Small Scale Brine inflow Experiments," by Novak, dated 17 June 1993; modified 26 July 1993. The results of the analyses are listed in Table 1, along with an arithmetic average of the element concentrations, in $\mathrm{mM}$ and $\mathrm{mg} / \mathrm{L}$. This average concentration was used by K.L. Robinson to develop a recipe from which to synthesize SB-139-Brine. Although it cannot be guaranteed that this brine will not cause dissolution of MB139 material, this is a reasonable brine composition for simulating brine from Marker Bed 139.

\section{Reference}

Finley, S.J., D.J. Hanson, and R. Parsons. 1992. Small-Scale Brine Inflow Experiments-Data Report Through 6/6/91. SAND91-1956. Albuquerque, New Mexico: Sandia National Laboratories. 
Table 1. Concentrations used to determine "standard" MB139 composition.

\begin{tabular}{|c|c|c|c|c|c|c|c|c|c|c|}
\hline Borehole & $\begin{array}{c}\text { Collection } \\
\text { Date }\end{array}$ & $\begin{array}{l}\text { Analysis } \\
\text { Report" }\end{array}$ & B. $\mathrm{mM}$ & $\mathrm{Br}, \mathrm{mM}$ & $\mathrm{Ca}, \mathrm{mM}$ & $\mathrm{Cl}, \mathrm{mM}$ & $\mathrm{K}, \mathrm{mM}$ & $\mathrm{Mg}, \mathrm{mM}$ & $\mathrm{Na}, \mathrm{mM}$ & $\mathrm{SO} 4, \mathrm{mM}$ \\
\hline QPB 01 & 16-Aug-90 & UNCS & 138 & 18.1 & 7.53 & 5501 & 426 & 776 & 3463 & 160 \\
\hline QPB 01 & 20-Sep-90 & UNCS & 144 & 18.8 & 7.88 & 5614 & 448 & 817 & 3554 & 166 \\
\hline QPB 02 & $4-O c t-89$ & UNC2 & 139 & 18.5 & 7.93 & 5571 & 431 & 813 & 3596 & 166 \\
\hline QPB 02 & 13-Dec-89 & UNC1 & 149 & 18.8 & 9.43 & 5529 & 453 & 866 & 3676 & 177 \\
\hline QPB 02 & 20-Jul-90 & UNC3 & 144 & 18.8 & 7.83 & 5472 & 453 & 842 & 3493 & 165 \\
\hline QPB 02 & 16-Aug-90 & UNC3 & 145 & 18.7 & 7.98 & 5444 & 458 & 819 & 3443 & 164 \\
\hline QPB 02 & 20-Sep-90 & UNC3 & 144 & 18.6 & 10.17 & 5585 & 436 & 825 & 3637 & 166 \\
\hline APB 03 & 16-Jan-90 & UNC1 & 96 & 13.1 & 12.64 & 5487 & 312 & 611 & 4148 & 133 \\
\hline OPB 03 & 20-Jul-90 & UNCS & 140 & 18.6 & 7.78 & 5557 & 453 & 842 & 3633 & 169 \\
\hline QPB 03 & 16-Aug-90 & UNC3 & 141 & 18.6 & 7.88 & 5416 & 460 & 809 & 3391 & 164 \\
\hline QPB 03 & 20-Sep-90 & UNC3 & 146 & 18.6 & 7.68 & 5529 & 435 & 825 & 3493 & 165 \\
\hline QPB 04 & 27-Apr-90 & UNC2 & 130 & 17.5 & 9.88 & 5501 & 399 & 767 & 3650 & 171 \\
\hline QPB 04 & 20-Jul-90 & UNC3 & 143 & 18.6 & 7.58 & 5416 & 472 & 823 & 3435 & 165 \\
\hline QPB 04 & 16-Aug-90 & UNCS & 140 & 18.5 & 8.83 & 5501 & 451 & 821 & 3596 & 168 \\
\hline QPB 04 & 20-Sep-90 & UNCS & 144 & 18.6 & 11.17 & 5642 & 431 & 821 & 3639 & 169 \\
\hline QPB 05 & 27-Apr-90 & UNC2 & 132 & 17.4 & 9.53 & 5529 & 409 & 784 & 3678 & 177 \\
\hline QPB 05 & 15-Jun-90 & UNC2 & 137 & 18.2 & 8.03 & 5501 & 416 & 813 & 3537 & 164 \\
\hline QPB 05 & 20-Jul-90 & UNCO & 140 & 18.5 & 8.88 & 5585 & 451 & 821 & 3554 & 170 \\
\hline QPB 05 & 16-Aug-90 & UNC3 & 146 & 18.8 & 7.83 & 5501 & 427 & 825 & 3430 & 165 \\
\hline QPB 05 & $20-$ Sep-90 & UNC3 & 147 & 18.5 & 7.81 & 5360 & 460 & 823 & 3428 & 164 \\
\hline $\begin{array}{c}\text { average, } \\
\mathrm{mM}\end{array}$ & & & 139 & 18.2 & 8.71 & 5512 & 434 & 807 & 3574 & 165 \\
\hline $\begin{array}{c}\text { average, } \\
\text { mg/L }\end{array}$ & & & 1505 & 1453 & 349 & 195419 & 16971 & 19618 & 82159 & 15888 \\
\hline
\end{tabular}




\section{Sandia National Laboratories}

date: 17 June 1993, modified 26 July 1993

Albuquerque, New Mexico 87185

to: Elaine D. Gorham, 6119, and J. Foesch, 6119

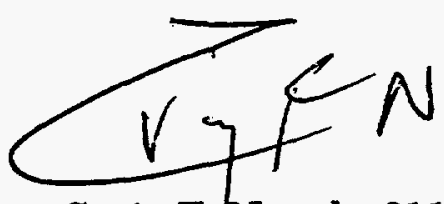

from: Craig F. Novak, 6119

subject: Evaluation of Chemical Analysis Data of Brine Samples from the Small Scale Brine Inflow Experiments

\section{Summary}

This memorandum documents the chemical compositions of 51 Salado brine samples collected as part of the Small Scale Brine Inflow Experiments. The compositions were examined for trends with time, location, and borehole size, but few trends were found. Most of the observed variations in compositions can be explained by the hypothesis of equilibrium evaporation during brine accumulation. Brine compositions are consistent with published data from previous studies of Salado Formation brines.

\section{Introduction}

Salado brine samples have been collected as part of the Small-Scale Brine Inflow Experiments (SSBIE), which were intended to provide some understanding of brine flow and transport mechanisms within the Salado Formation. Some of these brine samples have been analyzed for major chemical constituents under the supposition that transport information might be gleaned from the brine compositions. This memorandum examines the chemical analysis data of these brines to determine what can and cannot be learned about Salado transport from 
these chemical data. This memorandum does not examine brine inflow rates or borehole humidity data, and considers these quantities only peripherally as they impact interpretation of brine composition data. Comments and conclusions within this memorandum pertain only to the chemical component of the SSBIE, and have no implications for the strictly flow-related portion of these experiments.

The stratigraphic locations and orientations of the SSBIE boreholes are given in Figure 1. Ten boreholes are located in Room D in the north (experimental) end of the facility. Among the Room D boreholes, no brine has accumulated in DBT16 or DBT17. Brine has been withdrawn from all other Room D boreholes, including the nominally 4" diameter vertical boreholes DBT10, DBT11, DBT12, DBT13, DBT14, and DBT15, and from the vertical boreholes DBT31 and DBT32, which were nominally 4 " in diameter when drilled, but were later enlarged to nominally $36^{\text {" }}$ in diameter (Finley et al., 1992). Two subhorizontal boreholes from which brine has been sampled are located in the L4 drift, the nominally 4" diameter L4B01 and the nominally $36^{\prime \prime}$ diameter L4X01. Five boreholes are located in the Q-access drift approximately halfway between the Air Intake Shaft and the entrance to Room Q. Brine has been collected from all five of these nominally 2" diameter vertical boreholes numbered QPB01, QPB02, QPB03, QPB04, and QPB05. Details about borehole drilling history, brine sampling procedures, and brine inflow rates through 6 June 1991 can be found in Finley et al. (1992).

The stratigraphic units that the boreholes intersect are shown in Figure 1. The large diameter boreholes DBT31 and DBT 32 pass through several halite and argillaceous halite units, while the large borehole L4X01 is entirely within an argillaceous halite unit. The mineralogy of the Salado near the waste facility horizon is examined by Stein (1985), who in particular examined the residues remaining when the $\mathrm{NaCl}(\mathrm{s})$ in bulk halite samples was removed by dissolution. One conclusion in this report is "The non- $\mathrm{NaCl}$ components of halite [in the Salado Formation]...in the immediate vicinity (e.g., 100 vertical feet) of the WIPP facility horizon...consist[s] of quartz, anhydrite, gypsum, magnesite, polyhalite, and clays, with traces of...other minerals" (p. 20, Stein, 1985). The presence of these accessory minerals is an important consideration in understanding the measured chemical compositions of brines from the SSBIE, as discussed below. 
Selected brine samples were submitted for chemical analysis, as reported in UNC Geotech Analytical Laboratory (1990), Chem-Nuclear Geotech Analytical Laboratory (1990), and Chem-Nuclear Geotech Analytical Laboratory (1991). Table 1 gives a summary of these chemical analyses, along with sample collection and analysis dates.

\section{Analyzed Brine Compositions}

The composition data from Table 1 are plotted versus bromide concentration in Figure 2, with open symbols denoting small (2" and 4") diameter boreholes and filled symbols denoting large (36") diameter boreholes. Bromide concentration was chosen as the abscissa because dissolved bromide has been shown to act conservatively during seawater evaporation until the bromide concentration is approximately 90 times greater than that in seawater, i.e., up to bromide concentrations of about 65 to $70 \mathrm{mM}$ (McCaffrey et al., 1987). (Conservative behavior in this case means that bromide is not lost from the aqueous phase by precipitation but merely becomes more concentrated in solution as water is removed from the brine.) Because none of the measured bromide concentrations from the SSBIE exceeds $40 \mathrm{mM}$, it is reasonable to assume that bromide behaved conservatively when Salado brines were formed.

The concentrations of chloride, magnesium, potassium, sulfate, and boron (Figure 2) increase linearly as the bromide concentration increases, while the concentrations of sodium and calcium decrease linearly as the bromide concentration increases. This suggests that the brines are in chemical equilibrium with respect to halite, $\mathrm{NaCl}(\mathrm{s})$, because chemical thermodynamics indicates that the product of the activities ("effective" concentrations) of $\mathrm{Na}^{+}$and $\mathrm{Cl}^{-}$will be constant when in equilibrium with halite. A similar argument holds for $\mathrm{Ca}^{2+}$ and $\mathrm{SO}_{4}^{2-}$ and equilibrium with anhydrite, $\mathrm{CaSO}_{4}(\mathrm{~s})$. The presence of both these minerals in the map units that the boreholes intersect supports this suggestion. However, this trend is defined primarily by the data from the large diameter boreholes.

Much less concentration variation is seen when considering only the small diameter boreholes, i.e., the open symbols in Figure 2. This is emphasized by "zooming in" on the concentration ranges that represent all the 2 " and 4 " diameter 
boreholes, as is done in Figure 3. On this expanded scale, the compositions of the Room D small boreholes seem to form a cluster distinct from the Q-access and Room L small boreholes. In this representation, most of the element concentrations in the small diameter boreholes seem to form a linear trend over a very narrow (17-21 mM) bromide concentration range.

Close examination of the potassium and magnesium concentrations in the small diameter boreholes, Figure 3, shows that the Q-access and L4B01 data cluster around bromide concentrations of 17 to $19 \mathrm{mM}$ (with one outlying point at $13 \mathrm{mM}$ ), while the Room D data span the range from 19 to $21 \mathrm{mM}$ bromide. The concentrations of most, if not all, other elements exhibit small but distinct differences between the $\mathrm{Q}$-access/L4B01 and Room D populations. It is unlikely that these differences are caused by systematic sampling or analytical errors because samples from most locations were analyzed in the same batches.

In previous analyses of brine composition from the Salado (Stein and Krumhansl, 1988), data were presented in graphical form as $\mathrm{Na} / \mathrm{Cl}$ mass ratio versus $\mathrm{K} / \mathrm{Mg}$ mass ratio; the SSBIE data from the small diameter boreholes are plotted thus in Figure 4. In this representation, the samples from the Room D small diameter boreholes cluster tightly in an apparently distinct population from the $\mathrm{Q}$-access and L4B01 samples. The brine compositions from the small diameter boreholes seem to fall into two populations, one for the small DBT boreholes, and one for the small L4 and QPB boreholes. The sodium to chloride mass ratios are approximately the same for all the small boreholes, but the Room D samples are deficient in potassium and enriched in magnesium relative to the L4B01 and Q-access samples, as can be seen from Figure 3. However, the differences in the magnesium to potassium ratios observed for Room D and Room L4/Q-access are likely to be unimportant with regard to the solubility and migration behavior of actinides in Salado brines.

Figure 5 presents $\mathrm{Na} / \mathrm{Cl}$ versus $\mathrm{K} / \mathrm{Mg}$ mass ratios for all SSBIE samples, including analyses for brines from large diameter boreholes. Samples from the large diameter boreholes do not cluster with the small boreholes. Some of the brine analyses from the large DBT boreholes fall near the small borehole population, but others do not, generally being depleted in sodium relative to chloride. One of the analyses from the large L4X01 borehole appears to be depleted in sodium relative to 
chloride, but the other three L4X01 samples also have lower potassium to magnesium ratios, and appear on Figure 5 to look more similar to the large DBT borehole samples.

The accuracies of the chemical analyses summarized in Table 1 were not reported. However, it is worthwhile to consider approximate "error bars" on these data to determine whether the two data clusters in Figure 5 merge into one population when uncertainties are included. Assume one is interested in the ratio $\mathrm{R}=\mathrm{x} / \mathrm{y}$, where the quantities $\mathrm{x}$ and $\mathrm{y}$ have associated $\pm \mathrm{a} \%$ and $\pm b \%$ uncertainties, respectively. The associated ranges in values for $x$ and $y$ yield as uncertainty bounds on $R$ the smallest ratio $R_{s}=\frac{(1-a)}{(1+b)} R$ and the largest ratio $R_{\ell}=\frac{(1+a)}{(1-b)} R$. If $a$ and $b$ are both $5 \%$, then $R_{s} / R=0.90$ and $R_{\ell} / R=1.10$, giving about a $10 \%$ error bar on the final ratio. (A value of $5 \%$ may be conservative; if $a=b=10 \%$, the error on the final ratio will be about $20 \%$.) When this $5 \%$ error is included with the data (Figure 6), the brines still appear to fall into two distinct populations. The trends described above remain clearly distinguishable even after considering the possible magnitude of errors associated with sampling handling and analysis.

\section{Effects of Evaporation on Brine Compositions}

There are several reasons to suspect that water evaporation from the brine may have occurred while brine was accumulating in the boreholes. Figure 2 shows that concentrations in brines from the large diameter boreholes increase linearly with bromide concentration, while concentrations from the small diameter boreholes cluster at the low concentration end of this correlation. When bromide is assumed to behave conservatively during evaporation, this suggests that the brines were concentrated by evaporation in the large diameter boreholes.

The seals for the large boreholes also suggest evaporation may be occurring, as presented in the attached memorandum from Jim Foesch. The L4X01 borehole is closed with a brattice cloth seal that is considered to provide poor isolation of the gas phase within the L4X01 borehole. Humidity measurements within L4X01 and within Room L4 are correlated, suggesting that gas can flow out of and into the borehole as pressure changes within the drift. The DBT31 and DBT32 boreholes 
are sealed in a different manner from L4X01, using a $90 \mathrm{~cm}$ flange with a rubber gasket. This arrangement provides a better seal than for L4X01, but the seal is still not as good at those on the small diameter boreholes. Thus, loss of water vapor through exchange with the atmosphere is plausible for the L4X01 borehole and the large DBT boreholes.

The total void volume within boreholes relative to brine sample size provides a further suggestion that evaporation may be more important in the large boreholes. Table 2 shows the diameters and lengths of the SSBIE boreholes, along with calculated internal volumes (assuming cylindrical boreholes) and the mass of water needed to bring the air within the borehole to a relative humidity of $75 \%$, the relative humidity approximately in equilibrium with Salado brines. As shown, the mass of water necessary to reach 75\% relative humidity is about 1 gram for L4B01 and the small DBT boreholes, and about 0.1 gram for the Q-access boreholes, while it is on the order of 70 grams for DBT31, DBT32, and L4X01. A comparison of these masses of water to the mass of water in the brine samples is given in Table 3 . This calculation assumed that about three quarters of the brine mass was $\mathrm{H}_{2} \mathrm{O}$, estimated by assuming a brine density of $1200 \mathrm{~g} /$ liter and $300 \mathrm{~g} /$ liter total dissolved solids. As Table 3 shows, only for the large diameter boreholes is the mass of water in the air a significant fraction of the mass of water in the collected brine. This means that, if all boreholes were perfectly sealed, and contained dry air (relative humidity $0 \%$ ) at the start of a test interval, and all water in the air came from brine evaporation, the evaporation would affect the brine compositions only in the large diameter boreholes.

\section{Evaporation Path Modeling}

Equilibrium evaporation path modeling was performed to determine whether the hypothesis of water evaporation was consistent with the brine composition data. Three average brines, called $\overline{\mathrm{DBT}}, \overline{\mathrm{QPB}}$, and $\overline{\mathrm{L} 4 \mathrm{~B} 01}$, were defined as the arithmetic average of all samples for every small diameter borehole in a given room or drift. The equilibrium evaporation paths of these brines were calculated with the PHRQPITZ code (Plummer et al., 1988), using the Pitzer specific ion interaction approach and the data set of Felmy and Weare (1986) and Harvie et al. (1984). 
Using this code, the three average brines were sequentially evaporated to the following endpoints:

(1) halite, $\mathrm{NaCl}(\mathrm{s})$, saturation;

(2) anhydrite, $\mathrm{CaSO}_{4}$ (s), saturation in equilibrium with halite;

(3) polyhalite, $\mathrm{K}_{2} \mathrm{MgCa}_{2}\left(\mathrm{SO}_{4}\right)_{4} \bullet 2 \mathrm{H}_{2} \mathrm{O}(\mathrm{s})$, saturation in equilibrium with halite and anhydrite; and

(4) sylvite, $\mathrm{KCl}(\mathrm{s})$, saturation in equilibrium with halite and polyhalite, but not anhydrite.

Anhydrite equilibrium was not maintained in step 4 because more anhydrite would have needed to dissolve than had precipitated in the evaporation sequence. The modeling assumed a closed system, i.e., the model did not allow $\mathrm{CO}_{2}(\mathrm{~g})$ to dissolve or exsolve. The final solution for the average $L 4$ brine was slightly oversaturated with respect to a borate mineral that has not been observed near the repository horizon in the Salado. The calculated evaporation paths are plotted versus bromide concentration along with the measured concentrations for the large boreholes in Figure 7; three evaporation lines are shown for each element because the three average brine compositions are slightly different.

A comparison of the modeling results with composition data is shown in Figure 7, where the lines represent calculated evaporation paths for the three average brines, the solid squares represent data from the large DBT boreholes, and the filled circles represent data from the large L4 borehole. For all elements except total inorganic carbon (TIC), the calculated evaporation paths of the average brines agree very well with the observed compositions from the large boreholes, indicating that the hypothesis of water evaporation within boreholes is consistent with the data. Calculated evaporation paths are similar for the three different average brines.

The agreement between evaporation path modeling and the data points on Figure 7 provides partial verification and validation of both the chemical model (Felmy and Weare, 1986; Harvie et al., 1984; Harvie and Weare, 1980), and the PHRQPITZ implementation of this chemical model. The SSBIE brines are complex, highly concentrated chemical systems, and are independent from the data used to parameterize the chemical model. Nonetheless, excellent agreement 
between the model and data was achieved through straightforward analysis. This provides additional confidence that the methods used above can successfully describe the geochemical behavior of brine constituents in evaporite environments such as the Salado Formation.

The lack of agreement between the modeled and measured TIC data could be attributed to numerous causes. Carbon dioxide may have been lost during brine accumulation while water was evaporating. This explanation is consistent with the lower values measured in the large diameter boreholes relative to the TIC concentrations predicted by the model. Other possible explanations include $\mathrm{CO}_{2}(\mathrm{~g})$ outgassing during sample storage and handling, and errors or uncertainty in $\mathrm{pH}$ measurement.

\section{Compositional Trends over Multiple Samples from the Same Borehole}

As shown in Table 1, several different brine samples from each of the boreholes were chemically analyzed. The samples were withdrawn at different times to determine whether composition is a function of sampling date. This section examines the data for such variation for the eight boreholes from which three or more brine samples were analyzed. These small diameter boreholes include four from the Q-access drift, and four from Room D. Because the compositional variation

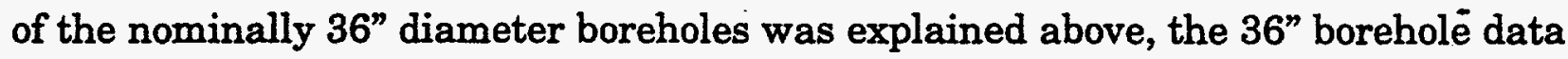
are not discussed here. The time spans represented by data for the boreholes with multiple samples range from five to thirty months. With so few samples, it would be difficult to define temporal trends. However, some regular variation in compositions might become apparent.

Plots of these data for the Room $D$ and Q-access boreholes are given in Figures 8 and 9, respectively. The data were reduced by calculating the average element concentrations for all samples from each borehole, and normalizing individual sample concentrations with respect to this average. This method of data reduction allows direct comparison of the magnitude of compositional variations among different elements. As shown in Figure 8 for boreholes DBT10, DBT11, DBT12, and DBT13, concentrations of all elements except boron and calcium vary by less than $\sim 5 \%$ from the borehole average. Boron varies by up to $\sim 10 \%$ in DBT12, 
and calcium varies by up to $20 \%$. Figure 9 shows similar plots for QPB02, QPB03, $\mathrm{QPB} 04$, and QPB05. Here there is more variation from the average, particularly seen in one QPB03 sample taken on 16 January 1990. However, if this data point is discounted, the variations appear about the same as discussed for the Room D samples. These variations are close to the 5 to $10 \%$ analytical variability proposed earlier in this memorandum. Overall, the concentrations in each borehole seem fairly constant across multiple samples; no trends are obvious from either Figure 8 or Figure 9.

\section{No Evidence for Contamination of Salado Brine in Q-access Boreholes}

The boreholes in the Q-access drift are located approximately 40 meters from the bottom of the Air Intake Shaft (AIS). Because brines from overlying formations collect in a sump at the bottom of the AIS, and because the sump has occasionally overflowed onto the floor of the excavations, there was concern that brines in Room $Q$ and the $Q$-access drift could become contaminated with nonSalado brines. Indeed, this was part of the reason for the location of the $\mathrm{Q}$-access boreholes.

The existing brine composition data provide no evidence that nonSalado brine has mixed with Salado brine, or that the brines collecting in the SSBIE boreholes have origins from other than the Salado Formation. Indeed, a comparison of the compositions of SSBIE samples in Figure 2 with those from brine weeps (Figure 2, Krumhansl et al., 1991) shows no significant differences. The brine weeps were located in drift walls one or several meters above the level at which the potential contamination mechanisms could be considered plausible. Thus, because the weep samples can be considered to represent uncontaminated Salado brines, and because the weep samples and the SSBIE samples have similar compositions, it appears that the SSBIE samples are also uncontaminated by brines from other formations.

\section{Conclusions}

The brines collected and analyzed as part of the Small Scale Brine Inflow Experiments do show compositional variation. However, much of the variation occurs in brines collected from the large (nominally $36^{\text {" }}$ diameter) boreholes, and 
appears to result from evaporation of water during the intervals between withdrawal of brine samples. This hypothesis, water evaporation during brine accumulation, accounts for the majority of compositional variation. Some small differences in element concentrations remain, and may be important if one is attempting to understand the origins of Salado brine. However, the SSBIE were originally intended to measure brine inflow rates; they were not intended to provide data for understanding brine origins or possible compositional variations. The study of brine origins and potential variations in brine compositions would be better conducted within a program specifically designed for those purposes.

The brine composition data examined in this memorandum appear to be an insufficient basis for drawing conclusions about transport in the Salado. Furthermore, these data do not suggest ways in which additional brine composition data may contribute to the development of inferences about brine transport in the Salado. Therefore, there do not appear to be any transport-related technical reasons for continuing to store and analyze brine samples from the SSBIE.

\section{Acknowledgment}

K.L. Robinson performed many of the PHRQPITZ simulations presented in this memorandum.

\section{References}

Chem-Nuclear Geotech Analytical Laboratory. 1990. "Analytical Report." Grand Junction, Colorado. Chem-Nuclear Geotech Analytical Laboratory. (copy on file at Quality Assurance Records/Notebook System, Sandia National Laboratories, Carlsbad, New Mexico.)

Chem-Nuclear Geotech Analytical Laboratory. 1991. “Analytical Report." Grand Junction, Colorado. Chem-Nuclear Geotech Analytical Laboratory. (copy on file at Quality Assurance Records/Notebook System, Sandia National Laboratories, Carlsbad, New Mexico.)

Felmy, A.R., and J.H. Weare. 1986. "The Prediction of Borate Mineral Equilibria in Natural Waters: Application to Searles Lake, California." Geochimica et Cosmochimica Acta vol. 50: 27712783.

Finley, S.J., D.J. Hanson, and R. Parsons. 1992. Small-Scale Brine Inflow Experiments-Data Report Through 6/6/91. SAND91-1956. Albuquerque, New Mexico: Sandia National Laboratories. 
Harvie, C.E., and J.H. Weare. 1980. "The Prediction of Mineral Solubilities in Natural Waters: The $\mathrm{Na}-\mathrm{K}-\mathrm{Mg}-\mathrm{Ca}-\mathrm{Cl}-\mathrm{SO}_{4}-\mathrm{H}_{2} \mathrm{O}$ System from Zero to High Concentration at $25^{\circ} \mathrm{C} . "$ Geochimica et Cosmochimica Acta vol. 44: 981-997.

Harvie, C.E., N. Møller, and J.H. Weare. 1984. "The Prediction of Mineral Solubilities in Natural Waters: The Na-K-Mg-Ca-H-Cl-SO $4-\mathrm{OH}-\mathrm{HCO}_{3}-\mathrm{CO}_{3}-\mathrm{CO}_{2}-\mathrm{H}_{2} \mathrm{O}$ System to High Ionic Strength at 25.C." Geochimica et Cosmochimica Acta vol. 48: 723-751.

Krumhansl, J.L., K.M Kimball, and C.L. Stein. 1991. Intergranular Fluid Compositions from the Waste Isolation Pilot Plant (WIPP), Southeastern New Mexico. SAND90-0584. Albuquerque, New Mexico: Sandia National Laboratories.

McCaffrey, M.A., B. Lazar, and H.D. Holland. 1987. "The Evaporation Path of Seawater and the Coprecipitation of $\mathrm{Br}^{-}$and $\mathrm{K}^{+}$with Halite." Journal of Sedimentary Petrology vol. 57 \#5: 928-937.

Perry, R.H., and C.H. Chilton, editors. 1973. Chemical Engineers Handbook. Fifth Edition. New York: McGraw-Hill.

Plummer, L.N., D.L. Parkhurst, G.W. Fleming, and S.A. Dunkel. 1988. A Computer Program Incorporating Pitzer's Equations for Calculation of Gecichemical Reactions in Brines. WaterResources Investigations Report 88-4153. Reston, Virginia: U.S. Geological Survey.

Stein, C.L. 1985. Mineralogy in the Waste Isolation Pilot Plant (WIPP) Facility Stratigraphic Horizon. SAND85-0321. Albuquerque, New Mexico: Sandia National Laboratories.

Stein, C.L., and J.L. Krumhansl. 1988. "A Model for the Evolution of Brines in Salt from the Lower Salado Formation, Southeastern New Mexico." Geochimica et Cosmochimica Acta vol. 52: 1037-1046.

UNC Geotech Analytical Laboratory. 1990. "Analytical Report." Grand Junction, Colorado. UNC Geotech Analytical Laboratory. (copy on file at Quality Assurance Records/Notebook Systsm, Sandia National Laboratories, Carlsbad, New Mexico.)

Copies to:

$\begin{array}{llll}6100 & \text { R.W. Lynch } & 6300 & \text { D.E. Ellis } \\ 6115 & \text { P.B. Davies } & 6303 & \text { W.D. Weart } \\ 6115 & \text { M.D. Siegel } & 6305 & \text { S.A. Goldstein } \\ 6118 & \text { H.R. Westrich } & 6341 & \text { A.L. Stevens } \\ 6119 & \text { F. Gelbard } & 6342 & \text { D.R. Anderson } \\ 6119 & \text { C.F. Novak } & 6345 & \text { R.C. Lincoln } \\ 6119 & \text { H.W. Papenguth } & 6345 & \text { E.J. Nowak } \\ 6119 & \text { K.L. Robinson } & 6348 & \text { J.T. Holmes } \\ 6119 & \text { File } & 6348 & \text { L.H. Brush } \\ 6121 & \text { J.R. Tillerson } & \text { SWCF } & \text { RC/BC 1.1.4.2 }\end{array}$


Table 1. Analyzed concentrations of brine samples from the Small Scale Brine Inflow Experiments

\begin{tabular}{|c|c|c|c|c|c|c|c|c|c|c|c|}
\hline Borehole & $\begin{array}{c}\text { Nominal } \\
\text { Diameter, } \\
\text { inches }\end{array}$ & $\begin{array}{c}\text { Collection } \\
\text { Date }\end{array}$ & $\begin{array}{l}\text { Analysis } \\
\text { Report" }\end{array}$ & $\begin{array}{l}\mathrm{B}, \\
\mathrm{mM}\end{array}$ & $\begin{array}{l}\mathrm{Br} \\
\mathrm{mM}\end{array}$ & $\begin{array}{l}\mathrm{Ca} \\
\mathrm{mM}\end{array}$ & $\begin{array}{l}\mathrm{Cl}, \\
\mathrm{mM}\end{array}$ & $\begin{array}{l}\mathrm{K} \\
\mathrm{mM}\end{array}$ & $\begin{array}{l}\mathrm{Mg} \\
\mathrm{mM}\end{array}$ & $\begin{array}{l}\mathrm{Na}, \\
\mathrm{mM}\end{array}$ & $\begin{array}{l}\mathrm{SO}_{4} \\
\mathrm{mM}\end{array}$ \\
\hline $\begin{array}{l}\text { DBT 10 } \\
\text { DBT 10 } \\
\text { DBT 10 } \\
\text { DBT 10 } \\
\end{array}$ & $\begin{array}{l}4 \\
4 \\
4 \\
4 \\
\end{array}$ & $\begin{array}{l}16 \text { Dec } 87 \\
15 \text { Feb } 90 \\
20 \text { Mar } 90 \\
21 \text { Jun } 90\end{array}$ & $\begin{array}{l}\text { UNC1 } \\
\text { UNC2 } \\
\text { UNC1 } \\
\text { UNC2 } \\
\end{array}$ & $\begin{array}{l}132 \\
136 \\
130 \\
133 \\
\end{array}$ & $\begin{array}{l}19.5 \\
20.4 \\
19.5 \\
20.2 \\
\end{array}$ & $\begin{array}{l}8.68 \\
6.86 \\
7.31 \\
7.13 \\
\end{array}$ & $\begin{array}{l}5571 \\
5571 \\
5543 \\
5557 \\
\end{array}$ & $\begin{array}{l}394 \\
409 \\
407 \\
402 \\
\end{array}$ & $\begin{array}{l}1037 \\
1043 \\
1053 \\
1008 \\
\end{array}$ & $\begin{array}{l}3509 \\
3226 \\
3326 \\
3278 \\
\end{array}$ & $\begin{array}{l}192 \\
197 \\
190 \\
192 \\
\end{array}$ \\
\hline $\begin{array}{l}\text { DBT } 11 \\
\text { DBT } 11 \\
\text { DBT } 11\end{array}$ & $\begin{array}{l}4 \\
4 \\
4 \\
\end{array}$ & $\begin{array}{l}11 \text { May } 88 \\
20 \text { Mar } 90 \\
21 \text { Jun } 90 \\
\end{array}$ & $\begin{array}{l}\text { UNC1 } \\
\text { UNC1 } \\
\text { UNC3 }\end{array}$ & $\begin{array}{l}131 \\
136 \\
134 \\
\end{array}$ & $\begin{array}{l}19.4 \\
19.4 \\
20.2 \\
\end{array}$ & \begin{tabular}{|l|}
7.33 \\
6.33 \\
6.63 \\
\end{tabular} & $\begin{array}{l}5614 \\
5571 \\
5529 \\
\end{array}$ & $\begin{array}{l}399 \\
399 \\
422 \\
\end{array}$ & $\begin{array}{l}1033 \\
1006 \\
1064 \\
\end{array}$ & $\begin{array}{l}5330 \\
3430 \\
3307 \\
\end{array}$ & $\begin{array}{l}192 \\
190 \\
190 \\
\end{array}$ \\
\hline $\begin{array}{l}\text { DBT } 12 \\
\text { DBT } 12 \\
\text { DBT } 12 \\
\end{array}$ & $\begin{array}{l}4 \\
4 \\
4 \\
\end{array}$ & $\begin{array}{l}18 \operatorname{Jan} 90 \\
20 \text { Mar } 90 \\
21 \text { Jun } 90 \\
\end{array}$ & $\begin{array}{l}\text { UNC1 } \\
\text { UNC2 } \\
\text { UNC2 }\end{array}$ & $\begin{array}{l}150 \\
133 \\
125 \\
\end{array}$ & $\begin{array}{l}20.9 \\
20.2 \\
19.5 \\
\end{array}$ & $\begin{array}{l}6.71 \\
7.06 \\
6.53 \\
\end{array}$ & $\begin{array}{l}5543 \\
5571 \\
5557 \\
\end{array}$ & $\begin{array}{l}412 \\
391 \\
380 \\
\end{array}$ & $\begin{array}{l}1084 \\
1012 \\
1004 \\
\end{array}$ & $\begin{array}{l}3189 \\
3298 \\
3389 \\
\end{array}$ & $\begin{array}{l}200 \\
191 \\
187 \\
\end{array}$ \\
\hline DBT 13 & 4 & 21 Jun 90 & UNC3 & 129 & 19.6 & 6.48 & 5529 & 399 & 1021 & 3313 & 186 \\
\hline $\begin{array}{l}\text { DBT } 14 \\
\text { DBT } 14 \\
\text { DBT } 14 \\
\text { DBT } 14 \\
\text { DBT } 14 \\
\end{array}$ & $\begin{array}{l}4 \\
4 \\
4 \\
4 \\
4 \\
\end{array}$ & $\begin{array}{c}28 \text { Sep } 88 \\
17 \text { May } 89 \\
1 \text { Nov } 89 \\
16 \text { May } 90 \\
16 \text { Aug } 90 \\
\end{array}$ & $\begin{array}{l}\text { UNC1 } \\
\text { UNC2 } \\
\text { UNC2 } \\
\text { UNC2 } \\
\text { UNC3 } \\
\end{array}$ & $\begin{array}{l}127 \\
130 \\
131 \\
129 \\
130 \\
\end{array}$ & $\begin{array}{c}19.5 \\
19.6 \\
20 \\
20 \\
20.1 \\
\end{array}$ & $\begin{array}{l}8.1 \\
6.68 \\
6.53 \\
6.63 \\
6.53 \\
\end{array}$ & $\begin{array}{l}5557 \\
\mathbf{5 5 5 7} \\
\mathbf{5 5 2 9} \\
\mathbf{5 5 2 9} \\
\mathbf{5 4 7 2} \\
\end{array}$ & $\begin{array}{l}395 \\
376 \\
381 \\
382 \\
398 \\
\end{array}$ & $\begin{array}{c}1021 \\
992 \\
1008 \\
1002 \\
1025 \\
\end{array}$ & $\begin{array}{l}3396 \\
3339 \\
3333 \\
3326 \\
3254 \\
\end{array}$ & $\begin{array}{l}188 \\
185 \\
188 \\
186 \\
184\end{array}$ \\
\hline $\begin{array}{l}\text { DBT } 15 \\
\text { DBT } 15 \\
\end{array}$ & $\begin{array}{l}4 \\
4 \\
\end{array}$ & $\begin{array}{l}27 \text { Jul } 88 \\
16 \text { Aug } 90\end{array}$ & $\begin{array}{l}\text { UNC1 } \\
\text { UNC3 }\end{array}$ & $\begin{array}{l}139 \\
139 \\
\end{array}$ & $\begin{array}{c}20.5 \\
20 \\
\end{array}$ & $\begin{array}{l}8.03 \\
6.43 \\
\end{array}$ & $\begin{array}{l}5585 \\
5585 \\
\end{array}$ & $\begin{array}{l}428 \\
409 \\
\end{array}$ & $\begin{array}{l}1097 \\
1029 \\
\end{array}$ & $\begin{array}{r}3289 \\
3283 \\
\end{array}$ & $\begin{array}{l}200 \\
188\end{array}$ \\
\hline $\begin{array}{l}\text { DBT } 31 \\
\text { DBT } 31 \\
\text { DBT } 31 \\
\end{array}$ & $\begin{array}{c}4 \\
36 \\
36 \\
\end{array}$ & $\begin{array}{l}16 \text { Mar } 88 \\
16 \text { May } 90 \\
16 \text { Aug } 90 \\
\end{array}$ & $\begin{array}{l}\text { UNC1 } \\
\text { UNC2 } \\
\text { UNC3 } \\
\end{array}$ & $\begin{array}{l}153 \\
241 \\
215 \\
\end{array}$ & $\begin{array}{c}22 \\
38.2 \\
31.8 \\
\end{array}$ & $\begin{array}{l}8.08 \\
2.52 \\
3.74 \\
\end{array}$ & $\begin{array}{r}5599 \\
5966 \\
5698 \\
\end{array}$ & $\begin{array}{l}471 \\
720 \\
664 \\
\end{array}$ & $\begin{array}{l}1163 \\
1994 \\
1621 \\
\end{array}$ & $\begin{array}{l}3217 \\
1757 \\
2185 \\
\end{array}$ & $\begin{array}{l}210 \\
335 \\
276\end{array}$ \\
\hline $\begin{array}{l}\text { DBT 32 } \\
\text { DBT } 32 \\
\text { DBT } 32 \\
\text { DBT } 32 \\
\end{array}$ & \begin{tabular}{r|r}
4 \\
36 \\
36 \\
36 \\
\end{tabular} & $\begin{array}{c}9 \text { Mar } 88 \\
6 \text { Apr } 89 \\
16 \text { May } 90 \\
16 \text { Aug } 90 \\
\end{array}$ & $\begin{array}{l}\text { UNC2 } \\
\text { UNC1 } \\
\text { UNC2 } \\
\text { UNC3 } \\
\end{array}$ & $\begin{array}{l}138 \\
188 \\
171 \\
167\end{array}$ & $\begin{array}{l}20.2 \\
26.8 \\
26.5 \\
25.4 \\
\end{array}$ & $\begin{array}{c}9 \\
6.16 \\
5.21 \\
5.09 \\
\end{array}$ & $\begin{array}{l}5491 \\
5698 \\
5684 \\
5585\end{array}$ & $\begin{array}{l}419 \\
541 \\
504 \\
490 \\
\end{array}$ & $\begin{array}{l}1056 \\
1533 \\
1374 \\
1288 \\
\end{array}$ & $\begin{array}{l}3057 \\
2687 \\
2678 \\
2759 \\
\end{array}$ & $\begin{array}{l}197 \\
251 \\
249 \\
232 \\
\end{array}$ \\
\hline $\begin{array}{l}\text { QPB } 01 \\
\text { QPB } 01\end{array}$ & $\begin{array}{l}2 \\
2 \\
\end{array}$ & $\begin{array}{l}16 \text { Aug } 90 \\
20 \text { Sep } 90\end{array}$ & $\begin{array}{l}\text { UNC3 } \\
\text { UNC3 }\end{array}$ & $\begin{array}{l}138 \\
144 \\
\end{array}$ & $\begin{array}{l}18.1 \\
18.8\end{array}$ & $\begin{array}{l}7.53 \\
7.88\end{array}$ & $\begin{array}{l}5501 \\
5614\end{array}$ & $\begin{array}{l}426 \\
448 \\
\end{array}$ & $\begin{array}{l}776 \\
817 \\
\end{array}$ & $\begin{array}{l}3463 \\
3554\end{array}$ & $\begin{array}{l}160 \\
166\end{array}$ \\
\hline $\begin{array}{l}\text { QPB } 02 \\
\text { QPB } 02 \\
\text { QPB } 02 \\
\text { QPB } 02 \\
\text { QPB } 02 \\
\end{array}$ & $\begin{array}{l}2 \\
2 \\
2 \\
2 \\
2 \\
\end{array}$ & $\begin{array}{c}4 \text { Oct } 89 \\
13 \text { Dec } 89 \\
20 \text { Jul } 90 \\
16 \text { Aug } 90 \\
20 \text { Sep } 90 \\
\end{array}$ & $\begin{array}{l}\text { UNC2 } \\
\text { UNC1 } \\
\text { UNC3 } \\
\text { UNC3 } \\
\text { UNC3 }\end{array}$ & $\begin{array}{l}139 \\
149 \\
144 \\
145 \\
144 \\
\end{array}$ & $\begin{array}{l}18.5 \\
18.8 \\
18.8 \\
18.7 \\
18.6 \\
\end{array}$ & $\begin{array}{c}7.93 \\
9.43 \\
7.83 \\
7.98 \\
10.17 \\
\end{array}$ & $\begin{array}{l}5571 \\
5529 \\
5472 \\
5444 \\
5585 \\
\end{array}$ & $\begin{array}{l}431 \\
453 \\
453 \\
458 \\
436 \\
\end{array}$ & $\begin{array}{l}813 \\
866 \\
842 \\
819 \\
825 \\
\end{array}$ & $\begin{array}{l}3596 \\
3676 \\
3493 \\
3443 \\
3637 \\
\end{array}$ & $\begin{array}{l}166 \\
177 \\
165 \\
164 \\
166 \\
\end{array}$ \\
\hline $\begin{array}{l}\text { QPB } 03 \\
\text { QPB } 03 \\
\text { QPB } 03 \\
\text { QPB } 03\end{array}$ & $\begin{array}{l}2 \\
2 \\
2 \\
2 \\
\end{array}$ & $\begin{array}{l}16 \text { Jan } 90 \\
20 \text { Jul } 90 \\
16 \text { Aug } 90 \\
20 \text { Sep } 90\end{array}$ & $\begin{array}{l}\text { UNC1 } \\
\text { UNC3 } \\
\text { UNC3 } \\
\text { UNC3 }\end{array}$ & $\begin{array}{c}96 \\
140 \\
141 \\
146 \\
\end{array}$ & $\begin{array}{l}13.1 \\
18.6 \\
18.6 \\
18.6 \\
\end{array}$ & $\begin{array}{c}12.64 \\
7.78 \\
7.88 \\
7.68\end{array}$ & $\begin{array}{l}5487 \\
5557 \\
5416 \\
5529\end{array}$ & $\begin{array}{l}312 \\
453 \\
460 \\
435 \\
\end{array}$ & $\begin{array}{l}611 \\
842 \\
809 \\
825\end{array}$ & $\begin{array}{l}4148 \\
3633 \\
3391 \\
3493\end{array}$ & $\begin{array}{l}133 \\
169 \\
164 \\
165\end{array}$ \\
\hline $\begin{array}{l}\text { QPB 04 } \\
\text { QPB 04 } \\
\text { QPB 04 } \\
\text { QPB 04 } \\
\end{array}$ & $\begin{array}{l}2 \\
2 \\
2 \\
2 \\
\end{array}$ & $\begin{array}{l}27 \text { Apr } 90 \\
20 \text { Jul } 90 \\
16 \text { Aug } 90 \\
20 \text { Sep } 90 \\
\end{array}$ & $\begin{array}{l}\text { UNC2 } \\
\text { UNC3 } \\
\text { UNC3 } \\
\text { UNC3 }\end{array}$ & $\begin{array}{l}130 \\
143 \\
140 \\
144 \\
\end{array}$ & $\begin{array}{l}17.5 \\
18.6 \\
18.5 \\
18.6 \\
\end{array}$ & $\begin{array}{r}9.88 \\
7.58 \\
8.83 \\
11.17 \\
\end{array}$ & $\begin{array}{l}5501 \\
5416 \\
5501 \\
5642 \\
\end{array}$ & $\begin{array}{l}399 \\
472 \\
451 \\
431 \\
\end{array}$ & $\begin{array}{l}767 \\
823 \\
821 \\
821 \\
\end{array}$ & $\begin{array}{l}3650 \\
3435 \\
3596 \\
3639 \\
\end{array}$ & $\begin{array}{l}171 \\
165 \\
168 \\
169 \\
\end{array}$ \\
\hline $\begin{array}{l}\text { QPB 05 } \\
\text { QPB 05 } \\
\text { QPB } 05 \\
\text { QPB } 05 \\
\text { QPB 05 } \\
\end{array}$ & $\begin{array}{l}2 \\
2 \\
2 \\
2 \\
2 \\
\end{array}$ & $\begin{array}{l}27 \text { Apr } 90 \\
15 \text { Jun } 90 \\
20 \text { Jul } 90 \\
16 \text { Aug } 90 \\
20 \text { Sep } 90\end{array}$ & $\begin{array}{l}\text { UNC2 } \\
\text { UNC2 } \\
\text { UNC3 } \\
\text { UNC3 } \\
\text { UNC3 }\end{array}$ & $\begin{array}{l}132 \\
137 \\
140 \\
146 \\
147 \\
\end{array}$ & $\begin{array}{l}17.4 \\
18.2 \\
18.5 \\
18.8 \\
18.5 \\
\end{array}$ & $\begin{array}{l}9.53 \\
8.03 \\
8.88 \\
7.83 \\
7.81 \\
\end{array}$ & $\begin{array}{l}5529 \\
5501 \\
5585 \\
5501 \\
5360\end{array}$ & $\begin{array}{l}409 \\
416 \\
451 \\
427 \\
460\end{array}$ & $\begin{array}{l}784 \\
813 \\
821 \\
825 \\
823\end{array}$ & $\begin{array}{l}3678 \\
3537 \\
3554 \\
3430 \\
3428\end{array}$ & $\begin{array}{l}177 \\
164 \\
170 \\
165 \\
164\end{array}$ \\
\hline $\begin{array}{l}4 \mathrm{BO01} \\
\text { L4B01 }\end{array}$ & $\begin{array}{l}4 \\
4 \\
\end{array}$ & $\begin{array}{l}21 \text { Jun } 89 \\
14 \text { Dec } 89 \\
\end{array}$ & $\begin{array}{l}\text { UNC1 } \\
\text { UNC2 }\end{array}$ & $\begin{array}{l}150 \\
137 \\
\end{array}$ & $\begin{array}{l}19.1 \\
18.2 \\
\end{array}$ & $\begin{array}{l}7.98 \\
7.78 \\
\end{array}$ & $\begin{array}{l}5529 \\
5543 \\
\end{array}$ & $\begin{array}{l}464 \\
428 \\
\end{array}$ & $\begin{array}{l}912 \\
829\end{array}$ & $\begin{array}{l}3559 \\
3628\end{array}$ & $\begin{array}{l}178 \\
171\end{array}$ \\
\hline $\begin{array}{l}L 4 \times 01 \\
L 4 X 01 \\
L 4 \times 01 \\
L 4 X 01\end{array}$ & $\begin{array}{l}36 \\
36 \\
36 \\
36 \\
\end{array}$ & $\begin{array}{l}13 \text { Jul } 89 \\
19 \text { Jul } 89 \\
15 \text { Jun } 90 \\
20 \text { Jul } 90\end{array}$ & $\begin{array}{l}\text { UNC2 } \\
\text { UNC1 } \\
\text { UNC2 } \\
\text { UNC3 }\end{array}$ & $\begin{array}{l}257 \\
242 \\
239 \\
201 \\
\end{array}$ & $\begin{array}{c}35.2 \\
32.7 \\
34.3 \\
29 \\
\end{array}$ & $\begin{array}{l}2.54 \\
2.99 \\
3.54 \\
5.19\end{array}$ & $\begin{array}{l}5853 \\
5797 \\
5825 \\
5755 \\
\end{array}$ & $\begin{array}{l}674 \\
726 \\
716 \\
646 \\
\end{array}$ & $\begin{array}{l}1809 \\
1831 \\
1576 \\
1265 \\
\end{array}$ & $\begin{array}{l}2015 \\
2309 \\
2304 \\
2728 \\
\end{array}$ & $\begin{array}{l}361 \\
337 \\
291 \\
245\end{array}$ \\
\hline
\end{tabular}

* UNC1: analysis date 20 Jun 90 (UNC Geotech Analytical Laboratory, 1990)

UNC2: analysis date 21 Nov 90 (Chem-Nuclear Geotech Analytical Laboratory, 1990)

UNC3: analysis date 29 Jan 91 (Chem-Nuclear Geotech Analytical Laboratory, 1991) 
Table 2. Final borehole diameters and lengths, calculated void volumes within boreholes, and the required mass of water necessary to provide $75 \%$ relative humidity in the borehole ( $75 \%$ is approximately equilibrium with the initial brines).

\begin{tabular}{|c|c|c|c|c|} 
Borehole & Diameter, cm & Length, $\mathrm{cm}$ & Volurne, liters & $\begin{array}{c}\mathrm{g} \mathrm{H}_{2} \mathrm{O} \text { needed to } \\
\text { saturate air to } 75 \% \\
\text { relative humidity }\end{array}$ \\
\hline DBT10 & 10.2 & 530 & 43.3 & 0.82 \\
DBT11 & 10.2 & 460 & 37.6 & 0.71 \\
DBT12 & 10.2 & 370 & 30.2 & 0.57 \\
DBT13 & 10.2 & 280 & 22.9 & 0.43 \\
DBT14 & 10.2 & 560 & 45.8 & 0.86 \\
DBT15 & 10.2 & 580 & 47.4 & 0.90 \\
DBT31 & 91.4 & 560 & 3670 & 69.44 \\
DBT32 & 91.4 & 570 & 3740 & 70.68 \\
QPB01 & 5.1 & 300 & 6.13 & 0.12 \\
QPB02 & 5.1 & 310 & 6.33 & 0.12 \\
QPB03 & 5.1 & 310 & 6.33 & 0.12 \\
QPB04 & 5.1 & 310 & 6.33 & 0.12 \\
QPB05 & 5.1 & 310 & 6.33 & 0.12 \\
L4B01 & 10.2 & 580 & 47.4 & 0.90 \\
L4X01 & 91.4 & 570 & 3740 & 70.68 \\
\hline
\end{tabular}

$\S$ A value of 0.0189 grams $\mathrm{H}_{2} \mathrm{O}$ per liter moist air ( $75 \%$ relative humidity) was estimated from the psychrometric chart on p. 20-6 of Perry and Chilton (1973). 
Table 3. Calculation of the mass of $\mathrm{H} 2 \mathrm{O}$ required to saturate the air in the SSBIE boreholes to $75 \%$ relative humidity, and the ratio of this mass to the mass of water in the brine sample.

\begin{tabular}{|c|c|c|c|c|c|}
\hline Borehole & $\begin{array}{l}\text { Nominal } \\
\text { Diameter, } \\
\text { inches }\end{array}$ & Collection Date & $\begin{array}{l}\text { g Brine in } \\
\text { Sample }\end{array}$ & $\begin{array}{c}\mathrm{g} \mathrm{H}_{2} \mathrm{O} \text { needed to } \\
\text { saturate air to } \\
75 \% \text { relative } \\
\text { humidity }\end{array}$ & $\begin{array}{l}\text { Ratio of } \mathrm{g} \mathrm{H}_{2} \mathrm{O} \\
\text { to reach } 75 \% \\
\text { relative humidity } \\
\text { to mass of water } \\
\text { in brine sample }\end{array}$ \\
\hline $\begin{array}{l}\text { DBT } 10 \\
\text { DBT } 10 \\
\text { DBT } 10 \\
\text { DBT } 10\end{array}$ & $\begin{array}{l}4 \\
4 \\
4 \\
4\end{array}$ & $\begin{array}{l}16 \text { Dec } 87 \\
15 \text { Feb } 90 \\
20 \text { Mar } 90 \\
21 \text { Jun } 90\end{array}$ & $\begin{array}{l}92 \\
194 \\
246 \\
871\end{array}$ & $\begin{array}{l}0.82 \\
0.82 \\
0.82 \\
0.82\end{array}$ & $\begin{array}{l}1.2 \% \\
0.6 \% \\
0.4 \% \\
0.1 \%\end{array}$ \\
\hline $\begin{array}{l}\text { DBT } 11 \\
\text { DBT } 11 \\
\text { DBT } 11\end{array}$ & $\begin{array}{l}4 \\
4 \\
4\end{array}$ & $\begin{array}{l}11 \text { May } 88 \\
20 \text { Mar } 90 \\
21 \text { Jun } 90\end{array}$ & $\begin{array}{c}69 \\
294 \\
967 \\
\end{array}$ & $\begin{array}{l}0.71 \\
0.71 \\
0.71 \\
\end{array}$ & $\begin{array}{l}1.4 \% \\
0.3 \% \\
0.1 \%\end{array}$ \\
\hline $\begin{array}{l}\text { DBT } 12 \\
\text { DBT } 12 \\
\text { DBT } 12\end{array}$ & $\begin{array}{l}4 \\
4 \\
4\end{array}$ & $\begin{array}{l}18 \text { Jan } 90 \\
20 \text { Mar } 90 \\
21 \text { Jun } 90\end{array}$ & $\begin{array}{l}173 \\
149 \\
510 \\
\end{array}$ & $\begin{array}{l}0.57 \\
0.57 \\
0.57\end{array}$ & $\begin{array}{l}0.4 \% \\
0.5 \% \\
0.1 \%\end{array}$ \\
\hline DBT 13 & 4 & 21 Jun 90 & 306 & 0.43 & $0.2 \%$ \\
\hline $\begin{array}{l}\text { DBT } 14 \\
\text { DBT } 14 \\
\text { DBT } 14 \\
\text { DBT } 14 \\
\text { DBT } 14\end{array}$ & $\begin{array}{l}4 \\
4 \\
4 \\
4 \\
4\end{array}$ & $\begin{array}{c}28 \text { Sep } 88 \\
17 \text { May } 89 \\
1 \text { Nov } 89 \\
16 \text { May } 90 \\
16 \text { Aug } 90\end{array}$ & $\begin{array}{l}78 \\
144 \\
200 \\
153 \\
514\end{array}$ & $\begin{array}{l}0.86 \\
0.86 \\
0.86 \\
0.86 \\
0.86\end{array}$ & $\begin{array}{l}1.5 \% \\
0.8 \% \\
0.6 \% \\
0.8 \% \\
0.2 \%\end{array}$ \\
\hline $\begin{array}{l}\text { DBT } 15 \\
\text { DBT } 15 \\
\end{array}$ & $\begin{array}{l}4 \\
4 \\
\end{array}$ & $\begin{array}{l}27 \text { Jul } 88 \\
16 \text { Aug } 90 \\
\end{array}$ & $\begin{array}{l}146 \\
120 \\
\end{array}$ & $\begin{array}{l}0.90 \\
0.90 \\
\end{array}$ & $\begin{array}{l}0.8 \% \\
1.0 \% \\
\end{array}$ \\
\hline DBT 31 & 4 & $16 \operatorname{Mar} 88$ & 88 & 0.86 & $1.3 \%$ \\
\hline 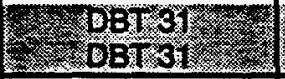 & 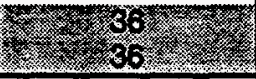 & $\begin{array}{l}\text { Tomay } 90 \\
16 \mathrm{Aug} 90\end{array}$ & 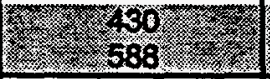 & (2) & (15\% \\
\hline DBT 32 & 4 & 9 Mar 88 & 79 & 0.87 & $1.5 \%$ \\
\hline 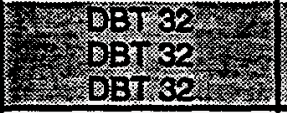 & 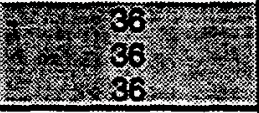 & 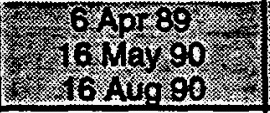 & 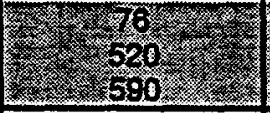 & 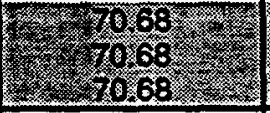 & 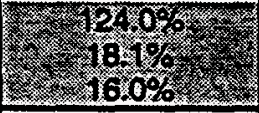 \\
\hline $\begin{array}{l}\text { QPB 01 } \\
\text { QPB } 01 \\
\end{array}$ & $\begin{array}{r}2 \\
2 \\
\end{array}$ & $\begin{array}{l}16 \text { Aug } 90 \\
20 \text { Sep } 90 \\
\end{array}$ & \begin{tabular}{|l|}
147 \\
207 \\
\end{tabular} & \begin{tabular}{l|}
0.12 \\
0.12 \\
\end{tabular} & $\begin{array}{l}0.1 \% \\
0.1 \% \\
\end{array}$ \\
\hline $\begin{array}{l}\text { QPB } 02 \\
\text { QPB } 02 \\
\text { QPB } 02 \\
\text { QPB } 02 \\
\text { QPB O2 }\end{array}$ & $\begin{array}{l}2 \\
2 \\
2 \\
2 \\
2 \\
\end{array}$ & $\begin{array}{c}4 \text { Oct } 89 \\
13 \text { Dec } 89 \\
20 \text { Jul } 90 \\
16 \text { Aug } 90 \\
20 \text { Sep } 90\end{array}$ & $\begin{array}{c}191 \\
56 \\
2486 \\
2164 \\
66 \\
\end{array}$ & $\begin{array}{l}0.12 \\
0.12 \\
0.12 \\
0.12 \\
0.12\end{array}$ & $\begin{array}{l}0.1 \% \\
0.3 \% \\
0.0 \% \\
0.0 \% \\
0.2 \%\end{array}$ \\
\hline $\begin{array}{l}\text { QPB 03 } \\
\text { QPB } 03 \\
\text { QPB } 03 \\
\text { QPB } 03\end{array}$ & $\begin{array}{l}2 \\
2 \\
2 \\
2 \\
\end{array}$ & $\begin{array}{l}16 \text { Jan } 90 \\
20 \text { Jul } 90 \\
16 \text { Aug } 90 \\
20 \text { Sep } 90\end{array}$ & $\begin{array}{r}235 \\
445 \\
666 \\
1027 \\
\end{array}$ & $\begin{array}{l}0.12 \\
0.12 \\
0.12 \\
0.12\end{array}$ & $\begin{array}{l}0.1 \% \\
0.0 \% \\
0.0 \% \\
0.0 \%\end{array}$ \\
\hline $\begin{array}{l}\text { QPB } 04 \\
\text { QPB } 04 \\
\text { QPB } 04 \\
\text { QPB } 04 \\
\end{array}$ & $\begin{array}{l}2 \\
2 \\
2 \\
2 \\
\end{array}$ & $\begin{array}{l}27 \text { Apr } 90 \\
20 \text { Jul } 90 \\
16 \text { Aug } 90 \\
20 \text { Sep } 90 \\
\end{array}$ & $\begin{array}{c}55 \\
103 \\
80 \\
107 \\
\end{array}$ & $\begin{array}{l}0.12 \\
0.12 \\
0.12 \\
0.12\end{array}$ & $\begin{array}{l}0.3 \% \\
0.1 \% \\
0.2 \% \\
0.1 \% \\
\end{array}$ \\
\hline $\begin{array}{l}\text { QPB } 05 \\
\text { QPB } 05 \\
\text { QPB } 05 \\
\text { QPB 05 } \\
\text { QPB } 05 \\
\end{array}$ & $\begin{array}{l}2 \\
2 \\
2 \\
2 \\
2 \\
\end{array}$ & $\begin{array}{l}27 \text { Apr } 90 \\
15 \text { Jun } 90 \\
20 \text { Jul } 90 \\
16 \text { Aug } 90 \\
20 \text { Sep } 90 \\
\end{array}$ & $\begin{array}{c}74 \\
1214 \\
2117 \\
1713 \\
2316 \\
\end{array}$ & $\begin{array}{l}0.12 \\
0.12 \\
0.12 \\
0.12 \\
0.12 \\
\end{array}$ & $\begin{array}{l}0.2 \% \\
0.0 \% \\
0.0 \% \\
0.0 \% \\
0.0 \% \\
\end{array}$ \\
\hline $\begin{array}{l}\mathrm{L} 4 \mathrm{BO} 1 \\
\mathrm{~L} 4 \mathrm{BO} 1\end{array}$ & $\begin{array}{l}4 \\
4 \\
\end{array}$ & $\begin{array}{l}21 \text { Jun } 89 \\
14 \text { Dec } 89\end{array}$ & $\begin{array}{l}30 \\
74 \\
\end{array}$ & $\begin{array}{l}0.90 \\
0.90 \\
\end{array}$ & $\begin{array}{l}4.0 \% \\
1.6 \% \\
\end{array}$ \\
\hline L4X01 & 36 & 13 Jul 89 & 115 & 70.68 & $81.9 \%$ \\
\hline 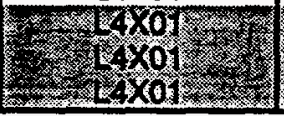 & 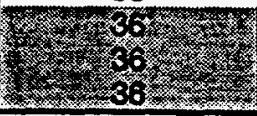 & (5) & 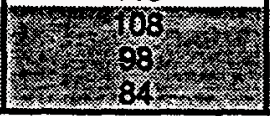 & 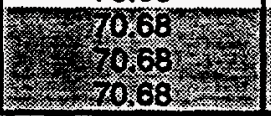 & (2) \\
\hline
\end{tabular}




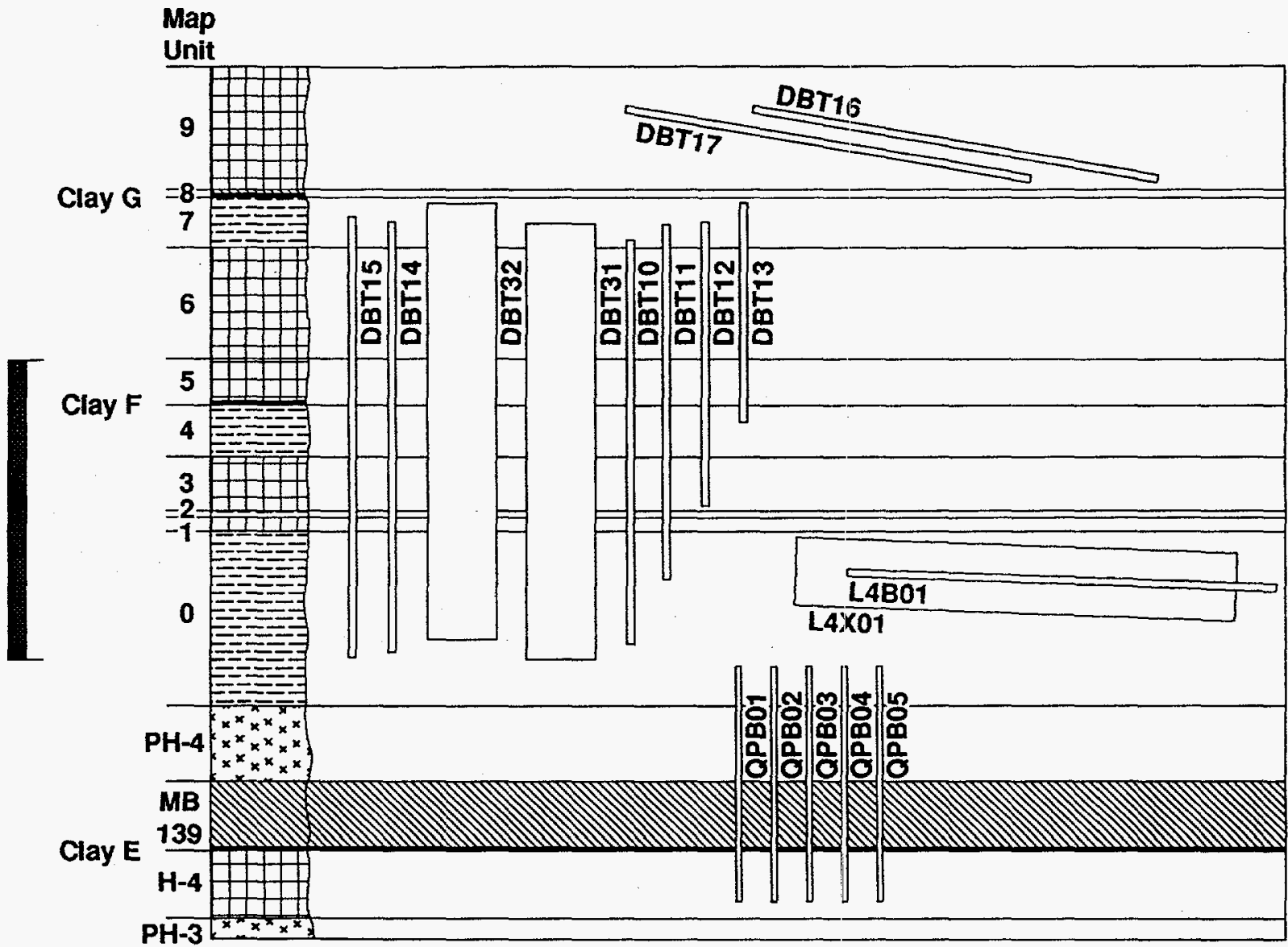

Legend

Halite

Anhydrite

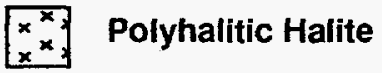

$E=-A$ Argillaceous Halite

Vertical Scale

(No Horizontal Scale)

Clay Seam

Waste
Facility
Horizon

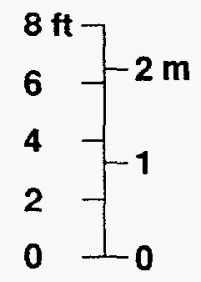

TRI-6344-553-0

Figure 1. A schematic representation of the small-scale brine inflow boreholes and the stratigraphic units tested in each borehole (p.3, Finley et al., 1992). 


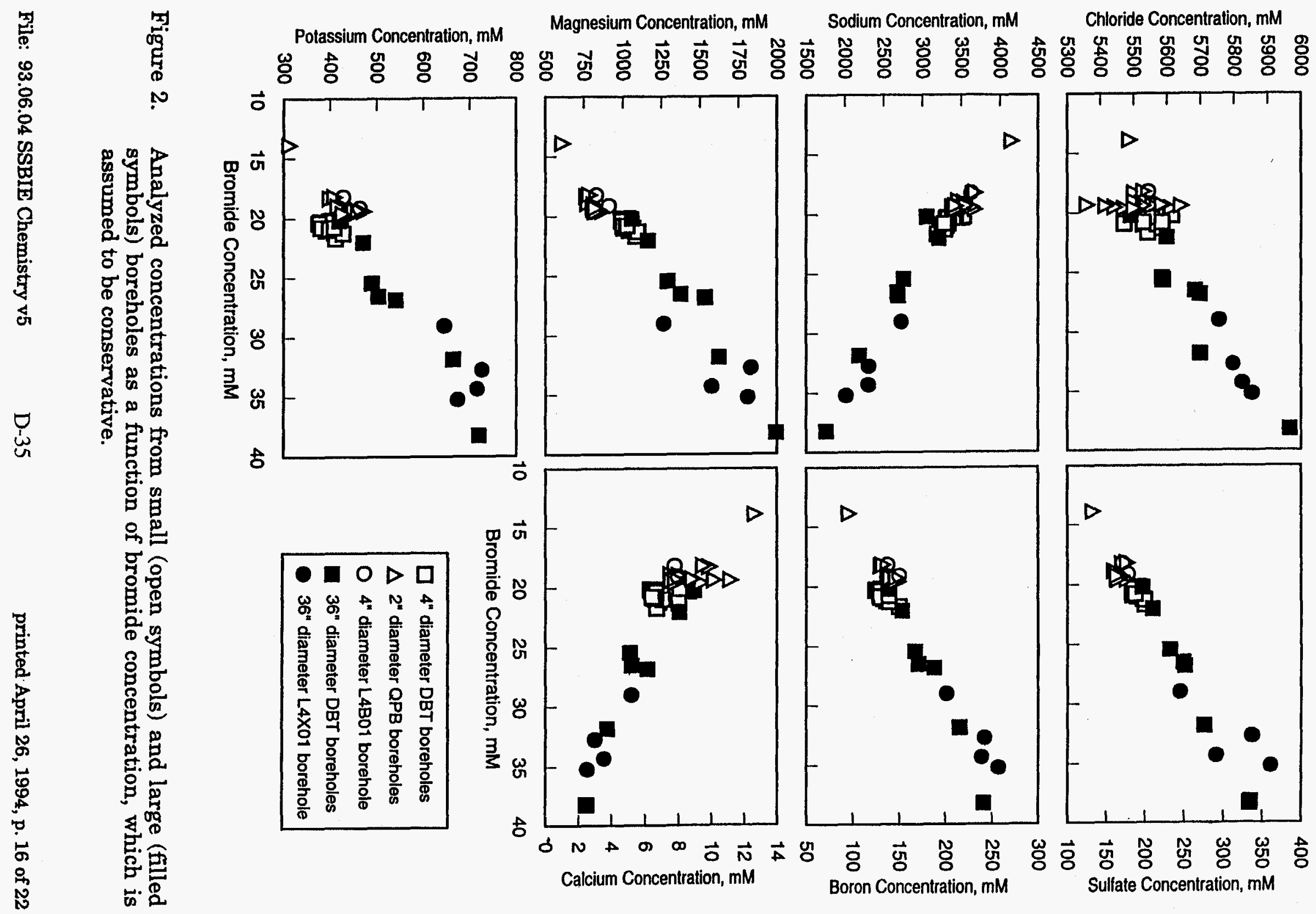



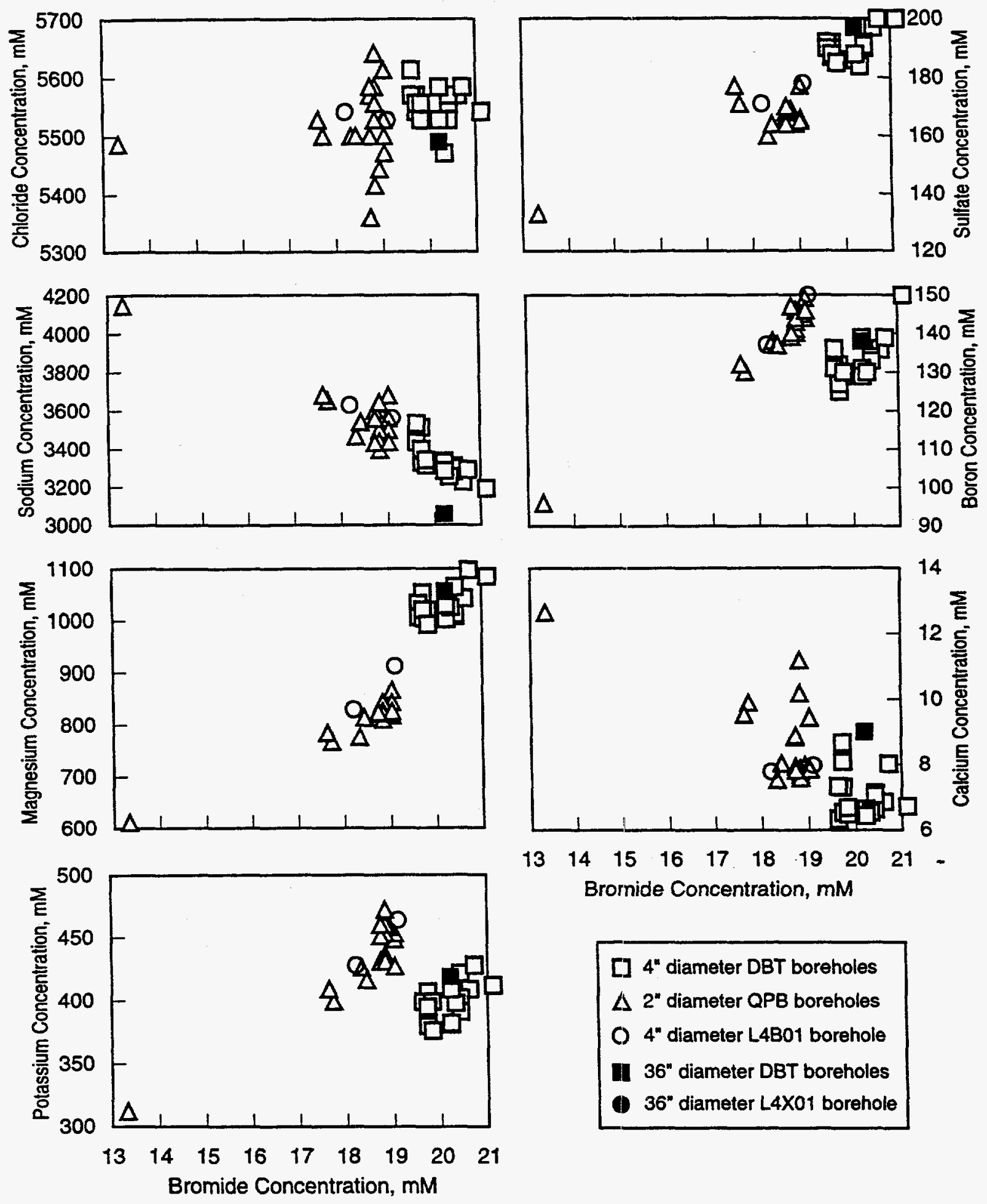

[] 4" diameter DBT boreholes
$\triangle 2^{*}$ diameter QPB boreholes
O $4^{\prime \prime}$ diameter L4BO1 borehole
II $36^{\prime \prime}$ diameter DBT boreholes
1) $36^{\prime \prime}$ diameter L $4 X 01$ borehole

Figure 3. Expanded view of analyzed concentrations from small (open symbols) and large (filled symbols) boreholes as a function of bromide concentration, c.f., Figure 2, showing two apparent populations of brine compositions. 


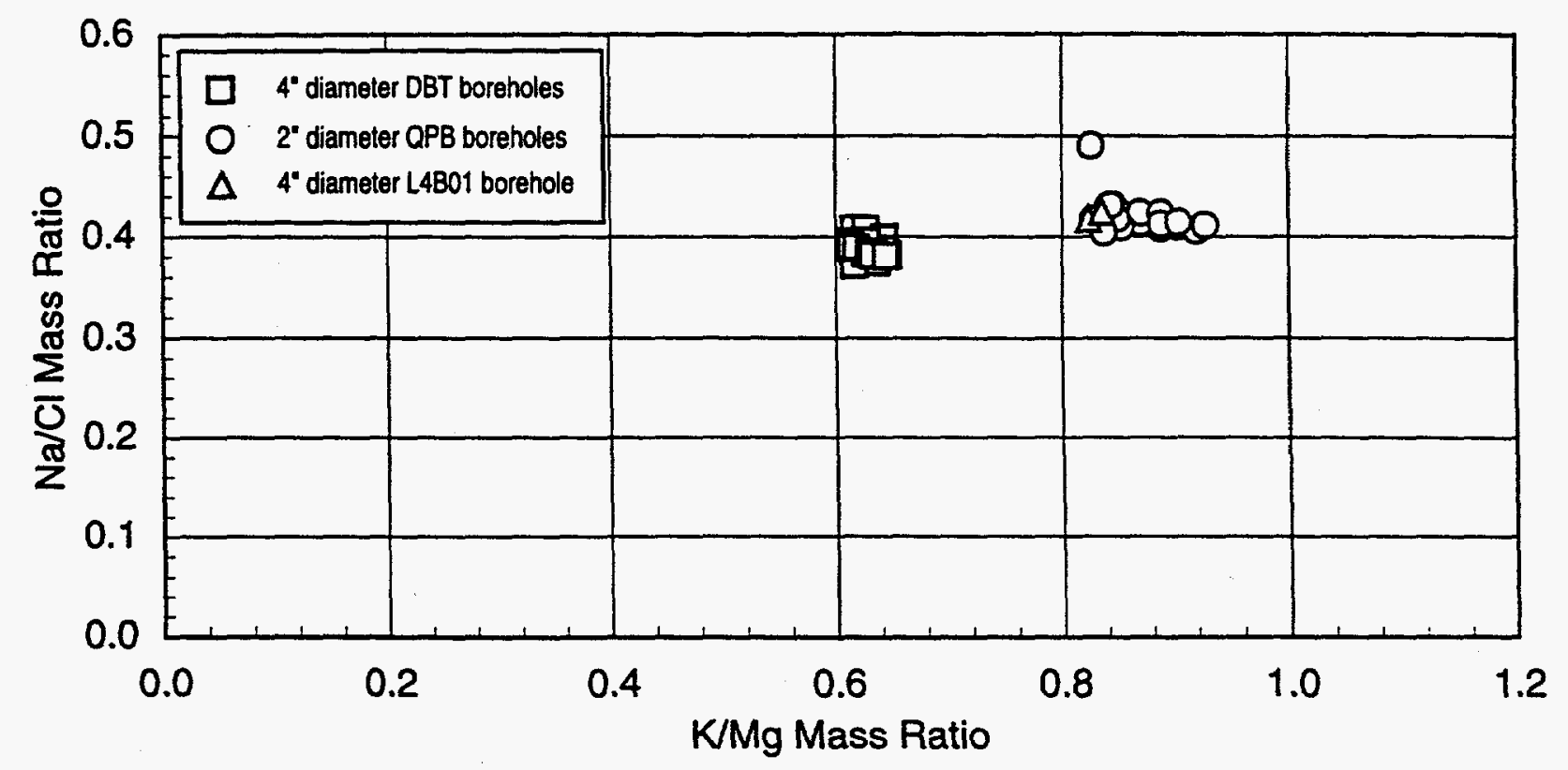

Figure 4. Plot of mass ratios of major solutes for the small diameter boreholes.

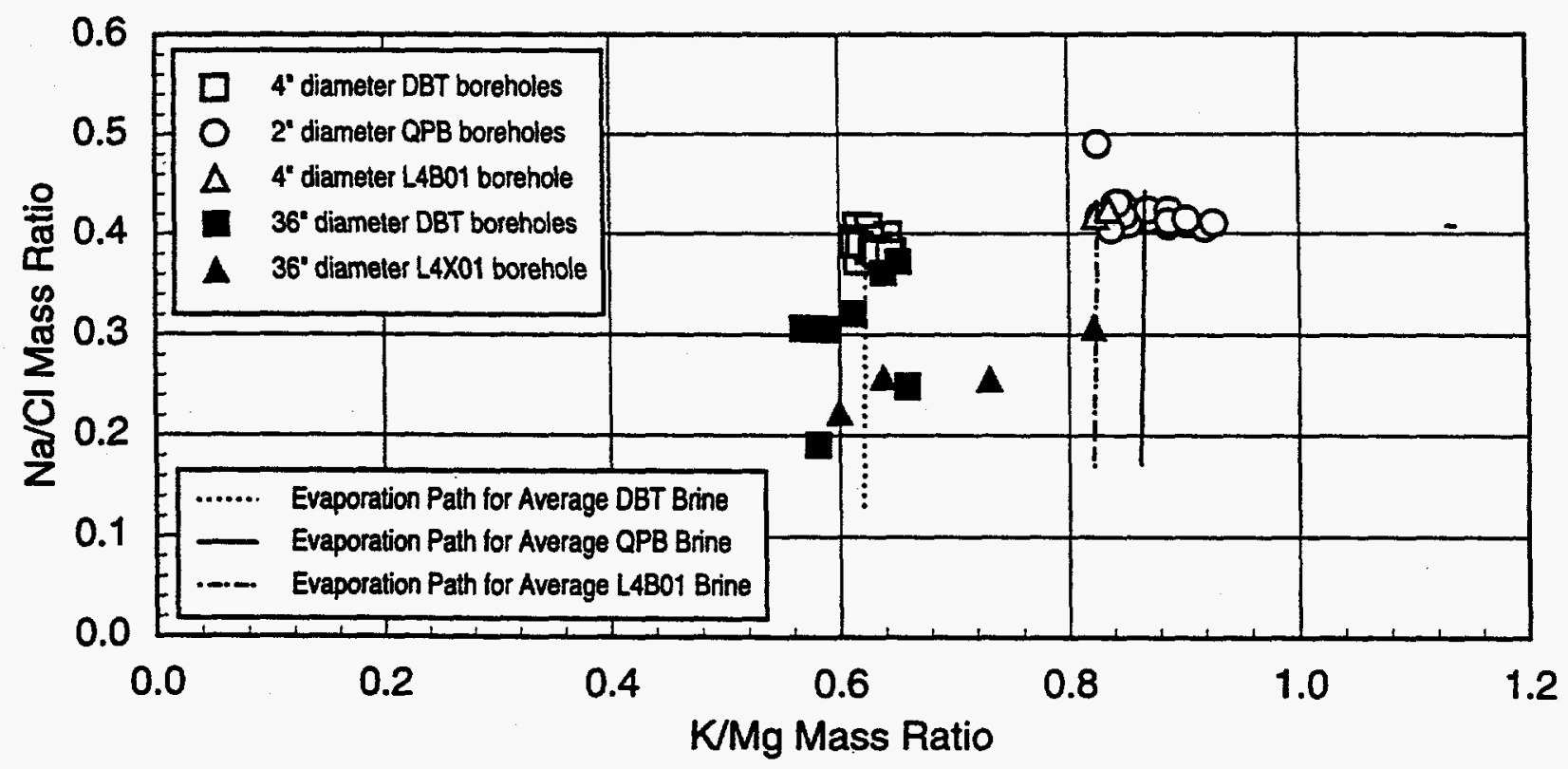

Figure 5. Plot of mass ratios of major solutes for small and large diameter boreholes, with calculated evaporation paths for average brines calculated from the small diameter borehole samples from Rooms D and $\mathrm{L} 4$, and the Q-access drift. 


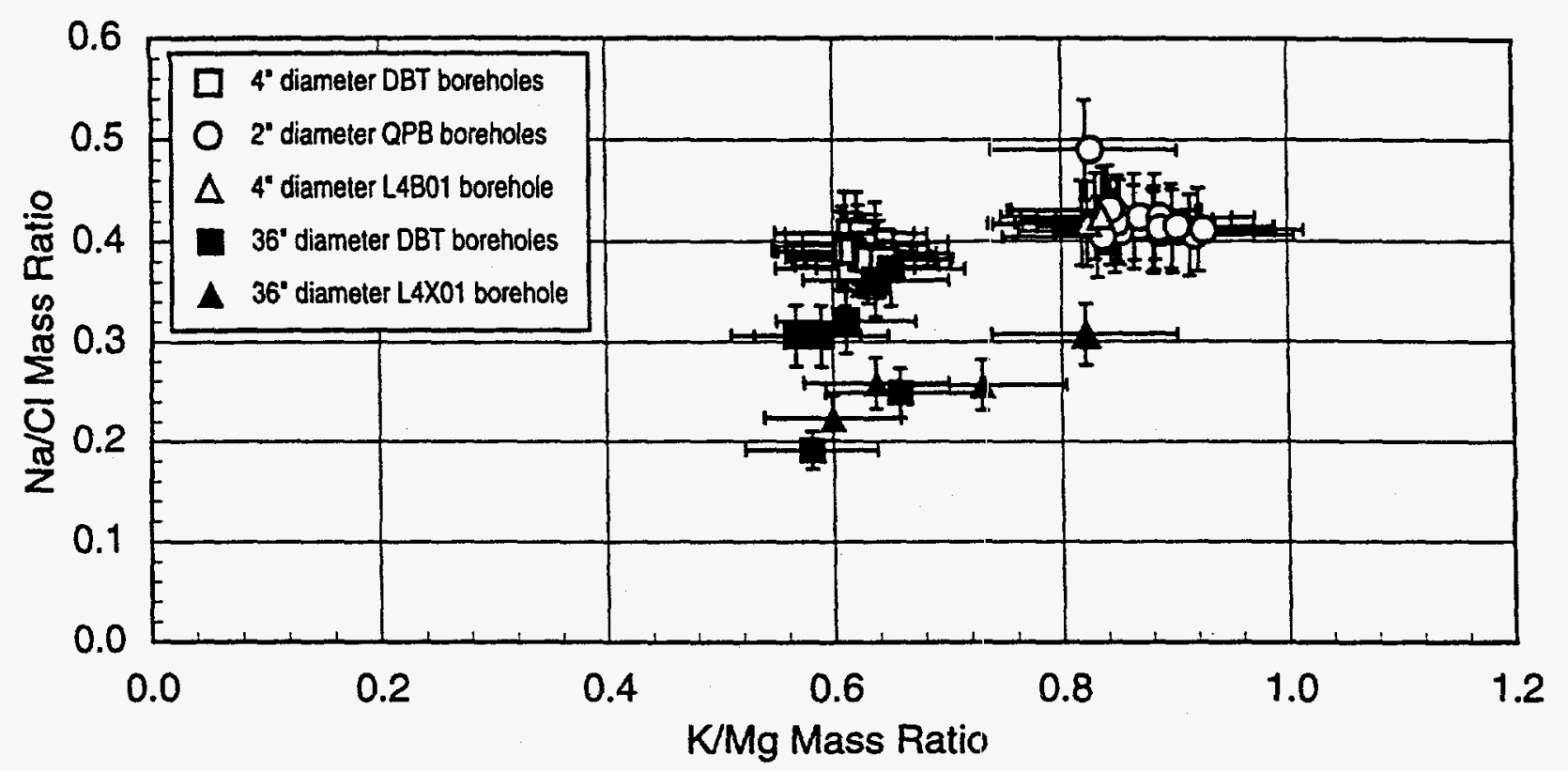

Figure 6. Plot of mass ratios of major solutes for both small and large diameter boreholes, showing the "error bars" that would result from a $\pm 5 \%$ analytical accuracy in concentrations. 


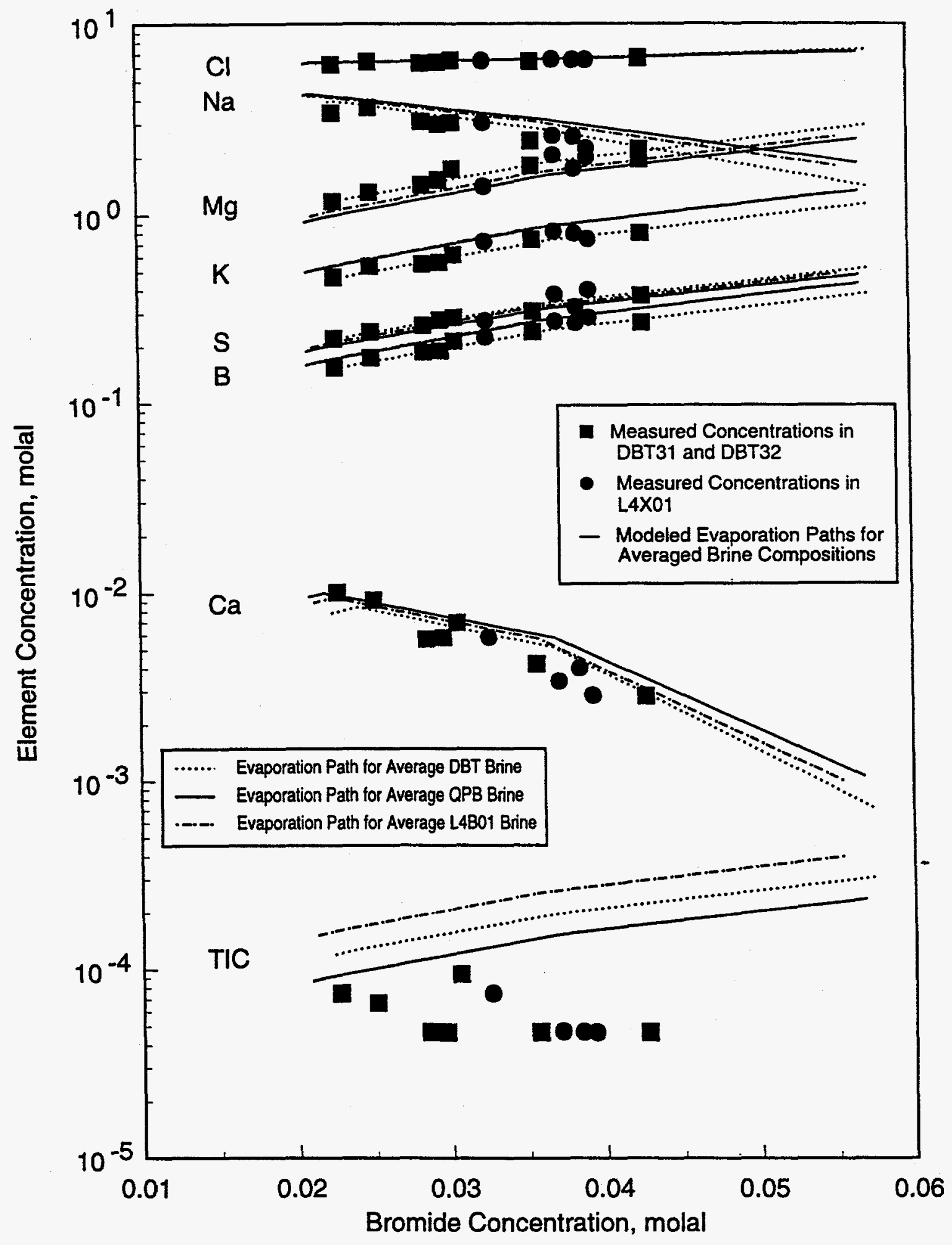

Figure 7. Comparison of modeled evaporation of average small borehole brines with concentration data from large boreholes. 

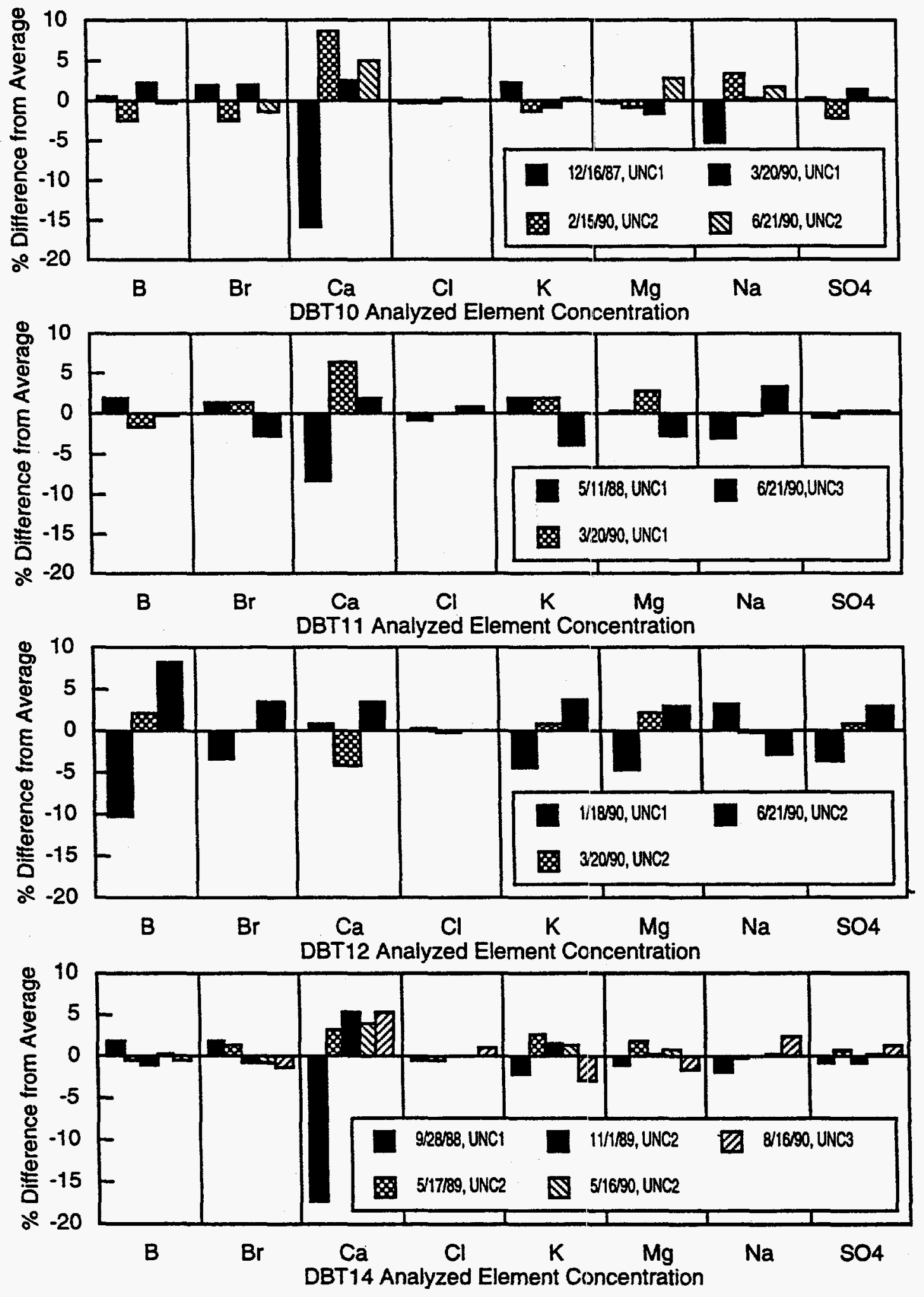

Figure 8. Percent difference in concentration of analyzed elements in DBT brines as a function of time. 


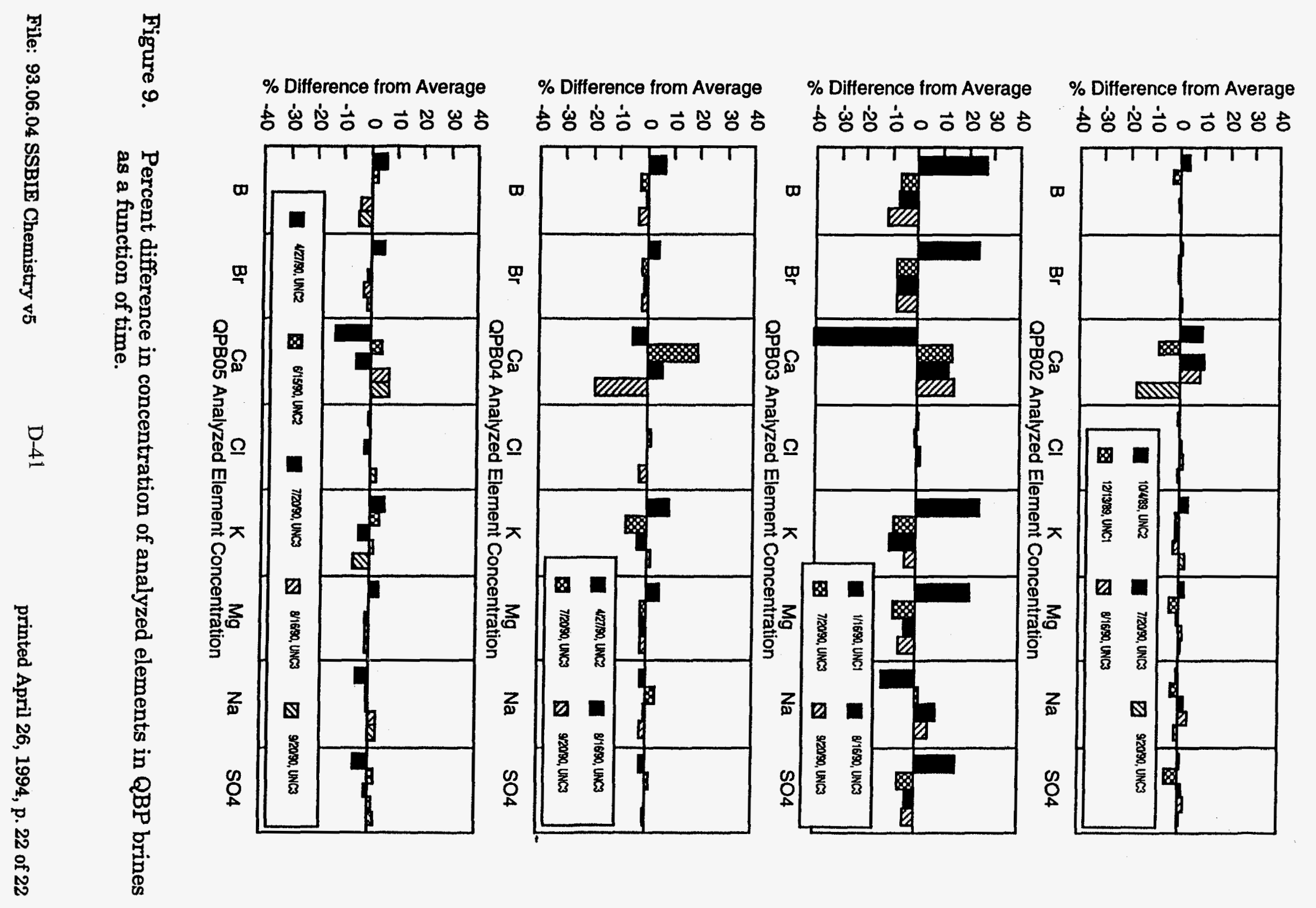


D-42 
Appendix E.

PA Parameter Package: Salado Data/Parameters: Anhydrite Two-Phase Parameters. Tracy Christian-Frear to SWCF-A, January 31, 1996.

The following information is provided as Appendix $\mathrm{E}$ of this document. 
E-2 


\section{Sandia National Laboratories}

Albuquerque, New Mexico 87185-1341

date: January 31,1996

to: SWCF-A Records Center, SWCF-A:WBS 1.2.07.1:PDD:QA:SALADO:PKG 10:Anh 2-Phase Parameters

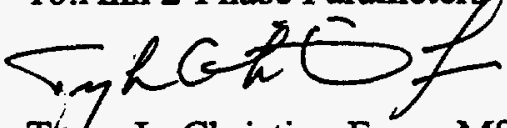

from: Tracy L. Christian-Frear, MS-1324 (6115)

subject: $\quad$ SALADO PARAMETER PRINCIPAL INVESTIGATOR

DOCUMENTATION PACKAGE FOR: ANHYDRITE TWO-PHASE PARAMETERS

The attached record package contains the anhydrite two-phase values for the capillary and relative permeability models, the residual gas and brine saturation, the initial brine saturation, pore size distribution parameter and the threshold pressure.

The parameter information provided in this record package was collected by Principal Investigators for input to the WIPP Data Entry Form and for use by Performance Assessment personnel making parameter estimates. The record package was prepared in accordance with WIPP Quality Assurance Procedure (QAP) 17-1, Rev. 1, WIPP QA Records Source Requirements.

Please call me at 848-0704 if you have any questions. 


\section{RECORD PACKAGE:}

\section{SALADO PARAMETER PRINCIPAL INVESTIGATOR DOCUMENTATION PACKAGE FOR:}

\section{ANHYDRITE TWO-PHASE PARAMETERS}

Purpose:

The parameter information in this package was collected by Principal Investigators for input to the WIPP Data Entry Form and for use by Performance Assessment personnel making parameter estimates.

Date of Record: $\quad$ January 31,1996

Author/Organization: Tracy L. Christian-Frear

SNL Department 6115 (MS 1324)

(505) 848-0704

Recipient:

SWCF-A Records Center

File Code:

SWCF-A:WBS 1.2.07.1:PDD:QA:SALADO:PKG 10:Anh 2-Phase Parameters 


\title{
RECORD PACKAGE:
}

\section{SALADO PARAMETER PRINCIPAL INVESTIGATOR DOCUMENTATION PACKAGE FOR:}

\author{
ANHYDRITE TWO-PHASE PARAMETERS
}

SWCF-A:WBS 1.2.07.1:PDD:QA:SALADO:PKG 10:Anh 2-Phase Parameters

\section{Table of Contents}

\begin{tabular}{|c|l|c|}
\hline $\begin{array}{c}\text { Record } \\
\text { Number }\end{array}$ & \multicolumn{1}{|c|}{ Contents } & $\begin{array}{c}\text { Number } \\
\text { of Pages }\end{array}$ \\
\hline $\mathbf{1}$ & $\begin{array}{l}\text { SALADO DATA/PARAMETERS : ANHYDRITE TWO-PHASE } \\
\text { PARAMETERS (Date: December 22, 1996) }\end{array}$ & $\mathbf{7}$ \\
\hline $\mathbf{2}$ & Attachment 1. Capillary Pressure Model Parameters Calc. Sheet & $\mathbf{3 7}$ \\
\hline $\mathbf{3}$ & General Correspondence & $\mathbf{0}$ \\
\hline $\mathbf{4}$ & Technical Review of Record Package & $\mathbf{1}$ \\
\hline $\mathbf{5}$ & Roadmap to Supporting Documentation & $\mathbf{0}$ \\
\hline $\mathbf{6}$ & $\begin{array}{l}\text { Memo to PA Parameter Task Leader (attachment not included--same as } \\
\text { record 1) }\end{array}$ & $\mathbf{1}$ \\
\hline
\end{tabular}

Total Number of Pages in Record Package 


\section{RECORD 1}

SALADO DATA/PARAMETERS : ANHYDRITE TWO-PHASE PARAMETERS

E-6 
Anhydrite Laboratory Data for two-phase parameters

I. Parameter No. (id):

CAP_MOD: $559,579,520$

PC_ $\overline{\mathrm{M} A X: ~ 561, ~ 582, ~} 522$

RELP_MOD: 575, 596, 536

SAT_IBRN: 576, 597, 537

SAT_RBRN: $\mathbf{5 7 7 , 5 9 8 , 5 3 8}$

SAT_RGAS:578, 599, 539

PTHRESH: 573, 594, 534

PCT_A: DPARAM Unknown at this time

PCT_EXP: IDPARAM Unknown at this time

II. Data/Parameter:

CAP_MOD: Capillary Pressure Model PC_MAX: Maximum Capillary Pressure

PORE_DIS: Brooks-Corey Pore Distribution Parameter (lambda)

RELP_MOD: Relative Permeability Model

SAT_IBRN: Initial Brine Saturation

SAT_RBRN: Residual Brine Saturation

SAT_RGAS: Residual Gas Saturation

PTHRESH: Threshold Pressure

PCT_A: Threshold Pressure Linear Parameter

PCT_EXP: Threshold Pressure Exponential parameter

III. Parameter id (idpram):
CAP_MOD
PC_M̄AX
PORE_DIS
RELP_MOD
SAT_BBRN
SAT_RBRN
SAT_RGAS
PTHRESH
PCT_A
PCT_EXP

IV. Material:

Anhydrite

V. Material Id (idmtrl):

S_MB138, S_MB139, S_ANH_AB 
VI. Units:

$$
\begin{aligned}
& \text { CAP_MOD: None } \\
& \text { PC_MAX: Pa } \\
& \text { PORE_DIS: None } \\
& \text { RELP_MOD: None } \\
& \text { SAT_IBRN: None } \\
& \text { SAT_RBRN: None } \\
& \text { SAT_RGAS: None } \\
& \text { PTHRESH: Pa } \\
& \text { PCT_A: Pa/(m2PCT_EXP) } \\
& \text { PCT_EXP: None }
\end{aligned}
$$

VII. Distribution Information:

The following are Model Parameters with recommendations:

CAP_MOD: Limit Capillary Pressure to PC_MAX

RELP_MOD: $50 \%$ mixed B/C and 50\% vG/P characteristic curves

PC_MAX: 1.0 E8 Pa

SAT__IBRN: 1.00

A. Category

PORE_DIS: Normal

SAT_RBRN: Normal

SAT_RGAS: Log Normal

PTHRESH: Normal

PCT_A: Constant

PCT_EXP: Constant

B. Mean

PORE_DIS: 0.6436

SAT RBRN: 0.084

SAT_RGAS: 0.077

PTHRESH: $5.418 \times 10^{5} \mathrm{~Pa}$

PCT_A: $0.26 \mathrm{~Pa} / \mathrm{m}^{-0.348^{*} 2}$

PCT_EXP: $\mathbf{- 0 . 3 4 8}$

C. Median

PORE_DIS: 0.6536

SAT_RBRN: 0.071

SAT_RGAS: 0.055

PTHRESH: $4.958 \times 10^{5} \mathrm{~Pa}$

PCT_A: NA

PCT_EXP: NA 
D. Std Deviation

PORE_DIS: 0.1189

SAT RBRN: 0.055

SAT_RGAS: 0.070

PTHRESH: $1.875 \times 10^{5} \mathrm{~Pa}$

PCT_A: NA

PCT_EXP: NA

E. Maximum

PORE DIS: 0.842

SAT RBRN: 0.174

SAT_RGAS: 0.197

PTHRESH: $7.8 \times 10^{5} \mathrm{~Pa}$

PCT_A: NA

PCT_EXP: NA

F. Minimum

PORE DIS: 0.491

SAT_RBRN: 0.008

SAT_RGAS: 0.014

PTHRESH: $3.29 \times 10^{5} \mathrm{~Pa}$

PCT_A: NA

PCT_EXP: NA

G. Number of data points

6

VIII. Data Collection and Interpretation Information:

A. Data Source Information:

1. Data Source:

WIPP Observational Data

2. Supporting Explanation/Justification for selection in \#1 if other than WIPP Observational Data:

NA

3. References for selection in \#1 above if other than WIPP Observational Data:

NA 
B. Data Collection (for WIPP Observational Data):

1. Data Collection and Test Method:

Unstressed capillary pressure tests were performed on 6 pairs of specimens. One specimen underwent centrifuge capillary pressure tests and the other specimen underwent mercury injection capillary pressure tests. Prior to the capillary pressure tests, the specimens underwent permeability and porosity testing. The specimens ranged in size from 12.05 to $12.65 \mathrm{cc}$ 's. The specimens were cut from whole core taken from 6 underground boreholes at the WIPP. Two of the cores were taken outside Room L3 and the other 4 from E140 Drift at the intersection of N1100 Drift.

2. Assumptions Made During Testing:

1) Cores were assumed to be $100 \%$ saturated at initiation of capillary pressure tests.

2) Used $140^{\circ}$ contact angle for correcting mercury-air data to brineair repository conditions

3) The data provided here was done using tests conducted at ambient conditions (no stress) and that this data is adequate to describe twophase conditions at stress.

3. Standard Error of Measurement of Tests Performed:

Errors are derived from formal propagation of random and systematic errors. Source of errors include pressure transducer accuracy, accuracy of injection rates (time and volume), caliper accuracy, uncertainty in fluid viscosity and uncertainty to the interpretive method. An approximate $10 \%$ error in capillary pressure measurements is assumed for these tests.

4. Form of Raw Data:

Pressures, time, lengths and volumes

5. References Related to Data Collection:

Contract numbers:

Rock Physics Assoc. (who contracted Core Labs) AF-3945

PI name(s):

Susan Howarth

Title of approved Test Plan under which data was collected:

Test Plan: Two-Phase Flow Laboratory Program for the Waste Isolation Pilot Plant 
SAND report number:

SAND94-0472

Sandia WIPP Central Files (SWCF) file code for non-SAND references:

SWCF-A:1.1.4.1:HYD/TPF

SWCF code for data package(s):

SWCF-A:1.1.4.1:HYD/TPF

6. QA Status of Data:

a. Are all of the data qualified (Yes or No?)

If Yes, answer questions below to identify method of qualification.

If No, list those data which are not yet qualified.

Yes

b. Was data qualified by QAP 20-3 (Yes or No?)

If Yes for "a" above, give SWCF of qualified data package.

No

c. Was the data the subject of audit/surveillance by SNL or DOE?

(Yes or No; and SNL or DOE?)

If Yes for "c" above, give audit reference number.

Yes - Core Labs audit 94-04

d. Was the data collected under an SNL approved QA Program?

(Yes or No?) If Yes, give title and approval dates of the QA

Program(s)

Yes

Test Procedures and Quality Assurance Plan: Porosity, Permeability, and Capillary Pressure Measurements in Anhydrite Samples from the WIPP Approved by Susan Pickering on 5/28/93

C. Interpretation of Data:

1. Was the interpretation made by reference to previous work (Yes or No?)

If yes, give reference and answer \#3 below.

Yes. Air-mercury to air-brine raw data corrected at $140^{\circ}$ contact angle made by Joel Walls as outlined in SAND94-0472.

2. Was the interpretation made by using newly performed calculations

(Yes or No?) If, yes, answer questions 3-9 below.

Yes 
3. Form of Interpreted Data: (Example: histogram, table of interpreted values)

Tables of data, Curve fits, Histograms, Probability graphs

4. Assumptions Made During Interpretation:

1) Cores were assumed to be $100 \%$ saturated at initiation of capillary pressure tests.

2) Used $140^{\circ}$ contact angle for correcting mercury-air data to brineair repository conditions

3) The data provided here was done using tests conducted at ambient conditions (no stress) and that this data is adequate to describe twophase conditions at stress.

4) The centrifuge data is inadequate to describe $\mathrm{Pt}$ and Slr, thus these test results are not used here to determine two-phase parameters.

5) The threshold pressures reported in SAND94-0472 are actually entry pressures (first gas bubble in to a $\mathbf{1 0 0} \%$ liquid saturated rock). The threshold pressures derived by curve fits to the data (as presented here) are the threshold pressure at the critical (residual) gas saturation. These threshold pressures are consistent with the Brooks and Corey and the vanGenuchten/Parker definition of threshold pressure.

5. Name of Code(s)/Software used to Interpret Data:

Microsoft Excel v. 4.0 and KaleidaGraph v. 3.0.3b2 on a Power Macintosh 8100 using system 7.5.

6. QA Status of Code(s) used to Interpret Data: For Sandia Codes:

a. Was the code qualified under QAP 19-1(Yes or No?) NA

b. QAP 9-1 (Yes or No?) NA

7. References Related to Data Interpretation:

Contract numbers:

Rock Physics Assoc. (who contracted Core Labs) AF-3945

SAND report number:

SAND94-0472

Sandia WIPP Central Files (SWCF) file code for non-SAND references: SWCF-A:1.1.4.1;HYD/TPF

SWCF code for data package(s):

SWCF-A:1.1.4.1;HYD/TPF

E-12 
8. For interpretations made by using newly performed calculations provide documentation that you followed the requirements of QAP 9-1 Appendix B.

NA

9. For routine calculations (not using code) did you follow requirements of QAP 9-5 (Yes or No?) Yes

IX Correlation with other Parameters (List only those not statistically independent of the parameter documented here):

\section{See Attachment 1:}

Sgr and $P t$

Pt and permeability

X. Limitations or qualifications for usage of data by Performance Assessment (PA):

Data was only measured on specimens from MB139 taken from intact rock.

XI. Attachments:

X11. Data

Attachment 1. Capillary Pressure Model Parameters Calc. Sheet Guidance dacuments are located in the following SWCF: SWCF-A:P.:1.07:PDD:QA:SALADO:CORR:Guidance Documents ffC $10 / 22 / 15$

\begin{tabular}{|c|c|c|c|c|c|}
\hline \multirow{7}{*}{$\begin{array}{l}\text { Mercury } \\
\text { Injection }\end{array}$} & Sample & $\begin{array}{c}\mathrm{Pt} \\
(\mathrm{MPa})\end{array}$ & $\begin{array}{l}\text { SIr } \\
(\%)\end{array}$ & $\begin{array}{l}\text { Sgr } \\
(\%) / \Delta\end{array}$ & Lambda \\
\hline & 5 & 0.54132 & 7.262 & $+2,1^{65}$ & 0.655 \\
\hline & 7 & 0.78 & 6.986 & 7.7729 & 0.66452 \\
\hline & 11 & 0.45026 & 17.401 & 1.3981 & 0.55775 \\
\hline & 13 & 0.75274 & 10.861 & 19.719 & 0.652 \\
\hline & 21 & 0.32914 & 0.77846 & 2.5201 & 0.49053 \\
\hline & 23 & 0.39724 & 6.8842 & 3.2177 & 0.84178 \\
\hline Statistics & & $\mathrm{Pt}(\mathrm{MPa})$ & SIr (\%) & Sgr(\%) & Lambda \\
\hline Minimum & & 0.329 & 0.778 & 1.398 & 0.491 \\
\hline Maximum & & 0.780 & 17.401 & 19.719 & 0.842 \\
\hline Sum & & 3.251 & 50.173 & 46.265 & 3.862 \\
\hline Points & & 6 & 6 & 6 & 6 \\
\hline Mean & & 0.5418 & 8.3621 & 7.7108 & 0.6436 \\
\hline Median & & 0.4958 & 7.1240 & 5.4953 & 0.6536 \\
\hline Std Deviatio & & 0.1875 & 5.4908 & 7.0228 & 0.1189 \\
\hline Variance & & 0.0351 & 30.1492 & 49.3195 & 0.0141 \\
\hline Std Error & & 0.0765 & 2.2416 & 2.8670 & 0.0486 \\
\hline Skewness & & 0.4327 & 0.5632 & 1.1346 & 0.6365 \\
\hline Kurtosis & & -1.8573 & 1.4753 & 0.5853 & 1.2579 \\
\hline
\end{tabular}

E-13 


\section{RECORD 2}

Attachment 1. Capillary Pressure Model Parameters Calc. Sheet

E-14 


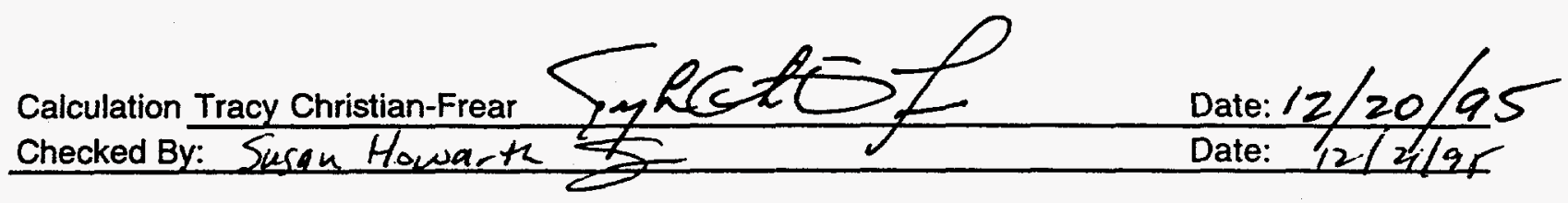

\section{Purpose:}

To determine the two-phase capillary pressure characteristic curve that best fits the data, and the parameters for BRAGFLO calculations.

Specific Parameters are: Threshold pressure (Pt), residual brine and gas saturation (Sbr and Sgr), and the pore size distribution parameter (lambda)

The following is a list of the paramters:

$$
\begin{aligned}
& \text { CAP_MOD } \\
& \text { PC_MAX } \\
& \text { PORE_DIS } \\
& \text { RELP_MOD } \\
& \text { SAT_IBRN } \\
& \text { SAT_RBRN } \\
& \text { SAT_RGAS } \\
& \text { PCT_A } \\
& \text { PCT_EXP }
\end{aligned}
$$

\section{Calculation Description:}

Using the 140 degree contact angle corrected mercury injection core data found in SAND94-0472

determine the following by using the standard mixed $B / C$ and $V G / P$ formulas added to the general curve fit equations of KaliedaGraph software (off the shelf software

a) The two-phase characteristic curve (either Brooks/Corey (B/C) or vanGenuchten (VG/P)) that best fits the data;

b) The Brooks and Corey $(\mathrm{B} / \mathrm{C})$ parameters of $\mathrm{Pt}$, Sgr, Sir, and lambda that best fit the data.

(BRAGFLO generates VGIP parameters from the BIC parameters)

c) Determine the initial brine saturation

d) Determine the maximum capillary pressure, Pc.

e) Based on the value of Pt determine PCT_A and PCT_EXP

I did not start with the values of $P t$ found in Table 12 of SAND94-0472 because the Pt values are actually entry pressure values ( $P e$ at Saturation=0.999), not threshold pressure as used in the Brooks and Corey or vanGenuchten/Parker characteristic curves ( $\mathrm{Pt}$ at liquid saturation $=1-\mathrm{Sgr}$ ).

I did not start with the values of Slr found in Table 12 of SAND94-0472 because those values may be the result of equipment limitations.

\section{Equations:}

Mixed Brooks and Corey (Appendix E of SAND94-0472):

$$
\begin{array}{ll}
\mathrm{Pc}=\mathrm{Pt} / \mathrm{Se} \mathrm{A}^{\wedge}(1 / \mathrm{lambda}) & \text { eqn } 1 \\
\mathrm{Se}=(\mathrm{S}-\mathrm{SIr}) /(1-\mathrm{Slr}-\mathrm{Sgr}) & \text { eqn } 2
\end{array}
$$

VG/P:

$$
\begin{array}{ll}
\mathrm{Pc}=1 / \text { alpha }\left(\left(\mathrm{Se}^{\prime} \wedge(-1 / \mathrm{m})\right)-1\right)^{\wedge}(1-\mathrm{m}) & \text { eqn } 3 \\
\mathrm{~m}=\text { lambda/(lambda+1) } & \text { eqn } 4 \\
\mathrm{Se}^{\prime}=(\mathrm{S}-\mathrm{SIr}) /(1-\mathrm{SIr}) & \text { eqn } 5 \\
\text { alpha }=\left(1 /\left(\mathrm{Pt} / 0.5^{\wedge}(1 / \text { lambda })\right)\right)^{\star}\left(\left(0.5^{\wedge}(-1 / \mathrm{m})\right)-1\right)^{\wedge}(1-\mathrm{m}) & \text { eqn } 6
\end{array}
$$

Threshold Pressure:

$P t=P C T \_A^{*} k \wedge P C T \_E X P$

if PCT_EXP=0, then PCT_A=Pt eqn 7 


Variables:
\begin{tabular}{|l|l|l|l|}
\hline $\mathrm{Pc}$ & Capillary Pressure & data & \\
\hline $\mathrm{Pt}$ & Threshold Pressure & data fit & $\mathrm{m} 1$ \\
\hline $\mathrm{S}$ & Saturation of brine & data & $\mathrm{m} 2$ \\
\hline Slr & Residual liquid saturation & data fit & $\mathrm{m3}$ \\
\hline $\mathrm{Sgr}$ & Residual gas saturation & data fit & $\mathrm{m} 4$ \\
\hline lambda & pore-size distribution param & data fit & $\mathrm{m} 6$ \\
\hline $\mathrm{m}$ & vanGenuchten parameter 1 & Fit and calculated from lambda & $\mathrm{m5}$ \\
\hline alpha & vanGenuchten parameter 2 & calc and fit & \\
\hline Se and Se & Effective Saturation & calculated & \\
\hline $\mathrm{k}$ & Permeability & data & \\
\hline
\end{tabular}

\section{Assumptions:}

1) The centrifuge data is assumed to be inadequate to define the threshold pressure and other parameters because the initial speed was too high to determine $\mathrm{Pt}$, and equipment limitations were such that Sir could not be adequately defined.

2) Cores were assumed to be $100 \%$ saturated with liquid at initiation of capillary pressure test.

3) 140 degree contact angle was best for correcting Mercury-air data to brine-air repository conditions

4) The data provided here was done using tests conducted at ambient conditions (no stress) and that this data is adequate to describe two-phase conditions at stress.

5) The threshold pressures reported in SAND94-0472 are actually entry pressures (first gas bubble in to a $100 \%$ liquid saturated rock). The threshold pressures derived by curve fits to the data (as presented here) are the threshold pressure at the critical (residual) gas saturation. These threshold pressures are consistent with the Brooks and Corey and the vanGenuchten/Parker definition of threshold pressure.

\section{Process:}

Verified that Kaliedagraph curve fits were working as required.

Curve fit steps:

1. Using KaliedaGraph general curve fit routine, define the VG/P equation for mixed relative permeability (eqn 3 except Se' is defined as in eqn 2) and solve for all 4 variables (Alpha, Sir, Sgr, m) based upon Pc vs Saturation data.

2. Check the curve fit $R 2$ and chisq. $R 2>0.99$ and chisq $<10$. (Also see if the curve fit looks adequate) If they ar OK-goto 3

If they are not OK-Eliminate data points (usually choose data with less than $40 \%$ saturation and one low sat. point) and goto 1.

3. Check for values with an error less than $12 \%$. Use that (those) values in subsequent curve fits until you get a Sir and Sgr that has less than $10 \%$ error.

4. Put the Sir and Sgr determined in the previous steps into the mixed B/C curve fit (eqn 1) and fit the data.

5. Obtain v: 3 variables ( $\mathrm{Pt}, \mathrm{SIr}$, lambda). If neither is negative, find the variable that has the least error and fix that variable

6. Determine the VG/P parameter $m$ from lambda (eqn 4), and alpha from $P t$, lambda, $m$ (eqn 6 ).

7. Using eqn 1 define $P c$ data that fit the parameters determined for $B / C$ and add the data to the graph.

8. Using eqn 3 define $P C$ data that fit the parameters determined for VG/P and add the data to the graph.

9. Curve fit the $B / C$ generated data to check the software.

10. Curve fit the VG/P generated data to check the software.

11. Equate PCT_A to the value determined for Pt, and equate PCT_EXP to 0 (see eqn 7). 
Solution: The values found are provided in Table 1.

Each curve fit data and errors sheet are provided as indicated in Table 1.

No definitive data exists to recommend one set of characteristic curves over the other. However the data does show that either $B / C$ or $V G / P$ can be used to describe the data.

Figure $1 \mathrm{a}, \mathrm{b}, \mathrm{c}, \mathrm{d}, \mathrm{e}$, f show that there is possibly a relationship between Pt and Sgr (Fig. 1b)

Figure 2 shows box diagrams indicating that there are data that may be considered statistical outliers.

Figures 3, 4, 5 and 6 show the histograms and probability distribution for Pt, SIr, Sgr and lambda, respectively.

Figure 7 shows the curve for the mean values

Figures 8 though 13 are the curve fits for each specimen (including data)

Figure 14 shows the ralationship between the Pt determined, the Davies equation and the entry pressures determined for the specimens.

\section{Recommendations:}

1. use $50 \%$ mixed $B / C$ and $50 \%$ mixed vG/P characteristic curves ( $\mathrm{PC}$ and rel perm).

2. The mean value of $\mathrm{Sgr}$ is: $\mathrm{Sgr}=7.71 \%$

3. The mean value of $\mathrm{Pt}$ is: $\mathrm{Pt}=0.54 \mathrm{MPa}$

4. The mean value of Sir is: $\operatorname{SI}=8.36 \%$

5. The mean value of lambda is: lambda $=0.644$

6. The maximum Pc should be $100 \mathrm{Mpa}$ (seems adequate to provide full data realization)

7. The inital brine saturation should be $100 \%$ (no evidence for anything else).

8. The Davies relatioship ( $\left.\mathrm{Pt}=0.26^{\bullet} \mathrm{k}^{\wedge}-0.348\right)$ appears to be adequate in relating threshold pressure to permeability for the anhydrites. $P C T \_A=.26$ Pa: $P C T \_E X P=-.348$

TABLE 1. Capillary Pressure Curve Fit Values

\begin{tabular}{|c|c|c|c|c|c|c|c|c|c|c|}
\hline & Sample & $\begin{array}{c}\mathrm{Pt} \\
(\mathrm{MPa})\end{array}$ & $\begin{array}{c}\text { Sir } \\
(\%)\end{array}$ & $\begin{array}{c}\text { Sgr } \\
(\%) \\
\end{array}$ & Lambda & $m$ & $\begin{array}{c}\text { Alpha } \\
(1 / \mathrm{MPa})\end{array}$ & $\begin{array}{c}\text { Pc Max } \\
(\mathrm{MPa})\end{array}$ & Figure & $\begin{array}{l}\text { Perm } \\
\text { (m2) }\end{array}$ \\
\hline \multirow{6}{*}{$\begin{array}{l}\text { Mercury } \\
\text { Injection }\end{array}$} & 5 & 0.54132 & 7.262 & $11.09+2 x^{5}$ & 0.655 & 0.3958 & 1.646 & 100 & 8 & $5.10 \mathrm{E}-19$ \\
\hline & 7 & 0.78 & 6.986 & 7.7729 & 0.66452 & 0.3992 & 1.141 & 100 & $\theta$ & 9.50E-19 \\
\hline & 11 & 0.45026 & 17.401 & 1.3981 & 0.55775 & 0.3580 & 2.010 & 100 & 10 & $1.80 \mathrm{E}-18$ \\
\hline & 13 & 0.75274 & 10.861 & 19.719 & 0.652 & 0.3947 & 1.184 & 100 & 11 & $1.60 \mathrm{E}-18$ \\
\hline & 21 & 0.32914 & 0.77846 & 2.5201 & 0.49053 & 0.3291 & 2.785 & 100 & 12 & $7.70 \mathrm{E}-19$ \\
\hline & 23 & 0.39724 & 6.8842 & 3.2177 & 0.84178 & 0.4570 & 2.201 & 100 & 13 & $1.30 \mathrm{E}-18$ \\
\hline Statistics & & $\mathrm{Pt}(\mathrm{MPa})$ & SIr $(\%)$ & Sgr $(\%)$ & Lambda & \multirow{12}{*}{0.391591} & \multirow{12}{*}{1.648} & \multirow{12}{*}{100} & & \\
\hline \multicolumn{2}{|l|}{ Minimum } & 0.329 & 0.778 & 1.398 & 0.491 & & & & & \\
\hline \multicolumn{2}{|l|}{ Maximum } & 0.780 & 17.401 & 19.719 & 0.842 & & & & & \\
\hline \multicolumn{2}{|l|}{ Sum } & 3.251 & 50.173 & 46.265 & 3.862 & & & & & \\
\hline \multicolumn{2}{|l|}{ Points } & 6 & 6 & 6 & 6 & & & & & \\
\hline \multicolumn{2}{|l|}{ Mean } & 0.5418 & 8.3621 & 7.7108 & 0.6436 & & & & & \\
\hline \multicolumn{2}{|l|}{ Median } & 0.4958 & 7.1240 & 5.4953 & 0.6536 & & & & & \\
\hline \multicolumn{2}{|l|}{ Std Deviation } & 0.1875 & 5.4908 & 7.0228 & 0.1189 & & & & & \\
\hline \multicolumn{2}{|l|}{ Variance } & 0.0351 & 30.1492 & 49.3195 & 0.0141 & & & & & \\
\hline \multicolumn{2}{|l|}{ Std Error } & 0.0765 & 2.2416 & 2.8670 & 0.0486 & & & & & \\
\hline \multicolumn{2}{|l|}{ Skewness } & 0.4327 & 0.5632 & 1.1346 & 0.6365 & & & & & \\
\hline \multicolumn{2}{|l|}{ Kurtosis } & -1.8573 & 1.4753 & 0.5853 & 1.2579 & & & & & \\
\hline
\end{tabular}




\section{Verification of Software as per QAP 9-1:}

1. Software used:

MicroMicrosoft Excel v. 4.0 and KaleidaGraph v. 3.0.3b2 on a PowerMacintosh with system 7.5.

2. All software is considered "off the shelf" and thus can be verifiecl in use.

3. Kaliedagraph was verified by computing the characteristic curve values and then applying the appropriate curve fit. The R2 value of the curve fit should equal 1. (shown on each graph)

4. Hand calculations of equations for Excell. An audit of the worksheet is provided from page 20 through 37 at the end of this calc sheet which includes a map, formulae, and contents. 
$12 / 20 / 95$

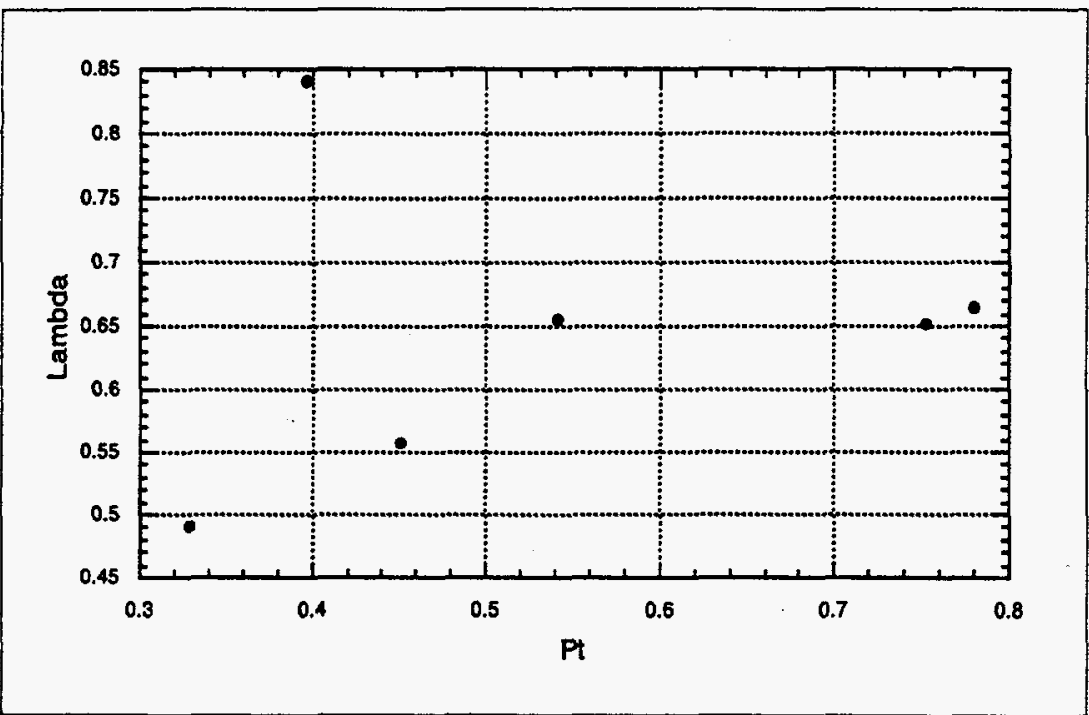

Figure 1a

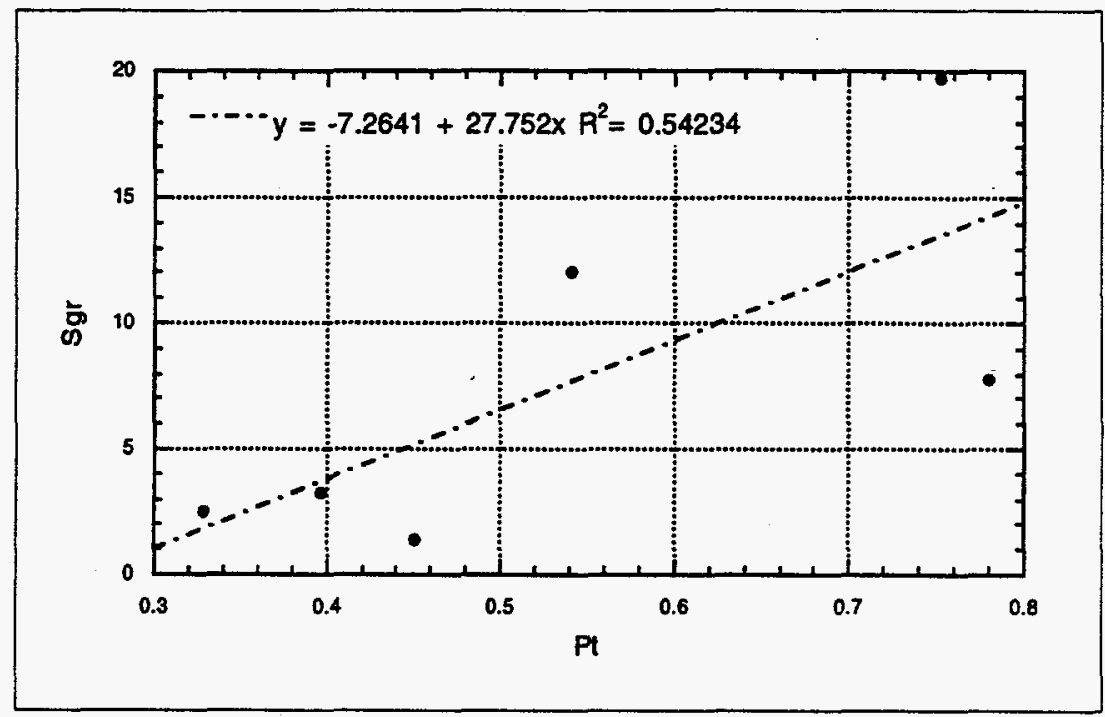

Figure 1b

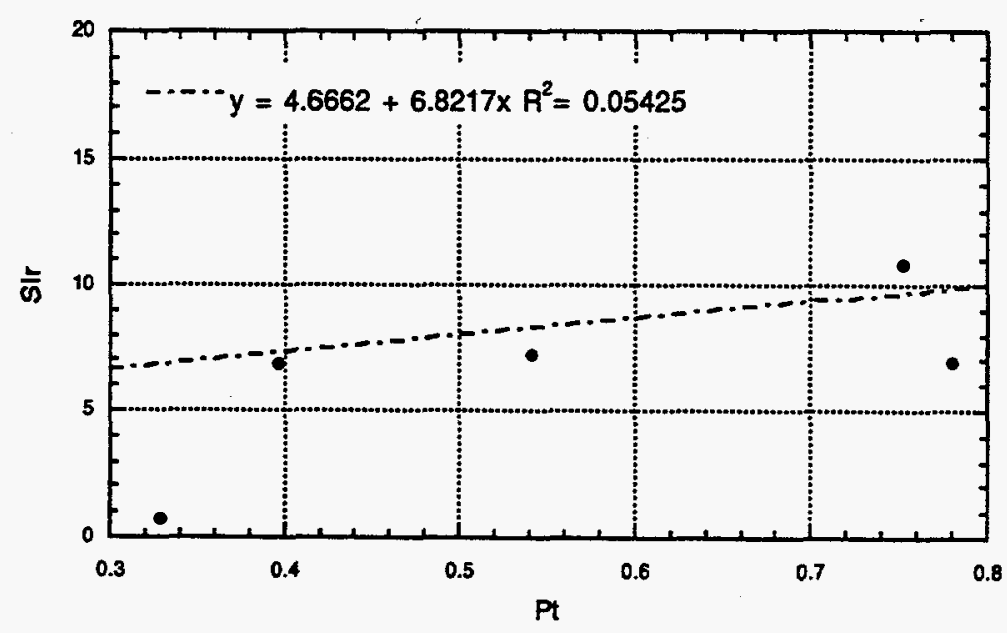

Figure 1c 


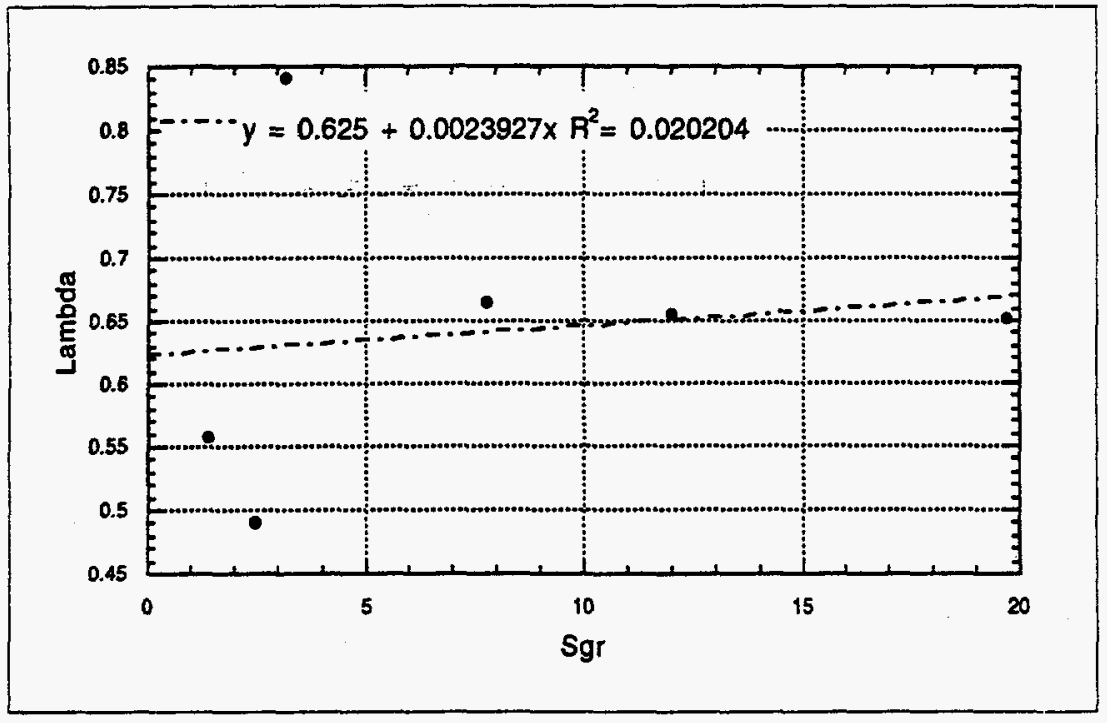

Figure 1d

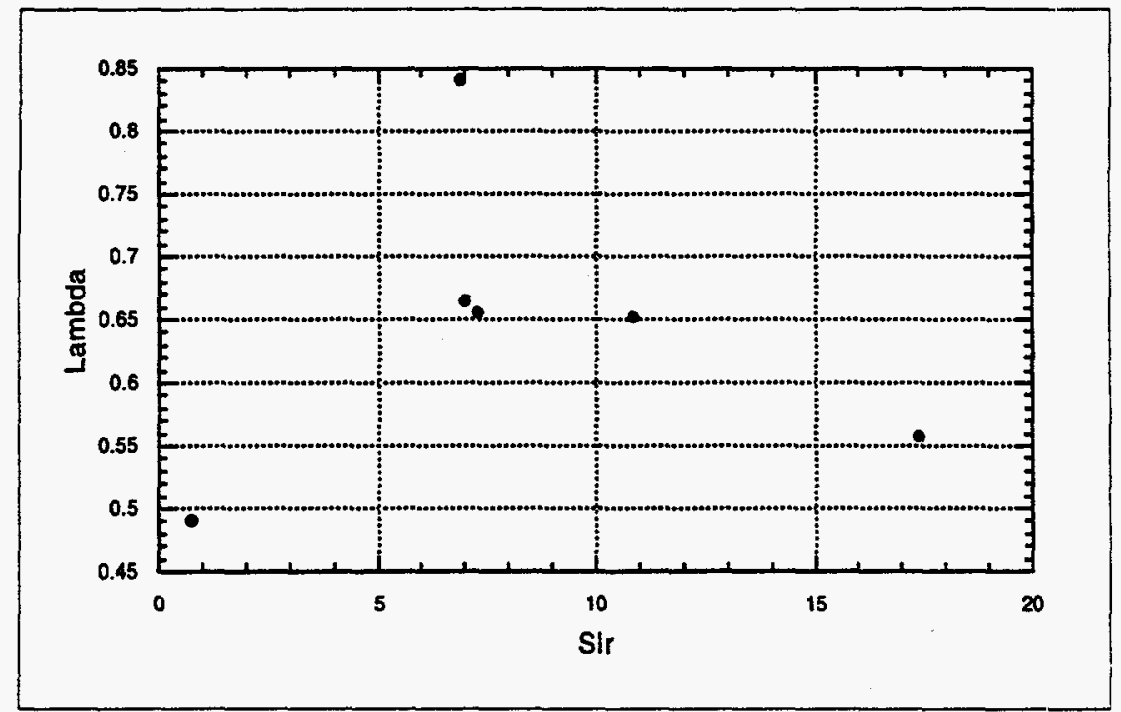

Figure 1e

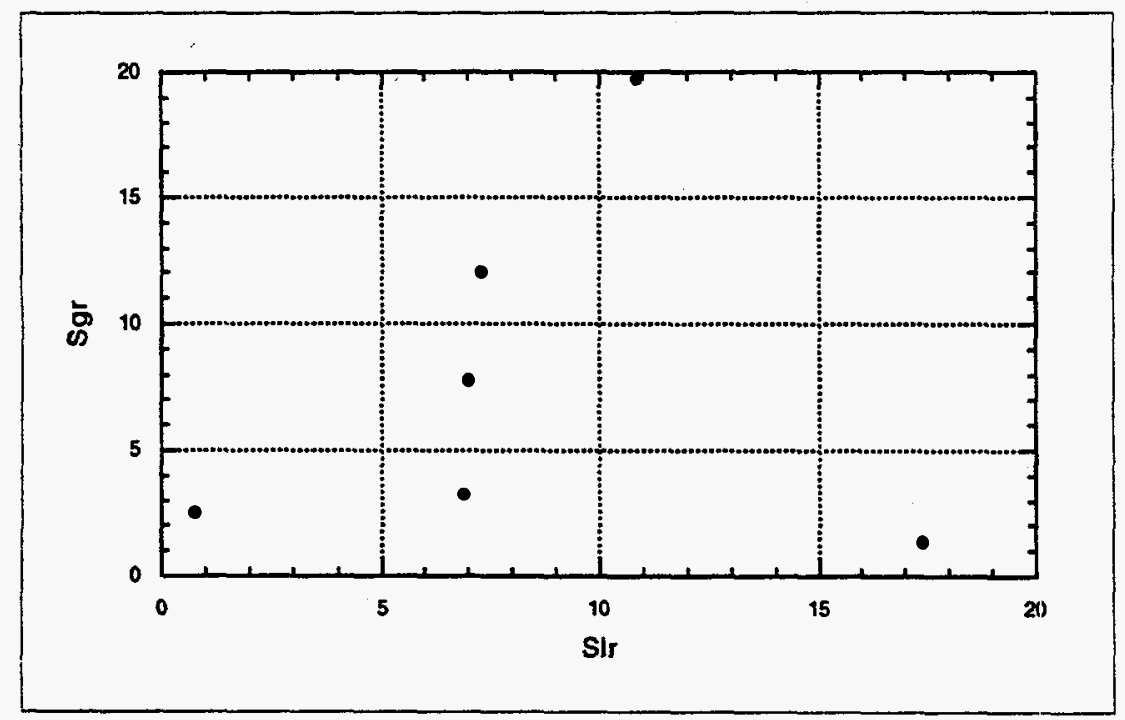

Figure if 


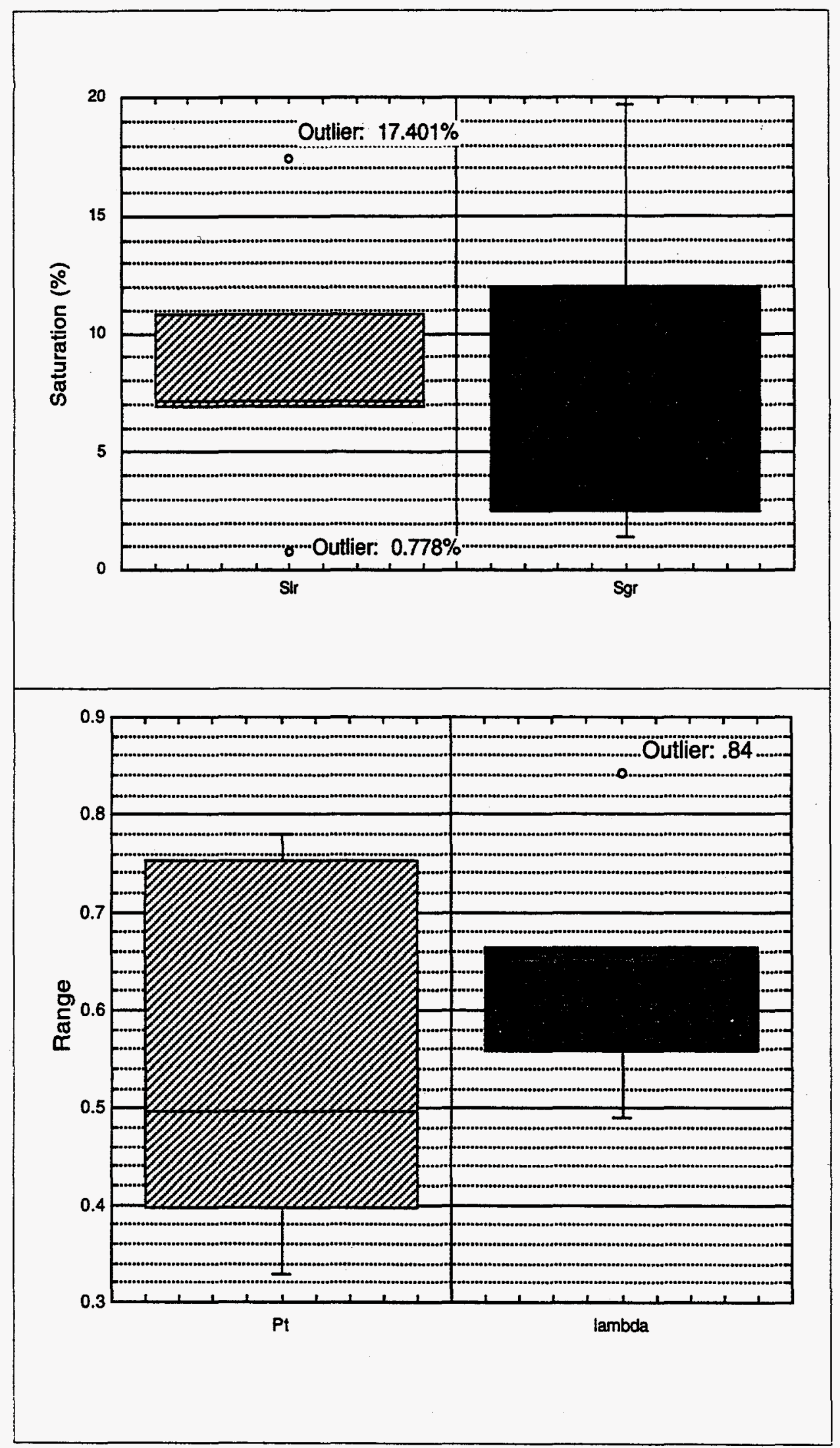

Figure 2. Box diagrams 


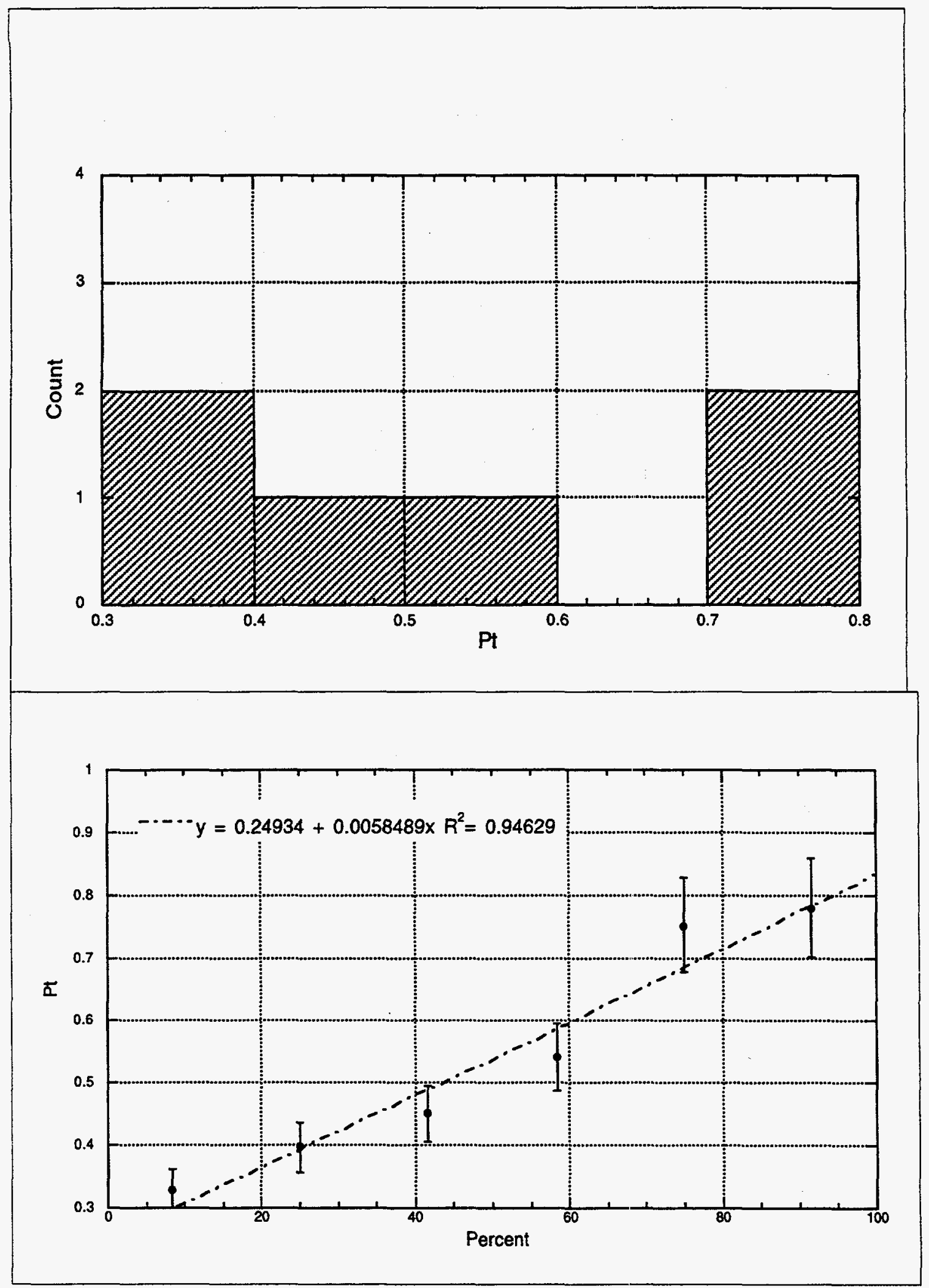

Figure 3. Pt histogram and probability graphs 

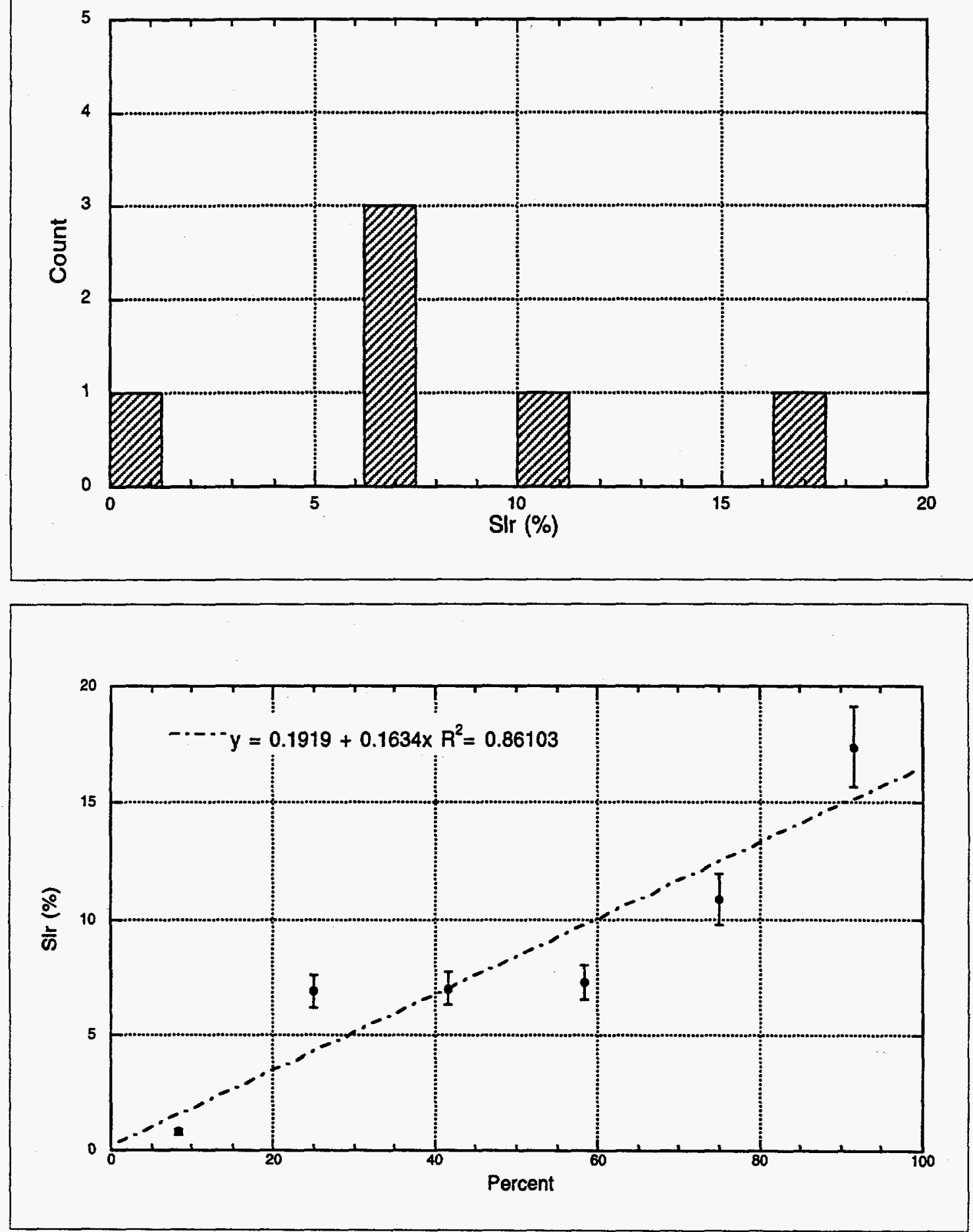

Figure 4. SIr histogram and probability graph 

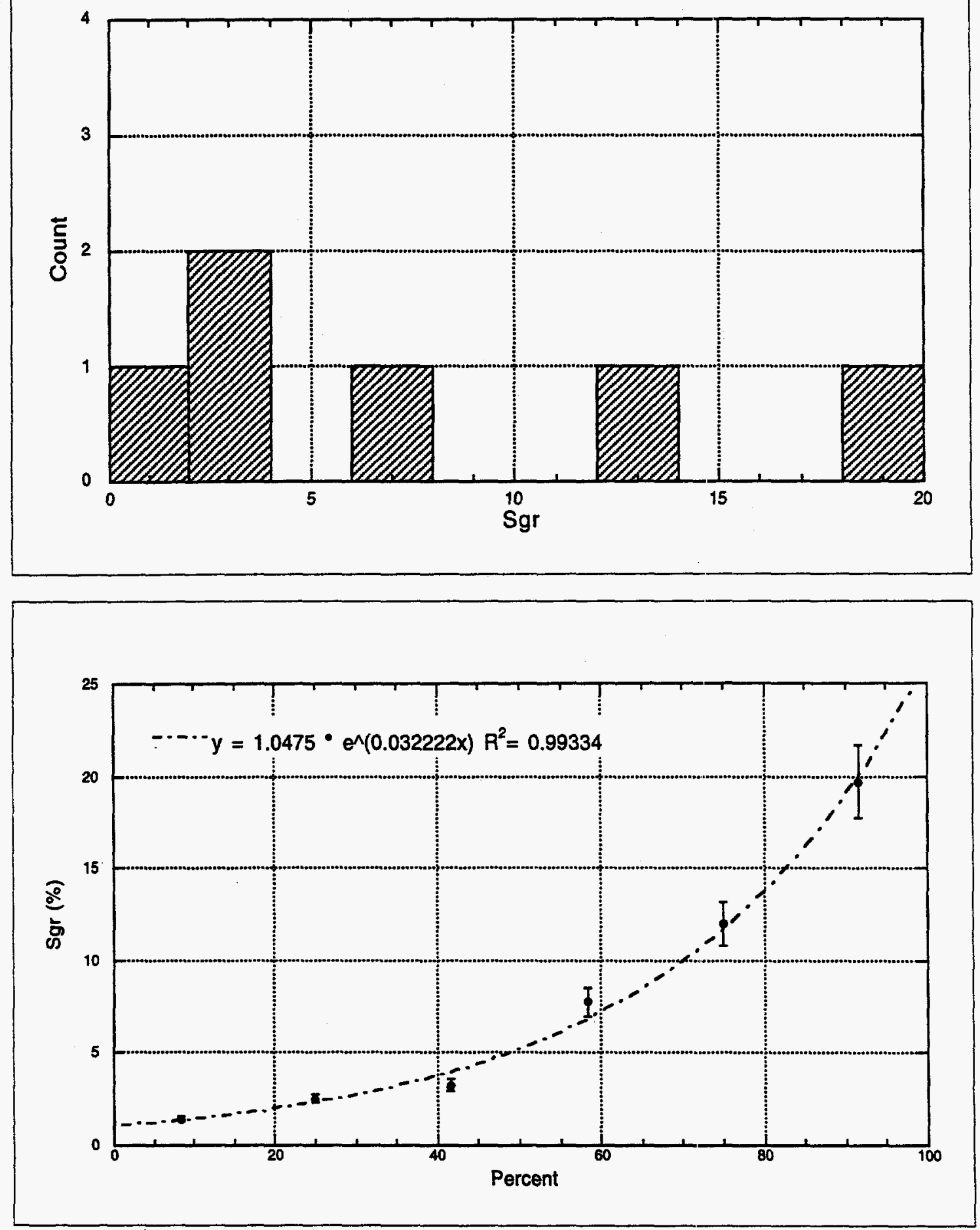

Figure 5. Sgr histogram and probability graphs 

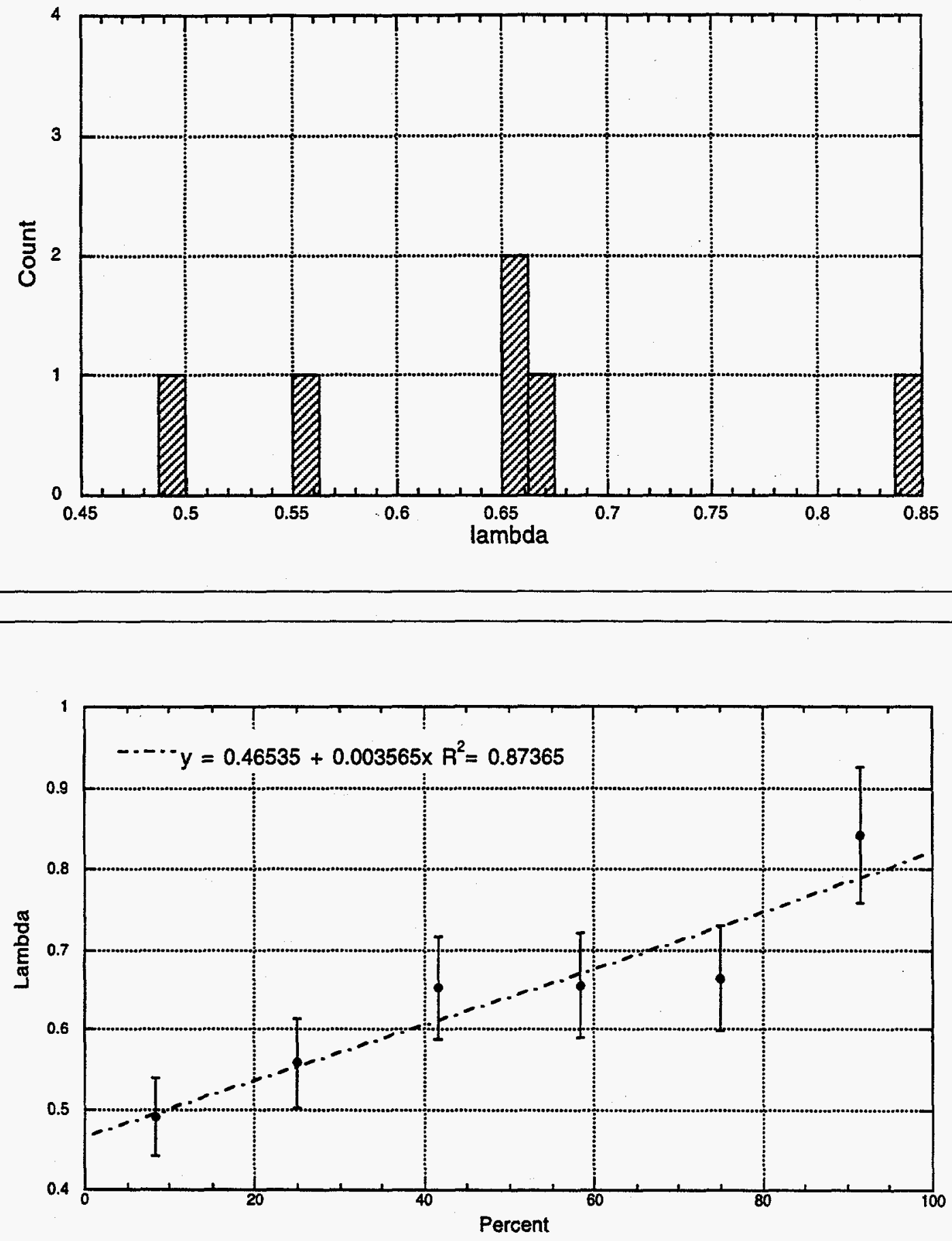

Figure 6. Lambda histogram and probability graphs 


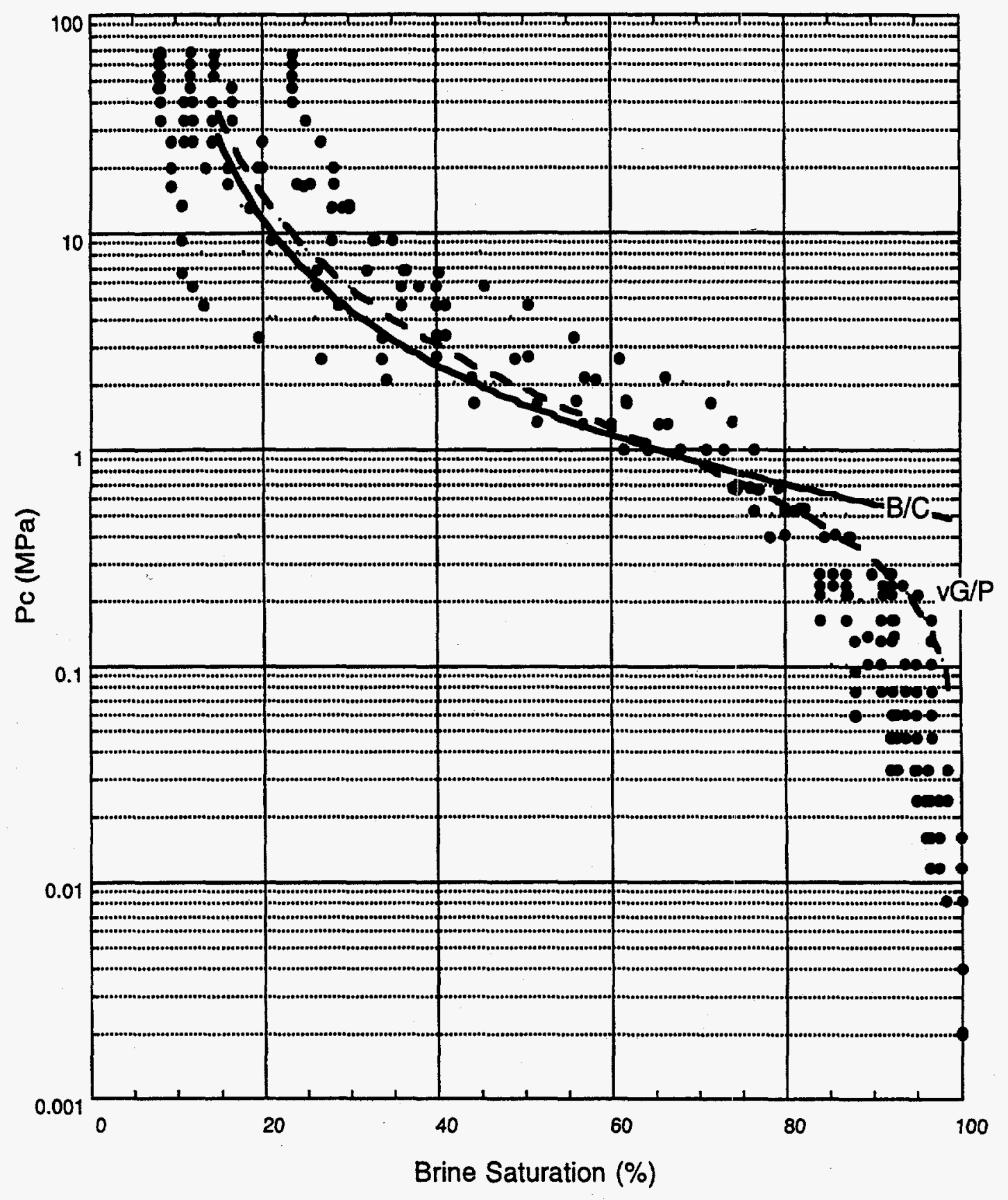

Figure 7. Mean values characteristic curves
Attachment 1
E-26

SWCF-A:1.2.07.1:PDD:QA:SALADO:PKG 10:Salado Anhydrite Two-Phase Parameters 
Sample 5 Data 11:57:09 AM 12/14/95 VG/P

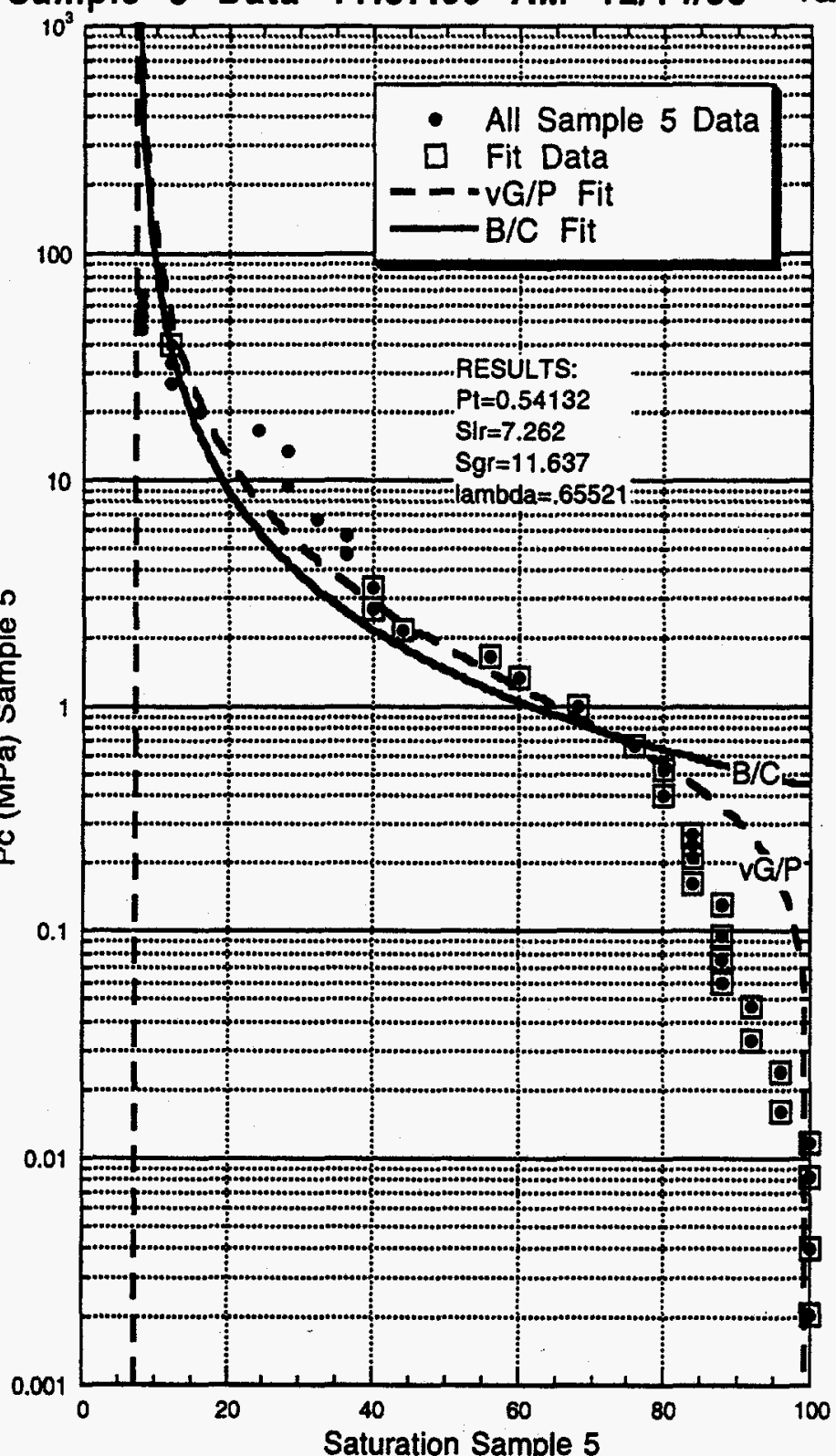

\begin{tabular}{|r|r|r|}
\hline$y=(1 / m 5)^{*}(((() \mathrm{m0}-\mathrm{m} 2) /(100-\ldots$ \\
\hline $\mathrm{m} 2$ & Value & Error \\
\hline $\mathrm{m} 3$ & 7.262 & 1.2759 \\
\hline $\mathrm{m} 5$ & 11.637 & 0.65159 \\
\hline $\mathrm{m} 6$ & 0.42977 & 0.027708 \\
\hline Chisq & 0.41455 & $\mathrm{NA}$ \\
\hline $\mathrm{R}^{2}$ & 0.99973 & $\mathrm{NA}$ \\
\hline$y=(1 / \mathrm{m5})^{*}(((((\mathrm{m0}-\mathrm{m} 2) /(100-\ldots$ \\
\hline & Value & Error \\
\hline $\mathrm{m} 2$ & 7.262 & 0.10451 \\
\hline $\mathrm{m} 5$ & 1.0531 & 0.028209 \\
\hline Chisq & 0.41455 & $\mathrm{NA}$ \\
\hline $\mathrm{R}^{2}$ & 0.99973 & $\mathrm{NA}$ \\
\hline
\end{tabular}

\begin{tabular}{|c|c|c|}
\hline \multicolumn{3}{|c|}{$h y=m 1 /((m 0-7.262) /(100-7.26 \ldots$} \\
\hline & Value & Error \\
\hline $\mathbf{m 1}$ & 0.5413 & 0.06774 \\
\hline $\mathrm{m} 4$ & 0.65521 & 0.019205 \\
\hline Chisq & 5.1848 & NA \\
\hline $\mathrm{R}^{2}$ & 0.99657 & NA \\
\hline \multicolumn{3}{|c|}{$y=m 1 /((m 0-7.262) /(100-7.26 \ldots$} \\
\hline & Value & Erro \\
\hline m1 & 0.54132 & 0.0061217 \\
\hline Chisq & 5.1848 & NA \\
\hline$R^{2}$ & 0.99657 & NA \\
\hline
\end{tabular}

VGIP: Validation of Software: $m 6$ should $=.3958$

\begin{tabular}{|r|r|r|}
\hline$y=(1 / 1.646) "(((() 0-7.262) \ldots$ \\
\hline & Value & Error \\
\hline$m 6$ & 0.3958 & $3.2804 e-12$ \\
\hline Chisq & $8.4361 e-13$ & NA \\
\hline$R^{2}$ & 1 & $N A$ \\
\hline
\end{tabular}

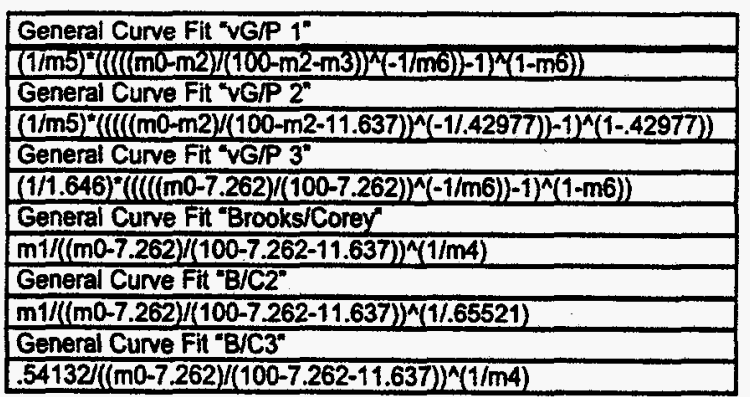

$B / C$ : Validation of Software: $\mathrm{m} 4$ should $=.65521$

\begin{tabular}{|r|r|r|}
\hline$y=$ & $.54132 /((\mathrm{mo}-7.262) /(100-.$. \\
\hline $\mathrm{m} 4$ & Value & Error \\
\hline Chisq & 0.65521 & $5.3358 \mathrm{e}-11$ \\
\hline $\mathrm{R}^{2}$ & $1.6523 \mathrm{e}-11$ & $\mathrm{NA}$ \\
\hline
\end{tabular}

Figure 8. Sample 5 curve fits

Attachment 1

E-27

SWCF-A:1.2.07.1:PDD:QA:SALADO:PKG 10:Salado Anhydrite Two-Phase Parameters 


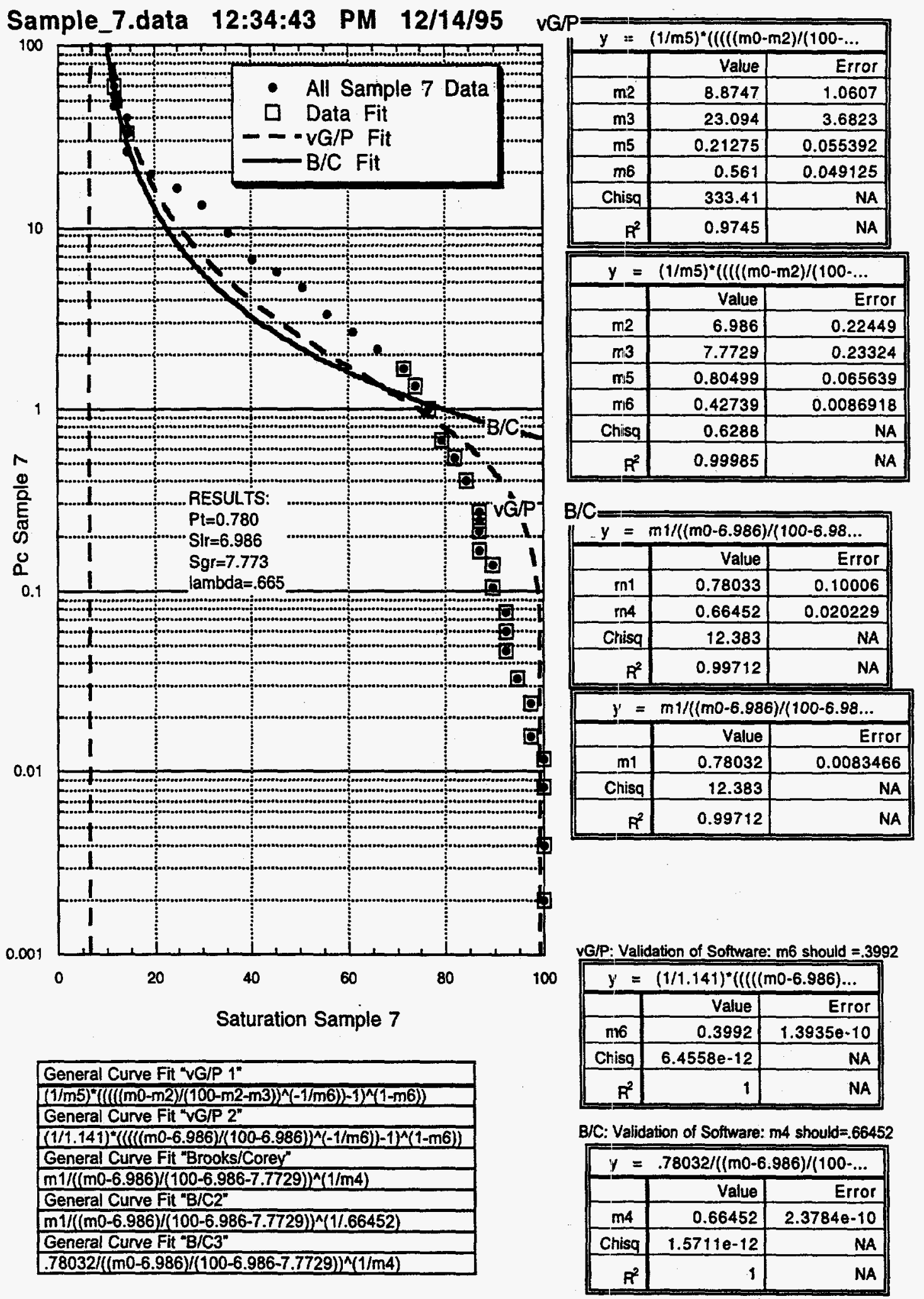

Figure 9. Sample 7 curve fits 


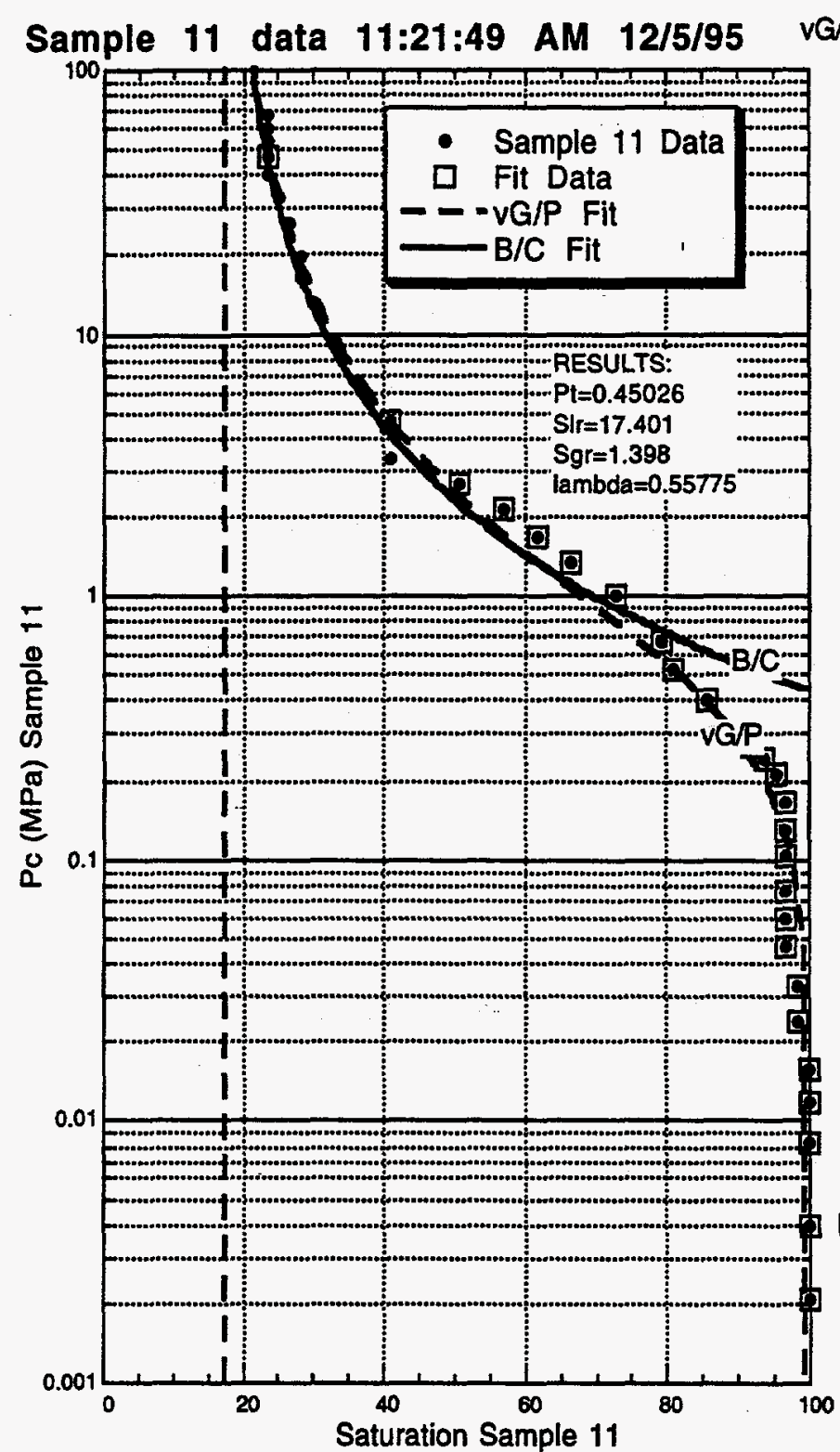

General Curve Fit "vG/P q" $^{\text {" }}$

$\left.(1 / m 5)^{\prime}\left(\left(((m 0-m 2) /(100-m 2-m 3))^{\lambda}(-1 / m 6)\right) 1\right)^{\lambda}(1-m 6)\right)$

General Curve Fit "vG/P 2"

$\left.(1 / m 5)^{*}\left(\left(\left((m 0-m 2) /\left(100-m_{2}-m 3\right)\right)^{\wedge}(-1 / .37669)\right)-1\right)^{\wedge}(1-.37669)\right)$

General Curve Fit " $v G / P$ 3'

$\left.(1 / 1.5532)^{*}\left(\left(((\mathrm{m0} 0-17.401) /(100-17.401-\mathrm{m} 3))^{\wedge}(-1 / .37669)\right)-1\right)^{\wedge}(1-.37669)\right)$

General Curve Fit " $\mathrm{VG} / \mathrm{P} 4$ "

$\left.(1 / 2.01)^{*}\left(\left(((m 0-17.401) /(100-17.401))^{\wedge}(-1 / m 6)\right)-1\right)^{\wedge}(1-m 6)\right)$

General Curve Fit "Brooks/Corey"

$\mathrm{m} 1 /((\mathrm{m0}-17.401) /(100-17.401-1.3981))^{\wedge}(1 / \mathrm{m} 4)$

General Curve Fit "BIC2"

$.45026 /((\mathrm{m0}-17.401) /(100-17.401-1.3981))^{\wedge}\left(1 / \mathrm{m}^{4}\right)$

$V G / P^{y}=(1 / m 5)^{*}(((((m 0-m 2) /(100-\ldots$

\begin{tabular}{|r|r|r|}
\hline & Value & Error \\
\hline $\mathrm{m} 2$ & 17.608 & 3.7007 \\
\hline $\mathrm{m} 3$ & -0.27798 & 25.642 \\
\hline $\mathrm{m} 5$ & 2.1116 & 2.7548 \\
\hline $\mathrm{m} 6$ & 0.35866 & 0.1025 \\
\hline Chisq & 456.65 & $\mathrm{NA}$ \\
\hline $\mathrm{R}^{2}$ & 0.96501 & $\mathrm{NA}$ \\
\hline
\end{tabular}

$y=(1 / \mathrm{m} 5)^{*}(((((\mathrm{m0}-\mathrm{m} 2)) /(100-\ldots$

\begin{tabular}{|r|r|r|}
\hline $\mathrm{m} 2$ & Value & Error \\
\hline $\mathrm{m} 3$ & 17.433 & 1.1487 \\
\hline $\mathrm{m} 5$ & 0.53421 & 1.7706 \\
\hline $\mathrm{m} 6$ & 1.5741 & 0.23392 \\
\hline Chisq & 0.37659 & 0.022446 \\
\hline $\mathrm{R}^{2}$ & 0.61342 & $\mathrm{NA}$ \\
\hline
\end{tabular}

$y=(1 / \mathrm{m} 5)^{*}(((((\mathrm{m0}-\mathrm{m} 2)) /(100-\ldots$

\begin{tabular}{|r|r|r|}
\hline & Value & Error \\
\hline $\mathrm{m} 2$ & 17.401 & 0.063272 \\
\hline $\mathrm{m} 3$ & 1.3932 & 0.51951 \\
\hline $\mathrm{m} 5$ & 1.5532 & 0.031652 \\
\hline Chisq & 0.1582 & $\mathrm{NA}$ \\
\hline $\mathrm{r}^{2}$ & 0.99992 & $\mathrm{NA}$ \\
\hline$y=(1 / 1.5532)^{*}(()((\mathrm{mo}-17.40 \ldots$ \\
\hline & Value & Error \\
\hline $\mathrm{m} 3$ & 1.3981 & 0.081404 \\
\hline Chisq & 0.1582 & $\mathrm{NA}$ \\
\hline$R^{2}$ & 0.99992 & $\mathrm{NA}$ \\
\hline
\end{tabular}

$B / C y=m 1 /(m 0-17.401) /(100-17 \ldots$

\begin{tabular}{|r|r|r|}
\hline & Value & Error \\
\hline $\mathrm{m} 1$ & 0.45026 & 0.042994 \\
\hline $\mathrm{m} 4$ & 0.55775 & 0.011568 \\
\hline Chisq & 3.3113 & $\mathrm{NA}$ \\
\hline $\mathrm{h}^{2}$ & 0.9984 & $\mathrm{NA}$ \\
\hline
\end{tabular}

VG/P: Validation of Software: $m 6$ should $=.358$

$y=\langle 1 / 2.01)^{*}((\langle((m 0-17.401\} \ldots$

\begin{tabular}{|r|r|r|}
\hline & Value & Error \\
\hline$m 6$ & 0.358 & $7.0637 e-11$ \\
\hline Chisq & $1.1011 e-12$ & NA \\
\hline$R^{2}$ & 1 & NA \\
\hline
\end{tabular}

B/C: Validation of Software: $\mathrm{m} 4$ should $=0.55775$

\begin{tabular}{|r|r|r|}
\hline$y=.45026 /((\mathrm{m0}-17.401) /(100 \ldots$ \\
\hline $\mathrm{m} 4$ & Value & Error \\
\hline Chisg & 0.55775 & $1.2818 \mathrm{e}-10$ \\
\hline$R^{2}$ & $1.3839 \mathrm{e}-13$ & $\mathrm{NA}$ \\
\hline
\end{tabular}

Figure 10. Sample 11 curve fits

Attachment 1

E-29

SWCF-A:1.2.07.1:PDD:QA:SALADO:PKG 10:Salado Anhydrite Two-Phase Parameters 
Sample 13 data 9:29:46 AM 12/14/95 vG/P

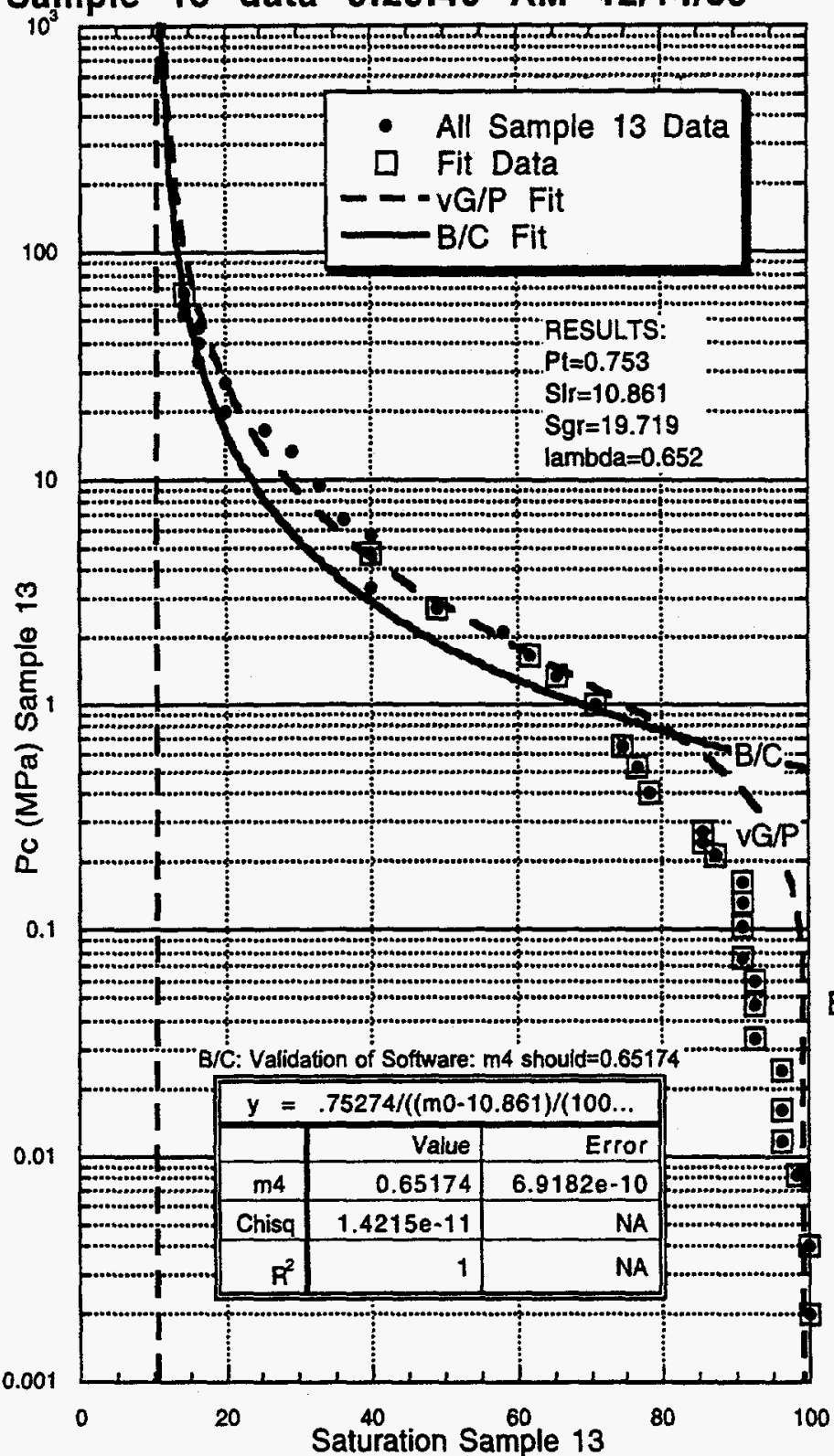

\begin{tabular}{|c|c|c|}
\hline \multicolumn{3}{|c|}{$y=(1 / \mathrm{m} 5)^{*}(l(((\mathrm{m0}-\mathrm{m} 2) /(100-\ldots$} \\
\hline & Value & Error \\
\hline$m 2$ & 10.002 & 1.4335 \\
\hline m3 & 25.068 & 6.2913 \\
\hline $\mathrm{m5}$ & 0.37471 & 0.1267 \\
\hline $\mathrm{m} 6$ & 0.45984 & 0.048036 \\
\hline Chisq & 225.57 & NA \\
\hline$R^{2}$ & 0.98271 & NA \\
\hline \multicolumn{3}{|c|}{$y=\langle 1 / \mathrm{m} 5)^{*}\langle(\langle((\mathrm{m0}-\mathrm{m} 2) /(100-\ldots$} \\
\hline & Value & Error \\
\hline $\mathrm{m} 2$ & 10.976 & 1.0028 \\
\hline $\mathrm{m} 3$ & 20.553 & 1.1045 \\
\hline $\mathrm{m} 5$ & 0.59574 & 0.062887 \\
\hline$m 6$ & 0.44388 & 0.027805 \\
\hline Chisq & 0.37516 & NA \\
\hline$R^{2}$ & 0.99991 & NA \\
\hline \multicolumn{3}{|c|}{$y=(1 / m 5)^{*}(((((m 0-m 2) /(100-\ldots$} \\
\hline & Value & Error \\
\hline $\mathrm{m} 2$ & 10.861 & 0.079001 \\
\hline $\mathrm{m} 3$ & 19.719 & 0.94012 \\
\hline m5 & 0.61652 & 0.02239 \\
\hline Chisq & 0.35013 & NA \\
\hline$R^{2}$ & 0.99992 & NA \\
\hline
\end{tabular}

$\mathrm{B} / \mathrm{C}$

\begin{tabular}{|r|r|r|}
\hline$y=m 1 /((m 0-10.861) /(100-10 \ldots$. \\
\hline$m 1$ & Value & Error \\
\hline$m 4$ & 0.75275 & 0.10968 \\
\hline Chisq & 0.65174 & 0.021272 \\
\hline$R^{2}$ & 8.4475 & $\mathrm{NA}$ \\
\hline
\end{tabular}

\begin{tabular}{|r|r|r|}
\hline$y=m 1 /((m 0-10.861) /(100-10 \ldots .$. \\
\hline & Value & Error \\
\hline$m 1$ & 0.75274 & 0.0066906 \\
\hline Chisq & 8.4475 & NA \\
\hline $\mathrm{A}^{2}$ & 0.99799 & $\mathrm{NA}$ \\
\hline
\end{tabular}

\begin{tabular}{|c|}
\hline 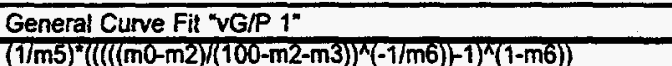 \\
\hline 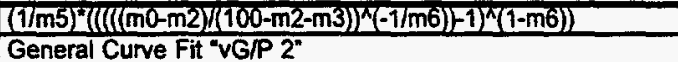 \\
\hline$\left.(1 / m 5)^{*}\left(\left(((m 0-m 2) /(100-m 2-m 3))^{\wedge}(-1 / .44021)\right)-1\right)^{\wedge}(1-.44021)\right)$ \\
\hline General Curve Fit " $v G / P 3^{\prime \prime}$ \\
\hline$\hat{x(1-m 6) 1}$ \\
\hline \\
\hline $\mathrm{m}^{1 /((\mathrm{m0} 0-10.861) /(100-10.861-19.719))^{\wedge}(1 / \mathrm{m} 4)}$ \\
\hline General Curve Fil "B/C2" \\
\hline \multirow{2}{*}{$\frac{\mathrm{m} 1 /((\mathrm{m} 0-10.861) /(100-10.861-19.719))^{\wedge}(1 / .65174)}{\text { General Curve Fit "B/C3" }}$} \\
\hline \\
\hline $.75274 /((\mathrm{m0} 0-10.861) /(100-10.861-19.719))^{\wedge}(1 / \mathrm{m}$ \\
\hline
\end{tabular}

VG/P: Validation of Software: $m 6$ should $=.3946$

\begin{tabular}{|r|r|r|}
\hline$y=(1 / 1.184)^{*}(((() \mathrm{m0}-10.861 \ldots$ \\
\hline & Value & Error \\
\hline$m 6$ & 0.3946 & $1.027 e-10$ \\
\hline Chisq & $7.0805 e-12$ & $\mathrm{NA}$ \\
\hline$R^{2}$ & 1 & $\mathrm{NA}$ \\
\hline
\end{tabular}

Figure 11. Sample 13 curve fits

Attachment 1 


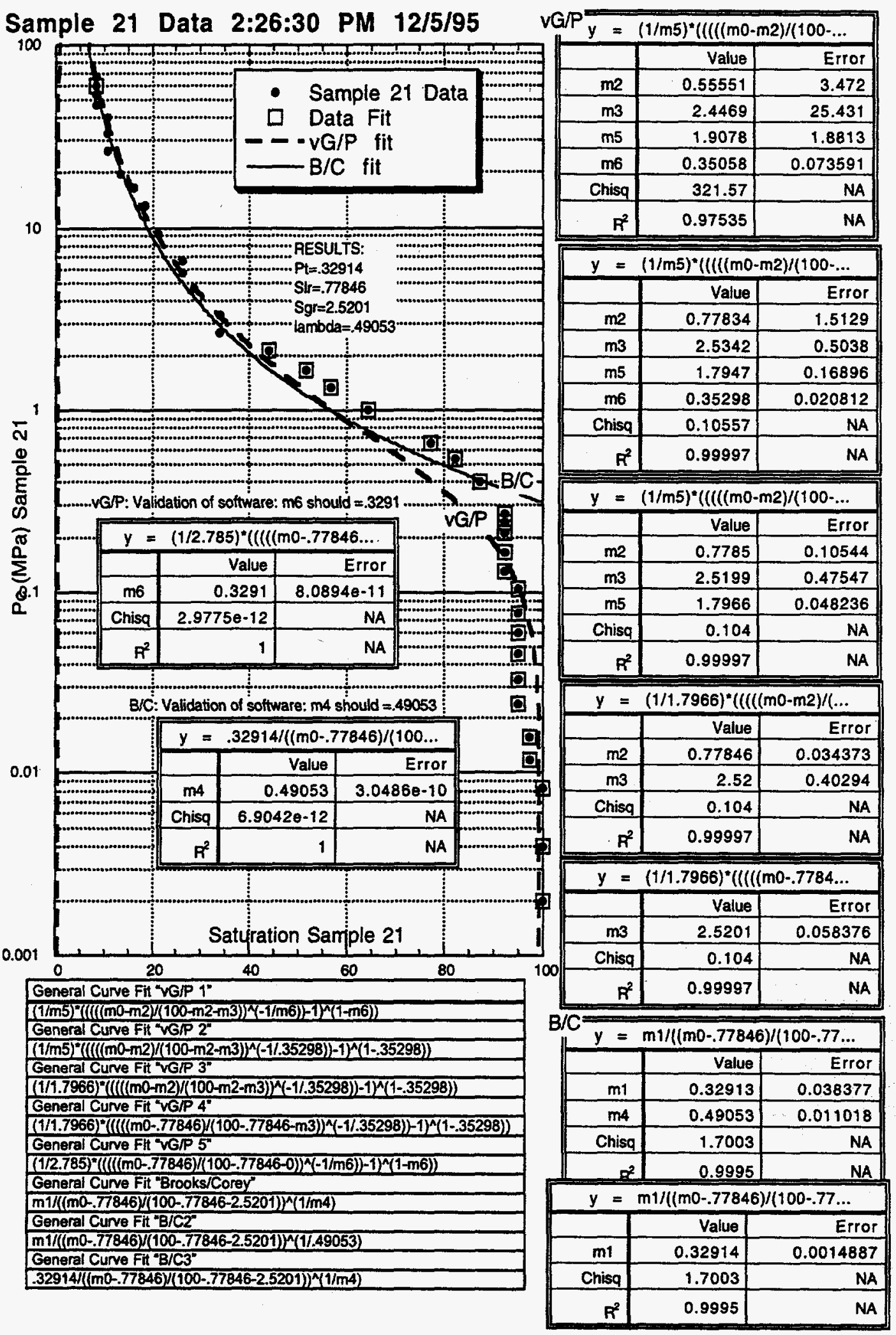

Figure 12. Sample 21 curve fits

Attachment 1 
Sample 23 data

100

10

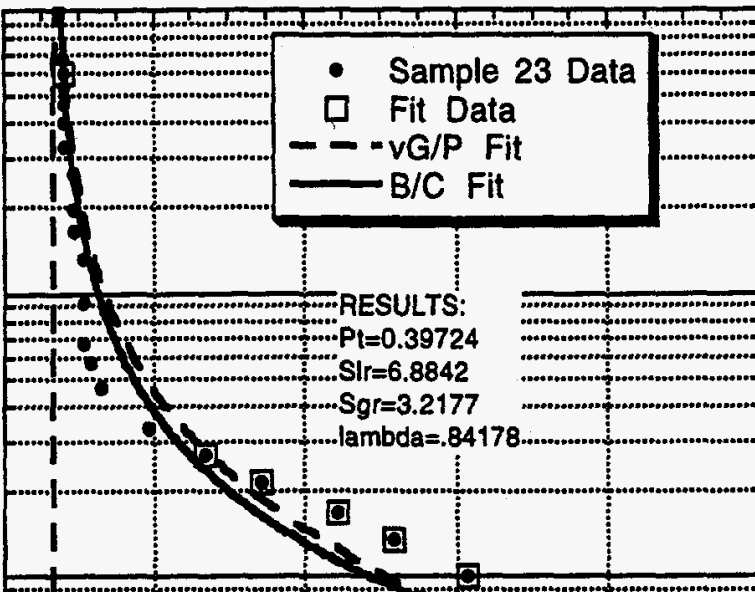

की

o

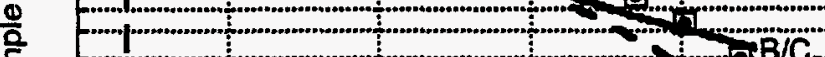

蛋

(I)

IVG/P:Validation of sottware: $m 6$ should $=.457$

0

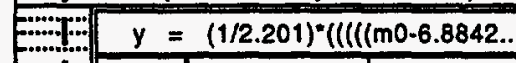

$y=(1 / 2.201)^{*}(((() \mathrm{m0}-6.8842 .$.

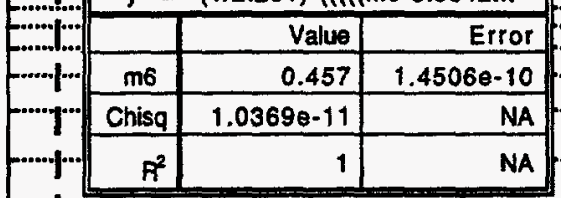

0.01

I B/C.Validation of soltware: $m 4$ should $=0.84178$

$y=.39724 /((m 0-6.8842) /(100 \ldots$

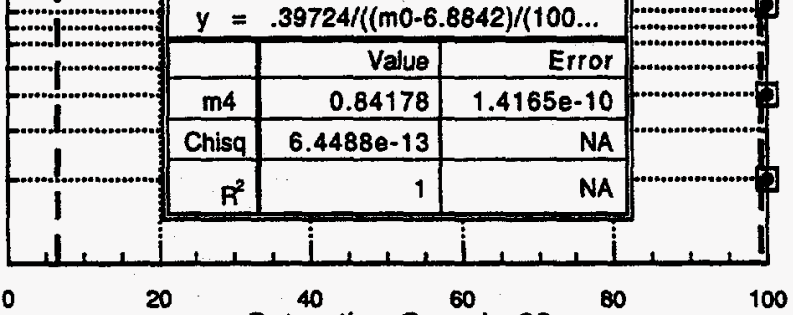

Saturation Sample 23

General Curve Fil "vGP 1":

$\left.\left.\left(1 / m^{5}\right)^{\prime}\left\{\left(\left(\left(m_{0} 0-m^{2}\right)\right)\left(100-m^{2}-m^{3}\right)\right)^{x}(-1 / m 6)\right)-1\right)^{x}\left(1-m^{6}\right)\right)$

General Curve Fit " $v G P$ " 2 "

$\left.(1 / m 5)^{*}\left(\left(\left((m 0-m 2) /\left(100-m 2-m^{3}\right)\right)^{n}(-1 / .47663)\right)^{1}\right)^{\wedge}(1-.47663)\right)$

General Cunve Fit 'vG/P 3 "

$\left((1 / 1.7222)^{k}\left(\left(\left(1\left(m 0-m^{2}\right) /\left(100-m^{2}-m^{3}\right)\right)^{M}(-1 / .47663)\right)-1\right)^{M}(1.47663)\right)$

General Curve Fit" "vGP" 4 "

$(1 / 1.7222)^{n}\left((((\operatorname{mo} 0-6.8842) /(100-6.8842-m 3) \wedge(-1 / .47663))-1)^{\wedge}(1-.47663)\right)$

General Curve Fit " $v G / P$ "

$\left.(1 / 2.201)^{\top}\left(\left(((m 0-6.8842) /(100-6.8842))^{\wedge}(-1 / m 6)\right)^{-1}\right)^{\wedge}(1-m 6)\right)$

General Curve Fit "Brooks/Corey"

mil/(m0-6.8842)/(100-6.8842-3.2177) $)^{\mathrm{N}}(1 / \mathrm{m} 4)$

General Curve Fit "B/C2"

$m 1 /((m 0-6.8842) /(100-6.8842-3.2177))(1 / .84178)$

General Curve Fit "B/C3"

$.39724 /(m 0-6.8842) /(100-6.8842-3.2177))^{2}\left(1 / \mathrm{m}^{4}\right)$ $v G / \bar{P} y=(1 / m 5)^{*}(((((m 0-m)) /(100-$

\begin{tabular}{|r|r|r|}
\hline & Value & Error \\
\hline$m 2$ & 6.4023 & 2.2757 \\
\hline$m 3$ & -67.739 & 10246 \\
\hline$m 5$ & 56.143 & 6370.3 \\
\hline$m 6$ & 0.36158 & 0.20869 \\
\hline Chisq & 881.58 & $\mathrm{NA}$ \\
\hline $\mathrm{R}^{2}$ & 0.93256 & $\mathrm{NA}$ \\
\hline $\mathrm{y}=$ & $(1 / \mathrm{m} 5)^{\circ}(((() \mathrm{m0}-\mathrm{m} 2) /(100-\ldots$ \\
\hline & Value & Error \\
\hline $\mathrm{m} 2$ & 6.8705 & 0.33304 \\
\hline $\mathrm{m} 3$ & 2.4815 & 0.26179 \\
\hline $\mathrm{m} 5$ & 1.7183 & 0.17424 \\
\hline $\mathrm{m} 6$ & 0.47663 & 0.01972 \\
\hline Chisq & 0.36215 & $\mathrm{NA}$ \\
\hline $\mathrm{R}^{2}$ & 0.99989 & $\mathrm{NA}$ \\
\hline & & \\
\hline
\end{tabular}

$y=(1 / m 5)^{*}(((((m 0-m 2) /(100-\ldots$

\begin{tabular}{|r|r|r|}
\hline & Value & Error \\
\hline $\mathrm{m} 2$ & 6.8732 & 0.038617 \\
\hline $\mathrm{m} 3$ & 2.4813 & 0.25583 \\
\hline $\mathrm{m} 5$ & 1.7222 & 0.05475 \\
\hline Chisq & 0.36206 & $\mathrm{NA}$ \\
\hline$R^{2}$ & 0.99989 & $\mathrm{NA}$ \\
\hline
\end{tabular}

$y=(1 / 1.7222)^{*}((()(\mathrm{m0} 0-\mathrm{m} 2) / / \ldots$

\begin{tabular}{|r|r|r|}
\hline & Value & Error \\
\hline $\mathrm{m} 2$ & 6.8842 & 0.016869 \\
\hline $\mathrm{m} 3$ & 3.2146 & 1.1258 \\
\hline Chisq & 0.35812 & $\mathrm{NA}$ \\
\hline $\mathrm{R}^{2}$ & 0.9999 & $\mathrm{NA}$ \\
\hline$y=$ & $(1 / 1.7222)^{*}(()(\mathrm{m} 0-6.884 \ldots$ \\
\hline $\mathrm{m} 3$ & Value & Error \\
\hline Chisg & 3.2177 & 0.16467 \\
\hline $\mathrm{F}^{2}$ & 0.35812 & $\mathrm{NA}$ \\
\hline
\end{tabular}

$B / C=m^{1 /((m 0-6.8842) /(100-6.8 \ldots}$

\begin{tabular}{|r|r|r|}
\hline & $y=m^{1 /(}(\mathrm{m0}-6.8842) /(100-6.8 \ldots$ \\
\hline $\mathrm{m} 1$ & 0.39723 & 0.044994 \\
\hline $\mathrm{m} 4$ & 0.84178 & 0.019069 \\
\hline Chisq & 2.6033 & $\mathrm{NA}$ \\
\hline $\mathrm{R}^{2}$ & 0.99924 & $\mathrm{NA}$ \\
\hline
\end{tabular}

\begin{tabular}{|r|r|r|}
\hline$y=m 1 /((m 0-6.8842) /(100-6.8 \ldots$ \\
\hline$m 1$ & Value & Error \\
\hline Chish & 0.39724 & 0.0021747 \\
\hline$f^{2}$ & 2.6033 & $\mathrm{NA}$ \\
\hline
\end{tabular}

Figure 13. Sample 23 curve fits

Attachment 1 


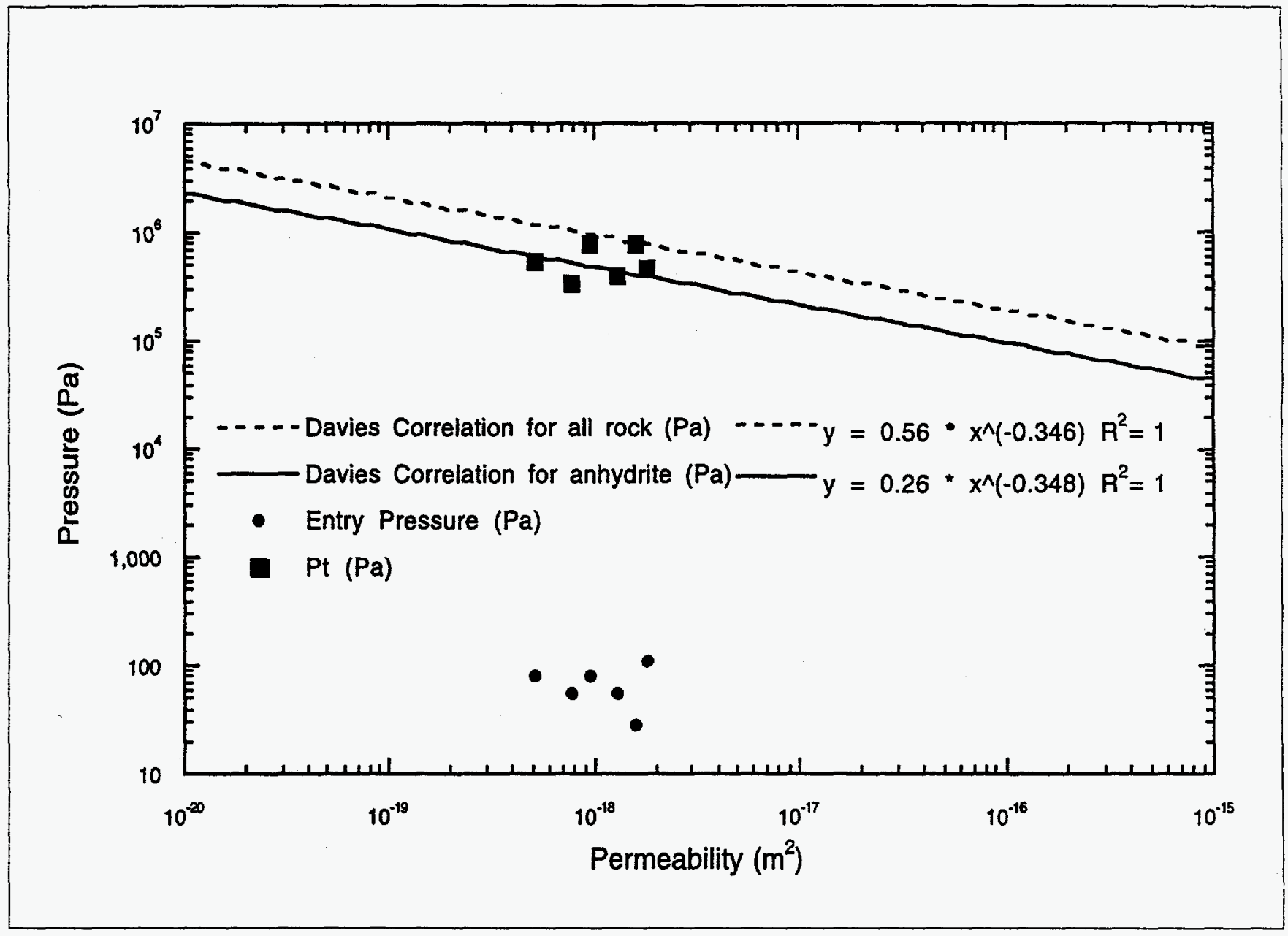

Figure 14. Permeability vs Threshold Pressure

Correlations found in SAND91-0893/3 page 2-13 


\section{Audit of Capillary Pressure Model Params}

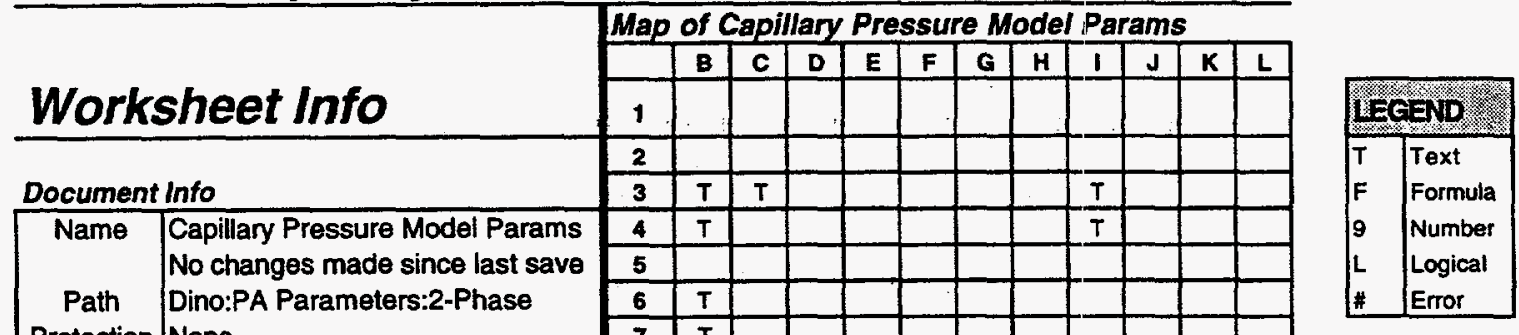

Protection None

Version Microsoft Excel version 4.0

System Macintosh 7.50

\section{Worksheet Info}

Active Area 830 rows by 11 columns

\begin{tabular}{c|l} 
Blanks & $\begin{array}{l}9,130 \text { cells } \\
8,812(96.5 \%)\end{array}$
\end{tabular}

Constants $259(2.8 \%)$
Numbers $48(0.5 \%)$
Text $211(2.3 \%)$
Logicais $0(0.0 \%)$
Errors $0(0.0 \%)$
Formulas $59(0.6 \%)$
Names 30 total
16 normal
14 hidden
Objects 24

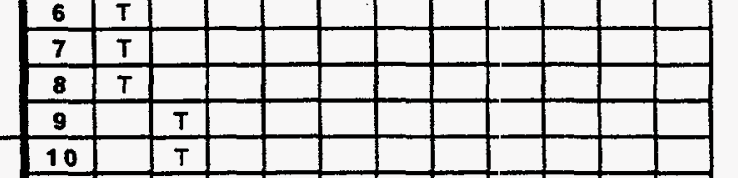

\begin{tabular}{|l|l|l|l|l|l|l|l|l|l|l|l|}
\hline 11 & & & $T$ & & & & & & & & \\
\hline 12 & & & $T$ & & & & & & & & \\
\hline 13 & & & $T$ & & & & & & & & \\
\hline 14 & & & & &
\end{tabular}

15

15

$\frac{16}{17}$

$\frac{18}{19}$

20

\begin{tabular}{l|l}
21 & $T$ \\
\hline 22 & $T$
\end{tabular}

\begin{tabular}{|l|l|l|l|l|l|l|l|l|l|l|l|}
\hline 22 & $T$ & & & & & & & & & & \\
\hline 23 & $T$ & & & & & & & & & & \\
\hline
\end{tabular}

24

2

27

27

2

\begin{tabular}{|c|c|}
30 & \\
\hline 31 & $T$ \\
\hline
\end{tabular}

\begin{tabular}{|l|l|l|l|l|l|l|l|l|l|l|l|}
\hline 31 & $\mathrm{~T}$ & & & & & & & & & & \\
\hline 32 & $\mathrm{~T}$ & & & & & & & & & & \\
\hline
\end{tabular}

\begin{tabular}{|l|l|}
\hline 32 & $T$ \\
\hline 33 & $T$ \\
\hline
\end{tabular}

\begin{tabular}{|l|l|}
\hline 33 & $T$ \\
\hline 34 & \\
\hline 35 & \\
\hline
\end{tabular}

\begin{tabular}{|l|l}
\hline 35 & $T$ \\
\hline
\end{tabular}

\begin{tabular}{|l|l|l|}
\hline 36 & & $T$ \\
\hline 37 & & \\
\hline
\end{tabular}

37

38

\begin{tabular}{l|l|l}
\hline 39 & & $T$ \\
\hline 40 & & \\
\hline
\end{tabular}

40

\begin{tabular}{|l|}
41 \\
\hline 42 \\
\hline 43
\end{tabular}

44

44

\begin{tabular}{|l|l|l|l|l|l|l|l|l|l|l|l|}
\hline 44 & & $\mathrm{~T}$ & & & & & & & & & \\
\hline 45 & & & $\mathrm{~T}$ & & & & & & & $\mathrm{~T}$ & \\
\hline 46 & & & $\mathrm{~T}$ & & & & & & & & \\
\hline 47 & & & & & & & & & & & \\
\hline 48 & & & & & & & & & & & \\
\hline 49 & $\mathrm{~T}$ & & $\mathrm{~T}$ & & & $\mathrm{~T}$ & & $\mathrm{~T}$ & & & \\
\hline 50 & & $\mathrm{~T}$ & $\mathrm{~T}$ & & & $\mathrm{~T}$ & & & & & \\
\hline 51 & & $\mathrm{~T}$ & $\mathrm{~T}$ & & & $\mathrm{~T}$ & & & $\mathrm{~T}$ & & \\
\hline 52 & & $\mathrm{~T}$ & $\mathrm{~T}$ & & & $\mathrm{~T}$ & & & & & \\
\hline 53 & & $\mathrm{~T}$ & $\mathrm{~T}$ & & & $\mathrm{~T}$ & & & $\mathrm{~T}$ & & \\
\hline 54 & & $\mathrm{~T}$ & $\mathrm{~T}$ & & & $\mathrm{~T}$ & & & $\mathrm{~T}$ & & \\
\hline 55 & & $\mathrm{~T}$ & $\mathrm{~T}$ & & & $\mathrm{~T}$ & & & $\mathrm{~T}$ & & \\
\hline 56 & & $\mathrm{~T}$ & $\mathrm{~T}$ & & & $\mathrm{~T}$ & & & $\mathrm{~T}$ & & \\
\hline 57 & & $\mathrm{~T}$ & $\mathrm{~T}$ & & & $\mathrm{~T}$ & & & $\mathrm{~T}$ & & \\
\hline 58 & & $\mathrm{~T}$ & $\mathrm{~T}$ & & & $\mathrm{~T}$ & & & & & \\
\hline
\end{tabular}

\begin{tabular}{|l|l|l|l|}
\hline 58 & & $T$ & $T$ \\
\hline
\end{tabular} 


\section{Audit of Capillary Pressure Model Params}

Map of Capillary Pressure Model Params

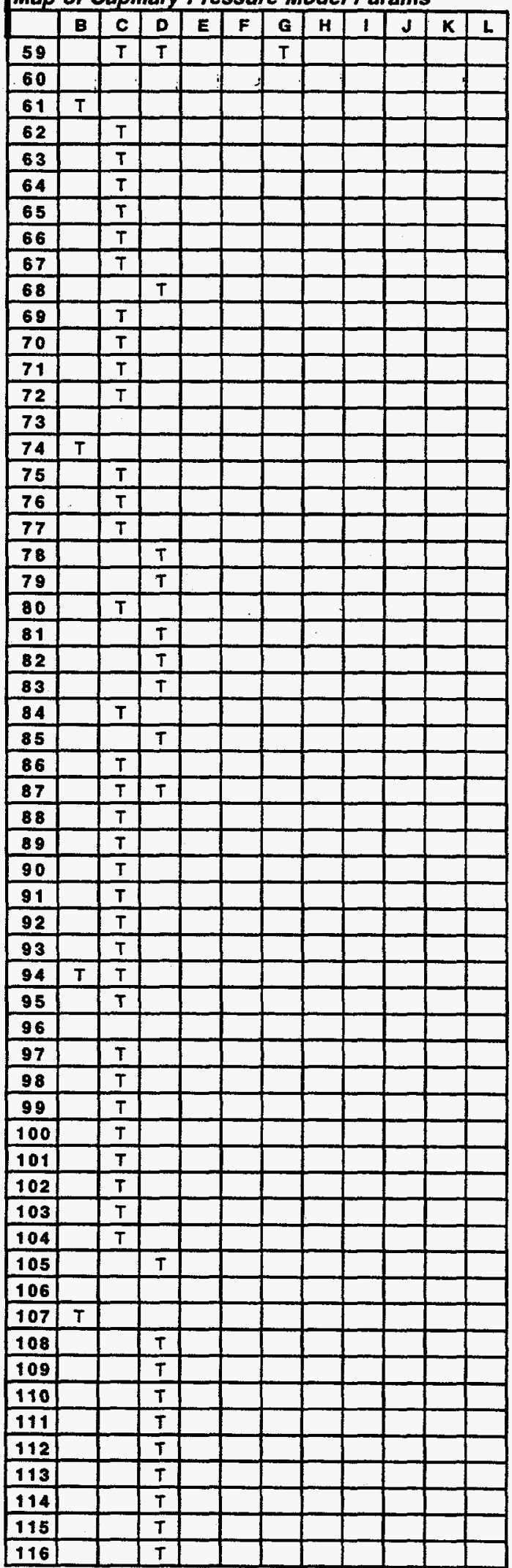




\section{Audit of Capillary Pressure Model Params}

Map of Caplllary Pressure Model Params

\begin{tabular}{|c|c|c|c|c|c|c|c|c|c|c|c|}
\hline & $B$ & c & 0 & $\bar{E}$ & $F$ & G & $H$ & 1 & $J$ & $K$ & $L$ \\
\hline 117 & & & & & & & & & & & \\
\hline 118 & & $T$ & & & & & & & & & \\
\hline 119 & & $T$ & $T$ & $T$ & $T$ & $T$ & $T$ & $T$ & $T$ & $\bar{T}$ & $\bar{T}$ \\
\hline 120 & & & $T$ & $T$ & $T$ & & & $T$ & $T$ & & $T$ \\
\hline 121 & $T$ & 9 & 9 & 9 & 9 & 9 & $F$ & $F$ & 9 & 9 & 9 \\
\hline 122 & $T$ & 9 & 9 & 9 & 9 & 9 & $F$ & $F$ & 9 & 9 & 9 \\
\hline 123 & & 9 & 9 & 9 & 9 & 9 & $F$ & $F$ & 9 & 9 & 9 \\
\hline 124 & & 9 & 9 & 9 & 9 & 9 & $F$ & $F$ & 9 & 9 & $\theta$ \\
\hline 125 & & 9 & 9 & 9 & 9 & 9 & $F$ & $F$ & 9 & 9 & 9 \\
\hline 126 & & 9 & 9 & 9 & 9 & 9 & $F$ & $F$ & 9 & 9 & 9 \\
\hline 127 & $T$ & & & & & & & & & & \\
\hline 128 & $T$ & & $T$ & $T$ & $T$ & $T$ & & & & & \\
\hline 129 & $T$ & & $F$ & $F$ & $F$ & $F$ & & & & & \\
\hline 130 & $T$ & & $F$ & $\bar{F}$ & $F$ & $\bar{F}$ & & & & & \\
\hline 131 & $T$ & & $F$ & $F$ & $F$ & $F$ & & & & & \\
\hline 132 & $T$ & & $F$ & $F$ & $F$ & $F$ & & & & & \\
\hline 133 & $T$ & & $F$ & $F$ & $F$ & $F$ & $F$ & $F$ & $F$ & & \\
\hline 134 & $T$ & & $F$ & $F$ & $F$ & $F$ & & & & & \\
\hline 135 & $T$ & & $F$ & $F$ & $\bar{F}$ & $F$ & & & & & \\
\hline 136 & $T$ & & $F$ & $F$ & $F$ & $F$ & & & & & \\
\hline 137 & $T$ & & $F$ & $F$ & $F$ & $\bar{F}$ & & & & & \\
\hline 138 & $T$ & & $F$ & $F$ & $F$ & $F$ & & & & & \\
\hline 139 & $T$ & & $F$ & $F$ & $F$ & $F$ & & & & & \\
\hline 140 & & & & & & & & & & & \\
\hline 141 & $T$ & & & & & & & & & & \\
\hline 142 & & $T$ & & & & & & & & & \\
\hline 143 & & & $T$ & & & & & & & & \\
\hline 144 & & & & & & & & & & & \\
\hline 145 & & $T$ & & & & & & & & & \\
\hline 146 & & $T$ & & & & & & & & & \\
\hline 147 & & & $T$ & & & & & & & & \\
\hline 148 & & $T$ & & & & & & & & & \\
\hline 149 & & & $T$ & & & & & & & & \\
\hline 150 & & & & & & & & & & & \\
\hline 151 & & & & & & & & & & & \\
\hline 152 & & & & & & & & & & & \\
\hline 153 & & & & & & & & & & & \\
\hline 154 & & & & & & & & & & & \\
\hline 155 & & & & & & & & & & & \\
\hline 156 & & & & & & & & & & & \\
\hline 157 & & & & & & & & & & & \\
\hline 158 & & & & & & & & & & & \\
\hline 159 & & & & & & & & & & & \\
\hline 160 & & & & & & & & & & & \\
\hline 161 & & & & & & & & & & & \\
\hline 162 & & & & & & & & & & & \\
\hline 163 & & & & & & & & $T$ & & & \\
\hline 164 & & & & & & & & & & & \\
\hline 165 & & & & & & & & & & & \\
\hline 166 & & & & & & & & & & & \\
\hline 167 & & & & & & & & & & & \\
\hline 168 & & & & & & & & & & & \\
\hline 169 & & & & & & & & & & & \\
\hline 170 & & & & & & & & & & & \\
\hline 171 & & & & & & & & & & & \\
\hline 172 & & & & & & & & & & & \\
\hline 173 & & & & & & & & & & & \\
\hline 174 & & & & & & & & & & & \\
\hline
\end{tabular}




\section{Audit of Capillary Pressure Model Params}

Map of Capillary Pressure Model Params

\begin{tabular}{|l|l|l|l|l|l|l|l|l|l|l|l|}
\hline & C & D & E & F & G & H & U & J & K & L \\
\hline 175 & & & & & & & & & & & \\
\hline 176 & & & & & & & & & & & \\
\hline 177 & & & & & & & & & & & \\
\hline 178 & & & & & & & & T & & & \\
\hline 179 & & & & & & & & & & & \\
\hline 180 & & & & & & & & & & & \\
\hline 181 & & & & & & & & & & & \\
\hline 182 & & & & & & & & & & & \\
\hline 183 & & & & & & & & & & & \\
\hline 184 & & & & & & & & & & & \\
\hline 185 & & & & & & & & & & & \\
\hline 186 & & & & & & & & & & & \\
\hline 187 & & & & & & & & & & & \\
\hline 188 & & & & & & & & & & & \\
\hline 189 & & & & & & & & & & & \\
\hline 190 & & & & & & & & & & & \\
\hline 191 & & & & & & & & & & & \\
\hline 192 & & & & & & & & & & & \\
\hline 193 & & & & & & & & T & & & \\
\hline 194 & & & & & & & & & & & \\
\hline 195 & & & & & & & & & & & \\
\hline 196 & & & & & & & & & & & \\
\hline 197 & & & & & & & & & & & \\
\hline 198 & & & & & & & & & & & \\
\hline 198 & & & & & & & & & & & \\
\hline 200 & & & & & & & & & & \\
\hline 201 & & & & & & & & & & \\
\hline 202 & & & & & & & & & & \\
\hline 203 & & & & & & & & & & \\
\hline 204 & & & & & & & & & & \\
\hline 205 & & & & & & & & & & \\
\hline 206 & & & & & & & & & & \\
\hline 207 & & & & & & & & & & \\
\hline 208 & & & & & & & T & & & \\
\hline 209 & & & & & & & & & & & \\
\hline 210 & & & & & & & & & & & \\
\hline 211 & & & & & & & & & & & \\
\hline 212 & & & & & & & & & & & \\
\hline 213 & & & & & & & & & & \\
\hline 214 & & & & & & & & & & & \\
\hline 215 & & & & & & & & & & \\
\hline 216 & & & & & & & & & & & \\
\hline 217 & & & & & & & & & & \\
\hline 218 & & & & & & & & & & \\
\hline 219 & & & & & & & & & & \\
\hline 220 & & & & & & & & & & & \\
\hline 221 & & & & & & & & & & & \\
\hline 222 & & & & & & & & & & \\
\hline 223 & & & & & & & & T & & & \\
\hline 224 & & & & & & & & & & & \\
\hline 225 & & & & & & & & & & & \\
\hline 226 & & & & & & & & & & & \\
\hline 227 & & & & & & & & & & & \\
\hline 228 & & & & & & & & & & & \\
\hline 229 & & & & & & & & & & & \\
\hline 231 & & & & & & & & & & & \\
\hline 232 & & & & & & & & & & & \\
\hline
\end{tabular}




\section{Audit of Capillary Pressure Model Params}

\begin{tabular}{|c|c|c|c|c|c|c|c|c|c|c|c|}
\hline Map & of $C$ & api & $\operatorname{ang}$ & Pre & $\mathrm{ssu}$ & $e M$ & ode & $P a$ & rams & & \\
\hline & 8 & $c$ & 0 & $E$ & $F$ & $\bar{G}$ & $\mathrm{H}$ & 1 & J & $K$ & $L$ \\
\hline 233 & & & & & & & & & & & \\
\hline 234 & & & & & & & & & & & \\
\hline 235 & & & & & & & & & & & \\
\hline 236 & & & & & & & & & & & \\
\hline 237 & & & & & & & & & & & \\
\hline 238 & & & & & & & & $T$ & 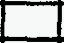 & & \\
\hline 239 & & & & & & & & - & & & \\
\hline 240 & & & & & & & & & & & \\
\hline 241 & & & & & & & & 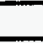 & 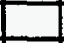 & & \\
\hline 242 & & & & & & & & & & & \\
\hline 243 & & & & & & & & & & & \\
\hline 244 & & & & & & & & & & & \\
\hline 245 & & & & & & & & & & & \\
\hline 246 & & & & & & & & & & & \\
\hline 247 & & & & & & & & & & & \\
\hline 248 & & & & & & & & & 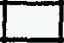 & & \\
\hline 249 & & & & & & & & & & & \\
\hline 250 & & & & & & & & & & & \\
\hline 251 & & & & & & & & & & & \\
\hline 252 & & & & & . & & & & & & \\
\hline 253 & & & & & & & & & & & \\
\hline 254 & & & & & & & & & & & \\
\hline 255 & & & & & & & & & & & \\
\hline 256 & & & & & & & & & & & \\
\hline 257 & & & & & & & & & & & \\
\hline 258 & & & & & & & & & & & \\
\hline 259 & & & & & & & & & & & \\
\hline 260 & & & & & & & & . & 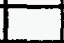 & & \\
\hline 261 & & & & & & & & & & & \\
\hline 262 & & & & & & & & & & & \\
\hline 263 & & & & & & & & & & & \\
\hline 264 & & & & & & & & & 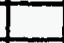 & & \\
\hline 265 & & & & & & & & & & & \\
\hline 266 & & & & & & & & & & & \\
\hline 267 & & & & & & & & & $E$ & - & \\
\hline 268 & & & & & & & & & & & \\
\hline 269 & & & & & & & & & . & & \\
\hline 270 & & & & & & & 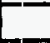 & & 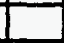 & & \\
\hline 271 & & & & & & & - & & $E$ & $\bar{Z}$ & \\
\hline 272 & & & & & & & . & & 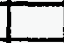 & 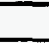 & \\
\hline 273 & & & & & & & & & & & \\
\hline 274 & & & & & & & & & & & \\
\hline 275 & & & & & & & & & & & \\
\hline 276 & & & & & & & 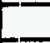 & & & 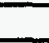 & \\
\hline 277 & & & & & & & & & 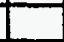 & 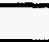 & \\
\hline 278 & & & & & & & & & $E$ & 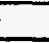 & \\
\hline 279 & & & & & & & & & - & & \\
\hline 280 & & & & & & & & & & & \\
\hline 281 & & & & & & & & & & & \\
\hline 282 & & & & & & & 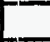 & & - & 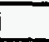 & \\
\hline 283 & & & & & & & & & & & \\
\hline 284 & & & & & & & 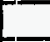 & & - & 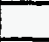 & \\
\hline 285 & & & & & & & & & & & \\
\hline 286 & & & & & & & & & & & \\
\hline 287 & & & & & & & & & & & \\
\hline $28 B$ & & & & & & & & & & & \\
\hline 289 & & & & & & & & & 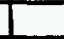 & 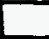 & \\
\hline 290 & & & & & & & - & 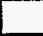 & - & 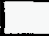 & \\
\hline
\end{tabular}




\section{Audit of Capillary Pressure Model Params}

Map of Capillary Pressure Model Params

\begin{tabular}{|l|l|l|l|l|l|l|l|l|l|l|l|}
\hline & B & C & D & E & F & G & H & I & J & K & L \\
\hline 291 & T & & & & & & & & & & \\
\hline 292 & & & & & & & & & & & \\
\hline 293 & T & & & & & & & & & & \\
\hline 294 & & & & & & & & & & & \\
\hline 295 & & & & & & & & & & & \\
\hline 296 & & & & & & & & & & & \\
\hline 297 & & & & & & & & & & & \\
\hline 298 & & & & & & & & & & & \\
\hline 299 & & & & & & & & & & & \\
\hline 300 & & & & & & & & & & & \\
\hline 301 & & & & & & & & & & & \\
\hline 302 & & & & & & & & & & & \\
\hline 303 & & & & & & & & & & & \\
\hline 304 & & & & & & & & & & & \\
\hline 305 & & & & & & & & & & & \\
\hline 306 & & & & & & & & & & \\
\hline 307 & & & & & & & & & & & \\
\hline 308 & & & & & & & & & & & \\
\hline 309 & & & & & & & & & & & \\
\hline 310 & & & & & & & & & & & \\
\hline 311 & & & & & & & & & & & \\
\hline 312 & & & & & & & & & & & \\
\hline 313 & & & & & & & & & & & \\
\hline 314 & & & & & & & & & & & \\
\hline 315 & & & & & & & & & & & \\
\hline 316 & & & & & & & & & & & \\
\hline 317 & & & & & & & & & & & \\
\hline 318 & & & & & & & & & & \\
\hline 319 & & & & & & & & & \\
\hline 320 & & & & & & & & & \\
\hline 321 & & & & & & & & & \\
\hline 322 & & & & & & & & & & \\
\hline 323 & & & & & & & & & & \\
\hline 324 & & & & & & & & & & & \\
\hline 325 & & & & & & & & & & & \\
\hline 326 & & & & & & & & & & & \\
\hline 327 & & & & & & & & & & & \\
\hline 328 & & & & & & & & & & & \\
\hline 329 & & & & & & & & & & & \\
\hline 330 & & & & & & & & & & & \\
\hline 331 & & & & & & & & & & & \\
\hline 332 & & & & & & & & & & & \\
\hline 333 & & & & & & & & & & & \\
\hline 334 & & & & & & & & & & \\
\hline 335 & & & & & & & & & & \\
\hline 336 & & & & & & & & & & \\
\hline 337 & & & & & & & & & & & \\
\hline 338 & T & & & & & & & & & & \\
\hline 339 & & & & & & & & & & & \\
\hline 340 & & & & & & & & & & & \\
\hline 341 & & & & & & & & & & & \\
\hline 342 & & & & & & & & & & & \\
\hline 343 & & & & & & & & & & & \\
\hline 344 & & & & & & & & & & & \\
\hline 346 & & & & & & & & & & & \\
\hline 348 & & & & & & & & & & & \\
\hline
\end{tabular}




\section{Audit of Capillary Pressure Model Params}

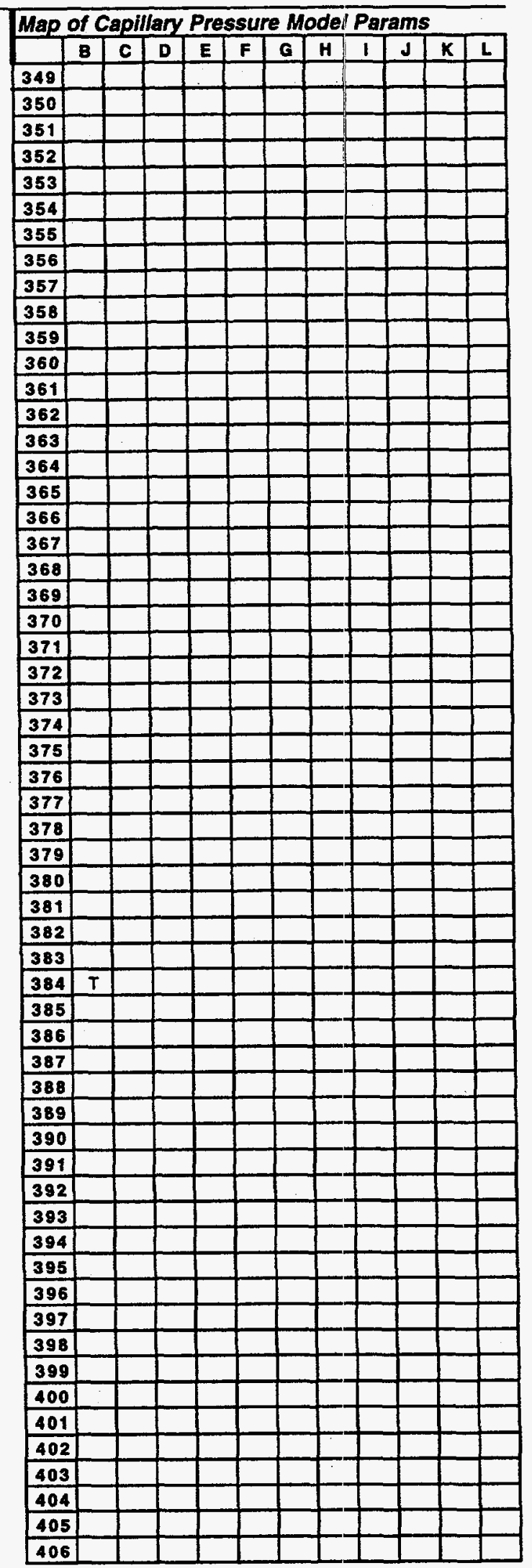




\section{Audit of Capillary Pressure Model Params}

Map of Capillary Pressure Model Params

\begin{tabular}{|l|l|l|l|l|l|l|l|l|l|l|l|}
\hline & O & C & E & F & G & H & I & J & K & $L$ \\
\hline 407 & & & & & & U & & & & & \\
\hline 408 & & & & & & & & & & & \\
\hline 409 & & & & & & & & & & & \\
\hline 410 & & & & & & & & & & & \\
\hline 411 & & & & & & & & & & & \\
\hline 412 & & & & & & & & & & & \\
\hline 413 & & & & & & & & & & & \\
\hline 414 & & & & & & & & & & & \\
\hline 415 & & & & & & & & & & & \\
\hline 416 & & & & & & & & & & & \\
\hline 417 & & & & & & & & & & & \\
\hline 418 & & & & & & & & & & & \\
\hline 419 & & & & & & & & & & & \\
\hline 420 & & & & & & & & & & & \\
\hline 421 & & & & & & & & & & & \\
\hline 422 & & & & & & & & & & & \\
\hline 423 & & & & & & & & & & & \\
\hline 424 & & & & & & & & & & & \\
\hline 425 & & & & & & & & & & & \\
\hline 426 & & & & & & & & & & & \\
\hline 427 & & & & & & & & & & & \\
\hline 428 & & & & & & & & & & & \\
\hline 429 & & & & & & & & & & & \\
\hline 430 & T & & & & & & & & & & \\
\hline 431 & & & & & & & & & & & \\
\hline 432 & & & & & & & & & & & \\
\hline 433 & & & & & & & & & & \\
\hline 434 & & & & & & & & & & & \\
\hline 435 & & & & & & & & & & \\
\hline 436 & & & & & & & & & & \\
\hline 437 & & & & & & & & & & & \\
\hline 438 & & & & & & & & & & \\
\hline 439 & & & & & & & & & & \\
\hline 440 & & & & & & & & & & & \\
\hline 441 & & & & & & & & & & & \\
\hline 442 & & & & & & & & & & & \\
\hline 443 & & & & & & & & & & & \\
\hline 444 & & & & & & & & & & & \\
\hline 445 & & & & & & & & & & \\
\hline 446 & & & & & & & & & & & \\
\hline 447 & & & & & & & & & & \\
\hline 448 & & & & & & & & & & & \\
\hline 449 & & & & & & & & & & \\
\hline 450 & & & & & & & & & & & \\
\hline 451 & & & & & & & & & & & \\
\hline 452 & & & & & & & & & & & \\
\hline 453 & & & & & & & & & & & \\
\hline 454 & & & & & & & & & & & \\
\hline 455 & & & & & & & & & & & \\
\hline 456 & & & & & & & & & & & \\
\hline 457 & & & & & & & & & & & \\
\hline 458 & & & & & & & & & & & \\
\hline 459 & & & & & & & & & & & \\
\hline 460 & & & & & & & & & & & \\
\hline 461 & & & & & & & & & & \\
\hline 462 & & & & & & & & & & & \\
\hline 469 & & & & & & & & & & & \\
\hline
\end{tabular}

Attachment 1

E-41

SWCF-A:1.2.07.1:PDD:QA:SALADO:PKG 10:Anh 2-Phase Parameters 


\section{Audit of Capillary Pressure Model Params}

Map of Capillany Pressure Model Params

\begin{tabular}{|c|c|c|c|c|c|c|c|c|c|c|c|}
\hline & 8 & C & D & $E$ & $\mathbf{F}$ & $\mathbf{a}$ & $\mathrm{H}$ & 1 & $J$ & $\mathbf{K}$ & L \\
\hline 465 & & & & & & & & & & & \\
\hline 466 & & & & & & & & & & & \\
\hline 467 & & & & & & & & & & & \\
\hline 468 & & & & & & & & & & & \\
\hline 469 & & & & & & & & & & & \\
\hline 470 & & & & & & & & & & & \\
\hline 471 & & & & & & & & & & & \\
\hline 472 & & & & & & & & & & & \\
\hline 473 & & & & & & & & & & & \\
\hline 474 & & & & & & & & & & & \\
\hline 475 & & & & & & & & & & & \\
\hline 476 & $T$ & & & & & & & & & & \\
\hline 477 & & & & & & & & & & & \\
\hline 478 & & & & & & & & & & & \\
\hline 479 & & & & & & & & & & & \\
\hline 480 & & & & & & & & & & & \\
\hline 481 & & & & & & & & & & & \\
\hline 482 & & & & & & & & & & & \\
\hline 483 & & & & & & & & & & & \\
\hline 484 & & & & & & & & & & & \\
\hline 485 & & & & & & & & & & & \\
\hline 486 & & & & & & & & & & & \\
\hline $4 B 7$ & & & & & & & & & & & \\
\hline 488 & & & & & & & & & & & \\
\hline 489 & & & & & & & & & & & \\
\hline 490 & & & & & & & & & & & \\
\hline 491 & & & & & & & & & & & \\
\hline 492 & & & & & & & & & & & \\
\hline 493 & & & & & & & & & & & \\
\hline 494 & & & & & & & & & & & \\
\hline 495 & & & & & & & & & & & \\
\hline 496 & & & & & & & & & & & \\
\hline 497 & & & & & & & & & & & \\
\hline 498 & & & & & & & & & & & \\
\hline 499 & & & & & & & & & & & \\
\hline 500 & & & & & & & & & & & \\
\hline 501 & & & & & & & & & & & \\
\hline 502 & & & & & & & & & & & \\
\hline 503 & & & & & & & & & & & \\
\hline 504 & & & & & & & & & & & \\
\hline 505 & & & & & & & & & & & \\
\hline 506 & & & & & & & & & & & \\
\hline 507 & & & & & & & & & & & \\
\hline 508 & & & & & & & & & & & \\
\hline 509 & & & & & & & & & & & \\
\hline 510 & & & & & & & & & & & \\
\hline 511 & & & & & & & & & & & \\
\hline 512 & & & & & & & & & & & \\
\hline 513 & & & & & & & & & & & \\
\hline 514 & & & & & & & & & & & \\
\hline 515 & & & & & & & & & & & \\
\hline 516 & & & & & & & & & & & \\
\hline 517 & & & & & & & & & & & \\
\hline 518 & & & & & & & & & & & \\
\hline 519 & & & & & & & & & & & \\
\hline 520 & & & & & & & & & & & \\
\hline 521 & & & & & & & & & & & \\
\hline 522 & & & & & & & & & & & \\
\hline
\end{tabular}




\section{Audit of Capillary Pressure Model Params}

\begin{tabular}{|c|c|c|c|c|c|c|c|c|c|c|c|}
\hline \multicolumn{12}{|c|}{ Map of Capillary Pressure Model Params } \\
\hline & & $\mathrm{c}$ & $\mathrm{D}$ & $E$ & $F$ & $G$ & $\mathrm{H}$ & & & $K$ & \\
\hline 523 & $T$ & & & & & & & & & & \\
\hline 524 & & & & & & & & & & & \\
\hline 525 & & & & & & & & & & & \\
\hline 526 & & & & & & & & & & & \\
\hline 527 & & & & & & & & & & & \\
\hline 528 & & & & & & & & & & & \\
\hline 529 & & & & & & & & & & & \\
\hline 530 & & & & & & & & & & & \\
\hline 531 & & & & & & & & & & & \\
\hline 532 & & & & & & & & & & & \\
\hline 533 & & & & & & & & & & & \\
\hline 534 & & & & & & & & & & & \\
\hline 535 & & & & & & & & & $\ldots$ & 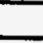 & \\
\hline 536 & & & & & & & & & & & \\
\hline 537 & & & & & & & & & 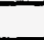 & 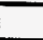 & \\
\hline 538 & & & & & & & & & & & \\
\hline 539 & & & & & & & & & L & . & \\
\hline 540 & & & & & & & & & & & \\
\hline 541 & & & & & & & & & . & & \\
\hline 542 & & & & & & & & & & & \\
\hline 543 & & & & & & & & & & & \\
\hline 544 & & & & & & & & & & & \\
\hline 545 & & & & & & & & & & & \\
\hline 546 & & & & & & & & & 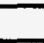 & & \\
\hline 547 & & & & & & & & & - & & \\
\hline 548 & & & & & & & & & 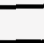 & & \\
\hline 549 & & & & & & & & & $E$ & & \\
\hline 550 & & & & & & & 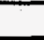 & & 7 & 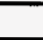 & \\
\hline 531 & & & & & & & & & 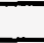 & & \\
\hline 552 & & & & & & & & & 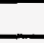 & 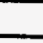 & \\
\hline 553 & & & & & & & & & 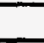 & & \\
\hline 554 & & & & & 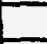 & & & & 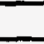 & & \\
\hline 555. & $T$ & & & & & & & & 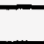 & & \\
\hline 556 & & & & & & & & & 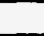 & & \\
\hline 557 & & & & & & & & & & - & \\
\hline 558 & & & & & & & & & & & \\
\hline 559 & & & & & & & & & 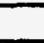 & & \\
\hline 560 & & & & & & & & & & & \\
\hline 561 & & & & & & & & & & & \\
\hline 562 & & & & & & & & & & & \\
\hline 563 & & & & & & 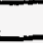 & & & 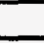 & 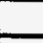 & \\
\hline 564 & & & & & & & & & 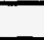 & & \\
\hline 565 & & & & & & & & . & 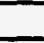 & & \\
\hline 566 & & & & & & & & & & & \\
\hline 567 & & & & & & . & 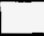 & & . & & \\
\hline 568 & & & & & & & & & & & \\
\hline 569 & $T$ & & & & & & & & & & \\
\hline 570 & & & & & & & & & & & \\
\hline 571 & & & & & & & & & . & & \\
\hline 572 & & & & & & & & & . & & \\
\hline 573 & & & & & & - & & & - & 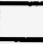 & \\
\hline 574 & & & & & & & & & & & \\
\hline 575 & & & & & & & & & & & \\
\hline 576 & & & & & & & & & & & \\
\hline 577 & & & & & & & & & & & \\
\hline 578 & & & & & & & & & - & 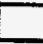 & \\
\hline 579 & & & & & & & & & 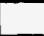 & & \\
\hline 580 & & & & & & & & & & & \\
\hline
\end{tabular}




\section{Audit of Capillary Pressure Model Params}

Map of Caplllary Pressure Model Params

\begin{tabular}{|c|c|c|c|c|c|c|c|c|c|c|c|}
\hline & $\mathrm{B}$ & C & $\mathbf{D}$ & $E$ & $F$ & a & $\mathrm{H}$ & 1 & $J$ & $K$ & $L$ \\
\hline 581 & & & & & & & & & & & \\
\hline 582 & & & & & & & & & & & \\
\hline 583 & & & & & & & & & & & \\
\hline 584 & & & & & & & & & & & \\
\hline 585 & & & & & & & & & & & \\
\hline 586 & & & & & & & & & & & \\
\hline 587 & & & & & & & & & & & \\
\hline 588 & & & & & & & & & & & \\
\hline 589 & & & & & & & & & & & \\
\hline 590 & & & & & & & & & & & \\
\hline 591 & & & & & & & & & & & \\
\hline 592 & & & & & & & & & & & \\
\hline 593 & & & & & & & & & & & \\
\hline 594 & & & & & & & & & & & \\
\hline 595 & & & & & & & & & & & \\
\hline 596 & & & & & & & & & & & \\
\hline 597 & & & & & & & & & & & \\
\hline 598 & & & & & & & & & & & \\
\hline 599 & & & & & & & & & & & \\
\hline 600 & & & & & & & & & & & \\
\hline 501 & & & & & & & & & & & \\
\hline 602 & & & & & & & & & & & \\
\hline 603 & & & & & & & & & & & \\
\hline 604 & & & & & & & & & & & \\
\hline 605 & & & & & & & & & & & \\
\hline 606 & & & & & & & & & & & \\
\hline 607 & & & & & & & & & & & \\
\hline 608 & & & & & & & & & & & \\
\hline 609 & & & & & & & & & & & \\
\hline 610 & & & & & & & & & & & \\
\hline 611 & & & & & & & & & & & \\
\hline 612 & & & & & & & & & & & \\
\hline 613 & & & & & & & & & & & \\
\hline 614 & & & & & & & & & & & \\
\hline 615 & $T$ & & & & & & & & & & \\
\hline 616 & & & & & & & & & & & \\
\hline 617 & & & & & & & & & & & \\
\hline 618 & & & & & & & & & & & \\
\hline 610 & & & & & & & & & & & \\
\hline 620 & & & & & & & & & & & \\
\hline 621 & & & & & & & & & & & \\
\hline 622 & & & & & & & & & & & \\
\hline 623 & & & & & & & & & & & \\
\hline 624 & & & & & & & & & & & \\
\hline 625 & & & & & & & & & & & \\
\hline 626 & & & & & & & & & & & \\
\hline 627 & & & & & & & & & & & \\
\hline 628 & & & & & & & & & & & \\
\hline 629 & & & & & & & & & & & \\
\hline 630 & & & & & & & & & & & \\
\hline 631 & & & & & & & & & & & \\
\hline 632 & & & & & & & & & & & \\
\hline 633 & & & & & & & & & & & \\
\hline 634 & & & & & & & & & & & \\
\hline 635 & & & & & & & & & & & \\
\hline 636 & & & & & & & & & & & \\
\hline 637 & & & & & & & & & & & \\
\hline 638 & & & & & & & & & & & \\
\hline
\end{tabular}




\section{Audit of Capillary Pressure Model Params}

\section{Map of Capillary Pressure Model Params}

\begin{tabular}{|c|c|c|c|c|c|c|c|c|c|c|}
\hline & B & C & D & $E$ & $F$ & G & H & \begin{tabular}{l|l}
1 & $\mathrm{~J}$ \\
\end{tabular} & $\mathbf{K}$ & $L$ \\
\hline 639 & & & & & & & & & & \\
\hline 640 & & & & & & & & & & \\
\hline 641 & & & & & & & & & & \\
\hline 642 & & & & & & & & & & \\
\hline 643 & & & & & & & & & & \\
\hline 644 & & & & & & & & & & \\
\hline 645 & & & & & & & & & & \\
\hline 646 & & & & & & & & & & \\
\hline 647 & & & & & & & & & & \\
\hline 648 & & & & & & & & & & \\
\hline 648 & & & & & & & & & & \\
\hline 650 & & & & & & & & & & \\
\hline 651 & & & & & & & & & & \\
\hline 652 & & & & & & & & & & \\
\hline 653 & & & & & & & & & & \\
\hline 654 & & & & & & & & & & \\
\hline 655 & & & & & & & & & & \\
\hline 656 & & & & & & & & & & \\
\hline 657 & & & & & & & & & & \\
\hline 658 & & & & & & & & & & \\
\hline 659 & & & & & & & & & & \\
\hline 660 & & & & & & & & & & \\
\hline 661 & $I$ & & & & & & & & & \\
\hline 6.62 & & & & & & & & & & \\
\hline 663 & & & & & & & & & & \\
\hline 664 & & & & & & & & & & \\
\hline 665 & & & & & & & & & & \\
\hline 666 & & & & & & & & & & \\
\hline 667 & & & & & & & & & & \\
\hline 668 & & & & & & & & & & \\
\hline 669 & & & & & & & & & & \\
\hline 670 & & & & & & & & & & \\
\hline 671 & & & & & & & & & & \\
\hline 672 & & & & & & & & & & \\
\hline 673 & & & & & & & & & & \\
\hline 674 & & & & & & & & & & \\
\hline 675 & & & & & & & & & & \\
\hline 676 & & & & & & & & & & \\
\hline 677 & & & & & & & & & & \\
\hline 678 & & & & & & & & & & \\
\hline 679 & & & & & & & & & & \\
\hline 680 & & & & & & & & & & \\
\hline 681 & & & & & & & & & & \\
\hline 682 & & & & & & & & & & \\
\hline 683 & & & & & & & & & & \\
\hline 684 & & & & & & & & & & \\
\hline 685 & & & & & & & & & & \\
\hline 686 & & & & & & & & & & \\
\hline 687 & & & & & & & & & & \\
\hline 688 & & & & & & & & & & \\
\hline 689 & & & & & & & & & & \\
\hline 690 & & & & & & & & & & \\
\hline 691 & & & & & & & & & & \\
\hline 692 & & & & & & & & & & \\
\hline 693 & & & & & & & & & & \\
\hline 694 & & & & & & & & & & \\
\hline 695 & & & & & & & & & & \\
\hline 696 & & & & & & & & & & \\
\hline
\end{tabular}




\section{Audit of Capillary Pressure Model Params}

\begin{tabular}{|l|l|l|l|l|l|l|l|l|l|l|l|}
\hline Map of Capilary Pressure Model Params \\
\hline & B & C & O & E & F & G & H & I & J & K & L \\
\hline 698 & & & & & & & & & & & \\
\hline 699 & & & & & & & & & & & \\
\hline 700 & & & & & & & & & & & \\
\hline 701 & & & & & & & & & & & \\
\hline 702 & & & & & & & & & & & \\
\hline 703 & & & & & & & & & & & \\
\hline 704 & & & & & & & & & & & \\
\hline 705 & & & & & & & & & & & \\
\hline 706 & & & & & & & & & & & \\
\hline 707 & T & & & & & & & & & & \\
\hline 708 & & & & & & & & & & & \\
\hline 709 & & & & & & & & & & & \\
\hline 710 & & & & & & & & & & & \\
\hline 711 & & & & & & & & & & & \\
\hline 712 & & & & & & & & & & & \\
\hline 713 & & & & & & & & & & & \\
\hline 714 & & & & & & & & & & & \\
\hline 715 & & & & & & & & & & & \\
\hline 716 & & & & & & & & & & & \\
\hline 717 & & & & & & & & & & & \\
\hline 718 & & & & & & & & & & & \\
\hline 719 & & & & & & & & & & & \\
\hline 720 & & & & & & & & & & & \\
\hline 721 & & & & & & & & & & & \\
\hline 722 & & & & & & & & & & & \\
\hline 723 & & & & & & & & & & & \\
\hline 724 & & & & & & & & & & \\
\hline 725 & & & & & & & & & \\
\hline 726 & & & & & & & & & & \\
\hline 727 & & & & & & & & & & \\
\hline 728 & & & & & & & & & & \\
\hline 729 & & & & & & & & & & & \\
\hline 730 & & & & & & & & & & & \\
\hline 731 & & & & & & & & & & & \\
\hline 732 & & & & & & & & & & & \\
\hline 733 & & & & & & & & & & & \\
\hline 734 & & & & & & & & & & & \\
\hline 735 & & & & & & & & & & & \\
\hline 736 & & & & & & & & & & & \\
\hline 737 & & & & & & & & & & & \\
\hline 738 & & & & & & & & & & & \\
\hline 739 & & & & & & & & & & & \\
\hline 740 & & & & & & & & & & & \\
\hline 741 & & & & & & & & & & & \\
\hline 742 & & & & & & & & & & & \\
\hline 743 & & & & & & & & & & & \\
\hline 744 & & & & & & & & & & & \\
\hline 745 & & & & & & & & & & & \\
\hline 746 & & & & & & & & & & & \\
\hline 748 & & & & & & & & & & & \\
\hline 759 & & & & & & & & & & & \\
\hline 750 & & & & & & & & & & & \\
\hline
\end{tabular}




\section{Audit of Capillary Pressure Model Params}

\section{Map of Capillary Pressure Model Params}

\begin{tabular}{|c|c|c|c|c|c|c|c|c|c|c|c|}
\hline & 8 & c & D & $\bar{E}$ & $F$ & G & $H$ & 1 & $\mathrm{~J}$ & $\mathbf{K}$ & $L$ \\
\hline 755 & & & & & & & & & & & \\
\hline 756 & & & & & & & & & & & \\
\hline 757 & & & & & & & & & & & \\
\hline 758 & & & & & & & & & & & \\
\hline 759 & & & & & & & & & & & \\
\hline 760 & & & & & & & & & & & \\
\hline 761 & & & & & & & & & & & \\
\hline 762 & & & & & & & & & & & \\
\hline 763 & & & & & & & & & & & \\
\hline 764 & & & & & & & & & & & \\
\hline 765 & & & & & & & & & & & \\
\hline 766 & & & & & & & & & & & \\
\hline 767 & & & & & & & & & & & \\
\hline 768 & & & & & & & & & & & \\
\hline 769 & & & & & & & & & & & \\
\hline 770 & & & & & & & & & & & \\
\hline 771 & & & & & & & & & & & \\
\hline 772 & & & & & & & & & & & \\
\hline 773 & & & & & & & & & & & \\
\hline 774 & & & & & & & & & & & \\
\hline 775 & & & & & & & & & & & \\
\hline 776 & & & & & & & & & & & \\
\hline 777 & & & & & & & & & & & \\
\hline 778 & & & & & & & & & & & . \\
\hline 779 & & & & & & & & & & & \\
\hline 780 & & & & & & & & & & & \\
\hline 781 & & & & & & & & & & & \\
\hline 782 & & & & & & & & & & & \\
\hline 783 & & & & & & & & & & & \\
\hline 784 & & & & & & & & & & & \\
\hline 785 & & & & & & & & & & & \\
\hline 786 & & & & & & & & & & & \\
\hline 787 & & & & & & & & & & & \\
\hline 788 & & & & & & & & & & & \\
\hline 789 & & & & & & & & & & & \\
\hline 790 & & & & & & & & & & & \\
\hline 791 & & & & & & & & & & & \\
\hline 792 & & & & & & & & & & & \\
\hline 793 & & & & & & & & & & & \\
\hline 794 & & & & & & & & & & & \\
\hline 795 & & & & & & & & & & & \\
\hline 796 & & & & & & & & & & & \\
\hline 797 & & & & & & & & & & & \\
\hline 798 & & & & & & & & & & & \\
\hline 799 & $T$ & & & & & & & & & & \\
\hline 800 & & & & & & & & & & & \\
\hline 801 & & & & & & & & & & & \\
\hline 802 & & & & & & & & & & & \\
\hline 803 & & & & & & & & & & & \\
\hline 804 & & & & & & & & & & & \\
\hline 805 & & & & & & & & & & & \\
\hline 806 & & & & & & & & & & & \\
\hline 807 & & & & & & & & & & & \\
\hline 808 & & & & & & & & & & & \\
\hline 809 & & & & & & & & & & & \\
\hline 810 & & & & & & & & & & & \\
\hline 811 & & & & & & & & & & & \\
\hline 812 & & & & & & & & & & & \\
\hline
\end{tabular}




\section{Audit of Capillary Pressure Model Params}

\begin{tabular}{|l|l|l|l|l|l|l|l|l|l|l|l|}
\hline \multicolumn{10}{|c|}{ Map of Caplliary Pressure Model Params } \\
\hline & B & C & D & E & F & G & H & I & J & K & L \\
\hline 813 & & & & & & & & & & & \\
\hline 814 & & & & & & & & & & & \\
\hline 815 & & & & & & & & & & & \\
\hline 816 & & & & & & & & & & & \\
\hline 817 & & & & & & & & & & & \\
\hline 818 & & & & & & & & & & & \\
\hline 819 & & & & & & & & & & & \\
\hline 820 & & & & & & & & & & & \\
\hline 821 & & & & & & & & & & & \\
\hline 822 & & & & & & & & & & & \\
\hline 823 & & & & & & & & & & & \\
\hline 824 & & & & & & & & & & & \\
\hline 825 & & & & & & & & & & & \\
\hline 826 & & & & & & & & & & & \\
\hline 827 & & & & & & & & & & & \\
\hline 828 & & & & & & & & & & & \\
\hline 829 & T & & & & & & & & & & \\
\hline 830 & T & & & & & & & & & & \\
\hline
\end{tabular}




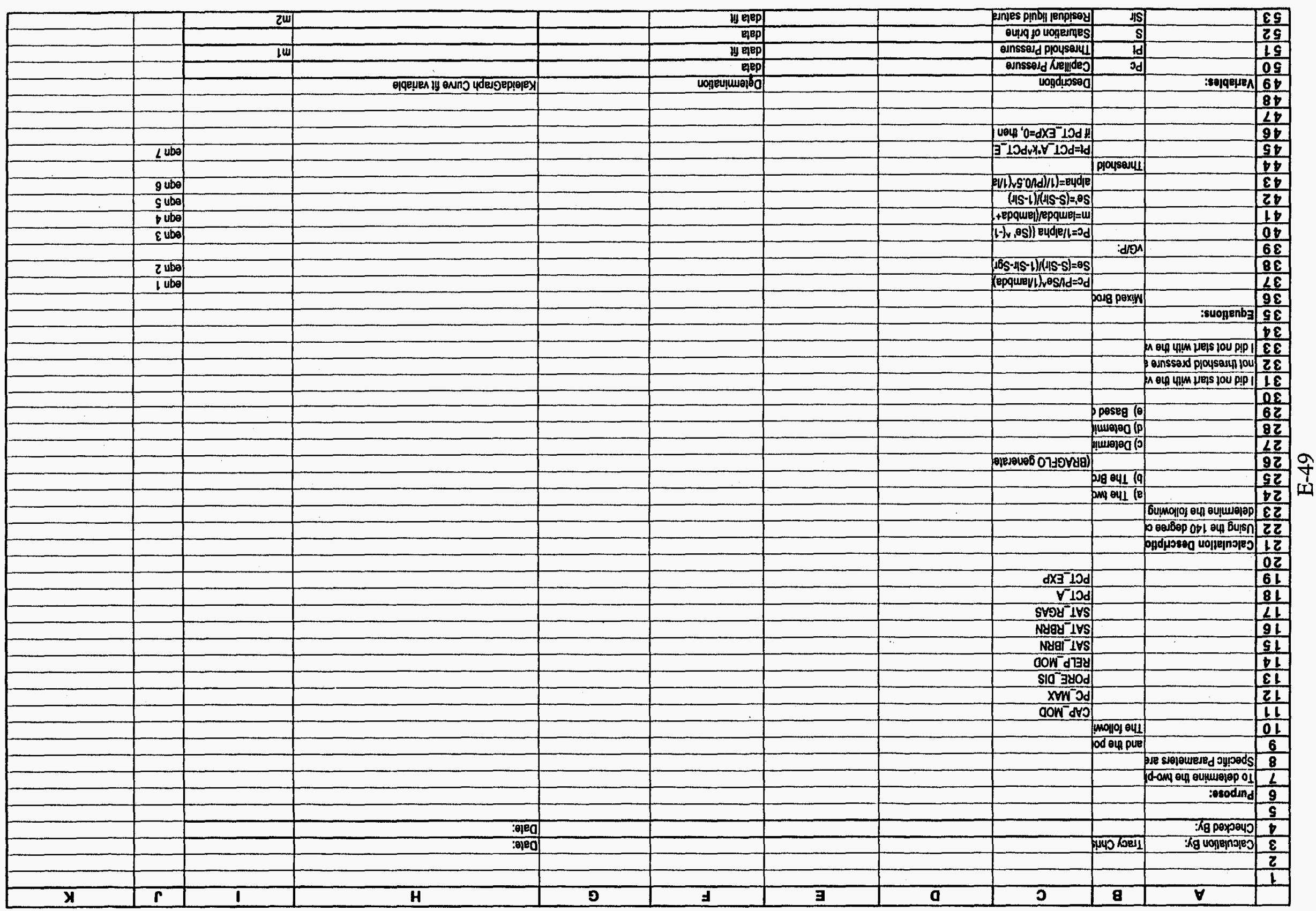

Irontor 

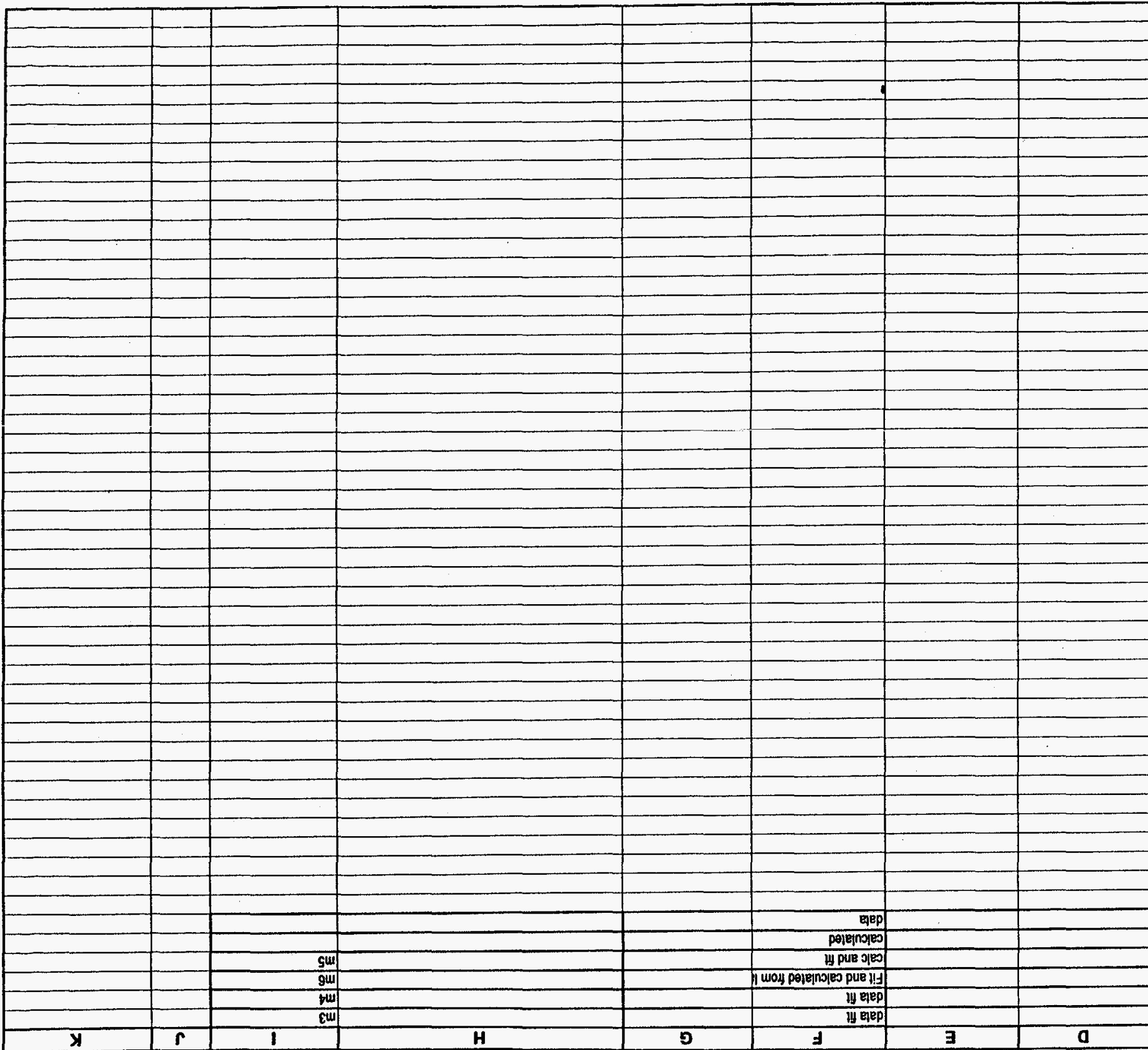

\begin{tabular}{|c|c|c|c|}
\hline \multirow{3}{*}{ 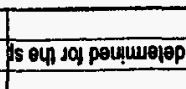 } & & & 901 \\
\hline & & & sol \\
\hline & $5+10 \ln \theta 6]$ & & 601 \\
\hline & $18 \operatorname{senn} 6 y$ & & 801 \\
\hline & $5 \operatorname{son} 6 y$ & & 201 \\
\hline & $\varepsilon \operatorname{sen} 6 \theta t$ & & 101 \\
\hline & $18 z$ oun $6 \mathrm{y}$ & & 001 \\
\hline & - 1 enn 6 & & 66 \\
\hline & Pein MO45 & & 86 \\
\hline & Mquyap ON & & $\angle 6$ \\
\hline & & & 96 \\
\hline & enno ype & & 96 \\
\hline & senjen a4l & :uagnjos & $\$ 6$ \\
\hline & Henb3 $\cdot 14$ & & 86 \\
\hline & founs of & & 26 \\
\hline & H4 OAnO 6 & & 16 \\
\hline & 6 6uls? 8 & & 06 \\
\hline & 2 Guss 2 & & 68 \\
\hline & 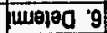 & & 88 \\
\hline 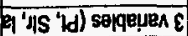 & tulemo $\mathrm{s}$ & & $\angle 8$ \\
\hline & ound in & & 98 \\
\hline bue dS e ye6 nol gun & & & 98 \\
\hline & 40040 & & 78 \\
\hline yod jes mol ouo pue & & & 88 \\
\hline 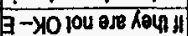 & & & 28 \\
\hline 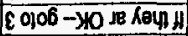 & & & 18 \\
\hline & $3 \times 190432$ & & 08 \\
\hline Es sn od uodn peseq & & & 61 \\
\hline 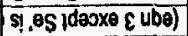 & & & $8 L$ \\
\hline & 4 Bussn in & & $\angle L$ \\
\hline & is & & 92 \\
\hline & 24p peypos & & SL \\
\hline & & $: 85050 d_{d}$ & $T L$ \\
\hline & & & $\varepsilon L$ \\
\hline & pur syoosg & & $2 L$ \\
\hline & tapiouselut & & $I L$ \\
\hline & injes panbu! & & $O L$ \\
\hline & องाम ब्या (9) & & 69 \\
\hline 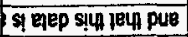 & & & 89 \\
\hline & 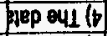 & & $\angle 9$ \\
\hline & Bep opl (E & & 99 \\
\hline & m sesoo (2) & & 99 \\
\hline & pinoosis & & 69 \\
\hline & sneseg & & 89 \\
\hline & 100 84! (1) & & 29 \\
\hline & & :suondunssy & 19 \\
\hline & & & 09 \\
\hline A.119e日uسed & $y$ & & 69 \\
\hline 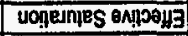 & es pue $\theta$ & & 85 \\
\hline 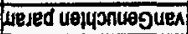 & eqdif & & $\angle G$ \\
\hline Lejed uejuonuaguen & $w$ & & 95 \\
\hline 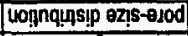 & epquiet & & SS \\
\hline ngejnjes se6 /enplsay & $16 \mathrm{~S}$ & & 79 \\
\hline 0 & $\mathbf{a}$ & $\forall$ & \\
\hline
\end{tabular}

Brrwusas 


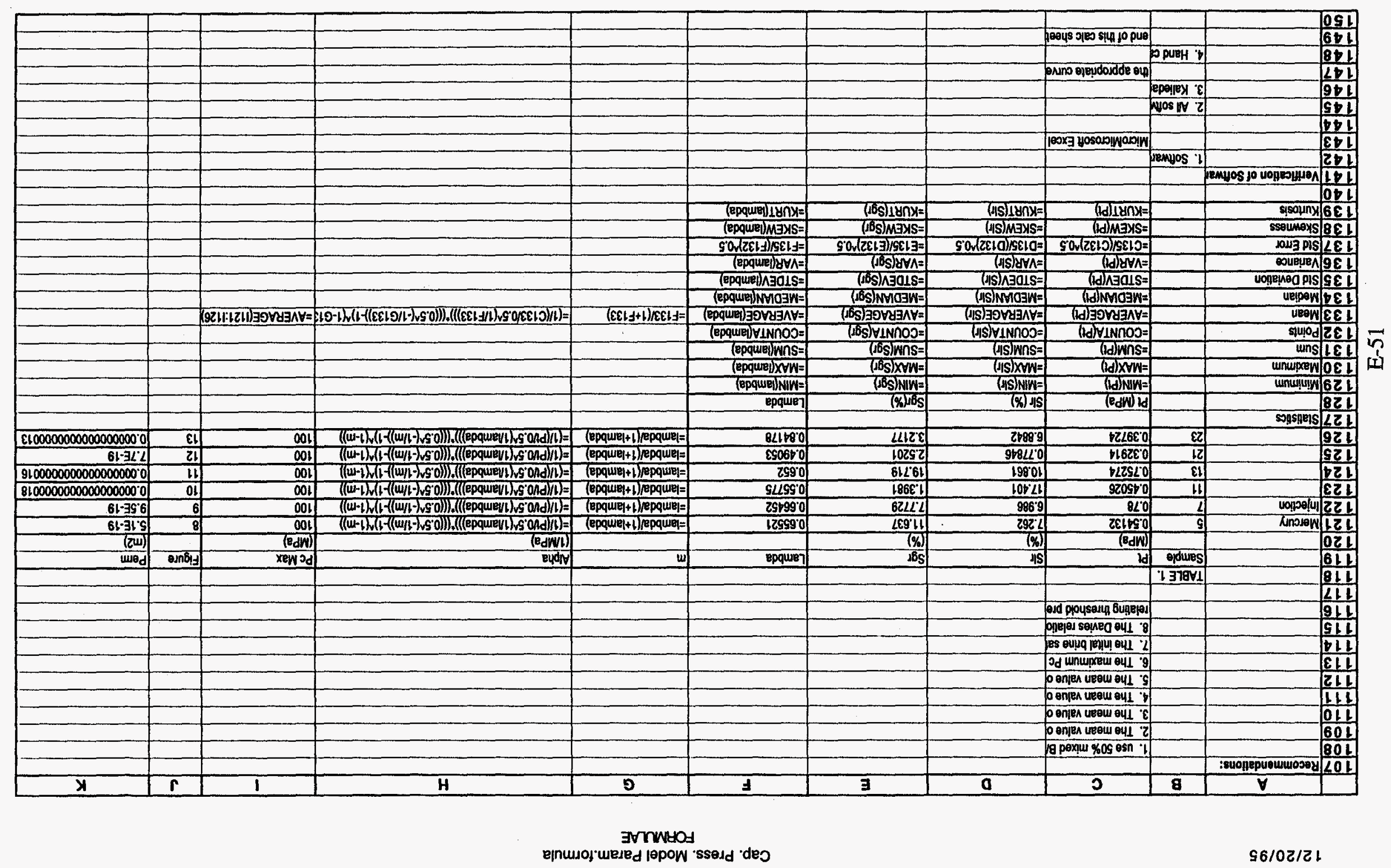




\section{RECORD 3}

General Correspondence

E-52 


\section{RECORD 4}

Technical Review of Record Package

E-53 


\section{Technical Review of Record Package}

\section{SALADO PARAMETER PRINCIPAL INVESTIGATOR DOCUMENTATION PACKAGE FOR: ANHYDRITE TWO-PHASE PARAMETERS}

Technical Review Status:

Scientific Notebooks:

Not Applicable ____ incomplete $\overline{\text { (Give Date) }} ;$ Complete $\frac{}{\text { (Give Date) }}$

Interpretive Analyses:

Not Applicable ; Incomplete Complete (Give Date) December 21, 1995 (Give Date)

Routine Calculations:

Capillary Pressure Model Parameters Calc. Sheet Incomplete ; Complete December 21, 1995 (Give Date) (Give Date)

Qualification Status:

Data: Used WIPP Observational Data

Not Applicable ; Incomplete $1 / 31 / 96$ ; Complete

$$
\text { (Give Date) (Give Date) }
$$

NOTES:

Data must be technically reviewed and forwarded to SWCF (TLCF-1/31/96)

\section{Codes}

Microsoft Excell; Incomplete ; Complete $12 / 21 / 95$ (in use verification) (Give Date) (Give Date)

KaleidaGraph; Incomplete ; Complete 12/21/95 (in use verification) (Give Date) (Give Date)

PARAMETER QUALIFIED: Incomplete: 1/31/96; Complete: (Give Date)

(Give Date) 


\section{RECORD 5}

Roadmap to Supporting Documentation 
January 31,1996

\section{RAODMAP FOR}

SALADO PARAMETER PRINCIPAL INVESTIGATOR DOCUMENTATION PACKAGE FOR: ANHYDRITE TWO-PHASE PARAMETERS 


\section{RECORD 6}

Memo to PA Parameter Task Leader 


\section{Sandia National Laboratories}

Albuquerque, New Mexico 87185-1341

date: January 31,1996

to: PA Parameter Task Leader

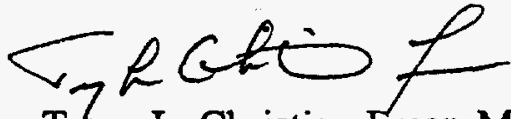

from: Tracy L. Christian-Frear, MS-1324 (6115)

subject: $\quad$ SALADO PARAMETER PRINCIPAL INVESTIGATOR DOCUMENTATION PACKAGE FOR: ANHYDRITE TWO-PHASE PARAMETERS

The attached record contains the anhydrite two-phase values for the capillary and relative permeability models, the residual gas and brine saturation, the initial brine saturation, pore size distribution parameter and the threshold pressure.

The title of the records package is: SALADO PARAMETER PRINCIPAL INVESTIGATOR DOCUMENTATION PACKAGE FOR: ANHYDRITE TWOPHASE PARAMETERS

The SWCF is: SWCF-A:WBS 1.2.07.1:PDD:QA:SALADO:PKG 10:Anh 2-Phase Parameters

The WPO is: 30643

The deficiencies in the parameter documentation are:

Data must be technically reviewed and forwarded to SWCF

Please call me at 848-0704 if you have any questions.

Attachment:

SALADO DATA/PARAMETERS : ANHYDRITE TWO-PHASE PARAMETERS 


\section{Appendix F.}

Memorandum: S.W. Webb to P. Vaughn, August 29, 1995

The following information is provided as Appendix $\mathrm{F}$ of this document. 


\section{Appendix F}

Memorandum: S.W. Webb to P. Vaughn, August 29, 1995.

\section{Errata Sheet}

The two citations in Appendix F:

Davies, SAND90-3246 on p. F-3 and

Davies (1991) on p. F-4

refer to the same report. A copy is on file in SWCF as WPO\#26169. 


\section{Sandia National Laboratories}

Albuquerque, New Mexico 87185

date: August 29, 1995

to: P. Vaughn, MS-1328 (6749)

from: S.W. Webb, MS-1324 (6115)

subject: Mixed Brooks and Corey Two-Phase Characteristic Curves

The mixed Brooks and Corey two-phase characteristic curves have been used in SPM-2 calculations and will be recommended for future studies including the compliance application. The mixed Brooks and Corey model uses two definitions of the effective saturation to reflect the different saturation ranges for the relative permeabilities of the wetting and nonwetting phases. However, the approach used in SPM-2 was inconsistent with the definition of threshold pressure used in the Davies' correlation (Davies, SAND90-3246), partially due to the use of displacement pressure instead of threshold pressure in the original specification. The correct form of the mixed Brooks and Corey curves consistent with Davies' threshold pressure definition is summarized below.

In the mixed Brooks and Corey model, two separate effective saturations are used which are defined as

$$
\begin{aligned}
& S_{e}=\frac{S-S_{r}}{1-S_{r}} \\
& S_{e}^{\prime}=\frac{S-S_{r}}{S_{c}-S_{r}} .
\end{aligned}
$$

where

$S_{\mathrm{e}} \quad=$ effective saturation

$S \quad=$ wetting phase saturation

$S_{\mathrm{r}} \quad=$ wetting phase residual saturation

$S_{c} \quad=$ critical gas saturation $=1-S_{\mathrm{r}, \mathrm{aw}}$

$S_{\mathrm{r}, \mathrm{aw}}=$ nonwetting phase residual saturation.

$S_{e}$ is the original Brooks and Corey definition while $S_{e}^{\prime}$ is a modified definition. 
The capillary pressure relationship is

$$
S_{e}^{\prime}=\left(\frac{P_{z}}{P_{c}}\right)^{\lambda}
$$

or

$$
P_{c}=\frac{P_{t}}{S_{e}^{1 / \lambda}}
$$

where $P_{t}$ and $P_{c}$ are the threshold pressure and the capillary pressure, respectively, and $\lambda$ is the pore-size distribution parameter.

The wetting phase relative permeability expression is given by

$$
k_{r, w}=S_{e}^{(2+3 \lambda) \lambda \lambda}
$$

while the nonwetting phase relationship is

$$
k_{r, m w}=\left(1-S_{e}^{\prime}\right)^{2}\left(1-S_{e}^{(2+18 \sqrt{2}}\right) .
$$

Therefore, consistent with Davies (1991), the threshold pressure is the capillary pressure when the saturation is equal to the critical gas saturation $\left(S_{c}\right)$, or when $S_{e}^{\prime}$ equals 1.0.

If there are any questions, please contact me.

cc:

MS-1324 P.B. Davies (6115)

MS-1324 A.R. Lappin (6115)

MS-1324 T.L. Christian-Frear (6115)

MS-1328 D.R. Anderson (6749)

MS-1341 K.W. Larson (6747) 


\section{WIPP \\ UC721 - DISTRIBUTION LIST \\ SAND94-0472}

Federal Agencies

US Department of Energy (4)

Office of Civilian Radioactive Waste Mgmt.

Attn: Deputy Director, RW-2

Acting Director, RW-10

Office of Human Resources \& Admin.

Director, RW-30

Office of Program Mgmt. \& Integ.

Director, RW-40

Office of Waste Accept., Stor., \& Tran.

Forrestal Building

Washington, DC 20585

Attn: Project Director

Yucca Mountain Site Characterization Office

Director, RW-3

Office of Quality Assurance

P.O. Box 30307

Las Vegas, NV 89036-0307

US Department of Energy

Albuquerque Operations Office

Attn: National Atomic Museum Library

P.O. Box 5400

Albuquerque, NM 87185-5400

US Department of Energy

Research \& Waste Management Division

Attn: Director

P.O. Box E

Oak Ridge, TN 37831

US Department of Energy (5)

Carlsbad Area Office

Attn: G. Dials

D. Galbraith

M. McFadden

R. Lark

J. A. Mewhinney

P.O. Box 3090

Carlsbad, NM 88221-3090

US Department of Energy

Office of Environmental Restoration and

Waste Management

Attn: M Frei, EM-30

Forrestal Building

Washington, DC 20585-0002
US Department of Energy (3)

Office of Environmental Restoration and

Waste Management

Attn: J. Juri, EM-34, Trevion II

Washington, DC 20585-0002

US Department of Energy

Office of Environmental Restoration and

Waste Management

Attn: S. Schneider, EM-342, Trevion II

Washington, DC 20585-0002

US Department of Energy (2)

Office of Environment, Safety \& Health

Attn: C. Borgstrom, EH-25

R. Pelletier, EH-231

Washington, DC 20585

US Department of Energy (2)

Idaho Operations Office

Fuel Processing \& Waste Mgmt. Division

785 DOE Place

Idaho Falls, ID 83402

US Environmental Protection Agency (2)

Radiation Protection Programs

Attn: M. Oge

ANR-460

Washington, DC 20460

Boards

Defense Nuclear Facilities Safety Board

Attn: D. Winters

625 Indiana Ave. NW, Suite 700

Washington, DC 20004

Nuclear Waste Technical Review Board (2)

Attn: Chairman

J. L. Cohon

1100 Wilson Blvd., Suite 910

Arlington, VA 22209-2297 


\section{State Agencies}

Attorney General of New Mexico

P.O. Drawer 1508

Santa Fe, NM 87504-1508

Environmental Evaluation Group (3)

Attn: Library

7007 Wyoming NE

Suite F-2

Albuquerque, NM 87109

NM Environment Department (3)

Secretary of the Environment

Attn: Mark Weidler

1190 St. Francis Drive

Santa Fe, NM 87503-0968

NM Bureau of Mines \& Mineral Resources

Socorro, NM 87801

\section{Laboratories/Corporations}

Battelle Pacific Northwest Laboratories

Battelle Blvd.

Richland, WA 99352

INTERA, Inc.

Attn: G. A. Freeze

1650 University Blvd. NE, Suite 300

Albuquerque, NM 87102

INTERA, Inc.

Attn: J. F. Pickens

6850 Austin Center Blvd., Suite 300

Austin, TX 78731

Los Alamos National Laboratory

Attn: B. Erdal, INC-12

P.O. Box 1663

Los Alamos, NM 87544

RE/SPEC, Inc.

Attn: A. Robb

4775 Indian School NE, Suite 300

Albuquerque, NM 87110-3927

RE/SPEC, Inc.

Attn: J. L. Ratigan

P. O. Box 725

Rapid City. SD 57709
Tech Reps, Inc. (3)

Attn: J. Chapman (1)

Loretta Robledo (2)

5000 Marble NE, Suite 222

Albuquerque, NM 87110

Westinghouse Electric Corporation (5)

Attn: Library

J. Epstein

J. Lee

B. A. Howard

R. Kehrman

P.O. Box 2078

Carlsbad, NM 88221

S. Cohen \& Associates

Attn: Bill Thurber

1355 Beverly Road

McLean, VA 22101

Rock Physics Associates

Attn: J. Walls

4320 Steven Creek Blvd., Ste 282

San Jose, CA 95129

National Academy of Sciences, WIPP Panel

Howard Adler

Oxyrase, Incorporated

7327 Oak Ridge Highway

Knoxville, TN 37931

Tom Kiess

Board of Radioactive Waste Management

GF456

2101 Constitution Ave.

Washington, DC 20418

Rodney C. Ewing

Department of Geology

University of New Mexico

Albuquerque, NM 87131

Charles Fairhurst

Department of Civil and Mineral Engineering

University of Minnesota

500 Pillsbury Dr. SE

Minneapolis, MN 55455-0220

B. John Garrick

PLG Incorporated

4590 MacArthur Blvd., Suite 400

Newport Beach, CA 92660-2027 
Leonard F. Konikow

US Geological Survey

431 National Center

Reston, VA 22092

Carl A. Anderson, Director

Board of Radioactive Waste Management

National Research Council

HA 456

2101 Constitution Ave. NW

Washington, DC 20418

Christopher G. Whipple

ICF Kaiser Engineers

1800 Harrison St., 7th Floor

Oakland, CA 94612-3430

John O. Blomeke

720 Clubhouse Way

Knoxville, TN 37909

Sue B. Clark

University of Georgia

Savannah River Ecology Lab

P.O. Drawer $\mathrm{E}$

Aiken, SC 29802

Konrad B. Krauskopf

Department of Geology

Stanford University

Stanford, CA 94305-2115

Della Roy

Pennsylvania State University

217 Materials Research Lab

Hastings Road

University Park, PA 16802

David A. Waite

$\mathrm{CH}_{2} \mathrm{M}$ Hill

P.O. Box 91500

Bellevue, WA 98009-2050

Thomas A. Zordon

Zordan Associates, Inc.

3807 Edinburg Drive

Murrysville, PA 15668

\section{Universities}

University of New Mexico

Geology Department

Attn: Library

141 Northrop Hall

Albuquerque, NM 87131

\section{Libraries}

Thomas Brannigan Library

Attn: D. Dresp

106 W. Hadley St.

Las Cruces, NM 88001

Government Publications Department

Zimmerman Library

University of New Mexico

Albuquerque, NM 87131

New Mexico Junior College

Pannell Library

Attn: R. Hill

Lovington Highway

Hobbs, NM 88240

New Mexico State Library

Attn: N. McCallan

325 Don Gaspar

Santa Fe, NM 87503

New Mexico Tech

Martin Speere Memorial Library

Campus Street

Socorro, NM 87810

WIPP Public Reading Room

Carlsbad Public Library

101 S. Halagueno St.

Carlsbad, NM 88220

Foreign Addresses

Atomic Energy of Canada, Ltd.

Whiteshell Laboratories

Attn: B. Goodwin

Pinawa, Manitoba, CANADA ROE ILO

Francois Chenevier (2)

ANDRA

Route de Panorama Robert Schumann

B. P. 38

92266 Fontenay-aux-Roses, Cedex

FRANCE 
Claude Sombret

Centre d'Etudes Nucleaires de la Vallee Rhone CEN/VALRHO

S.D.H.A. B.P. 171

30205 Bagnols-Sur-Ceze

FRANCE

Commissariat a L'Energie Atomique

Attn: D. Alexandre

Centre d'Etudes de Cadarache

13108 Saint Paul Lez Durance Cedex

FRANCE

Bundesanstalt fur Geowissenschaften und

Rohstoffe

Attn: M. Langer

Postfach 510153

D-30631 Hannover

GERMANY

Bundesministerium fur Forschung und

Technologie

Postfach 200706

5300 Bonn 2

GERMANY

Institut fur Tieflagerung

Attn: K. Kuhn

Theodor-Heuss-Strasse 4

D-3300 Braunschweig

GERMANY

Gesellschaft fur Anlagen und Reaktorsicherheit (GRS)

Attn: B. Baltes

Schwertnergasse 1

D-50667 Cologne

GERMANY

Shingo Tashiro

Japan Atomic Energy Research Institute

Tokai-Mura, Ibaraki-Ken, 319-11

JAPAN

Netherlands Energy Research Foundation ECN

Attn: J. Prij

3 Westerduinweg

P.O. Box 1

1755 ZG Petten

THE NETHERLANDS
Svensk Karnbransleforsorjning AB

Attn: F. Karlsson

Project KBS (Karnbranslesakerhet)

Box 5864

S-102 48 Stockholm

SWEDEN

Nationale Genossenschaft fur die Lagerung

Radioaktiver Abfalle (2)

Attn: S. Vomvoris

P. Zuidema

Hardstrasse 73

CH-5430 Wettingen

SWITZERLAND

AEA Technology

Attn: J. H. Rees

D5W/29 Culham Laboratory

Abington, Oxfordshire OX14 3DB

UNITED KINGDOM

AEA Technology

Attn: W. R. Rodwell

044/A31 Winfrith Technical Centre

Dorchester, Dorset DT2 8DH

UNITED KINGDOM

AEA Technology

Attn: J. E. Tinson

B4244 Harwell Laboratory

Didcot, Oxfordshire OXI1 ORA

UNITED KINGDOM

\section{Internal}

\begin{tabular}{|c|c|c|}
\hline MS & Org. & \\
\hline 1324 & 6115 & P. B. Davies \\
\hline 1324 & 6115 & T. L. Christian-Frear \\
\hline 1324 & 6115 & C. Boney \\
\hline 1324 & 6115 & R. L. Beauheim \\
\hline 1324 & 6115 & S. W. Webb \\
\hline 1320 & 6831 & E. J. Nowak \\
\hline 1322 & 6121 & J. R. Tillerson \\
\hline 1328 & 6849 & D. R. Anderson \\
\hline 1328 & 6848 & H. N. Jow \\
\hline 1335 & 6801 & M. Chu \\
\hline 1335 & 6801 & S. M. Howarth (15) \\
\hline 1341 & 6832 & J. T. Holmes \\
\hline 1395 & 6800 & L. Shephard \\
\hline 1395 & 6821 & M. Marietta \\
\hline 0751 & 6117 & L. S. Costin \\
\hline 0751 & 6117 & N. S. Brodsky \\
\hline 0751 & 6117 & J. T. Fredrich \\
\hline 0751 & 6117 & D. J. Holcomb \\
\hline
\end{tabular}


D. H. Zeuch

$0705 \quad 6116$

$1330 \quad 6811$

D. J. Borns

1330

4415

K. Hart (2)

9018 8940-2

NWM Library (20)

0899 4414

Central Technical Files

061912690 Review and Approval Desk (2), For DOE/OSTI

Distribution - 5 